\author{
Supporting Information
}

\title{
Modeling Decomposition of N-Nitrosoamides in a Self-Assembled Capsule
}

Oriana Brea ${ }^{\mathrm{a}}$, Henrik Daver ${ }^{\mathrm{a}}$, Julius Rebek, Jr. ${ }^{\mathrm{b}, \mathrm{c}}$, and Fahmi Himo ${ }^{\mathrm{a}}$

a. Department of Organic Chemistry, Arrhenius Laboratory, Stockholm University, SE-106 91 Stockholm, Sweden.

b. Center for Supramolecular Chemistry and Catalysis and Department of Chemistry, Shanghai University, Shanghai 200444, P.R. China.

c. The Skaggs Institute for Chemical Biology and Department of Chemistry, The Scripps Research Institute, 10550 North Torrey Pines Road, La Jolla, California 92037, United States.

* Corresponding author: fahmi.himo@su.se

\section{Contents}

1. Energy profiles of the reactions in the absence of capsule...................... S2

2. Optimized geometries involved in the formation of $\mathbf{4 b} @ \mathbf{1}_{2}$ and $\mathbf{4 c} @ \mathbf{1}_{2} \ldots \ldots \ldots \ldots \ldots . . . . . . . .33$

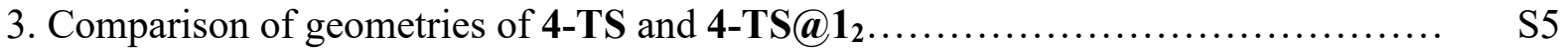

4. Comparison between rotamers of 4-TS in mesitylene.......................... S6

5. Details of energy decomposition analysis ................................ S7

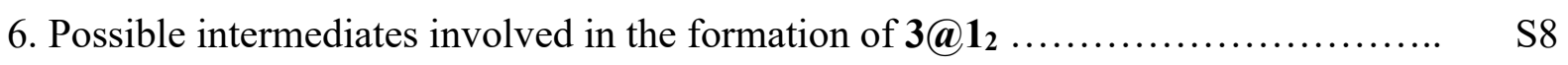

7. Sensitivity to methodology of the equilibrium $\mathbf{2 b} @ \mathbf{1}_{2}+\mathbf{3 b} \leftrightharpoons \mathbf{3 b} @ \mathbf{1}_{\mathbf{2}}+\mathbf{2 b} \ldots \ldots \ldots . . . \quad \mathrm{S} 9$

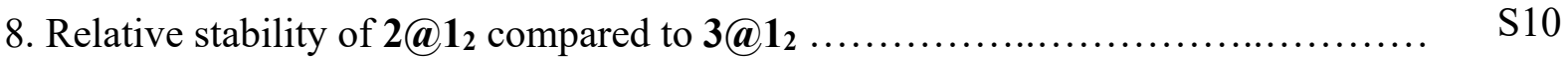

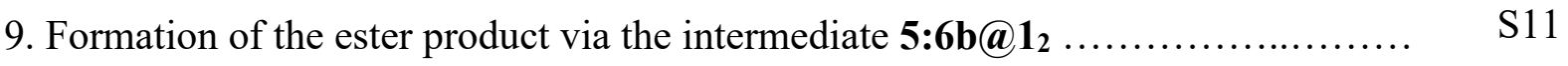

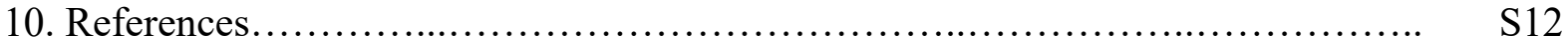

11. Calculated absolute energies and energy corrections........................ S13

12. Cartesian coordinates of optimized geometries............................ S15 


\section{Energy profiles of the reactions in the absence of capsule}

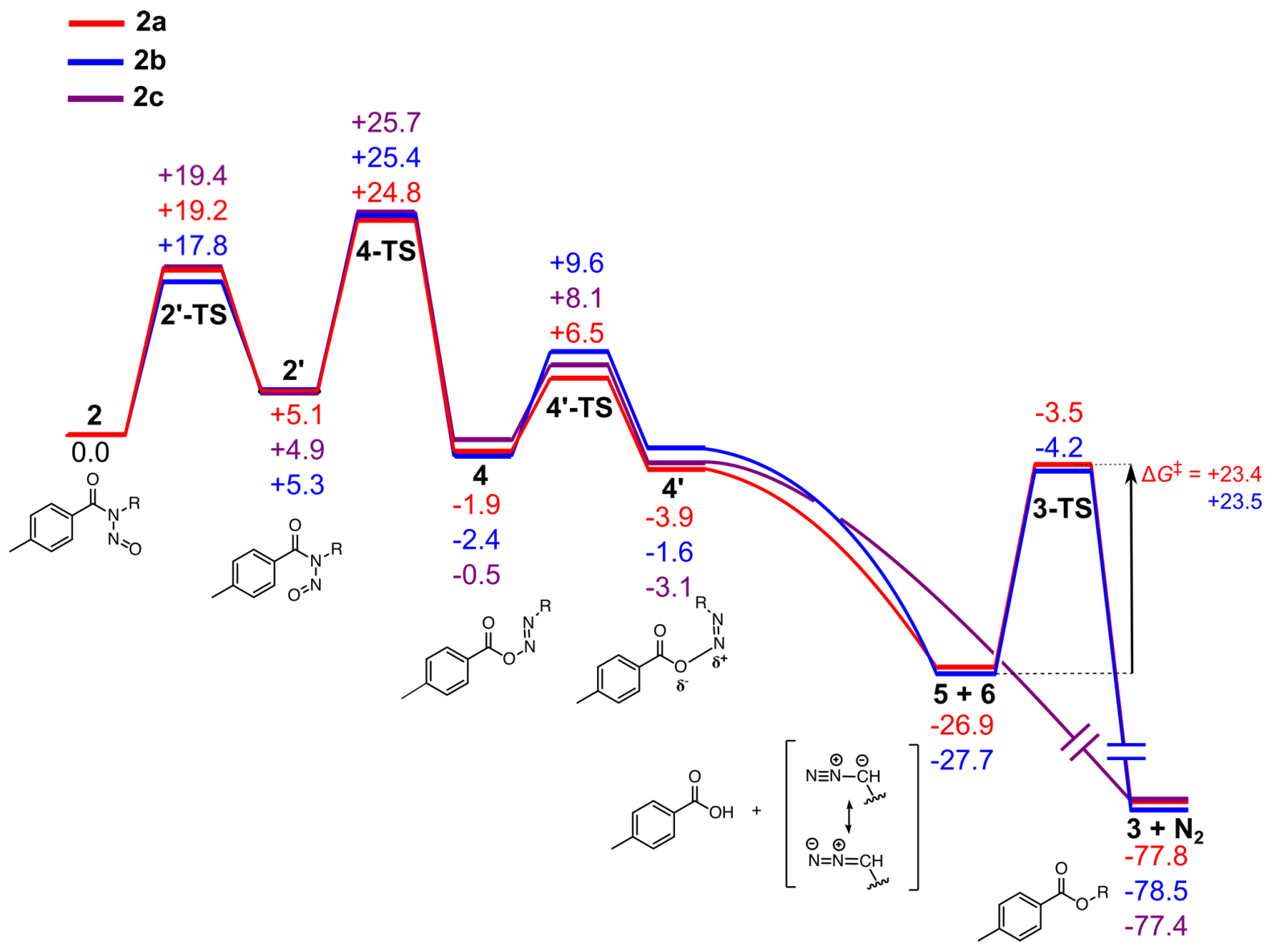

Figure S1. Free energy profile for the thermal decomposition of N-nitrosoamides $2 \boldsymbol{a}-\boldsymbol{c}$ in mesitylene. Energies are in $\mathrm{kcal} / \mathrm{mol}$. Note that the energies are slightly different compared to Reference [1]. This is because the solvent effects are now computed with COSMO-RS instead of SMD method. 
2. Optimized geometries involved in the formation of $4 b @ 1_{2}$ and $4 c @ 1_{2}$

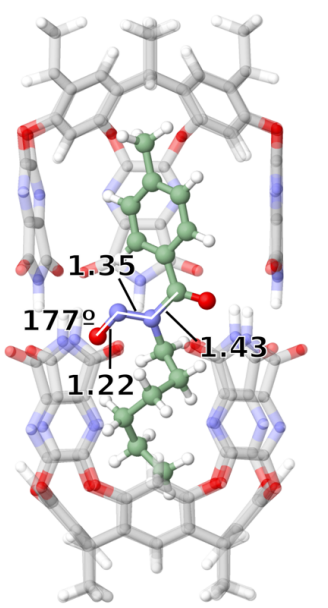

$\mathbf{2 b @ 1 _ { 2 }}$

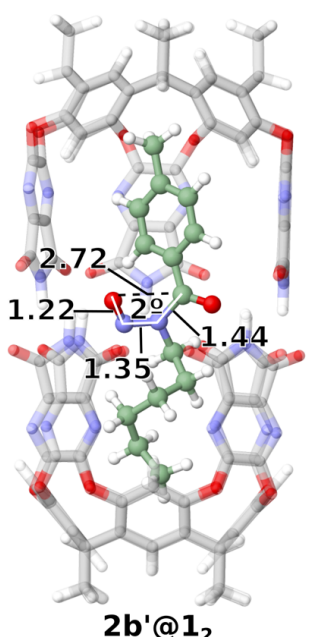

$\mathbf{2 b} @ \mathbf{1}_{2}$

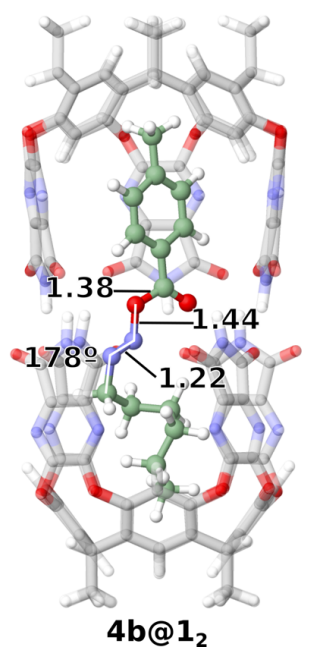

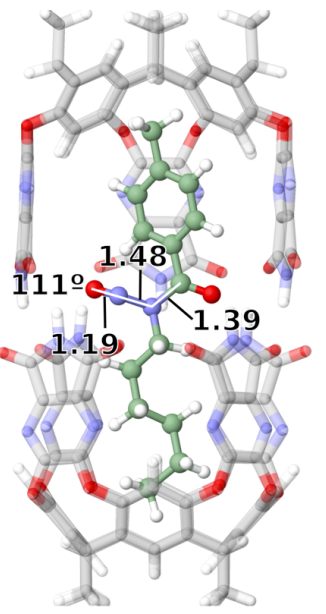

2b'-TS@1 2

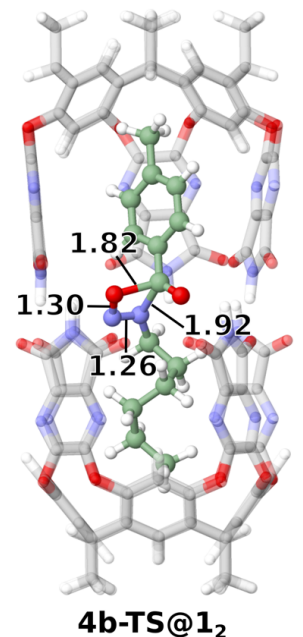

4b-TS@1

Figure $\mathbf{S}$ 2. Optimized geometries involved in the formation of $4 \boldsymbol{b} @ \mathbf{1}_{2}$. Relevant bond distances $(A)$ and dihedral angles (degrees) are indicated. 

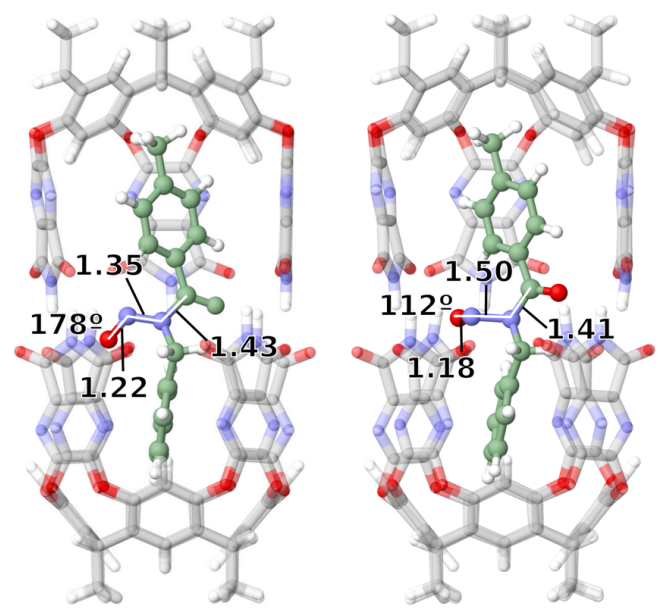

2c@1

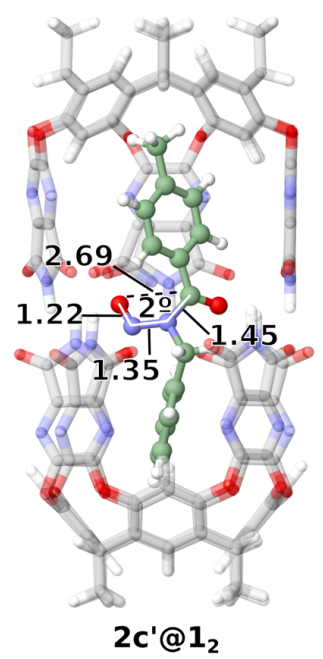

2c'-TS@1 2
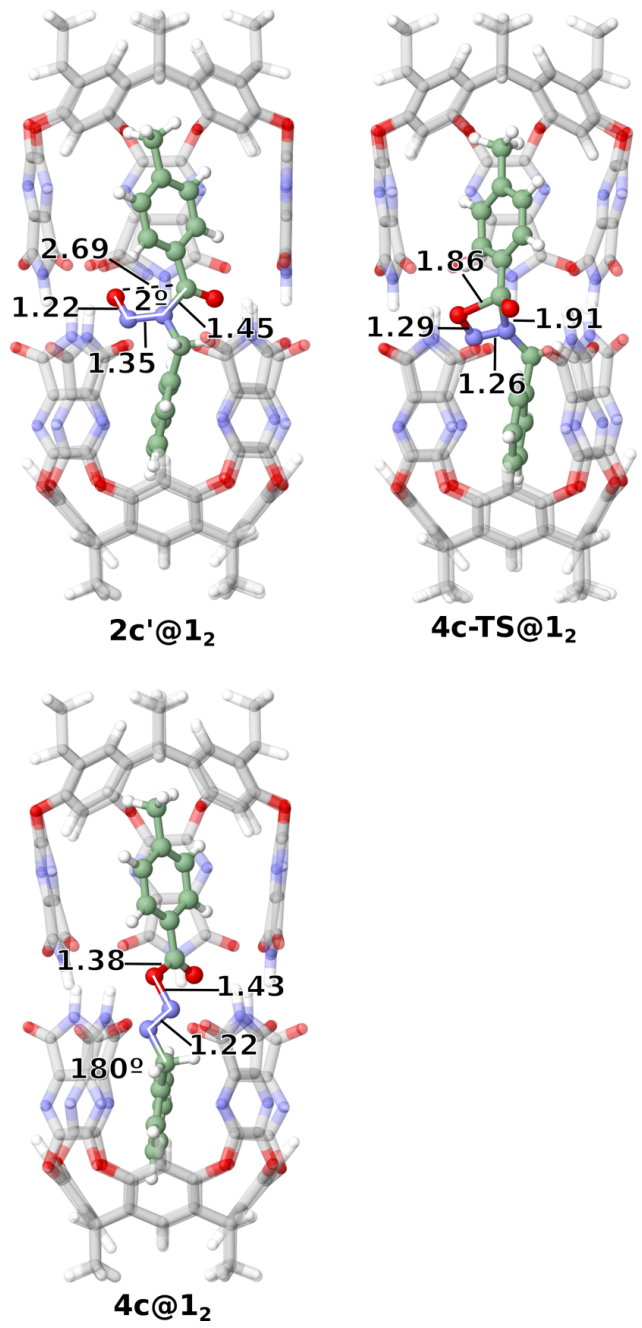

Figure S3. Optimized geometries involved in the formation of $4 c @ 11_{2}$. 
3. Comparison of geometries of 4-TS and 4-TS@1 1

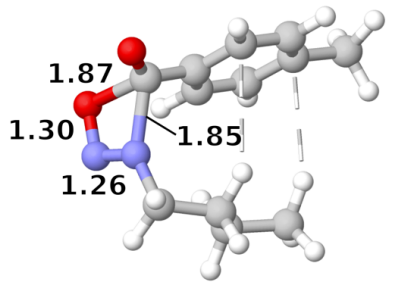

4a-TS

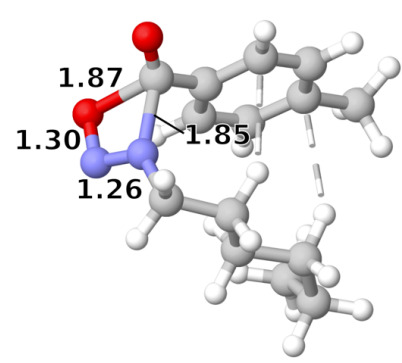

4b-TS

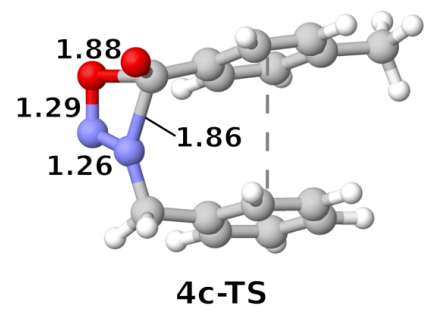

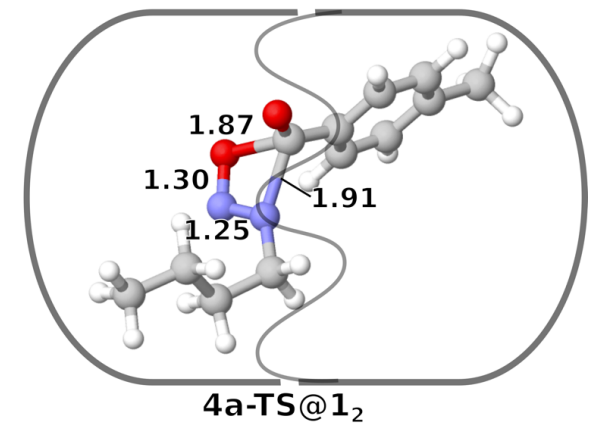
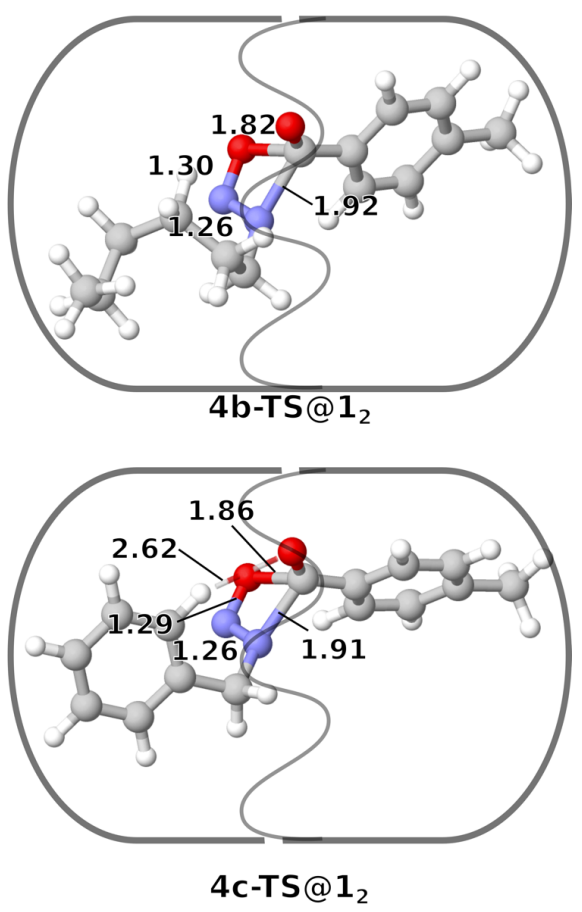

Figure S4. Optimized geometries of 4-TS (left) and 4-TS@12 (right). Relevant bond distances $(A)$ and dihedral angles (degrees) are indicated. 


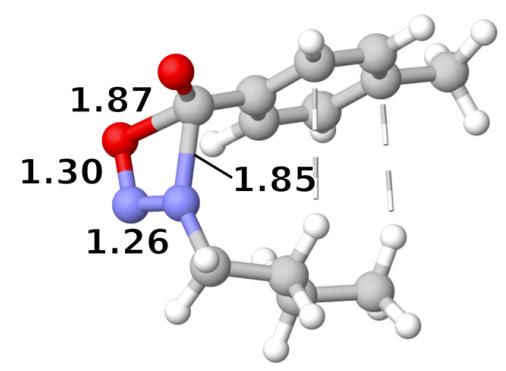

4a-TS

0.0

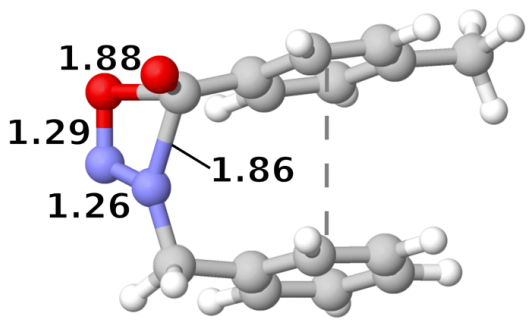

4c-TS

0.0

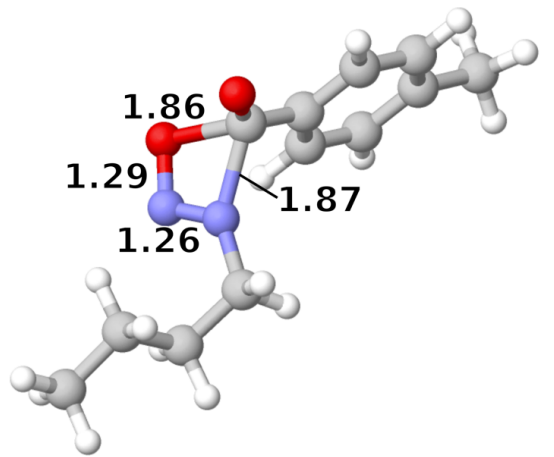

$4 a-T S(2)$

$+0.5$

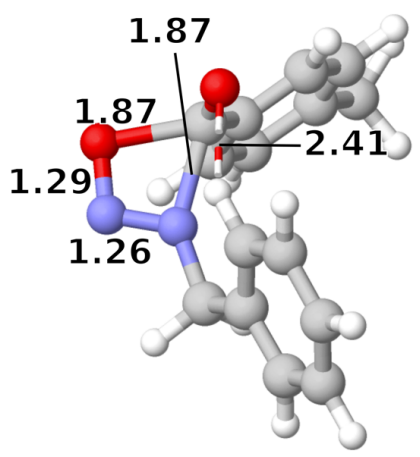

$4 \mathrm{c}-\mathrm{TS}(2)$

$+3.4$

Figure S5. Optimized geometries of 4-TS (left) and 4-TS(2) (right). In 4-TS(2), the CH- $\pi$ and $\pi-\pi$ interactions are broken. Energy differences are given in $\mathrm{kcal} / \mathrm{mol}$. 


\section{Details of energy decomposition analysis}

The effect of the capsule on an energy barrier $\left(\Delta G^{\ddagger}\right.$ caps $-\Delta G^{\ddagger}$ uncat $)$ can be analyzed by a distortion-interaction energy decomposition analysis. The protocol employed in the present work was proposed recently in Reference [2], and it is extended here by explicit consideration of the capsule distortion energy.

The energy components for the barrier associated with going from 2 to 4-TS were calculated as follows:

- $\Delta \Delta E_{\text {dist }}^{\ddagger}($ guest $)=\Delta E^{\ddagger}\left(2^{*} \rightarrow 4-\mathbf{T S}^{* *}\right)-\Delta E^{\ddagger}(\mathbf{2} \rightarrow 4$-TS $)$

- $\Delta E_{\text {dist }}{ }^{\ddagger}($ host $)=E\left(\mathbf{1}_{2}^{* *}\right)-E\left(\mathbf{1}_{2}^{*}\right)$

- $\Delta \Delta E_{\mathrm{int}^{*}}^{*}=\Delta E^{\ddagger}\left(2 @ 1_{2} \rightarrow 4-\mathbf{T S} @ 1_{2}\right)-\Delta E^{\ddagger}\left(\mathbf{2}^{*} \rightarrow\right.$ 4-TS**) $-\Delta E_{\mathrm{dist}^{\ddagger}}($ host $)$

- $\Delta \Delta G_{\text {corr }}^{\ddagger}=\Delta G_{\text {corr }}\left(2 @ 1_{2} \rightarrow 4-T S @ 11_{2}\right)-\Delta G_{\text {corr }}(2 \rightarrow 4-T S)$

- $\Delta G_{\text {caps }}^{\ddagger}-\Delta G^{\ddagger}$ uncat $=\Delta \Delta E_{\text {dist }}{ }^{\ddagger}$ (guest $)+\Delta E_{\text {dist }}{ }^{\ddagger}($ host $)+\Delta \Delta E_{\text {int }}+\Delta \Delta G_{\text {corr }}{ }^{\ddagger}$

$\mathbf{2}^{*}$ and $\mathbf{1}_{2} *$ denote the structures of $\mathbf{2}$ and $\mathbf{1}_{\mathbf{2}}$, respectively, in the host-guest complex $\mathbf{2} @ \mathbf{1}_{\mathbf{2}}$. Similarly, 4-TS** and $\mathbf{1}_{2}^{* *}$ are the corresponding structures in 4-TS@1. 


\section{Possible intermediates involved in the formation of $3 @ 1_{2}$}
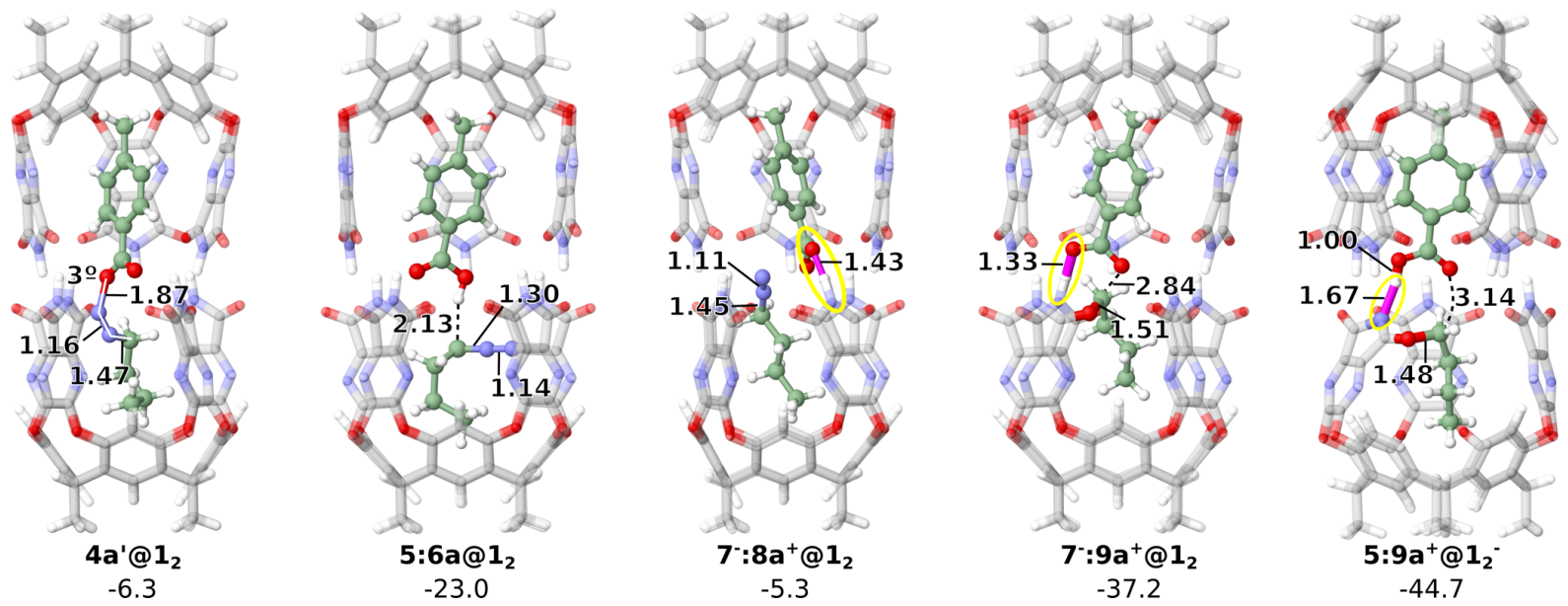

Figure S6. Optimized geometries of hypothetical intermediates identified by the calculations for the decomposition of $4 a @ 1_{2}$. Energies relative to 2a@1 2 are indicated in kcal/mol.
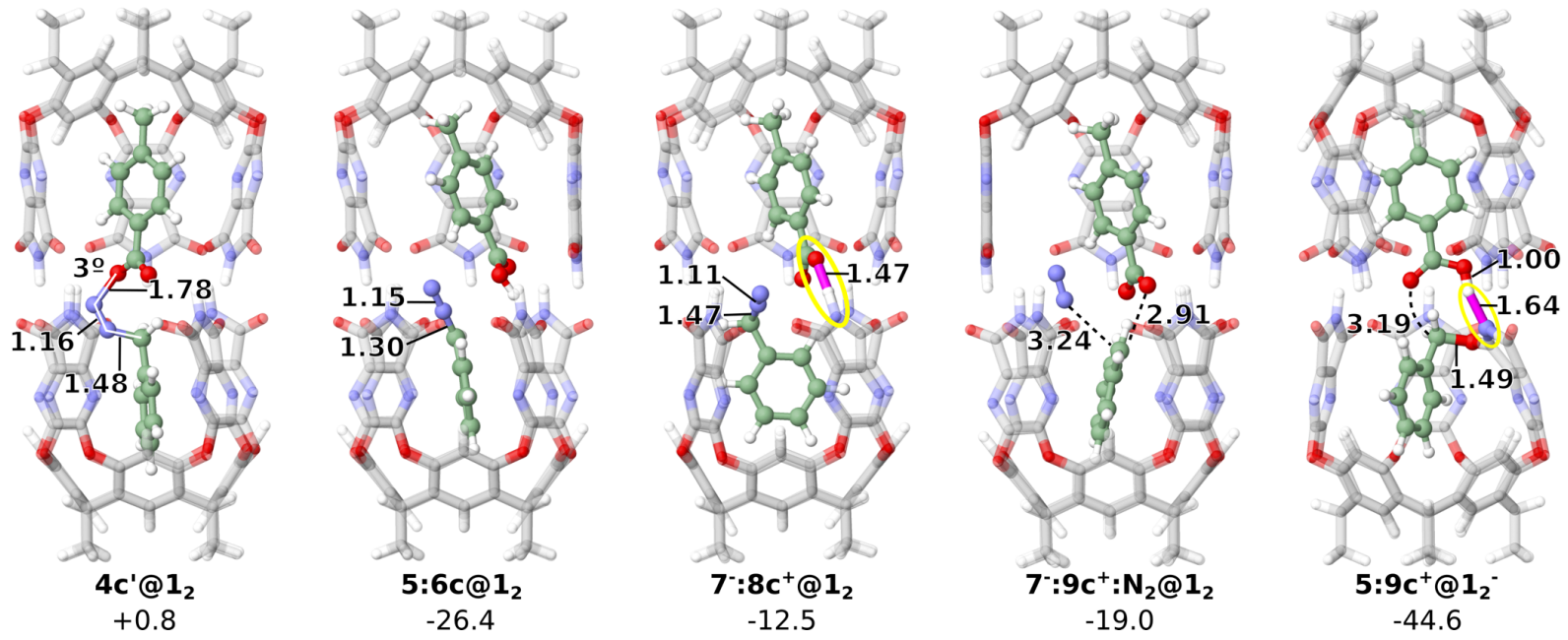

Figure S7. Optimized geometries of hypothetical intermediates identified by the calculations for the decomposition of $\mathbf{4 c @ 1}$. Energies relative to $2 c @ \mathbf{1}_{2}$ are indicated in $\mathrm{kcal} / \mathrm{mol}$.

Interestingly, the product of the dissociation of $\mathrm{N}_{2}$ from $7^{-}: \mathbf{8 \mathbf { c } ^ { + }} @ \mathbf{1}_{\mathbf{2}}$ is different compared to that from the aliphatic ion-pairs. The complex is named $7^{-}: 9 \mathbf{c}^{+}: \mathbf{N}_{2} @ \mathbf{1}_{2}$ (NBO charges: Rcarbocation $+0.8,7^{-}-0.8$ and neutral $\mathrm{N}_{2}$ ) and its optimized geometry is shown in Figure S7. The geometry optimization removing $\mathrm{N}_{2}$ from $7^{-}: 9 \mathbf{c}^{+}: \mathbf{N}_{2} @ 1_{2}$ results in the formation of the ester product $\mathbf{3 c} @ \mathbf{1}_{2}$. 
7. Sensitivity to methodology of the equilibrium $2 b @ 1_{2}+3 b \leq 3 b @ 1_{2}+2 b$

Table S1. Calculated free energy (in kcal/mol) as a function of computational protocol

\begin{tabular}{lc}
\hline Method & $\Delta \boldsymbol{G}$ \\
\hline B3LYP-D3(BJ)/6-311+G(2d,2p), COSMO-RS, quasi-RRHO & +2.0 \\
Solvation Model & \\
B3LYP-D3(BJ)/6-311+G(2d,2p) + SMD & +1.1 \\
Dispersion Model & \\
B3LYP-XDM ${ }^{[\mathrm{a}]} / 6-311+\mathrm{G}(2 \mathrm{~d}, 2 \mathrm{p})+$ COSMO-RS & +2.7 \\
Functional & \\
M06-2X/6-311+G(2d,2p) + COSMO-RS & +6.1 \\
PBE0-D3(BJ)/6-311+G(2d,2p) + COSMO-RS & +2.2 \\
TPSS0-D3(BJ)/6-311+G(2d,2p) + COSMO-RS & +0.8 \\
\hline
\end{tabular}

[a] The densities used by the WF-based dispersion corrections are calculated at the B3LYP$\mathrm{D} 3(\mathrm{BJ}) / 6-31+\mathrm{G}(\mathrm{d}, \mathrm{p})$ level of theory. 


\section{Relative stability of $2 @ 1_{2}$ compared to $3 @ 1_{2}$}

Equilibria (S1) and (S2) were calculated to be endergonic by $1.4 \mathrm{kcal} / \mathrm{mol}$ and $2.1 \mathrm{kcal} / \mathrm{mol}$, respectively. The optimized geometries of 3a-c@12 are shown in Figure S8.

$$
\begin{aligned}
& 2 \mathrm{a} @ 1_{2}+3 a \leftrightharpoons 3 a @ 1_{2}+2 a \\
& 2 c @ 1_{2}+3 c \leftrightharpoons 3 c @ 1_{2}+2 c
\end{aligned}
$$

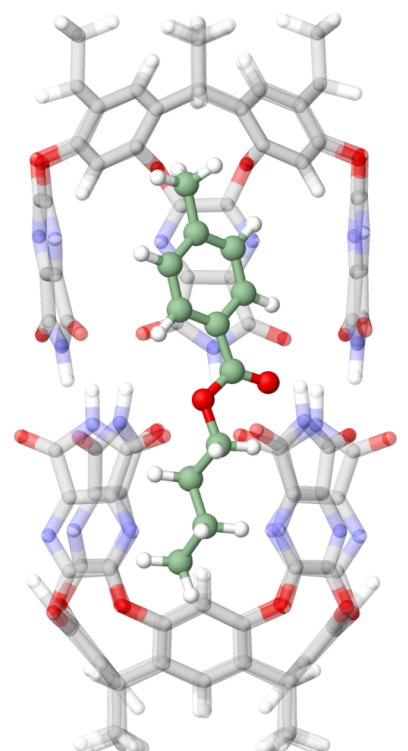

$3 a @ 1_{2}$

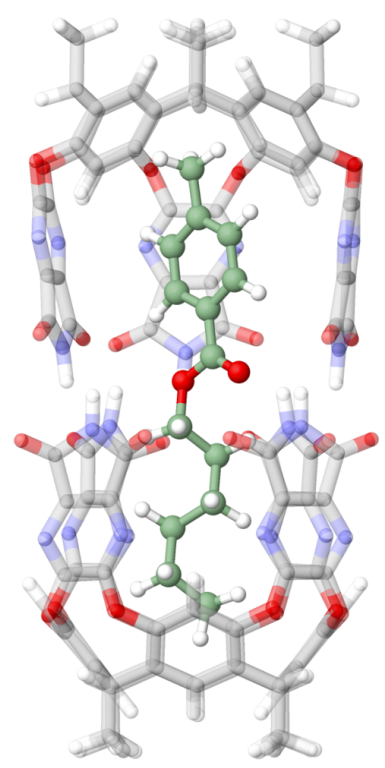

$\mathbf{3 b @ \mathbf { 1 } _ { 2 }}$

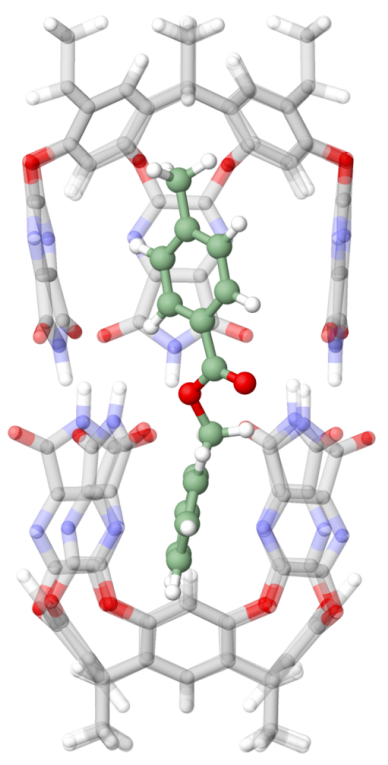

$3 c @ 1_{2}$

Figure S8. Optimized geometries of product complexes $\mathbf{3 a - c @ 1} \mathbf{1}_{2}$ 
9. Formation of ester product $3 b @ 1_{2}$ via intermediate 5:6b@12
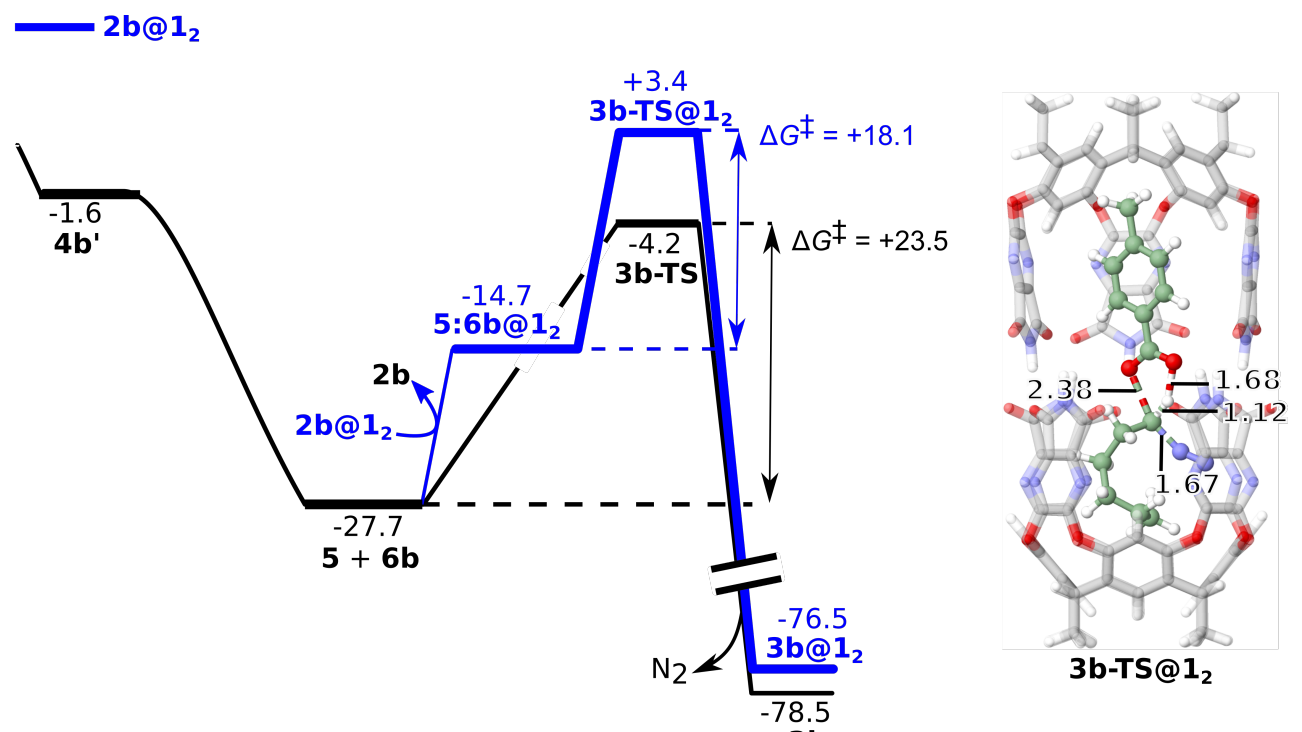

3b

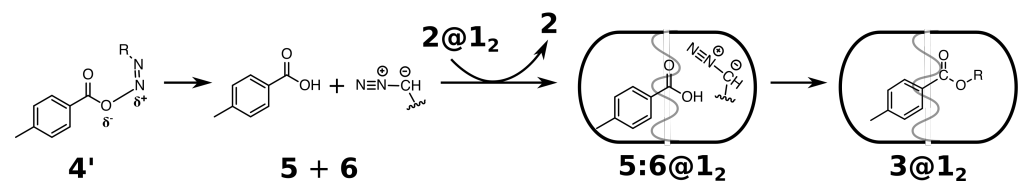

Figure S9. Calculated free energy profile (in $\mathrm{kcal} / \mathrm{mol}$ ) for the formation of $3 \boldsymbol{b} @ 1_{2}$ and $\mathrm{N}_{2}$ from 5:6b@12 in blue. The reaction in the absence of the capsule is shown in black. 
10. References

[1] O. Brea, H. Daver, J. Rebek and F. Himo, Tetrahedron, 2019, 8, 929-935.

[2] H. Daver, J. N. Harvey, J. Rebek and F. Himo, J. Am. Chem. Soc., 2017, 139, 1549415503. 


\section{Calculated absolute energies and energy corrections}

Table S2. Calculated absolute energies and energy corrections in mesitylene (au) in the absence of capsule.

\begin{tabular}{|c|c|c|c|c|c|}
\hline & $\begin{array}{l}\text { Absolute energy } \\
\text { B3LYP-D3(BJ)/ } \\
6-311+G(2 d, 2 p)\end{array}$ & $\begin{array}{l}\text { Three-body } \\
\text { dispersion } \\
\text { correction }\end{array}$ & 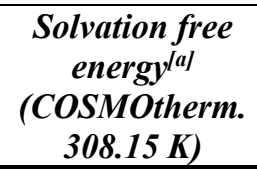 & $\begin{array}{c}\text { Solvation free } \\
\text { energy }^{j a J} \\
\text { (SMD) }\end{array}$ & $\begin{array}{c}\text { Thermal correction to } \\
\text { Gibbs free energy } \\
\text { (quasi-RRHO, } 308.15 \mathrm{~K} \text { ) }\end{array}$ \\
\hline $2 a$ & -727.095491 & 0.000098 & -0.013116 & -0.010570 & 0.221239 \\
\hline $2 a^{\prime}-T S$ & -727.062482 & 0.000033 & -0.013309 & -0.009983 & 0.219011 \\
\hline $2 a^{\prime}$ & -727.086562 & 0.000155 & -0.013512 & -0.010282 & 0.220699 \\
\hline 4a-TS & -727.053831 & 0.000361 & -0.014439 & -0.010187 & 0.220177 \\
\hline 4a-TS(2) & -727.049962 & 0.000078 & -0.015640 & -0.011630 & 0.218624 \\
\hline $4 a$ & -727.093398 & -0.000061 & -0.015385 & -0.009952 & 0.218589 \\
\hline $4 a^{\prime}-T S$ & -727.074197 & -0.000009 & -0.019098 & -0.012912 & 0.216407 \\
\hline $4 a^{\prime}$ & -727.096340 & 0.000064 & -0.015385 & -0.009084 & 0.218134 \\
\hline 5a-TS & -727.095244 & 0.000074 & -0.017028 & -0.009720 & 0.215420 \\
\hline $5+9 a$ & -727.112682 & 0.000011 & -0.011263 & -0.009986 & 0.193782 \\
\hline 3a-TS & -727.088264 & 0.000044 & -0.018394 & -0.009874 & 0.213781 \\
\hline $\mathbf{3 a}$ & -617.636749 & -0.000011 & -0.012433 & -0.009986 & 0.214523 \\
\hline $2 \mathbf{b}$ & -805.757226 & 0.000162 & -0.011991 & -0.014730 & $0.275115^{[b]}$ \\
\hline $2 b^{\prime}-T S$ & -805.724069 & 0.000022 & -0.011874 & -0.015290 & 0.271038 \\
\hline $2 b$ & -805.748600 & 0.000260 & -0.012090 & -0.015010 & $0.275072^{[b]}$ \\
\hline $4 b-T S$ & -805.716048 & 0.000534 & -0.011587 & -0.015683 & 0.274975 \\
\hline $4 b$ & -805.755012 & -0.000068 & -0.011859 & -0.017362 & 0.271887 \\
\hline $4 b^{\prime}-T S$ & -805.737382 & 0.000509 & -0.012666 & -0.017992 & 0.273441 \\
\hline $4 b^{\prime}$ & -805.757986 & 0.000467 & -0.009459 & -0.016497 & 0.274865 \\
\hline $5 b-T S$ & -805.757744 & 0.000390 & -0.012995 & -0.019402 & 0.272132 \\
\hline $5+9 b$ & -805.774176 & 0.000009 & -0.008548 & -0.013312 & 0.246696 \\
\hline 3b-TS & -805.749234 & -0.000027 & -0.012239 & -0.020355 & 0.266242 \\
\hline $\mathbf{3 b}$ & -696.298601 & 0.000054 & -0.011694 & -0.014369 & $0.267728^{[b]}$ \\
\hline $2 c$ & -840.241095 & 0.000122 & -0.016652 & -0.013128 & 0.215947 \\
\hline $2 c^{\prime}-T S$ & -840.208163 & 0.000049 & -0.016833 & -0.012993 & 0.214206 \\
\hline $2 c^{\prime}$ & -840.232232 & 0.000223 & -0.017123 & -0.013632 & 0.215215 \\
\hline $4 c-T S$ & -840.199530 & 0.000498 & -0.017760 & -0.012985 & 0.216132 \\
\hline $4 c-T S(2)$ & -840.196134 & 0.000106 & -0.012971 & -0.016978 & $0.213697^{[\mathrm{c}]}$ \\
\hline $4 c$ & -840.237462 & -0.000043 & -0.019001 & -0.013264 & $0.213970^{[b]}$ \\
\hline $4 c^{\prime}-T S$ & -840.216533 & 0.000024 & -0.023575 & -0.017393 & 0.211329 \\
\hline $4 c^{\prime}$ & -840.240413 & -0.000008 & -0.019522 & -0.011948 & 0.213248 \\
\hline $3 c$ & -730.781542 & 0.000041 & -0.016178 & -0.012666 & 0.209132 \\
\hline $\mathbf{N}_{2}$ & -109.563704 & 0.000000 & - & - & -0.005936 \\
\hline \multicolumn{6}{|c|}{$\begin{array}{l}\text { [a] Corrections for the transfer from ideal gas to solution, }+1.9 \mathrm{kcal} / \mathrm{mol} \text {, are included for all compounds } \\
\text { in solution. } \\
\text { [b] An imaginary frequency }\left(<15 i \mathrm{~cm}^{-1}\right) \text { was found in the vibrational analysis and could not be eliminated } \\
\text { by further geometry optimization. This was treated as real in the calculations of the thermal correction to } \\
\text { Gibbs free energy. } \\
\text { [c] Apart from the main imaginary frequency of the transition state }\left(-328 i \mathrm{~cm}^{-1}\right) \text {, a second imaginary } \\
\text { frequency }\left(11 i \mathrm{~cm}^{-1}\right) \text { was found in the vibrational analysis. It was treated as real in the calculations of the } \\
\text { thermal correction to Gibbs free energy. }\end{array}$} \\
\hline
\end{tabular}


Table S3. Calculated absolute energies and energy corrections

\begin{tabular}{|c|c|c|c|c|c|}
\hline & $\begin{array}{c}\text { Absolute energy } \\
B 3 L Y P-D 3(B J) / \\
6-311+G(2 d, 2 p)\end{array}$ & $\begin{array}{l}\text { Three-body } \\
\text { dispersion } \\
\text { correction }\end{array}$ & $\begin{array}{c}\text { Solvation free } \\
\text { energy [a] } \\
(\text { COSMOtherm. } \\
308.15 \mathrm{~K})\end{array}$ & $\begin{array}{c}\text { Solvation free } \\
\text { energy }^{[a]} \\
\text { (SMD) }\end{array}$ & $\begin{array}{c}\text { Thermal correction to } \\
\text { Gibbs free energy } \\
\text { (quasi-RRHO, } 308.15 \mathrm{~K} \text { ) }\end{array}$ \\
\hline $2 a @ 1_{2}$ & -8753.080982 & 0.015379 & -0.124742 & -0.078704 & 1.696878 \\
\hline 2a'-TS@12 & -8753.045708 & 0.015408 & -0.124256 & -0.079340 & 1.695936 \\
\hline 2a'@1 & -8753.071896 & 0.015445 & -0.123220 & -0.078564 & 1.696132 \\
\hline 4a-TS@12 & -8753.031809 & 0.015009 & -0.123611 & -0.079257 & 1.694140 \\
\hline $4 a @ 1_{2}$ & -8753.087374 & 0.015868 & -0.124264 & -0.073408 & 1.697356 \\
\hline $7^{-}: \mathbf{8 a ^ { + }} @ \mathbf{1}_{2}$ & -8753.084363 & 0.015509 & -0.125784 & -0.081060 & 1.692743 \\
\hline $7^{-}: 9 a^{+} @ 1_{2}$ & -8643.550095 & 0.014540 & -0.080772 & -0.127863 & 1.687162 \\
\hline $5: 9 a^{+} @ 1_{2}^{-}$ & -8643.569379 & 0.015659 & -0.125238 & -0.077457 & 1.690836 \\
\hline $4 a^{\prime} @ 1_{2}$ & -8753.089900 & 0.015879 & -0.124885 & -0.076933 & 1.695358 \\
\hline 5:6a@1 & -8753.114444 & 0.015842 & -0.124822 & -0.075602 & 1.693333 \\
\hline 3a-TS@12 & -8753.081562 & 0.015789 & -0.125070 & -0.077709 & 1.692246 \\
\hline $3 a @ 1_{2}$ & -8643.620578 & 0.014916 & -0.123301 & -0.078834 & 1.690399 \\
\hline $3 a: N_{2} @ 1_{2}$ & -8753.187396 & 0.015469 & -0.124114 & -0.075898 & 1.694713 \\
\hline $2 b @ 1_{2}$ & -8831.752735 & 0.016769 & -0.125134 & -0.076329 & 1.754086 \\
\hline 2b'-TS@12 & -8831.711076 & 0.016726 & -0.125199 & -0.077928 & 1.752792 \\
\hline $2 b^{\prime} @ 1_{2}$ & -8831.743363 & 0.016619 & -0.124165 & -0.077002 & 1.752820 \\
\hline 4b-TS@12 & -8831.705348 & 0.016404 & -0.124347 & -0.077341 & 1.752480 \\
\hline $4 b @ 1_{2}$ & -8831.743482 & 0.016940 & -0.124863 & -0.075280 & 1.756430 \\
\hline $7^{-}: 8 b^{+} @ \mathbf{1}_{2}$ & -8831.757641 & 0.016492 & -0.127083 & -0.080432 & 1.749841 \\
\hline $7^{-}: 8 b^{+}: \mathrm{N}_{2} @ 1_{2}$ & -8831.792211 & 0.015671 & -0.130342 & -0.079306 & 1.746860 \\
\hline $7^{-}: 9 b^{+} @ 1_{2}$ & -8722.222654 & 0.016058 & -0.080184 & -0.128485 & 1.743219 \\
\hline $5: 9 b^{+} @ 1_{2}^{-}$ & -8722.237794 & 0.016062 & -0.125506 & -0.079475 & 1.745637 \\
\hline $4 b^{\prime} @ 1_{2}$ & -8831.750828 & 0.016708 & -0.123860 & -0.077229 & 1.751743 \\
\hline $5: 6 b @ 1_{2}$ & -8831.775162 & 0.017142 & -0.123962 & -0.074978 & 1.751605 \\
\hline 3b-TS@12 & -8831.743585 & 0.017246 & -0.125204 & -0.076619 & 1.749945 \\
\hline $3 b @ 1_{2}$ & -8722.293288 & 0.016588 & -0.123941 & -0.076585 & 1.748259 \\
\hline $3 b: N_{2} @ 1_{2}$ & -8831.839596 & 0.016378 & -0.125287 & -0.075889 & 1.748553 \\
\hline $2 c @ 1_{2}$ & -8866.236869 & 0.015891 & -0.126914 & -0.078980 & 1.693654 \\
\hline 2c'-TS@12 & -8866.201277 & 0.016026 & -0.125961 & -0.078673 & 1.691997 \\
\hline $2 c^{\prime} @ 1_{2}$ & -8866.227598 & 0.015851 & -0.126050 & -0.079370 & 1.693642 \\
\hline 4c-TS@12 & -8866.189753 & 0.015967 & -0.126154 & -0.077567 & 1.691410 \\
\hline $4 c @ 1_{2}$ & -8866.227293 & 0.016191 & -0.127440 & -0.077683 & 1.693949 \\
\hline $7^{-}: 8 c^{+} @ 1_{2}$ & -8866.251721 & 0.015891 & -0.129489 & -0.081194 & 1.691202 \\
\hline $7^{-}: 9 c^{+}: \mathbf{N}_{2} @ 1_{2}$ & -8866.252778 & 0.015639 & -0.130414 & -0.080439 & 1.686855 \\
\hline $5: 9 c^{+} @ 1_{2}^{-}$ & -8756.722653 & 0.015668 & -0.128146 & -0.078869 & 1.686389 \\
\hline $4 c^{9} @ 1_{2}$ & -8866.233761 & 0.016550 & -0.127143 & -0.076813 & 1.691368 \\
\hline $3 c @ 1_{2}$ & -8756.774062 & 0.015460 & -0.078451 & -0.125389 & 1.686220 \\
\hline
\end{tabular}

${ }^{[a]}$ Corrections for the transfer from ideal gas to solution, $+1.9 \mathrm{kcal} / \mathrm{mol}$, are included for all compounds in solution. 


\section{S12. Cartesian coordinates}

2a

$6-0.171162-1.168568-0.221949$

$8-0.402314-2.056914-1.026218$

$\begin{array}{lllll}7 & -1.226842 & -0.735264 & 0.622081\end{array}$

$\begin{array}{lllll}7 & -0.897566 & -0.047164 & 1.758492\end{array}$

$\begin{array}{lllll}8 & -1.841348 & 0.253352 & 2.465363\end{array}$

$\begin{array}{llll}6 & -2.599776 & -1.182003 & 0.345645\end{array}$

$1-3.135193-1.112671 \quad 1.292916$

$\begin{array}{lllll}1 & -2.532518 & -2.223467 & 0.030418\end{array}$

$6-3.271350-0.330114-0.734584$

$1-4.268656-0.751277-0.914598$

$1-2.713441-0.443909-1.671793$

$\begin{array}{lllll}6 & -3.392778 & 1.152475 & -0.372141\end{array}$

$\begin{array}{lllll}1 & -2.392692 & 1.562555 & -0.183526\end{array}$

$\begin{array}{lllll}1 & -3.943452 & 1.253825 & 0.570534\end{array}$

$\begin{array}{lllll}6 & -4.077311 & 1.967455 & -1.470621\end{array}$

$\begin{array}{lllll}1 & -5.093418 & 1.603028 & -1.658308\end{array}$

$1-4.147702 \quad 3.024844-1.198070$

$1-3.523896 \quad 1.901128-2.413954$

$\begin{array}{lllll}6 & 1.162395 & -0.517710 & -0.122397\end{array}$

$\begin{array}{lllll}6 & 1.365104 & 0.827229 & 0.215611\end{array}$

$\begin{array}{lllll}6 & 2.264887 & -1.295993 & -0.504319\end{array}$

$\begin{array}{lllll}6 & 2.646397 & 1.367711 & 0.186645\end{array}$

$\begin{array}{lllll}1 & 0.527213 & 1.457030 & 0.484446\end{array}$

$6 \quad 3.542790-0.752709-0.509089$

$1 \quad 2.097015-2.325866-0.798378$

$\begin{array}{lllll}6 & 3.756455 & 0.588248 & -0.161272\end{array}$

$\begin{array}{lllll}1 & 2.788013 & 2.414966 & 0.437638\end{array}$

$14.388546-1.372030-0.794072$

$\begin{array}{lllll}6 & 5.146335 & 1.170115 & -0.145083\end{array}$

$1 \quad 5.7831890 .704245-0.902326$

$\begin{array}{lllll}1 & 5.130290 & 2.248420 & -0.325178\end{array}$

$1 \quad 5.6249391 .007845 \quad 0.828406$

\section{2a'-TS}

$\begin{array}{llll}6 & 0.017775 & 1.044988 & -0.047500\end{array}$

$\begin{array}{lllll}8 & 0.283310 & 2.225750 & -0.231065\end{array}$

$\begin{array}{lllll}7 & 1.024717 & 0.088052 & 0.168130\end{array}$

$\begin{array}{lllll}7 & 0.727750 & -0.932875 & 1.270664\end{array}$

$\begin{array}{lllll}8 & 0.650065 & -2.036520 & 0.856637\end{array}$

$\begin{array}{lllll}6 & 2.380707 & 0.584497 & 0.439103\end{array}$

$\begin{array}{lllll}1 & 2.561608 & 0.547576 & 1.524792\end{array}$

$\begin{array}{lllll}1 & 2.393194 & 1.636912 & 0.145785\end{array}$

$\begin{array}{lllll}6 & 3.435703 & -0.230516 & -0.305175\end{array}$

$\begin{array}{lllll}1 & 3.237451 & -0.163524 & -1.381493\end{array}$

$\begin{array}{lllll}1 & 3.337238 & -1.291327 & -0.039588\end{array}$

$\begin{array}{llll}6 & 4.857926 & 0.247625 & 0.000110\end{array}$

$1 \quad 5.038952 \quad 0.181511 \quad 1.081315$

$\begin{array}{lllll}1 & 4.945398 & 1.311009 & -0.258601\end{array}$

$\begin{array}{lllll}6 & 5.924895 & -0.553112 & -0.748601\end{array}$

$\begin{array}{lllll}1 & 5.786373 & -0.476050 & -1.832475\end{array}$

$\begin{array}{lllll}1 & 6.931783 & -0.194429 & -0.514183\end{array}$

$\begin{array}{lrrrr}1 & 5.878280 & -1.615301 & -0.484307\end{array}$

$\begin{array}{llll}6 & -1.378684 & 0.533112 & -0.100191\end{array}$

$\begin{array}{llll}6 & -1.706556 & -0.735239 & -0.596245\end{array}$

$\begin{array}{llll}6 & -2.407190 & 1.395784 & 0.303127\end{array}$

$\begin{array}{llll}6 & -3.037943 & -1.136380 & -0.660540\end{array}$

$\begin{array}{lllll}1 & -0.922861 & -1.393106 & -0.952649\end{array}$

$\begin{array}{llll}6 & -3.731371 & 0.980726 & 0.249048\end{array}$

$\begin{array}{llll}1 & -2.146258 & 2.388944 & 0.651637\end{array}$

$\begin{array}{llll}6 & -4.069480 & -0.293602 & -0.229429\end{array}$

$\begin{array}{llll}1 & -3.281811 & -2.117309 & -1.058648\end{array}$

$\begin{array}{llll}1 & -4.519545 & 1.653932 & 0.574666\end{array}$

$\begin{array}{llll}6 & -5.506216 & -0.748072 & -0.261437\end{array}$

$\begin{array}{llll}1 & -5.800365 & -1.180610 & 0.702699\end{array}$ $\begin{array}{llll}1 & -6.184205 & 0.086168 & -0.463136\end{array}$

$\begin{array}{llll}1 & -5.666902 & -1.513330 & -1.025575\end{array}$

\section{2a'}

$\begin{array}{llll}6 & 0.425789 & -1.031591 & 0.722775\end{array}$

$\begin{array}{llll}8 & 0.739802 & -1.373722 & 1.849629\end{array}$

$\begin{array}{lllll}7 & 1.435676 & -1.079305 & -0.303370\end{array}$

$\begin{array}{lllll}7 & 1.273900 & -1.609372 & -1.563697\end{array}$

$\begin{array}{lllll}8 & 0.148490 & -1.952182 & -1.865216\end{array}$

$\begin{array}{llll}6 & 2.840349 & -0.947560 & 0.108341\end{array}$

$\begin{array}{lllll}1 & 3.421890 & -1.175014 & -0.787975\end{array}$

$\begin{array}{lllll}1 & 3.053947 & -1.705573 & 0.866509\end{array}$

$\begin{array}{llll}6 & 3.165106 & 0.449025 & 0.638839\end{array}$

$\begin{array}{lllll}1 & 4.224718 & 0.453268 & 0.924134\end{array}$

$\begin{array}{lllll}1 & 2.599902 & 0.625576 & 1.561380\end{array}$

$\begin{array}{lllll}6 & 2.894301 & 1.572550 & -0.365380\end{array}$

$\begin{array}{lllll}1 & 1.829951 & 1.573983 & -0.628704\end{array}$

$\begin{array}{lllll}1 & 3.438622 & 1.367008 & -1.296681\end{array}$

$\begin{array}{lllll}6 & 3.286978 & 2.949012 & 0.173684\end{array}$

$\begin{array}{lllll}1 & 4.355118 & 2.989970 & 0.413955\end{array}$

$\begin{array}{lllll}1 & 3.078310 & 3.738219 & -0.555003\end{array}$

$\begin{array}{lllll}1 & 2.733635 & 3.185161 & 1.089297\end{array}$

$\begin{array}{lllll}6 & -0.899759 & -0.472091 & 0.387841\end{array}$

$\begin{array}{lllll}6 & -1.109360 & 0.373804 & -0.709380\end{array}$

$\begin{array}{llll}6 & -1.956639 & -0.721532 & 1.272859\end{array}$

$\begin{array}{lllll}6 & -2.355346 & 0.951072 & -0.914973\end{array}$

$\begin{array}{lllll}1 & -0.297000 & 0.584236 & -1.394688\end{array}$

$\begin{array}{llll}6 & -3.205274 & -0.155395 & 1.048365\end{array}$

$\begin{array}{llll}1 & -1.777434 & -1.359020 & 2.131328\end{array}$

$\begin{array}{lllll}6 & -3.426438 & 0.688361 & -0.048550\end{array}$

$\begin{array}{lllll}1 & -2.504939 & 1.614171 & -1.762112\end{array}$

$\begin{array}{llll}1 & -4.021300 & -0.363707 & 1.734349\end{array}$

$\begin{array}{llll}6 & -4.787640 & 1.279871 & -0.307328\end{array}$

$\begin{array}{lllll}1 & -5.361411 & 0.646165 & -0.994542\end{array}$

$\begin{array}{lllll}1 & -5.367015 & 1.370024 & 0.615399\end{array}$

$\begin{array}{lllll}1 & -4.712762 & 2.270340 & -0.765038\end{array}$

\section{4a-TS}

$\begin{array}{lllll}6 & -1.261696 & -1.593229 & -0.256267\end{array}$

$\begin{array}{lllll}8 & -1.723011 & -2.391094 & -1.007307\end{array}$

$\begin{array}{llll}7 & -2.231237 & -0.028203 & -0.046267\end{array}$

$\begin{array}{llll}7 & -2.597967 & -0.303150 & 1.126193\end{array}$

$\begin{array}{llll}8 & -2.092306 & -1.461903 & 1.412482\end{array}$

$\begin{array}{lllll}6 & -2.552847 & 1.237936 & -0.686625\end{array}$

$\begin{array}{llll}1 & -3.230319 & 1.796823 & -0.030876\end{array}$

$\begin{array}{lllll}1 & -3.082725 & 1.007826 & -1.617148\end{array}$

$\begin{array}{lllll}6 & -1.269779 & 2.025813 & -0.982868\end{array}$

$\begin{array}{lllll}1 & -1.519955 & 2.850722 & -1.660874\end{array}$

$\begin{array}{lllll}1 & -0.580013 & 1.370629 & -1.528698\end{array}$

$\begin{array}{llll}6 & -0.582962 & 2.567498 & 0.273962\end{array}$

$\begin{array}{llll}1 & -0.508981 & 1.768012 & 1.018226\end{array}$

$\begin{array}{lllll}1 & -1.211828 & 3.348458 & 0.720614\end{array}$

$\begin{array}{lllll}6 & 0.815460 & 3.114875 & -0.013285\end{array}$

$\begin{array}{lllll}1 & 0.785940 & 3.913488 & -0.762948\end{array}$

$\begin{array}{lllll}1 & 1.276630 & 3.523064 & 0.891303\end{array}$

$\begin{array}{lllll}1 & 1.467877 & 2.321184 & -0.392230\end{array}$

$\begin{array}{lllll}6 & 0.162697 & -1.220900 & -0.059214\end{array}$

$\begin{array}{lllll}6 & 0.663019 & -0.662542 & 1.121627\end{array}$

$\begin{array}{lllll}6 & 1.016151 & -1.407078 & -1.152344\end{array}$

$\begin{array}{lllll}6 & 1.996972 & -0.279144 & 1.193233\end{array}$

$\begin{array}{lllll}1 & 0.009447 & -0.553787 & 1.977993\end{array}$

$\begin{array}{lllll}6 & 2.348592 & -1.011230 & -1.071131\end{array}$

$\begin{array}{lllll}1 & 0.623659 & -1.857152 & -2.057681\end{array}$

$\begin{array}{lllll}6 & 2.858965 & -0.434279 & 0.097571\end{array}$

$\begin{array}{llll}1 & 2.380123 & 0.147903 & 2.115712\end{array}$ $\begin{array}{llll}1 & 3.002740 & -1.154014 & -1.926490\end{array}$

$\begin{array}{lllll}6 & 4.291638 & 0.027573 & 0.177375\end{array}$

$\begin{array}{lllll}1 & 4.900282 & -0.416704 & -0.614202\end{array}$

$\begin{array}{lllll}1 & 4.356372 & 1.117367 & 0.073352\end{array}$

$\begin{array}{llll}1 & 4.740448 & -0.232137 & 1.140859\end{array}$

\section{4a-TS(2)}

$\begin{array}{llll}6 & -0.135587 & 1.366946 & 0.067415\end{array}$

$\begin{array}{lllll}8 & 0.099841 & 2.446514 & -0.373372\end{array}$

$\begin{array}{lllll}7 & 1.247965 & 0.117479 & -0.099980\end{array}$

$\begin{array}{llll}7 & 1.532575 & 0.156202 & 1.125419\end{array}$

$\begin{array}{llll}8 & 0.697128 & 0.973634 & 1.681116\end{array}$

$\begin{array}{lllll}6 & 1.965366 & -0.734553 & -1.043042\end{array}$

$\begin{array}{lllll}1 & 2.074392 & -0.144755 & -1.960518\end{array}$

$\begin{array}{lllll}1 & 1.307242 & -1.578963 & -1.285026\end{array}$

$\begin{array}{lllll}6 & 3.329684 & -1.229890 & -0.555960\end{array}$

$13.690208 \quad-1.957466 \quad-1.294261$

$\begin{array}{lllll}1 & 3.202609 & -1.774135 & 0.386181\end{array}$

$\begin{array}{lllll}6 & 4.370233 & -0.119241 & -0.380877\end{array}$

$\begin{array}{llll}1 & 4.006413 & 0.609724 & 0.350054\end{array}$

$\begin{array}{lllll}1 & 4.482255 & 0.420785 & -1.330249\end{array}$

$\begin{array}{lllll}6 & 5.727556 & -0.662538 & 0.069118\end{array}$

$\begin{array}{lllll}1 & 6.129030 & -1.380380 & -0.655157\end{array}$

$\begin{array}{llll}1 & 6.459843 & 0.142365 & 0.182400\end{array}$

$\begin{array}{lllll}1 & 5.644671 & -1.175541 & 1.033437\end{array}$

$\begin{array}{llll}6 & -1.417394 & 0.623582 & 0.053883\end{array}$

$\begin{array}{llll}6 & -1.608373 & -0.595076 & 0.714058\end{array}$

$\begin{array}{lllll}6 & -2.469774 & 1.185970 & -0.676264\end{array}$

$\begin{array}{lllll}6 & -2.836899 & -1.238347 & 0.637260\end{array}$

$\begin{array}{llll}1 & -0.801735 & -1.026483 & 1.294121\end{array}$

$\begin{array}{lllll}6 & -3.696662 & 0.532360 & -0.746905\end{array}$

$\begin{array}{lllll}1 & -2.310361 & 2.131361 & -1.182912\end{array}$

$6-3.901578-0.687108 \quad-0.091865$

$\begin{array}{llll}1 & -2.978636 & -2.183281 & 1.154360\end{array}$

$\begin{array}{lllll}1 & -4.507219 & 0.974759 & -1.319030\end{array}$

$\begin{array}{llll}6 & -5.237894 & -1.382462 & -0.142026\end{array}$

$\begin{array}{lllll}1 & -5.842572 & -1.027082 & -0.980285\end{array}$

$\begin{array}{lllll}1 & -5.120322 & -2.465991 & -0.239037\end{array}$

$\begin{array}{llll}1 & -5.807426 & -1.199719 & 0.777130\end{array}$

\section{$4 a$}

$\begin{array}{llll}6 & 0.781458 & 1.027985 & 0.026673\end{array}$

$\begin{array}{lllll}8 & 0.536026 & 2.185969 & -0.209840\end{array}$

$\begin{array}{llll}7 & -2.238108 & -0.285038 & 0.776619\end{array}$

$\begin{array}{llll}7 & -1.488884 & 0.638784 & 0.485501\end{array}$

$\begin{array}{llll}8 & -0.163023 & 0.086332 & 0.383108\end{array}$

$\begin{array}{lllll}6 & -3.630535 & 0.176051 & 0.889188\end{array}$

$\begin{array}{llll}1 & -3.692188 & 1.267895 & 0.810096\end{array}$

$\begin{array}{llll}1 & -3.970048 & -0.126582 & 1.886361\end{array}$

$\begin{array}{llll}6 & -4.483487 & -0.508052 & -0.181647\end{array}$

$\begin{array}{llll}1 & -4.341141 & -1.593083 & -0.114311\end{array}$

$\begin{array}{llll}1 & -4.119844 & -0.203587 & -1.170783\end{array}$

$\begin{array}{llll}6 & -5.968846 & -0.161472 & -0.042493\end{array}$

$\begin{array}{lllll}1 & -6.094195 & 0.927968 & -0.090171\end{array}$

$\begin{array}{llll}1 & -6.320200 & -0.467223 & 0.951803\end{array}$

$\begin{array}{llll}6 & -6.829568 & -0.825289 & -1.118969\end{array}$

$\begin{array}{lllll}1 & -6.744085 & -1.916454 & -1.074692\end{array}$ 
$\begin{array}{llll}1 & -7.886110 & -0.567425 & -0.998943\end{array}$

$\begin{array}{lllll}1 & -6.519928 & -0.509260 & -2.120960\end{array}$

$\begin{array}{lllll}6 & 2.131399 & 0.409616 & -0.025382\end{array}$

$\begin{array}{lllll}6 & 2.363228 & -0.940488 & 0.265818\end{array}$

$\begin{array}{lllll}6 & 3.208535 & 1.233863 & -0.377286\end{array}$

$\begin{array}{lllll}6 & 3.656334 & -1.450961 & 0.203153\end{array}$

$\begin{array}{lllll}1 & 1.533468 & -1.579631 & 0.542337\end{array}$

$\begin{array}{lllll}6 & 4.494717 & 0.713883 & -0.436088\end{array}$

$\begin{array}{lllll}1 & 3.012312 & 2.277664 & -0.595487\end{array}$

$\begin{array}{lllll}6 & 4.740156 & -0.637186 & -0.149697\end{array}$

$\begin{array}{lllll}1 & 3.830117 & -2.498296 & 0.433398\end{array}$

$\begin{array}{lllll}1 & 5.324755 & 1.361051 & -0.705806\end{array}$

$\begin{array}{lllll}6 & 6.134665 & -1.201479 & -0.243933\end{array}$

$\begin{array}{lllll}1 & 6.391982 & -1.434351 & -1.284390\end{array}$

$\begin{array}{lllll}1 & 6.879080 & -0.487658 & 0.121106\end{array}$

$\begin{array}{lllll}1 & 6.232598 & -2.124489 & 0.333434\end{array}$

\section{4a'-TS}

$\begin{array}{llll}6 & -0.331948 & -0.619637 & -0.599172\end{array}$ $\begin{array}{lllll}8 & 0.554115 & 0.226655 & -0.793134\end{array}$

$\begin{array}{lllll}7 & 2.105343 & -1.407014 & 0.396794\end{array}$

$\begin{array}{lllll}7 & 1.668326 & -2.318987 & -0.143478\end{array}$

$\begin{array}{lllll}8 & -0.146568 & -1.913195 & -0.612130\end{array}$

$\begin{array}{lllll}6 & 3.406520 & -0.920163 & 0.855582\end{array}$

$\begin{array}{lllll}1 & 4.145120 & -1.724354 & 0.770903\end{array}$

$\begin{array}{lllll}1 & 3.269450 & -0.656849 & 1.907277\end{array}$

$\begin{array}{lllll}6 & 3.766286 & 0.315892 & 0.025783\end{array}$

$\begin{array}{lllll}1 & 2.919411 & 1.006960 & 0.052398\end{array}$

$\begin{array}{lllll}1 & 3.887652 & 0.015524 & -1.020367\end{array}$

$\begin{array}{lllll}6 & 5.047212 & 0.975453 & 0.545419\end{array}$

$\begin{array}{lllll}1 & 5.870743 & 0.249285 & 0.533261\end{array}$

$\begin{array}{lllll}1 & 4.907467 & 1.267321 & 1.594560\end{array}$

$\begin{array}{lllll}6 & 5.432003 & 2.203210 & -0.282711\end{array}$

$\begin{array}{lllll}1 & 4.635519 & 2.953868 & -0.263284\end{array}$

$\begin{array}{lllll}1 & 6.343932 & 2.668391 & 0.102409\end{array}$

$\begin{array}{lllll}1 & 5.608343 & 1.932787 & -1.328928\end{array}$

$\begin{array}{lllll}6 & -1.745256 & -0.193085 & -0.304896\end{array}$

$\begin{array}{llll}6 & -2.740174 & -1.118863 & 0.025157\end{array}$

$\begin{array}{llll}6 & -2.070028 & 1.166002 & -0.367342\end{array}$

$\begin{array}{llll}6 & -4.036442 & -0.687204 & 0.293597\end{array}$

$\begin{array}{llll}1 & -2.484148 & -2.171632 & 0.062837\end{array}$

$\begin{array}{lllll}6 & -3.367272 & 1.590453 & -0.099912\end{array}$

$\begin{array}{lllll}1 & -1.287524 & 1.869344 & -0.630349\end{array}$

$\begin{array}{llll}6 & -4.370838 & 0.671927 & 0.238409\end{array}$

$\begin{array}{llll}1 & -4.804120 & -1.413809 & 0.547468\end{array}$

$\begin{array}{llll}1 & -3.611019 & 2.648587 & -0.154474\end{array}$

$\begin{array}{llll}6 & -5.768490 & 1.139022 & 0.560202\end{array}$

$\begin{array}{llll}1 & -5.862902 & 1.398784 & 1.622032\end{array}$

$\begin{array}{lllll}1 & -6.034927 & 2.030126 & -0.015921\end{array}$

$\begin{array}{llll}1 & -6.508488 & 0.361947 & 0.348380\end{array}$

\section{$4 a^{\prime}$}

$\begin{array}{llll}6 & 0.007708 & -0.745900 & 0.572684\end{array}$ $\begin{array}{llll}8 & -0.662404 & -0.197150 & 1.445349\end{array}$

$\begin{array}{llll}7 & -2.889290 & -1.944757 & 0.093851\end{array}$

$\begin{array}{llll}7 & -1.913496 & -2.418162 & 0.522892\end{array}$

$\begin{array}{llll}8 & -0.428284 & -1.715992 & -0.211531\end{array}$

$\begin{array}{llll}6 & -3.082515 & -0.797618 & -0.809129\end{array}$

$\begin{array}{lllll}1 & -3.868638 & -1.089185 & -1.509340\end{array}$

$\begin{array}{llll}1 & -2.134886 & -0.646468 & -1.330312\end{array}$

$\begin{array}{llll}6 & -3.489581 & 0.425427 & 0.018976\end{array}$

$\begin{array}{lllll}1 & -2.732081 & 0.574408 & 0.793596\end{array}$

$\begin{array}{lllll}1 & -4.447607 & 0.228114 & 0.513434\end{array}$

$\begin{array}{lllll}6 & -3.595928 & 1.669522 & -0.869104\end{array}$

$\begin{array}{llll}1 & -4.331489 & 1.497960 & -1.666250\end{array}$

$\begin{array}{lllll}1 & -2.632524 & 1.839433 & -1.366273\end{array}$

$\begin{array}{llll}6 & -3.983535 & 2.912427 & -0.065432\end{array}$

$\begin{array}{llll}1 & -3.244211 & 3.117594 & 0.715339\end{array}$ $\begin{array}{llll}1 & -4.048715 & 3.794880 & -0.708761\end{array}$

$\begin{array}{llll}1 & -4.955387 & 2.780082 & 0.422053\end{array}$

$\begin{array}{llll}6 & 1.418482 & -0.332416 & 0.280081\end{array}$

$\begin{array}{lllll}6 & 2.224405 & -1.021543 & -0.632617\end{array}$

$\begin{array}{lllll}6 & 1.937935 & 0.781577 & 0.949570\end{array}$

$\begin{array}{lllll}6 & 3.529147 & -0.597640 & -0.869440\end{array}$

$\begin{array}{lllll}1 & 1.822852 & -1.889301 & -1.142949\end{array}$

$\begin{array}{lllll}6 & 3.241185 & 1.199071 & 0.706042\end{array}$

$\begin{array}{lllll}1 & 1.302220 & 1.297241 & 1.660561\end{array}$

$\begin{array}{lllll}6 & 4.057187 & 0.519125 & -0.209306\end{array}$

$\begin{array}{lllll}1 & 4.151321 & -1.142092 & -1.574738\end{array}$

$\begin{array}{lllll}1 & 3.637585 & 2.062583 & 1.233792\end{array}$

$\begin{array}{lllll}6 & 5.459876 & 0.995059 & -0.491818\end{array}$

$\begin{array}{lllll}1 & 5.460010 & 1.789219 & -1.248657\end{array}$

$\begin{array}{lllll}1 & 5.931162 & 1.404281 & 0.406662\end{array}$

$\begin{array}{lllll}1 & 6.089199 & 0.184275 & -0.868941\end{array}$

\section{5a-TS}

$\begin{array}{lllll}6 & 0.106154 & -0.717596 & 0.280428\end{array}$ $\begin{array}{lllll}8 & 0.702040 & -0.304237 & 1.334028\end{array}$

$\begin{array}{lllll}7 & 3.210381 & -1.834122 & 0.149125\end{array}$

$\begin{array}{lllll}7 & 2.584447 & -2.625925 & -0.349457\end{array}$

$\begin{array}{lllll}8 & 0.624133 & -1.380684 & -0.659044\end{array}$

$\begin{array}{lllll}6 & 3.274900 & -0.512142 & 0.693056\end{array}$

$\begin{array}{lllll}1 & 4.072415 & -0.475970 & 1.435844\end{array}$

$\begin{array}{lllll}1 & 2.170703 & -0.430128 & 1.140335\end{array}$

$\begin{array}{lllll}6 & 3.405198 & 0.521291 & -0.433293\end{array}$

$\begin{array}{lllll}1 & 2.640494 & 0.293007 & -1.181859\end{array}$

$\begin{array}{lllll}1 & 4.389224 & 0.468296 & -0.912894\end{array}$

$\begin{array}{lllll}6 & 3.160039 & 1.920081 & 0.148965\end{array}$

$\begin{array}{lllll}1 & 3.899431 & 2.127093 & 0.933708\end{array}$

$\begin{array}{lllll}1 & 2.176209 & 1.926417 & 0.629589\end{array}$

$\begin{array}{lllll}6 & 3.231388 & 3.003756 & -0.927952\end{array}$

$\begin{array}{lllll}1 & 2.481599 & 2.831996 & -1.707085\end{array}$

$\begin{array}{lllll}1 & 3.046958 & 3.992863 & -0.498930\end{array}$

$\begin{array}{lllll}1 & 4.215547 & 3.025220 & -1.408731\end{array}$

$\begin{array}{llll}6 & -1.350844 & -0.354136 & 0.154102\end{array}$

$\begin{array}{lllll}6 & -2.091724 & -0.798072 & -0.945717\end{array}$

$\begin{array}{llll}6 & -1.977660 & 0.434123 & 1.124984\end{array}$

$\begin{array}{lllll}6 & -3.436362 & -0.460930 & -1.068787\end{array}$

$\begin{array}{lllll}1 & -1.591897 & -1.403429 & -1.693179\end{array}$

$\begin{array}{llll}6 & -3.322120 & 0.769600 & 0.994984\end{array}$

$\begin{array}{llll}1 & -1.393758 & 0.775216 & 1.972074\end{array}$

$\begin{array}{lllll}6 & -4.074395 & 0.325469 & -0.100594\end{array}$

$\begin{array}{llll}1 & -4.002656 & -0.809688 & -1.928729\end{array}$

$\begin{array}{llll}1 & -3.799188 & 1.385197 & 1.753576\end{array}$

$\begin{array}{lllll}6 & -5.540065 & 0.661691 & -0.218956\end{array}$

$\begin{array}{lllll}1 & -5.862853 & 0.681737 & -1.263897\end{array}$

$\begin{array}{llll}1 & -6.158137 & -0.082410 & 0.298816\end{array}$

$\begin{array}{llll}1 & -5.764339 & 1.635472 & 0.226494\end{array}$

\section{5}

$\begin{array}{lllll}6 & 2.182081 & 0.111079 & 0.003055\end{array}$

$\begin{array}{llll}8 & 2.782277 & -1.107565 & 0.004664\end{array}$

$\begin{array}{lllll}8 & 2.825067 & 1.142863 & 0.004916\end{array}$

$\begin{array}{lllll}1 & 3.736263 & -0.927336 & 0.007570\end{array}$

$\begin{array}{lllll}6 & 0.702511 & 0.030693 & -0.000334\end{array}$

$\begin{array}{lllll}6 & -0.023328 & 1.228407 & -0.003856\end{array}$

$\begin{array}{lllll}6 & 0.018322 & -1.190667 & -0.004212\end{array}$

$\begin{array}{lllll}6 & -1.411982 & 1.201892 & -0.010105\end{array}$

$\begin{array}{lllll}1 & 0.522536 & 2.165262 & -0.005174\end{array}$

$\begin{array}{lllll}6 & -1.372991 & -1.206269 & -0.010412\end{array}$

$\begin{array}{lllll}1 & 0.578334 & -2.118316 & -0.006017\end{array}$

$\begin{array}{llll}6 & -2.109710 & -0.014742 & -0.010487\end{array}$

$\begin{array}{llll}1 & -1.968165 & 2.135182 & -0.016484\end{array}$

$\begin{array}{llll}1 & -1.898670 & -2.156973 & -0.016847\end{array}$

$\begin{array}{llll}6 & -3.616665 & -0.036828 & 0.014941\end{array}$

$\begin{array}{lllll}1 & -4.037715 & 0.790825 & -0.563171\end{array}$

$\begin{array}{llll}1 & -4.010193 & -0.973163 & -0.389522\end{array}$ $\begin{array}{llll}1 & -3.990565 & 0.060744 & 1.041459\end{array}$

\section{6a}

$\begin{array}{llll}7 & -1.964168 & -0.022148 & -0.046474\end{array}$

$\begin{array}{lllll}7 & -2.864478 & -0.735011 & 0.009696\end{array}$

$\begin{array}{llll}6 & -0.945195 & 0.773314 & -0.080497\end{array}$

$\begin{array}{lllll}1 & -1.086275 & 1.694842 & -0.631215\end{array}$

$\begin{array}{llll}6 & 0.377201 & 0.366466 & 0.515664\end{array}$

$\begin{array}{lllll}1 & 0.217105 & -0.469043 & 1.207358\end{array}$

$\begin{array}{lllll}1 & 0.762134 & 1.196441 & 1.123692\end{array}$

$\begin{array}{lllll}6 & 1.436774 & -0.026072 & -0.529082\end{array}$

$\begin{array}{lllll}1 & 1.567868 & 0.804008 & -1.234687\end{array}$

$\begin{array}{lllll}1 & 1.059111 & -0.871140 & -1.116927\end{array}$

$\begin{array}{lllll}6 & 2.780629 & -0.384407 & 0.107879\end{array}$

$\begin{array}{lllll}1 & 2.679283 & -1.232854 & 0.793833\end{array}$

$\begin{array}{lllll}1 & 3.521521 & -0.655734 & -0.650170\end{array}$

$\begin{array}{lllll}1 & 3.183323 & 0.457790 & 0.681779\end{array}$

\section{3a-TS}

$\begin{array}{llll}6 & -0.066947 & -0.466115 & 0.269909\end{array}$

$\begin{array}{lllll}8 & 0.550594 & -0.998733 & -0.709963\end{array}$

$\begin{array}{lllll}8 & 0.482613 & -0.092898 & 1.344932\end{array}$

$\begin{array}{lllll}7 & 4.011439 & -1.499983 & 0.173348\end{array}$

$\begin{array}{lllll}7 & 4.485050 & -2.234542 & -0.518291\end{array}$

$\begin{array}{llll}6 & 2.748919 & -0.647339 & 0.768098\end{array}$

$\begin{array}{lllll}1 & 1.953484 & -0.989962 & 0.005996\end{array}$

$\begin{array}{llll}1 & 2.664736 & -1.084184 & 1.759008\end{array}$

$\begin{array}{llll}6 & -1.552662 & -0.267923 & 0.125225\end{array}$

$\begin{array}{lllll}6 & -2.213187 & -0.669448 & -1.040186\end{array}$

$\begin{array}{lllll}6 & -2.288543 & 0.318769 & 1.159317\end{array}$

$\begin{array}{llll}6 & -3.586921 & -0.484950 & -1.166229\end{array}$

$\begin{array}{lllll}1 & -1.631921 & -1.127529 & -1.832349\end{array}$

$\begin{array}{llll}6 & -3.662008 & 0.501082 & 1.027173\end{array}$

$\begin{array}{llll}1 & -1.763961 & 0.620039 & 2.059069\end{array}$

$\begin{array}{llll}6 & -4.333009 & 0.105380 & -0.137469\end{array}$

$\begin{array}{llll}1 & -4.092664 & -0.804263 & -2.074137\end{array}$

$\begin{array}{lllll}1 & -4.226353 & 0.954950 & 1.838051\end{array}$

$\begin{array}{llll}6 & 3.150950 & 0.811620 & 0.690980\end{array}$

$\begin{array}{lllll}1 & 2.350728 & 1.323408 & 1.230143\end{array}$

$\begin{array}{lllll}1 & 4.083342 & 0.994527 & 1.238202\end{array}$

$\begin{array}{lllll}6 & -5.816239 & 0.333214 & -0.291521\end{array}$

$\begin{array}{llll}1 & -6.324903 & 0.325034 & 0.676912\end{array}$

$\begin{array}{lllll}1 & -6.020227 & 1.305025 & -0.758309\end{array}$

$\begin{array}{llll}1 & -6.274700 & -0.432619 & -0.924142\end{array}$

$\begin{array}{lllll}6 & 3.232015 & 1.331314 & -0.747942\end{array}$

$\begin{array}{lllll}1 & 2.295365 & 1.079439 & -1.256745\end{array}$

$\begin{array}{lllll}1 & 4.031585 & 0.806526 & -1.289335\end{array}$

$\begin{array}{lllll}6 & 3.483349 & 2.838020 & -0.804536\end{array}$

$\begin{array}{lllll}1 & 3.538843 & 3.190415 & -1.838442\end{array}$

$\begin{array}{lllll}1 & 2.677432 & 3.387189 & -0.306668\end{array}$

$\begin{array}{lllll}1 & 4.423842 & 3.104965 & -0.309314\end{array}$

\section{3a}

$\begin{array}{llll}6 & 0.284785 & 0.557662 & 0.473183\end{array}$

$\begin{array}{lllll}8 & 0.765134 & 1.674676 & 0.421163\end{array}$

$\begin{array}{lllll}8 & 1.000635 & -0.548641 & 0.783960\end{array}$

$\begin{array}{llll}6 & 2.407894 & -0.345077 & 1.034043\end{array}$

$\begin{array}{lllll}1 & 2.700850 & -1.189387 & 1.663379\end{array}$

$\begin{array}{lllll}1 & 2.538203 & 0.586055 & 1.591159\end{array}$

$\begin{array}{lllll}6 & 3.207051 & -0.317514 & -0.261652\end{array}$

$\begin{array}{lllll}1 & 2.859040 & 0.524181 & -0.869901\end{array}$

$\begin{array}{lllll}1 & 3.001295 & -1.234937 & -0.826948\end{array}$

$\begin{array}{lllll}6 & 4.711466 & -0.185970 & -0.004651\end{array}$

$\begin{array}{lllll}1 & 5.049376 & -1.017878 & 0.627961\end{array}$

$\begin{array}{lllll}1 & 4.902304 & 0.731245 & 0.567374\end{array}$

$\begin{array}{lllll}6 & 5.528805 & -0.162000 & -1.297865\end{array}$

$\begin{array}{lllll}1 & 5.232592 & 0.679131 & -1.933833\end{array}$ 
$\begin{array}{rrrr}1 & 6.599340 & -0.065630 & -1.092499 \\ 1 & 5.379801 & -1.081356 & -1.874666 \\ 6 & -1.144485 & 0.247463 & 0.211468 \\ 6 & -1.651012 & -1.057354 & 0.250290 \\ 6 & -2.003961 & 1.310073 & -0.091832 \\ 6 & -2.998102 & -1.287810 & -0.009495 \\ 1 & -0.985155 & -1.880816 & 0.478699 \\ 6 & -3.348549 & 1.069911 & -0.348052 \\ 1 & -1.594846 & 2.313678 & -0.123830 \\ 6 & -3.868678 & -0.231267 & -0.308523 \\ 1 & -3.383389 & -2.303384 & 0.018230 \\ 1 & -4.007968 & 1.900362 & -0.584730 \\ 6 & -5.334175 & -0.484630 & -0.554689 \\ 1 & -5.903017 & -0.425754 & 0.381395 \\ 1 & -5.758029 & 0.254787 & -1.239934 \\ 1 & -5.502786 & -1.479508 & -0.976195\end{array}$

\section{2a@12}

$\begin{array}{llll}6 & -9.027645 & 2.697414 & 2.479276\end{array}$

$\begin{array}{lllll}6 & -8.411646 & 1.360840 & 2.894251\end{array}$

$\begin{array}{lllll}6 & -8.440163 & 3.172767 & 1.154288\end{array}$

$\begin{array}{llll}1 & -8.717805 & 3.424016 & 3.231672\end{array}$

$\begin{array}{lllll}6 & -8.983204 & 0.117814 & 2.609848\end{array}$

$\begin{array}{lllll}6 & -7.209660 & 1.355058 & 3.611199\end{array}$

$\begin{array}{lllll}6 & -8.904779 & 2.656833 & -0.061602\end{array}$

$\begin{array}{lllll}6 & -7.409210 & 4.118388 & 1.089258\end{array}$

$\begin{array}{llll}6 & -8.418595 & -1.088458 & 3.043176\end{array}$

$\begin{array}{llll}1 & -9.905774 & 0.084124 & 2.042748\end{array}$

$\begin{array}{lllll}6 & -6.625677 & 0.196105 & 4.098141\end{array}$

$\begin{array}{lllll}8 & -6.645919 & 2.595513 & 3.898524\end{array}$

$6 \quad-8.377906 \quad 3.019856 \quad-1.302327$

$\begin{array}{lllll}1 & -9.712719 & 1.934678 & -0.036575\end{array}$

$\begin{array}{lllll}6 & -6.825729 & 4.486128 & -0.119795\end{array}$

$8 \quad-6.905406 \quad 4.713829 \quad 2.261472$

$\begin{array}{lllll}6 & -9.012310 & -2.450977 & 2.699825\end{array}$

$\begin{array}{lllll}6 & -7.250870 & -1.010069 & 3.809435\end{array}$

$\begin{array}{llll}-5.708817 & 0.226585 & 4.670519\end{array}$

$\begin{array}{lllll}6 & -5.538749 & 3.011480 & 3.239626\end{array}$

$\begin{array}{lllll}6 & -8.945376 & 2.489281 & -2.616355\end{array}$

$\begin{array}{lllll}6 & -7.302015 & 3.915602 & -1.289887\end{array}$

$\begin{array}{lllll}1 & -6.003975 & 5.190463 & -0.142578\end{array}$

$\begin{array}{lllll}6 & -5.647886 & 4.251351 & 2.547777\end{array}$

$1 \quad-8.688358 \quad-3.135009 \quad 3.484724$

$\begin{array}{lllll}6 & -8.420514 & -2.984921 & 1.396395\end{array}$

$8 \quad-6.725348 \quad-2.198089 \quad 4.331444$

$\begin{array}{lllll}6 & -8.355925 & 1.126612 & -2.960819\end{array}$

$\begin{array}{llll}-8.606197 & 3.175252 & -3.393105\end{array}$

$8 \quad-6.701478 \quad 4.260566 \quad-2.508847$

$\begin{array}{lllll}6 & -8.920968 & -2.606462 & 0.148530\end{array}$

$\begin{array}{lllll}6 & -7.349262 & -3.886269 & 1.395377\end{array}$

$\begin{array}{lllll}6 & -5.544838 & -2.622556 & 3.808257\end{array}$

$\begin{array}{lllll}6 & -3.366747 & 2.966408 & 2.760652\end{array}$

$\begin{array}{lllll}6 & -8.928602 & -0.073355 & -2.521639\end{array}$

$\begin{array}{lllll}6 & -7.200704 & 1.034751 & -3.744425\end{array}$

$6 \quad-5.503616 \quad 3.673023 \quad-2.774560$

$\begin{array}{lllll}6 & -3.436259 & 4.263494 & 2.272875\end{array}$

$\begin{array}{lllll}6 & -8.431155 & -3.105137 & -1.063035\end{array}$

$\begin{array}{llll}1 & -9.732759 & -1.889215 & 0.116991\end{array}$

$\begin{array}{lllll}6 & -6.830724 & -4.422203 & 0.223878\end{array}$

$\begin{array}{llll}8 & -6.790136 & -4.278644 & 2.620341\end{array}$ $\begin{array}{llll}6 & -5.579130 & -3.719575 & 2.902672\end{array}$

$\begin{array}{lllll}6 & -8.388342 & -1.323640 & -2.830650\end{array}$

$\begin{array}{lllll}1 & -9.835875 & -0.028797 & -1.931197\end{array}$

$6 \quad-6.608352 \quad-0.181095 \quad-4.062924$

$8 \quad-6.657484 \quad 2.216237 \quad-4.271587$

$\begin{array}{lllll}6 & -5.475335 & 2.624110 & -3.739683\end{array}$

$\begin{array}{lllll}6 & -9.015447 & -2.646390 & -2.395231\end{array}$

$6 \quad-7.382086 \quad-4.031156 \quad-0.991055$

$1 \quad-6.008338 \quad-5.125744 \quad 0.255306$

$6 \quad-3.331046 \quad-2.510716 \quad 3.569524$

$\begin{array}{lllll}6 & -7.211013 & -1.336416 & -3.586758\end{array}$

$1 \quad-5.709663 \quad-0.224801 \quad-4.663318$

$\begin{array}{lllll}6 & -3.290826 & 3.491391 & -2.550212\end{array}$

$1 \quad-8.713230 \quad-3.388114 \quad-3.135363$

$8 \quad-6.870729 \quad-4.614771 \quad-2.162965$

$\begin{array}{lllll}6 & -3.364316 & -3.574998 & 2.678887\end{array}$

$\begin{array}{lllll}8 & -6.694045 & -2.586229 & -3.922349\end{array}$

$\begin{array}{lllll}6 & -3.257388 & 2.508916 & -3.528985\end{array}$

$6 \quad-5.628123 \quad-4.166625 \quad-2.513927$

$6 \quad-5.545164 \quad-3.012297 \quad-3.343726$

$\begin{array}{lllll}6 & -3.398471 & -4.219890 & -2.405514\end{array}$

$6 \quad-3.333661 \quad-3.022429-3.103193$

$6 \begin{array}{llll}6 & -10.550237 & -2.605069 & -2.384659\end{array}$

$\begin{array}{llll}1 & -10.924959 & -2.324062 & -3.372527\end{array}$

$\begin{array}{lllll}1 & -10.955159 & -1.890696 & -1.662604\end{array}$

$\begin{array}{lllll}6 & -10.547620 & -2.455404 & 2.687283\end{array}$

$\begin{array}{lllll}1 & -10.916223 & -3.467677 & 2.502740\end{array}$

$\begin{array}{lllll}1 & -10.971871 & -1.807283 & 1.915495\end{array}$

$\begin{array}{lllll}6 & -10.562540 & 2.671344 & 2.466468\end{array}$

$\begin{array}{lllll}1 & -10.942307 & 2.368171 & 3.445817\end{array}$

$\begin{array}{lllll}1 & -10.972567 & 1.980811 & 1.724304\end{array}$

$\begin{array}{lllll}6 & -10.481207 & 2.500022 & -2.630504\end{array}$

$\begin{array}{lllll}1 & -10.848738 & 3.513361 & -2.449575\end{array}$

$\begin{array}{lllll}1 & -10.921139 & 1.851483 & -1.867987\end{array}$

$\begin{array}{lllll}1 & -10.849781 & 2.164278 & -3.603412\end{array}$

$\begin{array}{lllll}1 & -10.949781 & 3.667128 & 2.236183\end{array}$

$\begin{array}{lllll}1 & -10.932017 & -2.115601 & 3.652716\end{array}$

$1 \quad-10.947895 \quad-3.590491 \quad-2.128946$

$\begin{array}{lllll}6 & -1.907029 & -2.049808 & 3.704433\end{array}$

$6 \quad-1.966462 \quad-3.833481 \quad 2.192508$

$6 \quad-1.986186 \quad-4.664520 \quad-2.119326$

$6 \quad-1.898798 \quad-2.601572 \quad-3.200741$

$\begin{array}{llll}6 & -1.950292 & 2.484792 & 2.627423\end{array}$

$\begin{array}{lllll}6 & -2.037452 & 4.716986 & 1.948808\end{array}$

$6 \quad-1.890428 \quad 3.719120 \quad-2.056306$

$\begin{array}{lllll}6 & -1.827275 & 2.090739 & -3.730908\end{array}$

$7 \quad-1.178633 \quad-3.665816 \quad-2.701362$

$\begin{array}{lllll}7 & -1.185062 & -2.888591 & 2.848393\end{array}$

$\begin{array}{llll}7 & -1.236140 & 3.591688 & 2.220125\end{array}$

$\begin{array}{lllll}7 & -1.099507 & 2.874019 & -2.820728\end{array}$

$8 \quad-1.535727 \quad 4.465272 \quad-1.163501$

$8 \quad-1.369888 \quad 1.264211 \quad-4.484552$

$8 \quad-1.482249-1.524270 \quad-3.590985$

$8 \quad-1.587688 \quad-5.642093 \quad-1.535812$

$\begin{array}{lllll}8 & -1.598084 & -4.663288 & 1.386418\end{array}$

$\begin{array}{lllll}8 & -1.548164 & 1.346259 & 2.797837\end{array}$

$8 \quad-1.451270 \quad-1.159368 \quad 4.385254$

$\begin{array}{llll}8 & -1.648556 & 5.789753 & 1.557841\end{array}$ $\begin{array}{llll}1 & -0.086015 & 2.767172 & -2.744194\end{array}$

$\begin{array}{llll}-0.220903 & 3.631457 & 2.095720\end{array}$

$\begin{array}{llll}-0.173490 & -2.777980 & 2.744576\end{array}$

$\begin{array}{llll}-0.170454 & -3.642141 & -2.521348\end{array}$

$\begin{array}{llll}-4.421476 & -2.009311 & 4.150967\end{array}$

$\begin{array}{lll}-4.488828 & -4.200244 & 2.323886\end{array}$

$\begin{array}{lll}-4.412861 & 2.318302 & 3.282991\end{array}$

$\begin{array}{llll}-4.593051 & 4.914820 & 2.107554\end{array}$

$\begin{array}{llll}-4.554913 & -4.799537 & -2.064966\end{array}$

$\begin{array}{llll}-4.402163 & -2.396752 & -3.600798\end{array}$

$\begin{array}{llll}-4.412916 & 4.095552 & -2.151655\end{array}$

$\begin{array}{llll}-4.351840 & 2.041824 & -4.132527\end{array}$

$\begin{array}{llll}8.945543 & -0.168314 & -3.517336\end{array}$

$\begin{array}{llll}8.356105 & -1.412463 & -2.860767\end{array}$

$\begin{array}{llll}8.372781 & 1.112409 & -2.909204\end{array}$

$\begin{array}{llll}8.611554 & -0.185541 & -4.555497\end{array}$

$\begin{array}{llll}8.895289 & -1.952183 & -1.685544\end{array}$

$\begin{array}{llll}7.242005 & -2.062217 & -3.406906\end{array}$

$\begin{array}{llll}8.938612 & 1.754146 & -1.803873\end{array}$

$\begin{array}{llll}7.240678 & 1.713114 & -3.471924\end{array}$

$\begin{array}{llll}8.363772 & -3.074262 & -1.045381\end{array}$

$\begin{array}{llll}9.767566 & -1.474762 & -1.255099\end{array}$

$\begin{array}{llll}6.659054 & -3.167629 & -2.796479\end{array}$

$\begin{array}{llll}6.698648 & -1.612815 & -4.621084\end{array}$

$\begin{array}{llll}8.446442 & 2.960568 & -1.290997\end{array}$

$\begin{array}{llll}9.798059 & 1.301199 & -1.323352\end{array}$

$\begin{array}{llll}6.737346 & 2.927768 & -3.033671\end{array}$

$\begin{array}{llll}6.631681 & 1.072430 & -4.552773\end{array}$

$\begin{array}{llll}8.970095 & -3.676812 & 0.222621\end{array}$

$\begin{array}{llll}7.218954 & -3.641383 & -1.618980\end{array}$

$\begin{array}{llll}5.788382 & -3.643088 & -3.228822\end{array}$

$\begin{array}{llll}5.485330 & -0.999618 & -4.488064\end{array}$

$\begin{array}{llll}9.023340 & 3.609438 & -0.037395\end{array}$

$\begin{array}{llll}7.360339 & 3.541462 & -1.955481\end{array}$

$\begin{array}{llll}5.877236 & 3.379023 & -3.509919\end{array}$

$\begin{array}{llll}5.468302 & 0.412678 & -4.317889\end{array}$

$\begin{array}{llll}8.672148 & -4.725946 & 0.235593\end{array}$

$\begin{array}{llll}8.353131 & -3.037893 & 1.463258\end{array}$

$\begin{array}{llll}6.670422 & -4.763804 & -0.997443\end{array}$

$\begin{array}{llll}8.344083 & 3.033400 & 1.204364\end{array}$

$\begin{array}{llll}8.746695 & 4.663412 & -0.078164\end{array}$

$\begin{array}{llll}6.898648 & 4.794472 & -1.531825\end{array}$

$\begin{array}{llll}8.834346 & -1.830299 & 1.987349\end{array}$

$\begin{array}{llll}7.251980 & -3.617651 & 2.105750\end{array}$

$\begin{array}{llll}5.497908 & -4.628712 & -0.326170\end{array}$

$\begin{array}{llll}3.271427 & -1.034858 & -4.225944\end{array}$

$\begin{array}{llll}8.810521 & 1.883045 & 1.845373\end{array}$

$\begin{array}{llll}7.207936 & 3.644926 & 1.750563\end{array}$

$\begin{array}{llll}5.655948 & 4.797553 & -0.973251\end{array}$

$\begin{array}{llll}3.279623 & 0.317541 & -3.912800\end{array}$

$\begin{array}{llll}8.242192 & -1.181313 & 3.074164\end{array}$

$\begin{array}{llll}9.701193 & -1.375773 & 1.521805\end{array}$

$\begin{array}{lll}6.610550 & -2.999433 & 3.171837\end{array}$

$\begin{array}{llll}6.767321 & -4.864139 & 1.678473\end{array}$

$\begin{array}{llll}5.538537 & -4.801262 & 1.085547\end{array}$

$\begin{array}{llll}8.220401 & 1.351947 & 2.997895\end{array}$

$\begin{array}{llll}9.680050 & 1.381997 & 1.437339\end{array}$

$\begin{array}{llll}6.586011 & 3.165754 & 2.896447\end{array}$ 
$\begin{array}{llll}8 & 6.733203 & 4.813363 & 1.148810\end{array}$

$\begin{array}{lllll}6 & 5.569800 & 4.748084 & 0.445711\end{array}$

$\begin{array}{lllll}6 & 8.784265 & 0.110617 & 3.684149\end{array}$

$\begin{array}{lllll}6 & 7.100349 & -1.781950 & 3.615867\end{array}$

$\begin{array}{lllll}1 & 5.738656 & -3.448285 & 3.628449\end{array}$

$\begin{array}{lllll}6 & 3.284717 & -4.407716 & -0.208946\end{array}$

$\begin{array}{lllll}6 & 7.103874 & 2.027904 & 3.507202\end{array}$

$\begin{array}{lllll}1 & 5.718292 & 3.666426 & 3.306708\end{array}$

$\begin{array}{lllll}6 & 3.429025 & 4.793950 & -1.099921\end{array}$

$\begin{array}{llll}1 & 8.413094 & 0.138564 & 4.709327\end{array}$

$\begin{array}{lllll}8 & 6.452690 & -1.122523 & 4.662693\end{array}$

$\begin{array}{lllll}6 & 3.310639 & -4.691484 & 1.148092\end{array}$

$\begin{array}{lllll}8 & 6.493688 & 1.574552 & 4.688909\end{array}$

$\begin{array}{lllll}6 & 3.347969 & 4.699171 & 0.282407\end{array}$

$\begin{array}{lllll}6 & 5.303834 & -0.469643 & 4.345949\end{array}$

$\begin{array}{lllll}6 & 5.298577 & 0.945508 & 4.483078\end{array}$

$\begin{array}{llll}6 & 3.151820 & -0.403546 & 3.775594\end{array}$

$\begin{array}{lllll}6 & 3.109489 & 0.955558 & 4.053546\end{array}$

$\begin{array}{llll}6 & 10.319090 & 0.122017 & 3.751146\end{array}$

$\begin{array}{lllll}1 & 10.665544 & 1.031489 & 4.248690\end{array}$

$\begin{array}{lllll}1 & 10.791067 & 0.085130 & 2.765452\end{array}$

$\begin{array}{lllll}6 & 10.504449 & -3.631523 & 0.226827\end{array}$

$\begin{array}{lllll}1 & 10.889975 & -4.129911 & 1.119945\end{array}$

$\begin{array}{lllll}1 & 10.902721 & -2.613467 & 0.220949\end{array}$

$\begin{array}{lllll}6 & 10.481365 & -0.174853 & -3.528285\end{array}$

$\begin{array}{lllll}1 & 10.848126 & -1.074594 & -4.029082\end{array}$

$\begin{array}{lllll}1 & 10.916860 & -0.155161 & -2.525696\end{array}$

$\begin{array}{lllll}6 & 10.554730 & 3.534132 & 0.032145\end{array}$

$\begin{array}{lllll}1 & 10.992057 & 4.007838 & -0.850553\end{array}$

$\begin{array}{lllll}1 & 10.932470 & 2.509085 & 0.076243\end{array}$

$\begin{array}{lllll}1 & 10.916130 & 4.055968 & 0.922028\end{array}$

$\begin{array}{lllll}1 & 10.855344 & 0.700211 & -4.066029\end{array}$

$\begin{array}{lllll}1 & 10.897755 & -4.143946 & -0.654975\end{array}$

$\begin{array}{lllll}1 & 10.674523 & -0.742496 & 4.317860\end{array}$

$\begin{array}{lllll}6 & 1.862490 & -4.170099 & -0.621990\end{array}$

$\begin{array}{lllll}6 & 1.887634 & -4.728863 & 1.643891\end{array}$

$\begin{array}{llll}6 & 1.798405 & -0.835333 & 3.291684\end{array}$

$\begin{array}{llll}6 & 1.697830 & 1.431877 & 3.850289\end{array}$

$\begin{array}{lllll}6 & 1.853533 & -1.528032 & -4.164748\end{array}$

$\begin{array}{lllll}6 & 1.885043 & 0.724952 & -3.526244\end{array}$

$\begin{array}{lllll}6 & 2.026285 & 4.797567 & -1.653545\end{array}$

$\begin{array}{lllll}6 & 1.900977 & 4.595940 & 0.666493\end{array}$

$\begin{array}{lllll}7 & 1.006878 & 0.293973 & 3.398210\end{array}$

$\begin{array}{lllll}7 & 1.111678 & -4.426256 & 0.506293\end{array}$

$\begin{array}{lllll}7 & 1.114449 & -0.408307 & -3.735012\end{array}$

$\begin{array}{lllll}7 & 1.204177 & 4.716695 & -0.519991\end{array}$

$\begin{array}{lllll}8 & 1.649345 & 4.844232 & -2.800595\end{array}$

$\begin{array}{lllll}8 & 1.448525 & 4.398207 & 1.779052\end{array}$

$\begin{array}{lllll}8 & 1.226217 & 2.530350 & 4.020226\end{array}$

$\begin{array}{lllll}8 & 1.482130 & -1.932297 & 2.867062\end{array}$

$\begin{array}{lllll}8 & 1.462957 & -4.962568 & 2.748384\end{array}$

$\begin{array}{lllll}8 & 1.410230 & -2.620571 & -4.421924\end{array}$

$\begin{array}{lllll}8 & 1.478092 & -3.801588 & -1.719101\end{array}$

$\begin{array}{lllll}8 & 1.524620 & 1.798326 & -3.079728\end{array}$

$\begin{array}{lllll}1 & 0.183367 & 4.641858 & -0.603297\end{array}$

$\begin{array}{lllll}1 & 0.096117 & -0.456084 & -3.677271\end{array}$

$\begin{array}{lllll}1 & 0.086692 & -4.422815 & 0.552336\end{array}$

$\begin{array}{llll}1 & 0.001856 & 0.338764 & 3.222996\end{array}$ $\begin{array}{lllll}7 & 4.374217 & -4.382580 & -0.980840\end{array}$

$\begin{array}{llll}7 & 4.446284 & -4.864261 & 1.831517\end{array}$

$7 \quad 4.382477 \quad-1.731019 \quad-4.482000$

$\begin{array}{lllll}7 & 4.375877 & 1.076829 & -3.972475\end{array}$

$7 \quad 4.244396 \quad-1.150525 \quad 3.935200$

$\begin{array}{llll}7 & 4.192667 & 1.666522 & 4.375452\end{array}$

$\begin{array}{lllll}7 & 4.589109 & 4.831807 & -1.758521\end{array}$

$\begin{array}{lllll}7 & 4.413167 & 4.674315 & 1.086579\end{array}$

$\begin{array}{lllll}6 & 0.369844 & 0.797049 & -0.030026\end{array}$

$\begin{array}{lllll}8 & 0.013846 & 1.961372 & 0.051925\end{array}$

$\begin{array}{lllll}6 & -1.974909 & 0.168034 & -0.728480\end{array}$

$1 \quad-2.085083 \quad-0.287139 \quad-1.716474$

$\begin{array}{lllll}1 & -1.932560 & 1.248167 & -0.842622\end{array}$

$\begin{array}{lllll}6 & -3.156780 & -0.214975 & 0.169636\end{array}$

$\begin{array}{llll}1 & -2.999545 & 0.175212 & 1.180254\end{array}$

$\begin{array}{lllll}1 & -3.227873 & -1.302456 & 0.246155\end{array}$

$\begin{array}{lllll}6 & -4.462101 & 0.335424 & -0.427683\end{array}$

$\begin{array}{lllll}1 & -4.530666 & 0.040081 & -1.483968\end{array}$

$\begin{array}{lllll}1 & -4.431835 & 1.434047 & -0.420039\end{array}$

$\begin{array}{llll}6 & -5.708713 & -0.157023 & 0.313084\end{array}$

$\begin{array}{llll}1 & -5.666516 & 0.103167 & 1.375231\end{array}$

$1 \quad-6.620004 \quad 0.281435 \quad-0.104826$

$\begin{array}{lllll}1 & -5.803056 & -1.246827 & 0.239525\end{array}$

$\begin{array}{lllll}6 & 1.806304 & 0.407233 & 0.023088\end{array}$

$\begin{array}{lllll}6 & 2.364570 & -0.688906 & -0.651566\end{array}$

$\begin{array}{lllll}6 & 2.666686 & 1.292008 & 0.692637\end{array}$

$\begin{array}{lllll}6 & 3.743907 & -0.880879 & -0.659294\end{array}$

$1 \quad 1.738061 \quad-1.382870 \quad-1.191620$

$\begin{array}{llll}6 & 4.039249 & 1.077782 & 0.703198\end{array}$

$\begin{array}{llll}1 & 2.243380 & 2.138043 & 1.217329\end{array}$

$\begin{array}{lllll}6 & 4.604943 & -0.010062 & 0.022018\end{array}$

$1 \quad 4.154915 \quad-1.721446-1.210383$

$\begin{array}{lllll}1 & 4.681775 & 1.766497 & 1.243437\end{array}$

$\begin{array}{lllll}6 & 6.089546 & -0.251187 & 0.048467\end{array}$

$\begin{array}{llll}1 & 6.649688 & 0.686442 & 0.091160\end{array}$

$\begin{array}{lllll}1 & 6.368630 & -0.835577 & 0.931821\end{array}$

$1 \quad 6.420646 \quad-0.809879 \quad-0.828362$

$7 \quad-0.628774 \quad-0.198146 \quad-0.238517$

$\begin{array}{lllll}7 & -0.277631 & -1.473132 & 0.013484\end{array}$

$\begin{array}{lllll}8 & -1.146577 & -2.296927 & -0.241301\end{array}$

\section{2a'-TS@12}

$\begin{array}{lllll}6 & 9.031679 & -2.689464 & -2.477295\end{array}$

$6 \quad 8.428830 \quad-3.109856 \quad-1.137576$

$6 \quad 8.447415 \quad-1.357966 \quad-2.934675$

$1 \quad 8.708913 \quad-3.434821 \quad-3.204710$

$\begin{array}{lllll}6 & 8.930194 & -2.644736 & 0.079949\end{array}$

$6 \quad 7.344433 \quad-3.993018-1.073580$

$\begin{array}{llll}9.007543 & -0.124350 & -2.579784\end{array}$

$\begin{array}{llll}7.293120 & -1.333586 & -3.724618\end{array}$

$\begin{array}{lllll}6 & 8.431895 & -3.047507 & 1.323994\end{array}$

$\begin{array}{llll}9.749720 & -1.935878 & 0.061009\end{array}$

$\begin{array}{llll}6.822234 & -4.441540 & 0.131681\end{array}$

$\begin{array}{llll}6.782379 & -4.460174 & -2.269264\end{array}$

$\begin{array}{llll}8.443801 & 1.095837 & -2.960894\end{array}$

$\begin{array}{llll}9.919793 & -0.117591 & -1.995239\end{array}$

$6 \quad \begin{array}{llll}6.672990 & -0.149861 & -4.103708\end{array}$
6

6

$\begin{array}{lll}5.992451 & -5.136532 & 0.149911\end{array}$

$\begin{array}{llll}5.585656 & -3.907812 & -2.613350\end{array}$

$\begin{array}{llll}9.061651 & 2.456520 & -2.638473\end{array}$

$\begin{array}{llll}7.250833 & 1.040485 & -3.690629\end{array}$

$\begin{array}{llll}5.766346 & -0.158435 & -4.693113\end{array}$

$\begin{array}{llll}5.576194 & -2.923900 & -3.642077\end{array}$

$\begin{array}{llll}8.702997 & -3.177264 & 3.412564\end{array}$

$\begin{array}{llll}8.399738 & -1.137550 & 2.958021\end{array}$

$\begin{array}{llll}6.855615 & -4.461815 & 2.526616\end{array}$

$\begin{array}{llll}8.475103 & 3.027485 & -1.351686\end{array}$

$\begin{array}{llll}8.754209 & 3.128820 & -3.440663\end{array}$

$\begin{array}{llll}6.690769 & 2.256528 & -4.072968\end{array}$

$\begin{array}{llll}8.951030 & 0.082837 & 2.560542\end{array}$

$\begin{array}{llll}7.222870 & -1.083480 & 3.712204\end{array}$

$\begin{array}{llll}5.616223 & -3.966041 & 2.819858\end{array}$

$\begin{array}{llll}3.370153 & -3.713688 & -2.442730\end{array}$

$\begin{array}{llll}8.943356 & 2.608998 & -0.100191\end{array}$

$\begin{array}{llll}7.443247 & 3.974313 & -1.359114\end{array}$

$\begin{array}{llll}5.578759 & 2.716127 & -3.450452\end{array}$

$\begin{array}{llll}3.356668 & -2.780470 & -3.470569\end{array}$

$\begin{array}{llll}8.388842 & 1.316408 & 2.911917\end{array}$

$\begin{array}{llll}9.857134 & 0.076730 & 1.966561\end{array}$

$\begin{array}{llll}6.631554 & 0.107829 & 4.105815\end{array}$

$\begin{array}{llll}6.685807 & -2.302524 & 4.125932\end{array}$

$\begin{array}{llll}5.543661 & -2.743959 & 3.548354\end{array}$

$\begin{array}{lll}8.419592 & 3.069675 & 1.109095\end{array}$

$\begin{array}{llll}9.752304 & 1.888226 & -0.069998\end{array}$

$\begin{array}{llll}6.863724 & 4.437956 & -0.182096\end{array}$

$\begin{array}{llll}6.940641 & 4.476936 & -2.574321\end{array}$

$\begin{array}{llll}5.684858 & 3.994309 & -2.831949\end{array}$

$\begin{array}{llll}8.986725 & 2.643636 & 2.461274\end{array}$

$\begin{array}{llll}7.233680 & 1.292974 & 3.701740\end{array}$

$\begin{array}{llll}5.731628 & 0.116024 & 4.705666\end{array}$

$\begin{array}{llll}3.391972 & -3.957857 & 2.630316\end{array}$

$\begin{array}{llll}7.344318 & 3.962317 & 1.028163\end{array}$

$\begin{array}{llll}6.042425 & 5.142446 & -0.212461\end{array}$

$\begin{array}{llll}3.402802 & 2.702047 & -2.988601\end{array}$

$\begin{array}{llll}8.652183 & 3.391230 & 3.181058\end{array}$

$\begin{array}{llll}6.697567 & 2.511801 & 4.142084\end{array}$

$3.346072-2.698260 \quad 3.209426$

$\begin{array}{llll}6.750748 & 4.404586 & 2.217623\end{array}$

$\begin{array}{llll}3.471620 & 4.022283 & -2.568168\end{array}$

$\begin{array}{llll}5.515048 & 2.885763 & 3.582661\end{array}$

$\begin{array}{llll}5.549544 & 3.846630 & 2.531084\end{array}$

$\begin{array}{llll}3.294489 & 2.772504 & 3.387229\end{array}$

$\begin{array}{llll}3.336257 & 3.658386 & 2.320526\end{array}$

$\begin{array}{lll}10.522487 & 2.646022 & 2.473608\end{array}$

$\begin{array}{llll}10.896095 & 3.639565 & 2.212915\end{array}$

$\begin{array}{llll}10.957691 & 1.936317 & 1.764678\end{array}$

$\begin{array}{llll}10.550096 & -2.468841 & 2.614002\end{array}$

$10.924001-2.122081 \quad 3.581027$

$10.964607-1.811269 \quad 1.844979$

$10.566901-2.699827-2.455145$

$10.930145 \quad-3.695088-2.186479$

$\begin{array}{llll}10.989993 & -1.992106 & -1.736963\end{array}$ 
$\begin{array}{llll}6 & 10.596664 & 2.427553 & -2.623586\end{array}$

$\begin{array}{lllll}1 & 10.975689 & 2.059419 & -3.580687\end{array}$

$1 \quad 11.005520 \quad 1.786703 \quad-1.837530$

$1 \quad 10.985932 \quad 3.435562 \quad-2.459450$

$1 \quad 10.957630 \quad-2.441650 \quad-3.443018$

$\begin{array}{lllll}1 & 10.939054 & -3.473620 & 2.430322\end{array}$

$\begin{array}{lllll}1 & 10.890074 & 2.386119 & 3.469860\end{array}$

$\begin{array}{lllll}6 & 1.977392 & -4.376373 & 2.318344\end{array}$

$\begin{array}{lllll}6 & 1.930044 & -2.209803 & 3.195179\end{array}$

$\begin{array}{lllll}6 & 1.857675 & 2.394908 & 3.629605\end{array}$

$\begin{array}{lllll}6 & 1.938236 & 3.853709 & 1.806522\end{array}$

$\begin{array}{lllll}6 & 1.963995 & -3.895711 & -1.949262\end{array}$

$\begin{array}{lllll}6 & 1.932824 & -2.350665 & -3.701779\end{array}$

$\begin{array}{lllll}6 & 1.984613 & 2.229665 & -2.845647\end{array}$

$\begin{array}{lllll}6 & 2.071984 & 4.494163 & -2.275105\end{array}$

$\begin{array}{lllll}7 & 1.139797 & 3.092082 & 2.644441\end{array}$

$\begin{array}{lllll}7 & 1.191962 & -3.272447 & 2.709579\end{array}$

$\begin{array}{lllll}7 & 1.191265 & -3.087182 & -2.765763\end{array}$

$\begin{array}{lllll}7 & 1.270155 & 3.355180 & -2.486698\end{array}$

$\begin{array}{lllll}8 & 1.585149 & 1.084987 & -2.974241\end{array}$

$8 \quad 1.684835 \quad 5.588061 \quad-1.947330$

$\begin{array}{lllll}8 & 1.594351 & 4.520515 & 0.849127\end{array}$

$\begin{array}{lllll}8 & 1.389068 & 1.657260 & 4.464174\end{array}$

$\begin{array}{lllll}8 & 1.547873 & -1.093516 & 3.498258\end{array}$

$\begin{array}{lllll}8 & 1.586558 & -4.579410 & -1.014918\end{array}$

$\begin{array}{lllll}8 & 1.562694 & -5.407962 & 1.850807\end{array}$

$8 \quad 1.485068 \quad-1.554110 \quad-4.492276$

$\begin{array}{lllll}1 & 0.255093 & 3.406942 & -2.377850\end{array}$

$\begin{array}{lllll}1 & 0.187594 & -2.919416 & -2.636862\end{array}$

$\begin{array}{llll}0.177151 & -3.280048 & 2.579529\end{array}$

$\begin{array}{llll}0.125745 & 2.985423 & 2.568808\end{array}$

$\begin{array}{llll}4.538715 & -4.602908 & 2.388385\end{array}$

$\begin{array}{llll}4.416047 & -2.068677 & 3.698331\end{array}$

$\begin{array}{llll}4.482510 & -4.297577 & -1.992011\end{array}$

$\begin{array}{llll}4.462464 & -2.359393 & -4.086195\end{array}$

$\begin{array}{llll}4.387060 & 2.352168 & 4.028946\end{array}$

$\begin{array}{llll}4.462199 & 4.217398 & 1.871006\end{array}$

$\begin{array}{llll}4.451546 & 2.023922 & -3.465276\end{array}$

$\begin{array}{llll}4.628050 & 4.681244 & -2.433869\end{array}$

$\begin{array}{llll}-8.972875 & 3.632165 & -0.196230\end{array}$

$\begin{array}{llll}-8.395754 & 3.069220 & 1.098110\end{array}$

$\begin{array}{llll}-8.298798 & 2.965765 & -1.393994\end{array}$

$\begin{array}{llll}-8.691074 & 4.684986 & -0.230292\end{array}$

$\begin{array}{llll}-8.894878 & 1.905637 & 1.696120\end{array}$

$\begin{array}{llll}-7.304013 & 3.689254 & 1.716891\end{array}$

$\begin{array}{llll}-8.772683 & 1.774368 & -1.948455\end{array}$

$\begin{array}{llll}-7.158822 & 3.529714 & -1.982450\end{array}$

$\begin{array}{llll}-8.333697 & 1.342860 & 2.845874\end{array}$

$\begin{array}{llll}-9.756189 & 1.421937 & 1.250055\end{array}$

$\begin{array}{lll}-6.687084 & 3.153187 & 2.839128\end{array}$

$\begin{array}{llll}-6.838439 & 4.906522 & 1.205511\end{array}$

$\begin{array}{llll}-8.183144 & 1.153062 & -3.055221\end{array}$

$\begin{array}{llll}-9.647618 & 1.312829 & -1.506173\end{array}$

$\begin{array}{llll}-6.537002 & 2.959780 & -3.086528\end{array}$

$\begin{array}{llll}-6.683107 & 4.740260 & -1.473667\end{array}$

$\begin{array}{llll}-8.913378 & 0.111105 & 3.543832\end{array}$

$\begin{array}{llll}-7.200653 & 1.978483 & 3.366602\end{array}$

$\begin{array}{lll}-5.824034 & 3.632702 & 3.281382\end{array}$
$6 \quad-5.597516 \quad 4.879616 \quad 0.641670$

$\begin{array}{llll}-8.758487 & -0.127917 & -3.652838\end{array}$

$6 \quad-7.059143 \quad 1.779866 \quad-3.607865$

$\begin{array}{llll}-5.663861 & 3.421024 & -3.530359\end{array}$

$6 \quad-5.516011 \quad 4.737466 \quad-0.770970$

$\begin{array}{llll}-8.568325 & 0.159380 & 4.577326\end{array}$

$\begin{array}{llll}6 & -8.346275 & -1.182381 & 2.966547\end{array}$

$8 \quad-6.594663 \quad 1.412892 \quad 4.491222$

$\begin{array}{lllll}6 & -8.244719 & -1.383881 & -2.949872\end{array}$

$\begin{array}{llll}-8.379064 & -0.179800 & -4.674109\end{array}$

$\begin{array}{llll}-6.442547 & 1.224714 & -4.742657\end{array}$

$\begin{array}{llll}-8.915842 & -1.803698 & 1.846784\end{array}$

$\begin{array}{lll}-7.222380 & -1.797954 & 3.532724\end{array}$

$\begin{array}{llll}-5.429921 & 0.744527 & 4.289149\end{array}$

$\begin{array}{llll}-3.369362 & 4.930356 & 0.755890\end{array}$

$\begin{array}{llll}-8.856309 & -1.945821 & -1.826355\end{array}$

$\begin{array}{llll}-7.113748 & -2.044263 & -3.443549\end{array}$

$\begin{array}{llll}-5.261168 & 0.597382 & -4.468033\end{array}$

$\begin{array}{llll}-3.291899 & 4.748024 & -0.618121\end{array}$

$\begin{array}{llll}-8.404477 & -2.972648 & 1.278495\end{array}$

$\begin{array}{llll}-9.795326 & -1.354090 & 1.401396\end{array}$

$\begin{array}{lll}-6.661260 & -2.951406 & 2.994362\end{array}$

$\begin{array}{llll}-6.651960 & -1.265603 & 4.699133\end{array}$

$\begin{array}{llll}-5.442387 & -0.660278 & 4.510077\end{array}$

$\begin{array}{llll}-8.408953 & -3.129933 & -1.223272\end{array}$

$\begin{array}{llll}-9.709148 & -1.436466 & -1.393221\end{array}$

$\begin{array}{llll}-6.664449 & -3.247831 & -2.926666\end{array}$

$\begin{array}{llll}-6.436726 & -1.465281 & -4.519304\end{array}$

$\begin{array}{llll}-5.285455 & -0.804540 & -4.227626\end{array}$

$\begin{array}{llll}-9.035001 & -3.658580 & 0.064687\end{array}$

$\begin{array}{llll}-7.251547 & -3.506169 & 1.868457\end{array}$

$\begin{array}{llll}-5.784399 & -3.400135 & 3.442429\end{array}$

$\begin{array}{llll}-3.244457 & 0.641067 & 3.871233\end{array}$

$\begin{array}{llll}-7.329100 & -3.783682 & -1.829621\end{array}$

$\begin{array}{llll}-5.805337 & -3.749476 & -3.351661\end{array}$

$\begin{array}{llll}-3.081782 & 0.616442 & -3.996211\end{array}$

$\begin{array}{llll}-8.762226 & -4.712582 & 0.131415\end{array}$

$\begin{array}{llll}-6.733916 & -4.680679 & 1.322576\end{array}$

$\begin{array}{lll}-3.231850 & -0.703532 & 4.216512\end{array}$

$\begin{array}{llll}-6.881785 & -5.018209 & -1.330343\end{array}$

$\begin{array}{llll}-3.141509 & -0.719036 & -3.624994\end{array}$

$\begin{array}{llll}-5.570606 & -4.635935 & 0.626077\end{array}$

$\begin{array}{llll}-5.641983 & -4.949726 & -0.760166\end{array}$

$\begin{array}{llll}-3.354877 & -4.489320 & 0.451275\end{array}$

$\begin{array}{llll}-3.413931 & -4.918416 & -0.866280\end{array}$

$6 \quad-10.567566 \quad-3.576394 \quad 0.062183$

$1-10.969624 \quad-4.137766 \quad-0.785068$

$1 \quad-10.941564 \quad-2.551996 \quad-0.015978$

$\begin{array}{lllll}6 & -10.448959 & 0.125929 & 3.571164\end{array}$

$\begin{array}{llll}1 & -10.822489 & -0.736323 & 4.129710\end{array}$

$\begin{array}{lllll}1 & -10.895617 & 0.091669 & 2.573810\end{array}$

$\begin{array}{lllll}6 & -10.504709 & 3.560186 & -0.256839\end{array}$

$\begin{array}{lllll}1 & -10.937186 & 4.099517 & 0.589844\end{array}$

$\begin{array}{lllll}1 & -10.887840 & 2.536778 & -0.224783\end{array}$

$\begin{array}{lllll}6 & -10.292588 & -0.098587 & -3.734938\end{array}$

$\begin{array}{lllll}1 & -10.620815 & 0.777718 & -4.299842\end{array}$

$\begin{array}{lllll}1 & -10.773020 & -0.057394 & -2.753512\end{array}$

$1 \quad-10.656535 \quad-0.996398 \quad-4.241252$
$1 \quad-10.865743 \quad 4.017514 \quad-1.181710$

$\begin{array}{llll}-10.804712 & 1.037386 & 4.058376\end{array}$

$\begin{array}{llll}-10.968660 & -4.003952 & 0.984800\end{array}$

$\begin{array}{llll}-1.856595 & 1.041796 & 3.457251\end{array}$

$\begin{array}{llll}-1.814334 & -1.196897 & 4.136351\end{array}$

$\begin{array}{llll}-1.919257 & -4.235238 & 0.807208\end{array}$

$\begin{array}{llll}-2.003555 & -5.045179 & -1.381880\end{array}$

$\begin{array}{llll}-1.963346 & 5.005316 & 1.298670\end{array}$

$\begin{array}{llll}-1.842778 & 4.649266 & -1.002251\end{array}$

$\begin{array}{llll}-1.674217 & 1.098295 & -3.793679\end{array}$

$\begin{array}{llll}-1.796418 & -1.126069 & -3.095474\end{array}$

$\begin{array}{llll}-1.197752 & -4.658480 & -0.292560\end{array}$

$\begin{array}{llll}-1.082597 & -0.087509 & 3.678458\end{array}$

$\begin{array}{llll}-1.145022 & 4.893487 & 0.166459\end{array}$

$\begin{array}{llll}-0.995687 & -0.012000 & -3.257700\end{array}$

$\begin{array}{llll}-1.197920 & 2.184779 & -4.016853\end{array}$

$\begin{array}{llll}-1.488355 & -2.203330 & -2.611270\end{array}$

$\begin{array}{llll}-1.607177 & -5.394521 & -2.466152\end{array}$

$\begin{array}{llll}-1.501118 & -3.726306 & 1.832768\end{array}$

$\begin{array}{llll}-1.364403 & -2.288059 & 4.394430\end{array}$

$\begin{array}{llll}-1.585128 & 5.122456 & 2.440647\end{array}$

$\begin{array}{lll}-1.504737 & 2.105963 & 2.985191\end{array}$

$\begin{array}{llll}-1.390327 & 4.363996 & -2.094403\end{array}$

$\begin{array}{llll}0.000973 & 0.060555 & -3.041686\end{array}$

$\begin{array}{llll}-0.128799 & 4.784474 & 0.254986\end{array}$

$\begin{array}{llll}-0.066036 & -0.148784 & 3.586202\end{array}$

$\begin{array}{llll}-0.174915 & -4.630449 & -0.370884\end{array}$

$\begin{array}{llll}-4.340424 & 1.399679 & 3.917973\end{array}$

$\begin{array}{llll}-4.338700 & -1.391769 & 4.509311\end{array}$

$\begin{array}{llll}-4.527808 & 4.987294 & 1.416694\end{array}$

$\begin{array}{llll}-4.360539 & 4.646503 & -1.412067\end{array}$

$\begin{array}{llll}-4.429230 & -4.352526 & 1.233492\end{array}$

$\begin{array}{llll}-4.566313 & -5.126737 & -1.510845\end{array}$

$\begin{array}{llll}-4.149521 & 1.312628 & -4.390674\end{array}$

$\begin{array}{llll}-4.240066 & -1.465034 & -3.750808\end{array}$

$\begin{array}{llll}-0.500564 & 0.966371 & -0.011155\end{array}$

$\begin{array}{llll}-0.223717 & 2.150459 & -0.185900\end{array}$

$\begin{array}{llll}0.527976 & 0.041697 & 0.115807\end{array}$

$\begin{array}{llll}0.259821 & -1.305187 & 0.674948\end{array}$

$\begin{array}{llll}0.358970 & -2.141592 & -0.162458\end{array}$

$\begin{array}{llll}1.873246 & 0.488945 & 0.522182\end{array}$

$\begin{array}{llll}1.962108 & 0.397630 & 1.611677\end{array}$

$\begin{array}{llll}1.898141 & 1.546731 & 0.257021\end{array}$

$\begin{array}{llll}3.014260 & -0.269316 & -0.157964\end{array}$

$\begin{array}{llll}2.929337 & -0.154049 & -1.243824\end{array}$

$\begin{array}{llll}2.939517 & -1.343595 & 0.057643\end{array}$

$\begin{array}{llll}4.377306 & 0.241566 & 0.334960\end{array}$

$\begin{array}{llll}4.431751 & 0.135488 & 1.427165\end{array}$

$\begin{array}{llll}4.456306 & 1.318209 & 0.127206\end{array}$

$\begin{array}{llll}5.560163 & -0.486819 & -0.309967\end{array}$

$\begin{array}{llll}5.554194 & -0.357347 & -1.397056\end{array}$

$\begin{array}{llll}6.515147 & -0.106904 & 0.066065\end{array}$

$\begin{array}{llll}5.527968 & -1.562278 & -0.099698\end{array}$

$\begin{array}{llll}-1.928759 & 0.513639 & -0.023441\end{array}$

$\begin{array}{llll}-2.439488 & -0.580238 & 0.687343\end{array}$

$\begin{array}{llll}-2.826821 & 1.327254 & -0.730774\end{array}$

$\begin{array}{llll}-3.808487 & -0.840268 & 0.699018\end{array}$

$\begin{array}{llll}-1.795392 & -1.222804 & 1.272537\end{array}$ 
$\begin{array}{llll}6 & -4.188463 & 1.051579 & -0.731544\end{array}$

$\begin{array}{lllll}1 & -2.439363 & 2.167525 & -1.291190\end{array}$

$6 \quad-4.705946 \quad-0.033482-0.011324$

$1 \quad-4.179696 \quad-1.678465 \quad 1.280992$

$\begin{array}{lllll}1 & -4.860791 & 1.690702 & -1.294811\end{array}$

$\begin{array}{lllll}6 & -6.176232 & -0.343779 & -0.022136\end{array}$

$\begin{array}{lllll}1 & -6.503012 & -0.749972 & 0.935983\end{array}$

$\begin{array}{lllll}1 & -6.775355 & 0.542712 & -0.241680\end{array}$

$\begin{array}{lllll}1 & -6.404831 & -1.092291 & -0.787018\end{array}$

\section{2a'@1}

$\begin{array}{llll}6 & 9.018651 & 2.130329 & -2.852462\end{array}$

$\begin{array}{lllll}6 & 8.422839 & 0.731719 & -3.008503\end{array}$

$\begin{array}{lllll}6 & 8.430116 & 2.848018 & -1.642866\end{array}$

$\begin{array}{lllll}1 & 8.699373 & 2.696054 & -3.728933\end{array}$

$\begin{array}{lllll}6 & 9.003869 & -0.424048 & -2.480668\end{array}$

$\begin{array}{lllll}6 & 7.239757 & 0.566540 & -3.737547\end{array}$

$\begin{array}{lllll}6 & 8.912400 & 2.600504 & -0.352106\end{array}$

$\begin{array}{lllll}6 & 7.383080 & 3.770215 & -1.763947\end{array}$

$\begin{array}{lllll}6 & 8.473417 & -1.702537 & -2.695299\end{array}$

$\begin{array}{lllll}1 & 9.907452 & -0.329888 & -1.890211\end{array}$

$\begin{array}{lllll}6 & 6.698561 & -0.675832 & -4.024488\end{array}$

$\begin{array}{lllll}8 & 6.648272 & 1.714839 & -4.257305\end{array}$

$\begin{array}{llll}6 & 8.392889 & 3.208392 & 0.792704\end{array}$

$\begin{array}{lllll}1 & 9.728989 & 1.897559 & -0.235986\end{array}$

$6 \quad 6.811502 \quad 4.381509 \quad-0.652874$

$\begin{array}{lllll}8 & 6.862736 & 4.099837 & -3.029568\end{array}$

$\begin{array}{lllll}6 & 9.066684 & -2.957108 & -2.064470\end{array}$

$\begin{array}{lllll}6 & 7.338270 & -1.795055 & -3.507345\end{array}$

$1 \quad 5.802772 \quad-0.772128 \quad-4.622733$

$\begin{array}{lllll}6 & 5.530963 & 2.221403 & -3.682158\end{array}$

$\begin{array}{lllll}6 & 8.967210 & 2.950336 & 2.185225\end{array}$

$\begin{array}{lllll}6 & 7.310021 & 4.076392 & 0.604865\end{array}$

$\begin{array}{lllll}1 & 5.983207 & 5.069045 & -0.767230\end{array}$

$\begin{array}{lllll}6 & 5.616699 & 3.565491 & -3.219478\end{array}$

$\begin{array}{lllll}1 & 8.761210 & -3.792660 & -2.695042\end{array}$

$6 \quad 8.451724 \quad-3.212681 \quad-0.688316$

$8 \quad 6.841897 \quad-3.067346 \quad-3.823967$

$\begin{array}{lllll}6 & 8.365910 & 1.686896 & 2.790458\end{array}$

$\begin{array}{llll}1 & 8.635611 & 3.779364 & 2.811091\end{array}$

$\begin{array}{lllll}8 & 6.723287 & 4.671490 & 1.729474\end{array}$

$\begin{array}{lllll}6 & 8.941100 & -2.606354 & 0.470418\end{array}$

$6 \quad 7.362196 \quad-4.079328 \quad-0.530301$

$6 \quad 5.631834 \quad-3.361454 \quad-3.275034$

$\begin{array}{lllll}6 & 3.351505 & 2.229101 & -3.225982\end{array}$

$\begin{array}{lllll}6 & 8.925519 & 0.417238 & 2.599070\end{array}$

$\begin{array}{lllll}6 & 7.200358 & 1.761798 & 3.561235\end{array}$

$\begin{array}{lllll}6 & 5.519577 & 4.165034 & 2.112124\end{array}$

$\begin{array}{lllll}6 & 3.401755 & 3.592730 & -2.972253\end{array}$

$\begin{array}{lllll}6 & 8.427065 & -2.856796 & 1.748373\end{array}$

$\begin{array}{lllll}1 & 9.758529 & -1.901236 & 0.376009\end{array}$

$\begin{array}{lllll}6 & 6.836806 & -4.391411 & 0.714362\end{array}$

$\begin{array}{lllll}8 & 6.800333 & -4.671173 & -1.667777\end{array}$

$6 \quad 5.614448 \quad-4.156146 \quad-2.095660$

$\begin{array}{lllll}6 & 8.352609 & -0.746710 & 3.118582\end{array}$

$\begin{array}{lllll}1 & 9.847702 & 0.336208 & 2.036074\end{array}$

$\begin{array}{lllll}6 & 6.570965 & 0.634360 & 4.072459\end{array}$ $\begin{array}{lllll}8 & 6.675156 & 3.027785 & 3.856728\end{array}$

$\begin{array}{llll}6 & 5.489006 & 3.332295 & 3.266952\end{array}$

$\begin{array}{lllll}6 & 8.976082 & -2.135017 & 2.974887\end{array}$

$\begin{array}{lllll}6 & 7.382358 & -3.784465 & 1.840560\end{array}$

$1 \quad 6.007348 \quad-5.080705 \quad 0.806400$

$\begin{array}{lllll}6 & 3.411483 & -3.188794 & -3.181214\end{array}$

$\begin{array}{lllll}6 & 7.150908 & -0.599199 & 3.820213\end{array}$

$\begin{array}{lllll}1 & 5.657287 & 0.717313 & 4.645149\end{array}$

$\begin{array}{lllll}6 & 3.307385 & 3.950907 & 1.931689\end{array}$

$\begin{array}{lllll}1 & 8.645105 & -2.708691 & 3.841655\end{array}$

$8 \quad 6.837405 \quad-4.122852 \quad 3.094140$

$\begin{array}{lllll}6 & 3.399083 & -3.912995 & -1.996393\end{array}$

$\begin{array}{lllll}8 & 6.575865 & -1.755590 & 4.344308\end{array}$

$\begin{array}{llll}6 & 3.270889 & 3.185102 & 3.087382\end{array}$

$\begin{array}{lllll}6 & 5.582243 & -3.604633 & 3.257363\end{array}$

$\begin{array}{lllll}6 & 5.471814 & -2.275102 & 3.755849\end{array}$

$\begin{array}{lllll}6 & 3.377151 & -3.640959 & 2.928100\end{array}$

$\begin{array}{lllll}6 & 3.305772 & -2.292005 & 3.246001\end{array}$

$\begin{array}{lllll}6 & 10.511117 & -2.104129 & 3.001483\end{array}$

$\begin{array}{lllll}1 & 10.862187 & -1.622847 & 3.918168\end{array}$

$\begin{array}{lllll}1 & 10.942964 & -1.560587 & 2.156564\end{array}$

$\begin{array}{lllll}6 & 10.601614 & -2.944282 & -2.025314\end{array}$

$1 \quad 10.973923 \quad-3.890696 \quad-1.624883$

$\begin{array}{lllll}1 & 11.007620 & -2.142674 & -1.402222\end{array}$

$\begin{array}{lllll}6 & 10.554309 & 2.124663 & -2.841777\end{array}$

$\begin{array}{lllll}1 & 10.935211 & 1.647457 & -3.748583\end{array}$

$1 \quad 10.973905 \quad 1.589506 \quad-1.985430$

$\begin{array}{llll}6 & 10.502664 & 2.951086 & 2.192381\end{array}$

$\begin{array}{llll}1 & 10.877508 & 3.905930 & 1.815077\end{array}$

$\begin{array}{llll}1 & 10.934314 & 2.161555 & 1.571173\end{array}$

$\begin{array}{lllll}1 & 10.872768 & 2.809691 & 3.211282\end{array}$

$\begin{array}{lllll}1 & 10.929948 & 3.150314 & -2.803464\end{array}$

$\begin{array}{lllll}1 & 11.000432 & -2.812098 & -3.034563\end{array}$

$\begin{array}{llll}1 & 10.904319 & -3.123423 & 2.970590\end{array}$

$\begin{array}{lllll}6 & 1.994281 & -2.823129 & -3.528127\end{array}$

$\begin{array}{lllll}6 & 1.975836 & -4.015479 & -1.524530\end{array}$

$\begin{array}{lllll}6 & 1.985212 & -4.122039 & 2.614753\end{array}$

$\begin{array}{lllll}6 & 1.895681 & -1.826683 & 3.032899\end{array}$

$\begin{array}{lllll}6 & 1.931354 & 1.765597 & -3.043967\end{array}$

$6 \quad 1.995660 \quad 4.086476 \quad-2.766044$

$\begin{array}{lllll}6 & 1.909304 & 4.078104 & 1.399418\end{array}$

$\begin{array}{lllll}6 & 1.839774 & 2.809783 & 3.359766\end{array}$

$\begin{array}{llll}7 & 1.190378 & -2.959628 & 2.691298\end{array}$

$\begin{array}{lllll}7 & 1.225302 & -3.412729 & -2.517259\end{array}$

$\begin{array}{lllll}7 & 1.203254 & 2.925315 & -2.865733\end{array}$

$\begin{array}{lllll}7 & 1.115908 & 3.396179 & 2.309629\end{array}$

$\begin{array}{lllll}8 & 1.563265 & 4.639499 & 0.377771\end{array}$

$\begin{array}{lllll}8 & 1.381140 & 2.141517 & 4.256716\end{array}$

$8 \quad 1.481908 \quad-0.681302 \quad 3.109872$

$\begin{array}{lllll}8 & 1.588030 & -5.234159 & 2.373069\end{array}$

$8 \quad 1.569621 \quad-4.508893 \quad-0.487721$

$8 \quad 1.534106 \quad 0.612148 \quad-3.008003$

$8 \quad 1.571183 \quad-2.158990 \quad-4.446258$

$\begin{array}{lllll}8 & 1.599398 & 5.208621 & -2.573599\end{array}$

$\begin{array}{llll}0.101308 & 3.276376 & 2.250105\end{array}$

$\begin{array}{lllll}1 & 0.188709 & 2.977011 & -2.770017\end{array}$

$1 \quad 0.206883 \quad-3.284985 \quad-2.490455$

$\begin{array}{llll}1 & 0.175749 & -3.002805 & 2.528478\end{array}$ $\begin{array}{lllll}7 & 4.531563 & -2.882682 & -3.836472\end{array}$

$\begin{array}{lllll}7 & 4.498537 & -4.415081 & -1.428800\end{array}$

$\begin{array}{lllll}7 & 4.413781 & 1.514654 & -3.613368\end{array}$

$\begin{array}{lllll}7 & 4.547852 & 4.279410 & -2.912332\end{array}$

$\begin{array}{lllll}7 & 4.530606 & -4.315916 & 2.887010\end{array}$

$\begin{array}{lllll}7 & 4.346227 & -1.582270 & 3.692079\end{array}$

$\begin{array}{lllll}7 & 4.430111 & 4.460250 & 1.419111\end{array}$

$\begin{array}{lllll}7 & 4.365153 & 2.844768 & 3.771822\end{array}$

$\begin{array}{lllll}6 & -8.890446 & 0.479361 & 3.599502\end{array}$

$\begin{array}{lllll}6 & -8.315420 & -0.857588 & 3.141489\end{array}$

$\begin{array}{lllll}6 & -8.328596 & 1.641882 & 2.779794\end{array}$

$\begin{array}{lllll}1 & -8.537015 & 0.629457 & 4.620224\end{array}$

$\begin{array}{lllll}6 & -8.891165 & -1.583950 & 2.090320\end{array}$

$\begin{array}{lllll}6 & -7.182751 & -1.410772 & 3.753125\end{array}$

$\begin{array}{lllll}6 & -8.897139 & 2.079691 & 1.580468\end{array}$

$\begin{array}{lllll}6 & -7.200407 & 2.336504 & 3.229523\end{array}$

$\begin{array}{lllll}6 & -8.379935 & -2.800719 & 1.635096\end{array}$

$\begin{array}{lllll}1 & -9.777352 & -1.183696 & 1.612859\end{array}$

$\begin{array}{lllll}6 & -6.618073 & -2.606951 & 3.320300\end{array}$

$\begin{array}{llll}8 & -6.608243 & -0.768402 & 4.861411\end{array}$

$\begin{array}{lllll}6 & -8.407668 & 3.179022 & 0.864387\end{array}$

$\begin{array}{lllll}1 & -9.754297 & 1.546295 & 1.186758\end{array}$

$\begin{array}{lllll}6 & -6.693944 & 3.452081 & 2.580689\end{array}$

$\begin{array}{lllll}8 & -6.586483 & 1.888479 & 4.402566\end{array}$

$\begin{array}{lllll}6 & -9.019893 & -3.614474 & 0.509846\end{array}$

$\begin{array}{lllll}6 & -7.217433 & -3.268929 & 2.259170\end{array}$

$\begin{array}{lllll}1 & -5.733766 & -3.005529 & 3.799890\end{array}$

$\begin{array}{lllll}6 & -5.409819 & -0.164958 & 4.606131\end{array}$

$\begin{array}{lllll}6 & -8.998295 & 3.601758 & -0.476547\end{array}$

$\begin{array}{lllll}6 & -7.314879 & 3.863976 & 1.408983\end{array}$

$\begin{array}{lllll}1 & -5.830793 & 3.977731 & 2.966386\end{array}$

$\begin{array}{llll}6 & -5.417565 & 1.212978 & 4.254760\end{array}$

$\begin{array}{lllll}1 & -8.743118 & -4.654520 & 0.686724\end{array}$

$\begin{array}{lllll}6 & -8.412302 & -3.228056 & -0.835199\end{array}$

$\begin{array}{lllll}8 & -6.705737 & -4.488596 & 1.816778\end{array}$

$\begin{array}{lllll}6 & -8.340898 & 2.805327 & -1.601851\end{array}$

$\begin{array}{lllll}1 & -8.713033 & 4.643273 & -0.627448\end{array}$

$\begin{array}{lllll}8 & -6.848067 & 5.018789 & 0.768486\end{array}$

$\begin{array}{lllll}6 & -8.862308 & -2.104978 & -1.541064\end{array}$

$6 \begin{array}{llll}6 & -7.360709 & -3.957691 & -1.404213\end{array}$

$\begin{array}{lllll}6 & -5.561143 & -4.510495 & 1.090297\end{array}$

$\begin{array}{lllll}6 & -3.201323 & -0.210181 & 4.290246\end{array}$

$\begin{array}{lllll}6 & -8.828402 & 1.566796 & -2.026674\end{array}$

$\begin{array}{lllll}6 & -7.200888 & 3.296013 & -2.251440\end{array}$

$\begin{array}{lllll}6 & -5.613761 & 4.922765 & 0.196024\end{array}$

$\begin{array}{lllll}6 & -3.236836 & 1.094487 & 3.815648\end{array}$

$\begin{array}{lllll}6 & -8.293692 & -1.678740 & -2.743344\end{array}$

$\begin{array}{lllll}1 & -9.688374 & -1.537053 & -1.129877\end{array}$

$\begin{array}{lllll}6 & -6.737850 & -3.555188 & -2.580866\end{array}$

$8 \quad-6.901716 \quad-5.134513 \quad-0.786723$

$\begin{array}{lllll}6 & -5.654286 & -4.998539 & -0.244321\end{array}$

$\begin{array}{lllll}6 & -8.247557 & 0.832858 & -3.066370\end{array}$

$\begin{array}{lllll}1 & -9.706383 & 1.162758 & -1.537239\end{array}$

$\begin{array}{lllll}6 & -6.581949 & 2.608214 & -3.288364\end{array}$

$8 \quad-6.722394 \quad 4.553374 \quad-1.878485$

$\begin{array}{lllll}6 & -5.548530 & 4.621896 & -1.192248\end{array}$

$6 \quad-8.830360 \quad-0.493643 \quad-3.542276$

$\begin{array}{llll}6 & -7.198196 & -2.411297 & -3.214596\end{array}$ 
$\begin{array}{lllll}1 & -5.902708 & -4.113258 & -2.983385\end{array}$

$\begin{array}{lllll}6 & -3.353699 & -4.354678 & 0.848886\end{array}$

$\begin{array}{lllll}6 & -7.116408 & 1.385365 & -3.678821\end{array}$

$\begin{array}{lllll}1 & -5.707777 & 3.014654 & -3.780480\end{array}$

$\begin{array}{lllll}6 & -3.384009 & 4.976215 & 0.276494\end{array}$

$\begin{array}{lllll}1 & -8.473089 & -0.631766 & -4.563215\end{array}$

$\begin{array}{lllll}8 & -6.565286 & -1.974828 & -4.383751\end{array}$

$\begin{array}{lllll}6 & -3.427772 & -4.967064 & -0.392900\end{array}$

$\begin{array}{lllll}8 & -6.527031 & 0.704069 & -4.755132\end{array}$

$\begin{array}{lllll}6 & -3.323031 & 4.634048 & -1.067824\end{array}$

$\begin{array}{lllll}6 & -5.383245 & -1.322957 & -4.209385\end{array}$

$\begin{array}{lllll}6 & -5.352102 & 0.076872 & -4.466631\end{array}$

$\begin{array}{lllll}6 & -3.201757 & -1.267318 & -3.752192\end{array}$

$\begin{array}{lllll}6 & -3.155500 & 0.080178 & -4.076319\end{array}$

$\begin{array}{lllll}6 & -10.365950 & -0.478899 & -3.592318\end{array}$

$\begin{array}{lllll}1 & -10.712826 & 0.348059 & -4.217394\end{array}$

$\begin{array}{lllll}1 & -10.825436 & -0.363635 & -2.606874\end{array}$

$\begin{array}{lllll}6 & -10.553028 & -3.536482 & 0.514228\end{array}$

$\begin{array}{lllll}1 & -10.961208 & -4.186373 & -0.264005\end{array}$

$\begin{array}{lllll}1 & -10.931572 & -2.527207 & 0.330261\end{array}$

$\begin{array}{lllll}6 & -10.425720 & 0.483639 & 3.640198\end{array}$

$\begin{array}{llll}1 & -10.786689 & -0.327850 & 4.277513\end{array}$

$\begin{array}{lllll}1 & -10.881310 & 0.356757 & 2.654332\end{array}$

$\begin{array}{lllll}6 & -10.531106 & 3.531761 & -0.509085\end{array}$

$\begin{array}{lllll}1 & -10.949597 & 4.163439 & 0.278730\end{array}$

$\begin{array}{lllll}1 & -10.918463 & 2.520315 & -0.359016\end{array}$

$\begin{array}{lllll}1 & -10.902982 & 3.886524 & -1.473888\end{array}$

$\begin{array}{lllll}1 & -10.785124 & 1.432366 & 4.047042\end{array}$

$\begin{array}{lllll}1 & -10.943391 & -3.863825 & 1.481431\end{array}$

$\begin{array}{lllll}1 & -10.734155 & -1.415950 & -4.017993\end{array}$

$\begin{array}{lllll}6 & -1.920324 & -4.027670 & 1.134423\end{array}$

$\begin{array}{lllll}6 & -2.021347 & -5.153141 & -0.904416\end{array}$

$\begin{array}{lllll}6 & -1.826824 & -1.708602 & -3.344515\end{array}$

$\begin{array}{lllll}6 & -1.736953 & 0.552308 & -3.925748\end{array}$

$\begin{array}{lllll}6 & -1.779316 & -0.694582 & 4.229116\end{array}$

$\begin{array}{lllll}6 & -1.865179 & 1.463470 & 3.327779\end{array}$

$\begin{array}{lllll}6 & -1.972060 & 5.116020 & 0.788742\end{array}$

$\begin{array}{lllll}6 & -1.878737 & 4.489157 & -1.457826\end{array}$

$\begin{array}{lllll}7 & -1.033266 & -0.579594 & -3.486507\end{array}$

$\begin{array}{lllll}7 & -1.207395 & -4.581052 & 0.096111\end{array}$

$\begin{array}{lllll}7 & -1.071844 & 0.378456 & 3.658068\end{array}$

$\begin{array}{lllll}7 & -1.168009 & 4.855395 & -0.328712\end{array}$

$\begin{array}{lllll}8 & -1.579164 & 5.382617 & 1.900461\end{array}$

$\begin{array}{lllll}8 & -1.436599 & 4.091045 & -2.517859\end{array}$

$\begin{array}{lllll}8 & -1.274086 & 1.650994 & -4.123775\end{array}$

$\begin{array}{lllll}8 & -1.491821 & -2.814783 & -2.964958\end{array}$

$\begin{array}{lllll}8 & -1.631218 & -5.673860 & -1.919023\end{array}$

$\begin{array}{lllll}8 & -1.307746 & -1.751224 & 4.573925\end{array}$

$\begin{array}{lllll}8 & -1.499875 & -3.378695 & 2.080518\end{array}$

$\begin{array}{llll}8 & -1.538950 & 2.470072 & 2.726006\end{array}$

$\begin{array}{lllll}1 & -0.148348 & 4.781530 & -0.245706\end{array}$

$\begin{array}{lllll}1 & -0.069369 & 0.302276 & 3.483911\end{array}$

$\begin{array}{lllll}1 & -0.186743 & -4.514206 & -0.016627\end{array}$

$\begin{array}{lllll}1 & -0.024959 & -0.534775 & -3.344886\end{array}$

$\begin{array}{lllll}7 & -4.413963 & -4.130651 & 1.628976\end{array}$

$\begin{array}{lllll}7 & -4.589340 & -5.269369 & -0.982205\end{array}$

$\begin{array}{lllll}7 & -4.295648 & -0.879189 & 4.662302\end{array}$

$\begin{array}{lllll}7 & -4.340865 & 1.841046 & 3.806625\end{array}$ $\begin{array}{lllll}7 & -4.313785 & -2.000322 & -3.817886\end{array}$

$\begin{array}{lllll}7 & -4.233912 & 0.786334 & -4.419222\end{array}$

$\begin{array}{lllll}7 & -4.534064 & 5.114471 & 0.940249\end{array}$

$\begin{array}{lllll}7 & -4.401640 & 4.450672 & -1.832341\end{array}$

$\begin{array}{lllll}6 & -0.330159 & 0.930631 & -0.169769\end{array}$

$\begin{array}{lllll}8 & -0.056530 & 2.104792 & -0.358170\end{array}$

$\begin{array}{lllll}6 & 2.094618 & 0.639191 & 0.376889\end{array}$

$\begin{array}{lllll}1 & 2.119883 & 0.807749 & 1.455176\end{array}$

$\begin{array}{lllll}1 & 2.112599 & 1.606006 & -0.124770\end{array}$

$\begin{array}{lllll}6 & 3.300312 & -0.197327 & -0.045614\end{array}$

$\begin{array}{lllll}1 & 3.263392 & -0.366180 & -1.128201\end{array}$

$\begin{array}{lllll}1 & 3.269392 & -1.181927 & 0.429692\end{array}$

$\begin{array}{lllll}6 & 4.609468 & 0.507373 & 0.338215\end{array}$

$\begin{array}{lllll}1 & 4.628239 & 0.668732 & 1.424275\end{array}$

$1 \quad 4.644867 \quad 1.503222 \quad-0.124981$

$\begin{array}{lllll}6 & 5.845984 & -0.294860 & -0.077750\end{array}$

$\begin{array}{lllll}1 & 5.865330 & -0.453927 & -1.160945\end{array}$

$\begin{array}{lllll}1 & 6.769611 & 0.220772 & 0.199175\end{array}$

$\begin{array}{lllll}1 & 5.858010 & -1.278715 & 0.404804\end{array}$

$\begin{array}{lllll}6 & -1.743991 & 0.480793 & -0.116559\end{array}$

$\begin{array}{lllll}6 & -2.215740 & -0.508494 & 0.755495\end{array}$

$\begin{array}{llll}6 & -2.666599 & 1.202707 & -0.887034\end{array}$

$\begin{array}{lllll}6 & -3.579965 & -0.757460 & 0.860503\end{array}$

$\begin{array}{lllll}1 & -1.526930 & -1.080203 & 1.360520\end{array}$

$\begin{array}{lllll}6 & -4.027450 & 0.925450 & -0.801443\end{array}$

$\begin{array}{llll}1 & -2.309250 & 1.960822 & -1.571035\end{array}$

$\begin{array}{llll}6 & -4.509708 & -0.052576 & 0.077581\end{array}$

$\begin{array}{lllll}1 & -3.925512 & -1.514710 & 1.557575\end{array}$

$\begin{array}{lllll}1 & -4.723277 & 1.480884 & -1.422539\end{array}$

$\begin{array}{lllll}6 & -5.981353 & -0.361104 & 0.156524\end{array}$

$\begin{array}{lllll}1 & -6.588883 & 0.484716 & -0.175512\end{array}$

$1 \quad-6.235786 \quad-1.215563 \quad-0.481014$

$\begin{array}{lllll}1 & -6.280362 & -0.619109 & 1.174096\end{array}$

$\begin{array}{ccccc}7 & 0.776201 & 0.035653 & 0.057504\end{array}$

$\begin{array}{lllll}7 & 0.817544 & -1.289533 & -0.212898\end{array}$

$\begin{array}{lllll}8 & -0.218138 & -1.809372 & -0.597220\end{array}$

\section{4a-TS@12}

$\begin{array}{lllll}6 & -8.936722 & 3.004910 & -2.221498\end{array}$

$\begin{array}{lllll}6 & -8.333360 & 3.282968 & -0.847219\end{array}$

$\begin{array}{lllll}6 & -8.350180 & 1.710622 & -2.778815\end{array}$

$\begin{array}{lllll}1 & -8.599107 & 3.808084 & -2.877237\end{array}$

$\begin{array}{llll}6 & -8.834397 & 2.678673 & 0.312076\end{array}$

$\begin{array}{llll}-7.225086 & 4.124461 & -0.698216\end{array}$

$\begin{array}{llll}-8.952036 & 0.461285 & -2.586099\end{array}$

$\begin{array}{llll}-7.147133 & 1.740607 & -3.495985\end{array}$

$\begin{array}{llll}-8.278146 & 2.880519 & 1.577096\end{array}$

$\begin{array}{llll}-9.693432 & 2.024303 & 0.221711\end{array}$

$\begin{array}{llll}-6.620936 & 4.344390 & 0.534113\end{array}$

$\begin{array}{llll}-6.718406 & 4.798938 & -1.818756\end{array}$

$\begin{array}{llll}-8.399671 & -0.723815 & -3.079046\end{array}$

$\begin{array}{llll}-9.890178 & 0.413426 & -2.046447\end{array}$

$\begin{array}{llll}-6.547383 & 0.592755 & -3.996873\end{array}$

$\begin{array}{llll}-6.600994 & 2.991615 & -3.792599\end{array}$

$\begin{array}{llll}-8.847225 & 2.253317 & 2.847422\end{array}$

$\begin{array}{lll}-7.152478 & 3.708983 & 1.646093\end{array}$

$\begin{array}{llll}-5.755850 & 4.988619 & 0.621088\end{array}$ $\begin{array}{llll}-5.487502 & 4.392340 & -2.238887\end{array}$

$\begin{array}{llll}-9.040533 & -2.101844 & -2.907257\end{array}$

$\begin{array}{llll}-7.187462 & -0.617259 & -3.769145\end{array}$

$\begin{array}{llll}-5.620273 & 0.643317 & -4.551467\end{array}$

$\begin{array}{llll}-5.423682 & 3.387124 & -3.247053\end{array}$

$\begin{array}{llll}-8.514966 & 2.884037 & 3.672454\end{array}$

$\begin{array}{lll}-8.253622 & 0.871035 & 3.102612\end{array}$

$\begin{array}{llll}-6.578087 & 3.924010 & 2.902930\end{array}$

$\begin{array}{llll}-8.448663 & -2.814018 & -1.692696\end{array}$

$\begin{array}{llll}-8.750075 & -2.687754 & -3.780261\end{array}$

$\begin{array}{llll}-6.680881 & -1.795813 & -4.311892\end{array}$

$\begin{array}{lll}-8.789830 & -0.280858 & 2.519585\end{array}$

$\begin{array}{llll}-7.149627 & 0.698235 & 3.946947\end{array}$

$\begin{array}{llll}-5.397296 & 3.301186 & 3.152495\end{array}$

$\begin{array}{llll}-3.257956 & 4.465488 & -2.196561\end{array}$

$\begin{array}{llll}-8.896952 & -2.515385 & -0.399930\end{array}$

$\begin{array}{llll}-7.426416 & -3.765663 & -1.802964\end{array}$

$\begin{array}{llll}-5.566470 & -2.354221 & -3.788034\end{array}$

$\begin{array}{llll}-3.194226 & 3.461273 & -3.154083\end{array}$

$\begin{array}{llll}-8.284220 & -1.561784 & 2.754358\end{array}$

$\begin{array}{llll}-9.645829 & -0.175814 & 1.863807\end{array}$

$\begin{array}{llll}-6.596517 & -0.551636 & 4.201871\end{array}$

$\begin{array}{llll}-6.600876 & 1.819592 & 4.589171\end{array}$

$\begin{array}{llll}-5.400546 & 2.233407 & 4.094322\end{array}$

$\begin{array}{llll}-8.363473 & -3.095836 & 0.751666\end{array}$

$\begin{array}{llll}-9.695919 & -1.791894 & -0.289345\end{array}$

$\begin{array}{llll}-6.833897 & -4.342715 & -0.682668\end{array}$

$\begin{array}{llll}-6.949406 & -4.164117 & -3.066855\end{array}$

$\begin{array}{llll}-5.687652 & -3.684942 & -3.297594\end{array}$

$\begin{array}{llll}-8.912398 & -2.809154 & 2.145222\end{array}$

$\begin{array}{llll}-7.171896 & -1.662083 & 3.597974\end{array}$

$\begin{array}{lll}-5.738394 & -0.655726 & 4.853485\end{array}$

$\begin{array}{lll}-3.190915 & 3.079352 & 2.938481\end{array}$

$\begin{array}{llll}-7.295289 & -3.983904 & 0.574271\end{array}$

$\begin{array}{llll}-6.022313 & -5.050778 & -0.792283\end{array}$

$\begin{array}{llll}-3.387063 & -2.426045 & -3.367722\end{array}$

$\begin{array}{llll}-8.598114 & -3.643956 & 2.772233\end{array}$

$\begin{array}{lll}-6.653982 & -2.935218 & 3.874183\end{array}$

$\begin{array}{lll}-3.182146 & 2.091542 & 3.911367\end{array}$

$\begin{array}{llll}-6.710295 & -4.557006 & 1.709453\end{array}$

$\begin{array}{llll}-3.471884 & -3.776542 & -3.066964\end{array}$

$\begin{array}{llll}-5.472509 & -3.241416 & 3.269712\end{array}$

$\begin{array}{llll}-5.505361 & -4.060891 & 2.104819\end{array}$

$\begin{array}{llll}-3.247547 & -3.145908 & 3.121150\end{array}$

$\begin{array}{lll}-3.282571 & -3.908075 & 1.961900\end{array}$

$\begin{array}{llll}-10.448026 & -2.767358 & 2.170066\end{array}$

$\begin{array}{llll}-10.854134 & -3.708898 & 1.791318\end{array}$

$\begin{array}{llll}-10.863972 & -1.960793 & 1.560208\end{array}$

$\begin{array}{lll}-10.383221 & 2.247783 & 2.862636\end{array}$

$\begin{array}{llll}-10.746692 & 1.851420 & 3.814263\end{array}$

$\begin{array}{llll}-10.817494 & 1.638629 & 2.065192\end{array}$

$\begin{array}{llll}-10.470915 & 3.026800 & -2.215153\end{array}$

$\begin{array}{llll}-10.827785 & 4.001768 & -1.873477\end{array}$

$\begin{array}{llll}-10.909016 & 2.270199 & -1.558594\end{array}$

$\begin{array}{lllll}6 & -10.574297 & -2.053669 & -2.872764\end{array}$

$\begin{array}{llll}-10.956463 & -1.573911 & -3.777833\end{array}$

$\begin{array}{llll}-10.966553 & -1.501042 & -2.014546\end{array}$

$\begin{array}{llll}-10.977323 & -3.068194 & -2.820114\end{array}$ 
$\begin{array}{llll}1 & -10.853715 & 2.850429 & -3.223914\end{array}$

$\begin{array}{llll}-10.761669 & 3.266151 & 2.741517\end{array}$

$\begin{array}{lllll}1 & -10.801437 & -2.621878 & 3.194234\end{array}$

$\begin{array}{lllll}6 & -1.786446 & 3.279539 & 2.451278\end{array}$

$6 \quad-1.759910 \quad 1.662158 \quad 4.137150$

$6 \quad-1.810061 \quad-2.850597 \quad 3.449732$

$\begin{array}{lllll}6 & -1.870828 & -4.111597 & 1.484944\end{array}$

$6 \quad-1.855246 \quad 4.842183 \quad-1.801264$

$6 \quad-1.742778 \quad 3.145476 \quad-3.420574$

$6 \quad-1.975792 \quad-1.975533 \quad-3.164105$

$\begin{array}{llll}-2.074818 & -4.276988 & -2.804341\end{array}$

$\begin{array}{llll}-1.079954 & -3.500421 & 2.443268\end{array}$

$\begin{array}{lll}-1.005940 & 2.484733 & 3.280240\end{array}$

$\begin{array}{llll}-1.040950 & 4.024670 & -2.592041\end{array}$

$\begin{array}{llll}-1.263492 & -3.130531 & -2.933886\end{array}$

$\begin{array}{llll}-1.588510 & -0.817715 & -3.159089\end{array}$

$\begin{array}{llll}-1.691010 & -5.390840 & -2.547510\end{array}$

$\begin{array}{llll}-1.508912 & -4.689204 & 0.476099\end{array}$

$\begin{array}{llll}-1.351563 & -2.212709 & 4.367927\end{array}$

$\begin{array}{llll}-1.339554 & 0.793283 & 4.862741\end{array}$

$\begin{array}{llll}-1.484883 & 5.635217 & -0.965428\end{array}$

$\begin{array}{llll}-1.423015 & 3.951993 & 1.504721\end{array}$

$\begin{array}{llll}-1.270820 & 2.313964 & -4.162716\end{array}$

$\begin{array}{llll}-0.255109 & -3.190127 & -2.769746\end{array}$

$\begin{array}{llll}-0.024485 & 4.057389 & -2.528142\end{array}$

$\begin{array}{llll}0.011787 & 2.525044 & 3.292457\end{array}$

$\begin{array}{llll}-0.062354 & -3.418058 & 2.417812\end{array}$

$\begin{array}{llll}-4.297148 & 3.707987 & 2.537272\end{array}$

$\begin{array}{llll}-4.291329 & 1.627345 & 4.490694\end{array}$

$\begin{array}{llll}-4.406604 & 4.942816 & -1.709015\end{array}$

$\begin{array}{llll}-4.277569 & 2.900249 & -3.701245\end{array}$

$\begin{array}{llll}-4.343541 & -2.782569 & 3.789868\end{array}$

$\begin{array}{llll}-4.411478 & -4.380702 & 1.427676\end{array}$

$\begin{array}{llll}-4.425154 & -1.688338 & -3.764272\end{array}$

$\begin{array}{llll}-4.638542 & -4.426082 & -2.981924\end{array}$

$\begin{array}{llll}9.011900 & -3.543397 & -0.744979\end{array}$

$\begin{array}{llll}8.466583 & -3.157461 & 0.624927\end{array}$

$\begin{array}{llll}8.314298 & -2.729782 & -1.832629\end{array}$

$8.730211-4.583812-0.909384$

$\begin{array}{llll}8.960504 & -2.055854 & 1.333668\end{array}$

$\begin{array}{llll}7.420390 & -3.879248 & 1.210793\end{array}$

$\begin{array}{llll}8.778826 & -1.482389 & -2.254535\end{array}$

$\begin{array}{llll}7.158602 & -3.219401 & -2.454323\end{array}$

$\begin{array}{llll}8.442037 & -1.651369 & 2.566194\end{array}$

$\begin{array}{llll}9.783240 & -1.494303 & 0.906858\end{array}$

$\begin{array}{llll}6.851373 & -3.504451 & 2.421393\end{array}$

$\begin{array}{llll}6.937770 & -5.029095 & 0.568030\end{array}$

$\begin{array}{llll}8.169059 & -0.744439 & -3.275578\end{array}$

$\begin{array}{llll}9.660648 & -1.071608 & -1.777530\end{array}$

$\begin{array}{llll}6.519711 & -2.536416 & -3.480873\end{array}$

$\begin{array}{llll}6.690402 & -4.475513 & -2.059630\end{array}$

$\begin{array}{llll}9.019289 & -0.488696 & 3.372110\end{array}$

$\begin{array}{llll}7.359838 & -2.385710 & 3.063872\end{array}$

$\begin{array}{llll}6.023517 & -4.059742 & 2.841769\end{array}$

$\begin{array}{llll}5.680575 & -4.915178 & 0.051513\end{array}$

$\begin{array}{llll}8.715984 & 0.606816 & -3.723613\end{array}$

$\begin{array}{llll}7.041814 & -1.310287 & -3.883397\end{array}$

$\begin{array}{llll}5.640441 & -2.947605 & -3.960025\end{array}$ $\begin{array}{lllll}6 & 5.553177 & -4.547944 & -1.318776\end{array}$

$1 \quad 8.713841 \quad-0.655858 \quad 4.405451$

$\begin{array}{lllll}6 & 8.408590 & 0.847555 & 2.961209\end{array}$

$\begin{array}{lllll}8 & 6.793948 & -1.975193 & 4.275081\end{array}$

$\begin{array}{lllll}6 & 8.188045 & 1.752154 & -2.860855\end{array}$

$\begin{array}{lllll}8.319668 & 0.777057 & -4.725335\end{array}$

$8 \quad 6.425046 \quad-0.638363 \quad-4.952025$

$\begin{array}{llll}6 & 8.930519 & 1.605521 & 1.907220\end{array}$

$\begin{array}{lllll}6 & 7.306917 & 1.379798 & 3.642755\end{array}$

$\begin{array}{lllll}6 & 5.607235 & -1.318195 & 4.191868\end{array}$

$\begin{array}{lllll}6 & 3.455846 & -4.950465 & 0.236368\end{array}$

$6 \quad 8.815023 \quad 2.179661 \quad-1.687506$

$\begin{array}{lllll}6 & 7.037228 & 2.448602 & -3.248574\end{array}$

$6 \quad 5.228100 \quad-0.072233 \quad-4.614420$

$6 \quad 3.337121 \quad-4.530954 \quad-1.080608$

$\begin{array}{lllll}6 & 8.407406 & 2.842405 & 1.522568\end{array}$

$\begin{array}{llll}9.790330 & 1.219457 & 1.373498\end{array}$

$\begin{array}{llll}6.733385 & 2.597118 & 3.288499\end{array}$

$\begin{array}{llll}6.788241 & 0.687643 & 4.747755\end{array}$

$\begin{array}{llll}5.594478 & 0.064204 & 4.529116\end{array}$

$\begin{array}{llll}8.365101 & 3.275051 & -0.939267\end{array}$

$\begin{array}{llll}9.687716 & 1.640559 & -1.338576\end{array}$

$\begin{array}{llll}6.580471 & 3.572141 & -2.578747\end{array}$

$\begin{array}{llll}6.352264 & 2.004097 & -4.380077\end{array}$

$\begin{array}{llll}5.219490 & 1.279333 & -4.174553\end{array}$

$\begin{array}{llll}9.007196 & 3.670121 & 0.386235\end{array}$

$\begin{array}{llll}7.287168 & 3.301350 & 2.226921\end{array}$

$\begin{array}{lll}5.875940 & 2.981559 & 3.825204\end{array}$

$\begin{array}{llll}3.400701 & -1.246410 & 3.881192\end{array}$

$\begin{array}{llll}7.261566 & 3.976007 & -1.437070\end{array}$

$\begin{array}{llll}5.703379 & 4.104368 & -2.920880\end{array}$

$\begin{array}{llll}3.047198 & -0.204671 & -4.172079\end{array}$

$\begin{array}{llll}8.720577 & 4.705326 & 0.573783\end{array}$

$\begin{array}{llll}6.754593 & 4.545002 & 1.869181\end{array}$

$\begin{array}{llll}3.374568 & 0.068919 & 4.321000\end{array}$

$\begin{array}{llll}6.813346 & 5.122377 & -0.760310\end{array}$

$\begin{array}{llll}3.076095 & 1.058314 & -3.592644\end{array}$

$\begin{array}{llll}5.571310 & 4.572202 & 1.203039\end{array}$

$\begin{array}{llll}5.596546 & 4.961849 & -0.166947\end{array}$

$\begin{array}{llll}3.351363 & 4.410011 & 1.083071\end{array}$

$\begin{array}{llll}3.369013 & 4.849567 & -0.230589\end{array}$

$\begin{array}{llll}10.540750 & 3.613124 & 0.350674\end{array}$

$\begin{array}{llll}10.919545 & 4.271683 & -0.435193\end{array}$

$\begin{array}{llll}10.928636 & 2.609994 & 0.153021\end{array}$

$\begin{array}{lll}10.555228 & -0.469051 & 3.344927\end{array}$

$\begin{array}{lll}10.931181 & 0.338555 & 3.978466\end{array}$

$\begin{array}{llll}10.964064 & -0.319208 & 2.341862\end{array}$

$\begin{array}{llll}10.542623 & -3.460415 & -0.826327\end{array}$

$10.991474 \quad-4.106414 \quad-0.067358$

$\begin{array}{llll}10.927036 & -2.449454 & -0.666028\end{array}$

$10.248594 \quad 0.617201 \quad-3.828946$

$\begin{array}{llll}10.582341 & -0.175271 & -4.503747\end{array}$

$\begin{array}{llll}10.745183 & 0.462199 & -2.867290\end{array}$

$\begin{array}{llll}10.589785 & 1.577773 & -4.223694\end{array}$

$\begin{array}{llll}10.884670 & -3.790175 & -1.810807\end{array}$

$\begin{array}{llll}10.947029 & -1.417914 & 3.720267\end{array}$

$\begin{array}{lll}10.951546 & 3.942212 & 1.308710\end{array}$

$\begin{array}{lllll}6 & 1.997733 & -1.649022 & 3.518292\end{array}$ $\begin{array}{lllll}6 & 1.949280 & 0.538337 & 4.329939\end{array}$

$\begin{array}{lllll}6 & 1.934387 & 4.098366 & 1.459723\end{array}$

$\begin{array}{lllll}6 & 1.953270 & 4.878619 & -0.741721\end{array}$

$\begin{array}{lllll}6 & 2.067400 & -5.097173 & 0.807759\end{array}$

$6 \quad \begin{array}{llll}6 & 1.881945 & -4.346068 & -1.393761\end{array}$

$6 \quad 1.652150 \quad-0.746491 \quad-4.064527$

$\begin{array}{lllll}6 & 1.721126 & 1.329809 & -2.986092\end{array}$

$\begin{array}{lllll}7 & 1.180923 & 4.436123 & 0.340974\end{array}$

$7 \quad 1.210012 \quad-0.574229 \quad 3.909116$

$7 \quad 1.213449 \quad-4.713694 \quad-0.243361$

$\begin{array}{lllll}7 & 0.952205 & 0.238085 & -3.359019\end{array}$

$\begin{array}{lllll}8 & 1.203499 & -1.793308 & -4.470799\end{array}$

$\begin{array}{lllll}8 & 1.375554 & 2.258997 & -2.283775\end{array}$

$8 \quad 1.556625 \quad 5.189398 \quad-1.841564$

$\begin{array}{llll}8 & 1.537448 & 3.620092 & 2.502649\end{array}$

$8 \quad 1.525012 \quad 1.639666 \quad 4.595135$

$\begin{array}{lllll}8 & 1.718725 & -5.453128 & 1.906934\end{array}$

$\begin{array}{lllll}8 & 1.637525 & -2.667853 & 2.961498\end{array}$

$\begin{array}{lllll}8 & 1.408593 & -3.914058 & -2.429813\end{array}$

$1 \quad-0.042783 \quad 0.106782 \quad-3.165795$

$\begin{array}{llll}0.190139 & -4.699780 & -0.126445\end{array}$

$\begin{array}{llll}0.193825 & -0.578289 & 3.844051\end{array}$

$\begin{array}{llll}0.161721 & 4.421322 & 0.364225\end{array}$

$\begin{array}{llll}4.517116 & -1.972489 & 3.816363\end{array}$

$\begin{array}{llll}4.473667 & 0.764312 & 4.618548\end{array}$

$\begin{array}{llll}4.635138 & -5.134629 & 0.835599\end{array}$

$\begin{array}{llll}4.379122 & -4.322614 & -1.888728\end{array}$

$\begin{array}{llll}4.447449 & 4.262786 & 1.831189\end{array}$

$\begin{array}{llll}4.498015 & 5.118831 & -0.890171\end{array}$

$\begin{array}{llll}4.131821 & -0.812180 & -4.655446\end{array}$

$\begin{array}{llll}4.161050 & 1.836667 & -3.602127\end{array}$

$\begin{array}{llll}-0.064751 & -0.949937 & 0.106354\end{array}$

$\begin{array}{llll}-0.473403 & -1.956497 & -0.379694\end{array}$

$\begin{array}{llll}-1.389563 & 0.420726 & 0.138644\end{array}$

$\begin{array}{llll}-1.633071 & 0.253107 & 1.356486\end{array}$

$\begin{array}{llll}-0.784202 & -0.617795 & 1.810369\end{array}$

$\begin{array}{llll}-2.229862 & 1.312692 & -0.668564\end{array}$

$\begin{array}{llll}-2.099490 & 0.983446 & -1.701598\end{array}$

$\begin{array}{llll}-1.814945 & 2.319371 & -0.567383\end{array}$

$\begin{array}{llll}-3.712553 & 1.282928 & -0.274605\end{array}$

$\begin{array}{llll}-4.215520 & 2.089611 & -0.819365\end{array}$

$\begin{array}{llll}-3.812795 & 1.528725 & 0.785947\end{array}$

$\begin{array}{llll}-4.406256 & -0.048027 & -0.579531\end{array}$

$\begin{array}{llll}-3.841614 & -0.869191 & -0.119213\end{array}$

$\begin{array}{llll}-4.376521 & -0.218479 & -1.660929\end{array}$

$\begin{array}{llll}-5.855898 & -0.082262 & -0.089056\end{array}$

$\begin{array}{llll}-6.452993 & 0.708293 & -0.555932\end{array}$

$\begin{array}{llll}-6.334635 & -1.036617 & -0.326087\end{array}$

$\begin{array}{llll}-5.914450 & 0.057617 & 0.996463\end{array}$

$\begin{array}{llll}1.311239 & -0.416767 & 0.098021\end{array}$

$\begin{array}{llll}1.745853 & 0.630570 & 0.915020\end{array}$

$\begin{array}{llll}2.233653 & -1.073592 & -0.728559\end{array}$

$\begin{array}{lll}3.092588 & 0.984441 & 0.929057\end{array}$

$\begin{array}{lll}1.043770 & 1.147352 & 1.555346\end{array}$

$\begin{array}{llll}3.571615 & -0.694640 & -0.728720\end{array}$

$\begin{array}{llll}1.889251 & -1.866236 & -1.377328\end{array}$

$\begin{array}{llll}4.026774 & 0.336651 & 0.104243\end{array}$

$\begin{array}{lll}3.427400 & 1.762874 & 1.605699\end{array}$ 
$1 \quad 4.271995 \quad-1.214453 \quad-1.377291$

$\begin{array}{lllll}6 & 5.472671 & 0.761834 & 0.085078\end{array}$

$1 \quad 6.130412 \quad-0.055404 \quad-0.223659$

$1 \quad 5.619595 \quad 1.582528 \quad-0.627355$

$\begin{array}{llll}1 & 5.800828 & 1.116482 & 1.064738\end{array}$

\section{4a@12}

$6 \quad-9.014336 \quad-0.502400 \quad 3.582150$

$\begin{array}{lllll}6 & -8.406636 & -1.634719 & 2.748482\end{array}$

$\begin{array}{lllll}6 & -8.459735 & 0.857107 & 3.168878\end{array}$

$\begin{array}{lllll}1 & -8.680164 & -0.670758 & 4.606818\end{array}$

$\begin{array}{lllll}6 & -8.953292 & -2.079707 & 1.541203\end{array}$

$\begin{array}{lllll}6 & -7.265644 & -2.306945 & 3.202747\end{array}$

$\begin{array}{lllll}6 & -9.019984 & 1.590686 & 2.113846\end{array}$

$\begin{array}{lllll}6 & -7.345557 & 1.413607 & 3.809128\end{array}$

$\begin{array}{lllll}6 & -8.443171 & -3.172857 & 0.827452\end{array}$

$1 \quad-9.816685 \quad-1.560909 \quad 1.141654$

$\begin{array}{lllll}6 & -6.741620 & -3.417505 & 2.559995\end{array}$

$8 \quad-6.669191 \quad-1.856073 \quad 4.382282$

$\begin{array}{lllll}6 & -8.487404 & 2.798147 & 1.655295\end{array}$

$\begin{array}{lllll}1 & -9.897061 & 1.191260 & 1.618574\end{array}$

$\begin{array}{lllll}6 & -6.768002 & 2.603376 & 3.382800\end{array}$

$\begin{array}{lllll}8 & -6.783487 & 0.770880 & 4.926994\end{array}$

$\begin{array}{lllll}6 & -9.029945 & -3.611345 & -0.510939\end{array}$

$\begin{array}{lllll}6 & -7.350477 & -3.846069 & 1.386688\end{array}$

$\begin{array}{lllll}1 & -5.877224 & -3.933431 & 2.956124\end{array}$

$6 \quad-5.519826 \quad-1.142801 \quad 4.268831$

$\begin{array}{lllll}6 & -9.090560 & 3.598656 & 0.498688\end{array}$

$\begin{array}{lllll}6 & -7.329983 & 3.255684 & 2.296875\end{array}$

$\begin{array}{lllll}1 & -5.888040 & 2.997801 & 3.873290\end{array}$

$\begin{array}{lllll}6 & -5.559560 & 0.218059 & 4.684068\end{array}$

$\begin{array}{llll}-8.726704 & -4.648937 & -0.654892\end{array}$

$6 \quad-8.404974 \quad-2.817486-1.658012$

$8 \quad-6.853546 \quad-5.000808 \quad 0.761046$

$\begin{array}{lllll}6 & -8.476998 & 3.178341 & -0.836198\end{array}$

$\begin{array}{lllll}1 & -8.793275 & 4.636096 & 0.657985\end{array}$

$8 \quad-6.761091 \quad 4.442738 \quad 1.839174$

$6 \quad-8.917525 \quad-1.595640 \quad-2.096931$

$6 \quad-7.266264 \quad-3.296031 \quad-2.318002$

$\begin{array}{lllll}6 & -5.621486 & -4.840149 & 0.196489\end{array}$

$\begin{array}{lllll}6 & -3.337505 & -0.932812 & 3.871283\end{array}$

$\begin{array}{lllll}6 & -8.955610 & 2.070701 & -1.551120\end{array}$

$\begin{array}{lllll}6 & -7.395966 & 3.873649 & -1.392466\end{array}$

$\begin{array}{lllll}6 & -5.609187 & 4.378432 & 1.121311\end{array}$

$\begin{array}{lllll}6 & -3.348008 & 0.348075 & 4.405981\end{array}$

$6 \quad-8.366474 \quad-0.869011 \quad-3.159776$

$1 \quad-9.782163 \quad-1.186104 \quad-1.588782$

$\begin{array}{lllll}6 & -6.696235 & -2.635401 & -3.396537\end{array}$

$8 \quad-6.722507 \quad-4.508829 \quad-1.884835$

$6 \quad-5.562625 \quad-4.472955 \quad-1.177509$

$\begin{array}{lllll}6 & -8.382916 & 1.627432 & -2.746526\end{array}$

$\begin{array}{lllll}1 & -9.811483 & 1.535329 & -1.157038\end{array}$

$\begin{array}{lllll}6 & -6.765391 & 3.448483 & -2.556789\end{array}$

$8 \quad-6.906354 \quad 5.035739 \quad-0.772157$

$\begin{array}{lllll}6 & -5.669009 & 4.847385 & -0.219224\end{array}$

$\begin{array}{lllll}6 & -8.946237 & 0.476647 & -3.582418\end{array}$

$\begin{array}{lllll}6 & -7.256969 & -1.429669 & -3.806598\end{array}$ $\begin{array}{llll}1 & -5.823730 & -3.035901 & -3.895956\end{array}$

$\begin{array}{lllll}6 & -3.395512 & -4.702315 & 0.316833\end{array}$

$\begin{array}{lllll}6 & -7.251802 & 2.319629 & -3.193717\end{array}$

$\begin{array}{lllll}1 & -5.906947 & 3.980077 & -2.945181\end{array}$

$\begin{array}{lllll}6 & -3.414261 & 4.075513 & 0.890247\end{array}$

$1 \quad-8.602113 \quad 0.649661 \quad-4.602911$

$\begin{array}{lllll}8 & -6.684257 & -0.774747 & -4.910746\end{array}$

$6 \quad-3.352064 \quad-4.240183 \quad-0.990822$

$\begin{array}{lllll}8 & -6.630719 & 1.864750 & -4.359302\end{array}$

$\begin{array}{lllll}6 & -3.449544 & 4.680203 & -0.358708\end{array}$

$\begin{array}{lllll}6 & -5.477914 & -0.190947 & -4.639994\end{array}$

$\begin{array}{lllll}6 & -5.472148 & 1.169941 & -4.222758\end{array}$

$\begin{array}{lllll}6 & -3.268161 & -0.274414 & -4.327402\end{array}$

$\begin{array}{lllll}6 & -3.292474 & 1.002806 & -3.783626\end{array}$

$\begin{array}{lllll}6 & -10.482087 & 0.475783 & -3.610420\end{array}$

$1 \quad-10.849367 \quad 1.432401 \quad-3.990985$

$\begin{array}{lllll}1 & -10.928431 & 0.321874 & -2.624147\end{array}$

$6 \quad-10.564676 \quad-3.572143 \quad-0.531964$

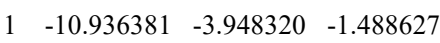

$\begin{array}{lllll}1 & -10.969674 & -2.565902 & -0.394672\end{array}$

$\begin{array}{lllll}6 & -10.549363 & -0.544504 & 3.587375\end{array}$

$\begin{array}{lllll}1 & -10.896239 & -1.513087 & 3.956616\end{array}$

$\begin{array}{lllll}1 & -10.985529 & -0.396408 & 2.595724\end{array}$

$\begin{array}{lllll}6 & -10.625112 & 3.554047 & 0.485375\end{array}$

$\begin{array}{lllll}1 & -11.020086 & 3.915414 & 1.438496\end{array}$

$\begin{array}{lllll}1 & -11.022793 & 2.548102 & 0.324421\end{array}$

$\begin{array}{lllll}1 & -11.010158 & 4.190818 & -0.315191\end{array}$

$\begin{array}{lllll}1 & -10.942622 & 0.238727 & 4.240622\end{array}$

$\begin{array}{lllll}1 & -10.965066 & -4.199751 & 0.268401\end{array}$

$1 \quad-10.844405 \quad-0.321762 \quad-4.264255$

$\begin{array}{lllll}6 & -1.983982 & -4.741363 & 0.847897\end{array}$

$\begin{array}{lllll}6 & -1.934595 & -3.877295 & -1.320435\end{array}$

$6 \begin{array}{llll}6 & -1.847393 & -0.771490 & -4.292440\end{array}$

$\begin{array}{lllll}6 & -1.917241 & 1.330159 & -3.270709\end{array}$

$\begin{array}{lllll}6 & -1.945870 & -1.241022 & 3.401413\end{array}$

$\begin{array}{lllll}6 & -1.933597 & 0.868422 & 4.401915\end{array}$

$\begin{array}{lllll}6 & -1.993850 & 3.683311 & 1.185839\end{array}$

$\begin{array}{lllll}6 & -2.035267 & 4.795403 & -0.865171\end{array}$

$\begin{array}{lllll}7 & -1.141329 & 0.240136 & -3.612869\end{array}$

$\begin{array}{lllll}7 & -1.201911 & -4.244414 & -0.208740\end{array}$

$7 \quad-1.190986 \quad-0.150259 \quad 3.768201$

$\begin{array}{lllll}7 & -1.255615 & 4.205634 & 0.144509\end{array}$

$\begin{array}{lllll}8 & -1.599059 & 3.034015 & 2.139738\end{array}$

$8 \quad-1.622720 \quad 5.281088-1.890211$

$\begin{array}{lllll}8 & -1.573125 & 2.327930 & -2.660678\end{array}$

$8 \quad-1.371631 \quad-1.783928 \quad-4.743334$

$8 \quad-1.536211 \quad-3.347971 \quad-2.341654$

$\begin{array}{lllll}8 & -1.577226 & -2.231742 & 2.788477\end{array}$

$\begin{array}{lllll}8 & -1.574854 & -5.108630 & 1.922265\end{array}$

$\begin{array}{lllll}8 & -1.485525 & 1.896011 & 4.844072\end{array}$

$1 \quad-0.238525 \quad 4.134336 \quad 0.048350$

$\begin{array}{llll}1 & -0.185636 & -0.040772 & 3.576814\end{array}$

$1-0.189626-4.111372-0.102030$

$1 \quad-0.129123 \quad 0.155591 \quad-3.462708$

$\begin{array}{llll}7 & -4.539279 & -4.986483 & 0.946541\end{array}$

$\begin{array}{lllll}7 & -4.430054 & -4.128561 & -1.770669\end{array}$

$7 \quad-4.418431 \quad-1.714142 \quad 3.806483$

$\begin{array}{llll}7 & -4.469533 & 0.962130 & 4.795415\end{array}$ $\begin{array}{lllll}7 & -4.370503 & -0.910784 & -4.734341\end{array}$

$\begin{array}{lllll}7 & -4.390395 & 1.760905 & -3.737775\end{array}$

$\begin{array}{lllll}7 & -4.490026 & 3.931239 & 1.669714\end{array}$

$\begin{array}{lllll}7 & -4.587876 & 5.043722 & -0.956778\end{array}$

$\begin{array}{lllll}6 & 8.986045 & 2.883565 & -2.176448\end{array}$

$\begin{array}{lllll}8.408961 & 1.608651 & -2.784306\end{array}$

$\begin{array}{lllll}6 & 8.366509 & 3.158832 & -0.806153\end{array}$

$\begin{array}{llll}8.669305 & 3.702099 & -2.823552\end{array}$

$\begin{array}{llll}8.958808 & 0.339919 & -2.557736\end{array}$

$\begin{array}{llll}7.265425 & 1.677104 & -3.589060\end{array}$

$\begin{array}{llll}8.870927 & 2.582885 & 0.362805\end{array}$

$\begin{array}{llll}7.259751 & 4.007142 & -0.662008\end{array}$

$\begin{array}{llll}8.395867 & -0.833176 & -3.072190\end{array}$

$\begin{array}{llll}9.858301 & 0.264076 & -1.957938\end{array}$

$\begin{array}{llll}6.652534 & 0.544428 & -4.104678\end{array}$

$\begin{array}{llll}6.751987 & 2.937957 & -3.915671\end{array}$

$\begin{array}{llll}8.350947 & 2.835417 & 1.637956\end{array}$

$\begin{array}{llll}9.707838 & 1.899711 & 0.278446\end{array}$

$\begin{array}{llll}6.722897 & 4.316273 & 0.580970\end{array}$

$\begin{array}{llll}6.704540 & 4.594778 & -1.803328\end{array}$

$\begin{array}{llll}9.000164 & -2.226793 & -2.866246\end{array}$

$\begin{array}{llll}7.219712 & -0.687640 & -3.817795\end{array}$

$\begin{array}{llll}5.755037 & 0.620351 & -4.703210\end{array}$

$\begin{array}{llll}5.536118 & 3.250480 & -3.388187\end{array}$

$\begin{array}{llll}8.936496 & 2.162615 & 2.877082\end{array}$

$\begin{array}{llll}7.277518 & 3.732639 & 1.716275\end{array}$

$\begin{array}{llll}5.879281 & 4.989921 & 0.664727\end{array}$

$\begin{array}{llll}5.514530 & 4.091894 & -2.241966\end{array}$

$\begin{array}{llll}8.674401 & -2.825111 & -3.718363\end{array}$

$8.424831-2.907546-1.624139$

$\begin{array}{llll}6.637940 & -1.844335 & -4.334435\end{array}$

$\begin{array}{llll}8.354180 & 0.764506 & 3.090580\end{array}$

$\begin{array}{llll}8.607220 & 2.758749 & 3.729296\end{array}$

$\begin{array}{llll}6.731356 & 4.078233 & 2.964542\end{array}$

$\begin{array}{llll}8.948566 & -2.666780 & -0.345611\end{array}$

$\begin{array}{llll}7.325992 & -3.774167 & -1.703368\end{array}$

$\begin{array}{llll}5.492399 & -2.297903 & -3.766768\end{array}$

$\begin{array}{llll}3.309667 & 3.113865 & -3.319986\end{array}$

$\begin{array}{llll}8.931809 & -0.406442 & 2.591222\end{array}$

$\begin{array}{llll}7.182727 & 0.615901 & 3.842177\end{array}$

$\begin{array}{llll}5.499379 & 3.522684 & 3.179291\end{array}$

$\begin{array}{llll}3.293171 & 3.896705 & -2.173729\end{array}$

$\begin{array}{llll}8.404066 & -3.202845 & 0.824903\end{array}$

$\begin{array}{llll}9.808622 & -2.013070 & -0.260379\end{array}$

$\begin{array}{llll}6.732716 & -4.315471 & -0.567702\end{array}$

$\begin{array}{llll}6.786901 & -4.129258 & -2.951544\end{array}$

$\begin{array}{llll}5.549474 & -3.587049 & -3.169809\end{array}$

$\begin{array}{llll}8.405071 & -1.683138 & 2.838390\end{array}$

$\begin{array}{llll}9.821952 & -0.325359 & 1.978459\end{array}$

$\begin{array}{llll}6.647131 & -0.620221 & 4.161249\end{array}$

$\begin{array}{llll}6.586996 & 1.773160 & 4.338495\end{array}$

$\begin{array}{llll}5.445547 & 2.225733 & 3.760792\end{array}$

$\begin{array}{llll}8.996967 & -2.944876 & 2.213079\end{array}$

$\begin{array}{llll}7.261829 & -4.000688 & 0.674290\end{array}$

$\begin{array}{llll}5.868607 & -4.961422 & -0.653256\end{array}$

$\begin{array}{llll}3.311770 & -2.215661 & -3.331269\end{array}$

$\begin{array}{llll}7.276205 & -1.754907 & 3.664309\end{array}$

$\begin{array}{llll}5.757324 & -0.701327 & 4.770755\end{array}$ 
$\begin{array}{llll}6 & 3.284098 & 3.473822 & 2.925094\end{array}$

$\begin{array}{lllll}1 & 8.672562 & -3.776660 & 2.839764\end{array}$

$\begin{array}{lllll}8 & 6.667130 & -4.535588 & 1.820498\end{array}$

$\begin{array}{lllll}6 & 3.335469 & -3.536834 & -2.905310\end{array}$

$8 \quad 6.754850 \quad-3.010336 \quad 4.017645$

$\begin{array}{llll}6 & 3.264024 & 2.144861 & 3.327736\end{array}$

$\begin{array}{lllll}6 & 5.497842 & -3.985997 & 2.244951\end{array}$

$\begin{array}{lllll}6 & 5.531333 & -3.266048 & 3.471342\end{array}$

$\begin{array}{lllll}6 & 3.301115 & -3.621349 & 2.125683\end{array}$

$\begin{array}{lllll}6 & 3.317579 & -3.008329 & 3.370158\end{array}$

$\begin{array}{lllll}6 & 10.532136 & -2.942309 & 2.205357\end{array}$

$\begin{array}{lllll}1 & 10.910273 & -2.819382 & 3.223535\end{array}$

$\begin{array}{lllll}1 & 10.955894 & -2.137874 & 1.597900\end{array}$

$\begin{array}{lllll}6 & 10.535304 & -2.210507 & -2.872702\end{array}$

$\begin{array}{lllll}1 & 10.920135 & -3.230429 & -2.793165\end{array}$

$1 \quad 10.960726 \quad-1.633662 \quad-2.046738$

$\begin{array}{lllll}6 & 10.520830 & 2.891233 & -2.138610\end{array}$

$\begin{array}{lllll}1 & 10.919506 & 2.747550 & -3.146271\end{array}$

$1 \quad 10.938296 \quad 2.104692 \quad-1.504010$

$\begin{array}{lllll}6 & 10.472223 & 2.168042 & 2.876957\end{array}$

$\begin{array}{llll}1 & 10.841732 & 3.192975 & 2.790332\end{array}$

$\begin{array}{lllll}1 & 10.902496 & 1.593117 & 2.052269\end{array}$

$\begin{array}{llll}1 & 10.848819 & 1.739061 & 3.809201\end{array}$

$\begin{array}{lllll}1 & 10.881981 & 3.848680 & -1.754490\end{array}$

$\begin{array}{lllll}1 & 10.902140 & -1.770989 & -3.803847\end{array}$

$\begin{array}{lllll}1 & 10.907347 & -3.888007 & 1.805933\end{array}$

$\begin{array}{lllll}6 & 1.914765 & -1.689559 & -3.168009\end{array}$

$\begin{array}{lllll}6 & 1.924530 & -3.952439 & -2.585179\end{array}$

$\begin{array}{lllll}6 & 1.900036 & -3.568286 & 1.591020\end{array}$

$\begin{array}{lllll}6 & 1.907647 & -2.605306 & 3.717087\end{array}$

$\begin{array}{lllll}6 & 1.889847 & 2.723195 & -3.645875\end{array}$

$6 \quad 1.876110 \quad 3.988066 \quad-1.680485$

$\begin{array}{lllll}6 & 1.870731 & 3.890899 & 2.613755\end{array}$

$\begin{array}{llll}1.865713 & 1.624719 & 3.157664\end{array}$

$\begin{array}{lllll}7 & 1.153362 & -2.969321 & 2.582458\end{array}$

$7 \quad 1.166840 \quad-2.778180 \quad-2.762343$

$\begin{array}{lllll}7 & 1.128884 & 3.279475 & -2.605042\end{array}$

$\begin{array}{llll}7 & 1.111075 & 2.720641 & 2.807057\end{array}$

$\begin{array}{lllll}8 & 1.438975 & 4.965550 & 2.277983\end{array}$

$\begin{array}{lllll}8 & 1.490223 & 0.466269 & 3.265058\end{array}$

$\begin{array}{lllll}8 & 1.472722 & -2.100681 & 4.721820\end{array}$

$\begin{array}{lllll}8 & 1.517554 & -3.943284 & 0.493715\end{array}$

$8 \quad 1.493182 \quad-5.031079 \quad-2.259812$

$\begin{array}{lllll}8 & 1.459500 & 2.085331 & -4.576377\end{array}$

$\begin{array}{llll}1.541455 & -0.538538 & -3.312561\end{array}$

$\begin{array}{lllll}8 & 1.481054 & 4.524559 & -0.662687\end{array}$

$\begin{array}{llll}0.107035 & 2.702615 & 2.574691\end{array}$

$1 \quad 0.122570 \quad 3.103410 \quad-2.525337$

$1 \quad 0.144612 \quad-2.794838 \quad-2.645820$

$\begin{array}{lllll}1 & 0.142054 & -2.790137 & 2.535197\end{array}$

$\begin{array}{lllll}7 & 4.385363 & -1.573902 & -3.797572\end{array}$

$7 \quad 4.467142 \quad-4.237921 \quad-2.775409$

$\begin{array}{lllll}7 & 4.435762 & 2.766689 & -3.946514\end{array}$

$\begin{array}{lllll}7 & 4.392568 & 4.406255 & -1.612107\end{array}$

$\begin{array}{lllll}7 & 4.385954 & -4.137912 & 1.541427\end{array}$

$\begin{array}{lllll}7 & 4.441122 & -2.799248 & 4.060829\end{array}$

$\begin{array}{llll}7 & 4.413391 & 4.177391 & 2.800521\end{array}$

$\begin{array}{llll}7 & 4.339416 & 1.498514 & 3.783020\end{array}$ $\begin{array}{lllll}6 & 0.831096 & 0.696180 & -0.031648\end{array}$

$\begin{array}{lllll}8 & 0.355489 & 1.806286 & 0.049100\end{array}$

$7 \quad-1.984362-1.113554 \quad-0.046474$

$\begin{array}{lllll}7 & -1.293606 & -0.169966 & 0.302227\end{array}$

$\begin{array}{lllll}8 & 0.067536 & -0.443555 & -0.109347\end{array}$

$\begin{array}{lllll}6 & -3.379481 & -0.919949 & 0.376165\end{array}$

$\begin{array}{lllll}1 & -3.452958 & -0.075303 & 1.069112\end{array}$

$\begin{array}{llll}1 & -3.670288 & -1.834272 & 0.902801\end{array}$

$6 \quad-4.252461 \quad-0.698848 \quad-0.858134$

$1 \quad-4.072973 \quad-1.501596 \quad-1.580309$

$\begin{array}{lllll}1 & -3.939990 & 0.237772 & -1.333189\end{array}$

$\begin{array}{lllll}6 & -5.738383 & -0.615832 & -0.505498\end{array}$

$\begin{array}{lllll}1 & -6.043994 & -1.525126 & 0.026801\end{array}$

$\begin{array}{lllll}1 & -6.312791 & -0.600600 & -1.433917\end{array}$

$\begin{array}{lllll}6 & -6.104365 & 0.615796 & 0.318319\end{array}$

$\begin{array}{lllll}1 & -5.820350 & 1.531866 & -0.210925\end{array}$

$\begin{array}{lllll}1 & -7.178620 & 0.656018 & 0.493027\end{array}$

$\begin{array}{lllll}1 & -5.614240 & 0.630404 & 1.296321\end{array}$

$\begin{array}{lllll}6 & 2.277481 & 0.401189 & -0.058950\end{array}$

$\begin{array}{lllll}6 & 2.782278 & -0.898168 & -0.187686\end{array}$

$\begin{array}{lllll}6 & 3.161999 & 1.478124 & 0.074737\end{array}$

$\begin{array}{lllll}6 & 4.155060 & -1.109075 & -0.192267\end{array}$

$\begin{array}{lllll}1 & 2.099273 & -1.729662 & -0.292344\end{array}$

$\begin{array}{lllll}6 & 4.533386 & 1.257527 & 0.079380\end{array}$

$\begin{array}{lllll}1 & 2.762237 & 2.477022 & 0.186824\end{array}$

$6 \quad 5.049361 \quad-0.037046 \quad-0.052490$

$1 \quad 4.542486 \quad-2.118088-0.299276$

$\begin{array}{lllll}1 & 5.213930 & 2.095225 & 0.189782\end{array}$

$\begin{array}{lllll}6 & 6.525433 & -0.286027 & -0.017839\end{array}$

$\begin{array}{lllll}1 & 6.822157 & -0.983733 & -0.800502\end{array}$

$\begin{array}{lllll}1 & 7.095581 & 0.634166 & -0.145067\end{array}$

$\begin{array}{llll}1 & 6.817276 & -0.729725 & 0.937173\end{array}$

\section{4a'@12}

$\begin{array}{lllll}6 & 8.951494 & -2.646853 & -2.363298\end{array}$

$\begin{array}{lllll}6 & 8.384808 & -3.040082 & -1.001515\end{array}$

$\begin{array}{lllll}6 & 8.342652 & -1.339489 & -2.855801\end{array}$

$1 \quad 8.626019 \quad-3.417550 \quad-3.062564$

$\begin{array}{lllll}6 & 8.915749 & -2.566244 & 0.198847\end{array}$

$6 \quad 7.292276 \quad-3.911132 \quad-0.907411$

$\begin{array}{lllll}6 & 8.877494 & -0.092577 & -2.511564\end{array}$

$6 \quad 7.213421 \quad-1.346831 \quad-3.683352$

$\begin{array}{lllll}6 & 8.435306 & -2.949594 & 1.457781\end{array}$

$\begin{array}{llll}9.735359 & -1.858420 & 0.156889\end{array}$

$\begin{array}{llll}6.808265 & -4.362707 & 0.310604\end{array}$

$\begin{array}{llll}6.705172 & -4.367359 & -2.090949\end{array}$

$\begin{array}{lllll}6 & 8.333746 & 1.114089 & -2.958053\end{array}$

$\begin{array}{llll}9.759871 & -0.064609 & -1.883221\end{array}$

$\begin{array}{llll}6.616600 & -0.174612 & -4.133812\end{array}$

$\begin{array}{llll}6.699658 & -2.576703 & -4.116091\end{array}$

$\begin{array}{llll}9.015128 & -2.347720 & 2.734200\end{array}$

$\begin{array}{llll}7.393943 & -3.886675 & 1.480245\end{array}$

$\begin{array}{llll}5.977214 & -5.055100 & 0.352316\end{array}$

$\begin{array}{llll}5.511079 & -3.825698 & -2.459826\end{array}$

$\begin{array}{llll}8.940950 & 2.478247 & -2.632436\end{array}$

$\begin{array}{llll}7.184878 & 1.032993 & -3.753077\end{array}$

$\begin{array}{llll}5.737661 & -0.205702 & -4.763964\end{array}$

$\begin{array}{lllll}6 & 5.501524 & -2.945003 & -3.579134\end{array}$ $\begin{array}{llll}8.706489 & -3.004297 & 3.548995\end{array}$

$\begin{array}{llll}8.400159 & -0.978956 & 3.039076\end{array}$

$\begin{array}{llll}6.878972 & -4.357147 & 2.704938\end{array}$

$\begin{array}{llll}8.359393 & 3.037661 & -1.338309\end{array}$

$\begin{array}{llll}8.621259 & 3.152139 & -3.427775\end{array}$

$\begin{array}{llll}6.643931 & 2.235199 & -4.217256\end{array}$

$\begin{array}{llll}8.952441 & 0.237097 & 2.628007\end{array}$

$\begin{array}{llll}7.227335 & -0.907998 & 3.799524\end{array}$

$\begin{array}{llll}5.630607 & -3.842791 & 2.937168\end{array}$

$\begin{array}{llll}3.287717 & -3.666060 & -2.349239\end{array}$

$\begin{array}{llll}8.883270 & 2.684104 & -0.089005\end{array}$

$\begin{array}{llll}7.274629 & 3.923841 & -1.347023\end{array}$

$\begin{array}{llll}5.483762 & 2.665060 & -3.663065\end{array}$

$\begin{array}{llll}3.271247 & -2.879275 & -3.492199\end{array}$

$\begin{array}{llll}8.406750 & 1.478330 & 2.985871\end{array}$

$\begin{array}{llll}9.843372 & 0.222881 & 2.011273\end{array}$

$\begin{array}{llll}6.674558 & 0.287729 & 4.226932\end{array}$

$\begin{array}{llll}6.646483 & -2.113464 & 4.182960\end{array}$

$\begin{array}{llll}5.545457 & -2.548511 & 3.522699\end{array}$

$\begin{array}{llll}8.367856 & 3.157946 & 1.119933\end{array}$

$\begin{array}{llll}9.731494 & 2.010393 & -0.060030\end{array}$

$\begin{array}{llll}6.704101 & 4.400762 & -0.171528\end{array}$

$\begin{array}{llll}6.752216 & 4.373362 & -2.571251\end{array}$

$\begin{array}{llll}5.527057 & 3.858932 & -2.887584\end{array}$

$\begin{array}{llll}8.979420 & 2.795266 & 2.473673\end{array}$

$\begin{array}{llll}7.285065 & 1.467854 & 3.822483\end{array}$

$\begin{array}{llll}5.786551 & 0.303201 & 4.843910\end{array}$

$\begin{array}{llll}3.428509 & -3.804289 & 2.597277\end{array}$

$\begin{array}{llll}7.247681 & 3.994068 & 1.039283\end{array}$

$\begin{array}{llll}5.851196 & 5.065971 & -0.202462\end{array}$

$\begin{array}{llll}3.279351 & 2.573556 & -3.354999\end{array}$

$\begin{array}{llll}8.653183 & 3.565727 & 3.172864\end{array}$

$\begin{array}{llll}6.755917 & 2.688511 & 4.277832\end{array}$

$\begin{array}{llll}3.396980 & -2.463757 & 2.953237\end{array}$

$\begin{array}{llll}6.679770 & 4.451787 & 2.235737\end{array}$

$\begin{array}{llll}3.300747 & 3.804043 & -2.714363\end{array}$

$\begin{array}{llll}5.540728 & 3.002135 & 3.748164\end{array}$

$\begin{array}{llll}5.514635 & 3.863914 & 2.613853\end{array}$

$\begin{array}{llll}3.327447 & 2.737461 & 3.585898\end{array}$

$\begin{array}{llll}3.322445 & 3.496832 & 2.425786\end{array}$

$\begin{array}{llll}10.514543 & 2.814677 & 2.448067\end{array}$

$\begin{array}{llll}10.871599 & 3.799179 & 2.135082\end{array}$

$\begin{array}{lll}10.941557 & 2.077586 & 1.762591\end{array}$

$\begin{array}{lll}10.550463 & -2.314816 & 2.724972\end{array}$

$\begin{array}{llll}10.924515 & -1.933840 & 3.678896\end{array}$

$\begin{array}{llll}10.960367 & -1.681845 & 1.933115\end{array}$

$10.487212 \quad-2.631880 \quad-2.374856$

$10.872740 \quad-3.611496 \quad-2.080827$

$10.914019-1.892719-1.691341$

$\begin{array}{llll}10.476417 & 2.459705 & -2.634110\end{array}$

$\begin{array}{llll}10.845259 & 2.112571 & -3.602748\end{array}$

$\begin{array}{llll}10.899262 & 1.804807 & -1.867364\end{array}$

$10.862098 \quad 3.466708 \quad-2.455287$

$10.851701-2.401446-3.379370$

$\begin{array}{llll}10.943289 & -3.323557 & 2.574614\end{array}$

$\begin{array}{lll}10.907300 & 2.603090 & 3.446004\end{array}$

$\begin{array}{llll}2.022672 & -4.232680 & 2.271512\end{array}$

$\begin{array}{llll}2.009918 & -1.944120 & 2.713780\end{array}$ 
$\begin{array}{llll}6 & 1.919793 & 2.253603 & 3.835811\end{array}$

$\begin{array}{lllll}6 & 1.949499 & 3.444563 & 1.828919\end{array}$

$\begin{array}{lllll}6 & 1.871475 & -3.852006 & -1.882285\end{array}$

$\begin{array}{lllll}6 & 1.834549 & -2.581272 & -3.837580\end{array}$

$\begin{array}{lllll}6 & 1.880823 & 2.032177 & -3.314231\end{array}$

$\begin{array}{lllll}6 & 1.881632 & 4.166209 & -2.356506\end{array}$

$\begin{array}{lllll}7 & 1.188300 & 2.729434 & 2.726824\end{array}$

$\begin{array}{lllll}7 & 1.262644 & -3.048979 & 2.394769\end{array}$

$\begin{array}{lllll}7 & 1.089545 & -3.257701 & -2.856033\end{array}$

$\begin{array}{lllll}7 & 1.128247 & 3.035593 & -2.728670\end{array}$

$\begin{array}{lllll}8 & 1.524370 & 0.923585 & -3.665853\end{array}$

$\begin{array}{lllll}8 & 1.446877 & 5.179814 & -1.867544\end{array}$

$\begin{array}{lllll}8 & 1.604129 & 3.878560 & 0.740777\end{array}$

$\begin{array}{lllll}8 & 1.476538 & 1.604307 & 4.749309\end{array}$

$\begin{array}{lllll}8 & 1.655788 & -0.770779 & 2.769631\end{array}$

$\begin{array}{lllll}8 & 1.504838 & -4.393847 & -0.855391\end{array}$

$\begin{array}{lllll}8 & 1.600465 & -5.322665 & 1.981668\end{array}$

$\begin{array}{lllll}8 & 1.383705 & -1.916121 & -4.738580\end{array}$

$\begin{array}{lllll}1 & 0.108071 & 3.031839 & -2.618096\end{array}$

$\begin{array}{lllll}1 & 0.079434 & -3.105395 & -2.783932\end{array}$

$\begin{array}{lllll}1 & 0.263103 & -3.0486675 & 2.176867\end{array}$

$\begin{array}{lllll}1 & 0.187656 & 2.515779 & 2.587209\end{array}$

$\begin{array}{lllll}7 & 4.563493 & -4.507889 & 2.529714\end{array}$

$\begin{array}{lllll}7 & 4.448545 & -1.811483 & 3.458634\end{array}$

$\begin{array}{lllll}7 & 4.405546 & -4.161419 & -1.811040\end{array}$

$\begin{array}{lllll}7 & 4.382679 & -2.480887 & -4.116064\end{array}$

$\begin{array}{lllll}7 & 4.446041 & 2.458345 & 4.260941\end{array}$

$\begin{array}{lllll}7 & 4.408221 & 4.086995 & 1.922465\end{array}$

$\begin{array}{lllll}7 & 4.365088 & 1.984746 & -3.859112\end{array}$

$\begin{array}{lllll}7 & 4.434002 & 4.457322 & -2.438336\end{array}$

$\begin{array}{lllll}6 & -8.998368 & 3.746212 & -0.048564\end{array}$

$\begin{array}{lllll}6 & -8.372874 & 3.206409 & 1.236332\end{array}$

$\begin{array}{lllll}6 & -8.396814 & 3.043184 & -1.268203\end{array}$

$\begin{array}{lllll}1 & -8.701301 & 4.793228 & -0.120548\end{array}$

$\begin{array}{lllll}6 & -8.854968 & 2.050894 & 1.868787\end{array}$

$\begin{array}{llll}6 & -7.255186 & 3.825012 & 1.810234\end{array}$

$\begin{array}{lllll}6 & -8.924898 & 1.865218 & -1.803208\end{array}$

$\begin{array}{lllll}6 & -7.254294 & 3.558938 & -1.893507\end{array}$

$\begin{array}{lllll}6 & -8.241571 & 1.483906 & 2.989321\end{array}$

$\begin{array}{lllll}1 & -9.736685 & 1.569215 & 1.462427\end{array}$

$\begin{array}{llll}6 & -6.589921 & 3.283413 & 2.902833\end{array}$

$\begin{array}{lllll}8 & -6.773241 & 5.032490 & 1.277491\end{array}$

$\begin{array}{lllll}6 & -8.380585 & 1.214104 & -2.917911\end{array}$

$\begin{array}{lllll}6 & -9.796377 & 1.429990 & -1.328556\end{array}$

$\begin{array}{lllll}6 & -6.684955 & 2.969151 & -3.013248\end{array}$

$\begin{array}{lllll}8 & -6.707570 & 4.740427 & -1.384503\end{array}$

$\begin{array}{lllll}6 & -8.782567 & 0.259116 & 3.729821\end{array}$

$\begin{array}{lllll}6 & -7.076710 & 2.108641 & 3.448466\end{array}$

$\begin{array}{llll}1 & -5.703675 & 3.757556 & 3.301962\end{array}$

$\begin{array}{lllll}6 & -5.551230 & 4.914586 & 0.680572\end{array}$

$\begin{array}{lllll}6 & -8.980879 & -0.079561 & -3.463631\end{array}$

$\begin{array}{lllll}6 & -7.258379 & 1.805226 & -3.513781\end{array}$

$\begin{array}{lllll}1 & -5.812526 & 3.400658 & -3.486355\end{array}$

$\begin{array}{lllll}6 & -5.526283 & 4.658790 & -0.718489\end{array}$

$\begin{array}{lllll}1 & -8.402038 & 0.333203 & 4.749540\end{array}$

$\begin{array}{llll}6 & -8.229316 & -1.047791 & 3.166930\end{array}$

$\begin{array}{lllll}8 & -6.410672 & 1.526940 & 4.528158\end{array}$

$\begin{array}{lllll}6 & -8.417625 & -1.314365 & -2.757160\end{array}$ $\begin{array}{lllll}1 & -8.652455 & -0.150804 & -4.501339\end{array}$

$\begin{array}{lllll}8 & -6.707626 & 1.245557 & -4.676761\end{array}$

$\begin{array}{lllll}6 & -8.823157 & -1.674531 & 2.064040\end{array}$

$\begin{array}{lllll}6 & -7.115490 & -1.684188 & 3.730821\end{array}$

$\begin{array}{lllll}6 & -5.280373 & 0.825447 & 4.257149\end{array}$

$\begin{array}{lllll}6 & -3.326812 & 4.739560 & 0.740973\end{array}$

$\begin{array}{lllll}6 & -8.973538 & -1.856287 & -1.594132\end{array}$

$\begin{array}{lllll}6 & -7.297542 & -1.970781 & -3.281284\end{array}$

$\begin{array}{lllll}6 & -5.505707 & 0.619092 & -4.499937\end{array}$

$\begin{array}{lllll}6 & -3.317432 & 4.394911 & -0.602624\end{array}$

$\begin{array}{llll}6 & -8.356301 & -2.870548 & 1.514655\end{array}$

$\begin{array}{llll}-9.692366 & -1.207579 & 1.616176\end{array}$

$\begin{array}{llll}6 & -6.596848 & -2.865153 & 3.208576\end{array}$

$8 \quad-6.492495 \quad-1.141248 \quad 4.869141$

$\begin{array}{llll}-5.288810 & -0.558009 & 4.586463\end{array}$

$\begin{array}{llll}-8.478132 & -3.014884 & -0.982324\end{array}$

$\begin{array}{llll}-9.822187 & -1.354485 & -1.143881\end{array}$

$\begin{array}{llll}-6.794684 & -3.145359 & -2.744578\end{array}$

$\begin{array}{llll}-6.697027 & -1.427910 & -4.417466\end{array}$

$\begin{array}{llll}-5.515486 & -0.778767 & -4.244848\end{array}$

$\begin{array}{llll}-9.042356 & -3.549549 & 0.329762\end{array}$

$\begin{array}{llll}-7.214066 & -3.431530 & 2.101559\end{array}$

$\begin{array}{llll}-5.725229 & -3.328254 & 3.653257\end{array}$

$\begin{array}{lll}-3.148118 & 0.643670 & 3.644595\end{array}$

$\begin{array}{llll}-7.403229 & -3.657820 & -1.606838\end{array}$

$\begin{array}{llll}-5.942369 & -3.640758 & -3.189863\end{array}$

$\begin{array}{llll}-3.286136 & 0.618488 & -4.276303\end{array}$

$\begin{array}{llll}-8.766068 & -4.603435 & 0.378681\end{array}$

$\begin{array}{lll}-6.727144 & -4.634766 & 1.586735\end{array}$

$\begin{array}{llll}-3.108176 & -0.656932 & 4.129205\end{array}$

$\begin{array}{llll}-6.934680 & -4.869371 & -1.081611\end{array}$

$\begin{array}{llll}-3.318434 & -0.713252 & -3.882273\end{array}$

$\begin{array}{llll}-5.574568 & -4.610406 & 0.860613\end{array}$

$\begin{array}{llll}-5.682669 & -4.814218 & -0.541959\end{array}$

$\begin{array}{llll}-3.355648 & -4.530828 & 0.652431\end{array}$

$\begin{array}{llll}6.457716 & -4.799741 & -0.706102\end{array}$

$\begin{array}{lllll}6 & -10.573311 & -3.468387 & 0.403942\end{array}$

$1 \quad-11.016736 \quad-4.019596 \quad-0.429355$

$\begin{array}{llll}-10.950807 & -2.443379 & 0.357840\end{array}$

$\begin{array}{lllll}6 & -10.316548 & 0.260300 & 3.811658\end{array}$

$\begin{array}{lllll}1 & -10.660838 & -0.592376 & 4.402599\end{array}$

$\begin{array}{lllll}1 & -10.797538 & 0.197582 & 2.831657\end{array}$

$\begin{array}{lllll}6 & -10.532418 & 3.700041 & -0.033084\end{array}$

$\begin{array}{lllll}1 & -10.914632 & 4.267299 & 0.819515\end{array}$

$\begin{array}{lllll}1 & -10.929275 & 2.684214 & 0.043830\end{array}$

$\begin{array}{lllll}6 & -10.516715 & -0.062527 & -3.467882\end{array}$

$\begin{array}{lllll}1 & -10.880791 & 0.799008 & -4.033670\end{array}$

$\begin{array}{lllll}1 & -10.946738 & -0.003606 & -2.464361\end{array}$

$\begin{array}{lllll}1 & -10.899128 & -0.973561 & -3.935433\end{array}$

$\begin{array}{lllll}1 & -10.930943 & 4.140010 & -0.950958\end{array}$

$\begin{array}{lllll}1 & -10.664554 & 1.179501 & 4.290081\end{array}$

$\begin{array}{lllll}1 & -10.928001 & -3.907547 & 1.340029\end{array}$

$\begin{array}{lllll}6 & -1.806472 & 0.971998 & 3.062487\end{array}$

$\begin{array}{lllll}6 & -1.702505 & -1.169766 & 3.979188\end{array}$

$\begin{array}{lllll}6 & -1.902574 & -4.339872 & 0.988633\end{array}$

$\begin{array}{lllll}6 & -2.065381 & -4.857946 & -1.281938\end{array}$

$\begin{array}{lllll}6 & -1.904902 & 4.710302 & 1.242712\end{array}$

$\begin{array}{lllll}6 & -1.914348 & 4.048569 & -0.997297\end{array}$ $\begin{array}{llll}-1.853787 & 1.076793 & -4.261932\end{array}$

$\begin{array}{llll}-1.925063 & -1.128997 & -3.493671\end{array}$

$\begin{array}{llll}-1.227508 & -4.579501 & -0.193376\end{array}$

$\begin{array}{llll}-1.019246 & -0.126830 & 3.321692\end{array}$

$\begin{array}{llll}-1.153617 & 4.284488 & 0.129568\end{array}$

$\begin{array}{llll}-1.135232 & -0.035030 & -3.795734\end{array}$

$\begin{array}{llll}-1.384278 & 2.146685 & -4.570868\end{array}$

$\begin{array}{llll}-1.575631 & -2.182934 & -2.994570\end{array}$

$\begin{array}{llll}-1.708320 & -5.090551 & -2.412626\end{array}$

$\begin{array}{llll}-1.427048 & -4.002469 & 2.056114\end{array}$

$\begin{array}{llll}-1.215943 & -2.215452 & 4.330997\end{array}$

$\begin{array}{llll}-1.460774 & 4.989936 & 2.328828\end{array}$

$\begin{array}{llll}-1.478843 & 1.993483 & 2.474786\end{array}$

$\begin{array}{llll}-1.543925 & 3.611628 & -2.073260\end{array}$

$\begin{array}{llll}-0.126356 & 0.030474 & -3.647656\end{array}$

$\begin{array}{llll}-0.139174 & 4.151106 & 0.186474\end{array}$

$\begin{array}{llll}-0.038784 & -0.244162 & 3.033726\end{array}$

$\begin{array}{llll}-0.208605 & -4.505735 & -0.301685\end{array}$

$\begin{array}{llll}-4.225839 & 1.425148 & 3.726226\end{array}$

$\begin{array}{llll}-4.191371 & -1.298550 & 4.574748\end{array}$

$\begin{array}{llll}-4.452106 & 4.989196 & 1.416546\end{array}$

$\begin{array}{llll}-4.411083 & 4.363182 & -1.366992\end{array}$

$\begin{array}{llll}-4.409631 & -4.438111 & 1.467328\end{array}$

$\begin{array}{llll}-4.627611 & -4.931323 & -1.334655\end{array}$

$\begin{array}{llll}-4.385312 & 1.323194 & -4.557746\end{array}$

$\begin{array}{llll}-4.431394 & -1.447969 & -3.881488\end{array}$

$\begin{array}{llll}-0.599211 & -0.636728 & -0.163679\end{array}$

$\begin{array}{llll}-0.131769 & -1.624816 & 0.410230\end{array}$

$\begin{array}{llll}2.602806 & -0.335861 & -0.796283\end{array}$

$\begin{array}{lll}1.648210 & -0.819883 & -1.230486\end{array}$

$\begin{array}{llll}0.118659 & 0.197991 & -0.876267\end{array}$

$\begin{array}{llll}2.864172 & 0.870174 & 0.007776\end{array}$

$\begin{array}{llll}2.667538 & 0.553515 & 1.039680\end{array}$

$\begin{array}{llll}-2.063691 & -0.329363 & -0.097051\end{array}$

$\begin{array}{llll}-2.642804 & 0.708025 & -0.835047\end{array}$

$\begin{array}{llll}-2.880268 & -1.131114 & 0.708014\end{array}$

$\begin{array}{llll}-4.019273 & 0.915053 & -0.791692\end{array}$

$\begin{array}{llll}-2.010861 & 1.342426 & -1.441325\end{array}$

$\begin{array}{llll}-4.252615 & -0.913292 & 0.757127\end{array}$

$\begin{array}{llll}-2.422771 & -1.911526 & 1.299709\end{array}$

$\begin{array}{llll}-4.845752 & 0.105913 & 0.001153\end{array}$

$\begin{array}{llll}-4.458020 & 1.710410 & -1.386259\end{array}$

$\begin{array}{llll}-4.873945 & -1.544048 & 1.385762\end{array}$

$\begin{array}{lll}-6.333510 & 0.309589 & 0.022429\end{array}$

$\begin{array}{llll}-6.592613 & 1.368099 & -0.024580\end{array}$

$\begin{array}{llll}-6.806216 & -0.176617 & -0.836526\end{array}$

$\begin{array}{llll}-6.783911 & -0.112031 & 0.922022\end{array}$

$\begin{array}{llll}4.291029 & 1.371417 & -0.199343\end{array}$

$\begin{array}{llll}4.519639 & 1.349063 & -1.269104\end{array}$

$\begin{array}{llll}4.303098 & 2.424237 & 0.092433\end{array}$

$\begin{array}{lll}5.366040 & 0.597372 & 0.572825\end{array}$

$\begin{array}{lll}5.117864 & 0.591365 & 1.641919\end{array}$

$\begin{array}{rrrr}6.311327 & 1.141889 & 0.476359\end{array}$

$\begin{array}{lll}5.570910 & -0.840867 & 0.089125\end{array}$

$\begin{array}{llll}4.687719 & -1.465357 & 0.254524\end{array}$

$\begin{array}{llll}5.795741 & -0.863012 & -0.982917\end{array}$

$\begin{array}{llll}2.095246 & 1.586324 & -0.289772\end{array}$

$\begin{array}{llll}6.407900 & -1.306879 & 0.611548\end{array}$ 


\section{5:6a@12}

$\begin{array}{llll}6 & -8.963294 & 2.657633 & 2.438269\end{array}$

$\begin{array}{lllll}6 & -8.341053 & 1.324673 & 2.855374\end{array}$

$\begin{array}{lllll}6 & -8.374808 & 3.139503 & 1.115537\end{array}$

$\begin{array}{lllll}1 & -8.661213 & 3.385298 & 3.192525\end{array}$

$\begin{array}{lllll}6 & -8.895625 & 0.079185 & 2.549461\end{array}$

$\begin{array}{lllll}6 & -7.153063 & 1.323494 & 3.595490\end{array}$

$\begin{array}{lllll}6 & -8.855942 & 2.651827 & -0.104098\end{array}$

$\begin{array}{lllll}6 & -7.329543 & 4.070581 & 1.057048\end{array}$

$\begin{array}{lllll}6 & -8.330899 & -1.127582 & 2.982020\end{array}$

$\begin{array}{llll}1 & -9.807352 & 0.043714 & 1.965182\end{array}$

$\begin{array}{lllll}6 & -6.564307 & 0.162303 & 4.074448\end{array}$

$\begin{array}{lllll}8 & -6.619610 & 2.568868 & 3.921886\end{array}$

$\begin{array}{lllll}6 & -8.342967 & 3.034058 & -1.345278\end{array}$

$\begin{array}{lllll}1 & -9.671267 & 1.938092 & -0.084590\end{array}$

$\begin{array}{lllll}6 & -6.757322 & 4.456024 & -0.151080\end{array}$

$\begin{array}{lllll}8 & -6.834218 & 4.658107 & 2.236848\end{array}$

$\begin{array}{lllll}6 & -8.923885 & -2.486760 & 2.627448\end{array}$

$\begin{array}{lllll}6 & -7.175343 & -1.047702 & 3.766899\end{array}$

$1 \quad-5.661962 \quad 0.196265 \quad 4.669720$

$\begin{array}{lllll}6 & -5.487272 & 3.008006 & 3.324189\end{array}$

$\begin{array}{lllll}6 & -8.939994 & 2.518810 & -2.654596\end{array}$

$\begin{array}{lllll}6 & -7.260842 & 3.923546 & -1.330498\end{array}$

$\begin{array}{lllll}1 & -5.929692 & 5.153883 & -0.171257\end{array}$

$\begin{array}{lllll}6 & -5.585495 & 4.214754 & 2.572295\end{array}$

$\begin{array}{llll}1 & -8.588317 & -3.178558 & 3.400462\end{array}$

$\begin{array}{lllll}6 & -8.357455 & -3.013295 & 1.309912\end{array}$

$8 \begin{array}{llll}8 & -6.646125 & -2.235499 & 4.297440\end{array}$

$\begin{array}{lllll}6 & -8.367314 & 1.150588 & -3.010368\end{array}$

$1 \quad-8.604884 \quad 3.204955 \quad-3.432870$

$8 \begin{array}{llll}8 & -6.685972 & 4.317324 & -2.547799\end{array}$

$\begin{array}{lllll}6 & -8.878628 & -2.632145 & 0.072412\end{array}$

$\begin{array}{lllll}6 & -7.291070 & -3.920561 & 1.288664\end{array}$

$\begin{array}{lllll}6 & -5.462864 & -2.638455 & 3.761476\end{array}$

$\begin{array}{lllll}6 & -3.290008 & 3.007959 & 2.983311\end{array}$

$6 \quad-8.945515 \quad-0.052659 \quad-2.585795$

$6 \begin{array}{lllll}6 & -7.196978 & 1.066310 & -3.772491\end{array}$

$\begin{array}{lllll}6 & -5.484326 & 3.745717 & -2.845672\end{array}$

$\begin{array}{lllll}6 & -3.361506 & 4.264262 & 2.400600\end{array}$

$\begin{array}{lllll}6 & -8.417832 & -3.136114 & -1.150040\end{array}$

$\begin{array}{lllll}1 & -9.680015 & -1.902560 & 0.055667\end{array}$

$6 \quad-6.824316 \quad-4.487606 \quad 0.112498$

$8 \quad-6.679776 \quad-4.264472 \quad 2.503955$

$\begin{array}{lllll}6 & -5.488894 & -3.659349 & 2.766271\end{array}$

$6 \begin{array}{llll}6 & -8.385110 & -1.300296 & -2.875709\end{array}$

$\begin{array}{lllll}1 & -9.867465 & -0.012183 & -2.017900\end{array}$

$6 \quad-6.584832 \quad-0.144893 \quad-4.069866$

$8 \quad-6.661622 \quad 2.254003 \quad-4.286031$

$6 \quad-5.471562 \quad 2.664620 \quad-3.772504$

$\begin{array}{lllll}6 & -9.007713 & -2.637178 & -2.467125\end{array}$

$\begin{array}{lllll}6 & -7.396137 & -4.094602 & -1.095247\end{array}$

$\begin{array}{lllll}1 & -6.005542 & -5.195488 & 0.126679\end{array}$

$\begin{array}{llll}6 & -3.251030 & -2.467428 & 3.512079\end{array}$

$6 \begin{array}{llll}6 & -7.185735 & -1.301825 & -3.597014\end{array}$

$1 \quad-5.669540 \quad-0.182183 \quad-4.644415$

$6 \quad-3.267776 \quad 3.570346 \quad-2.645785$

$\begin{array}{llll}1 & -8.709362 & -3.358328 & -3.229260\end{array}$ $\begin{array}{lllll}8 & -6.891313 & -4.676823 & -2.272764\end{array}$

$\begin{array}{lllll}6 & -3.287875 & -3.402623 & 2.488736\end{array}$

$8 \quad-6.647982 \quad-2.548686 \quad-3.905024$

$\begin{array}{lllll}6 & -3.254998 & 2.529085 & -3.563947\end{array}$

$6 \quad-5.634724 \quad-4.214006 \quad-2.571447$

$\begin{array}{lllll}6 & -5.531172 & -2.980240 & -3.273387\end{array}$

$\begin{array}{lllll}6 & -3.416080 & -4.231369 & -2.329257\end{array}$

$6 \quad-3.350515 \quad-2.941187 \quad-2.834315$

$\begin{array}{lllll}6 & -10.542374 & -2.598422 & -2.443935\end{array}$

$1-10.925411 \quad-2.287219 \quad-3.419453$

$\begin{array}{lllll}1 & -10.941698 & -1.907540 & -1.696237\end{array}$

$\begin{array}{lllll}6 & -10.459860 & -2.492783 & 2.638347\end{array}$

$\begin{array}{lllll}1 & -10.830407 & -3.503362 & 2.448525\end{array}$

$\begin{array}{lllll}1 & -10.895944 & -1.836652 & 1.880083\end{array}$

$\begin{array}{llll}6 & -10.497992 & 2.620121 & 2.422979\end{array}$

$\begin{array}{llll}1 & -10.876801 & 2.320681 & 3.403851\end{array}$

$\begin{array}{lllll}1 & -10.902397 & 1.921501 & 1.685336\end{array}$

$\begin{array}{lllll}6 & -10.475118 & 2.546224 & -2.643812\end{array}$

$\begin{array}{lllll}1 & -10.829208 & 3.561801 & -2.449333\end{array}$

$\begin{array}{lllll}1 & -10.910092 & 1.896406 & -1.879415\end{array}$

$\begin{array}{lllll}1 & -10.862885 & 2.222083 & -3.613271\end{array}$

$\begin{array}{lllll}1 & -10.892203 & 3.611429 & 2.185518\end{array}$

$1-10.829340 \quad-2.163665 \quad 3.613170$

$\begin{array}{lllll}1 & -10.937135 & -3.591761 & -2.216001\end{array}$

$\begin{array}{lllll}6 & -1.827867 & -2.008567 & 3.683110\end{array}$

$\begin{array}{lllll}6 & -1.903451 & -3.537946 & 1.920105\end{array}$

$6 \begin{array}{llll}6 & -2.011377 & -4.672754 & -2.004759\end{array}$

$6 \quad-1.941528 \quad-2.441015 \quad-2.689646$

$\begin{array}{lllll}6 & -1.881577 & 2.510070 & 2.876519\end{array}$

$\begin{array}{lllll}6 & -1.962120 & 4.681354 & 2.024773\end{array}$

$\begin{array}{lllll}6 & -1.868509 & 3.758797 & -2.127110\end{array}$

$\begin{array}{lllll}6 & -1.841633 & 2.040525 & -3.711526\end{array}$

$7 \quad-1.219354 \quad-3.539125 \quad-2.273422$

$\begin{array}{lllll}7 & -1.116934 & -2.689768 & 2.681105\end{array}$

$\begin{array}{lllll}7 & -1.162451 & 3.564689 & 2.358307\end{array}$

$\begin{array}{lllll}7 & -1.108460 & 2.814958 & -2.799105\end{array}$

$8 \quad-1.481675 \quad 4.546257 \quad-1.282786$

$8 \begin{array}{lllll}8 & -1.394019 & 1.181955 & -4.434198\end{array}$

$\begin{array}{lllll}8 & -1.563342 & -1.294199 & -2.859136\end{array}$

$8 \quad-1.615292 \quad-5.742886 \quad-1.614709$

$\begin{array}{lllll}8 & -1.561301 & -4.233376 & 0.981836\end{array}$

$\begin{array}{lllll}8 & -1.499381 & 1.376688 & 3.121221\end{array}$

$\begin{array}{lllll}8 & -1.364630 & -1.227317 & 4.479145\end{array}$

$\begin{array}{llll}8 & -1.565401 & 5.712885 & 1.544465\end{array}$

$\begin{array}{lllll}1 & -0.102810 & 2.655897 & -2.672744\end{array}$

$\begin{array}{lllll}1 & -0.151070 & 3.565244 & 2.182276\end{array}$

$\begin{array}{llll}1 & -0.106354 & -2.548688 & 2.579219\end{array}$

$\begin{array}{lllll}1 & -0.207022 & -3.560188 & -2.126355\end{array}$

$7 \quad-4.342943 \quad-2.050674 \quad 4.156362$

$\begin{array}{lllll}7 & -4.403524 & -4.024363 & 2.099208\end{array}$

$\begin{array}{llll}7 & -4.345349 & 2.356483 & 3.476395\end{array}$

$\begin{array}{llll}7 & -4.522239 & 4.877784 & 2.146845\end{array}$

$7 \quad-4.574009 \quad-4.876053 \quad-2.140758$

$7 \quad-4.401760 \quad-2.295059 \quad-3.344473$

$\begin{array}{lllll}7 & -4.382029 & 4.202970 & -2.270936\end{array}$

$7 \quad-4.357162 \quad 2.052920 \quad-4.145181$

$6 \quad 8.845678 \quad-0.166597 \quad-3.612239$

$\begin{array}{lllll}6 & 8.270832 & -1.405782 & -2.931119\end{array}$ $\begin{array}{llll}6 & 8.321923 & 1.133659 & -3.001810\end{array}$

$1 \quad 8.472470 \quad-0.187658 \quad-4.637001$

$\begin{array}{lllll}6 & 8.851427 & -1.941277 & -1.774997\end{array}$

$6 \quad 7.151726 \quad-2.074173 \quad-3.445431$

$\begin{array}{lllll}6 & 8.933408 & 1.786967 & -1.928283\end{array}$

$\begin{array}{lllll}6 & 7.183279 & 1.744104 & -3.539897\end{array}$

$\begin{array}{lllll}6 & 8.372464 & -3.086033 & -1.133846\end{array}$

$\begin{array}{lllll}1 & 9.719219 & -1.442614 & -1.360112\end{array}$

$\begin{array}{lllll}6 & 6.626624 & -3.210898 & -2.838782\end{array}$

$\begin{array}{lllll}8 & 6.540551 & -1.611379 & -4.623007\end{array}$

$\begin{array}{lllll}6 & 8.477297 & 3.011249 & -1.419372\end{array}$

$\begin{array}{lllll}1 & 9.793745 & 1.322748 & -1.459865\end{array}$

$\begin{array}{lllll}6 & 6.725659 & 2.980811 & -3.118947\end{array}$

$\begin{array}{lllll}8 & 6.512071 & 1.081649 & -4.568844\end{array}$

$\begin{array}{lllll}6 & 9.033124 & -3.655538 & 0.120910\end{array}$

$\begin{array}{lllll}6 & 7.238498 & -3.693640 & -1.689005\end{array}$

$\begin{array}{lllll}1 & 5.756277 & -3.704522 & -3.252105\end{array}$

$\begin{array}{lllll}6 & 5.349112 & -0.977810 & -4.404559\end{array}$

$\begin{array}{lllll}6 & 9.090282 & 3.649674 & -0.175698\end{array}$

$\begin{array}{lllll}6 & 7.391009 & 3.605898 & -2.071914\end{array}$

$\begin{array}{lllll}1 & 5.861244 & 3.439863 & -3.579429\end{array}$

$\begin{array}{lllll}6 & 5.363128 & 0.434600 & -4.240863\end{array}$

$\begin{array}{lllll}1 & 8.748899 & -4.707228 & 0.167826\end{array}$

$\begin{array}{llll}6 & 8.445677 & -2.992058 & 1.362587\end{array}$

$\begin{array}{lllll}8 & 6.749471 & -4.857018 & -1.088543\end{array}$

$\begin{array}{llll}6 & 8.446148 & 3.067912 & 1.084406\end{array}$

$\begin{array}{lllll}1 & 8.815209 & 4.704803 & -0.201873\end{array}$

$\begin{array}{lllll}8 & 6.941462 & 4.872836 & -1.664219\end{array}$

$\begin{array}{lllll}6 & 8.940565 & -1.785758 & 1.873662\end{array}$

$\begin{array}{lllll}6 & 7.345922 & -3.560211 & 2.016034\end{array}$

$\begin{array}{lllll}6 & 5.575902 & -4.777050 & -0.402204\end{array}$

$\begin{array}{lllll}6 & 3.161916 & -0.982262 & -3.970789\end{array}$

$\begin{array}{lllll}6 & 8.943750 & 1.936376 & 1.735778\end{array}$

$\begin{array}{lllll}6 & 7.297745 & 3.654284 & 1.632856\end{array}$

$\begin{array}{lllll}6 & 5.699685 & 4.848091 & -1.099044\end{array}$

$\begin{array}{lllll}6 & 3.213407 & 0.369567 & -3.659949\end{array}$

$\begin{array}{lllll}6 & 8.363851 & -1.130212 & 2.966173\end{array}$

$\begin{array}{lllll}1 & 9.807580 & -1.340639 & 1.399283\end{array}$

$\begin{array}{lllll}6 & 6.714626 & -2.934677 & 3.082198\end{array}$

$8 \quad 6.876854 \quad-4.810806 \quad 1.593515$

$\begin{array}{lllll}6 & 5.640960 & -4.808330 & 1.017755\end{array}$

$\begin{array}{lllll}6 & 8.368841 & 1.401926 & 2.895954\end{array}$

$\begin{array}{llll}1 & 9.817575 & 1.445226 & 1.324559\end{array}$

$\begin{array}{llll}6 & 6.700241 & 3.182675 & 2.792844\end{array}$

$\begin{array}{lllll}8 & 6.784062 & 4.793806 & 1.008586\end{array}$

$\begin{array}{lllll}6 & 5.622017 & 4.691658 & 0.313336\end{array}$

$\begin{array}{lllll}6 & 8.932599 & 0.154523 & 3.571617\end{array}$

$\begin{array}{lllll}6 & 7.220778 & -1.720436 & 3.518503\end{array}$

$\begin{array}{lllll}1 & 5.843527 & -3.375068 & 3.548309\end{array}$

$\begin{array}{lllll}6 & 3.351864 & -4.699955 & -0.272457\end{array}$

$\begin{array}{llll}6 & 7.249418 & 2.066150 & 3.413905\end{array}$

$\begin{array}{lllll}1 & 5.826502 & 3.669924 & 3.205492\end{array}$

$\begin{array}{lllll}6 & 3.473460 & 4.769548 & -1.219406\end{array}$

$\begin{array}{lllll}1 & 8.578102 & 0.183665 & 4.602706\end{array}$

$\begin{array}{lllll}8 & 6.595194 & -1.063394 & 4.579787\end{array}$

$\begin{array}{lllll}6 & 3.411433 & -4.777096 & 1.112397\end{array}$

$\begin{array}{lllll}8 & 6.668246 & 1.624259 & 4.613607\end{array}$

$\begin{array}{llll}6 & 3.406671 & 4.514719 & 0.142415\end{array}$ 
$\begin{array}{llll}6 & 5.442242 & -0.401345 & 4.300379\end{array}$

$\begin{array}{llll}6 & 5.457778 & 1.012159 & 4.449276\end{array}$

$\begin{array}{llll}6 & 3.271609 & -0.307144 & 3.809836\end{array}$

$\begin{array}{lllll}6 & 3.254726 & 1.049796 & 4.105491\end{array}$

$\begin{array}{lllll}6 & 10.467993 & 0.147738 & 3.613369\end{array}$

$\begin{array}{lllll}1 & 10.833156 & 1.050151 & 4.110506\end{array}$

$\begin{array}{lllll}1 & 10.923137 & 0.111556 & 2.619870\end{array}$

$\begin{array}{lllll}6 & 10.565624 & -3.590427 & 0.069204\end{array}$

$\begin{array}{lllll}1 & 10.989490 & -4.059236 & 0.961067\end{array}$

$\begin{array}{llll}1 & 10.950085 & -2.568055 & 0.021061\end{array}$

$\begin{array}{lllll}6 & 10.380252 & -0.196512 & -3.682094\end{array}$

$\begin{array}{lllll}1 & 10.714663 & -1.108028 & -4.184140\end{array}$

$1 \quad 10.853806 \quad-0.170068 \quad-2.696819$

$\begin{array}{lllll}6 & 10.622660 & 3.569846 & -0.152990\end{array}$

$1 \quad 11.034626 \quad 4.055230 \quad-1.041473$

$\begin{array}{lllll}1 & 10.997461 & 2.542957 & -0.136442\end{array}$

$\begin{array}{lllll}1 & 11.012840 & 4.077015 & 0.733229\end{array}$

$\begin{array}{lllll}1 & 10.745551 & 0.666085 & -4.245435\end{array}$

$1 \quad 10.934405 \quad-4.121918 \quad-0.811911$

$\begin{array}{lllll}1 & 10.823009 & -0.724166 & 4.168847\end{array}$

$\begin{array}{lllll}6 & 1.912928 & -4.563931 & -0.678865\end{array}$

$\begin{array}{lllll}6 & 2.000200 & -4.749224 & 1.646869\end{array}$

$\begin{array}{llll}6 & 1.896572 & -0.712293 & 3.359104\end{array}$

$\begin{array}{lllll}6 & 1.840610 & 1.542128 & 3.971818\end{array}$

$\begin{array}{lllll}6 & 1.747173 & -1.452660 & -3.785606\end{array}$

$\begin{array}{lllll}6 & 1.860611 & 0.795216 & -3.163913\end{array}$

$\begin{array}{lllll}6 & 2.068904 & 4.793343 & -1.763851\end{array}$

$\begin{array}{lllll}6 & 1.971111 & 4.284394 & 0.512186\end{array}$

$\begin{array}{lllll}7 & 1.122766 & 0.420559 & 3.521373\end{array}$

$\begin{array}{lllll}7 & 1.197715 & -4.637804 & 0.501579\end{array}$

$\begin{array}{lllll}7 & 1.062827 & -0.324165 & -3.296901\end{array}$

$\begin{array}{lllll}7 & 1.257232 & 4.509760 & -0.646780\end{array}$

$\begin{array}{lllll}8 & 1.678633 & 5.005935 & -2.884930\end{array}$

$\begin{array}{lllll}8 & 1.541801 & 3.932709 & 1.598262\end{array}$

$\begin{array}{lllll}8 & 1.380175 & 2.636241 & 4.193272\end{array}$

$\begin{array}{lllll}8 & 1.553239 & -1.791207 & 2.907329\end{array}$

$\begin{array}{lllll}8 & 1.606044 & -4.803988 & 2.787330\end{array}$

$\begin{array}{lllll}8 & 1.262911 & -2.536746 & -4.001244\end{array}$

$\begin{array}{lllll}8 & 1.476861 & -4.377416 & -1.798894\end{array}$

$\begin{array}{lllll}8 & 1.549614 & 1.886602 & -2.714411\end{array}$

$1 \quad 0.229816 \quad 4.510972 \quad-0.721754$

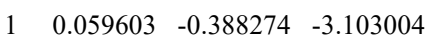

$\begin{array}{lllll}1 & 0.178817 & -4.521342 & 0.556377\end{array}$

$\begin{array}{lllll}1 & 0.122291 & 0.496710 & 3.325004\end{array}$

$\begin{array}{lllll}7 & 4.429301 & -4.700374 & -1.060607\end{array}$

$\begin{array}{lllll}7 & 4.562123 & -4.822088 & 1.787536\end{array}$

$7 \quad 4.238874 \quad-1.693214 \quad-4.312330$

$\begin{array}{lllll}7 & 4.309985 & 1.114078 & -3.810483\end{array}$

$\begin{array}{llll}7 & 4.361030 & -1.067972 & 3.924508\end{array}$

$\begin{array}{lllll}7 & 4.356800 & 1.745880 & 4.395563\end{array}$

$\begin{array}{lllll}7 & 4.628341 & 4.917303 & -1.873989\end{array}$

$\begin{array}{lllll}7 & 4.475939 & 4.483137 & 0.942727\end{array}$

$\begin{array}{llll}6 & 0.566399 & -0.694744 & 0.047309\end{array}$

$\begin{array}{lllll}8 & -0.184667 & 0.328686 & 0.492655\end{array}$

$\begin{array}{lllll}7 & -3.265763 & 1.214260 & 0.388341\end{array}$

$\begin{array}{lllll}7 & -3.254006 & 2.290122 & -0.000657\end{array}$

$\begin{array}{lllll}8 & 0.085385 & -1.775980 & -0.251790\end{array}$

$\begin{array}{llll}6 & -3.237702 & -0.027648 & 0.775223\end{array}$ $\begin{array}{llll}1 & -3.107533 & -0.142932 & 1.846442\end{array}$

$\begin{array}{lllll}1 & -1.119448 & 0.021040 & 0.527273\end{array}$

$\begin{array}{lllll}6 & -3.949376 & -1.034412 & -0.101215\end{array}$

$\begin{array}{lllll}1 & -3.490974 & -2.014342 & 0.062520\end{array}$

$\begin{array}{lllll}1 & -3.760829 & -0.778023 & -1.148660\end{array}$

$\begin{array}{lllll}6 & -5.464796 & -1.103302 & 0.152910\end{array}$

$\begin{array}{llll}1 & -5.645166 & -1.383788 & 1.195444\end{array}$

$\begin{array}{lllll}1 & -5.880121 & -1.910019 & -0.457991\end{array}$

$\begin{array}{lllll}6 & -6.204715 & 0.198801 & -0.148917\end{array}$

$\begin{array}{lllll}1 & -6.016563 & 0.533757 & -1.173816\end{array}$

$\begin{array}{llll}1 & -5.912278 & 1.004252 & 0.530968\end{array}$

$\begin{array}{lllll}6 & 2.015221 & -0.379709 & -0.013060\end{array}$

$\begin{array}{lllll}6 & 2.876503 & -1.304458 & -0.614019\end{array}$

$\begin{array}{lllll}6 & 2.546863 & 0.788104 & 0.548365\end{array}$

$\begin{array}{lllll}6 & 4.247931 & -1.079197 & -0.631623\end{array}$

$\begin{array}{lllll}1 & 2.454449 & -2.179543 & -1.088781\end{array}$

$\begin{array}{lllll}6 & 3.923529 & 0.994536 & 0.550371\end{array}$

$\begin{array}{lllll}1 & 1.883788 & 1.517483 & 0.993988\end{array}$

$\begin{array}{lllll}6 & 4.795702 & 0.063484 & -0.032954\end{array}$

$\begin{array}{lllll}1 & 4.903468 & -1.797250 & -1.114231\end{array}$

$\begin{array}{llll}1 & 4.327777 & 1.886928 & 1.017529\end{array}$

$\begin{array}{lllll}6 & 6.282862 & 0.275175 & 0.002040\end{array}$

$\begin{array}{lllll}1 & 6.786695 & -0.275414 & -0.793860\end{array}$

$\begin{array}{lllll}1 & 6.539744 & 1.331118 & -0.097224\end{array}$

$\begin{array}{lllll}1 & 6.702295 & -0.070968 & 0.952475\end{array}$

$\begin{array}{llll}1 & -7.281625 & 0.060143 & -0.040014\end{array}$

\section{3a-TS@12}

$\begin{array}{llll}6 & -9.022929 & 3.672388 & -0.107180\end{array}$

$\begin{array}{llll}6 & -8.395858 & 3.060113 & 1.147584\end{array}$

$\begin{array}{lllll}6 & -8.420050 & 3.050972 & -1.364507\end{array}$

$\begin{array}{lllll}1 & -8.730308 & 4.723151 & -0.112644\end{array}$

$\begin{array}{llll}6 & -8.911979 & 1.922372 & 1.772646\end{array}$

$\begin{array}{lllll}6 & -7.253027 & 3.628661 & 1.725072\end{array}$

$\begin{array}{lllll}6 & -8.907006 & 1.855873 & -1.913812\end{array}$

$\begin{array}{lllll}6 & -7.314957 & 3.636331 & -1.994221\end{array}$

$\begin{array}{llll}6 & -8.359865 & 1.362457 & 2.932498\end{array}$

$\begin{array}{llll}1 & -9.780755 & 1.443099 & 1.337080\end{array}$

$\begin{array}{llll}6 & -6.680142 & 3.134003 & 2.887929\end{array}$

$\begin{array}{llll}8 & -6.713990 & 4.771298 & 1.128182\end{array}$

$\begin{array}{lllll}6 & -8.307971 & 1.219524 & -3.004953\end{array}$

$\begin{array}{lllll}1 & -9.779582 & 1.398543 & -1.461716\end{array}$

$\begin{array}{lllll}6 & -6.665947 & 3.029886 & -3.061951\end{array}$

$\begin{array}{lllll}8 & -6.833596 & 4.876215 & -1.544747\end{array}$

$\begin{array}{llll}6 & -8.938152 & 0.098693 & 3.563567\end{array}$

$\begin{array}{lllll}6 & -7.245470 & 2.010610 & 3.481975\end{array}$

$\begin{array}{lllll}1 & -5.809889 & 3.608347 & 3.322612\end{array}$

$\begin{array}{lllll}6 & -5.544541 & 4.647406 & 0.446403\end{array}$

$\begin{array}{lllll}6 & -8.850231 & -0.058379 & -3.646974\end{array}$

$\begin{array}{lllll}6 & -7.155608 & 1.820301 & -3.524210\end{array}$

$\begin{array}{lllll}1 & -5.788685 & 3.480880 & -3.505510\end{array}$

$\begin{array}{lllll}6 & -5.599296 & 4.805592 & -0.965651\end{array}$

$\begin{array}{lllll}1 & -8.600915 & 0.096827 & 4.600890\end{array}$

$\begin{array}{llll}6 & -8.360106 & -1.168078 & 2.928934\end{array}$

$\begin{array}{lllll}8 & -6.683828 & 1.547853 & 4.683198\end{array}$

$\begin{array}{llll}6 & -8.270654 & -1.316681 & -3.005099\end{array}$

$\begin{array}{lllll}1 & -8.490481 & -0.052772 & -4.676833\end{array}$

$\begin{array}{llll}8 & -6.506727 & 1.179072 & -4.579295\end{array}$ $\begin{array}{llll}6 & -8.921582 & -1.797253 & 1.813831\end{array}$

$\begin{array}{lllll}6 & -7.220511 & -1.766761 & 3.478170\end{array}$

$\begin{array}{lllll}6 & -5.469943 & 0.937239 & 4.529599\end{array}$

$\begin{array}{lllll}6 & -3.334622 & 4.400714 & 0.305477\end{array}$

$\begin{array}{lllll}6 & -8.836138 & -1.874289 & -1.851834\end{array}$

$\begin{array}{lllll}6 & -7.158565 & -1.975392 & -3.545506\end{array}$

$\begin{array}{lllll}6 & -5.359003 & 0.510467 & -4.294992\end{array}$

$\begin{array}{lllll}6 & -3.375620 & 4.649531 & -1.058960\end{array}$

$\begin{array}{llll}6 & -8.413499 & -2.986340 & 1.274746\end{array}$

$\begin{array}{llll}1 & -9.786718 & -1.344882 & 1.342858\end{array}$

$\begin{array}{llll}6 & -6.701043 & -2.964387 & 3.013669\end{array}$

$\begin{array}{lllll}8 & -6.616103 & -1.136185 & 4.566869\end{array}$

$\begin{array}{lllll}6 & -5.455391 & -0.470363 & 4.333734\end{array}$

$\begin{array}{lllll}6 & -8.341955 & -3.023911 & -1.230407\end{array}$

$\begin{array}{lllll}1 & -9.702214 & -1.387170 & -1.419754\end{array}$

$\begin{array}{lllll}6 & -6.614521 & -3.111762 & -2.954958\end{array}$

$\begin{array}{lllll}8 & -6.566975 & -1.505054 & -4.731059\end{array}$

$\begin{array}{lllll}6 & -5.362233 & -0.892413 & -4.523623\end{array}$

$\begin{array}{lllll}6 & -8.991803 & -3.624597 & 0.015597\end{array}$

$\begin{array}{llll}6 & -7.315650 & -3.563883 & 1.922966\end{array}$

$\begin{array}{llll}1 & -5.831459 & -3.411065 & 3.476403\end{array}$

$\begin{array}{lllll}6 & -3.257522 & 0.976625 & 4.247108\end{array}$

$\begin{array}{lllll}6 & -7.203823 & -3.609432 & -1.800810\end{array}$

$\begin{array}{lllll}1 & -5.744787 & -3.592894 & -3.383795\end{array}$

$\begin{array}{lllll}6 & -3.202894 & 0.397194 & -3.751649\end{array}$

$\begin{array}{lllll}1 & -8.696653 & -4.674183 & 0.038689\end{array}$

$\begin{array}{lllll}8 & -6.826830 & -4.797910 & 1.470468\end{array}$

$\begin{array}{llll}6 & -3.274593 & -0.367827 & 3.898816\end{array}$

$\begin{array}{lllll}8 & -6.686180 & -4.766068 & -1.211310\end{array}$

$\begin{array}{lllll}6 & -3.166210 & -0.935622 & -4.140063\end{array}$

$\begin{array}{lllll}6 & -5.589101 & -4.748997 & 0.899573\end{array}$

$\begin{array}{lllll}6 & -5.518701 & -4.660472 & -0.517664\end{array}$

$\begin{array}{llll}6 & -3.362894 & -4.640062 & 1.008144\end{array}$

$\begin{array}{llll}6 & -3.301042 & -4.489131 & -0.370409\end{array}$

$\begin{array}{lllll}6 & -10.525253 & -3.575360 & -0.033527\end{array}$

$\begin{array}{lllll}1 & -10.889287 & -4.090088 & -0.926548\end{array}$

$\begin{array}{lllll}1 & -10.920371 & -2.556289 & -0.057486\end{array}$

$\begin{array}{llll}6 & -10.473917 & 0.094278 & 3.579351\end{array}$

$\begin{array}{lllll}1 & -10.839767 & -0.792325 & 4.103704\end{array}$

$\begin{array}{lllll}1 & -10.911992 & 0.087110 & 2.577683\end{array}$

$\begin{array}{lllll}6 & -10.556789 & 3.618723 & -0.089079\end{array}$

$\begin{array}{lllll}1 & -10.939976 & 4.118097 & 0.804591\end{array}$

$\begin{array}{lllll}1 & -10.948959 & 2.598274 & -0.089266\end{array}$

$\begin{array}{lllll}6 & -10.385405 & -0.078441 & -3.696947\end{array}$

$\begin{array}{lllll}1 & -10.753882 & 0.802100 & -4.229745\end{array}$

$\begin{array}{lllll}1 & -10.845669 & -0.078859 & -2.705106\end{array}$

$\begin{array}{lllll}1 & -10.731393 & -0.973239 & -4.220788\end{array}$

$\begin{array}{lllll}1 & -10.958625 & 4.125205 & -0.970479\end{array}$

$\begin{array}{llll}1 & -10.846211 & 0.983058 & 4.095504\end{array}$

$\begin{array}{lllll}1 & -10.943004 & -4.069167 & 0.847685\end{array}$

$\begin{array}{lllll}6 & -1.835865 & 1.462504 & 4.162688\end{array}$

$\begin{array}{lllll}6 & -1.892172 & -0.762538 & 3.467455\end{array}$

$\begin{array}{lllll}6 & -1.957262 & -4.583198 & 1.552206\end{array}$

$\begin{array}{lllll}6 & -1.869102 & -4.262707 & -0.759090\end{array}$

$\begin{array}{lllll}6 & -1.915991 & 4.107304 & 0.692443\end{array}$

$\begin{array}{lllll}6 & -1.964455 & 4.608436 & -1.587552\end{array}$

$\begin{array}{lllll}6 & -1.840348 & 0.780820 & -3.251382\end{array}$

$\begin{array}{lllll}6 & -1.749839 & -1.422035 & -4.010491\end{array}$ 
$\begin{array}{llll}7 & -1.159273 & -4.335808 & 0.422843\end{array}$

$\begin{array}{lllll}7 & -1.113575 & 0.350845 & 3.694138\end{array}$

$\begin{array}{lllll}7 & -1.184083 & 4.270192 & -0.465650\end{array}$

$\begin{array}{lllll}7 & -1.052591 & -0.327941 & -3.466915\end{array}$

$\begin{array}{lllll}8 & -1.502350 & 1.844644 & -2.754404\end{array}$

$\begin{array}{lllll}8 & -1.268280 & -2.489823 & -4.301548\end{array}$

$\begin{array}{lllll}8 & -1.435914 & -4.027418 & -1.872172\end{array}$

$\begin{array}{lllll}8 & -1.559631 & -4.725568 & 2.683212\end{array}$

$\begin{array}{lllll}8 & -1.539834 & -1.834478 & 3.001919\end{array}$

$\begin{array}{lllll}8 & -1.510988 & 3.761181 & 1.788796\end{array}$

$\begin{array}{llll}8 & -1.370235 & 2.544851 & 4.427002\end{array}$

$\begin{array}{lllll}8 & -1.548234 & 4.823987 & -2.699288\end{array}$

$\begin{array}{lllll}1 & -0.063545 & -0.426737 & -3.212092\end{array}$

$\begin{array}{llll}1 & -0.166905 & 4.156890 & -0.534767\end{array}$

$\begin{array}{llll}1 & -0.120884 & 0.440075 & 3.449957\end{array}$

$\begin{array}{lllll}1 & -0.153011 & -4.161494 & 0.501414\end{array}$

$\begin{array}{lllll}7 & -4.364463 & 1.666828 & 4.534828\end{array}$

$\begin{array}{lllll}7 & -4.367543 & -1.128528 & 3.963473\end{array}$

$\begin{array}{lllll}7 & -4.413472 & 4.410213 & 1.091976\end{array}$

$\begin{array}{lllll}7 & -4.516248 & 4.839268 & -1.727478\end{array}$

$\begin{array}{lllll}7 & -4.513406 & -4.762913 & 1.673533\end{array}$

$\begin{array}{lllll}7 & -4.373604 & -4.507274 & -1.164970\end{array}$

$\begin{array}{lllll}7 & -4.293119 & 1.159288 & -3.850825\end{array}$

$\begin{array}{lllll}7 & -4.256006 & -1.619395 & -4.497588\end{array}$

$\begin{array}{lllll}6 & 8.993210 & -2.561890 & -2.472525\end{array}$

$\begin{array}{lllll}6 & 8.381897 & -3.036701 & -1.157828\end{array}$

$\begin{array}{lllll}6 & 8.391638 & -1.224455 & -2.906160\end{array}$

$1 \quad 8.696966 \quad-3.289380 \quad-3.228969$

$\begin{array}{lllll}6 & 8.864152 & -2.560678 & 0.067315\end{array}$

$\begin{array}{lllll}6 & 7.326887 & -3.958120 & -1.112348\end{array}$

$\begin{array}{lllll}6 & 8.933773 & 0.018386 & -2.571176\end{array}$

$\begin{array}{lllll}6 & 7.239851 & -1.216368 & -3.700298\end{array}$

$\begin{array}{lllll}6 & 8.337577 & -2.945157 & 1.300646\end{array}$

$\begin{array}{lllll}1 & 9.695982 & -1.866431 & 0.056406\end{array}$

$\begin{array}{lllll}6 & 6.736423 & -4.338968 & 0.091567\end{array}$

$\begin{array}{lllll}8 & 6.845218 & -4.543027 & -2.299466\end{array}$

$\begin{array}{lllll}6 & 8.394636 & 1.228841 & -3.026971\end{array}$

$\begin{array}{lllll}1 & 9.812970 & 0.049679 & -1.938524\end{array}$

$\begin{array}{lllll}6 & 6.684075 & -0.053512 & -4.211558\end{array}$

$\begin{array}{lllll}8 & 6.697951 & -2.459701 & -4.024945\end{array}$

$\begin{array}{lllll}6 & 8.930435 & -2.478138 & 2.628116\end{array}$

$\begin{array}{lllll}6 & 7.235433 & -3.807946 & 1.273551\end{array}$

$\begin{array}{lllll}1 & 5.903478 & -5.030308 & 0.100787\end{array}$

$\begin{array}{lllll}6 & 5.614149 & -4.076247 & -2.672882\end{array}$

$\begin{array}{lllll}6 & 8.973605 & 2.578907 & -2.615998\end{array}$

$\begin{array}{lllll}6 & 7.281187 & 1.155814 & -3.872197\end{array}$

$\begin{array}{lllll}1 & 5.808267 & -0.081905 & -4.845799\end{array}$

$\begin{array}{lllll}6 & 5.555538 & -2.865444 & -3.423057\end{array}$

$\begin{array}{lllll}1 & 8.609048 & -3.201143 & 3.378057\end{array}$

$\begin{array}{lllll}6 & 8.341231 & -1.136178 & 3.043436\end{array}$

$\begin{array}{lllll}8 & 6.668932 & -4.176794 & 2.501688\end{array}$

$\begin{array}{lllll}6 & 8.368291 & 3.050579 & -1.294370\end{array}$

$\begin{array}{lllll}1 & 8.651333 & 3.294609 & -3.372680\end{array}$

$\begin{array}{lllll}8 & 6.760732 & 2.341362 & -4.417029\end{array}$

$\begin{array}{lllll}6 & 8.868432 & 0.077978 & 2.586615\end{array}$

$\begin{array}{lllll}6 & 7.233302 & -1.074004 & 3.896175\end{array}$

$\begin{array}{lllll}6 & 5.500197 & -3.586480 & 2.862763\end{array}$

$\begin{array}{lllll}6 & 3.386878 & -4.061094 & -2.523940\end{array}$ $\begin{array}{lllll}6 & 8.880754 & 2.665743 & -0.053372\end{array}$

$\begin{array}{lllll}6 & 7.253580 & 3.897672 & -1.280537\end{array}$

$\begin{array}{lllll}6 & 5.547384 & 2.714732 & -3.919595\end{array}$

$\begin{array}{lllll}6 & 3.359614 & -2.785260 & -3.070151\end{array}$

$\begin{array}{llll}6 & 8.329564 & 1.317833 & 2.936466\end{array}$

$\begin{array}{llll}1 & 9.739682 & 0.053158 & 1.942837\end{array}$

$\begin{array}{lllll}6 & 6.637557 & 0.131655 & 4.249362\end{array}$

$\begin{array}{lllll}8 & 6.725789 & -2.267645 & 4.440595\end{array}$

$\begin{array}{lllll}6 & 5.517696 & -2.658851 & 3.947610\end{array}$

$\begin{array}{lllll}6 & 8.358435 & 3.115066 & 1.165696\end{array}$

$1 \quad 9.724691 \quad 1.986357 \quad-0.032495$

$\begin{array}{lllll}6 & 6.715018 & 4.400914 & -0.104004\end{array}$

$\begin{array}{lllll}8 & 6.692213 & 4.263186 & -2.509795\end{array}$

$\begin{array}{lllll}6 & 5.522473 & 3.661531 & -2.855270\end{array}$

$\begin{array}{lllll}6 & 8.937295 & 2.651077 & 2.498783\end{array}$

$\begin{array}{lllll}6 & 7.189128 & 1.302630 & 3.747826\end{array}$

$\begin{array}{lllll}1 & 5.768269 & 0.155170 & 4.892978\end{array}$

$\begin{array}{lllll}6 & 3.288124 & -3.315289 & 2.712983\end{array}$

$\begin{array}{lllll}6 & 7.282261 & 4.010324 & 1.105359\end{array}$

$\begin{array}{lllll}1 & 5.866026 & 5.071795 & -0.123575\end{array}$

$\begin{array}{lllll}6 & 3.325226 & 2.519227 & -3.782444\end{array}$

$\begin{array}{lllll}1 & 8.622488 & 3.386208 & 3.240108\end{array}$

$\begin{array}{lllll}8 & 6.637560 & 2.538063 & 4.093698\end{array}$

$\begin{array}{lllll}6 & 3.288695 & -2.502201 & 3.835084\end{array}$

$\begin{array}{lllll}8 & 6.763515 & 4.556683 & 2.291965\end{array}$

$\begin{array}{lllll}6 & 3.317386 & 3.366706 & -2.684376\end{array}$

$\begin{array}{llll}6 & 5.488697 & 2.914543 & 3.477722\end{array}$

$\begin{array}{lllll}6 & 5.536710 & 4.064247 & 2.639338\end{array}$

$\begin{array}{lllll}6 & 3.296652 & 2.773024 & 3.112286\end{array}$

$\begin{array}{lllll}6 & 3.314447 & 3.982076 & 2.433097\end{array}$

$\begin{array}{lllll}6 & 10.472934 & 2.624849 & 2.499863\end{array}$

$\begin{array}{lllll}1 & 10.863552 & 3.613050 & 2.243772\end{array}$

$\begin{array}{llll}1 & 10.890353 & 1.910587 & 1.784767\end{array}$

$\begin{array}{lllll}6 & 10.466296 & -2.479450 & 2.615692\end{array}$

$\begin{array}{lllll}1 & 10.847672 & -2.198190 & 3.600733\end{array}$

$\begin{array}{lllll}1 & 10.890235 & -1.783381 & 1.886768\end{array}$

$\begin{array}{llll}6 & 10.528192 & -2.535644 & -2.436590\end{array}$

$\begin{array}{lllll}1 & 10.912873 & -3.526733 & -2.182949\end{array}$

$\begin{array}{lllll}1 & 10.928483 & -1.829954 & -1.703511\end{array}$

$\begin{array}{lllll}6 & 10.509068 & 2.590215 & -2.593693\end{array}$

$\begin{array}{lllll}1 & 10.899544 & 2.302175 & -3.573212\end{array}$

$\begin{array}{lllll}1 & 10.932410 & 1.904037 & -1.854995\end{array}$

$\begin{array}{lllll}1 & 10.872308 & 3.593269 & -2.355606\end{array}$

$\begin{array}{lllll}1 & 10.920389 & -2.250396 & -3.416283\end{array}$

$\begin{array}{llll}1 & 10.837559 & -3.477913 & 2.370782\end{array}$

$\begin{array}{lllll}1 & 10.841947 & 2.351056 & 3.491684\end{array}$

$\begin{array}{lllll}6 & 1.899135 & -3.347540 & 2.144047\end{array}$

$\begin{array}{lllll}6 & 1.863227 & -2.083560 & 4.106777\end{array}$

$\begin{array}{llll}6 & 1.914436 & 2.198863 & 3.034025\end{array}$

$\begin{array}{lllll}6 & 1.899076 & 4.305309 & 2.028043\end{array}$

$\begin{array}{lllll}6 & 1.977282 & -4.426649 & -2.132249\end{array}$

$\begin{array}{lllll}6 & 1.970505 & -2.235639 & -2.932333\end{array}$

$\begin{array}{lllll}6 & 1.908869 & 2.063043 & -4.033624\end{array}$

$\begin{array}{llll}6 & 1.926822 & 3.406418 & -2.124744\end{array}$

$\begin{array}{lllll}7 & 1.156956 & 3.170700 & 2.416476\end{array}$

$\begin{array}{lllll}7 & 1.128407 & -2.625265 & 3.030047\end{array}$

$7 \quad 1.220166 \quad-3.278473 \quad-2.438676$

$\begin{array}{llll}7 & 1.166388 & 2.643120 & -2.985099\end{array}$ $\begin{array}{llll}8 & 1.468070 & 1.363520 & -4.910946\end{array}$

$\begin{array}{lllll}8 & 1.567801 & 3.946883 & -1.091521\end{array}$

$\begin{array}{llll}8 & 1.458599 & 5.293528 & 1.496077\end{array}$

$\begin{array}{lllll}8 & 1.578772 & 1.082292 & 3.394923\end{array}$

$\begin{array}{lllll}8 & 1.412240 & -1.434397 & 5.016171\end{array}$

$\begin{array}{lllll}8 & 1.559765 & -5.445661 & -1.640998\end{array}$

$\begin{array}{lllll}8 & 1.563541 & -3.850088 & 1.083993\end{array}$

$\begin{array}{lllll}8 & 1.631405 & -1.082104 & -3.148798\end{array}$

$\begin{array}{lllll}1 & 0.162447 & 2.445864 & -2.853233\end{array}$

$\begin{array}{lllll}1 & 0.214169 & -3.251179 & -2.237985\end{array}$

$\begin{array}{lllll}1 & 0.117903 & -2.437242 & 2.923901\end{array}$

$\begin{array}{lllll}1 & 0.144087 & 3.137103 & 2.252877\end{array}$

$\begin{array}{lllll}7 & 4.386263 & -3.885523 & 2.212628\end{array}$

$\begin{array}{lllll}7 & 4.411869 & -2.138863 & 4.461700\end{array}$

$\begin{array}{lllll}7 & 4.527168 & -4.713035 & -2.269672\end{array}$

$\begin{array}{lllll}7 & 4.438633 & -2.168410 & -3.557158\end{array}$

$\begin{array}{llll}7 & 4.376075 & 2.224235 & 3.671464\end{array}$

$\begin{array}{lllll}7 & 4.445984 & 4.633982 & 2.149161\end{array}$

$\begin{array}{lllll}7 & 4.448300 & 2.159382 & -4.409762\end{array}$

$\begin{array}{lllll}7 & 4.409474 & 3.965892 & -2.205957\end{array}$

$\begin{array}{lllll}6 & -0.532325 & -0.395095 & 0.086450\end{array}$

$\begin{array}{lllll}8 & -0.036838 & -1.381494 & -0.540445\end{array}$

$\begin{array}{llll}8 & 0.139869 & 0.445122 & 0.756916\end{array}$

$\begin{array}{lllll}7 & 3.629878 & -1.531245 & 0.174728\end{array}$

$\begin{array}{lllll}7 & 4.158816 & -2.402224 & -0.264183\end{array}$

$\begin{array}{lllll}6 & 2.386383 & -0.431657 & 0.393443\end{array}$

$\begin{array}{lllll}1 & 1.560677 & -1.047944 & -0.061780\end{array}$

$\begin{array}{lllll}1 & 2.367340 & -0.367473 & 1.477697\end{array}$

$\begin{array}{lllll}6 & -2.030120 & -0.221561 & 0.036306\end{array}$

$\begin{array}{lllll}6 & -2.826887 & -1.100066 & -0.702356\end{array}$

$\begin{array}{lllll}6 & -2.647958 & 0.812018 & 0.747076\end{array}$

$\begin{array}{lllll}6 & -4.213078 & -0.958751 & -0.715915\end{array}$

$\begin{array}{lllll}1 & -2.345958 & -1.883101 & -1.270637\end{array}$

$\begin{array}{lllll}6 & -4.033152 & 0.945263 & 0.742595\end{array}$

$\begin{array}{llll}1 & -2.025435 & 1.496453 & 1.305492\end{array}$

$\begin{array}{lllll}6 & -4.838683 & 0.060348 & 0.011235\end{array}$

$\begin{array}{lllll}1 & -4.818142 & -1.650832 & -1.294406\end{array}$

$\begin{array}{lllll}1 & -4.496812 & 1.742937 & 1.316190\end{array}$

$\begin{array}{lllll}6 & 2.862376 & 0.797378 & -0.342428\end{array}$

$\begin{array}{lllll}1 & 2.868854 & 0.585312 & -1.416666\end{array}$

$\begin{array}{lllll}1 & 2.041007 & 1.494566 & -0.158838\end{array}$

$\begin{array}{lllll}6 & -6.331735 & 0.226329 & -0.009770\end{array}$

$\begin{array}{lllll}1 & -6.723137 & 0.424576 & 0.989626\end{array}$

$\begin{array}{lllll}1 & -6.619876 & 1.072109 & -0.640401\end{array}$

$\begin{array}{lllll}1 & -6.832487 & -0.661798 & -0.398671\end{array}$

$\begin{array}{lllll}6 & 4.201175 & 1.404445 & 0.119852\end{array}$

$\begin{array}{lllll}1 & 4.286219 & 1.310667 & 1.207144\end{array}$

$\begin{array}{lllll}1 & 4.169512 & 2.477013 & -0.089989\end{array}$

$\begin{array}{lllll}6 & 5.458168 & 0.818285 & -0.536778\end{array}$

$\begin{array}{lllll}1 & 6.325883 & 1.443335 & -0.318802\end{array}$

$\begin{array}{lllll}1 & 5.704908 & -0.183580 & -0.177512\end{array}$

$\begin{array}{lllll}1 & 5.353059 & 0.759099 & -1.624830\end{array}$

\section{$7^{-}: \mathbf{8} \mathbf{a}^{+} @ \mathbf{1}_{2}$}

$\begin{array}{llll}6 & -9.085236 & 2.244622 & 2.613185\end{array}$

$\begin{array}{lllll}6 & -8.445580 & 0.915023 & 3.001136\end{array}$

$\begin{array}{lllll}6 & -8.554846 & 2.796789 & 1.294214\end{array}$

$\begin{array}{llll}1 & -8.782773 & 2.958052 & 3.380337\end{array}$ 
$\begin{array}{llll}6 & -8.927460 & -0.310981 & 2.538491\end{array}$

$\begin{array}{lllll}6 & -7.361357 & 0.872163 & 3.885835\end{array}$

$\begin{array}{llll}6 & -9.078310 & 2.422979 & 0.055051\end{array}$

$\begin{array}{lllll}6 & -7.526960 & 3.747407 & 1.281628\end{array}$

$\begin{array}{lllll}6 & -8.402758 & -1.545869 & 2.938016\end{array}$

$\begin{array}{lllll}1 & -9.756800 & -0.308474 & 1.840838\end{array}$

$\begin{array}{lllll}6 & -6.814084 & -0.322925 & 4.331019\end{array}$

$\begin{array}{lllll}8 & -6.811932 & 2.084987 & 4.332614\end{array}$

$\begin{array}{lllll}6 & -8.644985 & 2.969641 & -1.159130\end{array}$

$\begin{array}{lllll}1 & -9.856293 & 1.668695 & 0.029041\end{array}$

$\begin{array}{lllll}6 & -7.080619 & 4.344361 & 0.112971\end{array}$

$\begin{array}{lllll}8 & -6.920555 & 4.091164 & 2.499341\end{array}$

$\begin{array}{lllll}6 & -8.980213 & -2.851009 & 2.399271\end{array}$

$\begin{array}{lllll}6 & -7.345693 & -1.518918 & 3.857547\end{array}$

$\begin{array}{lllll}1 & -5.978275 & -0.326812 & 5.018553\end{array}$

$\begin{array}{lllll}6 & -5.664834 & 2.437372 & 3.684705\end{array}$

$\begin{array}{lllll}6 & -9.222323 & 2.483535 & -2.485258\end{array}$

$6 \begin{array}{llll}6 & -7.649156 & 3.951746 & -1.094486\end{array}$

$\begin{array}{llll}1 & -6.289314 & 5.082141 & 0.135158\end{array}$

$\begin{array}{lllll}6 & -5.725172 & 3.471450 & 2.709130\end{array}$

$1 \quad-8.640723 \quad-3.636408 \quad 3.075877$

$\begin{array}{lllll}6 & -8.404753 & -3.196828 & 1.026330\end{array}$

$8 \quad-6.776070 \quad-2.718285 \quad 4.329685$

$\begin{array}{lllll}6 & -8.552766 & 1.172427 & -2.893746\end{array}$

$\begin{array}{lllll}1 & -8.940532 & 3.222974 & -3.235988\end{array}$

$\begin{array}{lllll}8 & -7.179318 & 4.570836 & -2.266277\end{array}$

$6 \quad-8.980108-2.807992-0.185416$

$\begin{array}{lllll}6 & -7.233633 & -3.958088 & 0.948526\end{array}$

$\begin{array}{lllll}6 & -5.549214 & -2.958187 & 3.772132\end{array}$

$\begin{array}{lllll}6 & -3.502303 & 2.147251 & 3.212832\end{array}$

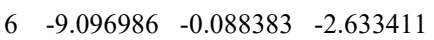

$\begin{array}{lllll}6 & -7.323810 & 1.211199 & -3.564169\end{array}$

$\begin{array}{lllll}6 & -5.902660 & 4.200173 & -2.586679\end{array}$

$\begin{array}{lllll}6 & -3.567108 & 3.134833 & 2.239951\end{array}$

$6 \quad-8.456486 \quad-3.182126 \quad-1.432107$

$1 \quad-9.866693 \quad-2.184970 \quad-0.164682$

$6 \begin{array}{lllll}6 & -6.701358 & -4.400547 & -0.248952\end{array}$

$8 \quad-6.620272 \quad-4.304423 \quad 2.150072$

$\begin{array}{lllll}6 & -5.504791 & -3.628089 & 2.517044\end{array}$

$6 \begin{array}{llll}6 & -8.475982 & -1.275882 & -3.036725\end{array}$

$\begin{array}{lllll}1 & -10.046212 & -0.149815 & -2.115282\end{array}$

$\begin{array}{lllll}6 & -6.665252 & 0.067534 & -3.995783\end{array}$

$8 \quad-6.839682 \quad 2.473574 \quad-3.895181$

$\begin{array}{lllll}6 & -5.725975 & 2.998485 & -3.334379\end{array}$

$\begin{array}{lllll}6 & -9.052340 & -2.660461 & -2.738003\end{array}$

$6 \quad-7.330468 \quad-4.013622 \quad-1.426040$

$1 \quad-5.811474 \quad-5.014845 \quad-0.271988$

$\begin{array}{lllll}6 & -3.345332 & -2.635667 & 3.642243\end{array}$

$6 \begin{array}{llll}6 & -7.261730 & -1.158238 & -3.723553\end{array}$

$1 \quad-5.732124 \quad 0.129013 \quad-4.539616$

$6 \quad-3.685946 \quad 4.425166 \quad-2.462020$

$1 \quad-8.697238 \quad-3.322371 \quad-3.528555$

$8 \begin{array}{lllll}8 & -6.797343 & -4.484447 & -2.637991\end{array}$

$\begin{array}{lllll}6 & -3.351739 & -3.089746 & 2.330339\end{array}$

$8 \quad-6.694676 \quad-2.334834 \quad-4.220875$

$\begin{array}{lllll}6 & -3.522605 & 3.197713 & -3.086528\end{array}$

$6 \quad-5.567962 \quad-3.973092 \quad-2.930663$

$\begin{array}{lllll}6 & -5.522977 & -2.782136 & -3.712038\end{array}$
$6 \begin{array}{llll}6 & -3.344080 & -3.921795 & -2.743989\end{array}$

$\begin{array}{lllll}6 & -3.312921 & -2.736878 & -3.462895\end{array}$

$\begin{array}{lllll}6 & -10.585839 & -2.698987 & -2.754945\end{array}$

$\begin{array}{lllll}1 & -10.960360 & -2.347504 & -3.719937\end{array}$

$\begin{array}{lllll}1 & -11.040683 & -2.078100 & -1.978260\end{array}$

$\begin{array}{lllll}6 & -10.516118 & -2.863203 & 2.413346\end{array}$

$\begin{array}{lllll}1 & -10.886276 & -3.837341 & 2.083082\end{array}$

$\begin{array}{lllll}1 & -10.956278 & -2.104291 & 1.760707\end{array}$

$\begin{array}{llll}6 & -10.621026 & 2.174743 & 2.631489\end{array}$

$\begin{array}{llll}1 & -10.969609 & 1.847181 & 3.614319\end{array}$

$\begin{array}{lllll}1 & -11.025017 & 1.480723 & 1.889359\end{array}$

$\begin{array}{lllll}6 & -10.754810 & 2.404274 & -2.466398\end{array}$

$\begin{array}{lllll}1 & -11.174183 & 3.387077 & -2.237230\end{array}$

$\begin{array}{lllll}1 & -11.138547 & 1.703458 & -1.719827\end{array}$

$\begin{array}{lllll}1 & -11.128640 & 2.086240 & -3.443357\end{array}$

$\begin{array}{lllll}1 & -11.042035 & 3.161656 & 2.422893\end{array}$

$\begin{array}{llll}1 & -10.881538 & -2.677091 & 3.426421\end{array}$

$\begin{array}{lllll}1 & -10.933760 & -3.723369 & -2.599616\end{array}$

$\begin{array}{lllll}6 & -1.923506 & -2.314235 & 4.013365\end{array}$

$\begin{array}{lllll}6 & -1.971961 & -2.913013 & 1.759008\end{array}$

$6 \begin{array}{lllll}6 & -1.930885 & -4.254389 & -2.328596\end{array}$

$\begin{array}{lllll}6 & -1.904293 & -2.239275 & -3.506612\end{array}$

$\begin{array}{lllll}6 & -2.114992 & 1.570963 & 3.190654\end{array}$

$\begin{array}{lllll}6 & -2.225860 & 3.185730 & 1.555103\end{array}$

$\begin{array}{lllll}6 & -2.318967 & 4.995591 & -2.178411\end{array}$

$\begin{array}{lllll}6 & -2.054920 & 2.889542 & -3.167979\end{array}$

$\begin{array}{lllll}7 & -1.158538 & -3.183989 & -2.834085\end{array}$

$\begin{array}{lllll}7 & -1.200384 & -2.501672 & 2.814445\end{array}$

$\begin{array}{llll}7 & -1.429130 & 2.272138 & 2.213840\end{array}$

$\begin{array}{lllll}7 & -1.424390 & 4.014971 & -2.656200\end{array}$

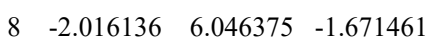

$8 \quad-1.537781 \quad 1.855156 \quad-3.544790$

$8 \quad-1.525829 \quad-1.175710 \quad-3.970723$

$\begin{array}{lllll}8 & -1.509426 & -5.195172 & -1.705340\end{array}$

$\begin{array}{lllll}8 & -1.629115 & -3.083089 & 0.592851\end{array}$

$\begin{array}{lllll}8 & -1.692844 & 0.643276 & 3.857307\end{array}$

$\begin{array}{lllll}8 & -1.459410 & -1.990881 & 5.076744\end{array}$

$\begin{array}{lllll}8 & -1.913518 & 3.839381 & 0.568655\end{array}$

$\begin{array}{lllll}1 & -0.405073 & 4.127595 & -2.561227\end{array}$

$\begin{array}{llll}1 & -0.411867 & 2.003044 & 1.872825\end{array}$

$\begin{array}{lllll}1 & -0.184262 & -2.341303 & 2.800916\end{array}$

$1 \quad-0.145285 \quad-3.101563 \quad-2.666123$

$\begin{array}{lllll}7 & -4.458648 & -2.511180 & 4.372232\end{array}$

$\begin{array}{lllll}7 & -4.427148 & -3.626602 & 1.747717\end{array}$

$\begin{array}{lllll}7 & -4.549964 & 1.766618 & 3.942109\end{array}$

$\begin{array}{lllll}7 & -4.680663 & 3.812643 & 1.964210\end{array}$

$\begin{array}{lllll}7 & -4.481649 & -4.561076 & -2.451826\end{array}$

$\begin{array}{lllll}7 & -4.395207 & -2.138519 & -3.965746\end{array}$

$\begin{array}{lllll}7 & -4.887234 & 4.937519 & -2.169320\end{array}$

$\begin{array}{lllll}7 & -4.537375 & 2.456747 & -3.546267\end{array}$

$\begin{array}{lllll}6 & 8.999940 & 0.154541 & -3.694947\end{array}$

$\begin{array}{lllll}6 & 8.438207 & -1.177439 & -3.187135\end{array}$

$\begin{array}{lllll}6 & 8.374256 & 1.352873 & -2.964039\end{array}$

$1 \quad 8.683434 \quad 0.239513 \quad-4.735827$

$\begin{array}{lllll}6 & 8.970674 & -1.824731 & -2.059770\end{array}$

$\begin{array}{lllll}6 & 7.331883 & -1.785365 & -3.797746\end{array}$

$\begin{array}{lllll}6 & 8.922658 & 1.932442 & -1.814380\end{array}$

$\begin{array}{lllll}6 & 7.191227 & 1.929066 & -3.443240\end{array}$ $\begin{array}{lllll}6 & 8.407271 & -2.973367 & -1.494299\end{array}$

$1 \quad 9.840772 \quad-1.389870 \quad-1.581770$

$\begin{array}{lllll}6 & 6.723915 & -2.915828 & -3.263255\end{array}$

$\begin{array}{lllll}8 & 6.785694 & -1.259022 & -4.981483\end{array}$

$\begin{array}{lllll}6 & 8.344005 & 3.029196 & -1.161700\end{array}$

$\begin{array}{lllll}1 & 9.831257 & 1.508316 & -1.403220\end{array}$

$\begin{array}{lllll}6 & 6.590664 & 3.033940 & -2.856283\end{array}$

$\begin{array}{lllll}8 & 6.617745 & 1.373648 & -4.585313\end{array}$

$\begin{array}{lllll}6 & 8.971824 & -3.685686 & -0.259815\end{array}$

$\begin{array}{lllll}6 & 7.247605 & -3.464412 & -2.106172\end{array}$

$\begin{array}{lllll}1 & 5.845919 & -3.340696 & -3.730977\end{array}$

$6 \quad 5.542842 \quad-0.717720 \quad-4.789040$

$\begin{array}{lllll}6 & 8.915925 & 3.619628 & 0.122678\end{array}$

$\begin{array}{lllll}6 & 7.175179 & 3.565909 & -1.716379\end{array}$

$1 \quad 5.682750 \quad 3.456686 \quad-3.264483$

$\begin{array}{lllll}6 & 5.478421 & 0.652960 & -4.425636\end{array}$

$\begin{array}{lllll}1 & 8.646964 & -4.724421 & -0.336960\end{array}$

$\begin{array}{llll}6 & 8.366989 & -3.146842 & 1.038402\end{array}$

$\begin{array}{lllll}8 & 6.631793 & -4.579549 & -1.534947\end{array}$

$\begin{array}{llll}6 & 8.317084 & 2.921853 & 1.342410\end{array}$

$\begin{array}{lllll}1 & 8.574317 & 4.654430 & 0.164537\end{array}$

$8 \quad 6.620625 \quad 4.711138 \quad-1.138011$

$\begin{array}{lllll}6 & 8.886202 & -2.003925 & 1.663667\end{array}$

$\begin{array}{lllll}6 & 7.262056 & -3.752006 & 1.652886\end{array}$

$\begin{array}{lllll}6 & 5.472180 & -4.401767 & -0.852647\end{array}$

$\begin{array}{lllll}6 & 3.335958 & -0.870566 & -4.507054\end{array}$

$\begin{array}{lllll}6 & 8.849615 & 1.732048 & 1.849761\end{array}$

$\begin{array}{lllll}6 & 7.199925 & 3.449181 & 2.003244\end{array}$

$\begin{array}{lllll}6 & 5.417229 & 4.613121 & -0.505525\end{array}$

$\begin{array}{lllll}6 & 3.308358 & 0.418639 & -3.989960\end{array}$

$\begin{array}{lllll}6 & 8.341438 & -1.441372 & 2.820544\end{array}$

$\begin{array}{lllll}1 & 9.748202 & -1.523271 & 1.216328\end{array}$

$\begin{array}{llll}6 & 6.674901 & -3.225795 & 2.800570\end{array}$

$\begin{array}{lllll}8 & 6.710773 & -4.933500 & 1.120888\end{array}$

$\begin{array}{lllll}6 & 5.493206 & -4.737531 & 0.530896\end{array}$

$\begin{array}{llll}6 & 8.342678 & 1.085498 & 2.981840\end{array}$

$\begin{array}{llll}1 & 9.697594 & 1.290644 & 1.339117\end{array}$

$\begin{array}{llll}6 & 6.670993 & 2.860943 & 3.143665\end{array}$

$\begin{array}{llll}8 & 6.637551 & 4.644280 & 1.540612\end{array}$

$\begin{array}{lllll}6 & 5.427326 & 4.574174 & 0.916854\end{array}$

$\begin{array}{lllll}6 & 8.941802 & -0.215935 & 3.511242\end{array}$

$\begin{array}{lllll}6 & 7.206864 & -2.069449 & 3.352251\end{array}$

$\begin{array}{lllll}1 & 5.810239 & -3.702295 & 3.244608\end{array}$

$\begin{array}{lllll}6 & 3.293822 & -3.960177 & -0.687515\end{array}$

$\begin{array}{lllll}6 & 7.256782 & 1.695044 & 3.621184\end{array}$

$\begin{array}{lllll}1 & 5.820169 & 3.299985 & 3.649064\end{array}$

$\begin{array}{lllll}6 & 3.183563 & 4.578716 & -0.473083\end{array}$

$\begin{array}{lllll}1 & 8.641621 & -0.283914 & 4.557712\end{array}$

$\begin{array}{lllll}8 & 6.648355 & -1.544490 & 4.519099\end{array}$

$\begin{array}{lllll}6 & 3.281149 & -4.444654 & 0.613705\end{array}$

$\begin{array}{lllll}8 & 6.755018 & 1.126663 & 4.800328\end{array}$

$\begin{array}{lllll}6 & 3.193133 & 4.530075 & 0.916099\end{array}$

$\begin{array}{lllll}6 & 5.476326 & -0.855773 & 4.435910\end{array}$

$\begin{array}{lllll}6 & 5.527830 & 0.547640 & 4.664307\end{array}$

$\begin{array}{lllll}6 & 3.263170 & -0.710217 & 4.225833\end{array}$

$\begin{array}{lllll}6 & 3.305048 & 0.646692 & 4.512017\end{array}$

$\begin{array}{lllll}6 & 10.477156 & -0.214011 & 3.472744\end{array}$

$\begin{array}{llll}1 & 10.863251 & 0.635523 & 4.041892\end{array}$ 
$\begin{array}{lllll}1 & 10.879589 & -0.139668 & 2.458877\end{array}$

$\begin{array}{lllll}6 & 10.507297 & -3.684030 & -0.236893\end{array}$

$\begin{array}{lllll}1 & 10.870129 & -4.256659 & 0.620518\end{array}$

$\begin{array}{lllll}1 & 10.932054 & -2.679103 & -0.164940\end{array}$

$\begin{array}{lllll}6 & 10.534314 & 0.194065 & -3.674111\end{array}$

$\begin{array}{lllll}1 & 10.938178 & -0.633995 & -4.262252\end{array}$

$\begin{array}{lllll}1 & 10.949430 & 0.114033 & -2.665763\end{array}$

$\begin{array}{lllll}6 & 10.450563 & 3.638168 & 0.148286\end{array}$

$\begin{array}{lllll}1 & 10.833475 & 4.190755 & -0.713853\end{array}$

$\begin{array}{lllll}1 & 10.891915 & 2.638257 & 0.121873\end{array}$

$\begin{array}{llll}1 & 10.802988 & 4.127469 & 1.059930\end{array}$

$\begin{array}{lllll}1 & 10.892277 & 1.133646 & -4.102989\end{array}$

$\begin{array}{lllll}1 & 10.896024 & -4.140359 & -1.150999\end{array}$

$\begin{array}{llll}1 & 10.865650 & -1.135341 & 3.914634\end{array}$

$\begin{array}{lllll}6 & 1.913765 & -3.470062 & -1.020295\end{array}$

$\begin{array}{lllll}6 & 1.866963 & -4.381282 & 1.129850\end{array}$

$\begin{array}{lllll}6 & 1.841313 & -1.086709 & 3.938528\end{array}$

$\begin{array}{lllll}6 & 1.895263 & 1.180943 & 4.492445\end{array}$

$\begin{array}{lllll}6 & 1.928837 & -1.403141 & -4.519549\end{array}$

$\begin{array}{lllll}6 & 1.912777 & 0.716462 & -3.514933\end{array}$

$\begin{array}{lllll}6 & 1.750774 & 4.565912 & -0.941745\end{array}$

$\begin{array}{lllll}6 & 1.766097 & 4.506732 & 1.403628\end{array}$

$\begin{array}{lllll}7 & 1.108231 & 0.060254 & 4.153233\end{array}$

$\begin{array}{lllll}7 & 1.144626 & -3.768796 & 0.084047\end{array}$

$\begin{array}{lllll}7 & 1.171473 & -0.390820 & -3.909122\end{array}$

$\begin{array}{lllll}7 & 0.999319 & 4.543927 & 0.219974\end{array}$

$\begin{array}{lllll}8 & 1.333142 & 4.565704 & -2.086319\end{array}$

$\begin{array}{lllll}8 & 1.331656 & 4.498668 & 2.526484\end{array}$

$\begin{array}{lllll}8 & 1.486608 & 2.290409 & 4.720964\end{array}$

$\begin{array}{llll}8 & 1.434713 & -2.166633 & 3.541210\end{array}$

$\begin{array}{lllll}8 & 1.404907 & -4.758621 & 2.177102\end{array}$

$\begin{array}{lllll}8 & 1.498947 & -2.440150 & -4.966507\end{array}$

$\begin{array}{lllll}8 & 1.539282 & -2.925276 & -2.047471\end{array}$

$\begin{array}{lllll}8 & 1.516337 & 1.681810 & -2.894982\end{array}$

$\begin{array}{lllll}1 & -0.016577 & 4.451682 & 0.239489\end{array}$

$\begin{array}{lllll}1 & 0.154039 & -0.470635 & -3.848146\end{array}$

$1 \quad 0.160011 \quad-3.500184 \quad 0.187853$

$\begin{array}{lllll}1 & 0.094273 & 0.135532 & 4.023861\end{array}$

$\begin{array}{lllll}7 & 4.382256 & -3.960717 & -1.461646\end{array}$

$\begin{array}{lllll}7 & 4.391527 & -4.811473 & 1.261759\end{array}$

$7 \quad 4.465739-1.480509 \quad-4.881786$

$\begin{array}{lllll}7 & 4.375536 & 1.219801 & -3.964628\end{array}$

$\begin{array}{lllll}7 & 4.343490 & -1.494437 & 4.189973\end{array}$

$\begin{array}{lllll}7 & 4.444852 & 1.307465 & 4.723626\end{array}$

$\begin{array}{lllll}7 & 4.296128 & 4.615582 & -1.212941\end{array}$

$\begin{array}{lllll}7 & 4.316935 & 4.527984 & 1.638238\end{array}$

$\begin{array}{lllll}6 & 1.066335 & 0.445161 & 1.016300\end{array}$

$\begin{array}{lllll}8 & 0.176989 & -0.439621 & 0.994504\end{array}$

$\begin{array}{lllll}7 & -0.938611 & 0.928428 & -0.886021\end{array}$

$\begin{array}{lllll}7 & -0.325148 & 1.851272 & -0.905089\end{array}$

$\begin{array}{lllll}8 & 0.867224 & 1.674287 & 1.323503\end{array}$

$\begin{array}{lllll}6 & -1.940491 & -0.114369 & -1.023256\end{array}$

$\begin{array}{lllll}1 & -1.433378 & -1.044861 & -0.762379\end{array}$

$\begin{array}{lllll}6 & 2.473065 & 0.165881 & 0.594265\end{array}$

$\begin{array}{lllll}6 & 3.144062 & 1.146222 & -0.148202\end{array}$

$\begin{array}{lllll}6 & 3.151492 & -0.991744 & 0.970728\end{array}$

$\begin{array}{lllll}6 & 4.476968 & 0.984054 & -0.484768\end{array}$

$\begin{array}{lllll}1 & 2.600528 & 2.019393 & -0.474065\end{array}$ $\begin{array}{lllll}6 & 4.508092 & -1.124048 & 0.679084\end{array}$

$\begin{array}{lllll}1 & 2.634082 & -1.754790 & 1.535880\end{array}$

$\begin{array}{lllll}6 & 5.186941 & -0.139601 & -0.042395\end{array}$

$\begin{array}{lllll}1 & 4.979182 & 1.731112 & -1.089089\end{array}$

$\begin{array}{lllll}1 & 5.052726 & -1.991305 & 1.033188\end{array}$

$\begin{array}{lllll}6 & 6.643703 & -0.265300 & -0.350533\end{array}$

$1 \quad 6.818422 \quad-0.185652-1.423156$

$\begin{array}{lllll}1 & 7.220632 & 0.531083 & 0.126893\end{array}$

$\begin{array}{lllll}1 & 7.039911 & -1.216696 & -0.010434\end{array}$

$\begin{array}{lllll}6 & -3.117260 & 0.192410 & -0.088116\end{array}$

$\begin{array}{lllll}1 & -2.778027 & 0.050038 & 0.940834\end{array}$

$\begin{array}{lllll}1 & -3.412414 & 1.240425 & -0.211369\end{array}$

$\begin{array}{lllll}6 & -4.310037 & -0.718743 & -0.410783\end{array}$

$\begin{array}{lllll}1 & -4.013870 & -1.767212 & -0.291631\end{array}$

$\begin{array}{lllll}1 & -4.596497 & -0.579738 & -1.460437\end{array}$

$\begin{array}{lllll}6 & -5.509738 & -0.409459 & 0.490303\end{array}$

$\begin{array}{llll}1 & -5.258168 & -0.530696 & 1.549328\end{array}$

$\begin{array}{llll}1 & -5.859360 & 0.619250 & 0.348108\end{array}$

$\begin{array}{lllll}1 & -2.203188 & -0.059381 & -2.084813\end{array}$

$\begin{array}{lllll}1 & -6.346391 & -1.076440 & 0.267501\end{array}$

\section{7:99 $\mathbf{a}^{+} @ \mathbf{1}_{2}$}

$\begin{array}{llll}6 & -9.110863 & -3.050316 & 1.535702\end{array}$

$\begin{array}{lllll}6 & -8.580218 & -3.072548 & 0.104443\end{array}$

$\begin{array}{lllll}6 & -8.463269 & -1.968056 & 2.394994\end{array}$

$\begin{array}{llll}1 & -8.813631 & -4.001553 & 1.978153\end{array}$

$\begin{array}{lllll}6 & -9.099424 & -2.253945 & -0.899588\end{array}$

$\begin{array}{lllll}6 & -7.562237 & -3.959017 & -0.271548\end{array}$

$\begin{array}{lllll}6 & -8.943922 & -0.658485 & 2.450899\end{array}$

$\begin{array}{lllll}6 & -7.364400 & -2.270727 & 3.207454\end{array}$

$\begin{array}{lllll}6 & -8.683143 & -2.305304 & -2.236463\end{array}$

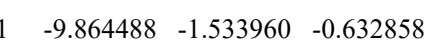

$\begin{array}{lllll}6 & -7.128711 & -4.070368 & -1.585302\end{array}$

$\begin{array}{lllll}8 & -6.967514 & -4.760029 & 0.710521\end{array}$

$\begin{array}{llll}6 & -8.392449 & 0.325847 & 3.279595\end{array}$

$\begin{array}{llll}1 & -9.791180 & -0.391269 & 1.830071\end{array}$

$\begin{array}{llll}6 & -6.785440 & -1.338512 & 4.056325\end{array}$

$\begin{array}{lllll}8 & -6.838684 & -3.569570 & 3.152197\end{array}$

$\begin{array}{lllll}6 & -9.275934 & -1.341460 & -3.261389\end{array}$

$\begin{array}{lllll}6 & -7.699425 & -3.249479 & -2.553464\end{array}$

$\begin{array}{lllll}1 & -6.348735 & -4.774141 & -1.845936\end{array}$

$\begin{array}{lllll}6 & -5.756921 & -4.300266 & 1.150260\end{array}$

$\begin{array}{llll}6 & -8.959587 & 1.740173 & 3.307795\end{array}$

$\begin{array}{lllll}6 & -7.308159 & -0.049935 & 4.083374\end{array}$

$\begin{array}{lllll}1 & -5.933475 & -1.600784 & 4.669581\end{array}$

$\begin{array}{llll}6 & -5.692068 & -3.673598 & 2.423612\end{array}$

$\begin{array}{lllll}1 & -8.995395 & -1.719734 & -4.245412\end{array}$

$\begin{array}{lllll}6 & -8.621290 & 0.032313 & -3.117672\end{array}$

$\begin{array}{lllll}8 & -7.226878 & -3.398557 & -3.883670\end{array}$

$\begin{array}{llll}6 & -8.403267 & 2.591176 & 2.168710\end{array}$

$\begin{array}{lllll}1 & -8.596396 & 2.195127 & 4.230190\end{array}$

$\begin{array}{lllll}8 & -6.716889 & 0.881618 & 4.956058\end{array}$

$\begin{array}{lllll}6 & -9.179488 & 1.098698 & -2.405740\end{array}$

$\begin{array}{lllll}6 & -7.379139 & 0.253952 & -3.721029\end{array}$

$\begin{array}{lllll}6 & -5.958638 & -2.925333 & -4.019281\end{array}$

$\begin{array}{lllll}6 & -3.576963 & -3.914110 & 0.873309\end{array}$

$\begin{array}{lllll}6 & -8.997169 & 2.686548 & 0.908609\end{array}$

$\begin{array}{lllll}6 & -7.240353 & 3.342470 & 2.374856\end{array}$ $\begin{array}{llll}6 & -5.498919 & 1.326087 & 4.516786\end{array}$

$\begin{array}{llll}6 & -3.516080 & -3.289818 & 2.110421\end{array}$

$\begin{array}{llll}6 & -8.554455 & 2.348020 & -2.311043\end{array}$

$\begin{array}{lllll}1 & -10.138410 & 0.958734 & -1.921941\end{array}$

$\begin{array}{lllll}6 & -6.719570 & 1.472312 & -3.680854\end{array}$

$\begin{array}{lllll}8 & -6.880005 & -0.810432 & -4.478590\end{array}$

$\begin{array}{llll}6 & -5.785834 & -1.504024 & -4.135146\end{array}$

$\begin{array}{lllll}6 & -8.506577 & 3.519493 & -0.107683\end{array}$

$\begin{array}{lllll}1 & -9.874978 & 2.085046 & 0.702284\end{array}$

$\begin{array}{lllll}6 & -6.745460 & 4.224386 & 1.431268\end{array}$

$\begin{array}{lllll}8 & -6.597600 & 3.216605 & 3.604495\end{array}$

$\begin{array}{lllll}6 & -5.467503 & 2.463660 & 3.662921\end{array}$

$\begin{array}{lllll}6 & -9.126172 & 3.516873 & -1.505525\end{array}$

$\begin{array}{llll}6 & -7.330899 & 2.502220 & -2.975140\end{array}$

$\begin{array}{lllll}1 & -5.772771 & 1.623632 & -4.180347\end{array}$

$\begin{array}{llll}6 & -3.744093 & -3.157370 & -3.872378\end{array}$

$\begin{array}{lllll}6 & -7.395354 & 4.311504 & 0.205232\end{array}$

$\begin{array}{llll}1 & -5.863685 & 4.817416 & 1.633665\end{array}$

$\begin{array}{llll}6 & -3.294808 & 1.097179 & 4.247870\end{array}$

$\begin{array}{llll}1 & -8.786311 & 4.428855 & -1.997719\end{array}$

$\begin{array}{lllll}8 & -6.768860 & 3.777452 & -2.974262\end{array}$

$\begin{array}{llll}6 & -3.610621 & -1.779595 & -3.791633\end{array}$

$\begin{array}{lllll}8 & -6.882672 & 5.223221 & -0.735733\end{array}$

$\begin{array}{llll}6 & -3.306626 & 2.082627 & 3.268702\end{array}$

$\begin{array}{lllll}6 & -5.598958 & 4.002940 & -2.328325\end{array}$

$\begin{array}{lllll}6 & -5.648764 & 4.859309 & -1.192245\end{array}$

$\begin{array}{lllll}6 & -3.398029 & 3.842614 & -2.078101\end{array}$

$\begin{array}{lllll}6 & -3.428122 & 4.733202 & -1.016931\end{array}$

$\begin{array}{lllll}6 & -10.659673 & 3.547181 & -1.480267\end{array}$

$\begin{array}{lllll}1 & -11.004586 & 4.438588 & -0.950454\end{array}$

$\begin{array}{lllll}1 & -11.098256 & 2.679870 & -0.979343\end{array}$

$\begin{array}{llll}6 & -10.808806 & -1.294925 & -3.206770\end{array}$

$\begin{array}{lllll}1 & -11.192821 & -0.614974 & -3.971975\end{array}$

$\begin{array}{lllll}1 & -11.192684 & -0.958754 & -2.239945\end{array}$

$\begin{array}{lllll}6 & -10.646102 & -2.983926 & 1.577020\end{array}$

$\begin{array}{lllll}1 & -11.072958 & -3.819392 & 1.016036\end{array}$

$\begin{array}{lllll}1 & -11.044738 & -2.060642 & 1.147977\end{array}$

$\begin{array}{lllll}6 & -10.494775 & 1.758741 & 3.359006\end{array}$

$\begin{array}{llll}1 & -10.846516 & 1.195271 & 4.227034\end{array}$

$\begin{array}{lllll}1 & -10.958015 & 1.318959 & 2.471612\end{array}$

$\begin{array}{rrrrr}1 & -10.854137 & 2.787757 & 3.442684\end{array}$

$\begin{array}{lllll}1 & -10.994138 & -3.043958 & 2.611347\end{array}$

$\begin{array}{lllll}1 & -11.217032 & -2.291632 & -3.391481\end{array}$

$\begin{array}{lllll}1 & -11.053749 & 3.575133 & -2.499741\end{array}$

$\begin{array}{lllll}6 & -2.381020 & -3.761585 & -3.670025\end{array}$

$\begin{array}{lllll}6 & -2.243648 & -1.501117 & -3.309123\end{array}$

$\begin{array}{lllll}6 & -1.994005 & 3.380671 & -2.277677\end{array}$

$\begin{array}{lllll}6 & -2.013960 & 4.912209 & -0.518804\end{array}$

$\begin{array}{llll}6 & -2.204488 & -3.840218 & 0.266999\end{array}$

$\begin{array}{lllll}6 & -2.106462 & -2.786541 & 2.289940\end{array}$

$\begin{array}{lllll}6 & -1.872118 & 0.639701 & 4.431878\end{array}$

$\begin{array}{lllll}6 & -1.923639 & 2.182048 & 2.682831\end{array}$

$\begin{array}{lllll}7 & -1.244750 & 4.052601 & -1.337780\end{array}$

$\begin{array}{lllll}7 & -1.547267 & -2.633071 & -3.285093\end{array}$

$\begin{array}{lllll}7 & -1.396747 & -3.211391 & 1.178830\end{array}$

$\begin{array}{llll}7 & -1.154612 & 1.322109 & 3.428315\end{array}$

$\begin{array}{lllll}8 & -1.402970 & -0.107172 & 5.253084\end{array}$

$\begin{array}{lllll}8 & -1.564839 & 2.874599 & 1.738854\end{array}$ 


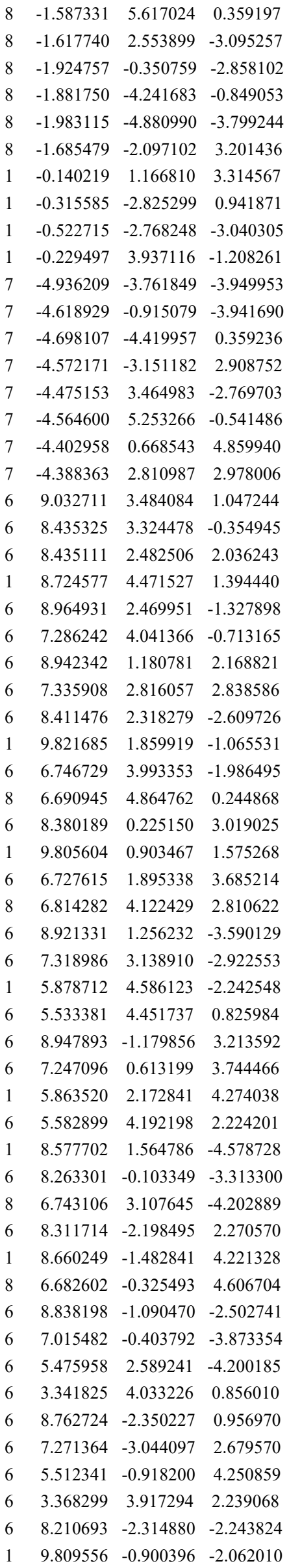

$\begin{array}{lllll}6 & 6.344080 & -1.597236 & -3.645248\end{array}$

$8 \quad 6.465817 \quad 0.525888 \quad-4.753580$

$\begin{array}{lllll}6 & 5.343101 & 1.187741 & -4.379783\end{array}$

$\begin{array}{lllll}6 & 8.245940 & -3.291580 & 0.061328\end{array}$

$\begin{array}{llll}9.557677 & -1.700181 & 0.610177\end{array}$

$\begin{array}{lllll}6 & 6.716846 & -3.992191 & 1.827654\end{array}$

$\begin{array}{lllll}8 & 6.757607 & -2.944546 & 3.981408\end{array}$

$\begin{array}{lllll}6 & 5.538500 & -2.322454 & 4.032172\end{array}$

$\begin{array}{lllll}6 & 8.803853 & -3.404601 & -1.355519\end{array}$

$\begin{array}{lllll}6 & 6.955787 & -2.530617 & -2.825788\end{array}$

$\begin{array}{llll}5.379239 & -1.790008 & -4.093815\end{array}$

$\begin{array}{lllll}6 & 3.273645 & 2.733249 & -3.888502\end{array}$

$\begin{array}{lllll}6 & 7.199954 & -4.099087 & 0.527961\end{array}$

$1 \quad 5.903244 \quad-4.622594 \quad 2.165074$

$\begin{array}{lllll}6 & 3.310284 & -0.928069 & 3.922518\end{array}$

$1 \quad 8.447569 \quad-4.356347 \quad-1.751866$

$8 \quad 6.388814 \quad-3.788966 \quad-2.648567$

$\begin{array}{lllll}6 & 3.164269 & 1.347818 & -3.951170\end{array}$

$\begin{array}{lllll}8 & 6.616045 & -5.061984 & -0.308757\end{array}$

$\begin{array}{lllll}6 & 3.316948 & -2.312268 & 3.819718\end{array}$

$\begin{array}{lllll}6 & 5.242629 & -3.989232 & -1.955159\end{array}$

$\begin{array}{lllll}6 & 5.361326 & -4.753019 & -0.757931\end{array}$

$6 \quad 3.024528 \quad-4.020756 \quad-1.740411$

$\begin{array}{lllll}6 & 3.135278 & -4.838712 & -0.622829\end{array}$

$\begin{array}{lllll}6 & 10.338077 & -3.440153 & -1.375720\end{array}$

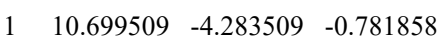

$1 \quad 10.792086 \quad-2.533756 \quad-0.965786$

$\begin{array}{lllll}6 & 10.453134 & 1.180522 & -3.633609\end{array}$

$\begin{array}{llll}10.773513 & 0.447243 & -4.378433\end{array}$

$\begin{array}{llll}10.894150 & 0.891062 & -2.675514\end{array}$

$\begin{array}{lllll}6 & 10.567744 & 3.457024 & 1.033672\end{array}$

$\begin{array}{lllll}1 & 10.950773 & 4.231291 & 0.363791\end{array}$

$\begin{array}{llll}1 & 10.974837 & 2.499841 & 0.696230\end{array}$

$\begin{array}{lllll}6 & 10.482312 & -1.209807 & 3.144425\end{array}$

$\begin{array}{llll}10.904802 & -0.517048 & 3.877108\end{array}$

$\begin{array}{llll}1 & 10.870935 & -0.928956 & 2.161584\end{array}$

$\begin{array}{lllll}1 & 10.846496 & -2.216314 & 3.365708\end{array}$

$\begin{array}{lllll}1 & 10.954519 & 3.643030 & 2.038963\end{array}$

$1 \quad 10.867882 \quad 2.155113 \quad-3.903491$

$\begin{array}{llll}10.698308 & -3.557299 & -2.401419\end{array}$

$\begin{array}{lllll}6 & 1.902228 & 3.311241 & -3.673135\end{array}$

$\begin{array}{lllll}6 & 1.728559 & 0.969534 & -3.690464\end{array}$

$6 \begin{array}{lllll}6 & 1.562054 & -3.813527 & -2.014640\end{array}$

$6 \quad \begin{array}{llll}6 & 1.751300 & -5.235431 & -0.184214\end{array}$

$\begin{array}{lllll}6 & 1.937849 & 3.769431 & 0.390694\end{array}$

$\begin{array}{lllll}6 & 1.960565 & 3.683237 & 2.723459\end{array}$

$\begin{array}{lllll}6 & 1.905487 & -0.444849 & 3.716582\end{array}$

$\begin{array}{lllll}6 & 1.897802 & -2.777651 & 3.614674\end{array}$

$7 \quad 0.902868 \quad-4.649194 \quad-1.151078$

$\begin{array}{llll}7 & 1.075221 & 2.188483 & -3.549308\end{array}$

$\begin{array}{llll}7 & 1.200214 & 3.585517 & 1.537472\end{array}$

$\begin{array}{lllll}7 & 1.141011 & -1.590065 & 3.612988\end{array}$

$\begin{array}{lllll}8 & 1.535771 & 0.713303 & 3.628892\end{array}$

$8 \quad 1.469404 \quad-3.895955 \quad 3.475782$

$\begin{array}{lllll}8 & 1.398433 & -5.918603 & 0.741709\end{array}$

$8 \quad 1.053173 \quad-3.061886 \quad-2.841189$

$8 \quad 1.222445 \quad-0.130727 \quad-3.612245$

$\begin{array}{llll}8 & 1.529719 & 3.708644 & -0.761160\end{array}$
$8 \quad 1.546623 \quad 4.465873 \quad-3.639353$

$\begin{array}{lllll}8 & 1.527830 & 3.617459 & 3.845486\end{array}$

$\begin{array}{lllll}1 & 0.130012 & -1.606338 & 3.443509\end{array}$

$\begin{array}{lllll}1 & 0.198778 & 3.351570 & 1.544667\end{array}$

$\begin{array}{llll}0.062076 & 2.270817 & -3.405088\end{array}$

$\begin{array}{llll}-0.116356 & -4.601086 & -1.031404\end{array}$

$\begin{array}{lllll}7 & 4.438391 & 3.381966 & -3.988146\end{array}$

$7 \quad 4.195796 \quad 0.546066 \quad-4.224657$

$7 \quad 4.418676 \quad 4.330373 \quad 0.121005$

$\begin{array}{lllll}7 & 4.498561 & 3.969357 & 2.949807\end{array}$

$7 \quad 4.076865 \quad-3.590925 \quad-2.442337$

$7 \quad 4.308183 \quad-5.200816 \quad-0.093813$

$\begin{array}{lllll}7 & 4.403125 & -0.200729 & 4.157054\end{array}$

$7 \quad 4.439163 \quad-3.035650 \quad 3.841470$

$\begin{array}{lllll}6 & 1.099591 & -1.141064 & 0.637029\end{array}$

$\begin{array}{llll}0.898032 & -2.415542 & 0.612439\end{array}$

$\begin{array}{lllll}8 & 0.249513 & -0.266638 & 0.890043\end{array}$

$\begin{array}{lllll}6 & -1.252332 & -0.266347 & -1.514331\end{array}$

$\begin{array}{llll}-0.484905 & -1.033873 & -1.467117\end{array}$

$\begin{array}{llll}2.505967 & -0.736049 & 0.335346\end{array}$

$\begin{array}{llll}2.763922 & 0.526217 & -0.204551\end{array}$

$\begin{array}{llll}3.566246 & -1.605688 & 0.606170\end{array}$

$\begin{array}{llll}4.071253 & 0.913085 & -0.473369\end{array}$

$\begin{array}{llll}1.927750 & 1.181547 & -0.416005\end{array}$

$\begin{array}{llll}4.875446 & -1.194861 & 0.385152\end{array}$

$\begin{array}{llll}3.351701 & -2.589290 & 1.004513\end{array}$

$\begin{array}{lllll}6.141857 & 0.067143 & -0.153602\end{array}$

$\begin{array}{llll}4.272882 & 1.883657 & -0.918168\end{array}$

$\begin{array}{llll}5.702180 & -1.851109 & 0.629710\end{array}$

$\begin{array}{llll}6.549645 & 0.531095 & -0.339936\end{array}$

$\begin{array}{llll}6.852738 & 1.164168 & 0.495932\end{array}$

$\begin{array}{llll}7.250058 & -0.302936 & -0.391544\end{array}$

$\begin{array}{llll}6.649289 & 1.120914 & -1.248151\end{array}$

$\begin{array}{llll}-2.363877 & -0.440330 & -0.492065\end{array}$

$\begin{array}{llll}-2.845625 & -1.411180 & -0.674148\end{array}$

$\begin{array}{llll}-1.854159 & -0.503291 & 0.472096\end{array}$

$\begin{array}{llll}6.425211 & 0.665433 & -0.477654\end{array}$

$\begin{array}{lllll}-3.779851 & 0.866197 & -1.494885\end{array}$

$\begin{array}{llll}-2.964369 & 1.588503 & -0.107274\end{array}$

$\begin{array}{llll}6 & -4.616095 & 0.293279 & 0.412913\end{array}$

$\begin{array}{llll}1 & -5.156551 & -0.570677 & 0.007562\end{array}$

$\begin{array}{lllll}1 & -4.290723 & 0.028087 & 1.425469\end{array}$

$1 \quad-0.797230 \quad 0.717463 \quad-1.513493$

$\begin{array}{lllll}1 & -5.327007 & 1.120837 & 0.498130\end{array}$

\section{5:9a ${ }^{+} @ 1^{-}$}

$\begin{array}{lllll}6 & -8.993888 & 0.646199 & -3.424801\end{array}$

$\begin{array}{lllll}6 & -8.403910 & 1.799470 & -2.617473\end{array}$

$\begin{array}{lllll}6 & -8.441933 & -0.717737 & -3.017362\end{array}$

$1 \quad-8.667065 \quad 0.805265 \quad-4.453193$

$\begin{array}{lllll}6 & -8.920455 & 2.151020 & -1.367924\end{array}$

$\begin{array}{lllll}6 & -7.359200 & 2.593821 & -3.110450\end{array}$

$\begin{array}{lllll}6 & -9.012308 & -1.526983 & -2.033291\end{array}$

$\begin{array}{lllll}6 & -7.313785 & -1.225180 & -3.670678\end{array}$

$\begin{array}{lllll}6 & -8.481212 & 3.248999 & -0.619409\end{array}$

$\begin{array}{lllll}1 & -9.713845 & 1.540172 & -0.954122\end{array}$

$\begin{array}{lllll}6 & -6.889326 & 3.700479 & -2.413715\end{array}$

$\begin{array}{lllll}8 & -6.767228 & 2.291589 & -4.350021\end{array}$ 
$6 \quad-8.523924 \quad-2.801883 \quad-1.710623$

$1 \quad-9.864354 \quad-1.148599 \quad-1.481070$

$6 \quad-6.828090 \quad-2.500228 \quad-3.442919$

$8 \quad-6.692229 \quad-0.401580 \quad-4.611716$

$\begin{array}{lllll}6 & -9.128971 & 3.570166 & 0.727856\end{array}$

$6 \quad-7.455490 \quad 4.023369 \quad-1.183335$

$\begin{array}{llll}-6.081603 & 4.297163 & -2.818633\end{array}$

$\begin{array}{lllll}6 & -5.561894 & 1.653609 & -4.249643\end{array}$

$\begin{array}{lllll}6 & -9.112503 & -3.601947 & -0.549895\end{array}$

$6 \quad-7.446863 \quad-3.279307 \quad-2.469217$

$\begin{array}{llll}-5.970595 & -2.875558 & -3.985404\end{array}$

$\begin{array}{lllll}6 & -5.547812 & 0.227969 & -4.246966\end{array}$

$\begin{array}{llll}-8.841273 & 4.594938 & 0.966984\end{array}$

$\begin{array}{lllll}6 & -8.568280 & 2.695609 & 1.850825\end{array}$

$8 \quad-6.920243 \quad 5.141228 \quad-0.495158$

$\begin{array}{llll}-8.500373 & -3.152761 & 0.780292\end{array}$

$\begin{array}{llll}-8.796101 & -4.635328 & -0.697341\end{array}$

$8 \quad-6.928869 \quad-4.569950 \quad-2.251507$

$\begin{array}{lllll}6 & -9.107205 & 1.466530 & 2.250190\end{array}$

$\begin{array}{lllll}6 & -7.435974 & 3.134911 & 2.539670\end{array}$

$\begin{array}{lllll}6 & -5.716333 & 4.793721 & 0.063553\end{array}$

$6 \quad-3.347504 \quad 1.644262 \quad-3.950157$

$\begin{array}{lllll}6 & -9.058612 & -2.169815 & 1.602238\end{array}$

$\begin{array}{lllll}6 & -7.310171 & -3.738444 & 1.225859\end{array}$

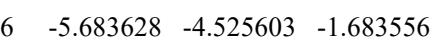

$\begin{array}{lllll}6 & -3.366357 & 0.267106 & -3.781271\end{array}$

$\begin{array}{lllll}6 & -8.544431 & 0.691802 & 3.275845\end{array}$

$\begin{array}{llll}-9.987862 & 1.092290 & 1.741356\end{array}$

$\begin{array}{lllll}6 & -6.862614 & 2.436718 & 3.585638\end{array}$

$\begin{array}{lllll}8 & -6.840955 & 4.322339 & 2.097763\end{array}$

$\begin{array}{lllll}6 & -5.740763 & 4.150779 & 1.331037\end{array}$

$\begin{array}{lllll}6 & -8.488599 & -1.780419 & 2.823133\end{array}$

$\begin{array}{llll}-9.973929 & -1.685416 & 1.284071\end{array}$

$\begin{array}{llll}-6.719107 & -3.419250 & 2.437814\end{array}$

$\begin{array}{llll}-6.745614 & -4.723573 & 0.417318\end{array}$

$6 \quad-5.612364 \quad-4.433789 \quad-0.265941$

$\begin{array}{lllll}6 & -9.097825 & -0.674428 & 3.683677\end{array}$

$\begin{array}{lllll}6 & -7.416877 & 1.212326 & 3.922845\end{array}$

$\begin{array}{lllll}1 & -5.977019 & 2.797790 & 4.087427\end{array}$

$6 \quad-3.591753 \quad 4.150151 \quad-0.196267$

$\begin{array}{lllll}6 & -7.319199 & -2.442065 & 3.221551\end{array}$

$\begin{array}{llll}1 & -5.808471 & -3.905363 & 2.760595\end{array}$

$6 \quad-3.471119 \quad-4.259410 \quad-1.791726$

$\begin{array}{lllll}1 & -8.749982 & -0.852954 & 4.701955\end{array}$

$\begin{array}{lllll}8 & -6.815957 & 0.471475 & 4.945284\end{array}$

$\begin{array}{lllll}6 & -3.728343 & 3.307042 & 0.901000\end{array}$

$8 \quad-6.751130 \quad-2.152669 \quad 4.470302$

$6 \quad-3.436849 \quad-3.998969 \quad-0.429190$

$6 \quad-5.602510 \quad-0.076650 \quad 4.655165$

$\begin{array}{lllll}6 & -5.563295 & -1.483297 & 4.452634\end{array}$

$\begin{array}{lllll}6 & -3.393348 & 0.015892 & 4.344368\end{array}$

$\begin{array}{lllll}6 & -3.344817 & -1.367154 & 4.222782\end{array}$

$\begin{array}{lllll}6 & -10.632168 & -0.708897 & 3.707986\end{array}$

$\begin{array}{lllll}1 & -10.980091 & -1.686141 & 4.052658\end{array}$

$\begin{array}{lllll}1 & -11.081440 & -0.526605 & 2.727867\end{array}$

$\begin{array}{lllll}6 & -10.663019 & 3.528180 & 0.657751\end{array}$

$\begin{array}{lllll}1 & -11.089534 & 3.828365 & 1.618545\end{array}$

$\begin{array}{lllll}1 & -11.051994 & 2.534337 & 0.419320\end{array}$ $\begin{array}{llll}6 & -10.530684 & 0.674357 & -3.424050\end{array}$

$1 \quad-10.887542 \quad 1.638486 \quad-3.795116$

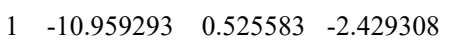

$6 \begin{array}{llll}6 & -10.647165 & -3.585298 & -0.534700\end{array}$

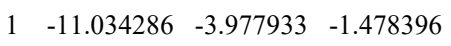

$\begin{array}{lllll}1 & -11.063285 & -2.582928 & -0.401114\end{array}$

$\begin{array}{lllll}1 & -11.021669 & -4.208709 & 0.281450\end{array}$

$\begin{array}{lllll}1 & -10.919477 & -0.114927 & -4.072718\end{array}$

$1 \quad-11.021964 \quad 4.215057 \quad-0.112832$

$1 \quad-11.012206 \quad 0.056120 \quad 4.389980$

$\begin{array}{lllll}6 & -2.263843 & 3.794586 & -0.824474\end{array}$

$\begin{array}{lllll}6 & -2.685279 & 2.266365 & 0.725491\end{array}$

$\begin{array}{lllll}6 & -2.003071 & 0.563552 & 4.139133\end{array}$

$\begin{array}{lllll}6 & -1.908033 & -1.775796 & 4.023509\end{array}$

$\begin{array}{lllll}6 & -1.917043 & 2.103652 & -3.869509\end{array}$

$6 \quad-1.968888 \quad-0.192263 \quad-3.459613$

$6 \quad-2.061185 \quad-4.167584 \quad-2.318094$

$\begin{array}{lllll}6 & -2.039098 & -3.606840 & -0.054917\end{array}$

$\begin{array}{llll}7 & -1.206931 & -0.557610 & 3.955124\end{array}$

$\begin{array}{lllll}7 & -1.876868 & 2.534530 & -0.269600\end{array}$

$7 \quad-1.189987 \quad 0.945176 \quad-3.544332$

$\begin{array}{lllll}7 & -1.295117 & -3.786383 & -1.197586\end{array}$

$\begin{array}{lllll}8 & -1.637608 & -4.375237 & -3.427553\end{array}$

$\begin{array}{llll}8 & -1.666824 & -3.177523 & 1.026724\end{array}$

$\begin{array}{lllll}8 & -1.425646 & -2.878983 & 3.943906\end{array}$

$\begin{array}{lllll}8 & -1.654510 & 1.729023 & 4.097688\end{array}$

$\begin{array}{lllll}8 & -2.748449 & 1.152465 & 1.397564\end{array}$

$\begin{array}{lllll}8 & -1.453290 & 3.202743 & -4.053963\end{array}$

$8 \quad-1.614252 \quad 4.425822 \quad-1.622248$

$8 \quad-1.608155 \quad-1.324191 \quad-3.185321$

$\begin{array}{lllll}1 & -0.274078 & -3.663901 & -1.265322\end{array}$

$\begin{array}{lllll}1 & -0.172222 & 0.997337 & -3.418131\end{array}$

$\begin{array}{llll}-0.317257 & 1.894314 & -0.230289\end{array}$

$\begin{array}{llll}-0.194533 & -0.538225 & 3.809446\end{array}$

$7 \quad-4.611188 \quad 4.872976-0.666450$

$\begin{array}{lllll}7 & -4.779297 & 3.323068 & 1.712618\end{array}$

$\begin{array}{lllll}-4.454036 & 2.370589 & -4.141739\end{array}$

$\begin{array}{llll}-4.464806 & -0.474263 & -3.946377\end{array}$

$\begin{array}{lllll}7 & -4.523706 & 0.688677 & 4.577917\end{array}$

$\begin{array}{lllll}7.432194 & -2.144451 & 4.257657\end{array}$

$\begin{array}{lllll}7 & -4.609715 & -4.487874 & -2.455732\end{array}$

$\begin{array}{llll}7 & -4.500691 & -4.103811 & 0.370857\end{array}$

$\begin{array}{lllll}6 & 8.881275 & -3.048840 & 2.247620\end{array}$

$\begin{array}{lllll}6 & 8.323740 & -1.751237 & 2.849284\end{array}$

$\begin{array}{llll}6 & 8.317020 & -3.326252 & 0.851138\end{array}$

$\begin{array}{lllll}1 & 8.514830 & -3.855347 & 2.884866\end{array}$

$\begin{array}{lllll}6 & 8.934145 & -0.498381 & 2.713217\end{array}$

$6 \quad 7.132706 \quad-1.785346 \quad 3.584626$

$\begin{array}{lllll}6 & 8.888904 & -2.756660 & -0.296810\end{array}$

$\begin{array}{lllll}6 & 7.186505 & -4.132990 & 0.655377\end{array}$

$\begin{array}{llll}6 & 8.406702 & 0.672820 & 3.278217\end{array}$

$\begin{array}{lllll}1 & 9.843673 & -0.423773 & 2.128123\end{array}$

$\begin{array}{lllll}6 & 6.599078 & -0.673296 & 4.213805\end{array}$

$8 \quad 6.485553 \quad-3.012325 \quad 3.715580$

$\begin{array}{lllll}6 & 8.364917 & -2.911932 & -1.583937\end{array}$

$1 \quad 9.770248 \quad-2.138390 \quad-0.173178$

$6 \quad 6.621439 \quad-4.313989 \quad-0.603311$

$\begin{array}{llll}8 & 6.581631 & -4.797687 & 1.735671\end{array}$ $\begin{array}{llll}6 & 9.010302 & 2.056302 & 3.042925\end{array}$

$\begin{array}{llll}7.250121 & 0.540930 & 4.056070\end{array}$

$\begin{array}{llll}5.689169 & -0.744620 & 4.793632\end{array}$

$\begin{array}{llll}5.341690 & -3.208458 & 3.011580\end{array}$

$\begin{array}{llll}9.005444 & -2.280740 & -2.825343\end{array}$

$\begin{array}{lllll}7.199605 & -3.683360 & -1.694446\end{array}$

$\begin{array}{llll}5.736281 & -4.925129 & -0.724459\end{array}$

$\begin{array}{lllll}6 & 5.361778 & -4.271510 & 2.069614\end{array}$

$1 \quad 8.689616 \quad 2.675262 \quad 3.881917$

$\begin{array}{llll}6 & 8.426220 & 2.712261 & 1.787543\end{array}$

$\begin{array}{lllll}8 & 6.737764 & 1.666539 & 4.713607\end{array}$

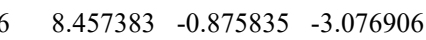

$1 \quad 8.684343 \quad-2.888763 \quad-3.672280$

$\begin{array}{lllll}8 & 6.633915 & -3.864814 & -2.959867\end{array}$

$\begin{array}{lllll}6 & 8.965455 & 2.497822 & 0.515489\end{array}$

$\begin{array}{llll}6 & 7.317988 & 3.568798 & 1.859982\end{array}$

$\begin{array}{lllll}6 & 5.527024 & 2.099745 & 4.257565\end{array}$

$\begin{array}{lllll}6 & 3.187908 & -2.869300 & 2.556720\end{array}$

$\begin{array}{lllll}6.013232 & 0.266635 & -2.481851\end{array}$

$\begin{array}{lllll}6.339123 & -0.680071 & -3.896816\end{array}$

$6 \quad 5.479419 \quad-3.204139 \quad-3.246602$

$\begin{array}{llll}6.164699 & -4.018952 & 1.776471\end{array}$

$\begin{array}{lllll}6.478525 & 3.108820 & -0.646687\end{array}$

$\begin{array}{llll}9.799584 & 1.812582 & 0.421396\end{array}$

$\begin{array}{lllll}6 & 6.821591 & 4.233617 & 0.747274\end{array}$

$\begin{array}{llll}6.715743 & 3.797172 & 3.099338\end{array}$

$\begin{array}{llll}5.521386 & 3.182094 & 3.338374\end{array}$

$\begin{array}{llll}8.468777 & 1.546331 & -2.631727\end{array}$

$\begin{array}{llll}9.897843 & 0.148469 & -1.867297\end{array}$

$\begin{array}{llll}6.741936 & 0.563867 & -4.056016\end{array}$

$\begin{array}{llll}6.805052 & -1.768424 & -4.603203\end{array}$

$\begin{array}{llll}5.561574 & -2.144484 & -4.191241\end{array}$

$\begin{array}{llll}9.080560 & 2.806911 & -2.017516\end{array}$

$\begin{array}{llll}7.410438 & 4.002331 & -0.490985\end{array}$

$\begin{array}{llll}5.980100 & 4.908411 & 0.840177\end{array}$

$\begin{array}{llll}3.304961 & 1.976532 & 4.108165\end{array}$

$\begin{array}{llll}7.301543 & 1.646954 & -3.399317\end{array}$

$\begin{array}{llll}5.859950 & 0.681912 & -4.671201\end{array}$

$\begin{array}{llll}3.279659 & -2.872356 & -3.112958\end{array}$

$\begin{array}{llll}8.785020 & 3.632676 & -2.666483\end{array}$

$\begin{array}{llll}6.912035 & 4.711871 & -1.594857\end{array}$

$\begin{array}{llll}3.304809 & 2.981879 & 3.149119\end{array}$

$\begin{array}{llll}6.746482 & 2.912410 & -3.565880\end{array}$

$\begin{array}{llll}3.347706 & -1.891993 & -4.092556\end{array}$

$\begin{array}{llll}5.668900 & 4.305099 & -1.995378\end{array}$

$\begin{array}{llll}5.589988 & 3.239729 & -2.934681\end{array}$

$\begin{array}{llll}3.442206 & 4.340212 & -1.883359\end{array}$

$\begin{array}{llll}3.378944 & 3.200735 & -2.674062\end{array}$

$\begin{array}{lllll}6 & 10.615208 & 2.766778 & -1.992859\end{array}$

$\begin{array}{llll}11.002381 & 2.606756 & -3.002573\end{array}$

$\begin{array}{llll}11.012858 & 1.970254 & -1.357917\end{array}$

$\begin{array}{lll}10.545503 & 2.047153 & 3.034562\end{array}$

$\begin{array}{llll}10.926658 & 3.065292 & 2.921374\end{array}$

$\begin{array}{llll}10.965470 & 1.447558 & 2.222146\end{array}$

$\begin{array}{llll}6 & 10.415464 & -3.095266 & 2.280582\end{array}$

$\begin{array}{llll}10.772590 & -2.960694 & 3.304863\end{array}$

$\begin{array}{llll}10.879369 & -2.318636 & 1.666214\end{array}$

$\begin{array}{llll}10.539072 & -2.323999 & -2.779312\end{array}$ 
$\begin{array}{lllll}1 & 10.881962 & -3.354839 & -2.658793\end{array}$

$\begin{array}{lllll}1 & 10.959087 & -1.740279 & -1.955500\end{array}$

$1 \quad 10.952743 \quad-1.925373 \quad-3.709244$

$\begin{array}{lllll}1 & 10.769519 & -4.061280 & 1.911614\end{array}$

$\begin{array}{llll}1 & 10.921775 & 1.636361 & 3.975113\end{array}$

$1 \quad 11.007875 \quad 3.714282 \quad-1.615156$

$\begin{array}{lllll}6 & 1.882691 & 1.557664 & 4.364354\end{array}$

$\begin{array}{lllll}6 & 1.882444 & 3.215736 & 2.699341\end{array}$

$\begin{array}{lllll}6 & 2.036308 & 4.780965 & -1.569852\end{array}$

$\begin{array}{lllll}6 & 1.937890 & 2.784119 & -2.783864\end{array}$

$\begin{array}{lllll}6 & 1.821572 & -2.237209 & 2.511381\end{array}$

$\begin{array}{lllll}6 & 1.748686 & -4.250820 & 1.321170\end{array}$

$\begin{array}{lllll}6 & 1.860912 & -2.962559 & -2.637303\end{array}$

$\begin{array}{lllll}6 & 1.954993 & -1.362778 & -4.329860\end{array}$

$\begin{array}{lllll}7 & 1.223953 & 3.805411 & -2.187193\end{array}$

$\begin{array}{lllll}7 & 1.128013 & 2.330935 & 3.476628\end{array}$

$\begin{array}{lllll}7 & 1.048680 & -3.120491 & 1.780751\end{array}$

$\begin{array}{lllll}7 & 1.155410 & -2.065827 & -3.408432\end{array}$

$\begin{array}{lllll}8 & 1.432862 & -3.656514 & -1.728985\end{array}$

$\begin{array}{lllll}8 & 1.564017 & -0.536923 & -5.116953\end{array}$

$\begin{array}{lllll}8 & 1.505299 & 1.746940 & -3.257199\end{array}$

$8 \quad 1.652611 \quad 5.747630 \quad-0.962925$

$8 \quad 1.465972 \quad 3.982856 \quad 1.862086$

$\begin{array}{lllll}8 & 1.471533 & -1.171214 & 2.984872\end{array}$

$\begin{array}{lllll}8 & 1.448694 & 0.753679 & 5.157952\end{array}$

$8 \quad 1.272515 \quad-5.183595 \quad 0.721603$

$1 \quad 0.158818 \quad-1.856434 \quad-3.274350$

$\begin{array}{lllll}1 & 0.044571 & -3.008718 & 1.600982\end{array}$

$\begin{array}{llll}0.107879 & 2.278232 & 3.483052\end{array}$

$\begin{array}{llll}0.204520 & 3.849805 & -2.133476\end{array}$

$\begin{array}{llll}4.419519 & 1.502687 & 4.669596\end{array}$

$\begin{array}{llll}4.414121 & 3.614875 & 2.753207\end{array}$

$\begin{array}{llll}4.271393 & -2.457865 & 3.220158\end{array}$

$\begin{array}{llll}4.264741 & -4.721161 & 1.482340\end{array}$

$7 \quad 4.595460 \quad 4.892241 \quad-1.492521$

$\begin{array}{lllll}7 & 4.451062 & 2.637698 & -3.239390\end{array}$

$\begin{array}{lllll}7 & 4.338378 & -3.560838 & -2.677177\end{array}$

$7 \quad 4.497860 \quad-1.497394 \quad-4.643871$

$\begin{array}{lllll}6 & 1.031978 & 0.493743 & -0.352177\end{array}$

$8 \quad 0.278778 \quad-0.382568 \quad-0.768118$

$\begin{array}{lllll}8 & 0.644714 & 1.722108 & -0.026120\end{array}$

$\begin{array}{lllll}6 & -2.508644 & -0.112017 & 0.661967\end{array}$

$\begin{array}{lllll}1 & -1.593439 & -0.043059 & 0.076110\end{array}$

$\begin{array}{lllll}6 & 2.487187 & 0.310945 & -0.166034\end{array}$

$\begin{array}{lllll}6 & 3.315473 & 1.403688 & 0.116285\end{array}$

$\begin{array}{lllll}6 & 3.037828 & -0.965744 & -0.299313\end{array}$

$\begin{array}{lllll}6 & 4.686298 & 1.217367 & 0.228734\end{array}$

$\begin{array}{lllll}1 & 2.879787 & 2.385374 & 0.246279\end{array}$

$\begin{array}{lllll}6 & 4.408357 & -1.146455 & -0.173466\end{array}$

$\begin{array}{lllll}1 & 2.374026 & -1.800451 & -0.477577\end{array}$

$\begin{array}{lllll}6 & 5.249650 & -0.054177 & 0.076789\end{array}$

$\begin{array}{llll}1 & 5.330430 & 2.063454 & 0.440179\end{array}$

$1 \quad 4.834991 \quad-2.139526 \quad-0.272863$

$\begin{array}{lllll}6 & 6.727952 & -0.241386 & 0.164762\end{array}$

$\begin{array}{llll}1 & 7.191163 & 0.531778 & 0.774154\end{array}$

$\begin{array}{lllll}1 & 6.972743 & -1.210857 & 0.589367\end{array}$

$1 \quad 7.182741 \quad-0.194622 \quad-0.826803$

$\begin{array}{lllll}6 & -3.746757 & -0.365856 & -0.180040\end{array}$
$1 \quad-3.840226 \quad 0.448812 \quad-0.911551$

$1 \quad-3.581086-1.271869-0.773331$

$\begin{array}{llll}6 & -5.031649 & -0.475863 & 0.649464\end{array}$

$\begin{array}{llll}1 & -5.051782 & 0.329289 & 1.392084\end{array}$

$1 \quad-5.015091 \quad-1.419643 \quad 1.206573$

$6 \quad-6.286425 \quad-0.393090 \quad-0.213840$

$\begin{array}{lllll}1 & -7.192098 & -0.472489 & 0.390798\end{array}$

$1 \quad-6.330362 \quad 0.561242 \quad-0.750671$

$\begin{array}{llll}1 & -2.381720 & -0.854556 & 1.445118\end{array}$

$1 \quad-6.308054 \quad-1.191636 \quad-0.959481$

\section{3a@12}

$\begin{array}{lllll}6 & 9.066613 & 2.744687 & -2.283640\end{array}$

$\begin{array}{lllll}6 & 8.432652 & 1.456223 & -2.809617\end{array}$

$\begin{array}{lllll}6 & 8.481719 & 3.125911 & -0.927559\end{array}$

$\begin{array}{lllll}1 & 8.772923 & 3.533520 & -2.977594\end{array}$

$\begin{array}{lllll}6 & 8.970410 & 0.183601 & -2.604234\end{array}$

$\begin{array}{lllll}6 & 7.255211 & 1.527343 & -3.562962\end{array}$

$\begin{array}{llll}6 & 8.945527 & 2.527642 & 0.250162\end{array}$

$\begin{array}{lllll}6 & 7.451300 & 4.065889 & -0.799139\end{array}$

$\begin{array}{lllll}6 & 8.403695 & -0.977375 & -3.147861\end{array}$

$1 \quad 9.870026 \quad 0.088336 \quad-2.007864$

$\begin{array}{lllll}6 & 6.677748 & 0.421125 & -4.164168\end{array}$

$8 \quad 6.705376 \quad 2.791726 \quad-3.759882$

$\begin{array}{llll}6 & 8.416023 & 2.804911 & 1.511518\end{array}$

$\begin{array}{llll}1 & 9.754626 & 1.810221 & 0.177353\end{array}$

$\begin{array}{lllll}6 & 6.861386 & 4.345697 & 0.429993\end{array}$

$\begin{array}{lllll}8 & 6.963703 & 4.751455 & -1.928427\end{array}$

$6 \quad 8.972551 \quad-2.364828 \quad-2.872026$

$\begin{array}{lllll}6 & 7.272140 & -0.817656 & -3.955747\end{array}$

$\begin{array}{lllll}1 & 5.783763 & 0.514711 & -4.765628\end{array}$

$\begin{array}{lllll}6 & 5.596299 & 3.155844 & -3.071622\end{array}$

$\begin{array}{lllll}6 & 8.981481 & 2.191067 & 2.790225\end{array}$

$\begin{array}{llll}6 & 7.335397 & 3.693703 & 1.558043\end{array}$

$\begin{array}{lllll}1 & 6.040568 & 5.047878 & 0.500843\end{array}$

$\begin{array}{lllll}6 & 5.707307 & 4.325228 & -2.266795\end{array}$

$1 \quad 8.628331 \quad-3.004281 \quad-3.685527$

$\begin{array}{lllll}6 & 8.388811 & -2.957797 & -1.589383\end{array}$

$8 \quad 6.715440 \quad-1.943240 \quad-4.588443$

$\begin{array}{lllll}6 & 8.384184 & 0.813330 & 3.048699\end{array}$

$\begin{array}{lllll}1 & 8.645489 & 2.829133 & 3.608067\end{array}$

$\begin{array}{lllll}8 & 6.737938 & 3.950808 & 2.798338\end{array}$

$6 \quad 8.923844 \quad-2.700359 \quad-0.325456$

$\begin{array}{lllll}6 & 7.279028 & -3.809353 & -1.637160\end{array}$

$\begin{array}{lllll}6 & 5.511967 & -2.319809 & -4.070867\end{array}$

$\begin{array}{lllll}6 & 3.420251 & 3.082510 & -2.615219\end{array}$

$\begin{array}{lllll}6 & 8.943807 & -0.357265 & 2.521492\end{array}$

$\begin{array}{llll}6 & 7.233729 & 0.676868 & 3.834114\end{array}$

$\begin{array}{llll}6 & 5.539132 & 3.354309 & 3.037665\end{array}$

$\begin{array}{lllll}6 & 3.493237 & 4.329825 & -2.012143\end{array}$

$\begin{array}{lllll}6 & 8.431401 & -3.279395 & 0.851042\end{array}$

$\begin{array}{lllll}1 & 9.758923 & -2.013810 & -0.249298\end{array}$

$\begin{array}{lllll}6 & 6.778904 & -4.447010 & -0.513026\end{array}$

$8 \quad 6.662221 \quad-4.025564 \quad-2.875344$

$6 \quad 5.502594 \quad-3.347232 \quad-3.086416$

$\begin{array}{lllll}6 & 8.395253 & -1.623219 & 2.741349\end{array}$

$\begin{array}{llll}1 & 9.848772 & -0.276862 & 1.931128\end{array}$

$\begin{array}{llll}6 & 6.633437 & -0.554460 & 4.065080\end{array}$ $\begin{array}{llll}8 & 6.698853 & 1.818582 & 4.449532\end{array}$

$\begin{array}{llll}6 & 5.511647 & 2.267545 & 3.959395\end{array}$

$\begin{array}{llll}6 & 9.015321 & -2.917723 & 2.213771\end{array}$

$\begin{array}{lllll}6 & 7.367898 & -4.181414 & 0.718206\end{array}$

$\begin{array}{lllll}1 & 5.931212 & -5.115391 & -0.585668\end{array}$

$\begin{array}{lllll}6 & 3.316380 & -2.043195 & -3.785586\end{array}$

$\begin{array}{llll}6 & 7.220629 & -1.677220 & 3.500189\end{array}$

$\begin{array}{lllll}1 & 5.736327 & -0.633131 & 4.664367\end{array}$

$\begin{array}{llll}6 & 3.321865 & 3.207127 & 2.837111\end{array}$

$\begin{array}{lllll}1 & 8.705680 & -3.706966 & 2.899923\end{array}$

$8 \quad 6.852240 \quad-4.846506 \quad 1.845482$

$\begin{array}{lllll}6 & 3.334358 & -2.958735 & -2.742393\end{array}$

$\begin{array}{lllll}8 & 6.677432 & -2.940389 & 3.733659\end{array}$

$\begin{array}{llll}6 & 3.287044 & 2.198931 & 3.789335\end{array}$

$\begin{array}{lllll}6 & 5.610373 & -4.396654 & 2.199878\end{array}$

$\begin{array}{lllll}6 & 5.537393 & -3.287780 & 3.089567\end{array}$

$\begin{array}{lllll}6 & 3.389163 & -4.345599 & 1.986388\end{array}$

$\begin{array}{lllll}6 & 3.345047 & -3.178316 & 2.734330\end{array}$

$\begin{array}{lllll}6 & 10.550229 & -2.884438 & 2.212058\end{array}$

$\begin{array}{lllll}1 & 10.922023 & -2.669732 & 3.217430\end{array}$

$\begin{array}{lllll}1 & 10.962263 & -2.127006 & 1.539679\end{array}$

$\begin{array}{lllll}6 & 10.508098 & -2.395862 & -2.878751\end{array}$

$\begin{array}{lllll}1 & 10.861655 & -3.421410 & -2.744723\end{array}$

$\begin{array}{lllll}1 & 10.951960 & -1.790049 & -2.084045\end{array}$

$\begin{array}{lllll}6 & 10.600979 & 2.689467 & -2.271227\end{array}$

$\begin{array}{lllll}1 & 10.976900 & 2.458893 & -3.271566\end{array}$

$\begin{array}{lllll}1 & 10.996903 & 1.933625 & -1.587450\end{array}$

$\begin{array}{lllll}6 & 10.517356 & 2.193316 & 2.805007\end{array}$

$\begin{array}{lllll}1 & 10.889697 & 3.213976 & 2.685608\end{array}$

$\begin{array}{lllll}1 & 10.954552 & 1.589957 & 2.004767\end{array}$

$\begin{array}{lllll}1 & 10.883810 & 1.797328 & 3.755787\end{array}$

$\begin{array}{lllll}1 & 11.006090 & 3.656137 & -1.961327\end{array}$

$1 \quad 10.886386 \quad-2.018450 \quad-3.832405$

$\begin{array}{lllll}1 & 10.944033 & -3.853464 & 1.894921\end{array}$

$\begin{array}{lllll}6 & 1.908662 & -1.534118 & -3.942751\end{array}$

$\begin{array}{lllll}6 & 1.965818 & -3.006136 & -2.132769\end{array}$

$\begin{array}{lllll}6 & 1.975268 & -4.707919 & 1.608816\end{array}$

$\begin{array}{lllll}6 & 1.931366 & -2.687604 & 2.780624\end{array}$

$\begin{array}{lllll}6 & 1.999489 & 2.605199 & -2.543526\end{array}$

$\begin{array}{lllll}6 & 2.094242 & 4.761408 & -1.660622\end{array}$

$\begin{array}{lllll}6 & 1.918311 & 3.472976 & 2.372149\end{array}$

$\begin{array}{lllll}6 & 1.850001 & 1.808854 & 4.011281\end{array}$

$\begin{array}{lllll}7 & 1.195147 & -3.650245 & 2.122167\end{array}$

$\begin{array}{lllll}7 & 1.196723 & -2.157560 & -2.898562\end{array}$

$\begin{array}{lllll}7 & 1.285473 & 3.679059 & -2.063299\end{array}$

$\begin{array}{lllll}7 & 1.122358 & 2.636771 & 3.138611\end{array}$

$\begin{array}{lllll}8 & 1.568753 & 4.235670 & 1.490485\end{array}$

$\begin{array}{lllll}8 & 1.386647 & 0.973998 & 4.750932\end{array}$

$\begin{array}{lllll}8 & 1.543399 & -1.641092 & 3.270786\end{array}$

$8 \quad 1.554778 \quad-5.674960 \quad 1.024085$

$8 \quad 1.611239 \quad-3.639605 \quad-1.150085$

$\begin{array}{lllll}8 & 1.597974 & 1.484251 & -2.821369\end{array}$

$8 \quad 1.444165 \quad-0.777331 \quad-4.759006$

$\begin{array}{lllll}8 & 1.709310 & 5.787550 & -1.158554\end{array}$

$\begin{array}{lllll}1 & 0.107560 & 2.539672 & 3.058503\end{array}$

$\begin{array}{lllll}1 & 0.275952 & 3.696849 & -1.899624\end{array}$

$1 \quad 0.191636 \quad-1.980808 \quad-2.754834$

$\begin{array}{lllll}1 & 0.173518 & -3.644048 & 2.017768\end{array}$ 
$7 \quad 4.413664 \quad-1.687029 \quad-4.456332$

$7 \quad 4.420594 \quad-3.645194 \quad-2.382985$

$\begin{array}{lllll}7 & 4.466229 & 2.477488 & -3.186815\end{array}$

$\begin{array}{lllll}7 & 4.652327 & 4.954387 & -1.778160\end{array}$

$\begin{array}{lllll}7 & 4.534785 & -4.960877 & 1.672985\end{array}$

$\begin{array}{lllll}7 & 4.412167 & -2.631846 & 3.319923\end{array}$

$\begin{array}{lllll}7 & 4.446547 & 3.809633 & 2.442390\end{array}$

$\begin{array}{lllll}7 & 4.385501 & 1.692054 & 4.354850\end{array}$

$\begin{array}{llll}6 & -8.901035 & -0.525643 & 3.490214\end{array}$

$\begin{array}{lllll}6 & -8.314744 & -1.714118 & 2.732816\end{array}$

$\begin{array}{lllll}6 & -8.335783 & 0.804399 & 2.990065\end{array}$

$\begin{array}{lllll}1 & -8.562197 & -0.628162 & 4.521745\end{array}$

$\begin{array}{llll}6 & -8.861696 & -2.153725 & 1.520545\end{array}$

$\begin{array}{lllll}6 & -7.206427 & -2.417584 & 3.223207\end{array}$

$\begin{array}{llll}6 & -8.905210 & 1.539645 & 1.946185\end{array}$

$\begin{array}{lllll}6 & -7.203705 & 1.355071 & 3.601112\end{array}$

$\begin{array}{lllll}6 & -8.350713 & -3.233121 & 0.795427\end{array}$

$\begin{array}{lllll}1 & -9.722821 & -1.627998 & 1.125853\end{array}$

$\begin{array}{llll}6 & -6.648147 & -3.488167 & 2.532146\end{array}$

$\begin{array}{lllll}8 & -6.644909 & -2.066412 & 4.461346\end{array}$

$\begin{array}{llll}6 & -8.409333 & 2.784218 & 1.537761\end{array}$

$\begin{array}{lllll}1 & -9.767005 & 1.131763 & 1.431045\end{array}$

$\begin{array}{llll}6 & -6.693217 & 2.598851 & 3.263565\end{array}$

$\begin{array}{lllll}8 & -6.599607 & 0.621035 & 4.622424\end{array}$

$\begin{array}{lllll}6 & -8.962473 & -3.710301 & -0.522756\end{array}$

$\begin{array}{llll}6 & -7.219342 & -3.866276 & 1.324831\end{array}$

$\begin{array}{lllll}1 & -5.782065 & -4.005324 & 2.923857\end{array}$

$\begin{array}{lllll}6 & -5.437752 & -1.430297 & 4.371794\end{array}$

$\begin{array}{lllll}6 & -8.989906 & 3.546391 & 0.352769\end{array}$

$\begin{array}{llll}6 & -7.314525 & 3.300957 & 2.239879\end{array}$

$\begin{array}{llll}1 & -5.828656 & 3.004061 & 3.771824\end{array}$

$\begin{array}{llll}6 & -5.434050 & -0.009513 & 4.326202\end{array}$

$\begin{array}{lllll}1 & -8.665434 & -4.753065 & -0.639367\end{array}$

$\begin{array}{lllll}6 & -8.347910 & -2.952020 & -1.696442\end{array}$

$\begin{array}{lllll}8 & -6.680743 & -4.943612 & 0.613834\end{array}$

$\begin{array}{lllll}6 & -8.324033 & 3.081354 & -0.940585\end{array}$

$\begin{array}{lllll}1 & -8.706638 & 4.590787 & 0.486319\end{array}$

$\begin{array}{llll}8 & -6.847332 & 4.580872 & 1.914720\end{array}$

$\begin{array}{lllll}6 & -8.834250 & -1.704673 & -2.111593\end{array}$

$\begin{array}{lllll}6 & -7.240757 & -3.464384 & -2.383280\end{array}$

$\begin{array}{lllll}6 & -5.506066 & -4.740900 & -0.037965\end{array}$

$\begin{array}{lllll}6 & -3.221955 & -1.417345 & 4.111403\end{array}$

$\begin{array}{lllll}6 & -8.793905 & 1.984577 & -1.665855\end{array}$

$\begin{array}{lllll}6 & -7.202590 & 3.746958 & -1.453299\end{array}$

$\begin{array}{llll}6 & -5.611628 & 4.630167 & 1.341591\end{array}$

$\begin{array}{llll}6 & -3.246575 & -0.043319 & 3.909683\end{array}$

$\begin{array}{lllll}6 & -8.238214 & -0.957590 & -3.131720\end{array}$

$\begin{array}{lllll}1 & -9.709144 & -1.301521 & -1.615332\end{array}$

$\begin{array}{lllll}6 & -6.589334 & -2.745823 & -3.378124\end{array}$

$\begin{array}{lllll}8 & -6.759805 & -4.745959 & -2.066342\end{array}$

$\begin{array}{lllll}6 & -5.537222 & -4.736888 & -1.460876\end{array}$

$\begin{array}{lllll}6 & -8.228395 & 1.562726 & -2.874419\end{array}$

$\begin{array}{lllll}1 & -9.646406 & 1.439140 & -1.279293\end{array}$

$\begin{array}{lllll}6 & -6.608933 & 3.379400 & -2.653536\end{array}$

$\begin{array}{lllll}8 & -6.711740 & 4.856766 & -0.759536\end{array}$

$\begin{array}{lllll}6 & -5.541185 & 4.720102 & -0.076191\end{array}$

$\begin{array}{lllll}6 & -8.792432 & 0.371722 & -3.643556\end{array}$

$\begin{array}{llll}6 & -7.082857 & -1.495388 & -3.710689\end{array}$ $\begin{array}{llll}1 & -5.710395 & -3.145647 & -3.865403\end{array}$

$\begin{array}{lllll}6 & -3.298484 & -4.453095 & -0.116771\end{array}$

$\begin{array}{lllll}6 & -7.136603 & 2.298141 & -3.353000\end{array}$

$\begin{array}{lllll}1 & -5.753856 & 3.921248 & -3.037055\end{array}$

$\begin{array}{llll}6 & -3.382402 & 4.615537 & 1.441917\end{array}$

$\begin{array}{lllll}1 & -8.430117 & 0.474415 & -4.667115\end{array}$

$\begin{array}{lllll}8 & -6.439810 & -0.739993 & -4.692877\end{array}$

$\begin{array}{lllll}6 & -3.312959 & -4.546522 & -1.500202\end{array}$

$\begin{array}{lllll}8 & -6.547777 & 1.956709 & -4.584280\end{array}$

$\begin{array}{llll}6 & -3.315032 & 4.657395 & 0.055666\end{array}$

$\begin{array}{llll}6 & -5.312105 & -0.076044 & -4.331170\end{array}$

$\begin{array}{lllll}6 & -5.336286 & 1.342953 & -4.431947\end{array}$

$\begin{array}{lllll}6 & -3.168537 & 0.026254 & -3.737368\end{array}$

$\begin{array}{lllll}6 & -3.148068 & 1.387991 & -4.005986\end{array}$

$\begin{array}{lllll}6 & -10.327690 & 0.381993 & -3.696500\end{array}$

$\begin{array}{lllll}1 & -10.680028 & 1.319583 & -4.134069\end{array}$

$\begin{array}{lllll}1 & -10.790503 & 0.284734 & -2.710684\end{array}$

$\begin{array}{lllll}6 & -10.496727 & -3.663182 & -0.515155\end{array}$

$\begin{array}{lllll}1 & -10.887745 & -4.068663 & -1.451923\end{array}$

$\begin{array}{lllll}1 & -10.893564 & -2.650471 & -0.404061\end{array}$

$\begin{array}{llll}6 & -10.436865 & -0.537367 & 3.507542\end{array}$

$\begin{array}{lllll}1 & -10.799421 & -1.476389 & 3.933764\end{array}$

$\begin{array}{lllll}1 & -10.877149 & -0.435979 & 2.511902\end{array}$

$\begin{array}{lllll}6 & -10.522313 & 3.485824 & 0.287204\end{array}$

$\begin{array}{lllll}1 & -10.951277 & 3.881366 & 1.211607\end{array}$

$\begin{array}{lllll}1 & -10.906535 & 2.470726 & 0.154200\end{array}$

$\begin{array}{lllll}1 & -10.885947 & 4.086749 & -0.550330\end{array}$

$\begin{array}{lllll}1 & -10.810165 & 0.289346 & 4.117499\end{array}$

$\begin{array}{lllll}1 & -10.886272 & -4.261247 & 0.312724\end{array}$

$\begin{array}{lllll}1 & -10.686948 & -0.446750 & -4.312220\end{array}$

$\begin{array}{llll}6 & -1.899315 & -4.162366 & 0.326622\end{array}$

$\begin{array}{lllll}6 & -1.893771 & -4.411779 & -1.993904\end{array}$

$\begin{array}{lllll}6 & -1.815373 & -0.375610 & -3.231726\end{array}$

$\begin{array}{lllll}6 & -1.746615 & 1.886643 & -3.793362\end{array}$

$\begin{array}{lllll}6 & -1.794278 & -1.879029 & 4.017870\end{array}$

$\begin{array}{lllll}6 & -1.858890 & 0.413369 & 3.553994\end{array}$

$\begin{array}{llll}6 & -1.975184 & 4.565436 & 1.980732\end{array}$

$\begin{array}{lllll}6 & -1.868813 & 4.588818 & -0.350920\end{array}$

$\begin{array}{lllll}7 & -1.040119 & 0.759181 & -3.333238\end{array}$

$\begin{array}{lllll}7 & -1.139876 & -4.186534 & -0.823190\end{array}$

$\begin{array}{llll}7 & -1.074362 & -0.718391 & 3.682024\end{array}$

$\begin{array}{lllll}7 & -1.163763 & 4.581640 & 0.838841\end{array}$

$\begin{array}{lllll}8 & -1.589165 & 4.512551 & 3.125134\end{array}$

$\begin{array}{lllll}8 & -1.420259 & 4.507192 & -1.478381\end{array}$

$\begin{array}{lllll}8 & -1.282788 & 2.987723 & -3.961311\end{array}$

$\begin{array}{lllll}8 & -1.485155 & -1.467121 & -2.792691\end{array}$

$\begin{array}{lllll}8 & -1.453432 & -4.480628 & -3.114029\end{array}$

$\begin{array}{llll}8 & -1.326561 & -2.978477 & 4.191849\end{array}$

$\begin{array}{lllll}8 & -1.530269 & -3.907760 & 1.461705\end{array}$

$\begin{array}{llll}8 & -1.513320 & 1.523335 & 3.191710\end{array}$

$\begin{array}{llll}1 & -0.144390 & 4.485352 & 0.906903\end{array}$

$\begin{array}{lllll}1 & -0.063418 & -0.766988 & 3.537814\end{array}$

$\begin{array}{lllll}1 & -0.124547 & -4.023560 & -0.849194\end{array}$

$\begin{array}{lllll}1 & -0.043587 & 0.846926 & -3.095436\end{array}$

$\begin{array}{lllll}7 & -4.387655 & -4.561676 & 0.646356\end{array}$

$\begin{array}{lllll}7 & -4.441782 & -4.670879 & -2.203470\end{array}$

$\begin{array}{llll}7 & -4.325178 & -2.145324 & 4.306688\end{array}$

$\begin{array}{llll}7 & -4.350148 & 0.694252 & 4.036875\end{array}$
$7 \quad-4.243269 \quad-0.742233 \quad-3.921181$

$\begin{array}{lllll}7 & -4.244419 & 2.082655 & -4.318236\end{array}$

$\begin{array}{llll}7 & -4.535128 & 4.588667 & 2.113492\end{array}$

$\begin{array}{lllll}7 & -4.390681 & 4.710557 & -0.733758\end{array}$

$\begin{array}{lllll}6 & -0.356750 & 0.865439 & 0.109882\end{array}$

$\begin{array}{lllll}8 & -0.007228 & 1.994672 & -0.189764\end{array}$

$\begin{array}{lllll}8 & 0.494672 & -0.078666 & 0.563314\end{array}$

$\begin{array}{lllll}6 & 1.859230 & 0.343813 & 0.827911\end{array}$

$\begin{array}{llll}1 & 2.025843 & 0.130441 & 1.884129\end{array}$

$\begin{array}{lllll}1 & 1.925327 & 1.415221 & 0.642351\end{array}$

$\begin{array}{lllll}6 & 2.870105 & -0.405999 & -0.023047\end{array}$

$\begin{array}{lllll}1 & 2.702670 & -0.167470 & -1.079618\end{array}$

$\begin{array}{lllll}1 & 2.728428 & -1.486052 & 0.099711\end{array}$

$\begin{array}{lllll}6 & 4.297789 & -0.013275 & 0.391725\end{array}$

$\begin{array}{lllll}1 & 4.448587 & -0.268500 & 1.449237\end{array}$

$\begin{array}{lllll}1 & 4.407374 & 1.078387 & 0.322173\end{array}$

$\begin{array}{lllll}6 & 5.381782 & -0.684722 & -0.454821\end{array}$

$\begin{array}{lllll}1 & 5.281473 & -0.409734 & -1.510544\end{array}$

$\begin{array}{lllll}1 & 6.383655 & -0.387998 & -0.129315\end{array}$

$\begin{array}{lllll}1 & 5.319378 & -1.775335 & -0.383083\end{array}$

$\begin{array}{lllll}6 & -1.772182 & 0.422101 & 0.042451\end{array}$

$\begin{array}{llll}6 & -2.220433 & -0.784912 & 0.584220\end{array}$

$\begin{array}{lllll}6 & -2.701843 & 1.304819 & -0.527255\end{array}$

$\begin{array}{lllll}6 & -3.584962 & -1.076500 & 0.600155\end{array}$

$\begin{array}{lllll}1 & -1.508413 & -1.476438 & 1.013281\end{array}$

$\begin{array}{lllll}6 & -4.054778 & 0.997356 & -0.531196\end{array}$

$\begin{array}{lllll}1 & -2.342373 & 2.220610 & -0.975474\end{array}$

$\begin{array}{lllll}6 & -4.522153 & -0.192046 & 0.049346\end{array}$

$\begin{array}{llll}1 & -3.927791 & -1.996894 & 1.061602\end{array}$

$\begin{array}{lllll}1 & -4.762675 & 1.689527 & -0.979511\end{array}$

$\begin{array}{lllll}6 & -5.995691 & -0.494940 & 0.067739\end{array}$

$\begin{array}{lllll}1 & -6.558146 & 0.307298 & 0.556682\end{array}$

$\begin{array}{lllll}1 & -6.390464 & -0.586196 & -0.949171\end{array}$

$\begin{array}{llll}1 & -6.206947 & -1.424763 & 0.594812\end{array}$

\section{3a: $\mathbf{N}_{2} @ \mathbf{1}_{2}$}

$\begin{array}{lllll}6 & -9.042457 & 3.033015 & 1.774326\end{array}$

$\begin{array}{lllll}6 & -8.406292 & 1.870247 & 2.535563\end{array}$

$\begin{array}{lllll}6 & -8.475132 & 3.145152 & 0.363287\end{array}$

$\begin{array}{llll}-8.738907 & 3.940201 & 2.297838\end{array}$

$\begin{array}{lllll}6 & -8.920373 & 0.573182 & 2.489166\end{array}$

$\begin{array}{lllll}6 & -7.280589 & 2.072744 & 3.344260\end{array}$

$\begin{array}{lllll}6 & -8.998347 & 2.420272 & -0.715456\end{array}$

$\begin{array}{lllll}6 & -7.388176 & 3.987540 & 0.099916\end{array}$

$\begin{array}{lllll}6 & -8.389719 & -0.487668 & 3.230850\end{array}$

$\begin{array}{llll}-9.776419 & 0.379218 & 1.854346\end{array}$

$\begin{array}{lllll}6 & -6.728733 & 1.063367 & 4.121707\end{array}$

$\begin{array}{llll}8 & -6.739001 & 3.360911 & 3.402686\end{array}$

$\begin{array}{lllll}6 & -8.469163 & 2.485123 & -2.008199\end{array}$

$\begin{array}{llll}-9.853174 & 1.778470 & -0.537887\end{array}$

$\begin{array}{llll}-6.802056 & 4.062915 & -1.156933\end{array}$

$\begin{array}{llll}-6.893391 & 4.809911 & 1.124769\end{array}$

$\begin{array}{lllll}6 & -8.973638 & -1.892022 & 3.138994\end{array}$

$\begin{array}{llll}-7.296529 & -0.204543 & 4.058061\end{array}$

$\begin{array}{llll}-5.869776 & 1.255700 & 4.751148\end{array}$

$\begin{array}{llll}-5.587018 & 3.602619 & 2.721485\end{array}$

$\begin{array}{llll}-9.069792 & 1.724743 & -3.195172\end{array}$

$\begin{array}{llll}-7.340499 & 3.295606 & -2.180702\end{array}$ 
$1 \quad-5.945726 \quad 4.700497 \quad-1.333428$

$\begin{array}{llll}-5.658617 & 4.463570 & 1.590220\end{array}$

$\begin{array}{lll}-8.643664 & -2.421180 & 4.033453\end{array}$

$\begin{array}{llll}-8.385419 & -2.650859 & 1.952171\end{array}$

$\begin{array}{llll}-6.766051 & -1.220933 & 4.874148\end{array}$

$\begin{array}{llll}-8.490149 & 0.315610 & -3.299880\end{array}$

$\begin{array}{llll}-8.744792 & 2.256723 & -4.090083\end{array}$

$\begin{array}{llll}-6.766182 & 3.362169 & -3.453238\end{array}$

$\begin{array}{llll}-8.932183 & -2.604309 & 0.667985\end{array}$

$\begin{array}{llll}-7.247677 & -3.447255 & 2.125593\end{array}$

$\begin{array}{llll}-5.547089 & -1.687168 & 4.478186\end{array}$

$\begin{array}{llll}-3.376331 & 3.480676 & 2.452725\end{array}$

$\begin{array}{llll}-9.027125 & -0.764638 & -2.585261\end{array}$

$\begin{array}{llll}-7.352339 & 0.061848 & -4.075803\end{array}$

$\begin{array}{llll}-5.584434 & 2.716964 & -3.637934\end{array}$

$\begin{array}{llll}-3.431334 & 4.429029 & 1.440504\end{array}$

$\begin{array}{llll}-8.423176 & -3.339180 & -0.411382\end{array}$

$\begin{array}{llll}-9.790264 & -1.965824 & 0.495465\end{array}$

$\begin{array}{llll}-6.726029 & -4.228791 & 1.107432\end{array}$

$\begin{array}{llll}-6.667816 & -3.486123 & 3.397972\end{array}$

$\begin{array}{llll}-5.507138 & -2.812004 & 3.601867\end{array}$

$\begin{array}{llll}-8.453657 & -2.039911 & -2.582776\end{array}$

$\begin{array}{llll}-9.916546 & -0.596717 & -1.989848\end{array}$

$\begin{array}{llll}-6.738947 & -1.185591 & -4.104581\end{array}$

$\begin{array}{llll}-6.811958 & 1.085062 & -4.870066\end{array}$

$\begin{array}{llll}-5.597782 & 1.558105 & -4.464519\end{array}$

$\begin{array}{llll}-9.030895 & -3.229525 & -1.810256\end{array}$

$\begin{array}{llll}-7.332453 & -4.176493 & -0.143981\end{array}$

$\begin{array}{llll}-5.863470 & -4.859743 & 1.276891\end{array}$

$\begin{array}{llll}-3.323137 & -1.530475 & 4.348669\end{array}$

$\begin{array}{llll}-7.286597 & -2.202667 & -3.339552\end{array}$

$\begin{array}{llll}-5.845886 & -1.349190 & -4.692811\end{array}$

$\begin{array}{llll}-3.374482 & 2.515879 & -3.428073\end{array}$

$\begin{array}{llll}-8.713150 & -4.122989 & -2.349557\end{array}$

$\begin{array}{llll}-6.814066 & -5.007241 & -1.155725\end{array}$

$\begin{array}{llll}-3.303180 & -2.537831 & 3.394827\end{array}$

$\begin{array}{llll}-6.698584 & -3.469020 & -3.348193\end{array}$

$\begin{array}{llll}-3.372309 & 1.448452 & -4.315368\end{array}$

$\begin{array}{llll}-5.586757 & -4.596021 & -1.589511\end{array}$

$\begin{array}{llll}-5.545000 & -3.660359 & -2.662846\end{array}$

$\begin{array}{llll}-3.372743 & -4.428510 & -1.378296\end{array}$

$\begin{array}{llll}-3.359221 & -3.414814 & -2.325047\end{array}$

$\begin{array}{llll}-10.566190 & -3.227294 & -1.786837\end{array}$

$\begin{array}{llll}-10.957191 & -3.209617 & -2.807497\end{array}$

$\begin{array}{llll}-10.984515 & -2.365953 & -1.258775\end{array}$

$\begin{array}{llll}-10.509436 & -1.899357 & 3.139849\end{array}$

$\begin{array}{llll}-10.878777 & -2.928197 & 3.135897\end{array}$

$\begin{array}{llll}-10.940288 & -1.391695 & 2.272583\end{array}$

$\begin{array}{llll}-10.577061 & 2.979502 & 1.796896\end{array}$

$\begin{array}{lll}-10.936617 & 2.958892 & 2.828913\end{array}$

$\begin{array}{llll}-10.982237 & 2.099564 & 1.289729\end{array}$

$\begin{array}{llll}-10.604987 & 1.739594 & -3.179245\end{array}$

$\begin{array}{llll}-10.969454 & 2.769986 & -3.169527\end{array}$

$\begin{array}{llll}-11.027925 & 1.233826 & -2.306780\end{array}$

$\begin{array}{llll}-10.994006 & 1.240329 & -4.070503\end{array}$

$\begin{array}{llll}-10.988413 & 3.863017 & 1.301841\end{array}$

$\begin{array}{llll}-10.885187 & -1.397499 & 4.035282\end{array}$

$\begin{array}{llll}-10.933550 & -4.127899 & -1.288264\end{array}$ $\begin{array}{lllll}6 & -1.915314 & -1.027535 & 4.533913\end{array}$

$\begin{array}{lllll}6 & -1.906654 & -2.655035 & 2.859756\end{array}$

$6 \quad-1.958864 \quad-4.651089 \quad-0.908561$

$6 \quad-1.963405 \quad-2.882991 \quad-2.433092$

$\begin{array}{lllll}6 & -1.953466 & 3.018050 & 2.590337\end{array}$

$\begin{array}{llll}-2.021612 & 4.712296 & 0.987898\end{array}$

$\begin{array}{lllll}6 & -1.966470 & 2.752260 & -2.959362\end{array}$

$6 \quad-1.943930 \quad 1.012074 \quad-4.522307$

$7 \quad-1.208284 \quad-3.663055 \quad-1.583446$

$\begin{array}{lllll}7 & -1.157830 & -1.763252 & 3.598578\end{array}$

$7 \quad-1.226965 \quad 3.842853 \quad 1.757229$

$7 \quad-1.195483 \quad 1.854510 \quad-3.676348$

$\begin{array}{lllll}8 & -1.596993 & 3.524666 & -2.092650\end{array}$

$8 \quad-1.495244 \quad 0.150328 \quad-5.237649$

$8 \quad-1.594888-1.932651 \quad-3.106320$

$\begin{array}{llll}-1.515584 & -5.473426 & -0.147106\end{array}$

$\begin{array}{llll}-1.528905 & -3.349693 & 1.928778\end{array}$

$\begin{array}{llll}-1.555512 & 2.076009 & 3.256820\end{array}$

$\begin{array}{lllll}8 & -1.484037 & -0.188932 & 5.285069\end{array}$

$8 \quad-1.622366 \quad 5.490124 \quad 0.155999$

$\begin{array}{llll}-0.182223 & 1.738529 & -3.572964\end{array}$

$\begin{array}{llll}-0.213666 & 3.800969 & 1.618566\end{array}$

$\begin{array}{llll}-0.154271 & -1.583054 & 3.463857\end{array}$

$\begin{array}{llll}-0.189917 & -3.598300 & -1.460180\end{array}$

$\begin{array}{llll}-4.452443 & -1.065849 & 4.889611\end{array}$

$\begin{array}{llll}-4.389652 & -3.216108 & 3.016655\end{array}$

$\begin{array}{llll}-4.447282 & 3.067057 & 3.132488\end{array}$

$\begin{array}{llll}-4.580580 & 4.916169 & 0.966407\end{array}$

$\begin{array}{llll}-4.498727 & -5.019859 & -0.966364\end{array}$

$\begin{array}{llll}-4.438978 & -3.020037 & -3.005325\end{array}$

$\begin{array}{llll}-4.478018 & 3.182495 & -3.079720\end{array}$

$\begin{array}{llll}-4.491055 & 0.931306 & -4.834178\end{array}$

$\begin{array}{llll}8.962601 & -1.027506 & -3.540071\end{array}$

$\begin{array}{llll}8.394032 & -2.107601 & -2.619776\end{array}$

$\begin{array}{llll}8.377111 & 0.352156 & -3.222613\end{array}$

$\begin{array}{llll}8.618890 & -1.275586 & -4.545258\end{array}$

$\begin{array}{llll}8.953044 & -2.374617 & -1.361545\end{array}$

$\begin{array}{llll}7.274298 & -2.865900 & -2.988254\end{array}$

$\begin{array}{llll}8.951149 & 1.243018 & -2.308974\end{array}$

$\begin{array}{llll}7.209555 & 0.781849 & -3.865008\end{array}$

$\begin{array}{lllll}6 & 8.423908 & -3.309455 & -0.465770\end{array}$

$\begin{array}{llll}9.828012 & -1.811044 & -1.059782\end{array}$

$\begin{array}{llll}6.700027 & -3.796464 & -2.129759\end{array}$

$\begin{array}{llll}6.704690 & -2.716911 & -4.262743\end{array}$

$\begin{array}{llll}8.415779 & 2.511019 & -2.043790\end{array}$

$\begin{array}{llll}9.843131 & 0.936098 & -1.775331\end{array}$

$\begin{array}{llll}6.660599 & 2.040290 & -3.673232\end{array}$

$8 \quad 6.607677 \quad-0.092185 \quad-4.769820$

$\begin{array}{llll}9.036908 & -3.592133 & 0.909116\end{array}$

$\begin{array}{llll}7.266639 & -3.983450 & -0.878188\end{array}$

$\begin{array}{llll}5.820878 & -4.351697 & -2.429285\end{array}$

$\begin{array}{llll}5.481054 & -2.106142 & -4.253744\end{array}$

$\begin{array}{lllll}6 & 8.998008 & 3.448939 & -0.991793\end{array}$

$\begin{array}{llll}7.279036 & 2.890256 & -2.767672\end{array}$

$\begin{array}{llll}5.768226 & 2.345865 & -4.202452\end{array}$

$\begin{array}{lllll}6 & 5.450052 & -0.692263 & -4.392080\end{array}$

$1 \quad 8.724297 \quad-4.602173 \quad 1.178021$

$\begin{array}{llll}6 & 8.450642 & -2.666085 & 1.974692\end{array}$ $\begin{array}{llll}8 & 6.691882 & -4.906540 & -0.001715\end{array}$

$\begin{array}{llll}8.378475 & 3.176002 & 0.379219\end{array}$

$\begin{array}{llll}8.685454 & 4.455738 & -1.271255\end{array}$

$\begin{array}{llll}6.771199 & 4.183151 & -2.596288\end{array}$

$\begin{array}{llll}6.970948 & -1.386550 & 2.218358\end{array}$

$6 \quad \begin{array}{llll}7.342812 & -3.053733 & 2.738710\end{array}$

$\begin{array}{lllll}6 & 5.535057 & -4.558054 & 0.620834\end{array}$

$\begin{array}{lllll}6.267285 & -2.104552 & -3.976163\end{array}$

$\begin{array}{llll}8.890792 & 2.207159 & 1.245515\end{array}$

$\begin{array}{llll}7.268918 & 3.903392 & 0.832295\end{array}$

$\begin{array}{llll}5.551884 & 4.272642 & -1.993932\end{array}$

$\begin{array}{llll}3.266089 & -0.715423 & -3.959034\end{array}$

$\begin{array}{llll}8.402187 & -0.492026 & 3.130393\end{array}$

$\begin{array}{llll}9.848249 & -1.072989 & 1.664871\end{array}$

$\begin{array}{llll}6.722463 & -2.192748 & 3.635885\end{array}$

$\begin{array}{llll}6.826817 & -4.354906 & 2.612926\end{array}$

$\begin{array}{llll}5.592500 & -4.387779 & 2.031615\end{array}$

$\begin{array}{llll}8.378382 & 1.966049 & 2.525874\end{array}$

$\begin{array}{llll}9.731968 & 1.612352 & 0.909879\end{array}$

$\begin{array}{llll}6.737266 & 3.724904 & 2.102623\end{array}$

$\begin{array}{llll}6.712945 & 4.876233 & -0.003739\end{array}$

$\begin{array}{llll}5.524497 & 4.587714 & -0.607308\end{array}$

$\begin{array}{llll}8.976504 & 0.892565 & 3.432886\end{array}$

$\begin{array}{llll}7.244646 & -0.920343 & 3.792109\end{array}$

$\begin{array}{llll}5.846267 & -2.505973 & 4.187465\end{array}$

$\begin{array}{llll}3.341710 & -4.167493 & 0.691180\end{array}$

$\begin{array}{llll}7.301492 & 2.763362 & 2.935385\end{array}$

$\begin{array}{llll}5.891678 & 4.313701 & 2.434933\end{array}$

$\begin{array}{llll}3.323097 & 4.158287 & -2.003011\end{array}$

$\begin{array}{llll}8.652847 & 1.137842 & 4.445347\end{array}$

$\begin{array}{llll}6.644585 & -0.035257 & 4.686551\end{array}$

$\begin{array}{llll}3.378307 & -4.122202 & 2.077656\end{array}$

$\begin{array}{llll}6.763572 & 2.612386 & 4.224825\end{array}$

$\begin{array}{llll}3.300127 & 4.415194 & -0.639182\end{array}$

$\begin{array}{llll}5.494419 & 0.586081 & 4.318899\end{array}$

$\begin{array}{llll}5.538210 & 2.004799 & 4.225926\end{array}$

$\begin{array}{llll}3.306035 & 0.647167 & 3.906028\end{array}$

$\begin{array}{llll}3.322161 & 2.034089 & 3.977343\end{array}$

$\begin{array}{lll}10.512185 & 0.906697 & 3.424189\end{array}$

$\begin{array}{llll}10.878383 & 1.896266 & 3.709177\end{array}$

$\begin{array}{llll}10.936870 & 0.668566 & 2.444939\end{array}$

$\begin{array}{llll}10.571642 & -3.573930 & 0.883532\end{array}$

$\begin{array}{llll}10.964969 & -3.842197 & 1.867409\end{array}$

$\begin{array}{llll}10.983754 & -2.595758 & 0.620035\end{array}$

$\begin{array}{llll}10.497712 & -1.021077 & -3.565639\end{array}$

$\begin{array}{llll}10.871475 & -2.005781 & -3.857875\end{array}$

$\begin{array}{llll}10.941058 & -0.775363 & -2.596484\end{array}$

$\begin{array}{llll}10.532731 & 3.433845 & -0.956176\end{array}$

$\begin{array}{llll}10.931479 & 3.694160 & -1.940164\end{array}$

$\begin{array}{llll}10.945848 & 2.459399 & -0.680649\end{array}$

$\begin{array}{llll}10.898244 & 4.162614 & -0.228186\end{array}$

$\begin{array}{llll}10.857402 & -0.283287 & -4.287487\end{array}$

$\begin{array}{llll}10.943572 & -4.294896 & 0.150948\end{array}$

$\begin{array}{llll}10.897884 & 0.173372 & 4.137247\end{array}$

$\begin{array}{llll}1.934249 & -3.896683 & 0.246525\end{array}$

$\begin{array}{llll}1.974262 & -3.910952 & 2.580319\end{array}$

$\begin{array}{llll}1.902078 & 0.209495 & 3.591660\end{array}$

$\begin{array}{lll}1.909959 & 2.530212 & 3.823689\end{array}$ 
$6 \quad 1.848520 \quad-2.577680 \quad-3.803390$

$\begin{array}{lllll}6 & 1.871414 & -0.243079 & -3.655117\end{array}$

$\begin{array}{lllll}6 & 1.902103 & 3.986744 & -2.476465\end{array}$

$\begin{array}{lllll}6 & 1.872409 & 4.364019 & -0.167476\end{array}$

$\begin{array}{lllll}7 & 1.149053 & 1.362411 & 3.631866\end{array}$

$\begin{array}{lllll}7 & 1.202587 & -3.777482 & 1.408128\end{array}$

$\begin{array}{lllll}7 & 1.110040 & -1.396172 & -3.609515\end{array}$

$7 \quad 1.129153 \quad 4.110596 \quad-1.310673$

$\begin{array}{lllll}8 & 1.480891 & 3.795987 & -3.592200\end{array}$

$8 \quad 1.467515 \quad 4.484320 \quad 0.971793$

$\begin{array}{lllll}8 & 1.480191 & 3.657205 & 3.849471\end{array}$

$\begin{array}{lllll}8 & 1.520365 & -0.919589 & 3.324232\end{array}$

$\begin{array}{lllll}8 & 1.553569 & -3.871282 & 3.709661\end{array}$

$\begin{array}{lllll}8 & 1.397205 & -3.696923 & -3.828839\end{array}$

$8 \quad 1.534181 \quad-3.770493 \quad-0.900490$

$8 \quad 1.499621 \quad 0.899862 \quad-3.460793$

$\begin{array}{lllll}1 & 0.110899 & 4.025752 & -1.343573\end{array}$

$1 \quad 0.097016 \quad-1.428002 \quad-3.447277$

$\begin{array}{lllll}1 & 0.185424 & -3.631379 & 1.455197\end{array}$

$1 \quad 0.141986 \quad 1.434697 \quad 3.443678$

$7 \quad 4.414528 \quad-4.406737 \quad-0.068099$

$7 \quad 4.513269 \quad-4.206702 \quad 2.776733$

$\begin{array}{llll}4.385087 & -2.829801 & -4.087059\end{array}$

$\begin{array}{lllll}7 & 4.353505 & 0.021813 & -4.192584\end{array}$

$\begin{array}{lllll}7 & 4.388102 & -0.109644 & 4.101493\end{array}$

$\begin{array}{lllll}7 & 4.447249 & 2.743190 & 4.097460\end{array}$

$7 \quad 4.453869 \quad 4.070339 \quad-2.707189$

$\begin{array}{lllll}7 & 4.398158 & 4.640114 & 0.087695\end{array}$

$\begin{array}{lllll}6 & 0.852907 & 0.753296 & 0.102392\end{array}$

$\begin{array}{lllll}8 & 0.396175 & 1.717586 & 0.695637\end{array}$

$\begin{array}{lllll}8 & 0.089458 & -0.096088 & -0.610909\end{array}$

$\begin{array}{lllll}6 & -1.299466 & 0.296746 & -0.715174\end{array}$

$\begin{array}{lllll}-1.650449 & -0.174977 & -1.631238\end{array}$

$\begin{array}{llll}-1.331142 & 1.380919 & -0.819870\end{array}$

$\begin{array}{llll}-2.128081 & -0.124860 & 0.485959\end{array}$

$\begin{array}{llll}-1.621906 & 0.185533 & 1.403868\end{array}$

$\begin{array}{llll}-2.226041 & -1.215816 & 0.510761\end{array}$

$\begin{array}{llll}-3.502952 & 0.542192 & 0.408709\end{array}$

$\begin{array}{llll}-3.982618 & 0.263323 & -0.536224\end{array}$

$\begin{array}{llll}-3.364983 & 1.629935 & 0.357721\end{array}$

$\begin{array}{llll}-4.413122 & 0.197028 & 1.582557\end{array}$

$\begin{array}{llll}-3.942960 & 0.467354 & 2.530359\end{array}$

$\begin{array}{llll}-5.360781 & 0.735577 & 1.533240\end{array}$

$\begin{array}{llll}-4.644867 & -0.872406 & 1.598395\end{array}$

$\begin{array}{llll}2.292495 & 0.426841 & 0.066578\end{array}$

$\begin{array}{llll}2.781767 & -0.727497 & -0.550848\end{array}$

$\begin{array}{llll}6.184595 & 1.335727 & 0.650945\end{array}$

$\begin{array}{lllll}6 & 4.152320 & -0.963002 & -0.590498\end{array}$

$\begin{array}{llll}2.088886 & -1.430833 & -0.991086\end{array}$

$\begin{array}{llll}6.549626 & 1.094209 & 0.606121\end{array}$

$\begin{array}{llll}2.792592 & 2.227153 & 1.123514\end{array}$

$\begin{array}{llll}5.052740 & -0.055280 & -0.018880\end{array}$

$\begin{array}{llll}4.529450 & -1.861568 & -1.069017\end{array}$

$\begin{array}{llll}5.238354 & 1.802876 & 1.053591\end{array}$

$\begin{array}{llll}6.529599 & -0.289936 & -0.085040\end{array}$

$\begin{array}{llll}7.003189 & 0.406680 & -0.781495\end{array}$

$\begin{array}{llll}6.996902 & -0.135242 & 0.888374\end{array}$

$\begin{array}{llll}6.755299 & -1.300035 & -0.417896\end{array}$
$7 \quad-6.914832 \quad-0.153755 \quad-0.328762$

$\begin{array}{lllll}7 & -6.256261 & 0.235242 & -1.126711\end{array}$

\section{2b}

$\begin{array}{lllll}6 & 0.747977 & -1.501921 & 0.011274\end{array}$

$\begin{array}{lllll}8 & 0.820156 & -2.587773 & -0.540849\end{array}$

$\begin{array}{lllll}7 & -0.471630 & -1.156924 & 0.651960\end{array}$

$\begin{array}{lllll}7 & -0.426117 & -0.189160 & 1.616540\end{array}$

$\begin{array}{lllll}8 & -1.478425 & 0.019409 & 2.192306\end{array}$

$\begin{array}{lllll}6 & -1.671949 & -1.964912 & 0.390872\end{array}$

$\begin{array}{lllll}1 & -2.375314 & -1.715532 & 1.183840\end{array}$

$\begin{array}{lllll}1 & -1.379632 & -3.012567 & 0.478167\end{array}$

$\begin{array}{lllll}6 & -2.253853 & -1.690718 & -1.002056\end{array}$

$\begin{array}{lllll}1 & -3.153507 & -2.310213 & -1.105623\end{array}$

$\begin{array}{lllll}1 & -1.539047 & -2.049185 & -1.749511\end{array}$

$\begin{array}{lllll}6 & -2.599247 & -0.222203 & -1.274690\end{array}$

$\begin{array}{lllll}1 & -2.892084 & -0.126997 & -2.327951\end{array}$

$1 \quad-1.694385 \quad 0.390545 \quad-1.161033$

$\begin{array}{lllll}6 & -3.711272 & 0.348602 & -0.389654\end{array}$

$\begin{array}{lllll}1 & -3.417924 & 0.281373 & 0.664141\end{array}$

$1 \quad-4.615875 \quad-0.265970 \quad-0.502940$

$\begin{array}{lllll}6 & 1.871953 & -0.529228 & 0.004253\end{array}$

$\begin{array}{lllll}6 & 1.712770 & 0.863296 & 0.010563\end{array}$

$\begin{array}{lllll}6 & 3.161101 & -1.065698 & -0.127808\end{array}$

$\begin{array}{lllll}6 & 2.824659 & 1.692251 & -0.094588\end{array}$

$\begin{array}{lllll}1 & 0.725723 & 1.301310 & 0.080886\end{array}$

$\begin{array}{lllll}6 & 4.266360 & -0.229499 & -0.211830\end{array}$

$\begin{array}{lllll}1 & 3.274005 & -2.143220 & -0.166423\end{array}$

$\begin{array}{lllll}6 & 4.117936 & 1.164347 & -0.193344\end{array}$

$1 \quad 2.686665 \quad 2.769581 \quad-0.101274$

$1 \quad 5.259831 \quad-0.659851 \quad-0.300590$

$\begin{array}{lllll}6 & 5.320571 & 2.069798 & -0.258556\end{array}$

$\begin{array}{lllll}1 & 6.120385 & 1.629045 & -0.860284\end{array}$

$\begin{array}{lllll}1 & 5.065442 & 3.043460 & -0.685447\end{array}$

$\begin{array}{llll}1 & 5.727436 & 2.248227 & 0.744275\end{array}$

$6 \quad-4.043786 \quad 1.806936 \quad-0.717277$

$\begin{array}{lllll}1 & -3.135385 & 2.414265 & -0.606020\end{array}$

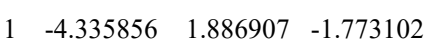

$\begin{array}{lllll}6 & -5.151964 & 2.378715 & 0.168964\end{array}$

$\begin{array}{lllll}1 & -4.868046 & 2.338990 & 1.226239\end{array}$

$\begin{array}{lllll}1 & -5.368816 & 3.422212 & -0.080382\end{array}$

$\begin{array}{llll}1 & -6.080766 & 1.808525 & 0.055140\end{array}$

\section{2b'-TS}

$\begin{array}{llll}6 & -0.976502 & 1.074556 & -0.050873\end{array}$

$\begin{array}{lllll}8 & -0.737270 & 2.250952 & -0.290320\end{array}$

$\begin{array}{llll}7 & 0.046745 & 0.163992 & 0.265195\end{array}$

$\begin{array}{lllll}7 & -0.270214 & -0.802286 & 1.410441\end{array}$

$8 \quad-0.295954 \quad-1.929459 \quad 1.057802$

$\begin{array}{lllll}6 & 1.373897 & 0.717361 & 0.567253\end{array}$

$\begin{array}{lllll}1 & 1.507422 & 0.740769 & 1.660134\end{array}$

$\begin{array}{lllll}1 & 1.368034 & 1.753497 & 0.220694\end{array}$

$\begin{array}{lllll}6 & 2.484371 & -0.100252 & -0.088240\end{array}$

$\begin{array}{lllll}1 & 2.334324 & -0.089008 & -1.174229\end{array}$

$\begin{array}{lllll}1 & 2.401228 & -1.149723 & 0.223165\end{array}$

$\begin{array}{lllll}6 & 3.876489 & 0.432771 & 0.258724\end{array}$

$1 \quad 4.012081 \quad 0.417840 \quad 1.349305$ $\begin{array}{lllll}1 & 3.948198 & 1.487225 & -0.041613\end{array}$

$\begin{array}{lllll}6 & 5.005975 & -0.360982 & -0.402514\end{array}$

$14.874296 \quad-0.339654 \quad-1.493241$

$14.928379 \quad-1.417182 \quad-0.108640$

$\begin{array}{lllll}6 & -2.353338 & 0.516166 & -0.137259\end{array}$

$6 \quad-2.619518 \quad-0.788508 \quad-0.570533$

$\begin{array}{lllll}6 & -3.425179 & 1.367491 & 0.167078\end{array}$

$\begin{array}{lllll}6 & -3.934433 & -1.236032 & -0.669759\end{array}$

$\begin{array}{lllll}1 & -1.800813 & -1.440377 & -0.850830\end{array}$

$\begin{array}{lllll}6 & -4.731986 & 0.907494 & 0.078577\end{array}$

$\begin{array}{lllll}1 & -3.210000 & 2.386949 & 0.467617\end{array}$

$\begin{array}{lllll}6 & -5.009161 & -0.403705 & -0.337293\end{array}$

$\begin{array}{lllll}1 & -4.130352 & -2.246263 & -1.017633\end{array}$

$\begin{array}{lllll}1 & -5.554253 & 1.572628 & 0.327822\end{array}$

$\begin{array}{lllll}6 & -6.430047 & -0.901328 & -0.410251\end{array}$

$1 \quad-6.812514 \quad-1.139695 \quad 0.589524$

$\begin{array}{lllll}1 & -7.095317 & -0.145211 & -0.838306\end{array}$

$1 \quad-6.506293 \quad-1.807146 \quad-1.017188$

$\begin{array}{lllll}6 & 6.401849 & 0.160634 & -0.050719\end{array}$

$\begin{array}{llll}6.531669 & 0.136999 & 1.039548\end{array}$

$\begin{array}{llll}6.476527 & 1.216739 & -0.342303\end{array}$

$\begin{array}{llll}7.524102 & -0.636640 & -0.718260\end{array}$

$\begin{array}{llll}8.510014 & -0.243664 & -0.450976\end{array}$

$\begin{array}{llll}7.492086 & -1.689917 & -0.417815\end{array}$

$\begin{array}{llll}7.436243 & -0.602856 & -1.809919\end{array}$

\section{2b'}

$\begin{array}{lllll}6 & 0.438959 & -1.733674 & -0.075583\end{array}$

$\begin{array}{lllll}8 & 0.525955 & -2.756453 & -0.732261\end{array}$

$\begin{array}{lllll}7 & -0.795647 & -1.480746 & 0.619357\end{array}$

$\begin{array}{lllll}7 & -0.908703 & -1.065482 & 1.924247\end{array}$

$\begin{array}{llll}8 & 0.128968 & -0.809692 & 2.502018\end{array}$

$\begin{array}{lllll}6 & -2.034580 & -2.046811 & 0.068600\end{array}$

$\begin{array}{lllll}1 & -2.802231 & -1.808848 & 0.806404\end{array}$

$\begin{array}{lllll}1 & -1.934083 & -3.134307 & 0.014798\end{array}$

$\begin{array}{lllll}6 & -2.381741 & -1.476819 & -1.310863\end{array}$

$\begin{array}{lllll}1 & -3.397400 & -1.814499 & -1.551883\end{array}$

$\begin{array}{lllll}1 & -1.719131 & -1.923440 & -2.058972\end{array}$

$\begin{array}{lllll}6 & -2.298946 & 0.052197 & -1.404598\end{array}$

$\begin{array}{lllll}1 & -2.614448 & 0.355943 & -2.410622\end{array}$

$\begin{array}{lllll}1 & -1.250564 & 0.363060 & -1.316981\end{array}$

$\begin{array}{lllll}6 & -3.130894 & 0.805885 & -0.363546\end{array}$

$\begin{array}{lllll}1 & -2.804319 & 0.531353 & 0.647669\end{array}$

$\begin{array}{lllll}1 & -4.183260 & 0.496931 & -0.438918\end{array}$

$\begin{array}{lllll}6 & 1.488217 & -0.693216 & -0.050179\end{array}$

$\begin{array}{lllll}6 & 1.218662 & 0.647315 & 0.258771\end{array}$

$\begin{array}{lllll}6 & 2.779386 & -1.057129 & -0.453027\end{array}$

$\begin{array}{lllll}6 & 2.226586 & 1.598101 & 0.170649\end{array}$

$\begin{array}{llll}1 & 0.221128 & 0.945792 & 0.557878\end{array}$

$\begin{array}{lllll}6 & 3.786361 & -0.102121 & -0.520153\end{array}$

$\begin{array}{lllll}1 & 2.971719 & -2.092339 & -0.711560\end{array}$

$\begin{array}{lllll}6 & 3.528375 & 1.239579 & -0.208556\end{array}$

$\begin{array}{llll}1 & 2.004906 & 2.636101 & 0.401194\end{array}$

$14.786946 \quad-0.396266 \quad-0.823705$

$\begin{array}{lllll}6 & 4.626812 & 2.269673 & -0.254622\end{array}$

$\begin{array}{lllll}1 & 5.087095 & 2.389839 & 0.733693\end{array}$

$\begin{array}{lllll}1 & 5.418053 & 1.980242 & -0.951274\end{array}$

$\begin{array}{llll}1 & 4.243791 & 3.248943 & -0.555460\end{array}$ 


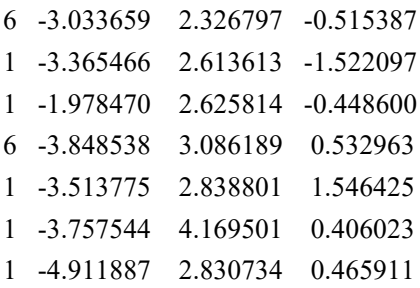

\section{4b-TS}

$\begin{array}{rrrrrrrrr}6 & -2.336769 & -0.956809 & -0.058992 & 6 & 3.412074 & -0.892081 & 0.440775 \\ 8 & -3.227500 & -1.422851 & -0.693712 & 6 & 4.287568 & 1.033417 & -0.734691 \\ 7 & -2.322193 & 0.890473 & 0.065928 & 6 & 4.674892 & -1.474921 & 0.406840 \\ 7 & -2.640358 & 0.903786 & 1.283321 & 1 & 2.582700 & -1.406207 & 0.911262 \\ 8 & -2.774902 & -0.334487 & 1.645600 & 6 & 5.543936 & 0.441986 & -0.762912 \\ 6 & -2.065774 & 2.107096 & -0.688641 & 1 & 4.115695 & 2.011687 & -1.169486 \\ 1 & -2.210092 & 2.965684 & -0.022724 & 6 & 5.758369 & -0.822408 & -0.195770 \\ 1 & -2.814618 & 2.159900 & -1.486938 & 1 & 4.825326 & -2.452357 & 0.856609 \\ 6 & -0.655081 & 2.082656 & -1.288150 & 1 & 6.374411 & 0.965118 & -1.228780 \\ 1 & -0.580883 & 2.899138 & -2.016635 & 6 & 7.116212 & -1.473718 & -0.257516 \\ 1 & -0.531610 & 1.149663 & -1.851369 & 1 & 7.233405 & -2.043538 & -1.187459 \\ 6 & 0.460730 & 2.210300 & -0.248700 & 1 & 7.917266 & -0.729649 & -0.229481 \\ 1 & 0.313207 & 1.459640 & 0.534108 & 1 & 7.264594 & -2.169648 & 0.572668 \\ 1 & 0.386993 & 3.191798 & 0.240491 & 6 & -7.295992 & -0.188083 & -0.287403 \\ 6 & 1.852481 & 2.047135 & -0.869569 & 1 & -7.600743 & -0.247089 & 0.765988 \\ 1 & 1.961562 & 1.014432 & -1.228545 & 1 & -7.387554 & 0.868723 & -0.571421 \\ 1 & 1.923682 & 2.690961 & -1.756607 & 6 & -8.241229 & -1.033470 & -1.143248 \\ 6 & -0.914359 & -1.380838 & -0.003825 & 1 & -8.193650 & -2.090013 & -0.856845 \\ 6 & -0.099496 & -1.213119 & 1.119135 & 1 & -9.280036 & -0.705756 & -1.037263 \\ 6 & -0.382137 & -1.947253 & -1.168488 & 1 & -7.977134 & -0.967608 & -2.204575\end{array}$

$\begin{array}{lllll}6 & 1.235055 & -1.601475 & 1.067241\end{array}$

$\begin{array}{llll}1 & -0.522489 & -0.802087 & 2.027029\end{array}$

$\begin{array}{lllll}6 & 0.959865 & -2.312142 & -1.214156\end{array}$

$\begin{array}{lllll}1 & -1.027128 & -2.093344 & -2.028225\end{array}$

$\begin{array}{lllll}6 & 1.791938 & -2.140217 & -0.100528\end{array}$

$\begin{array}{lllll}1 & 1.857758 & -1.483700 & 1.948817\end{array}$

$\begin{array}{lllll}1 & 1.366997 & -2.743665 & -2.124266\end{array}$

$6 \quad 3.253960 \quad-2.501100 \quad-0.162542$

$\begin{array}{lllll}1 & 3.854978 & -1.637370 & -0.472962\end{array}$

$\begin{array}{lllll}1 & 3.627811 & -2.822879 & 0.813364\end{array}$

$\begin{array}{lllll}1 & 3.438728 & -3.302935 & -0.882497\end{array}$

$\begin{array}{llll}6 & 3.010211 & 2.386893 & 0.078813\end{array}$

$\begin{array}{lllll}1 & 3.950284 & 2.354753 & -0.485923\end{array}$

$\begin{array}{lllll}1 & 2.897706 & 3.424005 & 0.422289\end{array}$

$\begin{array}{lllll}6 & 3.123385 & 1.457570 & 1.289505\end{array}$

$\begin{array}{llll}1 & 3.975488 & 1.731315 & 1.919635\end{array}$

$1 \quad 2.225143 \quad 1.492106 \quad 1.913611$

$\begin{array}{llll}1 & 3.259508 & 0.419305 & 0.970882\end{array}$

\section{4b}

$\begin{array}{lrrr}6 & 1.894133 & 1.059041 & -0.132828 \\ 8 & 1.678253 & 2.147850 & -0.607280 \\ 7 & -1.113186 & 0.110602 & 1.075335 \\ 7 & -0.352903 & 0.902254 & 0.532601 \\ 8 & 0.942448 & 0.273896 & 0.485972 \\ 6 & -2.478630 & 0.652919 & 1.152042 \\ 1 & -2.505951 & 1.700608 & 0.829971 \\ 1 & -2.767662 & 0.598659 & 2.207725 \\ 6 & -3.417666 & -0.212692 & 0.308431\end{array}$ $\begin{array}{llll}1 & -3.307657 & -1.260309 & 0.612299\end{array}$

$\begin{array}{lllll}1 & -3.102579 & -0.154465 & -0.740552\end{array}$

$\begin{array}{llll}6 & -4.878766 & 0.224528 & 0.439976\end{array}$

$\begin{array}{llll}1 & -4.972515 & 1.281041 & 0.154012\end{array}$

$\begin{array}{llll}1 & -5.183966 & 0.164841 & 1.493823\end{array}$

$\begin{array}{lllll}6 & -5.831498 & -0.617320 & -0.412719\end{array}$

$\begin{array}{lllll}1 & -5.736584 & -1.674435 & -0.128015\end{array}$

$1 \quad-5.524504-0.557898 \quad-1.466061$

$\begin{array}{lllll}6 & 3.210622 & 0.370193 & -0.132562\end{array}$

$\begin{array}{lllll}6 & 3.412074 & -0.892081 & 0.440775\end{array}$

$\begin{array}{lllll}6 & 4.287568 & 1.033417 & -0.734691\end{array}$

$\begin{array}{lllll}6 & 4.674892 & -1.474921 & 0.406840\end{array}$

$\begin{array}{lllll}1 & 2.582700 & -1.406207 & 0.911262\end{array}$

$\begin{array}{lllll}6 & 5.543936 & 0.441986 & -0.762912\end{array}$

$6 \quad 5.758369-0.822408 \quad-0.195770$

$\begin{array}{lllll}1 & 4.825326 & -2.452357 & 0.856609\end{array}$

$\begin{array}{lllll}1 & 6.374411 & 0.965118 & -1.228780\end{array}$

$6 \quad 7.116212 \quad-1.473718-0.257516$

$17.233405 \quad-2.043538-1.187459$

$17.917266-0.729649-0.229481$

$17.264594 \quad-2.169648 \quad 0.572668$

$\begin{array}{llll}6 & -7.295992 & -0.188083 & -0.287403\end{array}$

$\begin{array}{llll}1 & -7.600743 & -0.247089 & 0.765988\end{array}$

$\begin{array}{lllll}1 & -7.387554 & 0.868723 & -0.571421\end{array}$

$\begin{array}{lllll}6 & -8.241229 & -1.033470 & -1.143248\end{array}$

$1 \quad-8.193650-2.090013-0.856845$

$\begin{array}{llll}1 & -7.977134 & -0.967608 & -2.204575\end{array}$

\section{4b'-TS}

$\begin{array}{llll}6 & 1.257061 & -1.891235 & 0.485434\end{array}$

$8 \quad 1.798632-1.646157 \quad 1.560150$

$\begin{array}{lllll}7 & 3.105857 & -0.187914 & -0.164557\end{array}$

$\begin{array}{lllll}7 & 2.963361 & -0.903028 & -1.072183\end{array}$

$\begin{array}{lllll}8 & 1.934120 & -2.280980 & -0.591279\end{array}$

$\begin{array}{lllll}6 & 3.580467 & 1.210044 & -0.184525\end{array}$

$\begin{array}{lllll}1 & 3.856092 & 1.497915 & -1.204002\end{array}$

$\begin{array}{llll}1 & 4.458682 & 1.239051 & 0.463926\end{array}$

$\begin{array}{llll}6 & 2.431715 & 2.046382 & 0.393019\end{array}$

$\begin{array}{llll}1 & 2.789103 & 3.077125 & 0.499133\end{array}$

$\begin{array}{lllll}1 & 2.221923 & 1.665284 & 1.398011\end{array}$

$\begin{array}{lllll}6 & 1.155993 & 1.996321 & -0.456265\end{array}$

$1 \quad 0.952718 \quad 0.961796 \quad-0.754396$

$\begin{array}{lllll}1 & 1.307258 & 2.562315 & -1.385308\end{array}$

$\begin{array}{lllll}6 & -0.077020 & 2.515423 & 0.286472\end{array}$

$\begin{array}{lllll}1 & 0.082946 & 3.553927 & 0.608837\end{array}$

$\begin{array}{lllll}1 & -0.209053 & 1.921203 & 1.200167\end{array}$

$\begin{array}{lllll}6 & -0.200082 & -1.624839 & 0.263560\end{array}$

$6 \quad-0.788149-1.762699-0.997565$

$6 \begin{array}{llll}6 & -0.978836 & -1.165291 & 1.335007\end{array}$

$\begin{array}{lllll}6 & -2.132834 & -1.444150 & -1.181892\end{array}$

$1 \quad-0.178919-2.114607-1.822008$

$\begin{array}{lllll}6 & -2.317058 & -0.847330 & 1.143247\end{array}$

$1 \quad-0.506363 \quad-1.059895 \quad 2.305479$

$6-2.917044-0.980015-0.119540$

$1-2.581073-1.552891 \quad-2.165713$

$1 \quad-2.912123 \quad-0.489944 \quad 1.979811$ $\begin{array}{lllll}6 & -4.368195 & -0.622233 & -0.316260\end{array}$

$\begin{array}{lllll}1 & -4.552418 & 0.426736 & -0.060346\end{array}$

$\begin{array}{lllll}1 & -4.681471 & -0.776555 & -1.351859\end{array}$

$\begin{array}{lllll}1 & -5.016259 & -1.229095 & 0.325842\end{array}$

$\begin{array}{lllll}6 & -1.354073 & 2.422566 & -0.553156\end{array}$

$\begin{array}{lllll}1 & -1.249251 & 3.051293 & -1.447523\end{array}$

$\begin{array}{lllll}1 & -1.471152 & 1.392026 & -0.907835\end{array}$

$\begin{array}{lllll}6 & -2.604688 & 2.833184 & 0.225280\end{array}$

$\begin{array}{lllll}1 & -2.526540 & 3.861624 & 0.595736\end{array}$

$\begin{array}{lllll}1 & -3.501967 & 2.771919 & -0.398848\end{array}$

$\begin{array}{llll}1 & -2.752213 & 2.177691 & 1.090201\end{array}$

\section{$4 b$ '}

$\begin{array}{lllll}6 & 0.955065 & -1.271514 & 0.637389\end{array}$

$\begin{array}{lllll}8 & 1.723849 & -1.577405 & -0.388686\end{array}$

$\begin{array}{lllll}8 & 1.366682 & -0.969845 & 1.756240\end{array}$

$\begin{array}{lllll}6 & -0.507425 & -1.224843 & 0.301753\end{array}$

$\begin{array}{lllll}6 & -1.402272 & -0.805122 & 1.294006\end{array}$

$\begin{array}{lllll}6 & -0.991233 & -1.517214 & -0.976590\end{array}$

$\begin{array}{llll}6 & -2.753677 & -0.666273 & 1.004520\end{array}$

$\begin{array}{lllll}1 & -1.008705 & -0.584121 & 2.279667\end{array}$

$\begin{array}{lllll}6 & -2.348503 & -1.374449 & -1.259569\end{array}$

$\begin{array}{lllll}1 & -0.298424 & -1.845861 & -1.742683\end{array}$

$\begin{array}{lllll}6 & -3.248811 & -0.941479 & -0.279293\end{array}$

$\begin{array}{lllll}1 & -3.438891 & -0.333486 & 1.779735\end{array}$

$\begin{array}{lllll}1 & -2.715613 & -1.597826 & -2.257511\end{array}$

$\begin{array}{lllll}6 & -4.715093 & -0.769829 & -0.585548\end{array}$

$\begin{array}{lllll}1 & -5.035387 & 0.263519 & -0.411769\end{array}$

$\begin{array}{lllll}1 & -4.938830 & -1.021588 & -1.625143\end{array}$

$\begin{array}{lllll}1 & -5.331095 & -1.409366 & 0.056339\end{array}$

$\begin{array}{lllll}7 & 4.028800 & -0.714630 & -0.150470\end{array}$

$\begin{array}{lllll}7 & 3.435691 & -1.645271 & 0.221595\end{array}$

$\begin{array}{lllll}6 & 3.576416 & 0.475997 & -0.913110\end{array}$

$\begin{array}{lllll}1 & 3.015399 & 0.071973 & -1.758378\end{array}$

$\begin{array}{lllll}1 & 4.482173 & 0.967294 & -1.266617\end{array}$

$\begin{array}{lllll}6 & 2.687607 & 1.387267 & -0.035366\end{array}$

$\begin{array}{lllll}1 & 3.240587 & 2.297165 & 0.217074\end{array}$

$\begin{array}{lllll}1 & 2.471737 & 0.871507 & 0.903834\end{array}$

$\begin{array}{lllll}6 & 1.359608 & 1.724072 & -0.728748\end{array}$

$\begin{array}{lllll}1 & 0.947565 & 0.802585 & -1.153593\end{array}$

$\begin{array}{lllll}1 & 1.532880 & 2.407161 & -1.571097\end{array}$

$\begin{array}{lllll}6 & 0.326277 & 2.319344 & 0.231594\end{array}$

$\begin{array}{lllll}1 & 0.677566 & 3.289108 & 0.610837\end{array}$

$\begin{array}{lllll}1 & 0.239050 & 1.657857 & 1.101988\end{array}$

$\begin{array}{lllll}6 & -1.053340 & 2.481442 & -0.413425\end{array}$

$\begin{array}{lllll}1 & -0.976530 & 3.154007 & -1.278588\end{array}$

$\begin{array}{lllll}1 & -1.377860 & 1.509136 & -0.802662\end{array}$

$\begin{array}{lllll}6 & -2.106050 & 3.010358 & 0.561508\end{array}$

$\begin{array}{lllll}1 & -3.082478 & 3.114571 & 0.077275\end{array}$

$\begin{array}{lllll}1 & -2.225963 & 2.325861 & 1.407442\end{array}$

$\begin{array}{lllll}1 & -1.824277 & 3.991278 & 0.960813\end{array}$

\section{5b-TS}

$\begin{array}{lllll}6 & 0.195319 & -1.412257 & 0.416685\end{array}$

$\begin{array}{lllll}8 & 0.709989 & -1.314220 & 1.572664\end{array}$

$\begin{array}{lllll}7 & 3.109627 & -1.212486 & -0.761849\end{array}$

$\begin{array}{lllll}7 & 3.250897 & -1.303287 & -1.863301\end{array}$

$\begin{array}{lllll}8 & 0.840916 & -1.747296 & -0.623363\end{array}$ 
$\begin{array}{llll}6 & 3.270278 & -0.907510 & 0.636630\end{array}$

$\begin{array}{lllll}1 & 3.970511 & -1.668162 & 0.996337\end{array}$

$\begin{array}{lllll}1 & 2.251679 & -1.096334 & 1.118440\end{array}$

$\begin{array}{llll}6 & 3.794572 & 0.531077 & 0.810299\end{array}$

$\begin{array}{llll}1 & 4.865601 & 0.571902 & 0.583390\end{array}$

$\begin{array}{llll}1 & 3.681279 & 0.712895 & 1.885020\end{array}$

$\begin{array}{llll}6 & 3.045985 & 1.614634 & 0.019338\end{array}$

$\begin{array}{lllll}1 & 3.334556 & 1.559837 & -1.039721\end{array}$

$\begin{array}{llll}1 & 3.417731 & 2.583689 & 0.374236\end{array}$

$\begin{array}{llll}6 & 1.518796 & 1.559814 & 0.135185\end{array}$

$\begin{array}{llll}1 & 1.227657 & 1.397667 & 1.179746\end{array}$

$\begin{array}{lllll}1 & 1.148123 & 0.691140 & -0.416205\end{array}$

$\begin{array}{lllll}6 & -1.244269 & -1.007975 & 0.250893\end{array}$

$\begin{array}{lllll}6 & -1.847909 & -1.039928 & -1.009863\end{array}$

$\begin{array}{lllll}6 & -1.971526 & -0.524452 & 1.342506\end{array}$

$\begin{array}{lllll}6 & -3.155460 & -0.590975 & -1.174437\end{array}$

$\begin{array}{lllll}1 & -1.267144 & -1.406587 & -1.849082\end{array}$

$\begin{array}{lllll}6 & -3.278235 & -0.075850 & 1.173041\end{array}$

$\begin{array}{lllll}1 & -1.485999 & -0.496966 & 2.311650\end{array}$

$\begin{array}{lllll}6 & -3.891416 & -0.101789 & -0.086727\end{array}$

$1-3.614534 \quad-0.613091 \quad-2.159805$

$\begin{array}{lllll}1 & -3.833208 & 0.306001 & 2.026468\end{array}$

$\begin{array}{lllll}6 & -5.316681 & 0.359852 & -0.262741\end{array}$

$\begin{array}{lllll}1 & -5.488788 & 0.750992 & -1.269795\end{array}$

$1 \quad-6.021282 \quad-0.467219 \quad-0.109940$

$\begin{array}{lllll}1 & -5.575133 & 1.142927 & 0.456166\end{array}$

$\begin{array}{lllll}6 & 0.823904 & 2.811324 & -0.406604\end{array}$

$\begin{array}{lllll}1 & 1.142340 & 2.984129 & -1.444129\end{array}$

$\begin{array}{lllll}1 & 1.155005 & 3.690267 & 0.163566\end{array}$

$\begin{array}{lllll}6 & -0.701803 & 2.696428 & -0.349476\end{array}$

$\begin{array}{lllll}1 & -1.184044 & 3.602867 & -0.729144\end{array}$

$\begin{array}{lllll}1 & -1.057820 & 1.847392 & -0.940796\end{array}$

$\begin{array}{llll}1 & -1.046769 & 2.534835 & 0.676858\end{array}$

\section{$9 \mathbf{b}$}

$\begin{array}{llll}7 & -3.176706 & -0.205546 & -0.044840\end{array}$

$\begin{array}{llll}7 & -3.980946 & -0.988266 & 0.205476\end{array}$

$\begin{array}{llll}6 & -2.267349 & 0.678523 & -0.296530\end{array}$

$1-2.482982 \quad 1.355664-1.113494$

$\begin{array}{lllll}6 & -0.950055 & 0.667708 & 0.434685\end{array}$

$\begin{array}{lllll}1 & -1.049866 & 0.064540 & 1.344544\end{array}$

$\begin{array}{lllll}1 & -0.721981 & 1.688951 & 0.768620\end{array}$

$\begin{array}{lllll}6 & 0.223041 & 0.139046 & -0.408495\end{array}$

$1 \quad 0.293303 \quad 0.725589 \quad-1.334216$

$\begin{array}{lllll}1 & 0.005506 & -0.891704 & -0.715747\end{array}$

$\begin{array}{llll}6 & 1.560103 & 0.190832 & 0.334563\end{array}$

$1 \quad 1.485015 \quad-0.393882 \quad 1.262326$

$\begin{array}{lllll}1 & 1.765048 & 1.225345 & 0.644718\end{array}$

$\begin{array}{lllll}6 & 2.736069 & -0.329891 & -0.496910\end{array}$

$\begin{array}{lllll}1 & 2.530747 & -1.364120 & -0.803190\end{array}$

$\begin{array}{lllll}1 & 2.807187 & 0.253099 & -1.424772\end{array}$

$\begin{array}{lllll}6 & 4.069753 & -0.270482 & 0.250353\end{array}$

$14.035566 \quad-0.870213 \quad 1.166744$

$14.892080 \quad-0.648880 \quad-0.364814$

$\begin{array}{lllll}1 & 4.314568 & 0.757873 & 0.538829\end{array}$

\section{3b-TS}

$\begin{array}{llll}6 & -1.174018 & 0.623388 & -0.130057\end{array}$ $\begin{array}{llll}8 & -0.923617 & 1.710818 & 0.484512\end{array}$

$\begin{array}{lllll}8 & -0.324275 & -0.038098 & -0.792738\end{array}$

$\begin{array}{lllll}7 & 2.434753 & 2.779556 & -0.290527\end{array}$

$\begin{array}{lllll}7 & 2.555368 & 3.863137 & -0.058706\end{array}$

$\begin{array}{lllll}6 & 1.561573 & 1.396319 & -0.412452\end{array}$

$1 \quad 0.575243 \quad 1.772996 \quad 0.064981$

$\begin{array}{lllll}1 & 1.520721 & 1.293250 & -1.493718\end{array}$

$\begin{array}{lllll}6 & -2.582204 & 0.095061 & -0.053358\end{array}$

$\begin{array}{lllll}6 & -3.560263 & 0.778285 & 0.675948\end{array}$

$\begin{array}{lllll}6 & -2.923613 & -1.093312 & -0.706689\end{array}$

$\begin{array}{lllll}6 & -4.857606 & 0.278898 & 0.748055\end{array}$

$\begin{array}{lllll}1 & -3.281606 & 1.695407 & 1.182386\end{array}$

$\begin{array}{lllll}6 & -4.222373 & -1.587329 & -0.631269\end{array}$

$\begin{array}{lllll}1 & -2.153510 & -1.614812 & -1.263888\end{array}$

$\begin{array}{lllll}6 & -5.210782 & -0.908792 & 0.094210\end{array}$

$\begin{array}{lllll}1 & -5.610557 & 0.814605 & 1.320816\end{array}$

$1 \quad-4.477624 \quad-2.513915 \quad-1.139611$

$\begin{array}{llll}6 & 2.348764 & 0.340959 & 0.329604\end{array}$

$\begin{array}{llll}1 & 2.507040 & 0.661932 & 1.366043\end{array}$

$\begin{array}{llll}1 & 1.658703 & -0.506092 & 0.359743\end{array}$

$\begin{array}{lllll}6 & -6.625535 & -1.429882 & 0.146788\end{array}$

$\begin{array}{lllll}1 & -7.219977 & -1.043452 & -0.690599\end{array}$

$1 \quad-6.652063 \quad-2.521953 \quad 0.086122$

$\begin{array}{lllll}1 & -7.129246 & -1.127114 & 1.069427\end{array}$

$\begin{array}{lllll}6 & 3.669918 & -0.066649 & -0.326905\end{array}$

$\begin{array}{lllll}1 & 4.335790 & 0.805751 & -0.398534\end{array}$

$\begin{array}{lllll}1 & 3.472781 & -0.389761 & -1.357489\end{array}$

$\begin{array}{lllll}6 & 4.382993 & -1.186328 & 0.433966\end{array}$

$\begin{array}{lllll}1 & 4.566977 & -0.864403 & 1.468481\end{array}$

$\begin{array}{lllll}1 & 3.716887 & -2.057016 & 0.500289\end{array}$

$\begin{array}{lllll}6 & 5.707747 & -1.606496 & -0.209206\end{array}$

$1 \quad 5.521175 \quad-1.925716 \quad-1.243041$

$1 \quad 6.370452 \quad-0.733123 \quad-0.275001$

$\begin{array}{lllll}6 & 6.411515 & -2.728839 & 0.555775\end{array}$

$\begin{array}{lllll}1 & 5.783381 & -3.624896 & 0.606399\end{array}$

$\begin{array}{lllll}1 & 7.354727 & -3.009137 & 0.077061\end{array}$

$1 \quad 6.636290 \quad-2.424850 \quad 1.584114$

\section{3b}

$\begin{array}{lllll}6 & -0.656276 & -1.509917 & 0.178465\end{array}$

$\begin{array}{lllll}8 & -0.476466 & -2.275997 & 1.106675\end{array}$

$\begin{array}{lllll}8 & 0.177394 & -1.415522 & -0.883982\end{array}$

$\begin{array}{lllll}6 & 1.361298 & -2.244494 & -0.841683\end{array}$

$1 \quad 1.657950 \quad-2.342916 \quad-1.889135$

$\begin{array}{lllll}1 & 1.091695 & -3.224328 & -0.441809\end{array}$

$6 \quad 2.463192 \quad-1.597956 \quad-0.011844$

$\begin{array}{lllll}1 & 3.329632 & -2.272818 & -0.025930\end{array}$

$\begin{array}{lllll}1 & 2.127846 & -1.541800 & 1.029461\end{array}$

$\begin{array}{lllll}6 & 2.871932 & -0.211014 & -0.513842\end{array}$

$\begin{array}{lllll}1 & 1.988822 & 0.439100 & -0.531124\end{array}$

$\begin{array}{lllll}1 & 3.216011 & -0.286389 & -1.555249\end{array}$

$\begin{array}{lllll}6 & 3.965723 & 0.436490 & 0.338273\end{array}$

$1 \quad 4.849875 \quad-0.216473 \quad 0.358155$

$\begin{array}{llll}1 & 3.619704 & 0.508327 & 1.378871\end{array}$

$\begin{array}{lllll}6 & -1.805650 & -0.574301 & 0.073999\end{array}$

$\begin{array}{lllll}6 & -1.955217 & 0.316517 & -0.995985\end{array}$

$\begin{array}{lllll}6 & -2.760704 & -0.586856 & 1.097475\end{array}$

$\begin{array}{llll}6 & -3.047193 & 1.177538 & -1.035537\end{array}$ $\begin{array}{lllll}1 & -1.213061 & 0.330449 & -1.785092\end{array}$

$\begin{array}{lllll}6 & -3.848644 & 0.276321 & 1.048330\end{array}$

$\begin{array}{lllll}1 & -2.627034 & -1.278168 & 1.922032\end{array}$

$\begin{array}{lllll}6 & -4.011885 & 1.170145 & -0.019243\end{array}$

$\begin{array}{lllll}1 & -3.154997 & 1.869292 & -1.866467\end{array}$

$\begin{array}{lllll}1 & -4.583516 & 0.262374 & 1.848516\end{array}$

$\begin{array}{lllll}6 & -5.210181 & 2.082364 & -0.085311\end{array}$

$\begin{array}{lllll}1 & -6.044936 & 1.593423 & -0.602415\end{array}$

$\begin{array}{lllll}1 & -5.561148 & 2.353723 & 0.914280\end{array}$

$\begin{array}{lllll}1 & -4.983162 & 3.002504 & -0.630961\end{array}$

$\begin{array}{lllll}6 & 4.374460 & 1.826839 & -0.155823\end{array}$

$\begin{array}{lllll}1 & 3.488991 & 2.476078 & -0.176569\end{array}$

$\begin{array}{lllll}1 & 4.720614 & 1.752876 & -1.195496\end{array}$

$\begin{array}{llll}6 & 5.464034 & 2.471607 & 0.703159\end{array}$

$\begin{array}{lllll}1 & 6.371336 & 1.857276 & 0.715384\end{array}$

$\begin{array}{lllll}1 & 5.737773 & 3.463060 & 0.328796\end{array}$

$\begin{array}{llll}1 & 5.130842 & 2.586867 & 1.740616\end{array}$

\section{2b@12}

$\begin{array}{lllll}6 & 9.037228 & 3.616737 & 0.182289\end{array}$

$\begin{array}{lllll}6 & 8.385549 & 3.073076 & -1.088554\end{array}$

$\begin{array}{lllll}6 & 8.446962 & 2.922869 & 1.405886\end{array}$

$\begin{array}{lllll}1 & 8.748090 & 4.665869 & 0.251191\end{array}$

$\begin{array}{lllll}6 & 8.868964 & 1.933232 & -1.737266\end{array}$

$\begin{array}{lllll}6 & 7.263369 & 3.694574 & -1.653629\end{array}$

$\begin{array}{lllll}6 & 8.954526 & 1.721226 & 1.914578\end{array}$

$\begin{array}{lllll}6 & 7.327555 & 3.465266 & 2.046353\end{array}$

$\begin{array}{lllll}6 & 8.307856 & 1.416189 & -2.910278\end{array}$

$1 \quad 9.725760 \quad 1.424438 \quad-1.312060$

$\begin{array}{lllll}6 & 6.673029 & 3.230632 & -2.822794\end{array}$

$\begin{array}{lllll}8 & 6.761018 & 4.852831 & -1.052772\end{array}$

$\begin{array}{lllll}6 & 8.373326 & 1.049852 & 2.995160\end{array}$

$\begin{array}{llll}9.837966 & 1.296267 & 1.452276\end{array}$

$\begin{array}{llll}6.689387 & 2.823165 & 3.097659\end{array}$

$\begin{array}{lllll}8 & 6.857249 & 4.714166 & 1.625303\end{array}$

$\begin{array}{lllll}6 & 8.887791 & 0.182955 & -3.599550\end{array}$

$\begin{array}{lllll}6 & 7.203287 & 2.099458 & -3.435157\end{array}$

$\begin{array}{llll}5.814108 & 3.735746 & -3.246441\end{array}$

$\begin{array}{lllll}6 & 5.578131 & 4.763565 & -0.380983\end{array}$

$\begin{array}{lllll}6 & 8.957284 & -0.229112 & 3.599446\end{array}$

$\begin{array}{lllll}6 & 7.211888 & 1.616214 & 3.534917\end{array}$

$\begin{array}{llll}5.806326 & 3.247131 & 3.556424\end{array}$

$\begin{array}{llll}5.628819 & 4.723975 & 1.039570\end{array}$

$\begin{array}{llll}8.538305 & 0.224138 & -4.631928\end{array}$

$\begin{array}{llll}8.336965 & -1.120396 & -3.020619\end{array}$

$\begin{array}{llll}6.614105 & 1.655091 & -4.630128\end{array}$

$\begin{array}{llll}8.390547 & -1.473199 & 2.921412\end{array}$

$\begin{array}{llll}8.609587 & -0.261088 & 4.632817\end{array}$

$\begin{array}{llll}6.595386 & 0.955496 & 4.597909\end{array}$

$\begin{array}{llll}8.917647 & -1.793201 & -1.942003\end{array}$

$\begin{array}{llll}7.200857 & -1.709727 & -3.586760\end{array}$

$\begin{array}{llll}5.414266 & 1.027388 & -4.449713\end{array}$

$\begin{array}{llll}3.350580 & 4.732946 & -0.274518\end{array}$

$\begin{array}{llll}8.943129 & -1.988830 & 1.740602\end{array}$

$\begin{array}{llll}7.274237 & -2.138212 & 3.444524\end{array}$

$\begin{array}{llll}5.446018 & 0.276957 & 4.347916\end{array}$

$\begin{array}{llll}3.400213 & 4.712398 & 1.112345\end{array}$

$\begin{array}{llll}8.429287 & -3.010200 & -1.444985\end{array}$ 
$1 \quad 9.778393 \quad-1.348678 \quad-1.455883$

$6 \quad 6.704642 \quad-2.934123 \quad-3.170558$

$8 \quad 6.570771 \quad-1.036451 \quad-4.635158$

$\begin{array}{lllll}6 & 5.412957 & -0.390465 & -4.338380\end{array}$

$\begin{array}{lllll}6 & 8.414591 & -3.093015 & 1.067134\end{array}$

$\begin{array}{lllll}1 & 9.817976 & -1.499783 & 1.328654\end{array}$

$\begin{array}{lllll}6 & 6.694314 & -3.225539 & 2.800010\end{array}$

$\begin{array}{lllll}8 & 6.716807 & -1.720199 & 4.663168\end{array}$

$\begin{array}{llll}6 & 5.490040 & -1.133121 & 4.524514\end{array}$

$6 \quad 9.031550 \quad-3.671478 \quad-0.208103$

$\begin{array}{lllll}6 & 7.333260 & -3.575235 & -2.109869\end{array}$

$1 \quad 5.838089 \quad-3.373258 \quad-3.646616$

$\begin{array}{lllll}6 & 3.218444 & 1.035823 & -4.062613\end{array}$

$\begin{array}{lllll}6 & 7.260299 & -3.667450 & 1.614335\end{array}$

$\begin{array}{llll}5.819223 & -3.710170 & 3.212622\end{array}$

$\begin{array}{llll}6 & 3.263477 & 0.136384 & 3.918661\end{array}$

$\begin{array}{llll}8.731782 & -4.719652 & -0.243522\end{array}$

$\begin{array}{llll}6.838361 & -4.829235 & -1.715172\end{array}$

$\begin{array}{llll}3.245826 & -0.330972 & -3.827004\end{array}$

$\begin{array}{llll}6.707150 & -4.766669 & 0.956848\end{array}$

$\begin{array}{llll}3.280937 & -1.215396 & 4.239484\end{array}$

$\begin{array}{llll}5.601895 & -4.768701 & -1.136874\end{array}$

$\begin{array}{llll}5.544532 & -4.604404 & 0.275186\end{array}$

$\begin{array}{llll}3.376150 & -4.638302 & -1.226212\end{array}$

$\begin{array}{llll}3.335949 & -4.347585 & 0.129353\end{array}$

$\begin{array}{llll}10.565908 & -3.628662 & -0.195621\end{array}$

$\begin{array}{llll}10.949349 & -4.158613 & 0.680113\end{array}$

$\begin{array}{llll}10.965000 & -2.611351 & -0.165612\end{array}$

$\begin{array}{llll}10.423665 & 0.200075 & -3.633526\end{array}$

$\begin{array}{llll}10.794535 & -0.657290 & -4.201163\end{array}$

$\begin{array}{llll}10.873866 & 0.155077 & -2.638189\end{array}$

$\begin{array}{llll}10.570231 & 3.559970 & 0.135189\end{array}$

$\begin{array}{llll}10.940016 & 4.113297 & -0.731913\end{array}$

$\begin{array}{llll}10.959971 & 2.540851 & 0.065075\end{array}$

$\begin{array}{llll}10.492408 & -0.217105 & 3.629900\end{array}$

$\begin{array}{llll}10.849537 & 0.659068 & 4.177204\end{array}$

$\begin{array}{llll}10.940410 & -0.187067 & 2.632985\end{array}$

$\begin{array}{llll}10.863413 & -1.115377 & 4.130188\end{array}$

$\begin{array}{llll}10.988145 & 4.010270 & 1.039317\end{array}$

$\begin{array}{llll}10.777452 & 1.115828 & -4.114048\end{array}$

$\begin{array}{llll}10.960288 & -4.109961 & -1.094220\end{array}$

$\begin{array}{llll}1.808373 & 1.518217 & -3.873038\end{array}$

$\begin{array}{llll}1.873068 & -0.771307 & -3.405915\end{array}$

$\begin{array}{llll}1.959620 & -4.666511 & -1.739901\end{array}$

$\begin{array}{llll}1.910225 & -4.082759 & 0.518819\end{array}$

$\begin{array}{llll}1.908330 & 4.687733 & -0.696436\end{array}$

$\begin{array}{llll}1.986253 & 4.675646 & 1.632712\end{array}$

$\begin{array}{llll}1.862519 & 0.508016 & 3.507455\end{array}$

$\begin{array}{llll}1.875543 & -1.740725 & 4.162240\end{array}$

$\begin{array}{llll}1.171933 & -4.345518 & -0.615359\end{array}$

$\begin{array}{llll}1.092144 & 0.371000 & -3.487136\end{array}$

$\begin{array}{llll}1.188520 & 4.739464 & 0.487390\end{array}$

$\begin{array}{llll}1.120963 & -0.647660 & 3.704023\end{array}$

$\begin{array}{llll}1.467040 & 1.563146 & 3.051553\end{array}$

$\begin{array}{llll}1.447667 & -2.838206 & 4.429105\end{array}$

$\begin{array}{llll}1.512926 & -3.687153 & 1.602742\end{array}$

$\begin{array}{llll}1.546840 & -4.905132 & -2.847729\end{array}$

$\begin{array}{llll}1.536007 & -1.882795 & -3.042738\end{array}$ $\begin{array}{lllll}8 & 1.475277 & 4.582288 & -1.826288\end{array}$

$\begin{array}{lllll}8 & 1.361326 & 2.632758 & -4.001837\end{array}$

$\begin{array}{lllll}8 & 1.592384 & 4.589395 & 2.774668\end{array}$

$\begin{array}{lllll}1 & 0.100677 & -0.726941 & 3.634744\end{array}$

$\begin{array}{lllll}1 & 0.169832 & 4.660091 & 0.553284\end{array}$

$\begin{array}{lllll}0.075284 & 0.412021 & -3.400830\end{array}$

$\begin{array}{llll}0.147267 & -4.336600 & -0.674796\end{array}$

$7 \quad 4.310417 \quad 1.750702 \quad-4.343086$

$7 \quad 4.340700 \quad-1.078112 \quad-3.974581$

$7 \quad 4.436065 \quad 4.752664 \quad-1.052565$

$\begin{array}{lllll}7 & 4.543436 & 4.699355 & 1.799687\end{array}$

$7 \quad 4.518529 \quad-4.821404 \quad-1.895951$

$\begin{array}{llll}7.416339 & -4.339322 & 0.915316\end{array}$

$\begin{array}{llll}7 & 4.344316 & 0.918042 & 3.988128\end{array}$

$7 \quad 4.403293 \quad-1.887154 \quad 4.511682$

$\begin{array}{lllll}6 & -8.984047 & -2.709214 & 2.367054\end{array}$

$6 \quad-8.388220 \quad-3.128448 \quad 1.025266$

$6 \quad-8.366541 \quad-1.398606 \quad 2.851768$

$\begin{array}{llll}-8.686544 & -3.473007 & 3.086289\end{array}$

$6 \quad-8.871964 \quad-2.602349 \quad-0.176912$

$\begin{array}{llll}-7.336684 & -4.050212 & 0.934365\end{array}$

$\begin{array}{llll}-8.906843 & -0.141083 & 2.572987\end{array}$

$\begin{array}{llll}-7.197455 & -1.428579 & 3.619607\end{array}$

$\begin{array}{llll}-8.367478 & -2.955273 & -1.430770\end{array}$

$\begin{array}{llll}-9.685330 & -1.887530 & -0.134148\end{array}$

$\begin{array}{llll}-6.774902 & -4.409602 & -0.286064\end{array}$

$\begin{array}{llll}-6.832067 & -4.657342 & 2.097923\end{array}$

$\begin{array}{llll}-8.340042 & 1.050938 & 3.041904\end{array}$

$\begin{array}{llll}-9.808096 & -0.083373 & 1.974608\end{array}$

$\begin{array}{llll}-6.596830 & -0.282411 & 4.120311\end{array}$

$\begin{array}{lll}-6.682241 & -2.687457 & 3.931500\end{array}$

$\begin{array}{llll}-8.965737 & -2.404582 & -2.724012\end{array}$

$\begin{array}{llll}-7.291165 & -3.851006 & -1.448006\end{array}$

$\begin{array}{llll}-5.948115 & -5.107330 & -0.328715\end{array}$

$\begin{array}{llll}-5.596123 & -4.206777 & 2.465901\end{array}$

$\begin{array}{llll}-8.938953 & 2.415828 & 2.714969\end{array}$

$\begin{array}{llll}-7.183286 & 0.942662 & 3.824022\end{array}$

$\begin{array}{llll}-5.700449 & -0.338510 & 4.723595\end{array}$

$\begin{array}{llll}-5.527598 & -3.089965 & 3.347729\end{array}$

$\begin{array}{llll}-8.639239 & -3.073978 & -3.520261\end{array}$

$\begin{array}{llll}-8.381374 & -1.033703 & -3.046132\end{array}$

$\begin{array}{llll}-6.739172 & -4.225231 & -2.681609\end{array}$

$\begin{array}{llll}-8.378868 & 2.968214 & 1.405145\end{array}$

$\begin{array}{llll}-8.600787 & 3.093357 & 3.499603\end{array}$

$\begin{array}{lll}-6.635111 & 2.105022 & 4.387011\end{array}$

$\begin{array}{llll}-8.950248 & 0.160859 & -2.588723\end{array}$

$\begin{array}{llll}-7.212544 & -0.936302 & -3.809503\end{array}$

$\begin{array}{llll}-5.529262 & -3.673066 & -2.980743\end{array}$

$\begin{array}{llll}-3.367761 & -4.208536 & 2.326802\end{array}$

$\begin{array}{llll}-8.912641 & 2.641860 & 0.156385\end{array}$

$\begin{array}{llll}-7.284330 & 3.839465 & 1.411029\end{array}$

$\begin{array}{llll}-5.452591 & 2.542517 & 3.875933\end{array}$

$\begin{array}{llll}-3.319702 & -3.049988 & 3.087841\end{array}$

$\begin{array}{llll}-8.390659 & 1.413451 & -2.855220\end{array}$

$\begin{array}{llll}-9.865121 & 0.110308 & -2.010627\end{array}$

$\begin{array}{llll}-6.601109 & 0.280064 & -4.085920\end{array}$

$\begin{array}{llll}-6.689762 & -2.113274 & -4.358266\end{array}$

$\begin{array}{llll}-5.503377 & -2.560918 & -3.869982\end{array}$ $\begin{array}{llll}-8.426199 & 3.163436 & -1.048806\end{array}$

$\begin{array}{llll}-9.737626 & 1.940048 & 0.115970\end{array}$

$\begin{array}{llll}-6.784171 & 4.416099 & 0.253571\end{array}$

$\begin{array}{llll}-6.698901 & 4.157239 & 2.642668\end{array}$

$\begin{array}{llll}-5.492822 & 3.592135 & 2.912010\end{array}$

$\begin{array}{llll}-9.011621 & 2.732054 & -2.391475\end{array}$

$\begin{array}{llll}-7.199335 & 1.429902 & -3.590286\end{array}$

$\begin{array}{llll}-5.690595 & 0.328412 & -4.667686\end{array}$

$\begin{array}{llll}-3.305991 & -3.563949 & -2.816620\end{array}$

$\begin{array}{llll}-7.368055 & 4.077748 & -0.962773\end{array}$

$\begin{array}{llll}-5.949272 & 5.103990 & 0.292272\end{array}$

$\begin{array}{llll}-3.223860 & 2.512876 & 3.723408\end{array}$

$\begin{array}{llll}-8.704393 & 3.486724 & -3.116630\end{array}$

$\begin{array}{llll}-6.660968 & 2.680748 & -3.888721\end{array}$

$\begin{array}{llll}-3.280418 & -2.493852 & -3.701354\end{array}$

$\begin{array}{llll}-6.864115 & 4.691912 & -2.122640\end{array}$

$\begin{array}{llll}-3.272373 & 3.483299 & 2.732497\end{array}$

$\begin{array}{llll}-5.521442 & 3.089856 & -3.281579\end{array}$

$\begin{array}{llll}-5.614939 & 4.250016 & -2.461587\end{array}$

$\begin{array}{llll}-3.319025 & 3.066282 & -2.957203\end{array}$

$\begin{array}{llll}-3.390832 & 4.275555 & -2.281424\end{array}$

$\begin{array}{llll}-10.546317 & 2.698503 & -2.382681\end{array}$

$\begin{array}{llll}-10.939315 & 3.682494 & -2.114415\end{array}$

$\begin{array}{llll}-10.955064 & 1.976997 & -1.669863\end{array}$

$\begin{array}{lllll}6 & -10.500965 & -2.419057 & -2.708779\end{array}$

$\begin{array}{llll}-10.889078 & -2.067568 & -3.668521\end{array}$

$\begin{array}{llll}-10.928190 & -1.785018 & -1.927046\end{array}$

$\begin{array}{llll}6 & -10.518851 & -2.672090 & 2.343702\end{array}$

$\begin{array}{llll}-10.911568 & -3.651075 & 2.057383\end{array}$

$\begin{array}{llll}-10.919386 & -1.939176 & 1.638015\end{array}$

$\begin{array}{llll}-10.474582 & 2.416115 & 2.736054\end{array}$

$\begin{array}{llll}-10.837387 & 2.063030 & 3.704944\end{array}$

$\begin{array}{llll}-10.913802 & 1.776708 & 1.965410\end{array}$

$\begin{array}{llll}-10.849047 & 3.429803 & 2.571938\end{array}$

$\begin{array}{llll}-10.903103 & -2.419329 & 3.335529\end{array}$

$\begin{array}{llll}-10.862743 & -3.436296 & -2.538464\end{array}$

$\begin{array}{llll}-10.922293 & 2.431971 & -3.374045\end{array}$

$\begin{array}{llll}-1.903840 & -3.816914 & -2.336891\end{array}$

$\begin{array}{llll}-1.854143 & -2.044190 & -3.862352\end{array}$

$\begin{array}{llll}-1.890956 & 2.601544 & -2.958816\end{array}$

$\begin{array}{llll}-1.987305 & 4.699680 & -1.931355\end{array}$

$\begin{array}{llll}-1.951242 & -4.591435 & 1.978679\end{array}$

$\begin{array}{llll}-1.899590 & -2.585979 & 3.165954\end{array}$

$\begin{array}{llll}-1.778201 & 2.172064 & 3.981879\end{array}$

$\begin{array}{llll}-1.873049 & 3.763576 & 2.264973\end{array}$

$\begin{array}{llll}-1.178489 & 3.645207 & -2.395593\end{array}$

$\begin{array}{llll}-1.125622 & -2.886493 & -3.015587\end{array}$

$\begin{array}{llll}-1.160000 & -3.582919 & 2.569357\end{array}$

$\begin{array}{llll}-1.063397 & 2.993057 & 3.088193\end{array}$

$\begin{array}{llll}-1.302035 & 1.378842 & 4.757217\end{array}$

$\begin{array}{llll}-1.529660 & 4.476577 & 1.342338\end{array}$

$\begin{array}{llll}-1.603471 & 5.703162 & -1.382035\end{array}$

$\begin{array}{llll}-1.479609 & 1.516735 & -3.324071\end{array}$

$\begin{array}{llll}-1.400512 & -1.155582 & -4.546847\end{array}$

$\begin{array}{lll}-1.535526 & -5.532892 & 1.350590\end{array}$

$\begin{array}{llll}-1.529431 & -4.625974 & -1.512372\end{array}$

$\begin{array}{llll}-1.515567 & -1.516122 & 3.611799\end{array}$

$\begin{array}{llll}-0.046303 & 2.965741 & 3.024958\end{array}$ 
$\begin{array}{llll}-0.152559 & -3.539528 & 2.388027\end{array}$

$\begin{array}{llll}-0.115125 & -2.770698 & -2.914481\end{array}$

$\begin{array}{llll}-0.161393 & 3.685306 & -2.304142\end{array}$

$\begin{array}{llll}-4.430015 & -4.178039 & -2.440405\end{array}$

$\begin{array}{llll}-4.379273 & -1.965931 & -4.242072\end{array}$

$\begin{array}{llll}-4.516190 & -4.795725 & 1.974383\end{array}$

$\begin{array}{lll}-4.392222 & -2.472316 & 3.629787\end{array}$

$\begin{array}{llll}-4.379838 & 2.454156 & -3.489635\end{array}$

$\begin{array}{llll}-4.549875 & 4.875006 & -1.986413\end{array}$

$\begin{array}{llll}-4.317583 & 2.004212 & 4.299014\end{array}$

$\begin{array}{llll}-4.405720 & 4.045650 & 2.306651\end{array}$

$\begin{array}{llll}0.657714 & 0.895259 & -0.059123\end{array}$

$\begin{array}{llll}0.302549 & 2.056048 & -0.179961\end{array}$

$\begin{array}{llll}-0.342502 & -0.076494 & 0.229006\end{array}$

$\begin{array}{llll}-0.102391 & -1.341225 & -0.170076\end{array}$

$\begin{array}{llll}-1.004474 & -2.135807 & 0.061407\end{array}$

$\begin{array}{lll}-1.586720 & 0.341859 & 0.900201\end{array}$

$\begin{array}{lll}-1.267354 & 1.077799 & 1.633250\end{array}$

$\begin{array}{llll}-1.957843 & -0.532261 & 1.427873\end{array}$

$\begin{array}{llll}-2.648838 & 0.940483 & -0.025318\end{array}$

$\begin{array}{llll}-2.195001 & 1.782409 & -0.548749\end{array}$

$\begin{array}{llll}-3.424991 & 1.364597 & 0.623030\end{array}$

$\begin{array}{llll}-3.281552 & -0.040415 & -1.017623\end{array}$

$\begin{array}{llll}-2.492166 & -0.477348 & -1.637150\end{array}$

$\begin{array}{llll}-3.910504 & 0.524104 & -1.712264\end{array}$

$\begin{array}{llll}-4.114159 & -1.162262 & -0.378590\end{array}$

$\begin{array}{llll}-3.453731 & -1.867573 & 0.136292\end{array}$

$\begin{array}{llll}-4.590369 & -1.730340 & -1.184903\end{array}$

$\begin{array}{llll}2.078681 & 0.480976 & -0.116017\end{array}$

$\begin{array}{llll}2.589208 & -0.630577 & 0.572048\end{array}$

$\begin{array}{llll}2.970371 & 1.346688 & -0.765922\end{array}$

$\begin{array}{llll}3.959894 & -0.854128 & 0.617371\end{array}$

$\begin{array}{llll}1.928066 & -1.303158 & 1.098993\end{array}$

$\begin{array}{llll}4.337851 & 1.101926 & -0.735427\end{array}$

$\begin{array}{llll}2.578655 & 2.204882 & -1.297502\end{array}$

$\begin{array}{llll}4.856227 & 0.003540 & -0.036203\end{array}$

$\begin{array}{llll}4.340312 & -1.703023 & 1.177633\end{array}$

$\begin{array}{llll}5.013730 & 1.774554 & -1.252709\end{array}$

$\begin{array}{llll}6.331419 & -0.268852 & 0.005633\end{array}$

$\begin{array}{llll}6.915256 & 0.608096 & -0.277948\end{array}$

$\begin{array}{llll}6.644980 & -0.582269 & 1.003118\end{array}$

$\begin{array}{llll}6.591904 & -1.077785 & -0.682974\end{array}$

$\begin{array}{llll}-5.215797 & -0.693194 & 0.580526\end{array}$

$\begin{array}{llll}-5.779992 & -1.573318 & 0.908004\end{array}$

$\begin{array}{llll}-4.777406 & -0.269441 & 1.493046\end{array}$

$\begin{array}{llll}-6.190709 & 0.305770 & -0.042638\end{array}$

$\begin{array}{lll}-6.605446 & -0.086022 & -0.975698\end{array}$

$\begin{array}{llll}-7.024254 & 0.510049 & 0.630113\end{array}$

$\begin{array}{llll}-5.712232 & 1.264048 & -0.271854\end{array}$

\section{2b'-TS@12}

$\begin{array}{llll}6 & 9.020035 & 2.640217 & 2.572068 \\ 6 & 8.452489 & 3.073255 & 1.216384 \\ 6 & 8.423925 & 1.308375 & 3.023959 \\ 1 & 8.682487 & 3.386510 & 3.292464 \\ 6 & 8.995945 & 2.645591 & 0.001395 \\ 6 & 7.351134 & 3.935129 & 1.135392\end{array}$ $\begin{array}{llll}6 & 8.988944 & 0.071555 & 2.682118\end{array}$

$\begin{array}{llll}6 & 7.244643 & 1.281012 & 3.778019\end{array}$

$\begin{array}{lllll}6 & 8.508193 & 3.047818 & -1.249193\end{array}$

$\begin{array}{lllll}1 & 9.827412 & 1.950887 & 0.026913\end{array}$

$\begin{array}{lllll}6 & 6.855326 & 4.400917 & -0.072329\end{array}$

$\begin{array}{lllll}8 & 6.737846 & 4.365081 & 2.318193\end{array}$

$\begin{array}{lllll}6 & 8.401271 & -1.150698 & 3.021266\end{array}$

$\begin{array}{llll}1 & 9.917253 & 0.064526 & 2.123655\end{array}$

$\begin{array}{llll}6 & 6.605218 & 0.095931 & 4.120107\end{array}$

$\begin{array}{llll}8 & 6.701147 & 2.492010 & 4.230455\end{array}$

$\begin{array}{lllll}6 & 9.087935 & 2.493519 & -2.548405\end{array}$

$\begin{array}{lllll}6 & 7.443818 & 3.957971 & -1.250205\end{array}$

$1 \quad 6.017209 \quad 5.085188 \quad-0.097725$

$\begin{array}{lllll}6 & 5.538035 & 3.793990 & 2.611912\end{array}$

$\begin{array}{lllll}6 & 9.010645 & -2.516320 & 2.694578\end{array}$

$\begin{array}{lllll}6 & 7.184496 & -1.094088 & 3.711486\end{array}$

$\begin{array}{llll}5.681409 & 0.104580 & 4.682301\end{array}$

$\begin{array}{lllll}6 & 5.511599 & 2.843965 & 3.670477\end{array}$

$1 \quad 8.782445 \quad 3.181799 \quad-3.337776$

$\begin{array}{lllll}6 & 8.457491 & 1.142182 & -2.893198\end{array}$

$8 \quad 6.938087 \quad 4.462136 \quad-2.460871$

$\begin{array}{lllll}6 & 8.442970 & -3.083346 & 1.394886\end{array}$

$\begin{array}{llll}8.685212 & -3.190450 & 3.488444\end{array}$

$8 \quad 6.603693 \quad-2.308260 \quad 4.066962$

$\begin{array}{lllll}6 & 9.012104 & -0.084351 & -2.514480\end{array}$

$\begin{array}{lllll}6 & 7.263365 & 1.096939 & -3.623582\end{array}$

$\begin{array}{lllll}6 & 5.683236 & 4.007138 & -2.754206\end{array}$

$\begin{array}{lllll}6 & 3.332693 & 3.552631 & 2.369058\end{array}$

$\begin{array}{lllll}6 & 8.936960 & -2.665196 & 0.152204\end{array}$

$\begin{array}{lllll}6 & 7.407159 & -4.027614 & 1.377220\end{array}$

$\begin{array}{lllll}6 & 5.494719 & -2.750961 & 3.428186\end{array}$

$\begin{array}{lllll}6 & 3.299066 & 2.678455 & 3.446848\end{array}$

$6 \quad 8.439214 \quad-1.315586 \quad-2.856446$

$\begin{array}{llll}9.925344 & -0.082716 & -1.931716\end{array}$

$6 \quad 6.671010 \quad-0.092708 \quad-4.022225$

$\begin{array}{lllll}8 & 6.719832 & 2.313107 & -4.035394\end{array}$

$\begin{array}{lllll}6 & 5.577839 & 2.783995 & -3.477483\end{array}$

$6 \quad 8.430432 \quad-3.108244 \quad-1.072241$

$\begin{array}{lllll}1 & 9.749340 & -1.947592 & 0.141309\end{array}$

$\begin{array}{lllll}6 & 6.859287 & -4.491087 & 0.185091\end{array}$

$\begin{array}{lllll}8 & 6.859139 & -4.521902 & 2.576221\end{array}$

$\begin{array}{lllll}6 & 5.601516 & -4.024088 & 2.799169\end{array}$

$\begin{array}{lllll}6 & 9.020412 & -2.654739 & -2.409338\end{array}$

$\begin{array}{lllll}6 & 7.279093 & -1.280891 & -3.637919\end{array}$

$1 \quad 5.765492 \quad-0.094873 \quad-4.613799$

$\begin{array}{lllll}6 & 3.457850 & 4.083156 & -2.594318\end{array}$

$\begin{array}{lllll}6 & 7.355273 & -4.004703 & -1.014732\end{array}$

$1 \quad 6.033458 \quad-5.1908410 .195976$

$\begin{array}{lllll}6 & 3.327309 & -2.705952 & 2.917576\end{array}$

$\begin{array}{llll}8.695632 & -3.389620 & -3.146791\end{array}$

$8 \quad 6.744675 \quad-2.498491 \quad-4.081644$

$\begin{array}{lllll}6 & 3.369891 & 2.831068 & -3.183823\end{array}$

$\begin{array}{lllll}8 & 6.759933 & -4.433477 & -2.209514\end{array}$

$\begin{array}{lllll}6 & 3.393351 & -4.020902 & 2.480370\end{array}$

$6 \quad 5.553482 \quad-2.863802 \quad-3.539421$

$6 \quad 5.564163 \quad-3.857902 \quad-2.519076$

$\begin{array}{lllll}6 & 3.337630 & -2.705082 & -3.345537\end{array}$

$\begin{array}{lllll}6 & 3.350182 & -3.639060 & -2.319461\end{array}$
6

$\begin{array}{lll}11.029042 & 1.791893 & -1.769239\end{array}$

$\begin{array}{llll}6 & 10.555177 & 2.645221 & 2.585677\end{array}$

$\begin{array}{lllll}1 & 10.928754 & 3.638048 & 2.322381\end{array}$

$\begin{array}{lllll}1 & 10.991309 & 1.933447 & 1.879226\end{array}$

$\begin{array}{lllll}6 & 10.545907 & -2.500696 & 2.703320\end{array}$

$\begin{array}{llll}10.913358 & -2.140226 & 3.667797\end{array}$

$\begin{array}{llll}10.971540 & -1.859250 & 1.926643\end{array}$

$\begin{array}{llll}10.929635 & -3.510903 & 2.539947\end{array}$

$\begin{array}{lll}10.922454 & 2.387880 & 3.582675\end{array}$

$\begin{array}{llll}11.021030 & 3.452796 & -2.358538\end{array}$

$\begin{array}{llll}2.059280 & 4.566304 & -2.300484\end{array}$

$\begin{array}{llll}1.928629 & 2.407900 & -3.201444\end{array}$

$\begin{array}{llll}1.916995 & -2.274062 & -3.579328\end{array}$

$\begin{array}{llll}1.935611 & -3.830288 & -1.839260\end{array}$

$\begin{array}{llll}1.933028 & 3.701037 & 1.833791\end{array}$

$\begin{array}{llll}1.872434 & 2.263856 & 3.676579\end{array}$

$\begin{array}{lll}1.923325 & -2.205152 & 2.711673\end{array}$

$\begin{array}{llll}1.998087 & -4.469354 & 2.134001\end{array}$

$\begin{array}{llll}1.168422 & -3.010963 & -2.655335\end{array}$

$\begin{array}{llll}1.229182 & 3.496980 & -2.698055\end{array}$

$\begin{array}{llll}1.147479 & 2.932664 & 2.676739\end{array}$

$\begin{array}{llll}1.210149 & -3.309533 & 2.289555\end{array}$

$\begin{array}{llll}1.526092 & -1.058408 & 2.841430\end{array}$

$\begin{array}{llll}1.600240 & -5.561069 & 1.813635\end{array}$

$\begin{array}{llll}1.547943 & -4.539454 & -0.932027\end{array}$

$\begin{array}{llll}1.486147 & -1.459027 & -4.363593\end{array}$

$\begin{array}{llll}1.488552 & 1.326443 & -3.538547\end{array}$

$\begin{array}{llll}1.574033 & 4.328466 & 0.854560\end{array}$

$\begin{array}{llll}1.692881 & 5.618825 & -1.839656\end{array}$

$\begin{array}{llll}1.414459 & 1.520716 & 4.512278\end{array}$

$\begin{array}{llll}0.193911 & -3.353963 & 2.159360\end{array}$

$\begin{array}{llll}0.128423 & 2.835079 & 2.628267\end{array}$

$\begin{array}{llll}0.210809 & 3.581600 & -2.688911\end{array}$

$\begin{array}{llll}0.152240 & -2.914704 & -2.612788\end{array}$

$\begin{array}{llll}4.625919 & 4.683339 & -2.335242\end{array}$

$\begin{array}{llll}4.425466 & 2.158029 & -3.653965\end{array}$

$\begin{array}{llll}4.452939 & 4.133918 & 1.930668\end{array}$

$\begin{array}{llll}4.390949 & 2.291344 & 4.109945\end{array}$

$\begin{array}{llll}4.441040 & -2.285229 & -3.966345\end{array}$

$\begin{array}{llll}4.463798 & -4.235809 & -1.883831\end{array}$

$\begin{array}{llll}4.372669 & -2.050098 & 3.432102\end{array}$

$\begin{array}{llll}4.545729 & -4.693030 & 2.369538\end{array}$

$\begin{array}{llll}-9.077435 & -3.604381 & 0.116552\end{array}$

$\begin{array}{llll}-8.470749 & -3.040638 & -1.166294\end{array}$

$\begin{array}{llll}-8.427063 & -2.954779 & 1.337998\end{array}$

$\begin{array}{llll}-8.804883 & -4.659901 & 0.148265\end{array}$

$\begin{array}{llll}-8.936122 & -1.848419 & -1.734347\end{array}$

$\begin{array}{llll}-7.403751 & -3.678861 & -1.812390\end{array}$

$\begin{array}{llll}-8.925111 & -1.797698 & 1.944025\end{array}$

$\begin{array}{llll}-7.266085 & -3.510194 & 1.891270\end{array}$

$\begin{array}{llll}-8.374881 & -1.268307 & -2.875514\end{array}$

$\begin{array}{llll}-9.772721 & -1.347124 & -1.261787\end{array}$

$\begin{array}{llll}-6.794233 & -3.133065 & -2.937221\end{array}$

$\begin{array}{llll}-6.943986 & -4.915117 & -1.340200\end{array}$

$\begin{array}{llll}-8.329378 & -1.201962 & 3.063290\end{array}$

$\begin{array}{llll}-9.814919 & -1.338250 & 1.529957\end{array}$ 
$\begin{array}{llll}6 & -6.642795 & -2.973110 & 3.007745\end{array}$

$\begin{array}{lllll}8 & -6.788108 & -4.693675 & 1.329594\end{array}$

$6 \quad-8.947852 \quad-0.004190 \quad-3.521162$

$\begin{array}{lllll}6 & -7.270521 & -1.926538 & -3.432330\end{array}$

$\begin{array}{lllll}1 & -5.955033 & -3.630454 & -3.406455\end{array}$

$6 \quad-5.702176 \quad-4.895861 \quad-0.767868$

$\begin{array}{lllll}6 & -8.893267 & 0.067955 & 3.701827\end{array}$

$\begin{array}{lllll}6 & -7.188250 & -1.829363 & 3.579890\end{array}$

$\begin{array}{lllll}1 & -5.756032 & -3.432523 & 3.424596\end{array}$

$\begin{array}{lllll}6 & -5.619455 & -4.688791 & 0.636795\end{array}$

$\begin{array}{llll}-8.604801 & -0.016213 & -4.556402\end{array}$

$\begin{array}{lllll}6 & -8.381930 & 1.272920 & -2.904287\end{array}$

$8 \quad-6.649441 \quad-1.365099 \quad-4.553399$

$\begin{array}{lllll}6 & -8.338451 & 1.330990 & 3.039809\end{array}$

$\begin{array}{lllll}1 & -8.524436 & 0.078708 & 4.728242\end{array}$

$8 \quad-6.590361 \quad-1.319375 \quad 4.743171$

$\begin{array}{lllll}6 & -8.951519 & 1.888427 & -1.782466\end{array}$

$6 \quad-7.241941 \quad 1.878504 \quad-3.447124$

$6 \quad-5.468495 \quad-0.723075 \quad-4.334575$

$6 \quad-3.473638 \quad-4.982907 \quad-0.867941$

$\begin{array}{lllll}6 & -8.931844 & 1.934131 & 1.924818\end{array}$

$\begin{array}{lllll}6 & -7.177297 & 1.935520 & 3.536852\end{array}$

$\begin{array}{lllll}6 & -5.387267 & -0.709129 & 4.549596\end{array}$

$\begin{array}{lllll}6 & -3.395914 & -4.691499 & 0.487855\end{array}$

$6 \quad-8.427936 \quad 3.045574 \quad-1.195154$

$\begin{array}{lllll}1 & -9.832010 & 1.437899 & -1.339408\end{array}$

$\begin{array}{lllll}6 & -6.684166 & 3.031687 & -2.910140\end{array}$

$8 \quad-6.667628 \quad 1.321129 \quad-4.597384$

$\begin{array}{lllll}6 & -5.470953 & 0.698006 & -4.411258\end{array}$

$\begin{array}{lllll}6 & -8.424626 & 3.089963 & 1.316709\end{array}$

$\begin{array}{lllll}1 & -9.815479 & 1.472591 & 1.499577\end{array}$

$\begin{array}{lllll}6 & -6.652497 & 3.102772 & 2.999458\end{array}$

$8 \quad-6.555397 \quad 1.356532 \quad 4.646161$

$\begin{array}{lllll}6 & -5.380820 & 0.707924 & 4.426256\end{array}$

$\begin{array}{lllll}6 & -9.041144 & 3.687445 & 0.051519\end{array}$

$\begin{array}{lllll}6 & -7.282334 & 3.592224 & -1.789549\end{array}$

$\begin{array}{lllll}1 & -5.804895 & 3.481592 & -3.352976\end{array}$

$\begin{array}{lllll}6 & -3.270657 & -0.676839 & -3.955481\end{array}$

$\begin{array}{lllll}6 & -7.287675 & 3.665445 & 1.899295\end{array}$

$\begin{array}{lllll}1 & -5.769081 & 3.562071 & 3.423389\end{array}$

$\begin{array}{lllll}6 & -3.186242 & -0.735140 & 4.186271\end{array}$

$\begin{array}{lllll}1 & -8.744638 & 4.736940 & 0.032202\end{array}$

$8 \quad-6.770353 \quad 4.784598 \quad-1.273425$

$\begin{array}{lllll}6 & -3.263827 & 0.703821 & -4.091291\end{array}$

$\begin{array}{lllll}8 & -6.802914 & 4.875780 & 1.393106\end{array}$

$\begin{array}{lllll}6 & -3.199026 & 0.637332 & 3.985067\end{array}$

$\begin{array}{lllll}6 & -5.567417 & 4.763674 & -0.638082\end{array}$

$\begin{array}{lllll}6 & -5.585247 & 4.850963 & 0.781555\end{array}$

$\begin{array}{lllll}6 & -3.336903 & 4.810162 & -0.594650\end{array}$

$\begin{array}{lllll}6 & -3.354476 & 4.931214 & 0.789357\end{array}$

$\begin{array}{lllll}6 & -10.574896 & 3.639378 & 0.053602\end{array}$

$\begin{array}{lllll}1 & -10.964464 & 4.162728 & 0.930580\end{array}$

$\begin{array}{lllll}1 & -10.971589 & 2.620867 & 0.075923\end{array}$

$6 \quad-10.483861 \quad-0.015176 \quad-3.546085$

$\begin{array}{lllll}1 & -10.855575 & 0.864893 & -4.077320\end{array}$

$\begin{array}{lllll}1 & -10.927624 & -0.009004 & -2.546882\end{array}$

$6 \begin{array}{llll}6 & -10.609759 & -3.521151 & 0.138163\end{array}$

$\begin{array}{lllll}1 & -11.025214 & -4.054314 & -0.720801\end{array}$ $\begin{array}{llll}1 & -10.984080 & -2.494627 & 0.099473\end{array}$

$\begin{array}{lllll}6 & -10.427602 & 0.066618 & 3.764739\end{array}$

$\begin{array}{lllll}1 & -10.779518 & -0.819062 & 4.299940\end{array}$

$\begin{array}{lllll}1 & -10.897285 & 0.062580 & 2.777240\end{array}$

$\begin{array}{lllll}1 & -10.781161 & 0.956181 & 4.292556\end{array}$

$\begin{array}{lllll}1 & -10.997229 & -3.978501 & 1.052269\end{array}$

$\begin{array}{lllll}1 & -10.843746 & -0.910383 & -4.059616\end{array}$

$\begin{array}{lllll}1 & -10.966572 & 4.124900 & -0.843948\end{array}$

$6 \quad-1.868432 \quad-1.129714 \quad-3.655476$

$6 \quad-1.847402 \quad 1.190656 \quad-3.949921$

$\begin{array}{lllll}6 & -1.901161 & 4.799319 & -1.053801\end{array}$

$\begin{array}{lllll}6 & -1.931196 & 5.045389 & 1.274804\end{array}$

$\begin{array}{lllll}6 & -2.068345 & -5.108998 & -1.404312\end{array}$

$\begin{array}{lllll}6 & -1.945197 & -4.548793 & 0.857684\end{array}$

$\begin{array}{lllll}6 & -1.778777 & -1.226034 & 3.971209\end{array}$

$\begin{array}{lllll}6 & -1.832560 & 1.069940 & 3.543921\end{array}$

$\begin{array}{lllll}7 & -1.161919 & 5.024255 & 0.103028\end{array}$

$\begin{array}{lllll}7 & -1.099790 & 0.023078 & -3.717992\end{array}$

$\begin{array}{lllll}7 & -1.249143 & -4.905962 & -0.286019\end{array}$

$\begin{array}{lllll}7 & -1.066743 & -0.078737 & 3.567350\end{array}$

$\begin{array}{lllll}8 & -1.317402 & -2.331752 & 4.107628\end{array}$

$\begin{array}{lllll}8 & -1.506221 & 2.184901 & 3.171541\end{array}$

$\begin{array}{lllll}8 & -1.509453 & 5.130654 & 2.404073\end{array}$

$\begin{array}{lllll}8 & -1.473829 & 4.604761 & -2.172268\end{array}$

$8 \quad-1.422024 \quad 2.318433 \quad-4.019089$

$8 \quad-1.695550 \quad-5.318793 \quad-2.534565$

$8 \quad-1.496882 \quad-2.252553 \quad-3.367660$

$8 \quad-1.487788 \quad-4.168952 \quad 1.916761$

$\begin{array}{lllll}1 & -0.072163 & -0.139584 & 3.340662\end{array}$

$\begin{array}{lllll}1 & -0.234485 & -4.800375 & -0.381587\end{array}$

$\begin{array}{lllll}1 & -0.081688 & 0.047114 & -3.684213\end{array}$

$\begin{array}{lllll}1 & -0.143967 & 4.950559 & 0.144591\end{array}$

$7 \quad-4.372610 \quad-1.421065 \quad-4.076194$

$7 \quad-4.368106 \quad 1.422976 \quad-4.301647$

$7 \quad-4.631802 \quad-5.063620 \quad-1.530108$

$\begin{array}{lllll}7 & -4.466156 & -4.550220 & 1.272799\end{array}$

$7 \quad-4.444016 \quad 4.723473 \quad-1.338681$

$\begin{array}{lllll}7 & -4.481443 & 4.939586 & 1.508459\end{array}$

$7 \begin{array}{llll}7 & -4.287009 & -1.441330 & 4.456457\end{array}$

$\begin{array}{lllll}7 & -4.292057 & 1.391973 & 4.111678\end{array}$

$6 \quad 10.556043 \quad-2.664616 \quad-2.393730$

$1 \quad 10.921099 \quad-3.663362 \quad-2.141142$

$1 \quad 10.943088 \quad-2.391039 \quad-3.378885$

$1 \quad 10.980633 \quad-1.967301 \quad-1.666204$

$6 \quad-0.921534 \quad-0.951871 \quad-0.182341$

$8 \quad-0.515247 \quad-2.109765 \quad-0.245301$

$\begin{array}{lllll}7 & -0.027074 & 0.118572 & -0.168450\end{array}$

$\begin{array}{lllll}7 & -0.450027 & 1.345482 & -0.944645\end{array}$

$8 \quad-0.686598 \quad 2.260853 \quad-0.234231$

$\begin{array}{lllll}6 & 1.360072 & -0.165118 & -0.557452\end{array}$

$1 \quad 1.438165 \quad-0.207609 \quad-1.650426$

$\begin{array}{lllll}1 & 1.569990 & -1.158577 & -0.163999\end{array}$

$\begin{array}{lllll}6 & 2.313922 & 0.885205 & -0.003852\end{array}$

$\begin{array}{llll}1 & 2.221668 & 0.890865 & 1.086525\end{array}$

$\begin{array}{lllll}1 & 2.021173 & 1.878651 & -0.356382\end{array}$

$\begin{array}{lllll}6 & 3.760777 & 0.603788 & -0.406185\end{array}$

$\begin{array}{lllll}1 & 4.368759 & 1.480996 & -0.158988\end{array}$

$\begin{array}{lllll}1 & 3.830294 & 0.481204 & -1.495643\end{array}$ $\begin{array}{lllll}6 & 4.336376 & -0.626811 & 0.295384\end{array}$

$\begin{array}{llll}3.706429 & -1.503809 & 0.097584\end{array}$

$\begin{array}{llll}4.298082 & -0.461169 & 1.379138\end{array}$

$\begin{array}{lllll}6 & -2.365712 & -0.629774 & -0.090455\end{array}$

$\begin{array}{lllll}6 & -2.855308 & 0.478569 & 0.612099\end{array}$

$6 \quad-3.272156 \quad-1.503612 \quad-0.707835$

$\begin{array}{lllll}6 & -4.222573 & 0.714052 & 0.685362\end{array}$

$\begin{array}{llll}-2.172286 & 1.149725 & 1.108682\end{array}$

$\begin{array}{lllll}6 & -4.635329 & -1.254349 & -0.640879\end{array}$

$\begin{array}{llll}-2.891713 & -2.347057 & -1.267544\end{array}$

$\begin{array}{llll}6 & -5.130869 & -0.141884 & 0.053207\end{array}$

$\begin{array}{llll}-4.588541 & 1.570478 & 1.243967\end{array}$

$\begin{array}{llll}-5.328943 & -1.916708 & -1.144958\end{array}$

$\begin{array}{llll}6 & -6.596650 & 0.154520 & 0.071966\end{array}$

$\begin{array}{llll}-6.894596 & 0.634736 & -0.862567\end{array}$

$\begin{array}{llll}-7.189438 & -0.755279 & 0.169224\end{array}$

$\begin{array}{llll}-6.856061 & 0.827200 & 0.886359\end{array}$

$\begin{array}{llll}5.772748 & -0.945553 & -0.114866\end{array}$

$\begin{array}{llll}5.798264 & -1.229734 & -1.173371\end{array}$

$\begin{array}{llll}6.117023 & -1.817463 & 0.447288\end{array}$

$\begin{array}{llll}6.723091 & 0.221856 & 0.123344\end{array}$

$\begin{array}{llll}7.762698 & -0.079162 & -0.004247\end{array}$

$\begin{array}{llll}6.539387 & 1.045791 & -0.568939\end{array}$

$\begin{array}{llll}6.610767 & 0.607311 & 1.139891\end{array}$

\section{2b'@12}

$\begin{array}{lllll}6 & 9.015469 & 3.632478 & 0.002130\end{array}$

$\begin{array}{lllll}6 & 8.382056 & 2.996180 & -1.234211\end{array}$

$\begin{array}{lllll}6 & 8.413838 & 3.022704 & 1.264622\end{array}$

$1 \quad 8.719880 \quad 4.682032 \quad-0.006556$

$\begin{array}{lllll}6 & 8.880543 & 1.814816 & -1.791814\end{array}$

$\begin{array}{lllll}6 & 7.263649 & 3.567911 & -1.856259\end{array}$

$\begin{array}{lllll}6 & 8.925205 & 1.867750 & 1.868668\end{array}$

$\begin{array}{lllll}6 & 7.279789 & 3.598931 & 1.847197\end{array}$

$\begin{array}{lllll}6 & 8.331654 & 1.207925 & -2.926049\end{array}$

$\begin{array}{lllll}1 & 9.740190 & 1.349333 & -1.324399\end{array}$

$\begin{array}{lllll}6 & 6.682469 & 3.010609 & -2.989359\end{array}$

$\begin{array}{lllll}8 & 6.752227 & 4.769221 & -1.355806\end{array}$

$\begin{array}{lllll}6 & 8.335027 & 1.273562 & 2.988917\end{array}$

$\begin{array}{lllll}1 & 9.818456 & 1.416048 & 1.452938\end{array}$

$\begin{array}{lllll}6 & 6.635365 & 3.034157 & 2.938087\end{array}$

$\begin{array}{lllll}8 & 6.805270 & 4.806428 & 1.324908\end{array}$

$\begin{array}{lllll}6 & 8.922110 & -0.064225 & -3.528359\end{array}$

$\begin{array}{lllll}6 & 7.222870 & 1.837611 & -3.504442\end{array}$

$1 \quad 5.823064 \quad 3.475291 \quad-3.455754$

$\begin{array}{lllll}6 & 5.557646 & 4.726914 & -0.699304\end{array}$

$\begin{array}{lllll}6 & 8.916027 & 0.042526 & 3.689017\end{array}$

$\begin{array}{lllll}6 & 7.163917 & 1.869759 & 3.472915\end{array}$

$1 \quad 5.742746 \quad 3.483145 \quad 3.352188$

$\begin{array}{lllll}6 & 5.586262 & 4.768690 & 0.721685\end{array}$

$1 \quad 8.589047 \quad-0.088541 \quad-4.566523$

$\begin{array}{lllll}6 & 8.361928 & -1.327589 & -2.876932\end{array}$

$\begin{array}{lllll}8 & 6.654525 & 1.285821 & -4.661801\end{array}$

$\begin{array}{lllll}6 & 8.360401 & -1.247103 & 3.090796\end{array}$

$1 \quad 8.555677 \quad 0.081260 \quad 4.717863$

$\begin{array}{lllll}8 & 6.540611 & 1.285590 & 4.575997\end{array}$

$\begin{array}{lllll}6 & 8.919026 & -1.906718 & -1.734388\end{array}$ 
$\begin{array}{lllll}7 & 1.108271 & -0.031561 & -3.677094\end{array}$

$\begin{array}{lllll}7 & 1.155470 & 4.762681 & 0.107695\end{array}$

$\begin{array}{lllll}7 & 1.083823 & -0.387261 & 3.704490\end{array}$

$\begin{array}{llll}8 & 1.429612 & 1.772356 & 2.906164\end{array}$

$\begin{array}{lllll}8 & 1.402103 & -2.530478 & 4.559625\end{array}$

$\begin{array}{lllll}8 & 1.495564 & -3.515845 & 1.747771\end{array}$

$\begin{array}{lllll}8 & 1.611472 & -5.266906 & -2.513251\end{array}$

$\begin{array}{lllll}8 & 1.519813 & -2.323179 & -3.414407\end{array}$

$\begin{array}{lllll}8 & 1.466216 & 4.538448 & -2.199196\end{array}$

$\begin{array}{lllll}8 & 1.416907 & 2.257340 & -4.024159\end{array}$

$\begin{array}{llll}8 & 1.540549 & 4.746366 & 2.406576\end{array}$

$\begin{array}{lllll}1 & 0.068539 & -0.480223 & 3.596585\end{array}$

$\begin{array}{lllll}1 & 0.135378 & 4.747200 & 0.159310\end{array}$

$\begin{array}{lllll}1 & 0.090054 & 0.010919 & -3.625662\end{array}$

$\begin{array}{lllll}1 & 0.181913 & -4.385968 & -0.471961\end{array}$

$\begin{array}{lllll}7 & 4.354359 & 1.372060 & -4.368727\end{array}$

$\begin{array}{lllll}7 & 4.380571 & -1.465759 & -4.072434\end{array}$

$\begin{array}{lllll}7 & 4.426665 & 4.685793 & -1.388312\end{array}$

$\begin{array}{lllll}7 & 4.488994 & 4.784107 & 1.464096\end{array}$

$\begin{array}{lllll}7 & 4.569704 & -5.027387 & -1.539967\end{array}$

$\begin{array}{lllll}7 & 4.418329 & -4.250624 & 1.202417\end{array}$

$\begin{array}{lllll}7 & 4.295485 & 1.206120 & 3.948741\end{array}$

$\begin{array}{lllll}7 & 4.351181 & -1.560078 & 4.647616\end{array}$

$\begin{array}{lllll}6 & -9.000537 & -2.541943 & 2.628826\end{array}$

$\begin{array}{lllll}6 & -8.420011 & -3.054367 & 1.313358\end{array}$

$\begin{array}{lllll}6 & -8.370833 & -1.202696 & 3.010364\end{array}$

$\begin{array}{llll}1 & -8.696411 & -3.253972 & 3.396742\end{array}$

$\begin{array}{lllll}6 & -8.914141 & -2.614968 & 0.080011\end{array}$

$\begin{array}{lllll}6 & -7.368825 & -3.979157 & 1.280590\end{array}$

$\begin{array}{lllll}6 & -8.909931 & 0.032427 & 2.642336\end{array}$

$\begin{array}{lllll}6 & -7.190881 & -1.178695 & 3.762025\end{array}$

$\begin{array}{lllll}6 & -8.406165 & -3.044517 & -1.148121\end{array}$

$\begin{array}{llll}-9.737760 & -1.910658 & 0.078281\end{array}$

$\begin{array}{lll}-6.800328 & -4.408946 & 0.086114\end{array}$

$\begin{array}{lll}-6.864648 & -4.509811 & 2.482065\end{array}$

$\begin{array}{lll}-8.332670 & 1.254102 & 3.009373\end{array}$

$\begin{array}{lll}-9.818036 & 0.047154 & 2.052019\end{array}$

$\begin{array}{lll}-6.580025 & 0.000769 & 4.164840\end{array}$

$\begin{array}{lll}-6.672872 & -2.411773 & 4.164909\end{array}$

$\begin{array}{lll}-9.010704 & -2.601313 & -2.479385\end{array}$

$\begin{array}{llll}-7.316277 & -3.923082 & -1.106906\end{array}$

$\begin{array}{lll}-5.969314 & -5.102483 & 0.087805\end{array}$

$\begin{array}{lll}-5.620397 & -4.042235 & 2.795944\end{array}$

$\begin{array}{lll}-8.929075 & 2.592504 & 2.583417\end{array}$

$\begin{array}{lll}-7.165577 & 1.202064 & 3.782085\end{array}$

$\begin{array}{lll}-5.676979 & -0.012428 & 4.760516\end{array}$

$\begin{array}{lll}-5.532236 & -2.860601 & 3.586930\end{array}$

$\begin{array}{llll}-8.698893 & -3.341499 & -3.216754\end{array}$

$\begin{array}{llll}-8.418999 & -1.270649 & -2.928771\end{array}$

$\begin{array}{lll}-6.760924 & -4.360163 & -2.316151\end{array}$

$\begin{array}{lll}-8.363250 & 3.040902 & 1.236766\end{array}$

$\begin{array}{lll}-8.590110 & 3.325670 & 3.315897\end{array}$

$\begin{array}{lll}-6.616617 & 2.402226 & 4.254108\end{array}$

$\begin{array}{llll}-8.954488 & -0.038572 & -2.536407\end{array}$

$\begin{array}{llll}-7.286629 & -1.243022 & -3.749982\end{array}$

$\begin{array}{llll}-5.571764 & -3.806924 & -2.682139\end{array}$

$\begin{array}{llll}-3.399706 & -4.040156 & 2.573657\end{array}$

$\begin{array}{llll}-8.895957 & 2.619061 & 0.016321\end{array}$ $\begin{array}{llll}6 & -7.266446 & 3.906935 & 1.177492\end{array}$

$\begin{array}{llll}6 & -5.437854 & 2.822293 & 3.720834\end{array}$

$\begin{array}{lllll}6 & -3.337046 & -2.823940 & 3.236577\end{array}$

$\begin{array}{lllll}6 & -8.397375 & 1.184010 & -2.918743\end{array}$

$\begin{array}{llll}-9.841461 & -0.034027 & -1.914265\end{array}$

$\begin{array}{llll}-6.674113 & -0.056468 & -4.135186\end{array}$

$\begin{array}{llll}-6.786048 & -2.459332 & -4.235489\end{array}$

$\begin{array}{lll}-5.582021 & -2.846739 & -3.733830\end{array}$

$\begin{array}{llll}-8.401822 & 3.041273 & -1.223400\end{array}$

$\begin{array}{llll}-9.728886 & 1.925699 & 0.028534\end{array}$

$\begin{array}{llll}-6.747477 & 4.375023 & -0.021488\end{array}$

$\begin{array}{lll}-6.702840 & 4.341703 & 2.383127\end{array}$

$\begin{array}{llll}-5.488447 & 3.825910 & 2.709368\end{array}$

$\begin{array}{llll}-8.998228 & 2.534760 & -2.532621\end{array}$

$\begin{array}{llll}-7.234849 & 1.134486 & -3.696952\end{array}$

$\begin{array}{lll}-5.788779 & -0.062298 & -4.756821\end{array}$

$\begin{array}{lll}-3.351380 & -3.613589 & -2.561764\end{array}$

$\begin{array}{llll}-7.328029 & 3.940485 & -1.207953\end{array}$

$\begin{array}{llll}-5.908030 & 5.058528 & -0.034345\end{array}$

$\begin{array}{lll}-3.206505 & 2.833839 & 3.594025\end{array}$

$\begin{array}{lll}-8.686141 & 3.239488 & -3.304181\end{array}$

$\begin{array}{llll}-6.683875 & 2.351398 & -4.100056\end{array}$

$\begin{array}{llll}-3.356457 & -2.731131 & -3.634058\end{array}$

$\begin{array}{llll}-6.823781 & 4.449130 & -2.416648\end{array}$

$\begin{array}{lll}-3.264039 & 3.769867 & 2.571057\end{array}$

$\begin{array}{lll}-5.527091 & 2.778771 & -3.535570\end{array}$

$\begin{array}{lll}-5.590215 & 3.965523 & -2.750921\end{array}$

$\begin{array}{llll}-3.314776 & 2.744954 & -3.267073\end{array}$

$\begin{array}{llll}-3.361961 & 3.970997 & -2.619504\end{array}$

$\begin{array}{llll}-10.533461 & 2.519600 & -2.515558\end{array}$

$\begin{array}{llll}-10.913803 & 3.521442 & -2.300180\end{array}$

$\begin{array}{llll}-10.947205 & 1.842904 & -1.762809\end{array}$

$\begin{array}{llll}-10.546011 & -2.596222 & -.451418\end{array}$

$\begin{array}{llll}-10.937208 & -2.330506 & -.437062\end{array}$

$\begin{array}{llll}-10.959236 & -1.885889 & -.730059\end{array}$

$\begin{array}{lll}-10.535204 & -2.499858 & 2.620561\end{array}$

$\begin{array}{lll}-10.934133 & -3.494021 & 2.403496\end{array}$

$\begin{array}{lll}-10.941459 & -1.813254 & 1.872739\end{array}$

$\begin{array}{lll}-10.464451 & 2.598119 & 2.601559\end{array}$

$\begin{array}{lll}-10.829926 & 2.317951 & 3.593053\end{array}$

$\begin{array}{lll}-10.904345 & 1.905142 & 1.879019\end{array}$

$\begin{array}{lll}-10.836059 & 3.598028 & 2.363241\end{array}$

$\begin{array}{lll}-10.907553 & -2.181112 & 3.597782\end{array}$

$\begin{array}{llll}-10.918812 & -3.588543 & -.184810\end{array}$

$\begin{array}{llll}-10.916904 & 2.203476 & -3.489288\end{array}$

$\begin{array}{llll}-1.937948 & -3.744726 & -2.066349\end{array}$

$\begin{array}{llll}-1.936458 & -2.324235 & -3.919805\end{array}$

$\begin{array}{llll}-1.887103 & 2.272506 & -3.288994\end{array}$

$\begin{array}{llll}-1.949320 & 4.393393 & -2.307392\end{array}$

$\begin{array}{llll}-1.995966 & -4.426241 & 2.180437\end{array}$

$\begin{array}{lll}-1.928238 & -2.327145 & 3.196295\end{array}$

$\begin{array}{lll}-1.754992 & 2.536514 & 3.881522\end{array}$

$\begin{array}{lll}-1.867224 & 4.066014 & 2.109305\end{array}$

$\begin{array}{lll}-1.155626 & 3.338537 & -2.792017\end{array}$

$\begin{array}{lll}-1.180054 & -2.996931 & -2.950709\end{array}$

$\begin{array}{lll}-1.204227 & -3.329943 & 2.590158\end{array}$

$\begin{array}{lll}-1.049433 & 3.349411 & 2.974644\end{array}$

$\begin{array}{lll}-1.272952 & 1.777514 & 4.687134\end{array}$ 


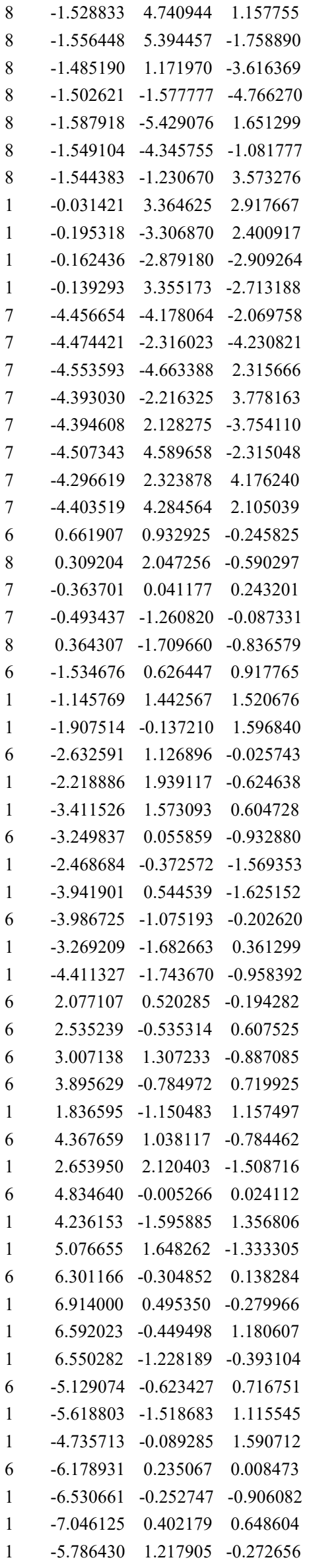

\section{4b-TS@12}

$\begin{array}{lllll}6 & -9.016615 & 3.562436 & -1.138501\end{array}$

$\begin{array}{lllll}6 & -8.391741 & 3.349145 & 0.240693\end{array}$

$\begin{array}{lllll}6 & -8.442555 & 2.565488 & -2.142955\end{array}$

$\begin{array}{lllll}1 & -8.698647 & 4.551261 & -1.470460\end{array}$

$\begin{array}{lllll}6 & -8.915741 & 2.456796 & 1.180982\end{array}$

$\begin{array}{lllll}6 & -7.234295 & 4.044267 & 0.609143\end{array}$

$\begin{array}{lllll}6 & -8.978064 & 1.281221 & -2.301545\end{array}$

$\begin{array}{lllll}6 & -7.333020 & 2.891587 & -2.931828\end{array}$

$\begin{array}{lllll}6 & -8.344640 & 2.254794 & 2.442781\end{array}$

$\begin{array}{lllll}1 & -9.804236 & 1.893866 & 0.920475\end{array}$

$\begin{array}{lllll}6 & -6.630456 & 3.892472 & 1.850540\end{array}$

$\begin{array}{lllll}8 & -6.709859 & 4.964402 & -0.305685\end{array}$

$\begin{array}{lllll}6 & -8.441815 & 0.335400 & -3.178412\end{array}$

$1 \quad-9.850049 \quad 1.010810 \quad-1.717750$

$\begin{array}{lllll}6 & -6.738984 & 1.977635 & -3.794562\end{array}$

$8 \quad-6.813212 \quad 4.195691 \quad-2.878020$

$\begin{array}{lllll}6 & -8.925685 & 1.269998 & 3.452920\end{array}$

$\begin{array}{lllll}6 & -7.199323 & 2.999649 & 2.750475\end{array}$

$\begin{array}{lllll}1 & -5.742420 & 4.453150 & 2.111270\end{array}$

$\begin{array}{lllll}6 & -5.539157 & 4.650773 & -0.915308\end{array}$

$6 \begin{array}{lllll}6 & -9.055551 & -1.046830 & -3.398655\end{array}$

$\begin{array}{lllll}6 & -7.291972 & 0.709289 & -3.883798\end{array}$

$\begin{array}{lllll}1 & -5.867186 & 2.247771 & -4.375614\end{array}$

$\begin{array}{lllll}6 & -5.587215 & 4.305694 & -2.296731\end{array}$

$\begin{array}{lllll}1 & -8.587319 & 1.604687 & 4.433968\end{array}$

$\begin{array}{lllll}6 & -8.346708 & -0.130065 & 3.256352\end{array}$

$8 \quad-6.647683 \quad 2.885900 \quad 4.034114$

$\begin{array}{lllll}6 & -8.462193 & -2.067008 & -2.433089\end{array}$

$\begin{array}{lllll}1 & -8.752261 & -1.359014 & -4.398697\end{array}$

$8 \quad-6.742510 \quad-0.225840 \quad-4.761324$

$\begin{array}{lllll}6 & -8.883432 & -1.042839 & 2.345479\end{array}$

$\begin{array}{lllll}6 & -7.242979 & -0.559686 & 4.002492\end{array}$

$\begin{array}{lllll}6 & -5.456378 & 2.236308 & 4.142784\end{array}$

$\begin{array}{lllll}6 & -3.324968 & 4.423459 & -0.961994\end{array}$

$6 \begin{array}{llll}6 & -8.955593 & -2.215668 & -1.131785\end{array}$

$6 \quad-7.390269 \quad-2.887491 \quad-2.804341$

$6 \begin{array}{lllll}6 & -5.591701 & -0.860380 & -4.421961\end{array}$

$\begin{array}{lllll}6 & -3.360506 & 4.147339 & -2.321061\end{array}$

$\begin{array}{lllll}6 & -8.384653 & -2.338968 & 2.179620\end{array}$

$\begin{array}{lllll}1 & -9.730018 & -0.735095 & 1.743015\end{array}$

$\begin{array}{lllll}6 & -6.711846 & -1.838077 & 3.886307\end{array}$

$8 \quad-6.676896 \quad 0.337372 \quad 4.916384$

$\begin{array}{lllll}6 & -5.477910 & 0.877113 & 4.569607\end{array}$

$6 \quad-8.419648 \quad-3.118204 \quad-0.211804$

$\begin{array}{lllll}1 & -9.796326 & -1.603701 & -0.827247\end{array}$

$\begin{array}{lllll}6 & -6.796773 & -3.777684 & -1.914830\end{array}$

$8 \quad-6.901378 \quad-2.838614 \quad-4.120811$

$6 \begin{array}{llll}6 & -5.665026 & -2.268257 & -4.223188\end{array}$

$\begin{array}{lllll}6 & -8.999561 & -3.316459 & 1.185210\end{array}$

$\begin{array}{lllll}6 & -7.291842 & -2.710328 & 2.972585\end{array}$

$\begin{array}{lllll}1 & -5.857938 & -2.142359 & 4.477689\end{array}$

$\begin{array}{lllll}6 & -3.228835 & 2.140384 & 4.010416\end{array}$

$\begin{array}{lllll}6 & -7.311297 & -3.865110 & -0.629392\end{array}$

$\begin{array}{lllll}1 & -5.954512 & -4.385646 & -2.219112\end{array}$

$\begin{array}{lllll}6 & -3.382403 & -0.963869 & -4.155670\end{array}$

$\begin{array}{lllll}1 & -8.690774 & -4.312001 & 1.505360\end{array}$

$\begin{array}{lllll}8 & -6.789593 & -4.015932 & 2.872863\end{array}$ $\begin{array}{llll}6 & -3.259809 & 0.798586 & 4.363139\end{array}$

$\begin{array}{lllll}8 & -6.758402 & -4.773570 & 0.277020\end{array}$

$6 \quad-3.439584 \quad-2.349202 \quad-4.102175$

$\begin{array}{lllll}6 & -5.582386 & -4.139243 & 2.248195\end{array}$

$\begin{array}{lllll}6 & -5.571710 & -4.458077 & 0.860573\end{array}$

$\begin{array}{lllll}6 & -3.350604 & -4.077538 & 2.231580\end{array}$

$\begin{array}{lllll}6 & -3.347270 & -4.291529 & 0.860347\end{array}$

$\begin{array}{lllll}6 & -10.535091 & -3.284517 & 1.196235\end{array}$

$\begin{array}{lllll}1 & -10.930155 & -4.042039 & 0.514287\end{array}$

$\begin{array}{lllll}1 & -10.946064 & -2.318913 & 0.888883\end{array}$

$\begin{array}{lllll}6 & -10.461353 & 1.270373 & 3.469893\end{array}$

$\begin{array}{lllll}1 & -10.826156 & 0.602698 & 4.254647\end{array}$

$\begin{array}{lllll}1 & -10.900580 & 0.936860 & 2.525560\end{array}$

$\begin{array}{lllll}6 & -10.551170 & 3.558830 & -1.102294\end{array}$

$\begin{array}{lllll}1 & -10.911389 & 4.324510 & -0.410261\end{array}$

$\begin{array}{lllll}1 & -10.970891 & 2.601656 & -0.780703\end{array}$

$\begin{array}{lllll}6 & -10.590569 & -1.024133 & -3.368978\end{array}$

$1 \quad-10.969883-0.320230 \quad-4.114435$

$\begin{array}{lllll}1 & -10.996497 & -0.726101 & -2.398336\end{array}$

$\begin{array}{lllll}1 & -10.982683 & -2.018501 & -3.597560\end{array}$

$\begin{array}{lllll}1 & -10.948925 & 3.774072 & -2.097434\end{array}$

$\begin{array}{lllll}1 & -10.833963 & 2.278599 & 3.669243\end{array}$

$\begin{array}{lllll}1 & -10.905876 & -3.494551 & 2.202878\end{array}$

$\begin{array}{lllll}6 & -1.788242 & 2.547170 & 3.822365\end{array}$

$\begin{array}{lllll}6 & -1.856596 & 0.264387 & 4.348126\end{array}$

$\begin{array}{lllll}6 & -1.920929 & -3.977993 & 2.696797\end{array}$

$\begin{array}{lllll}6 & -1.924204 & -4.238041 & 0.375611\end{array}$

$\begin{array}{lllll}6 & -1.912869 & 4.295352 & -0.483042\end{array}$

$\begin{array}{lllll}6 & -1.949612 & 3.887107 & -2.783587\end{array}$

$\begin{array}{lllll}6 & -1.951864 & -0.542562 & -3.976897\end{array}$

$6 \quad-2.031950 \quad-2.873565 \quad-3.996643$

$\begin{array}{llll}7 & -1.161404 & -4.160163 & 1.529233\end{array}$

$\begin{array}{llll}7 & -1.062053 & 1.365911 & 4.048650\end{array}$

$\begin{array}{lllll}7 & -1.172595 & 4.000259 & -1.612210\end{array}$

$\begin{array}{lllll}7 & -1.229405 & -1.717254 & -3.996613\end{array}$

$\begin{array}{lllll}8 & -1.545268 & 0.595417 & -3.805775\end{array}$

$8 \quad-1.639050 \quad-4.011845 \quad-3.917850$

$\begin{array}{lllll}8 & -1.543900 & -4.221752 & -0.780818\end{array}$

$\begin{array}{lllll}8 & -1.494628 & -3.779685 & 3.809256\end{array}$

$\begin{array}{lllll}8 & -1.501324 & -0.884302 & 4.515719\end{array}$

$\begin{array}{lllll}8 & -1.520482 & 4.370843 & 0.668507\end{array}$

$\begin{array}{lllll}8 & -1.332965 & 3.629918 & 3.541326\end{array}$

$\begin{array}{lllll}8 & -1.529293 & 3.626495 & -3.883747\end{array}$

$\begin{array}{lllll}1 & -0.216292 & -1.788984 & -3.885580\end{array}$

$\begin{array}{lllll}1 & -0.164019 & 3.809691 & -1.619044\end{array}$

$\begin{array}{lllll}1 & -0.043387 & 1.332304 & 4.030202\end{array}$

$\begin{array}{lllll}1 & -0.143087 & -4.105256 & 1.541338\end{array}$

$\begin{array}{lllll}7 & -4.331793 & 2.882757 & 3.871700\end{array}$

$\begin{array}{llll}7 & -4.382746 & 0.138624 & 4.653196\end{array}$

$\begin{array}{lllll}7 & -4.408131 & 4.685936 & -0.228857\end{array}$

$\begin{array}{lllll}7 & -4.499121 & 4.068670 & -3.015387\end{array}$

$\begin{array}{llll}7 & -4.471082 & -3.968012 & 2.949101\end{array}$

$\begin{array}{llll}7 & -4.455895 & -4.496210 & 0.146121\end{array}$

$\begin{array}{lllll}7 & -4.454517 & -0.187259 & -4.332840\end{array}$

$\begin{array}{lllll}7 & -4.589147 & -3.029083 & -4.098191\end{array}$

$\begin{array}{lllll}6 & 8.964646 & -3.164047 & -1.596945\end{array}$

$6 \quad 8.392242-3.160616-0.184106$

$\begin{array}{lllll}6 & 8.338472 & -2.059018 & -2.442278\end{array}$ 


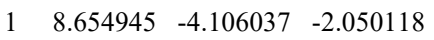

$\begin{array}{lllll}6 & 8.918948 & -2.367136 & 0.839783\end{array}$

$\begin{array}{lllll}6 & 7.294664 & -3.968375 & 0.134405\end{array}$

$\begin{array}{lllll}6 & 8.844044 & -0.756841 & -2.455937\end{array}$

$\begin{array}{lllll}6 & 7.227729 & -2.316813 & -3.256310\end{array}$

$\begin{array}{llll}6 & 8.403916 & -2.359514 & 2.140393\end{array}$

$\begin{array}{lllll}1 & 9.769781 & -1.733649 & 0.617410\end{array}$

$\begin{array}{lllll}6 & 6.734473 & -3.990161 & 1.403786\end{array}$

$\begin{array}{lllll}8 & 6.778881 & -4.813404 & -0.856333\end{array}$

$\begin{array}{lllll}6 & 8.317722 & 0.264339 & -3.254782\end{array}$

$\begin{array}{lllll}1 & 9.691998 & -0.527259 & -1.821527\end{array}$

$\begin{array}{lllll}6 & 6.676444 & -1.345955 & -4.082856\end{array}$

$\begin{array}{lllll}8 & 6.698307 & -3.610859 & -3.275296\end{array}$

$\begin{array}{lllll}6 & 9.016877 & -1.509893 & 3.255219\end{array}$

$\begin{array}{lllll}6 & 7.295013 & -3.179985 & 2.383232\end{array}$

$\begin{array}{lllll}1 & 5.880175 & -4.616572 & 1.625206\end{array}$

$\begin{array}{lllll}6 & 5.559823 & -4.478542 & -1.362851\end{array}$

$\begin{array}{lllll}6 & 8.913725 & 1.668568 & -3.245060\end{array}$

$\begin{array}{lllll}6 & 7.231877 & -0.069408 & -4.073692\end{array}$

$1 \quad 5.827404 \quad-1.574952-4.714485$

$\begin{array}{lllll}6 & 5.518415 & -3.819020 & -2.623917\end{array}$

$\begin{array}{lllll}1 & 8.695689 & -1.962906 & 4.193721\end{array}$

$\begin{array}{lllll}6 & 8.437722 & -0.097478 & 3.240867\end{array}$

$\begin{array}{lllll}8 & 6.754783 & -3.218399 & 3.674232\end{array}$

$\begin{array}{lllll}6 & 8.356335 & 2.516146 & -2.102370\end{array}$

$\begin{array}{lllll}1 & 8.577478 & 2.146315 & -4.165996\end{array}$

$\begin{array}{lllll}8 & 6.681633 & 0.900043 & -4.929800\end{array}$

$\begin{array}{lllll}6 & 8.992804 & 0.942347 & 2.483397\end{array}$

$\begin{array}{llll}6 & 7.285162 & 0.197235 & 3.979717\end{array}$

$\begin{array}{lllll}6 & 5.553257 & -2.598506 & 3.839689\end{array}$

$\begin{array}{lllll}6 & 3.330706 & -4.413593 & -1.270264\end{array}$

$\begin{array}{lllll}6 & 8.932841 & 2.577359 & -0.831256\end{array}$

$\begin{array}{lllll}6 & 7.215031 & 3.299043 & -2.313419\end{array}$

$\begin{array}{lllll}6 & 5.469341 & 1.366217 & -4.506217\end{array}$

$\begin{array}{lllll}6 & 3.291935 & -3.749046 & -2.487523\end{array}$

$\begin{array}{lllll}6 & 8.430640 & 2.221489 & 2.420600\end{array}$

$\begin{array}{lllll}1 & 9.894972 & 0.743715 & 1.917342\end{array}$

$\begin{array}{lllll}6 & 6.677431 & 1.445877 & 3.942886\end{array}$

$\begin{array}{lllll}8 & 6.757055 & -0.790643 & 4.816426\end{array}$

$\begin{array}{lllll}6 & 5.553100 & -1.318864 & 4.459990\end{array}$

$\begin{array}{lllll}6 & 8.439236 & 3.395927 & 0.195468\end{array}$

$\begin{array}{lllll}1 & 9.794393 & 1.954007 & -0.622648\end{array}$

$\begin{array}{lllll}6 & 6.716666 & 4.165945 & -1.356506\end{array}$

$\begin{array}{lllll}8 & 6.599262 & 3.225281 & -3.564173\end{array}$

$\begin{array}{lllll}6 & 5.445039 & 2.518233 & -3.670197\end{array}$

$\begin{array}{lllll}6 & 9.034358 & 3.366087 & 1.602126\end{array}$

$\begin{array}{llll}6 & 7.250662 & 2.426757 & 3.147086\end{array}$

$\begin{array}{lllll}1 & 5.779735 & 1.644084 & 4.512848\end{array}$

$\begin{array}{lllll}6 & 3.329048 & -2.509578 & 3.695012\end{array}$

$\begin{array}{lllll}6 & 7.344848 & 4.212659 & -0.116197\end{array}$

$\begin{array}{lllll}1 & 5.850425 & 4.781139 & -1.560622\end{array}$

$\begin{array}{lllll}6 & 3.258667 & 1.182133 & -4.277512\end{array}$

$\begin{array}{lllll}1 & 8.716363 & 4.289102 & 2.088684\end{array}$

$\begin{array}{llll}8 & 6.676948 & 3.697017 & 3.104760\end{array}$

$\begin{array}{llll}6 & 3.328316 & -1.272682 & 4.323911\end{array}$

$\begin{array}{lllll}8 & 6.839283 & 5.110890 & 0.838467\end{array}$

$\begin{array}{lllll}6 & 3.260422 & 2.214063 & -3.349126\end{array}$

$\begin{array}{lllll}6 & 5.527168 & 3.880503 & 2.406754\end{array}$ $\begin{array}{lllll}6 & 5.597477 & 4.752379 & 1.285565\end{array}$

$\begin{array}{llll}6 & 3.329949 & 3.684293 & 2.086207\end{array}$

$\begin{array}{llll}6 & 3.379914 & 4.639318 & 1.079833\end{array}$

$\begin{array}{llll}6 & 10.569160 & 3.349458 & 1.598132\end{array}$

$\begin{array}{lllll}1 & 10.950357 & 4.222982 & 1.063074\end{array}$

$\begin{array}{lllll}1 & 10.986620 & 2.461434 & 1.115655\end{array}$

$\begin{array}{llll}6 & 10.551664 & -1.530415 & 3.232953\end{array}$

$\begin{array}{lllll}1 & 10.946945 & -0.960214 & 4.077806\end{array}$

$\begin{array}{lllll}1 & 10.973179 & -1.101865 & 2.319540\end{array}$

$\begin{array}{lllll}6 & 10.499679 & -3.124215 & -1.618578\end{array}$

$\begin{array}{lllll}1 & 10.902519 & -3.970856 & -1.056667\end{array}$

$\begin{array}{lllll}1 & 10.911389 & -2.211858 & -1.178669\end{array}$

$\begin{array}{lllll}6 & 10.449623 & 1.654411 & -3.268385\end{array}$

$\begin{array}{lllll}1 & 10.805421 & 1.096082 & -4.138092\end{array}$

$\begin{array}{lllll}1 & 10.886850 & 1.192264 & -2.379238\end{array}$

$\begin{array}{lllll}1 & 10.832190 & 2.676507 & -3.330014\end{array}$

$\begin{array}{lllll}1 & 10.861794 & -3.185548 & -2.648120\end{array}$

$\begin{array}{lllll}1 & 10.912308 & -2.559376 & 3.308361\end{array}$

$\begin{array}{llll}1 & 10.948015 & 3.376238 & 2.623129\end{array}$

$\begin{array}{llll}6 & 1.904616 & -2.887962 & 3.383497\end{array}$

$\begin{array}{lllll}6 & 1.905486 & -0.809693 & 4.459612\end{array}$

$\begin{array}{lllll}6 & 1.906976 & 3.216849 & 2.229631\end{array}$

$\begin{array}{lllll}6 & 1.975818 & 4.897410 & 0.602234\end{array}$

$\begin{array}{lllll}6 & 1.916130 & -4.583550 & -0.781154\end{array}$

$\begin{array}{lllll}6 & 1.857474 & -3.432569 & -2.813299\end{array}$

$\begin{array}{lllll}6 & 1.849081 & 0.684537 & -4.427470\end{array}$

$\begin{array}{lllll}6 & 1.865701 & 2.363920 & -2.806393\end{array}$

$\begin{array}{lllll}7 & 1.184795 & 4.015954 & 1.358179\end{array}$

$\begin{array}{lllll}7 & 1.143912 & -1.824371 & 3.879700\end{array}$

$\begin{array}{lllll}7 & 1.126809 & -3.977972 & -1.768401\end{array}$

$\begin{array}{lllll}7 & 1.104775 & 1.459375 & -3.519684\end{array}$

$\begin{array}{lllll}8 & 1.411846 & -0.185355 & -5.140935\end{array}$

$\begin{array}{lllll}8 & 1.495875 & 3.106064 & -1.910894\end{array}$

$\begin{array}{lllll}8 & 1.574957 & 5.677810 & -0.227402\end{array}$

$\begin{array}{lllll}8 & 1.485498 & 2.309112 & 2.918938\end{array}$

$\begin{array}{llll}8 & 1.494236 & 0.219732 & 4.951205\end{array}$

$\begin{array}{lllll}8 & 1.516305 & -5.112659 & 0.229250\end{array}$

$\begin{array}{lllll}8 & 1.502583 & -3.881797 & 2.824781\end{array}$

$\begin{array}{lllll}8 & 1.442764 & -2.796279 & -3.761669\end{array}$

$\begin{array}{lllll}1 & 0.092717 & 1.305792 & -3.444856\end{array}$

$\begin{array}{lllll}1 & 0.109018 & -3.940098 & -1.666124\end{array}$

$1 \quad 0.123262 \quad-1.812352 \quad 3.926311$

$\begin{array}{lllll}1 & 0.162127 & 4.038111 & 1.287258\end{array}$

$\begin{array}{lllll}7 & 4.442889 & -3.198655 & 3.437128\end{array}$

$\begin{array}{lllll}7 & 4.441261 & -0.643359 & 4.708721\end{array}$

$\begin{array}{lllll}7 & 4.467383 & -4.783070 & -0.677281\end{array}$

$\begin{array}{lllll}7 & 4.383504 & -3.433279 & -3.188431\end{array}$

$\begin{array}{lllll}7 & 4.400993 & 3.296251 & 2.784980\end{array}$

$\begin{array}{lllll}7 & 4.523688 & 5.168213 & 0.634652\end{array}$

$\begin{array}{lllll}7 & 4.370571 & 0.711145 & -4.846314\end{array}$

$\begin{array}{llll}7 & 4.350334 & 2.919652 & -3.040265\end{array}$

$\begin{array}{lllll}6 & 0.755694 & -0.771272 & -0.634548\end{array}$

$\begin{array}{lllll}8 & 0.223836 & -1.049293 & -1.664309\end{array}$

$\begin{array}{lllll}7 & -0.475953 & 0.027101 & 0.595903\end{array}$

$\begin{array}{llll}7 & -0.556817 & -1.040505 & 1.253285\end{array}$

$\begin{array}{lllll}8 & 0.324760 & -1.858827 & 0.757127\end{array}$

$\begin{array}{llll}6 & -1.522139 & 1.031779 & 0.714276\end{array}$

$\begin{array}{lllll}1 & -1.046964 & 2.010263 & 0.771443\end{array}$ $\begin{array}{llll}1 & -2.058799 & 0.855721 & 1.647509\end{array}$

$\begin{array}{lllll}6 & -2.479776 & 0.948444 & -0.483887\end{array}$

$\begin{array}{lllll}1 & -1.934180 & 1.173401 & -1.403701\end{array}$

$\begin{array}{lllll}1 & -3.225820 & 1.739623 & -0.350370\end{array}$

$\begin{array}{lllll}6 & -3.178798 & -0.408316 & -0.636505\end{array}$

$\begin{array}{lllll}1 & -2.431124 & -1.181486 & -0.847159\end{array}$

$\begin{array}{lllll}1 & -3.813601 & -0.355061 & -1.526640\end{array}$

$\begin{array}{lllll}6 & -4.035632 & -0.838049 & 0.563114\end{array}$

$\begin{array}{llll}1 & -4.471254 & -1.815431 & 0.328488\end{array}$

$\begin{array}{llll}1 & -3.401631 & -0.993641 & 1.444576\end{array}$

$\begin{array}{lllll}6 & 2.147768 & -0.371814 & -0.384597\end{array}$

$\begin{array}{lllll}6 & 2.594988 & 0.085943 & 0.858947\end{array}$

$\begin{array}{lllll}6 & 3.061727 & -0.583090 & -1.425071\end{array}$

$\begin{array}{lllll}6 & 3.948719 & 0.329704 & 1.055326\end{array}$

$\begin{array}{lllll}1 & 1.886820 & 0.268402 & 1.657309\end{array}$

$\begin{array}{lllll}6 & 4.415928 & -0.349718 & -1.213173\end{array}$

$\begin{array}{lllll}1 & 2.708138 & -0.955698 & -2.378333\end{array}$

$\begin{array}{lllll}6 & 4.879281 & 0.108067 & 0.025934\end{array}$

$\begin{array}{lllll}1 & 4.289009 & 0.696521 & 2.018578\end{array}$

$\begin{array}{lllll}1 & 5.122460 & -0.534978 & -2.015539\end{array}$

$\begin{array}{lllll}6 & 6.338726 & 0.382038 & 0.242108\end{array}$

$\begin{array}{lllll}1 & 6.961036 & -0.160469 & -0.472174\end{array}$

$\begin{array}{lllll}1 & 6.550468 & 1.448219 & 0.119115\end{array}$

$\begin{array}{llll}1 & 6.649941 & 0.103813 & 1.249179\end{array}$

$\begin{array}{llll}6 & -5.182554 & 0.119223 & 0.914548\end{array}$

$\begin{array}{lllll}1 & -4.789202 & 1.091437 & 1.242384\end{array}$

$\begin{array}{llll}1 & -5.717960 & -0.295331 & 1.773743\end{array}$

$\begin{array}{lllll}6 & -6.172466 & 0.326492 & -0.233283\end{array}$

$\begin{array}{lllll}1 & -5.730697 & 0.878648 & -1.069074\end{array}$

$\begin{array}{lllll}1 & -6.522368 & -0.635040 & -0.623454\end{array}$

$\begin{array}{llll}1 & -7.048454 & 0.884141 & 0.100585\end{array}$

\section{$4 b @ 12$}

$\begin{array}{lllll}6 & 8.981289 & -1.798543 & -3.101634\end{array}$

$\begin{array}{lllll}6 & 8.354233 & -2.532583 & -1.916020\end{array}$

$\begin{array}{lllll}6 & 8.417978 & -0.386066 & -3.215957\end{array}$

$1 \quad 8.659052 \quad-2.333014 \quad-3.995779$

$\begin{array}{lllll}6 & 8.880637 & -2.446910 & -0.624049\end{array}$

$\begin{array}{lllll}6 & 7.222971 & -3.341794 & -2.083747\end{array}$

$\begin{array}{lllll}6 & 8.968184 & 0.700614 & -2.523995\end{array}$

$6 \quad 7.283500 \quad-0.139926 \quad-3.998392$

$\begin{array}{lllll}6 & 8.363620 & -3.155508 & 0.465825\end{array}$

$\begin{array}{llll}9.741306 & -1.809433 & -0.461007\end{array}$

$6.679397-4.083886-1.041563$

$\begin{array}{llll}6.685804 & -3.473311 & -3.369957\end{array}$

$\begin{array}{llll}8.416950 & 1.985227 & -2.557546\end{array}$

$\begin{array}{llll}9.854359 & 0.533817 & -1.923280\end{array}$

$\begin{array}{llll}6.689318 & 1.113807 & -4.062815\end{array}$

$\begin{array}{llll}6.768395 & -1.181244 & -4.785744\end{array}$

$\begin{array}{llll}8.961039 & -3.037657 & 1.865098\end{array}$

$\begin{array}{llll}7.270025 & -3.992926 & 0.215014\end{array}$

$\begin{array}{llll}5.831267 & -4.735726 & -1.209222\end{array}$

$\begin{array}{llll}5.506236 & -2.860896 & -3.654975\end{array}$

$\begin{array}{llll}9.020962 & 3.182760 & -1.811795\end{array}$

$\begin{array}{llll}7.257555 & 2.144964 & -3.326646\end{array}$

$\begin{array}{llll}5.804724 & 1.281428 & -4.662665\end{array}$

$\begin{array}{llll}5.546875 & -1.677881 & -4.449896\end{array}$

$\begin{array}{llll}8.654669 & -3.935298 & 2.403097\end{array}$ 
$\begin{array}{llll}6 & 8.361293 & -1.857932 & 2.629970\end{array}$

$\begin{array}{lllll}8 & 6.789054 & -4.813984 & 1.247550\end{array}$

$\begin{array}{lllll}6 & 8.437012 & 3.331977 & -0.404711\end{array}$

$\begin{array}{lllll}1 & 8.707457 & 4.069551 & -2.364343\end{array}$

$\begin{array}{lllll}8 & 6.692551 & 3.418996 & -3.384408\end{array}$

$\begin{array}{lllll}6 & 8.894257 & -0.568234 & 2.571911\end{array}$

$\begin{array}{lllll}6 & 7.234538 & -2.039164 & 3.442034\end{array}$

$\begin{array}{lllll}6 & 5.565948 & -4.471027 & 1.740743\end{array}$

$\begin{array}{lllll}6 & 3.276069 & -2.742160 & -3.639234\end{array}$

$\begin{array}{lllll}6 & 8.953609 & 2.626378 & 0.692004\end{array}$

$\begin{array}{lllll}7.329095 & 4.154661 & -0.159078\end{array}$

$\begin{array}{lllll}6 & 5.532739 & 3.634737 & -2.712495\end{array}$

$\begin{array}{lllll}6 & 3.312207 & -1.624604 & -4.461550\end{array}$

$\begin{array}{lllll}6 & 8.369982 & 0.512797 & 3.292064\end{array}$

$1 \quad 9.752896 \quad-0.392495 \quad 1.935016$

$\begin{array}{lllll}6 & 6.698167 & -1.016911 & 4.212153\end{array}$

$8 \quad 6.678496 \quad-3.320246 \quad 3.500155$

$\begin{array}{lllll}6 & 5.513077 & -3.579302 & 2.852490\end{array}$

$\begin{array}{llll}6 & 8.388648 & 2.671082 & 1.970349\end{array}$

$\begin{array}{lllll}1 & 9.820975 & 1.996605 & 0.533812\end{array}$

$\begin{array}{lllll}6 & 6.718908 & 4.222191 & 1.090076\end{array}$

$8 \quad 6.808281 \quad 4.955978 \quad-1.188510$

$\begin{array}{lllll}6 & 5.577978 & 4.554755 & -1.629708\end{array}$

$\begin{array}{lllll}6 & 8.962000 & 1.915974 & 3.171204\end{array}$

$\begin{array}{lllll}6 & 7.273336 & 0.248307 & 4.123783\end{array}$

$\begin{array}{llll}5.832135 & -1.187556 & 4.838184\end{array}$

$\begin{array}{lllll}6 & 3.334804 & -4.492840 & 1.668826\end{array}$

$\begin{array}{llll}6 & 7.243800 & 3.463184 & 2.124984\end{array}$

$\begin{array}{llll}5.848515 & 4.846287 & 1.244839\end{array}$

$\begin{array}{lllll}6 & 3.336681 & 3.420917 & -2.410078\end{array}$

$\begin{array}{lllll}1 & 8.632478 & 2.461570 & 4.056115\end{array}$

$\begin{array}{lllll}8 & 6.729905 & 1.285631 & 4.900537\end{array}$

$\begin{array}{llll}6 & 3.291321 & -3.524892 & 2.661836\end{array}$

$\begin{array}{llll}6.658573 & 3.525483 & 3.394585\end{array}$

$\begin{array}{lllll}6 & 3.357073 & 4.427309 & -1.454773\end{array}$

$\begin{array}{lllll}6 & 5.515277 & 1.736309 & 4.466776\end{array}$

$\begin{array}{lllll}6 & 5.489516 & 2.862515 & 3.594827\end{array}$

$\begin{array}{lllll}6 & 3.297172 & 1.550748 & 4.265924\end{array}$

$\begin{array}{lllll}6 & 3.291600 & 2.574873 & 3.329588\end{array}$

$\begin{array}{lllll}6 & 10.497887 & 1.908130 & 3.180010\end{array}$

$\begin{array}{lllll}1 & 10.877556 & 2.932919 & 3.159306\end{array}$

$\begin{array}{lllll}1 & 10.927393 & 1.380714 & 2.323789\end{array}$

$\begin{array}{lllll}6 & 10.496553 & -3.009748 & 1.853390\end{array}$

$\begin{array}{lllll}1 & 10.878685 & -2.988878 & 2.877261\end{array}$

$\begin{array}{lllll}1 & 10.905113 & -2.139680 & 1.332079\end{array}$

$\begin{array}{lllll}6 & 10.515711 & -1.833866 & -3.070737\end{array}$

$\begin{array}{lllll}1 & 10.866727 & -2.868663 & -3.044190\end{array}$

$\begin{array}{lllll}1 & 10.938737 & -1.321062 & -2.202552\end{array}$

$\begin{array}{lllll}6 & 10.556154 & 3.156794 & -1.812294\end{array}$

$\begin{array}{lllll}1 & 10.930954 & 3.112352 & -2.838135\end{array}$

$\begin{array}{lllll}1 & 10.968965 & 2.299011 & -1.273854\end{array}$

$110.945838 \quad 4.060827 \quad-1.337366$

$\begin{array}{lllll}1 & 10.918120 & -1.352057 & -3.965621\end{array}$

$\begin{array}{lllll}1 & 10.882687 & -3.902770 & 1.355201\end{array}$

$\begin{array}{llll}1 & 10.864828 & 1.418425 & 4.085746\end{array}$

$\begin{array}{lllll}6 & 1.920765 & -4.788763 & 1.238958\end{array}$

$\begin{array}{lllll}6 & 1.863771 & -3.093435 & 2.828792\end{array}$

$\begin{array}{lllll}6 & 1.886158 & 1.041461 & 4.415856\end{array}$ $\begin{array}{llll}6 & 1.911735 & 2.673610 & 2.741656\end{array}$

$\begin{array}{lllll}6 & 1.854569 & -2.969097 & -3.203901\end{array}$

$6 \quad 1.895984 \quad-1.140978 \quad-4.661120$

$\begin{array}{lllll}6 & 1.937648 & 2.883298 & -2.506143\end{array}$

$\begin{array}{lllll}6 & 1.939527 & 4.675665 & -1.009486\end{array}$

$\begin{array}{lllll}7 & 1.154577 & 1.756861 & 3.447823\end{array}$

$\begin{array}{lllll}7 & 1.125001 & -3.978476 & 2.067023\end{array}$

$\begin{array}{lllll}7 & 1.116965 & -2.006927 & -3.866304\end{array}$

$\begin{array}{lllll}7 & 1.183699 & 3.709863 & -1.699707\end{array}$

$\begin{array}{lllll}8 & 1.575549 & 1.903174 & -3.136407\end{array}$

$\begin{array}{lllll}8 & 1.506602 & 5.504694 & -0.247127\end{array}$

$\begin{array}{llll}8 & 1.552531 & 3.375225 & 1.812609\end{array}$

$\begin{array}{lllll}8 & 1.437137 & 0.220878 & 5.177253\end{array}$

$8 \quad 1.468283 \quad-2.122593 \quad 3.449739$

$8 \quad 1.458475 \quad-3.773240 \quad-2.378014$

$8 \quad 1.522318 \quad-5.522484 \quad 0.366554$

$\begin{array}{lllll}8 & 1.478912 & -0.223227 & -5.322596\end{array}$

$\begin{array}{llll}0.169976 & 3.652530 & -1.566276\end{array}$

$1 \quad 0.116289 \quad-1.843651 \quad-3.714167$

$\begin{array}{lllll}1 & 0.129384 & -3.866740 & 1.859853\end{array}$

$\begin{array}{llll}0.150806 & 1.593529 & 3.313683\end{array}$

$7 \quad 4.479111 \quad-4.957776 \quad 1.160605$

$\begin{array}{lllll}7 & 4.375774 & -3.054498 & 3.284506\end{array}$

$\begin{array}{lllll}7 & 4.368640 & -3.386307 & -3.222381\end{array}$

$7 \quad 4.451262 \quad-1.062280 \quad-4.873636$

$7 \quad 4.416294 \quad 1.096006 \quad 4.836783$

$\begin{array}{lllll}7 & 4.381383 & 3.269030 & 2.993443\end{array}$

$7 \quad 4.419695 \quad 3.018750 \quad-3.078589$

$\begin{array}{lllll}7 & 4.487557 & 4.992338 & -1.019424\end{array}$

$\begin{array}{lllll}6 & -9.036730 & 3.583629 & 0.919870\end{array}$

$\begin{array}{llll}6 & -8.465852 & 2.650182 & 1.985206\end{array}$

$\begin{array}{lllll}6 & -8.408436 & 3.297635 & -0.445908\end{array}$

$\begin{array}{lllll}1 & -8.720930 & 4.590904 & 1.194305\end{array}$

$\begin{array}{llll}6 & -9.010694 & 1.387051 & 2.255769\end{array}$

$\begin{array}{lllll}6 & -7.334769 & 3.027216 & 2.719582\end{array}$

$\begin{array}{lllll}6 & -8.919742 & 2.330608 & -1.316440\end{array}$

$\begin{array}{lllll}6 & -7.276103 & 3.999249 & -0.882750\end{array}$

$\begin{array}{lllll}6 & -8.448784 & 0.496795 & 3.177658\end{array}$

$\begin{array}{lllll}1 & -9.903248 & 1.084162 & 1.720810\end{array}$

$\begin{array}{lllll}6 & -6.722479 & 2.173816 & 3.626356\end{array}$

$\begin{array}{lllll}8 & -6.815781 & 4.318248 & 2.556697\end{array}$

$\begin{array}{lllll}6 & -8.372186 & 2.054297 & -2.575815\end{array}$

$\begin{array}{lllll}1 & -9.781032 & 1.756548 & -0.996006\end{array}$

$6 \quad-6.713004 \quad 3.791035 \quad-2.134854$

$8 \quad-6.722056 \quad 4.968885 \quad-0.042157$

$\begin{array}{lllll}6 & -9.050382 & -0.873901 & 3.510603\end{array}$

$\begin{array}{lllll}6 & -7.276308 & 0.918485 & 3.817098\end{array}$

$\begin{array}{llll}1 & -5.831370 & 2.476573 & 4.159200\end{array}$

$\begin{array}{lllll}6 & -5.588473 & 4.381899 & 1.966086\end{array}$

$\begin{array}{lllll}6 & -8.960297 & 0.971359 & -3.478825\end{array}$

$\begin{array}{lllll}6 & -7.267239 & 2.823054 & -2.966371\end{array}$

$\begin{array}{lllll}1 & -5.848070 & 4.359076 & -2.452837\end{array}$

$\begin{array}{lllll}6 & -5.544995 & 4.662052 & 0.573439\end{array}$

$\begin{array}{lllll}1 & -8.722126 & -1.106592 & 4.524737\end{array}$

$6 \quad-8.479041 \quad-1.975471 \quad 2.617182$

$8 \quad-6.680306 \quad 0.043401 \quad 4.724676$

$6 \quad-8.401729 \quad-0.415539 \quad-3.152294$

$\begin{array}{lllll}1 & -8.616967 & 1.202094 & -4.488217\end{array}$
$8 \quad-6.695742 \quad 2.646230 \quad-4.236935$

$\begin{array}{llll}-9.012941 & -2.246696 & 1.348893\end{array}$

$\begin{array}{llll}6 & -7.376959 & -2.744598 & 3.014402\end{array}$

$\begin{array}{llll}6 & -5.536819 & -0.586416 & 4.354555\end{array}$

$\begin{array}{lllll}6 & -3.364870 & 4.190640 & 1.988989\end{array}$

$\begin{array}{lllll}6 & -8.989992 & -1.296824 & -2.239948\end{array}$

$\begin{array}{llll}-7.243808 & -0.864105 & -3.798991\end{array}$

$\begin{array}{lllll}-5.481566 & 2.016485 & -4.214823\end{array}$

$\begin{array}{lllll}6 & -3.331297 & 4.397621 & 0.617036\end{array}$

$6 \quad-8.473170 \quad-3.190010 \quad 0.469940$

$\begin{array}{llll}-9.879051 & -1.680814 & 1.026862\end{array}$

$\begin{array}{llll}6 & -6.788516 & -3.680562 & 2.170317\end{array}$

$\begin{array}{llll}-6.830206 & -2.591788 & 4.299462\end{array}$

$\begin{array}{llll}6 & -5.594592 & -2.005235 & 4.283438\end{array}$

$6 \quad-8.481496 \quad-2.578158 \quad-1.977281$

$\begin{array}{llll}-9.869502 & -0.970231 & -1.697593\end{array}$

$\begin{array}{llll}6 & -6.729722 & -2.137164 & -3.620877\end{array}$

$\begin{array}{lllll}-6.626355 & 0.003703 & -4.697806\end{array}$

$\begin{array}{lllll}6 & -5.465327 & 0.599069 & -4.321496\end{array}$

$\begin{array}{llll}6.078261 & -3.488094 & -0.905198\end{array}$

$\begin{array}{llll}6 & -7.326018 & -3.867665 & 0.906258\end{array}$

$\begin{array}{llll}-5.922366 & -4.244064 & 2.492240\end{array}$

$\begin{array}{llll}6 & -3.358297 & -0.672369 & 3.899665\end{array}$

$\begin{array}{llll}6 & -7.363478 & -2.981292 & -2.718126\end{array}$

$\begin{array}{llll}-5.849293 & -2.461974 & -4.158770\end{array}$

$\begin{array}{lllll}6 & -3.266879 & 1.996283 & -3.946996\end{array}$

$\begin{array}{llll}-8.767149 & -4.502600 & -1.158567\end{array}$

$\begin{array}{llll}-6.744915 & -4.809433 & 0.054638\end{array}$

$\begin{array}{llll}6 & -3.384877 & -2.057829 & 3.995133\end{array}$

$\begin{array}{llll}-6.857585 & -4.281106 & -2.562906\end{array}$

$\begin{array}{lllll}6 & -3.279384 & 0.608188 & -3.893851\end{array}$

$\begin{array}{llll}-5.575442 & -4.496474 & -0.565330\end{array}$

$6 \quad-5.626046-4.325461 \quad-1.976366$

$\begin{array}{lllll}6 & -3.364917 & -4.202498 & -0.635893\end{array}$

$6 \quad-3.403841 \quad-4.139138 \quad-2.021419$

$\begin{array}{lllll}6 & -10.613046 & -3.464632 & -0.890613\end{array}$

$\begin{array}{lllll}1 & -10.999922 & -3.747262 & -1.873040\end{array}$

$\begin{array}{lllll}1 & -11.023968 & -2.481043 & -0.646106\end{array}$

$\begin{array}{lllll}6 & -10.585482 & -0.854670 & 3.515059\end{array}$

$\begin{array}{lllll}1 & -10.971090 & -1.830448 & 3.821434\end{array}$

$\begin{array}{lllll}1 & -11.013332 & -0.624945 & 2.535078\end{array}$

$\begin{array}{lllll}6 & -10.571055 & 3.575286 & 0.874374\end{array}$

$\begin{array}{lllll}1 & -10.975048 & 3.844089 & 1.853860\end{array}$

$\begin{array}{lllll}1 & -10.986658 & 2.600638 & 0.603155\end{array}$

$\begin{array}{lllll}6 & -10.495754 & 0.993889 & -3.498812\end{array}$

$\begin{array}{lllll}1 & -10.851726 & 1.983002 & -3.798119\end{array}$

$\begin{array}{lllll}1 & -10.940077 & 0.764947 & -2.526083\end{array}$

$\begin{array}{lllll}1 & -10.871714 & 0.256979 & -4.213282\end{array}$

$\begin{array}{lllll}1 & -10.928825 & 4.300480 & 0.138967\end{array}$

$\begin{array}{lllll}1 & -10.949154 & -0.099879 & 4.217040\end{array}$

$\begin{array}{lllll}1 & -10.992798 & -4.172631 & -0.149483\end{array}$

$\begin{array}{lllll}6 & -1.956199 & -0.250108 & 3.553289\end{array}$

$\begin{array}{lllll}6 & -1.980257 & -2.569600 & 3.836751\end{array}$

$6 \begin{array}{lllll}6 & -1.937528 & -4.027517 & -0.192539\end{array}$

$\begin{array}{lllll}6 & -1.993748 & -3.996057 & -2.529546\end{array}$

$\begin{array}{lllll}6 & -1.951726 & 3.961068 & 2.465072\end{array}$

$\begin{array}{lllll}6 & -1.913600 & 4.238573 & 0.146434\end{array}$

$\begin{array}{llll}6 & -1.843121 & 2.458643 & -3.787677\end{array}$ 
$6 \quad-1.886184 \quad 0.134034 \quad-3.580174$

$7 \quad-1.207238 \quad-3.935171 \quad-1.362104$

$7 \quad-1.217924 \quad-1.418371 \quad 3.568521$

$\begin{array}{lllll}7 & -1.179407 & 3.994544 & 1.291717\end{array}$

$\begin{array}{lllll}7 & -1.112434 & 1.274794 & -3.575942\end{array}$

$\begin{array}{lllll}8 & -1.384815 & 3.573837 & -3.830658\end{array}$

$\begin{array}{lllll}8 & -1.523669 & -1.008221 & -3.347584\end{array}$

$\begin{array}{lllll}8 & -1.585844 & -3.951768 & -3.663899\end{array}$

$8 \quad-1.529055 \quad-3.950015 \quad 0.952666$

$\begin{array}{lllll}8 & -1.555184 & -3.696618 & 3.918676\end{array}$

$\begin{array}{llll}8 & -1.533909 & 3.790442 & 3.584813\end{array}$

$\begin{array}{lllll}8 & -1.561803 & 0.870042 & 3.278854\end{array}$

$\begin{array}{lllll}8 & -1.514306 & 4.270073 & -1.002666\end{array}$

$1 \quad-0.102395 \quad 1.316798 \quad-3.380411$

$\begin{array}{lllll}1 & -0.167855 & 3.823850 & 1.310806\end{array}$

$1 \quad-0.195524 \quad-1.486239 \quad 3.476814$

$\begin{array}{lllll}1 & -0.187969 & -3.878956 & -1.429152\end{array}$

$\begin{array}{lllll}7 & -4.430699 & 0.096868 & 4.104235\end{array}$

$7 \quad-4.514190 \quad-2.756220 \quad 4.148133$

$7 \quad-4.500271 \quad 4.165904 \quad 2.690946$

$\begin{array}{llll}-4.416599 & 4.647350 & -0.119662\end{array}$

$\begin{array}{llll}-4.447924 & -4.398223 & 0.123366\end{array}$

$\begin{array}{llll}-4.541455 & -4.175607 & -2.720286\end{array}$

$\begin{array}{llll}-4.376355 & 2.730667 & -4.069313\end{array}$

$7 \quad-4.375660 \quad-0.122637 \quad-4.106098$

$\begin{array}{lllll}6 & -0.932013 & 0.387745 & -0.204109\end{array}$

$\begin{array}{llll}-0.350629 & 1.393461 & -0.545081\end{array}$

$\begin{array}{llll}1.635174 & -1.745481 & 0.090091\end{array}$

$\begin{array}{llll}1.058075 & -0.775632 & -0.374312\end{array}$

$\begin{array}{lllll}8 & -0.295793 & -0.793947 & 0.102879\end{array}$

$\begin{array}{lllll}6 & 3.027745 & -1.831891 & -0.378776\end{array}$

$\begin{array}{llll}3.097177 & -2.790880 & -0.898326\end{array}$

$\begin{array}{llll}3.618980 & -1.947939 & 0.531096\end{array}$

$\begin{array}{llll}3.547829 & -0.664818 & -1.211967\end{array}$

$\begin{array}{llll}2.893300 & -0.481593 & -2.065112\end{array}$

$\begin{array}{llll}4.521259 & -0.970217 & -1.607668\end{array}$

$\begin{array}{lllll}6 & -2.394672 & 0.243652 & -0.078857\end{array}$

$\begin{array}{lllll}6 & -2.986473 & -0.881417 & 0.511085\end{array}$

$\begin{array}{lllll}6 & -3.199902 & 1.265480 & -0.592854\end{array}$

$\begin{array}{lllll}6 & -4.368818 & -0.969190 & 0.590693\end{array}$

$\begin{array}{llll}-2.362311 & -1.667564 & 0.913543\end{array}$

$6 \quad-4.583407 \quad 1.156547 \quad-0.530031$

$\begin{array}{lllll}1 & -2.730967 & 2.127381 & -1.048444\end{array}$

$\begin{array}{lllll}6 & -5.185578 & 0.040560 & 0.061658\end{array}$

$\begin{array}{llll}-4.825615 & -1.835470 & 1.058727\end{array}$

$\begin{array}{llll}-5.204273 & 1.943097 & -0.944014\end{array}$

$\begin{array}{lllll}6 & -6.673359 & -0.099939 & 0.112786\end{array}$

$\begin{array}{lllll}1 & -6.999678 & -0.422583 & 1.100390\end{array}$

$\begin{array}{lllll}1 & -7.178223 & 0.833824 & -0.128285\end{array}$

$\begin{array}{llll}-7.009544 & -0.856895 & -0.598847\end{array}$

$\begin{array}{lllll}6 & 3.693476 & 0.645579 & -0.430715\end{array}$

$1 \quad 4.260959 \quad 1.338254 \quad-1.056611$

$\begin{array}{lllll}1 & 2.709199 & 1.096095 & -0.274469\end{array}$

$\begin{array}{lllll}6 & 4.406698 & 0.489556 & 0.913304\end{array}$

$\begin{array}{llll}3.751790 & -0.008589 & 1.640629\end{array}$

$\begin{array}{llll}1 & 4.594131 & 1.492252 & 1.303661\end{array}$

$\begin{array}{lllll}6 & 5.747834 & -0.244029 & 0.830926\end{array}$

$\begin{array}{llll}1 & 6.222542 & -0.194832 & 1.808935\end{array}$ $\begin{array}{llll}1 & 5.608606 & -1.311296 & 0.620029\end{array}$

$\begin{array}{lllll}6 & 6.678480 & 0.358693 & -0.210925\end{array}$

$1 \quad 6.708407 \quad 1.443888 \quad-0.111835$

$1 \quad 7.695887 \quad-0.014280 \quad-0.093477$

$\begin{array}{llll}6.364666 & 0.130899 & -1.231770\end{array}$

\section{4b'@1}

$\begin{array}{lllll}6 & -8.873567 & -0.835037 & -3.497318\end{array}$

$6 \quad-8.307286 \quad 0.543773 \quad-3.160114$

$6 \quad-8.295194 \quad-1.940824 \quad-2.617370$

$\begin{array}{llll}-8.541269 & -1.055097 & -4.512338\end{array}$

$\begin{array}{llll}-8.868725 & 1.387801 & -2.199342\end{array}$

$\begin{array}{llll}-7.192333 & 1.032512 & -3.851561\end{array}$

$\begin{array}{llll}-8.839463 & -2.247065 & -1.366033\end{array}$

$\begin{array}{llll}-7.212612 & -2.721754 & -3.044288\end{array}$

$\begin{array}{llll}-8.384189 & 2.675944 & -1.940784\end{array}$

$\begin{array}{llll}-9.722796 & 1.033730 & -1.634475\end{array}$

$\begin{array}{llll}-6.684392 & 2.308637 & -3.653802\end{array}$

$\begin{array}{llll}-6.600220 & 0.190386 & -4.798183\end{array}$

$\begin{array}{llll}-8.364504 & -3.277536 & -0.549763\end{array}$

$\begin{array}{llll}-9.673439 & -1.654507 & -1.009157\end{array}$

$\begin{array}{llll}-6.698128 & -3.757065 & -2.271921\end{array}$

$\begin{array}{llll}-6.641505 & -2.475473 & -4.303830\end{array}$

$\begin{array}{llll}-8.992866 & 3.562432 & -0.860989\end{array}$

$\begin{array}{llll}-7.296271 & 3.117222 & -2.702784\end{array}$

$\begin{array}{llll}-5.827269 & 2.660519 & -4.212177\end{array}$

$\begin{array}{llll}-5.437046 & -0.409303 & -4.432920\end{array}$

$\begin{array}{llll}-8.979434 & -3.562868 & 0.819613\end{array}$

$\begin{array}{llll}-7.275098 & -4.014160 & -1.033070\end{array}$

$\begin{array}{llll}-5.851391 & -4.335619 & -2.618630\end{array}$

$\begin{array}{llll}-5.441894 & -1.823352 & -4.267320\end{array}$

$\begin{array}{llll}-8.692894 & 4.584363 & -1.094178\end{array}$

$\begin{array}{llll}-8.383250 & 3.233493 & 0.499005\end{array}$

$\begin{array}{llll}-6.820725 & 4.427066 & -2.522228\end{array}$

$\begin{array}{llll}-8.390107 & -2.633121 & 1.878854\end{array}$

$\begin{array}{llll}-8.671040 & -4.572950 & 1.090350\end{array}$

$\begin{array}{llll}-6.746828 & -5.047624 & -0.243752\end{array}$

$\begin{array}{llll}-8.895871 & 2.236089 & 1.330433\end{array}$

$\begin{array}{llll}-7.262392 & 3.932814 & 0.963894\end{array}$

$\begin{array}{llll}-5.600679 & 4.513111 & -1.917492\end{array}$

$\begin{array}{llll}-3.252884 & -0.383449 & -3.992481\end{array}$

$\begin{array}{llll}-8.930954 & -1.376598 & 2.174798\end{array}$

$\begin{array}{lll}-7.245280 & -3.015299 & 2.586512\end{array}$

$\begin{array}{llll}-5.542201 & -4.796982 & 0.344746\end{array}$

$\begin{array}{llll}-3.231509 & -1.770609 & -3.955656\end{array}$

$\begin{array}{llll}-8.369996 & 1.947172 & 2.594681\end{array}$

$\begin{array}{llll}-9.750638 & 1.667161 & 0.985451\end{array}$

$\begin{array}{lll}-6.711892 & 3.703954 & 2.217891\end{array}$

$\begin{array}{llll}-6.731627 & 4.931827 & 0.140387\end{array}$

$\begin{array}{llll}-5.560972 & 4.677240 & -0.502301\end{array}$

$\begin{array}{lll}-8.370410 & -0.514853 & 3.123396\end{array}$

$\begin{array}{llll}-9.825194 & -1.060228 & 1.651328\end{array}$

$\begin{array}{lll}-6.642096 & -2.198246 & 3.535550\end{array}$

$\begin{array}{llll}-6.733633 & -4.296212 & 2.348392\end{array}$

$\begin{array}{llll}-5.537931 & -4.392054 & 1.711673\end{array}$

$\begin{array}{llll}-8.965844 & 0.848838 & 3.472194\end{array}$

$\begin{array}{lll}-7.280657 & 2.718638 & 3.019451\end{array}$

$\begin{array}{lll}-5.861880 & 4.276232 & 2.565964\end{array}$ $\begin{array}{lllll}6 & -3.370395 & 4.390325 & -1.951654\end{array}$

$\begin{array}{llll}-7.212126 & -0.954965 & 3.775705\end{array}$

$\begin{array}{llll}-5.754983 & -2.518908 & 4.065422\end{array}$

$\begin{array}{llll}-3.309722 & -4.685536 & 0.327446\end{array}$

$\begin{array}{llll}-8.649472 & 1.067684 & 4.492519\end{array}$

$\begin{array}{llll}-6.770606 & 2.536115 & 4.315117\end{array}$

$\begin{array}{llll}-3.344655 & 4.432607 & -0.565292\end{array}$

$\begin{array}{llll}-6.679984 & -0.119909 & 4.762211\end{array}$

$\begin{array}{llll}-3.310069 & -4.290009 & 1.657469\end{array}$

$\begin{array}{llll}-5.548245 & 1.937405 & 4.389193\end{array}$

$\begin{array}{llll}-5.505716 & 0.520082 & 4.541523\end{array}$

$\begin{array}{llll}-3.315392 & 1.984094 & 4.339301\end{array}$

$\begin{array}{llll}-3.282080 & 0.599229 & 4.408768\end{array}$

$\begin{array}{llll}-10.501207 & 0.848133 & 3.458588\end{array}$

$\begin{array}{llll}-10.881103 & 0.090981 & 4.149527\end{array}$

$\begin{array}{llll}-10.922673 & 0.634225 & 2.472483\end{array}$

$\begin{array}{lllll}-10.527780 & 3.523406 & -0.849629\end{array}$

$\begin{array}{llll}-10.914694 & 4.214567 & -0.096265\end{array}$

$\begin{array}{llll}-10.930754 & 2.532135 & -0.624296\end{array}$

$6 \quad-10.410221 \quad-0.844900 \quad-3.507107$

$\begin{array}{llll}-10.784730 & -0.093277 & -4.206889\end{array}$

$\begin{array}{llll}-10.845924 & -0.628809 & -2.527646\end{array}$

$\begin{array}{lllll}6 & -10.514355 & -3.539888 & 0.790167\end{array}$

$\begin{array}{llll}-10.884889 & -4.267098 & 0.063185\end{array}$

$\begin{array}{llll}-10.923366 & -2.563776 & 0.514443\end{array}$

$\begin{array}{llll}-10.912219 & -3.798097 & 1.775124\end{array}$

$\begin{array}{llll}-10.775807 & -1.826214 & -3.820352\end{array}$

$\begin{array}{llll}-10.915385 & 3.821209 & -1.827320\end{array}$

$\begin{array}{llll}-10.877795 & 1.825352 & 3.771404\end{array}$

$\begin{array}{llll}-1.952193 & 4.245407 & -2.441663\end{array}$

$\begin{array}{llll}-1.937180 & 4.185160 & -0.107350\end{array}$

$\begin{array}{llll}-1.896442 & 2.482248 & 4.207451\end{array}$

$\begin{array}{llll}-1.860500 & 0.143103 & 4.258108\end{array}$

$\begin{array}{llll}-1.869086 & 0.124337 & -3.704163\end{array}$

$\begin{array}{llll}-1.806354 & -2.207725 & -3.750854\end{array}$

$\begin{array}{llll}-1.881663 & -4.706701 & -0.158231\end{array}$

$\begin{array}{llll}-1.888429 & -4.060011 & 2.088502\end{array}$

$\begin{array}{llll}-1.116362 & 1.310387 & 4.183440\end{array}$

$\begin{array}{llll}-1.187997 & 4.117101 & -1.263311\end{array}$

$\begin{array}{llll}-1.082978 & -1.010268 & -3.638280\end{array}$

$\begin{array}{llll}-1.124654 & -4.349606 & 0.959207\end{array}$

$\begin{array}{llll}-1.470914 & -4.926278 & -1.276103\end{array}$

$\begin{array}{llll}-1.478224 & -3.678425 & 3.163181\end{array}$

$\begin{array}{llll}-1.469007 & -1.000644 & 4.141281\end{array}$

$\begin{array}{llll}-1.483479 & 3.612746 & 4.118960\end{array}$

$\begin{array}{llll}-1.562060 & 4.020198 & 1.042823\end{array}$

$\begin{array}{llll}-1.536417 & 1.283273 & -3.534191\end{array}$

$\begin{array}{llll}-1.520422 & 4.241769 & -3.567177\end{array}$

$\begin{array}{llll}-1.349865 & -3.324902 & -3.687771\end{array}$

$\begin{array}{llll}-0.104805 & -4.275865 & 0.989335\end{array}$

$\begin{array}{llll}-0.071502 & -1.038973 & -3.488113\end{array}$

$\begin{array}{llll}-0.168267 & 3.982495 & -1.310886\end{array}$

$\begin{array}{llll}-0.110110 & 1.334863 & 3.997634\end{array}$

$\begin{array}{llll}-4.505731 & 4.407543 & -2.655571\end{array}$

$\begin{array}{llll}-4.433463 & 4.603208 & 0.187037\end{array}$

$\begin{array}{llll}-4.351313 & 0.326617 & -4.249195\end{array}$

$\begin{array}{llll}-4.333466 & -2.518128 & -4.057775\end{array}$

$\begin{array}{llll}-4.455270 & 2.681423 & 4.307228\end{array}$ 
$1 \quad 8.688676 \quad 2.605203 \quad-3.879349$

$8 \quad 6.705763 \quad 3.697906 \quad-3.199096$

$\begin{array}{lllll}6 & 3.344303 & 4.408156 & 1.567092\end{array}$

$\begin{array}{lllll}8 & 6.773199 & 1.475813 & -4.698778\end{array}$

$\begin{array}{lllll}6 & 3.370827 & -3.370029 & -2.580379\end{array}$

$\begin{array}{lllll}6 & 5.529099 & 3.046421 & -3.408878\end{array}$

$\begin{array}{lllll}6 & 5.555982 & 1.916822 & -4.271982\end{array}$

$\begin{array}{lllll}6 & 3.324621 & 2.797397 & -3.193426\end{array}$

$\begin{array}{lllll}6 & 3.338462 & 1.749890 & -4.103820\end{array}$

$\begin{array}{lllll}6 & 10.550530 & 2.058208 & -2.990202\end{array}$

$\begin{array}{llll}10.937807 & 1.590303 & -3.899032\end{array}$

$\begin{array}{llll}10.975866 & 1.522911 & -2.136731\end{array}$

$\begin{array}{llll}6 & 10.534674 & 3.099641 & 2.026352\end{array}$

$\begin{array}{llll}1 & 10.921167 & 4.032564 & 1.608092\end{array}$

$\begin{array}{llll}10.961936 & 2.277700 & 1.445214\end{array}$

$\begin{array}{llll}6 & 10.521485 & -1.936012 & 3.077296\end{array}$

$\begin{array}{llll}10.899593 & -1.465700 & 3.988798\end{array}$

$\begin{array}{llll}10.952527 & -1.400809 & 2.226642\end{array}$

$\begin{array}{lllll}6 & 10.589784 & -2.950276 & -1.905415\end{array}$

$10.977708-3.875536-1.471746$

$\begin{array}{llll}10.992768 & -2.119207 & -1.319957\end{array}$

$\begin{array}{llll}10.976184 & -2.853713 & -2.923438\end{array}$

$\begin{array}{llll}10.888519 & -2.964768 & 3.037583\end{array}$

$\begin{array}{llll}10.897738 & 3.000906 & 3.052685\end{array}$

$\begin{array}{llll}10.914235 & 3.087987 & -2.945836\end{array}$

$\begin{array}{llll}1.877850 & 2.830006 & 2.498700\end{array}$

$\begin{array}{llll}1.941585 & 4.718790 & 1.118783\end{array}$

$\begin{array}{llll}1.916378 & 2.980699 & -2.706419\end{array}$

$\begin{array}{llll}1.922915 & 1.270142 & -4.287922\end{array}$

$\begin{array}{llll}1.864699 & -1.280591 & 4.242145\end{array}$

$\begin{array}{llll}1.892268 & -3.069933 & 2.733673\end{array}$

$2.003001-4.788868-1.306482$

$\begin{array}{llll}1.944720 & -2.920956 & -2.710790\end{array}$

$\begin{array}{llll}1.165887 & 2.067960 & -3.412132\end{array}$

$\begin{array}{llll}1.157422 & 3.709455 & 1.710038\end{array}$

$\begin{array}{llll}1.118692 & -2.137196 & 3.410131\end{array}$

$\begin{array}{llll}1.210180 & -3.853872 & -1.997165\end{array}$

$\begin{array}{llll}1.603171 & -5.656918 & -0.568902\end{array}$

$\begin{array}{llll}1.543054 & -1.923973 & -3.279360\end{array}$

$\begin{array}{llll}1.487420 & 0.407945 & -5.011879\end{array}$

$\begin{array}{llll}1.533704 & 3.752209 & -1.840277\end{array}$

$\begin{array}{llll}1.531766 & 5.621620 & 0.431659\end{array}$

$\begin{array}{llll}1.431677 & -0.407528 & 4.954477\end{array}$

$\begin{array}{llll}1.458383 & 1.856451 & 3.096320\end{array}$

$\begin{array}{llll}1.522805 & -3.906632 & 1.931613\end{array}$

$\begin{array}{llll}0.191739 & -3.897440 & -1.977868\end{array}$

$\begin{array}{llll}0.099942 & -2.150754 & 3.430662\end{array}$

$\begin{array}{llll}0.137810 & 3.699109 & 1.587896\end{array}$

$\begin{array}{llll}0.158634 & 1.904128 & -3.292564\end{array}$

$\begin{array}{llll}4.359397 & 2.867316 & 3.100290\end{array}$

$\begin{array}{llll}4.487576 & 5.000303 & 1.206562\end{array}$

$\begin{array}{llll}4.396750 & -1.208397 & 4.589765\end{array}$

$\begin{array}{llll}4.407972 & -3.479767 & 2.861945\end{array}$

$\begin{array}{llll}4.415748 & 3.477827 & -2.834901\end{array}$

$\begin{array}{llll}4.460140 & 1.274470 & -4.648655\end{array}$

$\begin{array}{llll}4.562906 & -4.972726 & -1.262057\end{array}$

$\begin{array}{llll}4.447522 & -2.870250 & -3.190176\end{array}$

$\begin{array}{llll}0.678790 & -0.436450 & -0.406417\end{array}$ $\begin{array}{llll}0.008638 & 0.479445 & -0.893750\end{array}$

$\begin{array}{llll}-2.331900 & -1.339815 & -0.064867\end{array}$

$\begin{array}{llll}-1.523053 & -1.787784 & -0.758340\end{array}$

$\begin{array}{llll}0.201392 & -1.599887 & -0.033360\end{array}$

$\begin{array}{lll}-2.245006 & -0.541888 & 1.170207\end{array}$

$\begin{array}{llll}-1.221336 & -0.688663 & 1.514351\end{array}$

$\begin{array}{llll}2.153652 & -0.289824 & -0.184613\end{array}$

$\begin{array}{llll}2.977951 & -1.392740 & 0.066611\end{array}$

$\begin{array}{llll}2.723105 & 0.983696 & -0.272371\end{array}$

$\begin{array}{llll}4.355024 & -1.225736 & 0.174732\end{array}$

$\begin{array}{llll}2.532960 & -2.372701 & 0.173522\end{array}$

$\begin{array}{llll}4.100137 & 1.146432 & -0.154510\end{array}$

$\begin{array}{llll}2.070454 & 1.831442 & -0.431699\end{array}$

$\begin{array}{llll}4.938941 & 0.042641 & 0.051039\end{array}$

$\begin{array}{llll}4.987796 & -2.090088 & 0.353643\end{array}$

$\begin{array}{llll}4.533912 & 2.138897 & -0.239816\end{array}$

$\begin{array}{llll}6.429622 & 0.207315 & 0.114022\end{array}$

$\begin{array}{llll}6.868376 & -0.420385 & 0.891615\end{array}$

$\begin{array}{llll}6.893592 & -0.084584 & -0.833348\end{array}$

$\begin{array}{llll}6.708828 & 1.241769 & 0.312123\end{array}$

$\begin{array}{llll}-2.557958 & 0.935527 & 0.882735\end{array}$

$\begin{array}{lll}-1.954839 & 1.491148 & 1.603819\end{array}$

$\begin{array}{llll}-2.128609 & 1.190707 & -0.090151\end{array}$

$\begin{array}{lll}-4.025640 & 1.373912 & 1.007362\end{array}$

$\begin{array}{llll}-4.025388 & 2.412731 & 1.347210\end{array}$

$\begin{array}{llll}-4.514281 & 0.805291 & 1.806598\end{array}$

$\begin{array}{llll}-4.878302 & 1.300878 & -0.265505\end{array}$

$\begin{array}{llll}-5.839997 & 1.777869 & -0.044261\end{array}$

$\begin{array}{llll}-4.405043 & 1.897925 & -1.054481\end{array}$

$\begin{array}{llll}-2.943173 & -0.993790 & 1.875548\end{array}$

$\begin{array}{llll}-5.161929 & -0.097923 & -0.819740\end{array}$

$\begin{array}{llll}-4.255900 & -0.520691 & -1.265932\end{array}$

$\begin{array}{llll}-5.884914 & 0.004748 & -1.634294\end{array}$

$\begin{array}{llll}-5.722987 & -1.065544 & 0.215482\end{array}$

$\begin{array}{llll}-5.979612 & -2.014118 & -0.252508\end{array}$

$\begin{array}{llll}-5.012877 & -1.277046 & 1.019071\end{array}$

$\begin{array}{lll}-6.628918 & -0.666293 & 0.679902\end{array}$

\section{5:6b@12}

$\begin{array}{lllll}6 & 8.990279 & 3.567539 & 0.900423\end{array}$

$\begin{array}{lllll}6 & 8.362387 & 3.307605 & -0.470643\end{array}$

$\begin{array}{lllll}6 & 8.413162 & 2.617879 & 1.949948\end{array}$

$\begin{array}{lllll}1 & 8.682583 & 4.572163 & 1.191847\end{array}$

$\begin{array}{lllll}6 & 8.844675 & 2.306348 & -1.319019\end{array}$

$\begin{array}{lllll}6 & 7.253680 & 4.036888 & -0.924588\end{array}$

$\begin{array}{lllll}6 & 8.951172 & 1.352498 & 2.216183\end{array}$

$\begin{array}{lllll}6 & 7.269202 & 2.984207 & 2.666779\end{array}$

$\begin{array}{lllll}6.301087 & 2.028707 & -2.578340\end{array}$

$\begin{array}{llll}9.684865 & 1.711337 & -0.981141\end{array}$

$\begin{array}{llll}6.679969 & 3.810251 & -2.170840\end{array}$

$\begin{array}{llll}6.718117 & 5.045255 & -0.107819\end{array}$

$\begin{array}{llll}8.373328 & 0.459077 & 3.125207\end{array}$

$\begin{array}{llll}9.847715 & 1.048465 & 1.688905\end{array}$

$\begin{array}{lll}6.648679 & 2.135459 & 3.575531\end{array}$

$\begin{array}{llll}6.765191 & 4.275747 & 2.471982\end{array}$

$\begin{array}{llll}8.885883 & 0.946447 & -3.485554\end{array}$

$\begin{array}{llll}7.214174 & 2.815328 & -2.983593\end{array}$

$\begin{array}{llll}5.825736 & 4.389167 & -2.498697\end{array}$ 
$\begin{array}{lllll}6 & 5.525566 & 4.788211 & 0.501488\end{array}$

$\begin{array}{lllll}6 & 8.956512 & -0.918589 & 3.440189\end{array}$

$\begin{array}{lllll}6 & 7.202886 & 0.878500 & 3.769818\end{array}$

$\begin{array}{lllll}1 & 5.755004 & 2.440936 & 4.102938\end{array}$

$\begin{array}{llll}6 & 5.552942 & 4.388153 & 1.870060\end{array}$

$\begin{array}{lllll}1 & 8.550361 & 1.189172 & -4.494510\end{array}$

$\begin{array}{lllll}6 & 8.328264 & -0.445042 & -3.181447\end{array}$

$\begin{array}{lllll}8 & 6.660575 & 2.627043 & -4.263077\end{array}$

$\begin{array}{llll}6 & 8.367948 & -2.001940 & 2.537063\end{array}$

$\begin{array}{lllll}1 & 8.631904 & -1.160436 & 4.452931\end{array}$

$\begin{array}{lllll}8 & 6.636576 & 0.002437 & 4.699276\end{array}$

$\begin{array}{lllll}6 & 8.900361 & -1.315913 & -2.250362\end{array}$

$\begin{array}{lllll}6 & 7.197170 & -0.910837 & -3.863384\end{array}$

$\begin{array}{lllll}6 & 5.451779 & 1.990184 & -4.260071\end{array}$

$\begin{array}{lllll}6 & 3.293155 & 4.672348 & 0.540080\end{array}$

$\begin{array}{lllll}6 & 8.893466 & -2.254411 & 1.264281\end{array}$

$\begin{array}{lllll}6 & 7.296694 & -2.805862 & 2.948611\end{array}$

$\begin{array}{lllll}6 & 5.478810 & -0.639598 & 4.408349\end{array}$

$\begin{array}{lllll}6 & 3.323838 & 4.286024 & 1.872878\end{array}$

$\begin{array}{lllll}6 & 8.409588 & -2.606426 & -2.012435\end{array}$

$\begin{array}{lllll}1 & 9.763540 & -0.979517 & -1.688365\end{array}$

$\begin{array}{lllll}6 & 6.690537 & -2.191654 & -3.695149\end{array}$

$\begin{array}{lllll}8 & 6.587128 & -0.037310 & -4.766864\end{array}$

$\begin{array}{lllll}6 & 5.435846 & 0.570690 & -4.379456\end{array}$

$\begin{array}{lllll}6 & 8.402790 & -3.247231 & 0.413348\end{array}$

$\begin{array}{lllll}1 & 9.729098 & -1.653708 & 0.925663\end{array}$

$\begin{array}{lllll}6 & 6.751164 & -3.789748 & 2.129012\end{array}$

$\begin{array}{lllll}8 & 6.766366 & -2.650776 & 4.241311\end{array}$

$\begin{array}{lllll}6 & 5.537272 & -2.059289 & 4.284943\end{array}$

$\begin{array}{lllll}6 & 9.017009 & -3.524442 & -0.957167\end{array}$

$\begin{array}{lllll}6 & 7.312396 & -3.026097 & -2.773296\end{array}$

$\begin{array}{lllll}1 & 5.822372 & -2.525006 & -4.247596\end{array}$

$\begin{array}{lllll}6 & 3.240116 & 1.968784 & -3.960483\end{array}$

$\begin{array}{lllll}6 & 7.300740 & -3.983521 & 0.868560\end{array}$

$\begin{array}{llll}5.907688 & -4.379737 & 2.463300\end{array}$

$\begin{array}{lllll}6 & 3.266862 & -0.735666 & 4.145233\end{array}$

$\begin{array}{lllll}1 & 8.722791 & -4.539377 & -1.225752\end{array}$

$\begin{array}{lllll}8 & 6.840329 & -4.340234 & -2.615937\end{array}$

$\begin{array}{lllll}6 & 3.258808 & 0.581916 & -3.900868\end{array}$

$\begin{array}{lllll}8 & 6.767310 & -4.963775 & 0.022207\end{array}$

$\begin{array}{lllll}6 & 3.309322 & -2.122563 & 4.146709\end{array}$

$\begin{array}{lllll}6 & 5.623267 & -4.449409 & -2.009188\end{array}$

$\begin{array}{lllll}6 & 5.590317 & -4.685743 & -0.604039\end{array}$

$\begin{array}{lllll}6 & 3.393827 & -4.295923 & -2.023010\end{array}$

$\begin{array}{lllll}6 & 3.373253 & -4.416617 & -0.640819\end{array}$

$\begin{array}{lllll}6 & 10.551751 & -3.478766 & -0.935599\end{array}$

$\begin{array}{lllll}1 & 10.937595 & -4.194467 & -0.204985\end{array}$

$1 \quad 10.947946 \quad-2.494298 \quad-0.671694$

$\begin{array}{lllll}6 & 10.422226 & 0.965880 & -3.496899\end{array}$

$\begin{array}{lllll}1 & 10.800900 & 0.233429 & -4.214610\end{array}$

$1 \quad 10.860196 \quad 0.729313 \quad-2.523143$

$\begin{array}{lllll}6 & 10.524712 & 3.547921 & 0.855780\end{array}$

$\begin{array}{lllll}1 & 10.887929 & 4.283634 & 0.133680\end{array}$

$\begin{array}{lllll}1 & 10.932445 & 2.575396 & 0.565913\end{array}$

$\begin{array}{lllll}6 & 10.492277 & -0.925677 & 3.437419\end{array}$

$1 \quad 10.871596 \quad-0.185377 \quad 4.146757$

$\begin{array}{lllll}1 & 10.920605 & -0.693243 & 2.458441\end{array}$

$\begin{array}{llll}1 & 10.861761 & -1.911403 & 3.731416\end{array}$ $\begin{array}{llll}1 & 10.931056 & 3.795450 & 1.840040\end{array}$

$\begin{array}{lllll}1 & 10.781512 & 1.956619 & -3.786818\end{array}$

$\begin{array}{lllll}1 & 10.946945 & -3.739349 & -1.920920\end{array}$

$\begin{array}{lllll}6 & 1.829188 & 2.429687 & -3.709366\end{array}$

$\begin{array}{lllll}6 & 1.897819 & 0.104593 & -3.485882\end{array}$

$\begin{array}{lllll}6 & 1.977891 & -4.091782 & -2.499007\end{array}$

$\begin{array}{lllll}6 & 1.968572 & -4.166647 & -0.165078\end{array}$

$\begin{array}{lllll}6 & 1.860702 & 4.625598 & 0.069099\end{array}$

$\begin{array}{lllll}6 & 1.915011 & 4.023749 & 2.330628\end{array}$

$\begin{array}{lllll}6 & 1.851782 & -0.304087 & 3.890545\end{array}$

$\begin{array}{lllll}6 & 1.899878 & -2.636384 & 3.992493\end{array}$

$\begin{array}{lllll}7 & 1.219796 & -4.024114 & -1.315186\end{array}$

$\begin{array}{lllll}7 & 1.120480 & 1.243033 & -3.432080\end{array}$

$\begin{array}{llll}7 & 1.133176 & 4.218319 & 1.184630\end{array}$

$\begin{array}{lllll}7 & 1.118053 & -1.471836 & 3.850455\end{array}$

$\begin{array}{lllll}8 & 1.457103 & 0.831315 & 3.687332\end{array}$

$\begin{array}{lllll}8 & 1.487116 & -3.769393 & 3.980587\end{array}$

$\begin{array}{lllll}8 & 1.587831 & -4.056684 & 0.988948\end{array}$

$\begin{array}{lllll}8 & 1.545954 & -4.001557 & -3.621927\end{array}$

$\begin{array}{lllll}8 & 1.571397 & -1.036082 & -3.196326\end{array}$

$\begin{array}{lllll}8 & 1.426432 & 4.833336 & -1.045048\end{array}$

$\begin{array}{lllll}8 & 1.360418 & 3.541111 & -3.724208\end{array}$

$\begin{array}{llll}8 & 1.510105 & 3.697523 & 3.422514\end{array}$

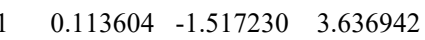

$1 \quad 0.112651 \quad 4.131547 \quad 1.234381$

$\begin{array}{lllll}1 & 0.114330 & 1.277760 & -3.243601\end{array}$

$\begin{array}{llll}0.202936 & -3.872027 & -1.343822\end{array}$

$\begin{array}{llll}4.347208 & 2.701798 & -4.101628\end{array}$

$\begin{array}{llll}4.350396 & -0.148091 & -4.139855\end{array}$

$\begin{array}{lllll}7 & 4.394716 & 4.928775 & -0.173742\end{array}$

$\begin{array}{lllll}7 & 4.455281 & 4.133086 & 2.564976\end{array}$

$\begin{array}{lllll}7 & 4.525194 & -4.293095 & -2.733531\end{array}$

$\begin{array}{llll}4.465595 & -4.642866 & 0.093061\end{array}$

$\begin{array}{lllll}7 & 4.345942 & 0.037464 & 4.293659\end{array}$

$\begin{array}{lllll}7 & 4.452705 & -2.812886 & 4.189764\end{array}$

$\begin{array}{llll}6 & -9.033213 & -3.166614 & 1.820964\end{array}$

$\begin{array}{lllll}6 & -8.435635 & -3.331662 & 0.424425\end{array}$

$\begin{array}{llll}6 & -8.440279 & -1.949648 & 2.535489\end{array}$

$\begin{array}{lllll}1 & -8.714531 & -4.039313 & 2.392921\end{array}$

$\begin{array}{lllll}6 & -8.940108 & -2.622608 & -0.673806\end{array}$

$\begin{array}{lllll}6 & -7.349112 & -4.182861 & 0.181508\end{array}$

$\begin{array}{llll}6 & -9.015660 & -0.675218 & 2.505233\end{array}$

$\begin{array}{lllll}6 & -7.255211 & -2.087014 & 3.268778\end{array}$

$\begin{array}{lllll}6 & -8.393914 & -2.695688 & -1.959273\end{array}$

$\begin{array}{lllll}1 & -9.789493 & -1.968907 & -0.513656\end{array}$

$\begin{array}{lllll}6 & -6.756092 & -4.276857 & -1.072793\end{array}$

$\begin{array}{llll}8 & -6.825429 & -4.983585 & 1.210372\end{array}$

$\begin{array}{llll}6 & -8.466853 & 0.426134 & 3.178477\end{array}$

$\begin{array}{lllll}1 & -9.920184 & -0.528858 & 1.927118\end{array}$

$\begin{array}{lllll}6 & -6.691798 & -1.045800 & 3.988343\end{array}$

$\begin{array}{lllll}8 & -6.677857 & -3.353464 & 3.323292\end{array}$

$\begin{array}{lllll}6 & -8.981566 & -1.930460 & -3.147798\end{array}$

$\begin{array}{lllll}6 & -7.267878 & -3.515365 & -2.113303\end{array}$

$\begin{array}{lllll}1 & -5.901188 & -4.921639 & -1.230997\end{array}$

$\begin{array}{llll}6 & -5.586384 & -4.577838 & 1.626026\end{array}$

$\begin{array}{llll}6 & -9.058803 & 1.830332 & 3.076529\end{array}$

$\begin{array}{llll}6 & -7.314364 & 0.194842 & 3.939872\end{array}$

$\begin{array}{lllll}1 & -5.788622 & -1.191607 & 4.565457\end{array}$ $\begin{array}{lllll}6 & -5.524605 & -3.592863 & 2.650523\end{array}$

$\begin{array}{lllll}1 & -8.635045 & -2.450271 & -4.041957\end{array}$

$\begin{array}{lllll}6 & -8.417315 & -0.513291 & -3.232906\end{array}$

$\begin{array}{lllll}8 & -6.657096 & -3.595288 & -3.368453\end{array}$

$\begin{array}{lllll}6 & -8.477040 & 2.599878 & 1.887093\end{array}$

$\begin{array}{lllll}1 & -8.730743 & 2.364310 & 3.969411\end{array}$

$\begin{array}{llll}8 & -6.781466 & 1.240524 & 4.706322\end{array}$

$\begin{array}{lllll}6 & -9.002285 & 0.575198 & -2.571189\end{array}$

$\begin{array}{lllll}6 & -7.259893 & -0.256724 & -3.978147\end{array}$

$\begin{array}{lllll}6 & -5.477144 & -2.929136 & -3.514691\end{array}$

$\begin{array}{llll}6 & -3.364974 & -4.492972 & 1.440678\end{array}$

$\begin{array}{lllll}6 & -9.021751 & 2.527876 & 0.601491\end{array}$

$\begin{array}{llll}6 & -7.360392 & 3.431568 & 2.045779\end{array}$

$\begin{array}{llll}6 & -5.572424 & 1.705444 & 4.282783\end{array}$

$\begin{array}{lllll}6 & -3.329776 & -3.422723 & 2.324047\end{array}$

$\begin{array}{lllll}6 & -8.460304 & 1.864371 & -2.599947\end{array}$

$\begin{array}{lllll}1 & -9.912452 & 0.409013 & -2.007292\end{array}$

$\begin{array}{lllll}6 & -6.666012 & 0.997527 & -4.020950\end{array}$

$\begin{array}{lllll}8 & -6.697721 & -1.292293 & -4.737920\end{array}$

$\begin{array}{lllll}6 & -5.489496 & -1.744814 & -4.301271\end{array}$

$\begin{array}{lllll}6 & -8.525600 & 3.251171 & -0.492619\end{array}$

$\begin{array}{lllll}1 & -9.860194 & 1.861558 & 0.435605\end{array}$

$\begin{array}{llll}6 & -6.861988 & 4.210786 & 1.013088\end{array}$

$\begin{array}{lllll}8 & -6.753621 & 3.515848 & 3.301645\end{array}$

$\begin{array}{llll}6 & -5.565933 & 2.869715 & 3.466689\end{array}$

$\begin{array}{llll}6 & -9.098002 & 3.068063 & -1.898184\end{array}$

$\begin{array}{lllll}6 & -7.266649 & 2.027275 & -3.314530\end{array}$

$\begin{array}{llll}-5.757988 & 1.163230 & -4.584736\end{array}$

$\begin{array}{lllll}6 & -3.281254 & -2.679376 & -3.219286\end{array}$

$\begin{array}{lllll}6 & -7.455777 & 4.120408 & -0.240974\end{array}$

$\begin{array}{lllll}1 & -6.019038 & 4.869363 & 1.178091\end{array}$

$\begin{array}{lllll}6 & -3.354644 & 1.573197 & 4.091919\end{array}$

$\begin{array}{lllll}1 & -8.793953 & 3.947663 & -2.467607\end{array}$

$\begin{array}{lllll}8 & -6.716304 & 3.306666 & -3.362575\end{array}$

$\begin{array}{lllll}6 & -3.279771 & -1.569530 & -4.052639\end{array}$

$\begin{array}{lllll}8 & -6.944089 & 4.938136 & -1.262550\end{array}$

$\begin{array}{lllll}6 & -3.358194 & 2.658454 & 3.226964\end{array}$

$\begin{array}{lllll}6 & -5.581983 & 3.575023 & -2.670121\end{array}$

$\begin{array}{lllll}6 & -5.687167 & 4.566717 & -1.654611\end{array}$

$\begin{array}{lllll}6 & -3.390111 & 3.463632 & -2.300545\end{array}$

$\begin{array}{lllll}6 & -3.468701 & 4.540389 & -1.428829\end{array}$

$\begin{array}{lllll}6 & -10.632057 & 3.016887 & -1.910071\end{array}$

$\begin{array}{lllll}1 & -11.039711 & 3.927382 & -1.463549\end{array}$

$\begin{array}{lllll}1 & -11.036002 & 2.168126 & -1.351121\end{array}$

$\begin{array}{lllll}6 & -10.516582 & -1.964722 & -3.158083\end{array}$

$\begin{array}{lllll}1 & -10.896539 & -1.459772 & -4.050015\end{array}$

$\begin{array}{lllll}1 & -10.960595 & -1.474848 & -2.287028\end{array}$

$\begin{array}{lllll}6 & -10.568337 & -3.157658 & 1.809041\end{array}$

$\begin{array}{lllll}1 & -10.943725 & -4.077174 & 1.352841\end{array}$

$\begin{array}{llll}1 & -10.987021 & -2.318082 & 1.247107\end{array}$

$\begin{array}{lllll}6 & -10.594245 & 1.832060 & 3.074183\end{array}$

$\begin{array}{lllll}1 & -10.970695 & 1.329456 & 3.968937\end{array}$

$\begin{array}{lllll}1 & -11.020419 & 1.322376 & 2.205583\end{array}$

$\begin{array}{llll}1 & -10.967936 & 2.859125 & 3.067854\end{array}$

$\begin{array}{lrrrr}1 & -10.951388 & -3.093947 & 2.830866\end{array}$

$\begin{array}{lllll}1 & -10.868155 & -2.999581 & -3.166906\end{array}$

$\begin{array}{lllll}1 & -10.997539 & 2.938582 & -2.937276\end{array}$

$\begin{array}{lllll}6 & -1.887706 & -2.880271 & -2.696371\end{array}$ 
$6 \quad-1.866016 \quad-1.060216 \quad-4.155771$

$\begin{array}{lllll}6 & -1.961327 & 3.004591 & -2.361077\end{array}$

$6 \quad-2.072621 \quad 4.900967 \quad-0.994646$

$\begin{array}{lllll}6 & -1.950868 & -4.803714 & 1.022063\end{array}$

$\begin{array}{lllll}6 & -1.915312 & -2.924534 & 2.410647\end{array}$

$\begin{array}{lllll}6 & -1.932175 & 1.113826 & 4.271409\end{array}$

$\begin{array}{lllll}6 & -1.948197 & 2.896483 & 2.759579\end{array}$

$\begin{array}{lllll}7 & -1.254717 & 3.930095 & -1.610304\end{array}$

$\begin{array}{lllll}7 & -1.130979 & -1.893662 & -3.293645\end{array}$

$\begin{array}{lllll}7 & -1.175773 & -3.805483 & 1.647668\end{array}$

$\begin{array}{llll}7 & -1.184870 & 1.963717 & 3.433972\end{array}$

$\begin{array}{lllll}8 & -1.491093 & 0.238233 & 4.974286\end{array}$

$\begin{array}{llll}8 & -1.574801 & 3.715429 & 1.936436\end{array}$

$\begin{array}{lllll}8 & -1.693495 & 5.803588 & -0.290722\end{array}$

$\begin{array}{lllll}8 & -1.536625 & 2.006916 & -2.912541\end{array}$

$8 \quad-1.416714 \quad-0.156974 \quad-4.818295$

$\begin{array}{lllll}8 & -1.529411 & -5.695687 & 0.329161\end{array}$

$\begin{array}{lllll}8 & -1.517137 & -3.715397 & -1.888552\end{array}$

$\begin{array}{lllll}8 & -1.521271 & -1.937742 & 3.008564\end{array}$

$\begin{array}{lllll}1 & -0.170703 & 1.872584 & 3.369902\end{array}$

$\begin{array}{lllll}1 & -0.159249 & -3.761490 & 1.509154\end{array}$

$\begin{array}{lllll}1 & -0.128092 & -1.742613 & -3.138589\end{array}$

$1 \quad-0.235510 \quad 4.015488 \quad-1.580071$

$\begin{array}{lllll}7 & -4.375696 & -3.392193 & -2.942777\end{array}$

$7 \quad-4.389375 \quad-1.069131 \quad-4.599806$

$\begin{array}{llll}-4.504986 & -5.069050 & 1.043099\end{array}$

$\begin{array}{llll}-4.404781 & -2.965726 & 2.971826\end{array}$

$\begin{array}{llll}-4.441906 & 2.970486 & -2.961016\end{array}$

$\begin{array}{llll}-4.630730 & 5.087761 & -1.053669\end{array}$

$\begin{array}{llll}-4.466735 & 1.060881 & 4.623430\end{array}$

$\begin{array}{lllll}7 & -4.461928 & 3.338796 & 2.903836\end{array}$

$\begin{array}{lllll}6 & -0.905014 & -0.154732 & 0.070355\end{array}$

$\begin{array}{llll}-0.324737 & 1.002272 & 0.433318\end{array}$

$\begin{array}{llll}2.849005 & 1.471923 & -0.211253\end{array}$

$\begin{array}{lllll}7 & 3.070353 & 2.192572 & -1.071965\end{array}$

$8 \quad-0.273827 \quad-1.171374 \quad-0.176634$

$\begin{array}{lllll}6 & 2.564020 & 0.635183 & 0.739087\end{array}$

$\begin{array}{llll}2.567747 & 1.073154 & 1.732923\end{array}$

$\begin{array}{llll}0.650541 & 0.848170 & 0.500803\end{array}$

$\begin{array}{lllll}6 & 2.712886 & -0.858763 & 0.520328\end{array}$

$\begin{array}{llll}2.448486 & -1.097679 & -0.514834\end{array}$

$\begin{array}{lllll}1 & 1.940283 & -1.340805 & 1.121847\end{array}$

$\begin{array}{lllll}6 & 4.099815 & -1.431277 & 0.874651\end{array}$

$\begin{array}{llll}3.969080 & -2.463966 & 1.208750\end{array}$

$\begin{array}{llll}4.513204 & -0.891290 & 1.730890\end{array}$

$\begin{array}{lllll}6 & 5.112517 & -1.412875 & -0.271921\end{array}$

$1 \quad 4.718628 \quad-1.998168 \quad-1.110072$

$\begin{array}{lllll}1 & 6.017831 & -1.917999 & 0.074656\end{array}$

$\begin{array}{lllll}6 & -2.380429 & -0.062774 & 0.015935\end{array}$

$6 \quad-3.117768 \quad-1.233219 \quad-0.187706$

$\begin{array}{lllll}6 & -3.047510 & 1.156607 & 0.176977\end{array}$

$6 \quad-4.505496 \quad-1.187054 \quad-0.218799$

$1 \quad-2.587669 \quad-2.167276 \quad-0.313728$

$\begin{array}{lllll}6 & -4.435357 & 1.196874 & 0.139913\end{array}$

$\begin{array}{lllll}1 & -2.473766 & 2.061048 & 0.325703\end{array}$

$6 \quad-5.182952 \quad 0.027350 \quad-0.051104$

$1 \quad-5.073351 \quad-2.099261 \quad-0.373465$

$\begin{array}{llll}1 & -4.950135 & 2.143982 & 0.270262\end{array}$ $\begin{array}{lllll}6 & -6.677757 & 0.075339 & -0.037566\end{array}$

$\begin{array}{lllll}1 & -7.111234 & -0.743738 & -0.609411\end{array}$

$\begin{array}{llll}1 & -7.051815 & -0.002713 & 0.985624\end{array}$

$\begin{array}{lllll}1 & -7.045437 & 1.014596 & -0.445842\end{array}$

$\begin{array}{lllll}6 & 5.513378 & -0.036197 & -0.800523\end{array}$

$1 \quad 6.328383 \quad-0.173751 \quad-1.513386$

$\begin{array}{lllll}1 & 4.695930 & 0.402075 & -1.375703\end{array}$

$\begin{array}{lllll}6 & 5.973981 & 0.927533 & 0.283639\end{array}$

$\begin{array}{llll}1 & 6.853291 & 0.534825 & 0.798214\end{array}$

$1 \quad 6.242932 \quad 1.887081 \quad-0.151032$

$1 \quad 5.207335 \quad 1.106750 \quad 1.040791$

\section{3b-TS@12}

$\begin{array}{llll}6 & -9.100744 & 3.670840 & 0.474752\end{array}$

$\begin{array}{lllll}6 & -8.461916 & 2.884955 & 1.624705\end{array}$

$\begin{array}{lllll}6 & -8.512900 & 3.235511 & -0.867120\end{array}$

$\begin{array}{lllll}1 & -8.806402 & 4.711812 & 0.616188\end{array}$

$\begin{array}{lllll}6 & -8.979292 & 1.678309 & 2.103582\end{array}$

$\begin{array}{lllll}6 & -7.296785 & 3.355703 & 2.244646\end{array}$

$\begin{array}{lllll}6 & -9.003760 & 2.123910 & -1.569177\end{array}$

$\begin{array}{lllll}6 & -7.417051 & 3.904873 & -1.426233\end{array}$

$\begin{array}{lllll}6 & -8.403363 & 0.955508 & 3.157848\end{array}$

$\begin{array}{lllll}1 & -9.863745 & 1.272828 & 1.627017\end{array}$

$\begin{array}{lllll}6 & -6.704608 & 2.702339 & 3.314871\end{array}$

$\begin{array}{lllll}8 & -6.752972 & 4.559755 & 1.792767\end{array}$

$\begin{array}{lllll}6 & -8.415140 & 1.639566 & -2.741269\end{array}$

$\begin{array}{lllll}1 & -9.868327 & 1.605531 & -1.171457\end{array}$

$\begin{array}{lllll}6 & -6.779379 & 3.447450 & -2.572389\end{array}$

$\begin{array}{lllll}8 & -6.925229 & 5.075687 & -0.824955\end{array}$

$\begin{array}{llll}6 & -8.975386 & -0.384363 & 3.618779\end{array}$

$\begin{array}{lllll}6 & -7.268651 & 1.512988 & 3.762444\end{array}$

$\begin{array}{lllll}1 & -5.818141 & 3.103157 & 3.788752\end{array}$

$\begin{array}{lllll}6 & -5.598743 & 4.522356 & 1.076425\end{array}$

$\begin{array}{lllll}6 & -8.966571 & 0.460536 & -3.547664\end{array}$

$\begin{array}{lllll}6 & -7.268957 & 2.308479 & -3.187110\end{array}$

$\begin{array}{lllll}1 & -5.909960 & 3.958353 & -2.963470\end{array}$

$\begin{array}{lllll}6 & -5.679489 & 4.917855 & -0.286910\end{array}$

$\begin{array}{lllll}1 & -8.622387 & -0.528456 & 4.640928\end{array}$

$\begin{array}{lllll}6 & -8.410844 & -1.555695 & 2.808744\end{array}$

$\begin{array}{lllll}8 & -6.682495 & 0.888340 & 4.874859\end{array}$

$\begin{array}{lllll}6 & -8.385404 & -0.879015 & -3.097958\end{array}$

$\begin{array}{lllll}1 & -8.618914 & 0.610578 & -4.570888\end{array}$

$8 \quad-6.628710 \quad 1.823348 \quad-4.327288$

$\begin{array}{lllll}6 & -8.988154 & -2.026346 & 1.624148\end{array}$

$\begin{array}{lllll}6 & -7.261859 & -2.221634 & 3.251846\end{array}$

$\begin{array}{lllll}6 & -5.474687 & 0.302142 & 4.616538\end{array}$

$\begin{array}{lllll}6 & -3.396061 & 4.273298 & 0.846631\end{array}$

$\begin{array}{lllll}6 & -8.943219 & -1.598354 & -2.033146\end{array}$

$\begin{array}{lllll}6 & -7.275267 & -1.451907 & -3.731836\end{array}$

$6 \begin{array}{lllll}6 & -5.472089 & 1.130707 & -4.155087\end{array}$

$\begin{array}{lllll}6 & -3.459009 & 4.773314 & -0.446485\end{array}$

$\begin{array}{lllll}6 & -8.482994 & -3.123681 & 0.913970\end{array}$

$\begin{array}{lllll}1 & -9.858971 & -1.513611 & 1.232593\end{array}$

$\begin{array}{lllll}6 & -6.747061 & -3.339611 & 2.615872\end{array}$

$8 \quad-6.637370 \quad-1.750141 \quad 4.407639$

$\begin{array}{lllll}6 & -5.471843 & -1.069923 & 4.248163\end{array}$

$\begin{array}{lllll}6 & -8.438177 & -2.819427 & -1.577958\end{array}$

$\begin{array}{lllll}1 & -9.805812 & -1.180058 & -1.528397\end{array}$
$6 \begin{array}{llll}6 & -6.728635 & -2.661218 & -3.314456\end{array}$

$8 \begin{array}{llll}8 & -6.687965 & -0.811234 & -4.835281\end{array}$

$\begin{array}{lllll}6 & -5.481549 & -0.233802 & -4.551641\end{array}$

$\begin{array}{lllll}6 & -9.073493 & -3.584534 & -0.416316\end{array}$

$\begin{array}{lllll}6 & -7.374456 & -3.781496 & 1.459747\end{array}$

$\begin{array}{lllll}1 & -5.869831 & -3.843167 & 2.998662\end{array}$

$\begin{array}{lllll}6 & -3.266933 & 0.368788 & 4.301714\end{array}$

$\begin{array}{lllll}6 & -7.303339 & -3.316338 & -2.233727\end{array}$

$\begin{array}{lllll}1 & -5.859793 & -3.073111 & -3.811906\end{array}$

$\begin{array}{lllll}6 & -3.300006 & 0.962802 & -3.686362\end{array}$

$\begin{array}{lllll}1 & -8.777888 & -4.627157 & -0.539376\end{array}$

$\begin{array}{lllll}8 & -6.889215 & -4.936409 & 0.832442\end{array}$

$\begin{array}{lllll}6 & -3.295725 & -0.926016 & 3.798455\end{array}$

$\begin{array}{lllll}8 & -6.769842 & -4.538728 & -1.817853\end{array}$

$\begin{array}{lllll}6 & -3.280313 & -0.323905 & -4.210550\end{array}$

$\begin{array}{lllll}6 & -5.654945 & -4.809454 & 0.265142\end{array}$

$\begin{array}{lllll}6 & -5.594184 & -4.528799 & -1.126864\end{array}$

$\begin{array}{lllll}6 & -3.427360 & -4.736349 & 0.368828\end{array}$

$6 \begin{array}{lllll}6 & -3.371858 & -4.401846 & -0.977580\end{array}$

$\begin{array}{lllll}6 & -10.607371 & -3.532420 & -0.441020\end{array}$

$\begin{array}{lllll}1 & -10.981743 & -3.919626 & -1.392302\end{array}$

$\begin{array}{lllll}1 & -11.003592 & -2.520653 & -0.319189\end{array}$

$\begin{array}{lllll}6 & -10.510446 & -0.390936 & 3.657686\end{array}$

$\begin{array}{lllll}1 & -10.869828 & -1.338793 & 4.066714\end{array}$

$\begin{array}{lllll}1 & -10.964232 & -0.267301 & 2.670516\end{array}$

$\begin{array}{lllll}6 & -10.634326 & 3.615796 & 0.500245\end{array}$

$\begin{array}{lllll}1 & -11.008343 & 3.982799 & 1.459614\end{array}$

$\begin{array}{lllll}1 & -11.028440 & 2.605754 & 0.358354\end{array}$

$\begin{array}{lllll}6 & -10.502241 & 0.449764 & -3.582113\end{array}$

$1 \quad-10.874188 \quad 1.395808 \quad-3.984130$

$\begin{array}{lllll}1 & -10.951150 & 0.313676 & -2.594317\end{array}$

$\begin{array}{lllll}1 & -10.857154 & -0.364023 & -4.219773\end{array}$

$\begin{array}{lllll}1 & -11.043594 & 4.242526 & -0.296305\end{array}$

$\begin{array}{lllll}1 & -10.873446 & 0.422416 & 4.291439\end{array}$

$\begin{array}{lllll}1 & -11.014340 & -4.143953 & 0.368491\end{array}$

$\begin{array}{lllll}6 & -1.843271 & 0.856098 & 4.259047\end{array}$

$\begin{array}{lllll}6 & -1.917347 & -1.276462 & 3.315305\end{array}$

$\begin{array}{lllll}6 & -2.018577 & -4.787314 & 0.903495\end{array}$

$\begin{array}{lllll}6 & -1.934041 & -4.161845 & -1.348564\end{array}$

$\begin{array}{lllll}6 & -1.970523 & 3.909493 & 1.145078\end{array}$

$\begin{array}{lllll}6 & -2.057506 & 4.838833 & -0.996343\end{array}$

$\begin{array}{lllll}6 & -1.915156 & 1.293975 & -3.198851\end{array}$

$\begin{array}{lllll}6 & -1.866734 & -0.830294 & -4.163441\end{array}$

$\begin{array}{lllll}7 & -1.221403 & -4.410712 & -0.188337\end{array}$

$\begin{array}{lllll}7 & -1.133009 & -0.195596 & 3.654665\end{array}$

$\begin{array}{lllll}7 & -1.258111 & 4.299540 & 0.028929\end{array}$

$\begin{array}{lllll}7 & -1.144974 & 0.206418 & -3.548354\end{array}$

$\begin{array}{lllll}8 & -1.546127 & 2.303264 & -2.617783\end{array}$

$8 \quad-1.405056 \quad-1.872246 \quad-4.561788$

$\begin{array}{lllll}8 & -1.498392 & -3.809539 & -2.427090\end{array}$

$\begin{array}{lllll}8 & -1.621064 & -5.094128 & 2.001853\end{array}$

$\begin{array}{lllll}8 & -1.564007 & -2.295324 & 2.744010\end{array}$

$\begin{array}{llll}8 & -1.543089 & 3.356431 & 2.143410\end{array}$

$\begin{array}{lllll}8 & -1.366929 & 1.894728 & 4.649460\end{array}$

$\begin{array}{lllll}8 & -1.660206 & 5.262829 & -2.053841\end{array}$

$\begin{array}{lllll}1 & -0.149363 & 0.078244 & -3.348362\end{array}$

$\begin{array}{lllll}1 & -0.238783 & 4.239323 & -0.064413\end{array}$

$\begin{array}{lllll}1 & -0.145182 & -0.089809 & 3.399799\end{array}$ 
$\begin{array}{llll}1 & -0.212600 & -4.289010 & -0.090658\end{array}$

$\begin{array}{llll}-4.365161 & 1.022174 & 4.691110\end{array}$

$\begin{array}{lllll}7 & -4.392269 & -1.684731 & 3.789100\end{array}$

$\begin{array}{llll}-4.460533 & 4.156950 & 1.645194\end{array}$

$\begin{array}{llll}-4.610126 & 5.083080 & -1.049893\end{array}$

$\begin{array}{lllll}7 & -4.574359 & -4.938180 & 1.020728\end{array}$

$7 \begin{array}{llll}7.451656 & -4.302849 & -1.757899\end{array}$

$\begin{array}{lllll}7 & -4.392232 & 1.729048 & -3.672464\end{array}$

$\begin{array}{lllll}7 & -4.377623 & -0.961090 & -4.623974\end{array}$

$\begin{array}{llll}6 & 8.958387 & -2.155232 & -2.776090\end{array}$

$\begin{array}{lllll}6 & 8.358220 & -2.805419 & -1.531188\end{array}$

$\begin{array}{lllll}6 & 8.363674 & -0.768399 & -3.024478\end{array}$

$\begin{array}{lllll}1 & 8.649289 & -2.773293 & -3.619718\end{array}$

$\begin{array}{lllll}6 & 8.866649 & -2.535126 & -0.254425\end{array}$

$\begin{array}{lllll}6 & 7.286881 & -3.705908 & -1.612033\end{array}$

$\begin{array}{lllll}6 & 8.919921 & 0.411644 & -2.523634\end{array}$

$\begin{array}{lllll}6 & 7.205032 & -0.639172 & -3.799243\end{array}$

$\begin{array}{lllll}6 & 8.355052 & -3.101444 & 0.915633\end{array}$

$\begin{array}{lllll}1 & 9.705538 & -1.854351 & -0.171976\end{array}$

$\begin{array}{lllll}6 & 6.711941 & -4.265473 & -0.472620\end{array}$

$\begin{array}{lllll}8 & 6.801872 & -4.107246 & -2.870747\end{array}$

$\begin{array}{lllll}6 & 8.383527 & 1.676528 & -2.796158\end{array}$

$\begin{array}{llll}9.804484 & 0.347152 & -1.901057\end{array}$

$\begin{array}{lllll}6 & 6.651238 & 0.589505 & -4.132504\end{array}$

$\begin{array}{lllll}8 & 6.658540 & -1.817897 & -4.307738\end{array}$

$\begin{array}{lllll}6 & 8.963578 & -2.828055 & 2.293099\end{array}$

$\begin{array}{llll}7.241180 & -3.940178 & 0.771089\end{array}$

$\begin{array}{llll}5.873395 & -4.945237 & -0.555909\end{array}$

$\begin{array}{lllll}6 & 5.571987 & -3.608787 & -3.198693\end{array}$

$\begin{array}{lllll}6 & 8.953450 & 2.955341 & -2.195659\end{array}$

$\begin{array}{lllll}6 & 7.261904 & 1.734026 & -3.630910\end{array}$

$1 \quad 5.771744 \quad 0.655218 \quad-4.759118$

$\begin{array}{lllll}6 & 5.507194 & -2.316404 & -3.800450\end{array}$

$\begin{array}{llll}8.646905 & -3.651518 & 2.933638\end{array}$

$\begin{array}{lllll}6 & 8.384560 & -1.557440 & 2.910081\end{array}$

$\begin{array}{lllll}8 & 6.681415 & -4.496024 & 1.929412\end{array}$

$\begin{array}{lllll}6 & 8.321322 & 3.233347 & -0.832603\end{array}$

$\begin{array}{lllll}1 & 8.641738 & 3.769549 & -2.849869\end{array}$

$\begin{array}{lllll}8 & 6.776266 & 2.998329 & -3.999621\end{array}$

$\begin{array}{lllll}6 & 8.904932 & -0.285724 & 2.635293\end{array}$

$\begin{array}{lllll}6 & 7.268467 & -1.622487 & 3.752762\end{array}$

$\begin{array}{lllll}6 & 5.508841 & -3.980669 & 2.388032\end{array}$

$\begin{array}{lllll}6 & 3.340028 & -3.655394 & -3.117719\end{array}$

$\begin{array}{lllll}6 & 8.807993 & 2.648272 & 0.338754\end{array}$

$\begin{array}{lllll}6 & 7.227665 & 4.099668 & -0.701082\end{array}$

$\begin{array}{lllll}6 & 5.557151 & 3.337381 & -3.496715\end{array}$

$\begin{array}{lllll}6 & 3.296032 & -2.329682 & -3.523908\end{array}$

$\begin{array}{lllll}6 & 8.334410 & 0.890792 & 3.131041\end{array}$

$\begin{array}{lllll}1 & 9.781016 & -0.211648 & 2.001733\end{array}$

$\begin{array}{lllll}6 & 6.655400 & -0.484371 & 4.263472\end{array}$

$\begin{array}{llll}6.758502 & -2.883546 & 4.103115\end{array}$

$\begin{array}{lllll}6 & 5.540883 & -3.193879 & 3.577447\end{array}$

$\begin{array}{llll}6 & 8.295151 & 2.924375 & 1.611181\end{array}$

$\begin{array}{lllll}1 & 9.636069 & 1.953841 & 0.259908\end{array}$

$\begin{array}{lllll}6 & 6.691603 & 4.426799 & 0.538736\end{array}$

$\begin{array}{lllll}8 & 6.684708 & 4.671018 & -1.861320\end{array}$

$\begin{array}{lllll}6 & 5.512887 & 4.155292 & -2.328559\end{array}$

$\begin{array}{llll}6 & 8.897101 & 2.287292 & 2.863715\end{array}$ $\begin{array}{llll}6 & 7.185673 & 0.750360 & 3.919274\end{array}$

$\begin{array}{lllll}1 & 5.775494 & -0.560089 & 4.888027\end{array}$

$\begin{array}{lllll}6 & 3.287254 & -3.737388 & 2.327982\end{array}$

$\begin{array}{llll}6 & 7.235805 & 3.840255 & 1.679356\end{array}$

$\begin{array}{lllll}1 & 5.852506 & 5.106263 & 0.617217\end{array}$

$\begin{array}{lllll}6 & 3.329837 & 3.159938 & -3.448782\end{array}$

$\begin{array}{lllll}1 & 8.573615 & 2.907195 & 3.700643\end{array}$

$\begin{array}{rrrrr}8 & 6.596677 & 1.921698 & 4.397761\end{array}$

$\begin{array}{llll}6 & 3.308849 & -3.039118 & 3.524893\end{array}$

$\begin{array}{lllll}8 & 6.702538 & 4.200503 & 2.930520\end{array}$

$\begin{array}{lllll}6 & 3.295310 & 3.885327 & -2.266713\end{array}$

$\begin{array}{lllll}6 & 5.451525 & 2.355896 & 3.812584\end{array}$

$\begin{array}{lllll}6 & 5.483886 & 3.632255 & 3.181486\end{array}$

$\begin{array}{llll}6 & 3.270349 & 2.226216 & 3.376441\end{array}$

$\begin{array}{llll}6 & 3.267480 & 3.536930 & 2.922681\end{array}$

$\begin{array}{llll}6 & 10.433181 & 2.306816 & 2.844480\end{array}$

$\begin{array}{lllll}1 & 10.792028 & 3.331619 & 2.720164\end{array}$

$\begin{array}{lllll}1 & 10.859020 & 1.709118 & 2.033869\end{array}$

$\begin{array}{lllll}6 & 10.499064 & -2.829984 & 2.263743\end{array}$

$\begin{array}{lllll}1 & 10.891254 & -2.695050 & 3.275053\end{array}$

$\begin{array}{lllll}1 & 10.916846 & -2.035138 & 1.639811\end{array}$

$\begin{array}{lllll}6 & 10.493924 & -2.144601 & -2.753532\end{array}$

$\begin{array}{lllll}1 & 10.872141 & -3.163683 & -2.639926\end{array}$

$\begin{array}{lllll}1 & 10.907690 & -1.547849 & -1.936035\end{array}$

$\begin{array}{llll}6 & 10.488203 & 2.966296 & -2.144189\end{array}$

$\begin{array}{lllll}1 & 10.896333 & 2.817149 & -3.147291\end{array}$

$\begin{array}{lllll}1 & 10.902142 & 2.185833 & -1.499954\end{array}$

$\begin{array}{lllll}1 & 10.842885 & 3.927522 & -1.763652\end{array}$

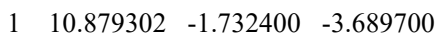

$\begin{array}{llll}1 & 10.865788 & -3.782176 & 1.871965\end{array}$

$\begin{array}{lllll}1 & 10.824620 & 1.912184 & 3.785746\end{array}$

$\begin{array}{lllll}6 & 1.879755 & -3.733181 & 1.796082\end{array}$

$\begin{array}{lllll}6 & 1.888099 & -2.657571 & 3.870000\end{array}$

$\begin{array}{lllll}6 & 1.906033 & 1.642547 & 3.153290\end{array}$

$\begin{array}{llll}6 & 1.855555 & 3.892412 & 2.535651\end{array}$

$\begin{array}{lllll}6 & 1.934123 & -4.084062 & -2.773059\end{array}$

$\begin{array}{lllll}6 & 1.890470 & -1.826338 & -3.358293\end{array}$

$\begin{array}{lllll}6 & 1.925159 & 2.720619 & -3.779973\end{array}$

$\begin{array}{lllll}6 & 1.888881 & 3.854607 & -1.738885\end{array}$

$\begin{array}{lllll}7 & 1.138282 & 2.686138 & 2.686608\end{array}$

$\begin{array}{lllll}7 & 1.128210 & -3.102963 & 2.766414\end{array}$

$\begin{array}{lllll}7 & 1.155683 & -2.924905 & -2.967540\end{array}$

$\begin{array}{lllll}7 & 1.152301 & 3.184765 & -2.697541\end{array}$

$\begin{array}{lllll}8 & 1.515476 & 2.102217 & -4.730510\end{array}$

$\begin{array}{lllll}8 & 1.499404 & 4.264875 & -0.659281\end{array}$

$\begin{array}{lllll}8 & 1.400107 & 4.947175 & 2.171336\end{array}$

$\begin{array}{llll}8 & 1.586356 & 0.472039 & 3.299604\end{array}$

$\begin{array}{lllll}8 & 1.457995 & -2.098640 & 4.846807\end{array}$

$\begin{array}{lllll}8 & 1.538415 & -5.150803 & -2.374053\end{array}$

$\begin{array}{lllll}8 & 1.514123 & -4.128112 & 0.701410\end{array}$

$\begin{array}{lllll}8 & 1.528361 & -0.665675 & -3.466583\end{array}$

$\begin{array}{lllll}1 & 0.148390 & 2.967863 & -2.615375\end{array}$

$\begin{array}{lllll}1 & 0.147757 & -2.927747 & -2.769797\end{array}$

$1 \quad 0.115806 \quad-2.913150 \quad 2.689065$

$\begin{array}{llll}1 & 0.131071 & 2.657181 & 2.489514\end{array}$

$\begin{array}{lllll}7 & 4.381330 & -4.229724 & 1.740540\end{array}$

$\begin{array}{lllll}7 & 4.443337 & -2.739337 & 4.165598\end{array}$

$\begin{array}{lllll}7 & 4.488960 & -4.308777 & -2.905420\end{array}$ $\begin{array}{lllll}7 & 4.375131 & -1.636897 & -3.899129\end{array}$

$\begin{array}{lllll}7 & 4.355658 & 1.613739 & 3.853479\end{array}$

$\begin{array}{lllll}7 & 4.386384 & 4.252260 & 2.775916\end{array}$

$\begin{array}{lllll}7 & 4.467307 & 2.860537 & -4.080641\end{array}$

$\begin{array}{lllll}7 & 4.379997 & 4.412945 & -1.692506\end{array}$

$6 \begin{array}{llll}6 & -0.845808 & -0.501985 & -0.103333\end{array}$

$\begin{array}{lllll}8 & -0.386359 & -1.383098 & -0.892367\end{array}$

$\begin{array}{lllll}8 & -0.143562 & 0.218440 & 0.669704\end{array}$

$\begin{array}{lllll}7 & 3.239414 & -1.716803 & -0.159392\end{array}$

$\begin{array}{lllll}7 & 3.697071 & -2.666088 & -0.505102\end{array}$

$\begin{array}{lllll}6 & 2.019397 & -0.603325 & 0.117409\end{array}$

$\begin{array}{lllll}1 & 1.201902 & -1.187328 & -0.383437\end{array}$

$\begin{array}{lllll}1 & 2.013048 & -0.617074 & 1.203287\end{array}$

$\begin{array}{lllll}6 & -2.334397 & -0.299702 & -0.082263\end{array}$

$\begin{array}{lllll}6 & -3.164463 & -1.030168 & -0.935397\end{array}$

$\begin{array}{lllll}6 & -2.904947 & 0.618884 & 0.805200\end{array}$

$\begin{array}{lllll}6 & -4.545229 & -0.854397 & -0.890603\end{array}$

$\begin{array}{lllll}1 & -2.716406 & -1.725349 & -1.632810\end{array}$

$\begin{array}{lllll}6 & -4.283604 & 0.783082 & 0.856335\end{array}$

$\begin{array}{llll}1 & -2.248577 & 1.193349 & 1.443766\end{array}$

$\begin{array}{lllll}6 & -5.123613 & 0.046970 & 0.008525\end{array}$

$\begin{array}{lllll}1 & -5.182648 & -1.422770 & -1.559050\end{array}$

$\begin{array}{lllll}1 & -4.716371 & 1.492309 & 1.555497\end{array}$

$\begin{array}{lllll}6 & 2.460857 & 0.673920 & -0.553578\end{array}$

$\begin{array}{lllll}1 & 2.656207 & 0.481833 & -1.613263\end{array}$

$\begin{array}{lllll}1 & 1.540215 & 1.260550 & -0.508954\end{array}$

$\begin{array}{lllll}6 & -6.608577 & 0.237902 & 0.071348\end{array}$

$\begin{array}{lllll}1 & -6.998410 & -0.043988 & 1.051192\end{array}$

$\begin{array}{lllll}1 & -6.873329 & 1.284459 & -0.087814\end{array}$

$\begin{array}{lllll}1 & -7.125588 & -0.359381 & -0.678113\end{array}$

$\begin{array}{lllll}6 & 3.613834 & 1.439640 & 0.120731\end{array}$

$\begin{array}{lllll}1 & 3.727635 & 1.091994 & 1.150373\end{array}$

$\begin{array}{lllll}1 & 3.336718 & 2.494095 & 0.196778\end{array}$

$\begin{array}{lllll}6 & 4.968360 & 1.363783 & -0.590389\end{array}$

$\begin{array}{lllll}1 & 5.624999 & 2.102418 & -0.124451\end{array}$

$\begin{array}{lllll}1 & 4.838278 & 1.668395 & -1.631486\end{array}$

$\begin{array}{lllll}6 & 5.684422 & 0.014317 & -0.577898\end{array}$

$\begin{array}{lllll}1 & 6.675809 & 0.153517 & -1.016813\end{array}$

$\begin{array}{lllll}1 & 5.175961 & -0.687730 & -1.244308\end{array}$

$\begin{array}{lllll}6 & 5.854940 & -0.565709 & 0.821342\end{array}$

$\begin{array}{llll}1 & 4.908329 & -0.701287 & 1.354234\end{array}$

$\begin{array}{lllll}1 & 6.355666 & -1.530800 & 0.782539\end{array}$

$\begin{array}{llll}1 & 6.462703 & 0.102710 & 1.431580\end{array}$

\section{$7^{-}: \mathbf{8 b} \mathbf{b}^{+} @ \mathbf{1}_{2}$}

$\begin{array}{llll}6 & -9.157753 & 2.637563 & 2.208370\end{array}$

$\begin{array}{lllll}6 & -8.537761 & 1.388503 & 2.834612\end{array}$

$\begin{array}{lllll}6 & -8.623653 & 2.955380 & 0.810290\end{array}$

$\begin{array}{lllll}1 & -8.845605 & 3.470685 & 2.839569\end{array}$

$\begin{array}{lllll}6 & -9.040398 & 0.102523 & 2.616898\end{array}$

$\begin{array}{lllll}6 & -7.429595 & 1.490381 & 3.684776\end{array}$

$\begin{array}{lllll}6 & -9.160249 & 2.393470 & -0.352537\end{array}$

$\begin{array}{lllll}6 & -7.577776 & 3.870842 & 0.634176\end{array}$

$6 \quad-8.497296 \quad-1.050181 \quad 3.199666$

$\begin{array}{lllll}1 & -9.882564 & -0.012989 & 1.944828\end{array}$

$\begin{array}{lllll}6 & -6.880459 & 0.389498 & 4.325788\end{array}$

$\begin{array}{llll}8 & -6.848030 & 2.750501 & 3.890647\end{array}$

$\begin{array}{lllll}6 & -8.711457 & 2.714103 & -1.640757\end{array}$ 
$\begin{array}{llll}1 & -9.951477 & 1.659184 & -0.252766\end{array}$

$\begin{array}{lllll}6 & -7.133715 & 4.265773 & -0.618060\end{array}$

$\begin{array}{llll}8 & -6.942001 & 4.400911 & 1.764914\end{array}$

$\begin{array}{llll}6 & -9.046559 & -2.438671 & 2.874022\end{array}$

$\begin{array}{lllll}6 & -7.419132 & -0.868049 & 4.077323\end{array}$

$\begin{array}{lllll}1 & -6.030528 & 0.502868 & 4.985947\end{array}$

$\begin{array}{llll}6 & -5.698551 & 2.945055 & 3.182783\end{array}$

$\begin{array}{lllll}6 & -9.253973 & 2.006384 & -2.878919\end{array}$

$\begin{array}{lllll}6 & -7.708380 & 3.684301 & -1.740637\end{array}$

$\begin{array}{lllll}1 & -6.334028 & 4.987647 & -0.717376\end{array}$

$\begin{array}{lllll}6 & -5.750531 & 3.801276 & 2.050639\end{array}$

$\begin{array}{lllll}1 & -8.707093 & -3.094739 & 3.677047\end{array}$

$\begin{array}{lllll}6 & -8.438479 & -2.998249 & 1.585200\end{array}$

$\begin{array}{lllll}8 & -6.840393 & -1.965052 & 4.741755\end{array}$

$\begin{array}{lllll}6 & -8.545889 & 0.665241 & -3.067609\end{array}$

$\begin{array}{lllll}1 & -8.976254 & 2.623846 & -3.734181\end{array}$

$\begin{array}{lllll}8 & -7.244073 & 4.090848 & -3.003065\end{array}$

$\begin{array}{llll}6 & -9.002321 & -2.825738 & 0.317511\end{array}$

$\begin{array}{lllll}6 & -7.247670 & -3.731790 & 1.644972\end{array}$

$\begin{array}{lllll}6 & -5.598643 & -2.283928 & 4.261768\end{array}$

$\begin{array}{lllll}6 & -3.550151 & 2.529920 & 2.745597\end{array}$

$\begin{array}{lllll}6 & -9.056796 & -0.538394 & -2.574800\end{array}$

$\begin{array}{lllll}6 & -7.330985 & 0.603360 & -3.762461\end{array}$

$\begin{array}{lllll}6 & -5.959148 & 3.700336 & -3.253379\end{array}$

$\begin{array}{lllll}6 & -3.605050 & 3.347562 & 1.625070\end{array}$

$\begin{array}{llll}6 & -8.439669 & -3.366751 & -0.847736\end{array}$

$\begin{array}{lllll}1 & -9.906082 & -2.234853 & 0.226409\end{array}$

$\begin{array}{lllll}6 & -6.675328 & -4.332360 & 0.536404\end{array}$

$\begin{array}{lllll}8 & -6.649490 & -3.891965 & 2.890640\end{array}$

$\begin{array}{llll}6 & -5.524440 & -3.182368 & 3.162644\end{array}$

$\begin{array}{lllll}6 & -8.426984 & -1.771591 & -2.772677\end{array}$

$\begin{array}{lllll}6 & -9.993416 & -0.517269 & -2.031497\end{array}$

$\begin{array}{lllll}6 & -6.662798 & -0.592196 & -3.994285\end{array}$

$\begin{array}{lllll}8 & -6.853197 & 1.787596 & -4.320343\end{array}$

$\begin{array}{lllll}6 & -5.754220 & 2.413366 & -3.830653\end{array}$

$\begin{array}{lllll}6 & -9.005562 & -3.082186 & -2.237158\end{array}$

$\begin{array}{lllll}6 & -7.287627 & -4.144998 & -0.694581\end{array}$

$\begin{array}{lllll}1 & -5.770774 & -4.918480 & 0.626333\end{array}$

$\begin{array}{lllll}6 & -3.388249 & -2.004138 & 4.142792\end{array}$

$\begin{array}{lllll}6 & -7.225325 & -1.759784 & -3.492459\end{array}$

$\begin{array}{lllll}1 & -5.738572 & -0.614746 & -4.556642\end{array}$

$\begin{array}{lllll}6 & -3.748759 & 3.980096 & -3.132774\end{array}$

$\begin{array}{lllll}1 & -8.634864 & -3.872639 & -2.890853\end{array}$

$\begin{array}{lllll}8 & -6.732444 & -4.782285 & -1.816049\end{array}$

$\begin{array}{lllll}6 & -3.351912 & -2.732000 & 2.960214\end{array}$

$\begin{array}{lllll}8 & -6.630317 & -2.990729 & -3.792727\end{array}$

$\begin{array}{lllll}6 & -3.555037 & 2.685365 & -3.592635\end{array}$

$\begin{array}{lllll}6 & -5.506515 & -4.334516 & -2.193456\end{array}$

$\begin{array}{lllll}6 & -5.456425 & -3.338660 & -3.214635\end{array}$

$\begin{array}{lllll}6 & -3.279000 & -4.301351 & -2.066242\end{array}$

$\begin{array}{lllll}6 & -3.234925 & -3.318874 & -3.044982\end{array}$

$\begin{array}{lllll}6 & -10.538526 & -3.127730 & -2.281179\end{array}$

$\begin{array}{lllll}1 & -10.891641 & -2.951070 & -3.300529\end{array}$

$\begin{array}{lllll}1 & -11.009490 & -2.381911 & -1.635162\end{array}$

$\begin{array}{lllll}6 & -10.581454 & -2.477650 & 2.863923\end{array}$

$\begin{array}{lllll}1 & -10.930003 & -3.498863 & 2.688710\end{array}$

$\begin{array}{lllll}1 & -11.020204 & -1.842545 & 2.089412\end{array}$

$\begin{array}{lllll}6 & -10.694009 & 2.591281 & 2.234605\end{array}$ $\begin{array}{llll}1 & -11.046618 & 2.441849 & 3.258378\end{array}$

$\begin{array}{lllll}1 & -11.105617 & 1.783496 & 1.623145\end{array}$

$\begin{array}{lllll}6 & -10.783974 & 1.887191 & -2.871301\end{array}$

$\begin{array}{lllll}1 & -11.232931 & 2.882368 & -2.820786\end{array}$

$\begin{array}{lllll}1 & -11.165358 & 1.313984 & -2.021760\end{array}$

$\begin{array}{lllll}1 & -11.129707 & 1.395757 & -3.784643\end{array}$

$\begin{array}{lllll}1 & -11.104191 & 3.531649 & 1.857523\end{array}$

$\begin{array}{lllll}1 & -10.970408 & -2.137144 & 3.826879\end{array}$

$\begin{array}{lllll}1 & -10.890466 & -4.110250 & -1.956517\end{array}$

$\begin{array}{lllll}6 & -1.977226 & -1.615967 & 4.494610\end{array}$

$\begin{array}{lllll}6 & -1.940806 & -2.710936 & 2.433146\end{array}$

$\begin{array}{lllll}6 & -1.868147 & -4.606634 & -1.632106\end{array}$

$\begin{array}{lllll}6 & -1.805024 & -2.944140 & -3.278984\end{array}$

$\begin{array}{llll}6 & -2.157537 & 1.970174 & 2.841002\end{array}$

$\begin{array}{llll}6 & -2.249141 & 3.303379 & 0.964136\end{array}$

$\begin{array}{llll}6 & -2.399832 & 4.614182 & -2.912036\end{array}$

$\begin{array}{lllll}6 & -2.079924 & 2.398711 & -3.605495\end{array}$

$\begin{array}{lllll}7 & -1.071553 & -3.737679 & -2.417242\end{array}$

$\begin{array}{lllll}7 & -1.208904 & -2.066486 & 3.400457\end{array}$

$\begin{array}{lllll}7 & -1.465817 & 2.495399 & 1.762007\end{array}$

$\begin{array}{lllll}7 & -1.480254 & 3.597647 & -3.242586\end{array}$

$\begin{array}{lllll}8 & -2.122088 & 5.732041 & -2.556888\end{array}$

$\begin{array}{lllll}8 & -1.523996 & 1.340846 & -3.831603\end{array}$

$\begin{array}{lllll}8 & -1.374693 & -2.087687 & -4.036404\end{array}$

$\begin{array}{lllll}8 & -1.456040 & -5.389902 & -0.816331\end{array}$

$\begin{array}{lllll}8 & -1.545444 & -3.155818 & 1.362828\end{array}$

$\begin{array}{llll}8 & -1.727613 & 1.206352 & 3.685393\end{array}$

$\begin{array}{lllll}8 & -1.553324 & -1.074989 & 5.484221\end{array}$

$\begin{array}{lllll}8 & -1.905689 & 3.837194 & -0.082542\end{array}$

$\begin{array}{lllll}1 & -0.466165 & 3.741488 & -3.156445\end{array}$

$\begin{array}{lllll}1 & -0.408687 & 2.261354 & 1.542864\end{array}$

$\begin{array}{llll}1 & -0.191623 & -1.918749 & 3.391672\end{array}$

$\begin{array}{lllll}1 & -0.060557 & -3.647296 & -2.281659\end{array}$

$\begin{array}{lllll}7 & -4.522806 & -1.728432 & 4.794905\end{array}$

$\begin{array}{llll}7 & -4.417867 & -3.362979 & 2.457988\end{array}$

$\begin{array}{lllll}7 & -4.596203 & 2.299508 & 3.538576\end{array}$

$\begin{array}{llll}7 & -4.709246 & 3.995550 & 1.250493\end{array}$

$\begin{array}{lllll}7 & -4.421632 & -4.827534 & -1.615483\end{array}$

$\begin{array}{lllll}7 & -4.318749 & -2.812408 & -3.641054\end{array}$

$\begin{array}{lllll}7 & -4.961055 & 4.504958 & -2.927249\end{array}$

$\begin{array}{lllll}7 & -4.552164 & 1.875187 & -3.964330\end{array}$

$\begin{array}{lllll}6 & 9.213894 & -0.458868 & -3.574100\end{array}$

$\begin{array}{lllll}6 & 8.657048 & -1.696006 & -2.862163\end{array}$

$\begin{array}{lllll}6 & 8.522799 & 0.822908 & -3.081938\end{array}$

$\begin{array}{lllll}1 & 8.940273 & -0.564642 & -4.625135\end{array}$

$\begin{array}{lllll}6 & 9.121451 & -2.109184 & -1.602762\end{array}$

$\begin{array}{lllll}6 & 7.602113 & -2.434805 & -3.413430\end{array}$

$\begin{array}{lllll}6 & 9.034914 & 1.627157 & -2.058723\end{array}$

$\begin{array}{lllll}6 & 7.317283 & 1.246624 & -3.656667\end{array}$

$\begin{array}{lllll}6 & 8.520368 & -3.131502 & -0.859453\end{array}$

$\begin{array}{lllll}1 & 9.962275 & -1.580769 & -1.168136\end{array}$

$\begin{array}{lllll}6 & 6.948071 & -3.433736 & -2.700470\end{array}$

$\begin{array}{lllll}8 & 7.140401 & -2.156959 & -4.713074\end{array}$

$\begin{array}{lllll}6 & 8.401347 & 2.794767 & -1.613537\end{array}$

$\begin{array}{lllll}1 & 9.962157 & 1.332895 & -1.582657\end{array}$

$\begin{array}{llll}6 & 6.658607 & 2.408348 & -3.274645\end{array}$

$\begin{array}{lllll}8 & 6.808589 & 0.489430 & -4.711064\end{array}$

$\begin{array}{lllll}6 & 9.033873 & -3.609167 & 0.502107\end{array}$ $\begin{array}{llll}6 & 7.383770 & -3.726954 & -1.421227\end{array}$

$1 \quad 6.101698 \quad-3.954155 \quad-3.128386$

$\begin{array}{lllll}6 & 5.863411 & -1.668559 & -4.694314\end{array}$

$\begin{array}{lllll}6 & 8.941534 & 3.630776 & -0.458818\end{array}$

$\begin{array}{lllll}6 & 7.208745 & 3.165807 & -2.248614\end{array}$

$\begin{array}{lllll}1 & 5.741392 & 2.712846 & -3.761299\end{array}$

$\begin{array}{lllll}6 & 5.700636 & -0.266410 & -4.519755\end{array}$

$\begin{array}{lllll}1 & 8.693079 & -4.640504 & 0.604674\end{array}$

$\begin{array}{lllll}6 & 8.406887 & -2.840332 & 1.664718\end{array}$

$\begin{array}{lllll}8 & 6.705658 & -4.694770 & -0.677658\end{array}$

$\begin{array}{lllll}6 & 8.332050 & 3.161688 & 0.861299\end{array}$

$\begin{array}{lllll}1 & 8.582329 & 4.647901 & -0.618714\end{array}$

$\begin{array}{lllll}8 & 6.621991 & 4.384785 & -1.900938\end{array}$

$\begin{array}{lllll}6 & 8.915518 & -1.603878 & 2.084353\end{array}$

$\begin{array}{lllll}6 & 7.293218 & -3.331229 & 2.357748\end{array}$

$\begin{array}{lllll}6 & 5.544278 & -4.363621 & -0.054574\end{array}$

$\begin{array}{lllll}6 & 3.657075 & -1.926073 & -4.537458\end{array}$

$\begin{array}{lllll}6 & 8.856197 & 2.079322 & 1.575959\end{array}$

$\begin{array}{lllll}6 & 7.215438 & 3.802821 & 1.410241\end{array}$

$\begin{array}{lllll}6 & 5.427745 & 4.434629 & -1.247851\end{array}$

$\begin{array}{lllll}6 & 3.518555 & -0.582245 & -4.215342\end{array}$

$\begin{array}{lllll}6 & 8.355236 & -0.853130 & 3.120661\end{array}$

$\begin{array}{lllll}1 & 9.780720 & -1.204939 & 1.568376\end{array}$

$\begin{array}{lllll}6 & 6.695258 & -2.622220 & 3.396440\end{array}$

$\begin{array}{lllll}8 & 6.743773 & -4.579845 & 2.007289\end{array}$

$\begin{array}{lllll}6 & 5.542518 & -4.467357 & 1.366576\end{array}$

$\begin{array}{lllll}6 & 8.340606 & 1.652735 & 2.803594\end{array}$

$\begin{array}{llll}1 & 9.706116 & 1.550692 & 1.160038\end{array}$

$\begin{array}{llll}6 & 6.666955 & 3.424724 & 2.628561\end{array}$

$\begin{array}{lllll}8 & 6.668739 & 4.902865 & 0.737878\end{array}$

$\begin{array}{lllll}6 & 5.454682 & 4.726196 & 0.146678\end{array}$

$\begin{array}{lllll}6 & 8.939630 & 0.481664 & 3.579006\end{array}$

$\begin{array}{lllll}6 & 7.220510 & -1.386181 & 3.747887\end{array}$

$\begin{array}{lllll}1 & 5.826088 & -3.017383 & 3.906723\end{array}$

$\begin{array}{lllll}6 & 3.365875 & -3.872248 & 0.002606\end{array}$

$\begin{array}{lllll}6 & 7.244520 & 2.361399 & 3.310517\end{array}$

$\begin{array}{lllll}1 & 5.811268 & 3.946882 & 3.037871\end{array}$

$\begin{array}{lllll}6 & 3.194453 & 4.472670 & -1.187315\end{array}$

$\begin{array}{lllll}1 & 8.622486 & 0.610351 & 4.614421\end{array}$

$8 \quad 6.655295 \quad-0.674206 \quad 4.806793$

$\begin{array}{lllll}6 & 3.336040 & -4.138303 & 1.365683\end{array}$

$\begin{array}{llll}8 & 6.736241 & 2.008577 & 4.566851\end{array}$

$\begin{array}{lllll}6 & 3.221158 & 4.746447 & 0.174524\end{array}$

$\begin{array}{lllll}6 & 5.475512 & -0.018981 & 4.609960\end{array}$

$\begin{array}{lllll}6 & 5.514769 & 1.401531 & 4.547554\end{array}$

$\begin{array}{lllll}6 & 3.255545 & 0.063870 & 4.426198\end{array}$

$\begin{array}{lllll}6 & 3.287695 & 1.450838 & 4.416319\end{array}$

$\begin{array}{lllll}6 & 10.475208 & 0.489866 & 3.563130\end{array}$

$\begin{array}{lllll}1 & 10.846786 & 1.433290 & 3.971307\end{array}$

$\begin{array}{lllll}1 & 10.893781 & 0.379847 & 2.558964\end{array}$

$\begin{array}{lllll}6 & 10.568258 & -3.622243 & 0.571139\end{array}$

$\begin{array}{lllll}1 & 10.895898 & -4.038505 & 1.527178\end{array}$

$\begin{array}{lllll}1 & 11.007504 & -2.625390 & 0.478480\end{array}$

$6 \begin{array}{lllll}6 & 10.744082 & -0.372223 & -3.498101\end{array}$

$\begin{array}{lllll}1 & 11.190414 & -1.270329 & -3.932530\end{array}$

$\begin{array}{lllll}1 & 11.115914 & -0.284445 & -2.473412\end{array}$

$\begin{array}{lllll}6 & 10.474669 & 3.684977 & -0.411170\end{array}$

$\begin{array}{llll}1 & 10.868252 & 4.060343 & -1.359674\end{array}$ 
$\begin{array}{lllll}1 & 10.933396 & 2.709967 & -0.224782\end{array}$

$\begin{array}{lllll}1 & 10.797866 & 4.355284 & 0.389271\end{array}$

$1 \quad 11.101454 \quad 0.498462 \quad-4.054034$

$\begin{array}{lllll}1 & 10.975070 & -4.237775 & -0.235414\end{array}$

$\begin{array}{lllll}1 & 10.862180 & -0.330462 & 4.173380\end{array}$

$\begin{array}{lllll}6 & 1.982902 & -3.436675 & -0.414422\end{array}$

$\begin{array}{lllll}6 & 1.922507 & -3.988609 & 1.852464\end{array}$

$\begin{array}{lllll}6 & 1.832244 & -0.376449 & 4.249134\end{array}$

$\begin{array}{lllll}6 & 1.870693 & 1.958364 & 4.304697\end{array}$

$\begin{array}{lllll}6 & 2.294880 & -2.561329 & -4.536566\end{array}$

$\begin{array}{lllll}6 & 2.075937 & -0.322259 & -3.887464\end{array}$

$\begin{array}{lllll}6 & 1.756284 & 4.385883 & -1.624859\end{array}$

$\begin{array}{lllll}6 & 1.801912 & 4.873163 & 0.667441\end{array}$

$\begin{array}{lllll}7 & 1.090083 & 0.786635 & 4.234421\end{array}$

$\begin{array}{lllll}7 & 1.208430 & -3.552573 & 0.715905\end{array}$

$\begin{array}{lllll}7 & 1.426971 & -1.511304 & -4.199539\end{array}$

$7 \quad 1.018441 \quad 4.676596 \quad-0.493660$

$\begin{array}{lllll}8 & 1.325861 & 4.089155 & -2.728561\end{array}$

$\begin{array}{lllll}8 & 1.383312 & 5.116107 & 1.768318\end{array}$

$\begin{array}{lllll}8 & 1.459278 & 3.090361 & 4.286566\end{array}$

$\begin{array}{lllll}8 & 1.436924 & -1.519173 & 4.095034\end{array}$

$\begin{array}{lllll}8 & 1.452119 & -4.191419 & 2.942772\end{array}$

$\begin{array}{lllll}8 & 1.970400 & -3.701226 & -4.769511\end{array}$

$8 \begin{array}{lllll}8 & 1.609566 & -3.051681 & -1.518349\end{array}$

$\begin{array}{lllll}8 & 1.574724 & 0.676164 & -3.408999\end{array}$

$\begin{array}{lllll}1 & 0.004597 & 4.571251 & -0.441067\end{array}$

$1 \quad 0.414905 \quad-1.655170 \quad-4.153763$

$\begin{array}{lllll}1 & 0.209503 & -3.322001 & 0.794335\end{array}$

$\begin{array}{lllll}1 & 0.082085 & 0.820030 & 4.051823\end{array}$

$7 \quad 4.467400 \quad-4.011513 \quad-0.744065$

$\begin{array}{lllll}7 & 4.430821 & -4.405119 & 2.081339\end{array}$

$\begin{array}{lllll}7 & 4.842190 & -2.505942 & -4.748931\end{array}$

$\begin{array}{lllll}7 & 4.535478 & 0.284915 & -4.220080\end{array}$

$\begin{array}{llll}7 & 4.344496 & -0.702119 & 4.529490\end{array}$

$\begin{array}{lllll}7 & 4.424096 & 2.148879 & 4.464428\end{array}$

$\begin{array}{lllll}7 & 4.296261 & 4.304060 & -1.926323\end{array}$

$\begin{array}{lllll}7 & 4.354137 & 4.879825 & 0.867651\end{array}$

$\begin{array}{lllll}6 & 1.212403 & 0.715464 & 1.125852\end{array}$

$\begin{array}{lllll}8 & 0.413255 & -0.207909 & 1.394446\end{array}$

$\begin{array}{lllll}7 & -0.160248 & 0.928238 & -1.429474\end{array}$

$\begin{array}{lllll}7 & 0.049785 & 1.995075 & -1.238122\end{array}$

$\begin{array}{lllll}8 & 0.949969 & 1.971453 & 1.151263\end{array}$

$\begin{array}{llll}6 & -0.541126 & -0.455221 & -1.568358\end{array}$

$\begin{array}{lllll}1 & 0.348544 & -1.029972 & -1.298440\end{array}$

$\begin{array}{lllll}6 & 2.604751 & 0.385502 & 0.688621\end{array}$

$\begin{array}{lllll}6 & 3.228232 & 1.170702 & -0.286520\end{array}$

$\begin{array}{llll}6 & 3.304325 & -0.674052 & 1.266973\end{array}$

$\begin{array}{lllll}6 & 4.539197 & 0.920312 & -0.658325\end{array}$

$\begin{array}{lllll}1 & 2.673861 & 1.970169 & -0.755970\end{array}$

$\begin{array}{lllll}6 & 4.639830 & -0.882712 & 0.936250\end{array}$

$\begin{array}{lllll}1 & 2.812768 & -1.296803 & 2.003347\end{array}$

$\begin{array}{lllll}6 & 5.273408 & -0.088481 & -0.022625\end{array}$

$\begin{array}{lllll}1 & 5.002652 & 1.508564 & -1.441485\end{array}$

$\begin{array}{lllll}1 & 5.203218 & -1.662197 & 1.434668\end{array}$

$\begin{array}{lllll}6 & 6.702526 & -0.318083 & -0.391332\end{array}$

$1 \quad 6.777534 \quad-0.694391 \quad-1.412659$

$\begin{array}{lllll}1 & 7.279273 & 0.607061 & -0.345063\end{array}$

$\begin{array}{lllll}1 & 7.171147 & -1.041333 & 0.269385\end{array}$ $\begin{array}{llll}6 & -1.740916 & -0.716947 & -0.656730\end{array}$

$\begin{array}{lllll}1 & -1.905074 & -1.795744 & -0.702052\end{array}$

$\begin{array}{lllll}1 & -1.434855 & -0.496828 & 0.368071\end{array}$

$\begin{array}{lllll}6 & -3.001269 & 0.040427 & -1.058261\end{array}$

$\begin{array}{lllll}1 & -3.247056 & -0.148823 & -2.109977\end{array}$

$\begin{array}{lllll}1 & -2.835051 & 1.123303 & -0.972438\end{array}$

$\begin{array}{lllll}6 & -4.172179 & -0.358728 & -0.165387\end{array}$

$\begin{array}{lllll}1 & -4.339300 & -1.442187 & -0.251601\end{array}$

$\begin{array}{lllll}1 & -3.908544 & -0.184325 & 0.887982\end{array}$

$\begin{array}{lllll}1 & -0.772125 & -0.546010 & -2.629635\end{array}$

$\begin{array}{lllll}6 & -5.468262 & 0.376971 & -0.496958\end{array}$

$\begin{array}{lllll}1 & -5.686267 & 0.257794 & -1.563487\end{array}$

$\begin{array}{lllll}1 & -5.338887 & 1.454548 & -0.326014\end{array}$

$\begin{array}{lllll}6 & -6.631533 & -0.156374 & 0.328146\end{array}$

$\begin{array}{lllll}1 & -7.566277 & 0.347481 & 0.083453\end{array}$

$\begin{array}{lllll}1 & -6.770842 & -1.224501 & 0.146064\end{array}$

$\begin{array}{llll}1 & -6.448077 & -0.022642 & 1.398219\end{array}$

\section{$\mathbf{7}^{-}: \mathbf{8 b ^ { + }}: \mathrm{N}_{2} @ \mathbf{1}_{2}$}

$\begin{array}{llll}6 & 9.200437 & -3.391683 & 0.317397\end{array}$

$\begin{array}{lllll}6 & 8.675640 & -2.607630 & 1.516467\end{array}$

$\begin{array}{lllll}6 & 8.521908 & -2.982501 & -0.985082\end{array}$

$\begin{array}{lllll}1 & 8.918465 & -4.430612 & 0.491185\end{array}$

$\begin{array}{lllll}6 & 9.202632 & -1.378581 & 1.916187\end{array}$

$\begin{array}{lllll}6 & 7.634050 & -3.130229 & 2.293003\end{array}$

$\begin{array}{lllll}6 & 8.958733 & -1.883129 & -1.727947\end{array}$

$\begin{array}{lllll}6 & 7.449997 & -3.717346 & -1.507565\end{array}$

$\begin{array}{lllll}6 & 8.767266 & -0.688765 & 3.056086\end{array}$

$\begin{array}{lllll}1 & 9.985876 & -0.931441 & 1.315674\end{array}$

$\begin{array}{lllll}6 & 7.197465 & -2.516900 & 3.456299\end{array}$

$\begin{array}{lllll}8 & 7.041414 & -4.324373 & 1.880819\end{array}$

$\begin{array}{lllll}6 & 8.395756 & -1.510245 & -2.951793\end{array}$

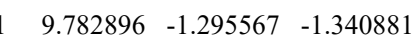

$\begin{array}{lllll}6 & 6.849188 & -3.386369 & -2.716448\end{array}$

$\begin{array}{lllll}8 & 6.969894 & -4.828620 & -0.792661\end{array}$

$\begin{array}{lllll}6 & 9.332765 & 0.685698 & 3.405680\end{array}$

$\begin{array}{lllll}6 & 7.779643 & -1.309931 & 3.827864\end{array}$

$\begin{array}{lllll}1 & 6.408738 & -2.960086 & 4.049395\end{array}$

$\begin{array}{lllll}6 & 5.845603 & -4.210725 & 1.233244\end{array}$

$\begin{array}{lllll}6 & 8.936319 & -0.323919 & -3.744487\end{array}$

$\begin{array}{lllll}6 & 7.330192 & -2.288794 & -3.423017\end{array}$

$\begin{array}{lllll}1 & 6.014393 & -3.962481 & -3.094907\end{array}$

$\begin{array}{lllll}6 & 5.795624 & -4.578725 & -0.138423\end{array}$

$\begin{array}{lllll}1 & 9.070261 & 0.874341 & 4.447556\end{array}$

$\begin{array}{lllll}6 & 8.635831 & 1.767841 & 2.579214\end{array}$

$\begin{array}{lllll}8 & 7.316435 & -0.706724 & 5.024231\end{array}$

$\begin{array}{lllll}6 & 8.355316 & 0.998234 & -3.249624\end{array}$

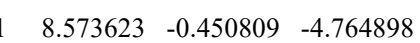

$\begin{array}{lllll}8 & 6.729760 & -1.971540 & -4.653069\end{array}$

$\begin{array}{llll}6 & 9.138774 & 2.267611 & 1.374890\end{array}$

$\begin{array}{lllll}6 & 7.424174 & 2.306245 & 3.025554\end{array}$

$\begin{array}{llll}6 & 6.044974 & -0.247789 & 4.895394\end{array}$

$\begin{array}{lllll}6 & 3.658690 & -3.773176 & 1.176204\end{array}$

$\begin{array}{lllll}6 & 8.940377 & 1.771211 & -2.243849\end{array}$

$\begin{array}{lllll}6 & 7.179471 & 1.493883 & -3.825414\end{array}$

$\begin{array}{lllll}6 & 5.505327 & -1.374930 & -4.527016\end{array}$

$\begin{array}{lllll}6 & 3.589054 & -4.241974 & -0.128218\end{array}$

$\begin{array}{llll}6 & 8.498618 & 3.277572 & 0.647054\end{array}$ $\begin{array}{llll}1 & 10.071093 & 1.867500 & 0.995525\end{array}$

$\begin{array}{llll}6 & 6.749341 & 3.317152 & 2.357662\end{array}$

$\begin{array}{lllll}8 & 6.954790 & 1.835033 & 4.258482\end{array}$

$\begin{array}{llll}6 & 5.862116 & 1.057619 & 4.330615\end{array}$

$\begin{array}{lllll}6 & 8.416422 & 3.002701 & -1.829758\end{array}$

$\begin{array}{lllll}1 & 9.838131 & 1.403396 & -1.760867\end{array}$

$\begin{array}{lllll}6 & 6.632666 & 2.718292 & -3.475865\end{array}$

$\begin{array}{lllll}8 & 6.576656 & 0.725397 & -4.820755\end{array}$

$\begin{array}{lllll}6 & 5.446586 & 0.045559 & -4.491244\end{array}$

$\begin{array}{lllll}6 & 9.036247 & 3.800557 & -0.683921\end{array}$

$\begin{array}{lllll}6 & 7.306780 & 3.789967 & 1.175111\end{array}$

$\begin{array}{lllll}1 & 5.829012 & 3.734495 & 2.743304\end{array}$

$\begin{array}{lllll}6 & 3.835654 & -0.548365 & 4.871459\end{array}$

$\begin{array}{lllll}6 & 7.266766 & 3.457093 & -2.484902\end{array}$

$\begin{array}{lllll}1 & 5.731420 & 3.082338 & -3.950662\end{array}$

$\begin{array}{lllll}6 & 3.304194 & -1.448010 & -4.169531\end{array}$

$\begin{array}{lllll}1 & 8.672922 & 4.823663 & -0.785147\end{array}$

$\begin{array}{lllll}8 & 6.718417 & 4.876065 & 0.524338\end{array}$

$\begin{array}{lllll}6 & 3.685145 & 0.651610 & 4.193493\end{array}$

$\begin{array}{lllll}8 & 6.737394 & 4.713592 & -2.148854\end{array}$

$\begin{array}{lllll}6 & 3.277019 & -0.071907 & -3.995940\end{array}$

$\begin{array}{lllll}6 & 5.521142 & 4.726660 & -0.098455\end{array}$

$\begin{array}{lllll}6 & 5.529838 & 4.685940 & -1.522977\end{array}$

$\begin{array}{lllll}6 & 3.297338 & 4.612306 & -0.131567\end{array}$

$\begin{array}{lllll}6 & 3.301066 & 4.593213 & -1.518395\end{array}$

$\begin{array}{lllll}6 & 10.568519 & 3.845895 & -0.746033\end{array}$

$\begin{array}{lllll}1 & 10.888097 & 4.290753 & -1.691751\end{array}$

$\begin{array}{lllll}1 & 11.031732 & 2.857903 & -0.677418\end{array}$

$\begin{array}{llll}6 & 10.863127 & 0.739138 & 3.300647\end{array}$

$\begin{array}{lllll}1 & 11.226733 & 1.727675 & 3.593425\end{array}$

$\begin{array}{lllll}1 & 11.230784 & 0.536447 & 2.291144\end{array}$

$\begin{array}{lllll}6 & 10.733239 & -3.340782 & 0.217104\end{array}$

$\begin{array}{lllll}1 & 11.182775 & -3.706947 & 1.143932\end{array}$

$\begin{array}{lllll}1 & 11.116224 & -2.331879 & 0.039891\end{array}$

$\begin{array}{lllll}6 & 10.471310 & -0.309648 & -3.800393\end{array}$

$\begin{array}{lllll}1 & 10.837793 & -1.246182 & -4.228477\end{array}$

$\begin{array}{lllll}1 & 10.934813 & -0.195250 & -2.816588\end{array}$

$\begin{array}{lllll}1 & 10.816135 & 0.517891 & -4.426065\end{array}$

$\begin{array}{lllll}1 & 11.073720 & -3.971071 & -0.608316\end{array}$

$\begin{array}{lllll}1 & 11.306927 & -0.005810 & 3.965783\end{array}$

$\begin{array}{lllll}1 & 10.961565 & 4.453103 & 0.073798\end{array}$

$\begin{array}{lllll}6 & 2.476856 & -1.173699 & 5.012747\end{array}$

$\begin{array}{llll}6 & 2.277474 & 0.741574 & 3.766824\end{array}$

$\begin{array}{lllll}6 & 1.878612 & 4.522914 & 0.344658\end{array}$

$\begin{array}{lllll}6 & 1.874986 & 4.501053 & -1.991909\end{array}$

$\begin{array}{lllll}6 & 2.289938 & -3.273596 & 1.547667\end{array}$

$\begin{array}{lllll}6 & 2.157889 & -4.108451 & -0.583514\end{array}$

$\begin{array}{lllll}6 & 1.906172 & -1.970149 & -3.981906\end{array}$

$\begin{array}{lllll}6 & 1.887205 & 0.327637 & -3.582862\end{array}$

$\begin{array}{lllll}7 & 1.113131 & 4.457963 & -0.802094\end{array}$

$\begin{array}{lllll}7 & 1.600478 & -0.284432 & 4.273174\end{array}$

$\begin{array}{lllll}7 & 1.472253 & -3.527683 & 0.485836\end{array}$

$\begin{array}{lllll}7 & 1.149316 & -0.833747 & -3.643308\end{array}$

$\begin{array}{lllll}8 & 1.502732 & -3.100974 & -4.095469\end{array}$

$\begin{array}{lllll}8 & 1.523119 & 1.441888 & -3.239665\end{array}$

$\begin{array}{lllll}8 & 1.431518 & 4.467726 & -3.111889\end{array}$

$\begin{array}{lllll}8 & 1.496771 & 4.490181 & 1.504638\end{array}$

$\begin{array}{lllll}8 & 1.878131 & 1.648347 & 2.967572\end{array}$ 
$\begin{array}{lllll}8 & 1.978246 & -2.714681 & 2.603868\end{array}$

$\begin{array}{lllll}8 & 2.105923 & -2.147113 & 5.599497\end{array}$

$\begin{array}{lllll}8 & 1.695844 & -4.420495 & -1.658969\end{array}$

$\begin{array}{lllll}1 & 0.170686 & -0.931562 & -3.367197\end{array}$

$1 \quad 0.377484 \quad-3.195250 \quad 0.409890$

$\begin{array}{lllll}1 & 0.575234 & -0.507281 & 4.124873\end{array}$

$\begin{array}{lllll}1 & 0.090032 & 4.370865 & -0.846039\end{array}$

$7 \quad 5.030562 \quad-1.035516 \quad 5.216936$

$\begin{array}{lllll}7 & 4.682714 & 1.495441 & 3.922384\end{array}$

$\begin{array}{lllll}7 & 4.783908 & -3.762346 & 1.891700\end{array}$

$\begin{array}{lllll}7 & 4.659366 & -4.627997 & -0.820950\end{array}$

$\begin{array}{lllll}7 & 4.404772 & 4.681011 & 0.611824\end{array}$

$\begin{array}{lllll}7 & 4.421046 & 4.624595 & -2.245184\end{array}$

$\begin{array}{lllll}7 & 4.426307 & -2.132461 & -4.403696\end{array}$

$\begin{array}{lllll}7 & 4.344197 & 0.708536 & -4.172684\end{array}$

$\begin{array}{lllll}6 & -8.990720 & 2.141040 & -2.895718\end{array}$

$\begin{array}{lllll}6 & -8.440432 & 2.831223 & -1.646865\end{array}$

$\begin{array}{lllll}6 & -8.367092 & 0.759130 & -3.086436\end{array}$

$\begin{array}{lllll}1 & -8.663833 & 2.742862 & -3.744309\end{array}$

$\begin{array}{llll}6 & -8.981196 & 2.632539 & -0.370804\end{array}$

$\begin{array}{lllll}6 & -7.325756 & 3.674998 & -1.735435\end{array}$

$\begin{array}{lllll}6 & -8.893047 & -0.385688 & -2.473045\end{array}$

$\begin{array}{lllll}6 & -7.226864 & 0.586483 & -3.880937\end{array}$

$\begin{array}{lllll}6 & -8.472491 & 3.248028 & 0.778862\end{array}$

$\begin{array}{lllll}1 & -9.816420 & 1.950066 & -0.265918\end{array}$

$\begin{array}{lllll}6 & -6.814376 & 4.352860 & -0.637861\end{array}$

$\begin{array}{lllll}8 & -6.732238 & 3.865138 & -2.988439\end{array}$

$\begin{array}{lllll}6 & -8.327538 & -1.653961 & -2.621800\end{array}$

$\begin{array}{lllll}1 & -9.782673 & -0.281128 & -1.863221\end{array}$

$\begin{array}{lllll}6 & -6.614901 & -0.651088 & -4.050585\end{array}$

$\begin{array}{lllll}8 & -6.711018 & 1.694616 & -4.577392\end{array}$

$\begin{array}{llll}6 & -8.998345 & 2.950123 & 2.184373\end{array}$

$\begin{array}{lllll}6 & -7.404743 & 4.134720 & 0.599698\end{array}$

$\begin{array}{lllll}1 & -5.970710 & 5.022253 & -0.742010\end{array}$

$\begin{array}{lllll}6 & -5.533302 & 3.263375 & -3.216682\end{array}$

$\begin{array}{lllll}6 & -8.900800 & -2.920171 & -1.987377\end{array}$

$\begin{array}{lllll}6 & -7.177461 & -1.748967 & -3.415001\end{array}$

$\begin{array}{lllll}1 & -5.731191 & -0.757276 & -4.666357\end{array}$

$\begin{array}{lllll}6 & -5.517220 & 2.167725 & -4.127681\end{array}$

$\begin{array}{lllll}1 & -8.721445 & 3.803706 & 2.804053\end{array}$

$\begin{array}{llll}6 & -8.270530 & 1.737254 & 2.770179\end{array}$

$\begin{array}{llll}8 & -6.938482 & 4.847293 & 1.711171\end{array}$

$\begin{array}{lllll}6 & -8.245656 & -3.216912 & -0.639347\end{array}$

$\begin{array}{lllll}1 & -8.623038 & -3.743131 & -2.646661\end{array}$

$\begin{array}{lllll}8 & -6.673267 & -3.026973 & -3.631034\end{array}$

$\begin{array}{lllll}6 & -8.756281 & 0.433841 & 2.616516\end{array}$

$\begin{array}{llll}6 & -7.070890 & 1.884331 & 3.481617\end{array}$

$\begin{array}{llll}6 & -5.671686 & 4.572656 & 2.119979\end{array}$

$\begin{array}{lllll}6 & -3.317883 & 3.080912 & -2.995274\end{array}$

$\begin{array}{llll}6 & -8.676769 & -2.576639 & 0.524895\end{array}$

$\begin{array}{lllll}6 & -7.201302 & -4.143652 & -0.502357\end{array}$

$\begin{array}{lllll}6 & -5.483480 & -3.398918 & -3.089506\end{array}$

$\begin{array}{lllll}6 & -3.292799 & 2.084954 & -3.962797\end{array}$

$\begin{array}{llll}6 & -8.097787 & -0.688704 & 3.124104\end{array}$

$\begin{array}{lllll}1 & -9.695148 & 0.291520 & 2.095643\end{array}$

$\begin{array}{llll}6 & -6.357020 & 0.796806 & 3.977779\end{array}$

$\begin{array}{llll}8 & -6.637835 & 3.180540 & 3.788637\end{array}$

$\begin{array}{llll}6 & -5.510443 & 3.669733 & 3.210210\end{array}$ $\begin{array}{llll}6 & -8.130011 & -2.818309 & 1.787864\end{array}$

$\begin{array}{lllll}1 & -9.476890 & -1.849706 & 0.445684\end{array}$

$\begin{array}{lllll}6 & -6.622049 & -4.419909 & 0.733192\end{array}$

$\begin{array}{lllll}8 & -6.730646 & -4.831191 & -1.625746\end{array}$

$\begin{array}{lllll}6 & -5.510802 & -4.413529 & -2.095317\end{array}$

$\begin{array}{lllll}6 & -8.659981 & -2.102672 & 3.024135\end{array}$

$\begin{array}{llll}6 & -6.875539 & -0.474986 & 3.772199\end{array}$

$\begin{array}{lllll}1 & -5.434495 & 0.942224 & 4.524311\end{array}$

$\begin{array}{lllll}6 & -3.458628 & 4.816728 & 2.008343\end{array}$

$\begin{array}{llll}6 & -7.080638 & -3.741570 & 1.857765\end{array}$

$\begin{array}{lllll}1 & -5.805471 & -5.127823 & 0.808348\end{array}$

$\begin{array}{lllll}6 & -3.259327 & -3.418879 & -3.029808\end{array}$

$\begin{array}{lllll}1 & -8.272965 & -2.648320 & 3.885209\end{array}$

$\begin{array}{lllll}8 & -6.258913 & -1.591402 & 4.342788\end{array}$

$\begin{array}{llll}6 & -3.299505 & 3.936141 & 3.070405\end{array}$

$\begin{array}{lllll}8 & -6.469841 & -3.999526 & 3.097661\end{array}$

$\begin{array}{lllll}6 & -3.279831 & -4.467321 & -2.119588\end{array}$

$\begin{array}{llll}6 & -5.129631 & -2.140875 & 3.832462\end{array}$

$\begin{array}{lllll}6 & -5.232296 & -3.447048 & 3.260865\end{array}$

$\begin{array}{lllll}6 & -2.913805 & -2.231789 & 3.580146\end{array}$

$\begin{array}{llll}6 & -3.003702 & -3.528187 & 3.094179\end{array}$

$\begin{array}{llll}6 & -10.193379 & -2.146830 & 3.103395\end{array}$

$\begin{array}{lllll}1 & -10.534242 & -3.185231 & 3.110494\end{array}$

$\begin{array}{lllll}1 & -10.679685 & -1.649292 & 2.259856\end{array}$

$\begin{array}{lllll}6 & -10.526122 & 2.819219 & 2.233012\end{array}$

$\begin{array}{lllll}1 & -10.857253 & 2.643033 & 3.259827\end{array}$

$\begin{array}{llll}1 & -10.906988 & 1.998873 & 1.618546\end{array}$

$\begin{array}{lllll}6 & -10.525851 & 2.109036 & -2.916852\end{array}$

$\begin{array}{lllll}1 & -10.922795 & 3.125098 & -2.845544\end{array}$

$\begin{array}{lllll}1 & -10.952970 & 1.531081 & -2.092634\end{array}$

$\begin{array}{lllll}6 & -10.433610 & -2.900360 & -1.894666\end{array}$

$\begin{array}{lllll}1 & -10.866979 & -2.740731 & -2.885611\end{array}$

$\begin{array}{lllll}1 & -10.813096 & -2.112819 & -1.237552\end{array}$

$\begin{array}{lllll}1 & -10.797022 & -3.854792 & -1.505203\end{array}$

$\begin{array}{lllll}1 & -10.878087 & 1.661352 & -3.849766\end{array}$

$\begin{array}{llll}1 & -10.989896 & 3.740525 & 1.871237\end{array}$

$\begin{array}{lllll}1 & -10.538071 & -1.660406 & 4.019925\end{array}$

$\begin{array}{llll}6 & -2.098496 & 5.257488 & 1.540530\end{array}$

$\begin{array}{llll}6 & -1.826780 & 3.745881 & 3.318737\end{array}$

$\begin{array}{llll}6 & -1.466281 & -1.854333 & 3.628714\end{array}$

$\begin{array}{llll}6 & -1.608057 & -4.043355 & 2.854490\end{array}$

$\begin{array}{lllll}6 & -1.930058 & 3.228899 & -2.430755\end{array}$

$\begin{array}{lllll}6 & -1.868894 & 1.634189 & -4.142298\end{array}$

$\begin{array}{lllll}6 & -1.830206 & -3.037587 & -3.294345\end{array}$

$\begin{array}{lllll}6 & -1.854369 & -4.849105 & -1.806949\end{array}$

$\begin{array}{lllll}7 & -0.771581 & -2.969754 & 3.265475\end{array}$

$\begin{array}{llll}7 & -1.208036 & 4.570856 & 2.379761\end{array}$

$\begin{array}{lllll}7 & -1.146390 & 2.373248 & -3.182147\end{array}$

$\begin{array}{lllll}7 & -1.082823 & -3.969696 & -2.585777\end{array}$

$\begin{array}{lllll}8 & -1.430403 & -2.098174 & -3.950647\end{array}$

$\begin{array}{lllll}8 & -1.442784 & -5.711538 & -1.070527\end{array}$

$\begin{array}{lllll}8 & -1.231183 & -5.113827 & 2.462074\end{array}$

$\begin{array}{llll}8 & -0.994339 & -0.760483 & 3.946842\end{array}$

$\begin{array}{llll}8 & -1.273538 & 3.024679 & 4.121826\end{array}$

$\begin{array}{llll}8 & -1.585657 & 3.911271 & -1.479678\end{array}$

$\begin{array}{llll}8 & -1.792906 & 6.019096 & 0.654079\end{array}$

$\begin{array}{lllll}8 & -1.402699 & 0.828471 & -4.908108\end{array}$

$\begin{array}{lllll}1 & -0.065227 & -3.944234 & -2.512638\end{array}$ $\begin{array}{llll}1 & -0.138608 & 2.216735 & -3.069931\end{array}$

$\begin{array}{lllll}1 & -0.197040 & 4.616515 & 2.230243\end{array}$

$\begin{array}{llll}1 & 0.238098 & -2.949111 & 3.065225\end{array}$

$\begin{array}{lllll}7 & -4.650742 & 5.156141 & 1.512398\end{array}$

$\begin{array}{llll}7 & -4.321924 & 3.346139 & 3.696676\end{array}$

$\begin{array}{lllll}7 & -4.437520 & 3.705473 & -2.615929\end{array}$

$\begin{array}{lllll}7 & -4.394509 & 1.595330 & -4.534897\end{array}$

$\begin{array}{llll}7 & -3.969481 & -1.517197 & 3.975933\end{array}$

$\begin{array}{llll}7 & -4.168524 & -4.154247 & 2.909875\end{array}$

$\begin{array}{llll}7 & -4.359229 & -2.869052 & -3.544317\end{array}$

$\begin{array}{lllll}7 & -4.410032 & -4.970586 & -1.614991\end{array}$

$\begin{array}{lllll}6 & -1.126597 & -1.833406 & -0.477280\end{array}$

$\begin{array}{lllll}8 & -0.923914 & -2.773309 & 0.375372\end{array}$

$\begin{array}{llll}8 & -0.261632 & -1.310297 & -1.207175\end{array}$

$\begin{array}{llll}6 & 0.618729 & 1.440089 & 2.185891\end{array}$

$\begin{array}{lllll}1 & -0.165444 & 1.181860 & 2.893199\end{array}$

$\begin{array}{lllll}6 & -2.543878 & -1.333417 & -0.526668\end{array}$

$\begin{array}{lllll}6 & -2.830454 & -0.110903 & -1.136944\end{array}$

$\begin{array}{lllll}6 & -3.576230 & -2.046653 & 0.093577\end{array}$

$\begin{array}{lllll}6 & -4.110171 & 0.428062 & -1.051908\end{array}$

$\begin{array}{lllll}1 & -2.019154 & 0.408458 & -1.631953\end{array}$

$\begin{array}{lllll}6 & -4.853348 & -1.506015 & 0.166464\end{array}$

$\begin{array}{lllll}1 & -3.357981 & -3.019512 & 0.513277\end{array}$

$\begin{array}{lllll}6 & -5.126542 & -0.240322 & -0.365267\end{array}$

$\begin{array}{lllll}1 & -4.324906 & 1.404001 & -1.466385\end{array}$

$\begin{array}{lllll}1 & -5.647027 & -2.048749 & 0.661062\end{array}$

$\begin{array}{lllll}6 & -6.448134 & 0.420154 & -0.121357\end{array}$

$\begin{array}{lllll}1 & -6.651319 & 1.187937 & -0.863981\end{array}$

$\begin{array}{lllll}1 & -7.270377 & -0.296467 & -0.133074\end{array}$

$\begin{array}{lllll}1 & -6.449872 & 0.901345 & 0.861684\end{array}$

$\begin{array}{lllll}6 & 0.853218 & 0.394314 & 1.116877\end{array}$

$\begin{array}{lllll}1 & 1.195670 & -0.541091 & 1.570998\end{array}$

$\begin{array}{lllll}1 & -0.135403 & 0.170486 & 0.711919\end{array}$

$\begin{array}{lllll}6 & 1.741023 & 0.813426 & -0.062917\end{array}$

$\begin{array}{lllll}1 & 1.264727 & 1.665827 & -0.559690\end{array}$

$\begin{array}{lllll}1 & 1.683854 & -0.006623 & -0.781726\end{array}$

$\begin{array}{lllll}6 & 3.207141 & 1.173700 & 0.232353\end{array}$

$\begin{array}{lllll}1 & 3.623806 & 1.640722 & -0.670508\end{array}$

$\begin{array}{lllll}1 & 3.259746 & 1.934505 & 1.017415\end{array}$

$\begin{array}{lllll}1 & 0.438522 & 2.429297 & 1.778586\end{array}$

$\begin{array}{lllll}6 & 4.117648 & -0.008194 & 0.609554\end{array}$

$\begin{array}{lllll}1 & 5.044680 & 0.386596 & 1.044163\end{array}$

$\begin{array}{lllll}1 & 3.650767 & -0.617776 & 1.395445\end{array}$

$\begin{array}{lllll}6 & 4.473320 & -0.898431 & -0.585871\end{array}$

$\begin{array}{lllll}1 & 4.952321 & -0.307664 & -1.374594\end{array}$

$\begin{array}{lllll}1 & 5.172895 & -1.690344 & -0.304342\end{array}$

$\begin{array}{lllll}1 & 3.586477 & -1.371329 & -1.018551\end{array}$

$\begin{array}{llll}7 & -3.639783 & 0.846040 & 2.094004\end{array}$

$\begin{array}{llll}7 & -2.558996 & 0.929973 & 1.880272\end{array}$

\section{7-:9b+@ $\mathbf{1}_{2}$}

$\begin{array}{lllll}6 & -9.071515 & -3.449766 & -0.120273\end{array}$

$\begin{array}{lllll}6 & -8.498895 & -2.769366 & -1.364496\end{array}$

$\begin{array}{lllll}6 & -8.454973 & -2.931639 & 1.176909\end{array}$

$\begin{array}{lllll}1 & -8.781077 & -4.498332 & -0.194504\end{array}$

$\begin{array}{lllll}6 & -9.012994 & -1.581472 & -1.888851\end{array}$

$\begin{array}{lllll}6 & -7.431996 & -3.352602 & -2.060636\end{array}$

$\begin{array}{lllll}6 & -8.925794 & -1.781214 & 1.813706\end{array}$ 
$\begin{array}{lllll}6 & -7.388053 & -3.598944 & 1.791847\end{array}$

$\begin{array}{lllll}6 & -8.542126 & -0.992499 & -3.070828\end{array}$

$\begin{array}{lllll}1 & -9.818512 & -1.088734 & -1.357171\end{array}$

$\begin{array}{lllll}6 & -6.947542 & -2.831474 & -3.252068\end{array}$

$\begin{array}{lllll}8 & -6.853901 & -4.510288 & -1.532640\end{array}$

$\begin{array}{lllll}6 & -8.390606 & -1.287787 & 3.008505\end{array}$

$\begin{array}{lllll}1 & -9.743911 & -1.236724 & 1.356692\end{array}$

$\begin{array}{lllll}6 & -6.827033 & -3.159618 & 2.983654\end{array}$

$\begin{array}{lllll}8 & -6.849906 & -4.737434 & 1.170278\end{array}$

$\begin{array}{lllll}6 & -9.119856 & 0.326501 & -3.576196\end{array}$

$\begin{array}{lllll}6 & -7.517601 & -1.663425 & -3.747130\end{array}$

$1 \quad-6.135271 \quad-3.318610 \quad-3.775143$

$\begin{array}{lllll}6 & -5.675093 & -4.321560 & -0.870789\end{array}$

$\begin{array}{lllll}6 & -8.944568 & -0.023692 & 3.656435\end{array}$

$\begin{array}{lllll}6 & -7.330284 & -2.006973 & 3.576350\end{array}$

$\begin{array}{lllll}1 & -5.994426 & -3.688331 & 3.429549\end{array}$

$\begin{array}{llll}6 & -5.663251 & -4.492886 & 0.539425\end{array}$

$\begin{array}{llll}1 & -8.820149 & 0.416464 & -4.621200\end{array}$

$\begin{array}{lllll}6 & -8.483940 & 1.505198 & -2.840157\end{array}$

$\begin{array}{lllll}8 & -7.023290 & -1.161871 & -4.977377\end{array}$

$\begin{array}{lllll}6 & -8.358611 & 1.237546 & 3.024805\end{array}$

$\begin{array}{lllll}1 & -8.595615 & -0.033788 & 4.689808\end{array}$

$8 \begin{array}{llll}8 & -6.746221 & -1.578394 & 4.781314\end{array}$

$\begin{array}{lllll}6 & -9.039497 & 2.091641 & -1.699731\end{array}$

$\begin{array}{lllll}6 & -7.281289 & 2.048159 & -3.305760\end{array}$

$\begin{array}{lllll}6 & -5.770471 & -0.648192 & -4.860868\end{array}$

$\begin{array}{lllll}6 & -3.502018 & -3.830998 & -0.808626\end{array}$

$\begin{array}{llll}6 & -8.946793 & 1.915343 & 1.953945\end{array}$

$\begin{array}{lllll}6 & -7.179626 & 1.783614 & 3.545847\end{array}$

$\begin{array}{llll}6 & -5.511519 & -1.009232 & 4.615429\end{array}$

$\begin{array}{lllll}6 & -3.475288 & -4.045078 & 0.561414\end{array}$

$\begin{array}{lllll}6 & -8.461263 & 3.190202 & -1.053538\end{array}$

$\begin{array}{lllll}1 & -9.963605 & 1.686075 & -1.306087\end{array}$

$\begin{array}{lllll}6 & -6.674672 & 3.151784 & -2.725897\end{array}$

$\begin{array}{lllll}8 & -6.740787 & 1.469772 & -4.462095\end{array}$

$\begin{array}{lllll}6 & -5.637048 & 0.703429 & -4.400809\end{array}$

$\begin{array}{llll}6 & -8.433710 & 3.112630 & 1.437500\end{array}$

$\begin{array}{lllll}1 & -9.843683 & 1.502713 & 1.506833\end{array}$

$\begin{array}{lllll}6 & -6.650816 & 2.985067 & 3.103910\end{array}$

$\begin{array}{lllll}8 & -6.572603 & 1.107751 & 4.600433\end{array}$

$\begin{array}{lllll}6 & -5.446194 & 0.388670 & 4.358103\end{array}$

$\begin{array}{lllll}6 & -9.049116 & 3.799240 & 0.219229\end{array}$

$\begin{array}{lllll}6 & -7.283211 & 3.707703 & -1.606149\end{array}$

$\begin{array}{lllll}1 & -5.758457 & 3.567087 & -3.122518\end{array}$

$\begin{array}{lllll}6 & -3.555134 & -0.888984 & -4.732654\end{array}$

$\begin{array}{lllll}6 & -7.298768 & 3.639923 & 2.063879\end{array}$

$\begin{array}{lllll}1 & -5.757104 & 3.398305 & 3.551824\end{array}$

$\begin{array}{llll}6 & -3.296230 & -1.128325 & 4.361848\end{array}$

$\begin{array}{llll}1 & -8.719246 & 4.838095 & 0.247747\end{array}$

$8 \begin{array}{lllll}8 & -6.745261 & 4.862947 & -1.031726\end{array}$

$\begin{array}{lllll}6 & -3.462768 & 0.344612 & -4.102965\end{array}$

$\begin{array}{lllll}8 & -6.799245 & 4.883926 & 1.644800\end{array}$

$\begin{array}{lllll}6 & -3.266723 & 0.193254 & 3.936569\end{array}$

$\begin{array}{lllll}6 & -5.556359 & 4.766583 & -0.381606\end{array}$

$\begin{array}{lllll}6 & -5.581354 & 4.828614 & 1.040789\end{array}$

$\begin{array}{lllll}6 & -3.341656 & 4.578096 & -0.306472\end{array}$

$\begin{array}{llll}6 & -3.355951 & 4.677101 & 1.076235\end{array}$

$\begin{array}{llll}6 & -10.583197 & 3.799457 & 0.238851\end{array}$ $\begin{array}{llll}1 & -10.943186 & 4.300913 & 1.140735\end{array}$

$\begin{array}{lllll}1 & -11.013463 & 2.794518 & 0.229725\end{array}$

$\begin{array}{lllll}6 & -10.654508 & 0.350727 & -3.536275\end{array}$

$\begin{array}{lllll}1 & -11.027032 & 1.295335 & -3.941254\end{array}$

$\begin{array}{lllll}1 & -11.058249 & 0.240680 & -2.526467\end{array}$

$\begin{array}{lllll}6 & -10.607463 & -3.397949 & -0.092333\end{array}$

$\begin{array}{lllll}1 & -11.015586 & -3.839200 & -1.005425\end{array}$

$\begin{array}{lllll}1 & -10.997639 & -2.379539 & -0.014296\end{array}$

$\begin{array}{llll}6 & -10.480177 & -0.007612 & 3.689491\end{array}$

$\begin{array}{lllll}1 & -10.850896 & -0.891115 & 4.215344\end{array}$

$\begin{array}{lllll}1 & -10.929447 & -0.006556 & 2.692628\end{array}$

$\begin{array}{lllll}1 & -10.835372 & 0.883960 & 4.213018\end{array}$

$\begin{array}{lllll}1 & -10.983910 & -3.960916 & 0.765481\end{array}$

$\begin{array}{lllll}1 & -11.054362 & -0.468221 & -4.139373\end{array}$

$\begin{array}{lllll}1 & -10.970973 & 4.332611 & -0.633220\end{array}$

$6 \quad-2.184032-1.505840 \quad-4.740713$

$\begin{array}{lllll}6 & -2.122676 & 0.408209 & -3.482627\end{array}$

$\begin{array}{lllll}6 & -1.939639 & 4.328692 & -0.754737\end{array}$

$\begin{array}{lllll}6 & -1.936799 & 4.547567 & 1.571786\end{array}$

$6 \begin{array}{llll}6 & -2.120785 & -3.426639 & -1.230808\end{array}$

$\begin{array}{lllll}6 & -2.061394 & -3.784158 & 1.027136\end{array}$

$\begin{array}{lllll}6 & -1.880158 & -1.641903 & 4.377969\end{array}$

$\begin{array}{llll}6 & -1.857489 & 0.529215 & 3.524238\end{array}$

$\begin{array}{llll}7 & -1.178613 & 4.321672 & 0.399043\end{array}$

$\begin{array}{lllll}7 & -1.397236 & -0.624348 & -3.898893\end{array}$

$7-1.333273-3.466362-0.112695$

$\begin{array}{lllll}7 & -1.118473 & -0.588816 & 3.834104\end{array}$

$\begin{array}{lllll}8 & -1.450136 & -2.693506 & 4.781421\end{array}$

$\begin{array}{lllll}8 & -1.474998 & 1.567186 & 3.004962\end{array}$

$\begin{array}{lllll}8 & -1.498396 & 4.613095 & 2.691403\end{array}$

$\begin{array}{lllll}8 & -1.570973 & 4.125429 & -1.900512\end{array}$

$\begin{array}{lllll}8 & -1.853296 & 1.255011 & -2.565601\end{array}$

$8 \begin{array}{llll}8 & -1.779040 & -3.102437 & -2.367464\end{array}$

$8 \begin{array}{llll}8 & -1.751676 & -2.470434 & -5.299243\end{array}$

$\begin{array}{llll}8 & -1.642815 & -3.813935 & 2.168062\end{array}$

$\begin{array}{llll}1 & -0.100027 & -0.694425 & 3.721104\end{array}$

$1-0.227759-3.119807 \quad-0.087390$

$1 \quad-0.378031 \quad-0.845317 \quad-3.684851$

$1-0.164625 \quad 4.155330 \quad 0.442675$

$7 \quad-4.724456 \quad-1.425391 \quad-5.094081$

$\begin{array}{lllll}7 & -4.489690 & 1.184579 & -3.952658\end{array}$

$7 \quad-4.597751 \quad-3.962708 \quad-1.555634$

$\begin{array}{lllll}7 & -4.560558 & -4.363691 & 1.266675\end{array}$

$\begin{array}{lllll}7 & -4.435108 & 4.631461 & -1.069592\end{array}$

$\begin{array}{lllll}7 & -4.483055 & 4.801502 & 1.781837\end{array}$

$\begin{array}{lllll}7 & -4.429429 & -1.769174 & 4.667914\end{array}$

$\begin{array}{llll}7 & -4.339056 & 0.990101 & 3.950878\end{array}$

$\begin{array}{llll}6 & 9.026814 & 2.619529 & 2.501586\end{array}$

$\begin{array}{llll}6 & 8.452440 & 3.130291 & 1.176830\end{array}$

$\begin{array}{lllll}6 & 8.434818 & 1.267750 & 2.903977\end{array}$

$\begin{array}{llll}1 & 8.700373 & 3.329332 & 3.263185\end{array}$

$\begin{array}{lllll}6 & 9.003696 & 2.825758 & -0.072567\end{array}$

$\begin{array}{lllll}6 & 7.306121 & 3.935587 & 1.174338\end{array}$

$\begin{array}{lllll}6 & 8.966960 & 0.058089 & 2.433943\end{array}$

$\begin{array}{lllll}6 & 7.327324 & 1.179831 & 3.757410\end{array}$

$\begin{array}{lllll}6 & 8.475509 & 3.294113 & -1.286108\end{array}$

$\begin{array}{lllll}1 & 9.858125 & 2.159847 & -0.108729\end{array}$

$\begin{array}{llll}6 & 6.792056 & 4.492409 & 0.017033\end{array}$ $\begin{array}{llll}8 & 6.681829 & 4.210256 & 2.392950\end{array}$

$\begin{array}{lllll}6 & 8.430030 & -1.188649 & 2.762321\end{array}$

$\begin{array}{llll}1 & 9.834826 & 0.094648 & 1.786146\end{array}$

$\begin{array}{lllll}6 & 6.739717 & -0.035763 & 4.094148\end{array}$

$\begin{array}{lllll}8 & 6.783693 & 2.349837 & 4.318056\end{array}$

$\begin{array}{lllll}6 & 9.001898 & 2.806042 & -2.639899\end{array}$

$\begin{array}{lllll}6 & 7.386484 & 4.171321 & -1.198101\end{array}$

$\begin{array}{lllll}1 & 5.925197 & 5.138587 & 0.052365\end{array}$

$\begin{array}{llll}6 & 5.526314 & 3.554508 & 2.684929\end{array}$

$\begin{array}{lllll}6 & 9.025817 & -2.515660 & 2.299578\end{array}$

$\begin{array}{lllll}6 & 7.292197 & -1.196683 & 3.578285\end{array}$

$\begin{array}{llll}1 & 5.871869 & -0.071455 & 4.739449\end{array}$

$\begin{array}{llll}6 & 5.562486 & 2.670359 & 3.799122\end{array}$

$1 \quad 8.667920 \quad 3.535413 \quad-3.379539$

$\begin{array}{lllll}6 & 8.341845 & 1.473120 & -3.020289\end{array}$

$\begin{array}{lllll}8 & 6.832835 & 4.738472 & -2.357466\end{array}$

$\begin{array}{lllll}6 & 8.389782 & -2.993520 & 0.997763\end{array}$

$\begin{array}{lllll}1 & 8.754109 & -3.250891 & 3.057902\end{array}$

$\begin{array}{lllll}8 & 6.774689 & -2.439129 & 3.937710\end{array}$

$\begin{array}{lllll}6 & 8.914261 & 0.223463 & -2.746757\end{array}$

$\begin{array}{lllll}6 & 7.089416 & 1.465783 & -3.648502\end{array}$

$\begin{array}{lllll}6 & 5.563309 & 4.295526 & -2.608012\end{array}$

$\begin{array}{lllll}6 & 3.344482 & 3.139354 & 2.460352\end{array}$

$\begin{array}{lllll}6 & 8.823291 & -2.507429 & -0.237874\end{array}$

$\begin{array}{lllll}6 & 7.360330 & -3.944498 & 0.975993\end{array}$

$\begin{array}{lllll}6 & 5.598797 & -2.861177 & 3.401951\end{array}$

$\begin{array}{lllll}6 & 3.357093 & 2.386504 & 3.626744\end{array}$

$\begin{array}{lllll}6 & 8.275952 & -0.983001 & -3.057767\end{array}$

$\begin{array}{lllll}1 & 9.890339 & 0.189240 & -2.277612\end{array}$

$\begin{array}{lllll}6 & 6.406157 & 0.300632 & -3.968871\end{array}$

$\begin{array}{lllll}8 & 6.549461 & 2.694545 & -4.025185\end{array}$

$\begin{array}{lllll}6 & 5.420876 & 3.138073 & -3.418768\end{array}$

$6 \quad 8.303026 \quad-2.927598 \quad-1.466124$

$\begin{array}{lllll}1 & 9.609831 & -1.761529 & -0.247207\end{array}$

$\begin{array}{lllll}6 & 6.803376 & -4.392217 & -0.216183\end{array}$

$\begin{array}{lllll}8 & 6.874090 & -4.478222 & 2.177174\end{array}$

$\begin{array}{lllll}6 & 5.645884 & -4.000503 & 2.551580\end{array}$

$\begin{array}{lllll}6 & 8.859988 & -2.364850 & -2.772943\end{array}$

$\begin{array}{lllll}6 & 7.014252 & -0.904006 & -3.658267\end{array}$

$\begin{array}{lllll}1 & 5.436894 & 0.334518 & -4.447021\end{array}$

$\begin{array}{lllll}6 & 3.355336 & 4.328708 & -2.306759\end{array}$

$\begin{array}{lllll}6 & 7.270549 & -3.874559 & -1.420290\end{array}$

$\begin{array}{lllll}1 & 6.000447 & -5.119507 & -0.206321\end{array}$

$\begin{array}{lllll}6 & 3.377834 & -2.854161 & 3.239447\end{array}$

$\begin{array}{lllll}1 & 8.501098 & -3.021185 & -3.566921\end{array}$

$\begin{array}{lllll}8 & 6.437479 & -2.107639 & -4.047490\end{array}$

$\begin{array}{lllll}6 & 3.226824 & 3.146889 & -3.029246\end{array}$

$\begin{array}{lllll}8 & 6.671634 & -4.341279 & -2.601857\end{array}$

$\begin{array}{lllll}6 & 3.413566 & -4.022211 & 2.491153\end{array}$

$\begin{array}{lllll}6 & 5.301736 & -2.587059 & -3.489677\end{array}$

$\begin{array}{lllll}6 & 5.417072 & -3.840749 & -2.822004\end{array}$

$\begin{array}{lllll}6 & 3.096441 & -2.655079 & -3.207128\end{array}$

$\begin{array}{lllll}6 & 3.194011 & -3.927565 & -2.658929\end{array}$

$\begin{array}{lllll}6 & 10.394315 & -2.395176 & -2.807419\end{array}$

$\begin{array}{lllll}1 & 10.749891 & -3.419665 & -2.671720\end{array}$

$\begin{array}{lllll}1 & 10.851789 & -1.784689 & -2.023975\end{array}$

$\begin{array}{lllll}6 & 10.534090 & 2.755420 & -2.694422\end{array}$

$\begin{array}{lllll}1 & 10.866698 & 2.444568 & -3.688354\end{array}$ 
$\begin{array}{llll}1 & 10.964320 & 2.058471 & -1.969631\end{array}$

$\begin{array}{llll}6 & 10.562491 & 2.613585 & 2.504281\end{array}$

$\begin{array}{llll}1 & 10.941640 & 3.614254 & 2.280864\end{array}$

$\begin{array}{lllll}1 & 10.988008 & 1.928702 & 1.765568\end{array}$

$\begin{array}{lllll}6 & 10.559248 & -2.482314 & 2.218617\end{array}$

$1 \quad 10.979726 \quad-2.190344 \quad 3.184654$

$\begin{array}{lllll}1 & 10.932617 & -1.778773 & 1.469296\end{array}$

$\begin{array}{lllll}1 & 10.941135 & -3.472568 & 1.957344\end{array}$

$\begin{array}{lllll}1 & 10.934446 & 2.310769 & 3.486485\end{array}$

$\begin{array}{lllll}1 & 10.948143 & 3.744459 & -2.482326\end{array}$

$\begin{array}{lllll}1 & 10.756717 & -2.025757 & -3.770649\end{array}$

$\begin{array}{lllll}6 & 1.989007 & 4.774311 & -1.867295\end{array}$

$\begin{array}{lllll}6 & 1.770349 & 2.752827 & -3.041258\end{array}$

$\begin{array}{lllll}6 & 1.644940 & -2.287744 & -3.262270\end{array}$

$\begin{array}{lllll}6 & 1.801613 & -4.449465 & -2.425507\end{array}$

$\begin{array}{llll}6 & 1.950025 & 3.101918 & 1.893878\end{array}$

$\begin{array}{lllll}6 & 1.953333 & 1.927217 & 3.910236\end{array}$

$\begin{array}{lllll}6 & 1.946392 & -2.427293 & 3.388616\end{array}$

$\begin{array}{lllll}6 & 1.993898 & -4.430860 & 2.185439\end{array}$

$\begin{array}{lllll}7 & 0.961679 & -3.420175 & -2.918283\end{array}$

$\begin{array}{llll}7 & 1.134542 & 3.771112 & -2.339362\end{array}$

$\begin{array}{lllll}7 & 1.207447 & 2.389281 & 2.806037\end{array}$

$\begin{array}{lllll}7 & 1.207311 & -3.436620 & 2.800925\end{array}$

$\begin{array}{lllll}8 & 1.543656 & -1.396998 & 3.896809\end{array}$

$\begin{array}{lllll}8 & 1.587877 & -5.385868 & 1.572812\end{array}$

$\begin{array}{lllll}8 & 1.431705 & -5.500717 & -1.973171\end{array}$

$8 \quad 1.164647 \quad-1.194140 \quad-3.555120$

$\begin{array}{lllll}8 & 1.234141 & 1.784119 & -3.538132\end{array}$

$\begin{array}{lllll}8 & 1.559363 & 3.576462 & 0.834780\end{array}$

$\begin{array}{lllll}8 & 1.655059 & 5.769009 & -1.267104\end{array}$

$\begin{array}{llll}8 & 1.511096 & 1.324492 & 4.855358\end{array}$

$1 \quad 0.188889 \quad-3.427926 \quad 2.697175$

$\begin{array}{llll}1 & 0.209693 & 2.156005 & 2.721313\end{array}$

$\begin{array}{lllll}1 & 0.114967 & 3.842618 & -2.242888\end{array}$

$1 \quad-0.049684 \quad-3.400208 \quad-2.737693$

$\begin{array}{lllll}7 & 4.530264 & 4.918049 & -2.065388\end{array}$

$\begin{array}{lllll}7 & 4.258630 & 2.535018 & -3.617184\end{array}$

$\begin{array}{lllll}7 & 4.424931 & 3.762098 & 1.977851\end{array}$

$\begin{array}{llll}7 & 4.473520 & 2.115805 & 4.306875\end{array}$

$7 \quad 4.146386 \quad-1.963134 \quad-3.656408$

$\begin{array}{lllll}7 & 4.362374 & -4.534879 & -2.427553\end{array}$

$\begin{array}{llll}7 & 4.466851 & -2.255233 & 3.724290\end{array}$

$\begin{array}{lllll}7 & 4.553949 & -4.604249 & 2.107644\end{array}$

$\begin{array}{llll}6 & 1.238895 & -1.525521 & 0.334122\end{array}$

$\begin{array}{lllll}8 & 1.050257 & -2.697445 & -0.162810\end{array}$

$8 \begin{array}{lllll}8 & 0.403324 & -0.850444 & 0.967393\end{array}$

$\begin{array}{lllll}6 & -1.064283 & 0.802732 & -1.369832\end{array}$

$\begin{array}{lllll}1 & -0.228845 & 0.196636 & -1.716841\end{array}$

$\begin{array}{lllll}6 & 2.609460 & -0.963137 & 0.123427\end{array}$

$\begin{array}{lllll}6 & 2.786594 & 0.421827 & 0.081742\end{array}$

$\begin{array}{lllll}6 & 3.716380 & -1.806827 & -0.009044\end{array}$

$\begin{array}{lllll}6 & 4.058248 & 0.959254 & -0.075777\end{array}$

$\begin{array}{llll}1 & 1.915536 & 1.059221 & 0.170670\end{array}$

$\begin{array}{lllll}6 & 4.992598 & -1.265135 & -0.113769\end{array}$

$\begin{array}{lllll}1 & 3.565809 & -2.879243 & -0.011115\end{array}$

$\begin{array}{lllll}6 & 5.178467 & 0.120443 & -0.138690\end{array}$

$14.192560 \quad 2.036360 \quad-0.125274$

$\begin{array}{llll}1 & 5.857982 & -1.914349 & -0.170254\end{array}$ $\begin{array}{llll}6 & 6.555812 & 0.698296 & -0.170199\end{array}$

$\begin{array}{llll}1 & 6.892599 & 0.924427 & 0.843122\end{array}$

$\begin{array}{lllll}1 & 7.277827 & 0.007341 & -0.605339\end{array}$

$1 \quad 6.582023 \quad 1.626954 \quad-0.736677$

$\begin{array}{lllll}6 & -2.014874 & 0.063298 & -0.447844\end{array}$

$1 \quad-2.472827 \quad-0.759248 \quad-1.009706$

$\begin{array}{lllll}1 & -1.351634 & -0.402684 & 0.289432\end{array}$

$\begin{array}{llll}6 & -3.074552 & 0.912146 & 0.268625\end{array}$

$\begin{array}{lllll}1 & -2.603877 & 1.833711 & 0.622795\end{array}$

$1-3.356245 \quad 0.384198 \quad 1.183823$

$\begin{array}{lllll}6 & -4.357603 & 1.239631 & -0.507265\end{array}$

$\begin{array}{llll}1 & -4.971124 & 1.899890 & 0.120920\end{array}$

$1 \quad-4.127464 \quad 1.805510 \quad-1.414854$

$1-0.687028 \quad 1.732688 \quad-0.952745$

$\begin{array}{lllll}6 & -5.213936 & 0.016745 & -0.866062\end{array}$

$1-6.101216 \quad 0.361501 \quad-1.408351$

$1 \quad-4.674473 \quad-0.643580-1.558908$

$\begin{array}{llll}6 & -5.667095 & -0.794568 & 0.347684\end{array}$

$\begin{array}{llll}1 & -6.170801 & -0.156415 & 1.081940\end{array}$

$\begin{array}{lllll}1 & -6.376113 & -1.568178 & 0.053042\end{array}$

$\begin{array}{lllll}1 & -4.830492 & -1.286030 & 0.853231\end{array}$

\section{5:9b ${ }^{+} @ 1^{-}$}

$\begin{array}{llll}6 & 8.986349 & 2.876661 & 2.118233\end{array}$

$\begin{array}{lllll}6 & 8.403902 & 3.132917 & 0.729796\end{array}$

$\begin{array}{lllll}6 & 8.412843 & 1.621593 & 2.772332\end{array}$

$\begin{array}{llll}1 & 8.663389 & 3.714314 & 2.737421\end{array}$

$\begin{array}{lllll}6 & 8.911832 & 2.494968 & -0.405960\end{array}$

$\begin{array}{lllll}6 & 7.354860 & 4.040798 & 0.527775\end{array}$

$\begin{array}{lllll}6 & 8.963045 & 0.348308 & 2.611723\end{array}$

$\begin{array}{lllll}6 & 7.277737 & 1.718452 & 3.585031\end{array}$

$\begin{array}{lllll}6 & 8.450327 & 2.739655 & -1.703757\end{array}$

$1 \quad 9.709574 \quad 1.773563 \quad-0.273257$

$\begin{array}{lllll}6 & 6.862895 & 4.325671 & -0.741519\end{array}$

$\begin{array}{lllll}8 & 6.787347 & 4.706233 & 1.629033\end{array}$

$\begin{array}{lllll}6 & 8.444514 & -0.792387 & 3.239630\end{array}$

$\begin{array}{lllll}1 & 9.823820 & 0.232902 & 1.963512\end{array}$

$\begin{array}{lllll}6 & 6.754919 & 0.636417 & 4.273741\end{array}$

$\begin{array}{lllll}8 & 6.681138 & 2.975598 & 3.711711\end{array}$

$\begin{array}{lllll}6 & 9.060113 & 2.030841 & -2.911433\end{array}$

$\begin{array}{lllll}6 & 7.419046 & 3.678166 & -1.840468\end{array}$

$1 \quad 6.053080 \quad 5.032651 \quad-0.870095$

$\begin{array}{lllll}6 & 5.571071 & 4.215265 & 2.016109\end{array}$

$\begin{array}{lllll}6 & 9.015172 & -2.183373 & 2.975333\end{array}$

$\begin{array}{lllll}6 & 7.352889 & -0.606404 & 4.096604\end{array}$

$\begin{array}{llll}1 & 5.887651 & 0.747615 & 4.910901\end{array}$

$\begin{array}{lllll}6 & 5.533416 & 3.209299 & 3.024796\end{array}$

$1 \quad 8.761803 \quad 2.610276 \quad-3.785826$

$\begin{array}{lllll}6 & 8.473336 & 0.633795 & -3.106205\end{array}$

$\begin{array}{lllll}8 & 6.908330 & 3.974143 & -3.123962\end{array}$

$\begin{array}{lllll}6 & 8.399764 & -2.790602 & 1.712623\end{array}$

$\begin{array}{lllll}1 & 8.689668 & -2.811468 & 3.805268\end{array}$

$\begin{array}{lllll}8 & 6.830010 & -1.700938 & 4.806887\end{array}$

$\begin{array}{lllll}6 & 9.018191 & -0.518845 & -2.532534\end{array}$

$\begin{array}{lllll}6 & 7.337700 & 0.462680 & -3.906030\end{array}$

$\begin{array}{lllll}6 & 5.688347 & 3.389291 & -3.322283\end{array}$

$\begin{array}{lllll}6 & 3.352037 & 4.056231 & 1.815469\end{array}$

$\begin{array}{lllll}6 & 8.959858 & -2.638227 & 0.441725\end{array}$ $\begin{array}{llll}6 & 7.229962 & -3.555748 & 1.796075\end{array}$

$\begin{array}{lllll}6 & 5.594407 & -2.091879 & 4.376796\end{array}$

$\begin{array}{llll}6.340396 & 2.970676 & 2.679947\end{array}$

$\begin{array}{lllll}6 & 8.484298 & -1.795559 & -2.744196\end{array}$

$\begin{array}{llll}9.893433 & -0.421830 & -1.901315\end{array}$

$\begin{array}{llll}6.780552 & -0.779166 & -4.170034\end{array}$

$\begin{array}{llll}6.770798 & 1.611571 & -4.471467\end{array}$

$\begin{array}{llll}5.646681 & 2.080192 & -3.877521\end{array}$

$\begin{array}{llll}8.423587 & -3.234554 & -0.705252\end{array}$

$\begin{array}{llll}9.861340 & -2.046017 & 0.340598\end{array}$

$\begin{array}{llll}6.654185 & -4.173667 & 0.693841\end{array}$

$\begin{array}{llll}6.679506 & -3.746521 & 3.065014\end{array}$

$\begin{array}{llll}5.528570 & -3.097224 & 3.371003\end{array}$

$\begin{array}{llll}9.055766 & -3.046040 & -2.081630\end{array}$

$\begin{array}{llll}7.365815 & -1.890902 & -3.578966\end{array}$

$\begin{array}{llll}5.905682 & -0.879991 & -4.796952\end{array}$

$\begin{array}{llll}3.512943 & 3.237233 & -2.843495\end{array}$

$\begin{array}{llll}7.266235 & -4.007515 & -0.543203\end{array}$

$\begin{array}{llll}5.757748 & -4.771248 & 0.794558\end{array}$

$\begin{array}{llll}3.374378 & -1.881961 & 4.287171\end{array}$

$\begin{array}{llll}8.733605 & -3.892586 & -2.688435\end{array}$

$\begin{array}{llll}6.849971 & -3.163125 & -3.853083\end{array}$

$\begin{array}{llll}3.531854 & 1.896389 & -3.207568\end{array}$

$\begin{array}{llll}6.755240 & -4.698497 & -1.651193\end{array}$

$\begin{array}{llll}3.331001 & -2.777521 & 3.228569\end{array}$

$\begin{array}{llll}5.624828 & -3.463749 & -3.346214\end{array}$

$\begin{array}{llll}5.573019 & -4.287893 & -2.183805\end{array}$

$\begin{array}{llll}3.396042 & -3.410983 & -3.384560\end{array}$

$\begin{array}{llll}3.340865 & -4.240989 & -2.273329\end{array}$

$\begin{array}{llll}10.590177 & -3.058598 & -2.048836\end{array}$

$\begin{array}{llll}10.948068 & -3.990967 & -1.604492\end{array}$

$\begin{array}{llll}11.015080 & -2.233961 & -1.470027\end{array}$

$\begin{array}{llll}10.595798 & 2.025645 & -2.865444\end{array}$

$\begin{array}{llll}10.997254 & 1.564537 & -3.771692\end{array}$

$\begin{array}{llll}10.995326 & 1.475361 & -2.009208\end{array}$

$\begin{array}{llll}10.523116 & 2.878995 & 2.107392\end{array}$

$\begin{array}{llll}10.893238 & 3.824267 & 1.702249\end{array}$

$\begin{array}{llll}10.946571 & 2.073226 & 1.501508\end{array}$

$\begin{array}{lll}10.550104 & -2.203400 & 2.955662\end{array}$

$\begin{array}{llll}10.939958 & -1.827274 & 3.904986\end{array}$

$\begin{array}{llll}10.977302 & -1.588329 & 2.158871\end{array}$

$\begin{array}{llll}10.910583 & -3.225315 & 2.811644\end{array}$

$\begin{array}{lll}10.904445 & 2.762129 & 3.125152\end{array}$

$\begin{array}{llll}10.970105 & 3.050406 & -2.800277\end{array}$

$\begin{array}{llll}10.985337 & -2.983312 & -3.065228\end{array}$

$\begin{array}{llll}2.169329 & 3.484819 & -2.200934\end{array}$

$\begin{array}{llll}2.318986 & 1.303757 & -2.582757\end{array}$

$\begin{array}{llll}1.995963 & -3.029979 & -3.766159\end{array}$

$\begin{array}{llll}1.890080 & -4.485571 & -1.931085\end{array}$

$\begin{array}{lll}1.931786 & 4.356832 & 1.421856\end{array}$

$\begin{array}{lll}1.927595 & 2.451142 & 2.770076\end{array}$

$\begin{array}{llll}1.966801 & -1.424948 & 4.570680\end{array}$

$\begin{array}{llll}1.924984 & -2.844279 & 2.716739\end{array}$

$\begin{array}{llll}1.180325 & -3.738373 & -2.891926\end{array}$

$\begin{array}{llll}1.571735 & 2.206958 & -1.997268\end{array}$

$\begin{array}{llll}1.173809 & 3.347626 & 2.033066\end{array}$

$\begin{array}{llll}1.190872 & -2.046671 & 3.570816\end{array}$

$\begin{array}{lllll}8 & 1.548091 & -0.705710 & 5.443201\end{array}$ 
$\begin{array}{llll}8 & 1.538079 & -3.434036 & 1.720737\end{array}$

$\begin{array}{lllll}8 & 1.410346 & -5.150356 & -1.046794\end{array}$

$\begin{array}{lllll}8 & 1.635810 & -2.221008 & -4.601784\end{array}$

$\begin{array}{lllll}8 & 2.183011 & 0.011555 & -2.531893\end{array}$

$\begin{array}{lllll}8 & 1.504876 & 5.260294 & 0.741819\end{array}$

$\begin{array}{lllll}8 & 1.669617 & 4.539347 & -1.891762\end{array}$

$\begin{array}{lllll}8 & 1.541815 & 1.453895 & 3.352866\end{array}$

$\begin{array}{lllll}1 & 0.168475 & -1.927281 & 3.546958\end{array}$

$\begin{array}{lllll}1 & 0.156676 & 3.328887 & 1.927464\end{array}$

$\begin{array}{lllll}1 & 0.037189 & 2.086101 & -1.359734\end{array}$

$\begin{array}{lllll}1 & 0.159495 & -3.719080 & -2.970958\end{array}$

$\begin{array}{lllll}7 & 4.603560 & 4.001758 & -2.864240\end{array}$

$\begin{array}{lllll}7 & 4.578210 & 1.300656 & -3.771307\end{array}$

$\begin{array}{lllll}7 & 4.474095 & 4.673536 & 1.433272\end{array}$

$\begin{array}{lllll}7 & 4.429071 & 2.538526 & 3.321097\end{array}$

$\begin{array}{llll}4.538145 & -3.006442 & -3.946538\end{array}$

$\begin{array}{llll}4.431783 & -4.686995 & -1.641669\end{array}$

$\begin{array}{lllll}7 & 4.516192 & -1.500290 & 4.867499\end{array}$

$\begin{array}{lllll}7 & 4.401917 & -3.419143 & 2.757558\end{array}$

$\begin{array}{lllll}6 & -8.987687 & -3.736824 & 0.507730\end{array}$

$\begin{array}{lllll}6 & -8.387437 & -3.254206 & -0.822527\end{array}$

$\begin{array}{lllll}6 & -8.436270 & -2.960523 & 1.708658\end{array}$

$\begin{array}{lllll}1 & -8.645516 & -4.765430 & 0.632408\end{array}$

$\begin{array}{lllll}6 & -8.953543 & -2.254033 & -1.624703\end{array}$

$\begin{array}{lllll}6 & -7.195262 & -3.822262 & -1.289139\end{array}$

$\begin{array}{lllll}6 & -8.988495 & -1.732201 & 2.104505\end{array}$

$\begin{array}{lllll}6 & -7.325474 & -3.411896 & 2.437191\end{array}$

$\begin{array}{lllll}6 & -8.374005 & -1.827841 & -2.830338\end{array}$

$\begin{array}{lllll}6 & -9.860604 & -1.766291 & -1.286771\end{array}$

$\begin{array}{lllll}6 & -6.618886 & -3.489899 & -2.503235\end{array}$

$\begin{array}{lllll}8 & -6.577726 & -4.787603 & -0.497974\end{array}$

$\begin{array}{llll}6 & -8.452605 & -0.932463 & 3.119046\end{array}$

$\begin{array}{llll}-9.856486 & -1.367144 & 1.568531\end{array}$

$\begin{array}{llll}6 & -6.750532 & -2.651071 & 3.450971\end{array}$

$\begin{array}{llll}8 & -6.738470 & -4.658779 & 2.158210\end{array}$

$\begin{array}{lllll}6 & -8.897645 & -0.656135 & -3.657141\end{array}$

$\begin{array}{lllll}6 & -7.221989 & -2.496009 & -3.256475\end{array}$

$\begin{array}{lllll}1 & -5.708879 & -3.968485 & -2.836730\end{array}$

$\begin{array}{lllll}6 & -5.446392 & -4.433693 & 0.165795\end{array}$

$\begin{array}{lllll}6 & -9.056964 & 0.413097 & 3.532877\end{array}$

$\begin{array}{lllll}6 & -7.300145 & -1.415637 & 3.754092\end{array}$

$\begin{array}{llll}1 & -5.879343 & -3.013407 & 3.981202\end{array}$

$\begin{array}{lllll}6 & -5.501846 & -4.535787 & 1.580637\end{array}$

$\begin{array}{lllll}1 & -8.550903 & -0.823098 & -4.677611\end{array}$

$\begin{array}{lllll}6 & -8.271561 & 0.669451 & -3.210332\end{array}$

$\begin{array}{lllll}8 & -6.664039 & -2.146596 & -4.492222\end{array}$

$\begin{array}{llll}6 & -8.463964 & 1.568615 & 2.724674\end{array}$

$\begin{array}{lllll}1 & -8.747472 & 0.576531 & 4.566083\end{array}$

$\begin{array}{lllll}8 & -6.730146 & -0.648002 & 4.773244\end{array}$

$\begin{array}{lllll}6 & -8.827673 & 1.420536 & -2.171578\end{array}$

$\begin{array}{lllll}6 & -7.141034 & 1.215659 & -3.837391\end{array}$

$\begin{array}{lllll}6 & -5.432047 & -1.570507 & -4.430465\end{array}$

$\begin{array}{lllll}6 & -3.290495 & -3.879071 & 0.303261\end{array}$

$\begin{array}{llll}6 & -8.986407 & 1.955917 & 1.481734\end{array}$

$\begin{array}{llll}6 & -7.354665 & 2.284282 & 3.192472\end{array}$

$\begin{array}{llll}6 & -5.560030 & 0.001447 & 4.529520\end{array}$

$\begin{array}{lllll}6 & -3.303940 & -4.165297 & 1.664322\end{array}$

$\begin{array}{lllll}6 & -8.355523 & 2.677963 & -1.781976\end{array}$ $\begin{array}{llll}1 & -9.678896 & 1.008893 & -1.642850\end{array}$

$\begin{array}{lllll}6 & -6.650361 & 2.474647 & -3.512152\end{array}$

$\begin{array}{lllll}8 & -6.509627 & 0.504890 & -4.863925\end{array}$

$\begin{array}{lllll}6 & -5.355192 & -0.156105 & -4.560852\end{array}$

$\begin{array}{lllll}6 & -8.430583 & 2.979207 & 0.709109\end{array}$

$\begin{array}{lllll}1 & -9.858405 & 1.435391 & 1.103793\end{array}$

$\begin{array}{lllll}6 & -6.745228 & 3.289309 & 2.449376\end{array}$

$\begin{array}{llll}8 & -6.846126 & 2.006211 & 4.471004\end{array}$

$\begin{array}{lllll}6 & -5.614897 & 1.421557 & 4.463281\end{array}$

$\begin{array}{lllll}6 & -9.004817 & 3.438263 & -0.629955\end{array}$

$\begin{array}{lllll}6 & -7.265710 & 3.196221 & -2.494134\end{array}$

$\begin{array}{lllll}1 & -5.793985 & 2.881870 & -4.035316\end{array}$

$\begin{array}{lllll}6 & -3.218847 & -1.657880 & -4.185578\end{array}$

$\begin{array}{llll}6 & -7.283600 & 3.604411 & 1.212660\end{array}$

$\begin{array}{lllll}1 & -5.875671 & 3.810299 & 2.828146\end{array}$

$\begin{array}{lllll}6 & -3.351007 & 0.105156 & 4.247119\end{array}$

$\begin{array}{lllll}1 & -8.710248 & 4.481773 & -0.746873\end{array}$

$\begin{array}{lllll}8 & -6.792337 & 4.485991 & -2.218416\end{array}$

$\begin{array}{lllll}6 & -3.142420 & -0.272582 & -4.258618\end{array}$

$\begin{array}{lllll}8 & -6.748341 & 4.646297 & 0.461219\end{array}$

$\begin{array}{lllll}6 & -3.394621 & 1.491748 & 4.264273\end{array}$

$\begin{array}{lllll}6 & -5.577795 & 4.552567 & -1.592570\end{array}$

$\begin{array}{lllll}6 & -5.553934 & 4.514638 & -0.169557\end{array}$

$\begin{array}{lllll}6 & -3.349816 & 4.691868 & -1.601935\end{array}$

$\begin{array}{lllll}6 & -3.327989 & 4.519257 & -0.225029\end{array}$

$\begin{array}{lllll}6 & -10.538410 & 3.384500 & -0.671192\end{array}$

$\begin{array}{lllll}1 & -10.955729 & 3.988510 & 0.138759\end{array}$

$\begin{array}{lllll}1 & -10.935374 & 2.371431 & -0.564363\end{array}$

$\begin{array}{lllll}6 & -10.431077 & -0.589675 & -3.696571\end{array}$

$\begin{array}{lllll}1 & -10.756526 & 0.233862 & -4.337251\end{array}$

$\begin{array}{lllll}1 & -10.878662 & -0.435411 & -2.710767\end{array}$

$\begin{array}{lllll}6 & -10.522377 & -3.760163 & 0.478423\end{array}$

$\begin{array}{lllll}1 & -10.872673 & -4.378611 & -0.352030\end{array}$

$\begin{array}{lllll}1 & -10.961877 & -2.766275 & 0.355994\end{array}$

$\begin{array}{lllll}6 & -10.591842 & 0.399263 & 3.506954\end{array}$

$\begin{array}{lllll}1 & -10.970844 & -0.401960 & 4.146651\end{array}$

$\begin{array}{lllll}1 & -11.001391 & 0.241339 & 2.505351\end{array}$

$\begin{array}{lllll}1 & -10.980754 & 1.352430 & 3.874337\end{array}$

$\begin{array}{lllll}1 & -10.909248 & -4.177648 & 1.411556\end{array}$

$\begin{array}{lllll}1 & -10.833566 & -1.523573 & -4.097796\end{array}$

$\begin{array}{lllll}1 & -10.901069 & 3.780438 & -1.623275\end{array}$

$\begin{array}{lllll}6 & -1.832666 & -2.215407 & -4.059346\end{array}$

$\begin{array}{lllll}6 & -1.689285 & 0.126620 & -4.152457\end{array}$

$\begin{array}{lllll}6 & -1.930261 & 4.872763 & -2.078793\end{array}$

$\begin{array}{lllll}6 & -1.893656 & 4.456368 & 0.218929\end{array}$

$\begin{array}{lllll}6 & -1.902488 & -3.410062 & -0.068398\end{array}$

$\begin{array}{lllll}6 & -1.900993 & -4.041348 & 2.190383\end{array}$

$\begin{array}{lllll}6 & -1.934791 & -0.316112 & 3.986046\end{array}$

$\begin{array}{lllll}6 & -1.990120 & 2.010893 & 4.088980\end{array}$

$\begin{array}{lllll}7 & -1.157210 & 4.799368 & -0.903184\end{array}$

$\begin{array}{lllll}7 & -1.003389 & -1.096889 & -4.035722\end{array}$

$\begin{array}{lllll}7 & -1.165885 & -3.555000 & 1.098389\end{array}$

$\begin{array}{lllll}7 & -1.205379 & 0.851328 & 3.940387\end{array}$

$\begin{array}{lllll}8 & -1.532895 & -1.453483 & 3.795895\end{array}$

$\begin{array}{lllll}8 & -1.580646 & 3.145178 & 4.074128\end{array}$

$\begin{array}{lllll}8 & -1.478336 & 4.117508 & 1.310972\end{array}$

$\begin{array}{lllll}8 & -1.517005 & 5.054764 & -3.196484\end{array}$

$\begin{array}{lllll}8 & -1.184976 & 1.223223 & -4.174174\end{array}$ $\begin{array}{llll}8 & -1.496438 & -2.973355 & -1.127510\end{array}$

$8 \begin{array}{llll}8 & -1.481195 & -3.376852 & -4.008916\end{array}$

$\begin{array}{llll}8 & -1.459923 & -4.312554 & 3.281350\end{array}$

$\begin{array}{llll}1 & -0.205589 & 0.915786 & 3.715885\end{array}$

$\begin{array}{lllll}1 & -0.145655 & -3.452460 & 1.159014\end{array}$

$\begin{array}{llll}0.004705 & -1.200316 & -4.159442\end{array}$

$\begin{array}{llll}-0.137034 & 4.801409 & -0.931854\end{array}$

$\begin{array}{llll}-4.365882 & -2.334194 & -4.255681\end{array}$

$\begin{array}{llll}-4.212569 & 0.507269 & -4.451609\end{array}$

$\begin{array}{llll}-4.360379 & -4.039547 & -0.481920\end{array}$

$\begin{array}{llll}-4.421968 & -4.454215 & 2.339335\end{array}$

$\begin{array}{llll}-4.478249 & 4.672527 & -2.319863\end{array}$

$\begin{array}{llll}-4.430014 & 4.444942 & 0.526007\end{array}$

$\begin{array}{llll}-4.427915 & -0.672354 & 4.392674\end{array}$

$\begin{array}{llll}-4.534433 & 2.180242 & 4.357541\end{array}$

$\begin{array}{llll}6 & -1.200342 & 1.227182 & -0.114931\end{array}$

$\begin{array}{llll}8 & -0.375530 & 0.991502 & 0.760523\end{array}$

$\begin{array}{lllll}8 & -0.943033 & 1.913939 & -1.225857\end{array}$

$\begin{array}{lllll}6 & 1.374191 & -0.561934 & -1.434912\end{array}$

$\begin{array}{llll}0.425208 & -0.039463 & -1.412816\end{array}$

$\begin{array}{lllll}6 & -2.601627 & 0.760289 & -0.079655\end{array}$

$\begin{array}{lllll}6 & -3.538035 & 1.223127 & -1.012558\end{array}$

$\begin{array}{lllll}6 & -2.978113 & -0.175203 & 0.886354\end{array}$

$\begin{array}{lllll}6 & -4.854528 & 0.798576 & -0.925334\end{array}$

$\begin{array}{llll}-3.225931 & 1.893325 & -1.802925\end{array}$

$\begin{array}{llll}6 & -4.295843 & -0.607726 & 0.956665\end{array}$

$\begin{array}{llll}-2.217393 & -0.572574 & 1.544256\end{array}$

$\begin{array}{llll}6 & -5.252005 & -0.104418 & 0.065849\end{array}$

$\begin{array}{llll}-5.588386 & 1.160100 & -1.633796\end{array}$

$\begin{array}{llll}-4.589968 & -1.335117 & 1.706882\end{array}$

$\begin{array}{llll}6 & -6.682634 & -0.512331 & 0.164921\end{array}$

$\begin{array}{llll}-7.164510 & -0.506944 & -0.811357\end{array}$

$\begin{array}{llll}-6.777264 & -1.504799 & 0.595087\end{array}$

$\begin{array}{llll}-7.236809 & 0.178475 & 0.804205\end{array}$

$\begin{array}{llll}6.128316 & -0.483067 & -0.117363\end{array}$

$\begin{array}{llll}2.362012 & 0.564034 & 0.099130\end{array}$

$\begin{array}{llll}1.403280 & -0.771133 & 0.649236\end{array}$

$\begin{array}{llll}3.371078 & -1.377372 & -0.012369\end{array}$

$\begin{array}{llll}3.075253 & -2.418454 & -0.175684\end{array}$

$\begin{array}{llll}3.711148 & -1.344595 & 1.026945\end{array}$

$\begin{array}{llll}4.562800 & -1.030624 & -0.916509\end{array}$

$\begin{array}{llll}5.325582 & -1.805997 & -0.773744\end{array}$

$\begin{array}{llll}4.269852 & -1.066353 & -1.968265\end{array}$

$\begin{array}{llll}1.198767 & -1.588170 & -1.744495\end{array}$

$\begin{array}{llll}5.222452 & 0.322992 & -0.626824\end{array}$

$\begin{array}{llll}6.029831 & 0.475450 & -1.351097\end{array}$

$\begin{array}{llll}4.509640 & 1.142049 & -0.790445\end{array}$

$\begin{array}{llll}5.793564 & 0.436723 & 0.783148\end{array}$

$\begin{array}{llll}6.499209 & -0.374769 & 0.988582\end{array}$

$\begin{array}{llll}6.336087 & 1.375324 & 0.898493\end{array}$

$\begin{array}{llll}5.016102 & 0.407186 & 1.551613\end{array}$

\section{$3 b @ 1_{2}$}

$6 \quad 8.961618 \quad-0.734729 \quad 3.613884$

$\begin{array}{lllll}6 & 8.388674 & 0.618655 & 3.174151\end{array}$

$\begin{array}{lllll}6 & 8.389640 & -1.889077 & 2.789465\end{array}$

$1 \quad 8.611112 \quad-0.890380 \quad 4.635149$

$\begin{array}{llll}6 & 8.973488 & 1.428581 & 2.193572\end{array}$ 
$\begin{array}{llll}6 & 7.219917 & 1.107290 & 3.770961\end{array}$

$\begin{array}{lllll}6 & 8.949712 & -2.262274 & 1.558281\end{array}$

$\begin{array}{llll}6 & 7.260309 & -2.604149 & 3.212411\end{array}$

$\begin{array}{llll}6 & 8.450730 & 2.675996 & 1.824630\end{array}$

$\begin{array}{lllll}1 & 9.865198 & 1.073138 & 1.690676\end{array}$

$\begin{array}{llll}6 & 6.687146 & 2.353153 & 3.481044\end{array}$

$\begin{array}{lllll}8 & 6.599300 & 0.309746 & 4.732467\end{array}$

$\begin{array}{lllll}6 & 8.410861 & -3.255117 & 0.734886\end{array}$

$\begin{array}{lllll}1 & 9.834301 & -1.736934 & 1.218868\end{array}$

$\begin{array}{llll}6 & 6.677485 & -3.591930 & 2.425594\end{array}$

$\begin{array}{lllll}8 & 6.684568 & -2.348982 & 4.467868\end{array}$

$\begin{array}{lllll}6 & 9.042883 & 3.523245 & 0.700638\end{array}$

$\begin{array}{lllll}6 & 7.319215 & 3.124305 & 2.516627\end{array}$

$\begin{array}{llll}1 & 5.796567 & 2.708776 & 3.980997\end{array}$

$\begin{array}{lllll}6 & 5.443653 & -0.313225 & 4.387900\end{array}$

$\begin{array}{lllll}6 & 9.029678 & -3.657510 & -0.608510\end{array}$

$\begin{array}{lllll}6 & 7.243756 & -3.880783 & 1.193838\end{array}$

$\begin{array}{lllll}1 & 5.793639 & -4.115557 & 2.765747\end{array}$

$\begin{array}{lllll}6 & 5.464491 & -1.734290 & 4.391931\end{array}$

$\begin{array}{lllll}1 & 8.742039 & 4.552252 & 0.901231\end{array}$

$\begin{array}{lllll}6 & 8.418671 & 3.152022 & -0.646011\end{array}$

$\begin{array}{lllll}8 & 6.825220 & 4.406896 & 2.248359\end{array}$

$\begin{array}{lllll}6 & 8.449260 & -2.825577 & -1.752246\end{array}$

$\begin{array}{lllll}1 & 8.715306 & -4.686369 & -0.789622\end{array}$

$\begin{array}{lllll}8 & 6.669761 & -4.872759 & 0.395441\end{array}$

$\begin{array}{lllll}6 & 8.921899 & 2.115266 & -1.437103\end{array}$

$\begin{array}{lllll}6 & 7.304659 & 3.840280 & -1.147004\end{array}$

$\begin{array}{lllll}6 & 5.603166 & 4.454044 & 1.646133\end{array}$

$\begin{array}{lllll}6 & 3.270639 & -0.365741 & 3.905300\end{array}$

$\begin{array}{lllll}6 & 8.966541 & -1.566896 & -2.096761\end{array}$

$\begin{array}{lllll}6 & 7.339846 & -3.273439 & -2.480644\end{array}$

$\begin{array}{lllll}6 & 5.515418 & -4.589471 & -0.262371\end{array}$

$\begin{array}{llll}6 & 3.256455 & -1.744623 & 4.072134\end{array}$

$\begin{array}{lllll}6 & 8.386162 & 1.759705 & -2.681122\end{array}$

$\begin{array}{lllll}1 & 9.768849 & 1.551855 & -1.064373\end{array}$

$\begin{array}{lllll}6 & 6.754263 & 3.552238 & -2.389068\end{array}$

$\begin{array}{lllll}8 & 6.757563 & 4.880710 & -0.391055\end{array}$

$\begin{array}{lllll}6 & 5.570566 & 4.643161 & 0.237765\end{array}$

$\begin{array}{lllll}6 & 8.390911 & -0.745886 & -3.070950\end{array}$

$\begin{array}{lllll}1 & 9.844397 & -1.209847 & -1.571412\end{array}$

$\begin{array}{lllll}6 & 6.713223 & -2.484208 & -3.437277\end{array}$

$\begin{array}{lllll}8 & 6.824218 & -4.560650 & -2.252966\end{array}$

$\begin{array}{lllll}6 & 5.584349 & -4.544894 & -1.682624\end{array}$

$\begin{array}{lllll}6 & 8.966918 & 0.606343 & -3.495893\end{array}$

$\begin{array}{lllll}6 & 7.300319 & 2.516794 & -3.140678\end{array}$

$\begin{array}{lllll}1 & 5.903468 & 4.110664 & -2.758362\end{array}$

$\begin{array}{lllll}6 & 3.375417 & 4.330131 & 1.671838\end{array}$

$\begin{array}{lllll}6 & 7.230422 & -1.225946 & -3.690248\end{array}$

$\begin{array}{lllll}1 & 5.835975 & -2.839973 & -3.960640\end{array}$

$\begin{array}{lllll}6 & 3.319643 & -4.228889 & -0.386988\end{array}$

$\begin{array}{lllll}1 & 8.632377 & 0.764342 & -4.522081\end{array}$

$\begin{array}{lllll}8 & 6.738924 & 2.254901 & -4.401268\end{array}$

$\begin{array}{llll}6 & 3.347983 & 4.450515 & 0.289015\end{array}$

$\begin{array}{lllll}8 & 6.622082 & -0.414618 & -4.645613\end{array}$

$\begin{array}{lllll}6 & 3.369389 & -4.297177 & -1.771866\end{array}$

$\begin{array}{lllll}6 & 5.513462 & 1.650360 & -4.341585\end{array}$

$\begin{array}{lllll}6 & 5.470911 & 0.229102 & -4.322671\end{array}$

$\begin{array}{lllll}6 & 3.295755 & 1.694889 & -4.108164\end{array}$ $\begin{array}{llll}6 & 3.279965 & 0.317151 & -3.928276\end{array}$

$\begin{array}{lllll}6 & 10.502643 & 0.610952 & -3.506986\end{array}$

$\begin{array}{lllll}1 & 10.875023 & -0.185747 & -4.156204\end{array}$

$\begin{array}{lllll}1 & 10.938312 & 0.458336 & -2.515728\end{array}$

$\begin{array}{llll}6 & 10.577198 & 3.487842 & 0.666435\end{array}$

$\begin{array}{lllll}1 & 10.950854 & 4.152829 & -0.116460\end{array}$

$\begin{array}{lllll}1 & 10.978799 & 2.489981 & 0.468551\end{array}$

$\begin{array}{llll}6 & 10.496448 & -0.736237 & 3.647740\end{array}$

$\begin{array}{llll}1 & 10.857546 & 0.062394 & 4.300809\end{array}$

$\begin{array}{lllll}1 & 10.946506 & -0.583495 & 2.662872\end{array}$

$\begin{array}{lllll}6 & 10.564171 & -3.640322 & -0.578976\end{array}$

$\begin{array}{lllll}1 & 10.932180 & -4.291183 & 0.218329\end{array}$

$\begin{array}{lllll}1 & 10.977702 & -2.642800 & -0.405994\end{array}$

$\begin{array}{lllll}1 & 10.960157 & -3.999065 & -1.532500\end{array}$

$\begin{array}{lllll}1 & 10.861934 & -1.692294 & 4.031404\end{array}$

$\begin{array}{lllll}1 & 10.979741 & 3.819794 & 1.626984\end{array}$

$\begin{array}{lllll}1 & 10.870999 & 1.568549 & -3.883813\end{array}$

$\begin{array}{lllll}6 & 1.958090 & 4.186758 & 2.162900\end{array}$

$\begin{array}{lllll}6 & 1.921366 & 4.318406 & -0.171767\end{array}$

$\begin{array}{lllll}6 & 1.879311 & 2.196753 & -4.022277\end{array}$

$\begin{array}{lllll}6 & 1.870296 & -0.095574 & -3.600332\end{array}$

$\begin{array}{lllll}6 & 1.892685 & 0.078211 & 3.500162\end{array}$

$\begin{array}{lllll}6 & 1.838362 & -2.223434 & 3.908769\end{array}$

$\begin{array}{lllll}6 & 1.903693 & -3.948559 & 0.021110\end{array}$

$\begin{array}{lllll}6 & 1.968382 & -4.139664 & -2.304082\end{array}$

$\begin{array}{lllll}7 & 1.115167 & 1.043425 & -3.764603\end{array}$

$\begin{array}{llll}7 & 1.183897 & 4.171645 & 0.991993\end{array}$

$\begin{array}{lllll}7 & 1.119978 & -1.065595 & 3.555050\end{array}$

$\begin{array}{lllll}7 & 1.181579 & -3.932004 & -1.152438\end{array}$

$\begin{array}{lllll}8 & 1.489627 & -3.744687 & 1.151540\end{array}$

$\begin{array}{lllll}8 & 1.560103 & -4.186458 & -3.437442\end{array}$

$\begin{array}{lllll}8 & 1.481860 & -1.194346 & -3.230518\end{array}$

$\begin{array}{lllll}8 & 1.447366 & 3.316458 & -4.143393\end{array}$

$\begin{array}{lllll}8 & 1.511973 & 4.302174 & -1.316392\end{array}$

$\begin{array}{lllll}8 & 1.541333 & 1.194818 & 3.161688\end{array}$

$\begin{array}{lllll}8 & 1.538709 & 4.108639 & 3.292864\end{array}$

$\begin{array}{lllll}8 & 1.373690 & -3.328342 & 4.048086\end{array}$

$\begin{array}{lllll}1 & 0.165079 & -3.791815 & -1.212410\end{array}$

$\begin{array}{lllll}1 & 0.113767 & -1.109444 & 3.357052\end{array}$

$\begin{array}{lllll}1 & 0.164525 & 4.078306 & 1.036722\end{array}$

$\begin{array}{lllll}1 & 0.115103 & 1.127278 & -3.539666\end{array}$

$\begin{array}{lllll}7 & 4.507708 & 4.316780 & 2.378414\end{array}$

$\begin{array}{lllll}7 & 4.443165 & 4.618582 & -0.457290\end{array}$

$\begin{array}{lllll}7 & 4.359258 & 0.384287 & 4.088211\end{array}$

$\begin{array}{lllll}7 & 4.365041 & -2.462897 & 4.280420\end{array}$

$\begin{array}{lllll}7 & 4.420915 & 2.394730 & -4.277110\end{array}$

$\begin{array}{lllll}7 & 4.364952 & -0.450362 & -4.056741\end{array}$

$\begin{array}{lllll}7 & 4.386677 & -4.391692 & 0.400756\end{array}$

$\begin{array}{lllll}7 & 4.511745 & -4.433868 & -2.450622\end{array}$

$\begin{array}{lllll}6 & -9.056190 & -2.093252 & -2.930141\end{array}$

$\begin{array}{lllll}6 & -8.477535 & -0.697953 & -3.137712\end{array}$

$\begin{array}{lllll}6 & -8.451522 & -2.766604 & -1.697642\end{array}$

$\begin{array}{lllll}1 & -8.736633 & -2.687515 & -3.786954\end{array}$

$\begin{array}{lllll}6 & -9.009071 & 0.435647 & -2.507926\end{array}$

$\begin{array}{lllll}6 & -7.366547 & -0.504268 & -3.966327\end{array}$

$\begin{array}{lllll}6 & -8.973752 & -2.596459 & -0.413599\end{array}$

$\begin{array}{lllll}6 & -7.335847 & -3.605270 & -1.813576\end{array}$

$\begin{array}{lllll}6 & -8.457649 & 1.712706 & -2.645343\end{array}$ $\begin{array}{lllll}1 & -9.889987 & 0.315408 & -1.888510\end{array}$

$\begin{array}{lllll}6 & -6.760136 & 0.736686 & -4.113328\end{array}$

$\begin{array}{lllll}8 & -6.848326 & -1.594102 & -4.688573\end{array}$

$\begin{array}{lllll}6 & -8.465042 & -3.246954 & 0.717956\end{array}$

$\begin{array}{lllll}1 & -9.814544 & -1.925460 & -0.283881\end{array}$

$\begin{array}{lllll}6 & -6.823808 & -4.316483 & -0.739766\end{array}$

$\begin{array}{lllll}8 & -6.738979 & -3.744046 & -3.070950\end{array}$

$\begin{array}{lllll}6 & -9.061452 & 2.967228 & -2.007558\end{array}$

$\begin{array}{llll}6 & -7.302339 & 1.813825 & -3.429454\end{array}$

$\begin{array}{lllll}1 & -5.883589 & 0.857944 & -4.735580\end{array}$

$\begin{array}{lllll}6 & -5.625824 & -2.010016 & -4.253602\end{array}$

$\begin{array}{llll}6 & -9.041957 & -2.989246 & 2.108123\end{array}$

$\begin{array}{lllll}6 & -7.400586 & -4.134648 & 0.513077\end{array}$

$\begin{array}{lllll}1 & -5.973086 & -4.973251 & -0.865299\end{array}$

$\begin{array}{lllll}6 & -5.581977 & -3.067608 & -3.299637\end{array}$

$\begin{array}{lllll}1 & -8.746290 & 3.804411 & -2.631938\end{array}$

$\begin{array}{lllll}6 & -8.474606 & 3.224454 & -0.621986\end{array}$

$\begin{array}{lllll}8 & -6.724217 & 3.074626 & -3.567568\end{array}$

$\begin{array}{lllll}6 & -8.426615 & -1.739924 & 2.741806\end{array}$

$\begin{array}{lllll}1 & -8.728599 & -3.830020 & 2.728412\end{array}$

$\begin{array}{lllll}8 & -6.874299 & -4.869785 & 1.591311\end{array}$

$\begin{array}{lllll}6 & -8.984998 & 2.594106 & 0.520678\end{array}$

$\begin{array}{lllll}6 & -7.379302 & 4.078692 & -0.439429\end{array}$

$\begin{array}{lllll}6 & -5.572288 & 3.340573 & -2.901088\end{array}$

$\begin{array}{lllll}6 & -3.408942 & -1.802257 & -4.107622\end{array}$

$\begin{array}{lllll}6 & -8.976398 & -0.460520 & 2.624475\end{array}$

$\begin{array}{llll}6 & -7.254206 & -1.852089 & 3.498295\end{array}$

$\begin{array}{llll}6 & -5.632737 & -4.438606 & 1.967074\end{array}$

$\begin{array}{lllll}6 & -3.385052 & -2.743217 & -3.087712\end{array}$

$\begin{array}{lllll}6 & -8.428873 & 2.743727 & 1.793594\end{array}$

$\begin{array}{lllll}1 & -9.845382 & 1.945054 & 0.407145\end{array}$

$\begin{array}{lllll}6 & -6.776407 & 4.247459 & 0.801952\end{array}$

$\begin{array}{lllll}8 & -6.859271 & 4.805671 & -1.524655\end{array}$

$\begin{array}{lllll}6 & -5.625589 & 4.371478 & -1.922058\end{array}$

$\begin{array}{lllll}6 & -8.420398 & 0.670817 & 3.237678\end{array}$

$\begin{array}{llll}1 & -9.871662 & -0.334365 & 2.027495\end{array}$

$\begin{array}{llll}6 & -6.681695 & -0.777646 & 4.160432\end{array}$

$\begin{array}{lllll}8 & -6.693988 & -3.124341 & 3.627124\end{array}$

$\begin{array}{llll}6 & -5.557187 & -3.406542 & 2.945551\end{array}$

$\begin{array}{llll}6 & -9.004028 & 2.068441 & 3.041329\end{array}$

$\begin{array}{llll}6 & -7.294193 & 3.558377 & 1.887377\end{array}$

$\begin{array}{lllll}1 & -5.913009 & 4.890137 & 0.914143\end{array}$

$\begin{array}{lllll}6 & -3.376291 & 3.175961 & -2.573277\end{array}$

$\begin{array}{llll}6 & -7.280310 & 0.469843 & 4.026533\end{array}$

$\begin{array}{lllll}1 & -5.788376 & -0.901730 & 4.757810\end{array}$

$\begin{array}{llll}6 & -3.417902 & -4.333878 & 1.712402\end{array}$

$\begin{array}{llll}1 & -8.664022 & 2.662676 & 3.890424\end{array}$

$\begin{array}{llll}8 & -6.691410 & 3.705747 & 3.139685\end{array}$

$\begin{array}{lllll}6 & -3.403874 & 4.284103 & -1.738874\end{array}$

$\begin{array}{lllll}8 & -6.726120 & 1.549997 & 4.731807\end{array}$

$\begin{array}{lllll}6 & -3.372597 & -3.230931 & 2.552509\end{array}$

$\begin{array}{lllll}6 & -5.512107 & 3.064070 & 3.346963\end{array}$

$\begin{array}{llll}6 & -5.517392 & 1.978087 & 4.266744\end{array}$

$\begin{array}{lllll}6 & -3.308471 & 2.823114 & 3.111932\end{array}$

$\begin{array}{llll}6 & -3.297503 & 1.830795 & 4.082388\end{array}$

$\begin{array}{llll}6 & -10.539454 & 2.079937 & 3.055241\end{array}$

$\begin{array}{llll}1 & -10.909234 & 1.645356 & 3.987600\end{array}$

$\begin{array}{llll}1 & -10.978776 & 1.512489 & 2.230121\end{array}$ 


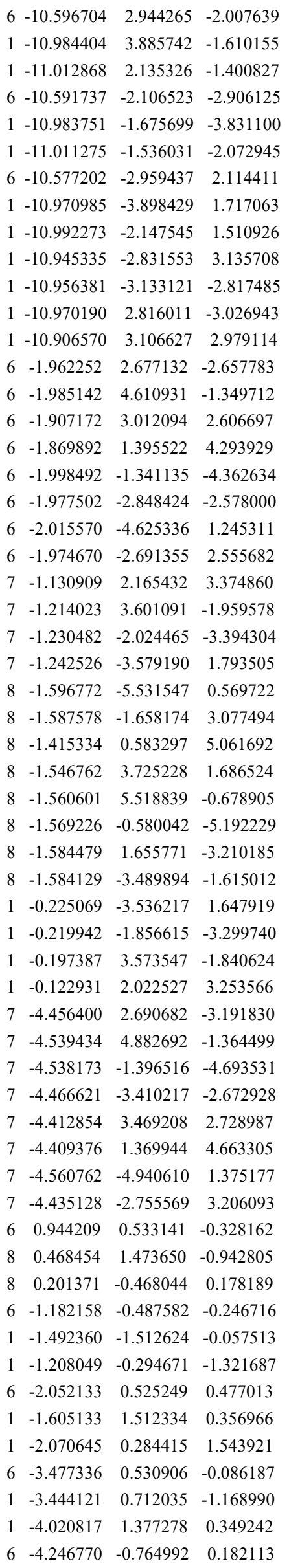

$\begin{array}{llll}1 & -4.308292 & -0.919010 & 1.267123\end{array}$

$\begin{array}{lllll}1 & -3.695469 & -1.623938 & -0.220714\end{array}$

$\begin{array}{lllll}6 & 2.394053 & 0.344149 & -0.122878\end{array}$

$\begin{array}{lllll}6 & 2.910290 & -0.760202 & 0.564929\end{array}$

$\begin{array}{lllll}6 & 3.265197 & 1.273243 & -0.700800\end{array}$

$\begin{array}{lllll}6 & 4.283994 & -0.925695 & 0.673144\end{array}$

$\begin{array}{lllll}1 & 2.231501 & -1.476923 & 1.005165\end{array}$

$\begin{array}{lllll}6 & 4.638956 & 1.093902 & -0.597830\end{array}$

$\begin{array}{lllll}1 & 2.854418 & 2.118395 & -1.237850\end{array}$

$\begin{array}{llll}6 & 5.165870 & -0.008615 & 0.083116\end{array}$

$\begin{array}{lllll}1 & 4.681520 & -1.784410 & 1.205459\end{array}$

$\begin{array}{lllll}1 & 5.311363 & 1.810135 & -1.055649\end{array}$

$\begin{array}{llll}6 & 6.641759 & -0.235108 & 0.160251\end{array}$

$\begin{array}{lllll}1 & 6.929745 & -1.086463 & -0.459772\end{array}$

$1 \quad 7.204742 \quad 0.632744 \quad-0.179632$

$\begin{array}{lllll}1 & 6.947073 & -0.463408 & 1.179980\end{array}$

$\begin{array}{lllll}6 & -5.660474 & -0.770700 & -0.401389\end{array}$

$\begin{array}{lllll}1 & -6.108729 & -1.750562 & -0.221168\end{array}$

$\begin{array}{lllll}1 & -5.610687 & -0.647800 & -1.491488\end{array}$

$\begin{array}{lllll}6 & -6.559774 & 0.306891 & 0.195237\end{array}$

$\begin{array}{lllll}1 & -6.258845 & 1.314057 & -0.105501\end{array}$

$\begin{array}{lllll}1 & -7.595194 & 0.171795 & -0.119066\end{array}$

$\begin{array}{lllll}1 & -6.536612 & 0.264318 & 1.287474\end{array}$

\section{3b: $\mathbf{N}_{2} @ \mathbf{1}_{2}$}

$\begin{array}{lllll}6 & 8.903879 & 0.247256 & -3.750466\end{array}$

$\begin{array}{lllll}6 & 8.341674 & -1.041889 & -3.138066\end{array}$

$\begin{array}{lllll}6 & 8.355586 & 1.494761 & -3.056921\end{array}$

$\begin{array}{lllll}1 & 8.527971 & 0.281246 & -4.774101\end{array}$

$\begin{array}{lllll}6 & 8.949576 & -1.722467 & -2.076476\end{array}$

$\begin{array}{lllll}6 & 7.168275 & -1.608567 & -3.650564\end{array}$

$\begin{array}{lllll}6 & 8.948201 & 2.018745 & -1.898576\end{array}$

$\begin{array}{lllll}6 & 7.210448 & 2.149068 & -3.531024\end{array}$

$\begin{array}{lllll}6 & 8.447082 & -2.921610 & -1.553838\end{array}$

$\begin{array}{lllll}1 & 9.845885 & -1.301932 & -1.635162\end{array}$

$\begin{array}{lllll}6 & 6.649735 & -2.812489 & -3.200427\end{array}$

$\begin{array}{lllll}8 & 6.516993 & -0.939191 & -4.687763\end{array}$

$\begin{array}{lllll}6 & 8.428984 & 3.107736 & -1.192240\end{array}$

$\begin{array}{lllll}6 & 9.842481 & 1.537683 & -1.521121\end{array}$

$\begin{array}{lllll}6 & 6.642668 & 3.224659 & -2.856260\end{array}$

$\begin{array}{lllll}8 & 6.602303 & 1.731942 & -4.725867\end{array}$

$\begin{array}{lllll}6 & 9.063753 & -3.627504 & -0.349324\end{array}$

$\begin{array}{lllll}6 & 7.305726 & -3.456113 & -2.162276\end{array}$

$\begin{array}{lllll}1 & 5.751905 & -3.230064 & -3.635143\end{array}$

$\begin{array}{lllll}6 & 5.371315 & -0.280694 & -4.376567\end{array}$

$\begin{array}{lllll}6 & 9.086178 & 3.670866 & 0.071628\end{array}$

$\begin{array}{lllll}6 & 7.243990 & 3.668834 & -1.689118\end{array}$

$\begin{array}{lllll}1 & 5.746148 & 3.699833 & -3.232626\end{array}$

$\begin{array}{lllll}6 & 5.389069 & 1.131365 & -4.532193\end{array}$

$1 \quad 8.770050 \quad-4.675221 \quad-0.422023$

$\begin{array}{lllll}6 & 8.447934 & -3.097170 & 0.945400\end{array}$

$\begin{array}{lllll}8 & 6.832033 & -4.697744 & -1.724716\end{array}$

$\begin{array}{lllll}6 & 8.522763 & 2.994644 & 1.320830\end{array}$

$\begin{array}{lllll}1 & 8.786568 & 4.718265 & 0.129089\end{array}$

$\begin{array}{lllll}8 & 6.697120 & 4.763233 & -1.016336\end{array}$

$\begin{array}{lllll}6 & 8.948621 & -1.959609 & 1.584913\end{array}$

$\begin{array}{lllll}6 & 7.346316 & -3.724358 & 1.544418\end{array}$

$\begin{array}{lllll}6 & 5.619573 & -4.695753 & -1.103906\end{array}$ $\begin{array}{lll}3.218536 & -0.174778 & -3.815187\end{array}$ $\begin{array}{llll}9.020402 & 1.773530 & 1.799620\end{array}$

$\begin{array}{llll}7.442285 & 3.552206 & 2.014660\end{array}$

$\begin{array}{llll}5.561800 & 4.606418 & -0.286858\end{array}$ $\begin{array}{llll}3.200076 & 1.180988 & -4.115784\end{array}$ $\begin{array}{llll}8.421578 & -1.446967 & 2.775256\end{array}$

$\begin{array}{llll}9.790445 & -1.446420 & 1.135428\end{array}$

$\begin{array}{llll}6.794062 & -3.267609 & 2.734801\end{array}$

$\begin{array}{llll}6.812983 & -4.872015 & 0.950054\end{array}$

$\begin{array}{llll}5.610395 & -4.751493 & 0.316571\end{array}$

$\begin{array}{llll}8.453886 & 1.089120 & 2.879486\end{array}$

$\begin{array}{llll}9.874080 & 1.334038 & 1.296635\end{array}$

$\begin{array}{llll}6.823789 & 2.898153 & 3.073227\end{array}$

$\begin{array}{llll}6.949990 & 4.813309 & 1.640632\end{array}$

$\begin{array}{llll}5.689022 & 4.759135 & 1.122349\end{array}$

$\begin{array}{llll}9.018881 & -0.212950 & 3.446830\end{array}$

$\begin{array}{llll}7.335524 & -2.134273 & 3.332037\end{array}$

$\begin{array}{llll}5.946936 & -3.773992 & 3.180056\end{array}$

$\begin{array}{llll}3.389331 & -4.639777 & -1.107554\end{array}$

$\begin{array}{llll}7.320577 & 1.666766 & 3.464691\end{array}$

$\begin{array}{llll}5.967623 & 3.333922 & 3.570756\end{array}$

$\begin{array}{llll}3.357609 & 4.365267 & -0.033060\end{array}$

$\begin{array}{llll}8.694675 & -0.251258 & 4.487655\end{array}$

$\begin{array}{llll}6.777105 & -1.692408 & 4.541134\end{array}$

$\begin{array}{llll}3.380326 & -4.663589 & 0.280381\end{array}$

$\begin{array}{llll}6.713056 & 0.991539 & 4.522961\end{array}$

$\begin{array}{llll}3.473808 & 4.620468 & 1.326018\end{array}$

$\begin{array}{llll}5.564583 & -1.077075 & 4.403465\end{array}$

$\begin{array}{llll}5.544824 & 0.339382 & 4.281194\end{array}$

$\begin{array}{llll}3.348883 & -1.107175 & 4.153297\end{array}$

$\begin{array}{llll}3.347025 & 0.261050 & 3.916619\end{array}$

$\begin{array}{llll}10.555018 & -0.231947 & 3.442959\end{array}$

$\begin{array}{lll}10.941431 & 0.627979 & 3.996227\end{array}$

$\begin{array}{llll}10.980003 & -0.193887 & 2.436411\end{array}$

$\begin{array}{llll}10.597748 & -3.575877 & -0.339826\end{array}$

$\begin{array}{llll}10.987266 & -4.137693 & 0.513048\end{array}$

$\begin{array}{llll}10.991987 & -2.558440 & -0.270564\end{array}$

$10.437580 \quad 0.237228 \quad-3.822665$

$\begin{array}{llll}10.780258 & -0.634803 & -4.385564\end{array}$

$\begin{array}{llll}10.912066 & 0.199390 & -2.838117\end{array}$

$\begin{array}{llll}10.619173 & 3.628955 & 0.011865\end{array}$

$\begin{array}{llll}10.976576 & 4.163846 & -0.871855\end{array}$

$\begin{array}{llll}11.017191 & 2.611724 & -0.035315\end{array}$

$\begin{array}{llll}11.040179 & 4.104905 & 0.901173\end{array}$

$\begin{array}{llll}10.795058 & 1.139424 & -4.325769\end{array}$

$\begin{array}{llll}10.991725 & -4.019376 & -1.257997\end{array}$

$\begin{array}{llll}10.919193 & -1.145629 & 3.919642\end{array}$

$\begin{array}{llll}1.963309 & -4.580433 & -1.590177\end{array}$

$\begin{array}{llll}1.951860 & -4.577096 & 0.749956\end{array}$

$\begin{array}{llll}1.929854 & -1.600212 & 4.083500\end{array}$

$\begin{array}{llll}1.935284 & 0.688454 & 3.611846\end{array}$

$\begin{array}{llll}1.851594 & -0.571492 & -3.325065\end{array}$

$\begin{array}{llll}1.796322 & 1.684395 & -3.937311\end{array}$

$\begin{array}{llll}1.904478 & 4.126175 & -0.347762\end{array}$

$\begin{array}{llll}2.097765 & 4.619501 & 1.932861\end{array}$

$\begin{array}{llll}1.174193 & -0.451048 & 3.789754\end{array}$

$\begin{array}{llll}1.198623 & -4.542298 & -0.418285\end{array}$

$\begin{array}{lll}1.085286 & 0.576213 & -3.453864\end{array}$ 
$\begin{array}{lllll}7 & 1.242899 & 4.326749 & 0.852987\end{array}$

$\begin{array}{lllll}8 & 1.419049 & 3.800857 & -1.414669\end{array}$

$\begin{array}{lllll}8 & 1.757100 & 4.820533 & 3.072660\end{array}$

$\begin{array}{lllll}8 & 1.554808 & 1.792067 & 3.259165\end{array}$

$\begin{array}{lllll}8 & 1.496383 & -2.714722 & 4.244510\end{array}$

$\begin{array}{lllll}8 & 1.549029 & -4.519821 & 1.892458\end{array}$

$\begin{array}{lllll}8 & 1.498272 & -1.646951 & -2.881878\end{array}$

$\begin{array}{lllll}8 & 1.537834 & -4.568503 & -2.722520\end{array}$

$\begin{array}{lllll}8 & 1.341253 & 2.783406 & -4.154869\end{array}$

$\begin{array}{lllll}1 & 0.228646 & 4.370231 & 0.977517\end{array}$

$\begin{array}{llll}0.076335 & 0.642732 & -3.290376\end{array}$

$\begin{array}{llll}0.177203 & -4.525782 & -0.459050\end{array}$

$\begin{array}{llll}0.161410 & -0.532070 & 3.670294\end{array}$

$\begin{array}{lllll}7 & 4.512039 & -4.647492 & -1.829381\end{array}$

$\begin{array}{lllll}7 & 4.490049 & -4.719231 & 1.022695\end{array}$

$\begin{array}{llll}4.300269 & -0.941830 & -3.964194\end{array}$

$\begin{array}{llll}4.295588 & 1.871594 & -4.444864\end{array}$

$\begin{array}{llll}4.462762 & -1.810242 & 4.369619\end{array}$

$\begin{array}{llll}4.444173 & 1.018021 & 3.989742\end{array}$

$\begin{array}{lllll}7 & 4.398613 & 4.365901 & -0.873725\end{array}$

$\begin{array}{llll}7 & 4.647952 & 4.796438 & 1.937407\end{array}$

$\begin{array}{lllll}6 & -8.937141 & 2.396372 & 2.670425\end{array}$

$\begin{array}{lllll}6 & -8.389174 & 1.017729 & 3.018812\end{array}$

$\begin{array}{lllll}6 & -8.301275 & 2.919190 & 1.382720\end{array}$

$\begin{array}{lllll}1 & -8.614227 & 3.069330 & 3.464835\end{array}$

$\begin{array}{lllll}6 & -8.962027 & -0.180912 & 2.577471\end{array}$

$\begin{array}{lllll}6 & -7.229346 & 0.928269 & 3.793167\end{array}$

$\begin{array}{lllll}6 & -8.816697 & 2.557661 & 0.137061\end{array}$

$\begin{array}{lllll}6 & -7.214571 & 3.805700 & 1.381338\end{array}$

$\begin{array}{lllll}6 & -8.400766 & -1.431257 & 2.859227\end{array}$

$\begin{array}{llll}-9.872524 & -0.136846 & 1.991477\end{array}$

$\begin{array}{lllll}6 & -6.620136 & -0.281176 & 4.093297\end{array}$

$\begin{array}{lllll}8 & -6.725353 & 2.123114 & 4.317754\end{array}$

$\begin{array}{lllll}6 & -8.362051 & 3.076653 & -1.078103\end{array}$

$\begin{array}{llll}-9.633317 & 1.846333 & 0.112216\end{array}$

$\begin{array}{lllll}6 & -6.732701 & 4.366030 & 0.201483\end{array}$

$\begin{array}{lllll}8 & -6.660254 & 4.224374 & 2.604231\end{array}$

$\begin{array}{lllll}6 & -9.011003 & -2.758032 & 2.398043\end{array}$

$\begin{array}{lllll}6 & -7.213881 & -1.436100 & 3.601860\end{array}$

$\begin{array}{llll}-5.715092 & -0.325194 & 4.683743\end{array}$

$\begin{array}{lllll}6 & -5.513620 & 2.558632 & 3.900017\end{array}$

$\begin{array}{lllll}6 & -9.008441 & 2.632615 & -2.388882\end{array}$

$\begin{array}{lllll}6 & -7.315583 & 4.008341 & -1.013509\end{array}$

$\begin{array}{lllll}1 & -5.923588 & 5.085152 & 0.228655\end{array}$

$\begin{array}{lllll}6 & -5.479570 & 3.677153 & 3.012248\end{array}$

$\begin{array}{lllll}1 & -8.695099 & -3.506803 & 3.125759\end{array}$

$\begin{array}{lllll}6 & -8.430369 & -3.198701 & 1.053918\end{array}$

$\begin{array}{lllll}8 & -6.661682 & -2.678500 & 3.906395\end{array}$

$\begin{array}{lllll}6 & -8.413701 & 1.306443 & -2.862626\end{array}$

$\begin{array}{lllll}1 & -8.734038 & 3.378252 & -3.135815\end{array}$

$\begin{array}{lllll}8 & -6.862268 & 4.657312 & -2.181386\end{array}$

$\begin{array}{lllll}6 & -8.946013 & -2.717534 & -0.156514\end{array}$

$\begin{array}{lllll}6 & -7.348165 & -4.085972 & 0.970252\end{array}$

$\begin{array}{lllll}6 & -5.508934 & -3.059972 & 3.303042\end{array}$

$\begin{array}{lllll}6 & -3.281718 & 2.507423 & 3.920140\end{array}$

$\begin{array}{lllll}6 & -8.960398 & 0.051865 & -2.584391\end{array}$

$\begin{array}{lllll}6 & -7.244272 & 1.330485 & -3.629249\end{array}$

$\begin{array}{lllll}6 & -5.644590 & 4.236166 & -2.637625\end{array}$ $\begin{array}{lll}-3.242818 & 3.616614 & 3.088810\end{array}$

$\begin{array}{llll}-8.415294 & -3.048487 & -1.406227\end{array}$

$\begin{array}{llll}-9.787358 & -2.035367 & -0.121054\end{array}$

$\begin{array}{llll}-6.770691 & -4.431784 & -0.246376\end{array}$

$\begin{array}{lll}-6.819095 & -4.674668 & 2.131245\end{array}$

$\begin{array}{lll}-5.578114 & -4.208883 & 2.466923\end{array}$

$\begin{array}{llll}-8.397539 & -1.142275 & -3.057040\end{array}$

$\begin{array}{llll}-9.859246 & -0.004038 & -1.981967\end{array}$

$\begin{array}{llll}-6.650770 & 0.187052 & -4.139110\end{array}$

$\begin{array}{lll}-6.745014 & 2.594501 & -3.935044\end{array}$

$\begin{array}{llll}-5.584005 & 3.032943 & -3.404267\end{array}$

$\begin{array}{llll}-8.988568 & -2.509714 & -2.717397\end{array}$

$\begin{array}{llll}-7.297662 & -3.891052 & -1.409320\end{array}$

$\begin{array}{llll}-5.920292 & -5.100495 & -0.281748\end{array}$

$\begin{array}{llll}-3.304678 & -2.998454 & 3.002621\end{array}$

$\begin{array}{llll}-7.245493 & -1.035912 & -3.846739\end{array}$

$\begin{array}{llll}-5.756177 & 0.238642 & -4.744920\end{array}$

$\begin{array}{llll}-3.412748 & 4.325331 & -2.675534\end{array}$

$\begin{array}{llll}-8.651073 & -3.190658 & -3.499492\end{array}$

$\begin{array}{llll}-6.713883 & -4.217323 & -2.639232\end{array}$

$\begin{array}{llll}-3.351975 & -4.198025 & 2.307249\end{array}$

$\begin{array}{llll}-6.697800 & -2.196914 & -4.411379\end{array}$

$\begin{array}{llll}-3.368329 & 3.048192 & -3.212916\end{array}$

$\begin{array}{llll}-5.516269 & -3.640404 & -2.926184\end{array}$

$\begin{array}{llll}-5.500978 & -2.608239 & -3.907099\end{array}$

$\begin{array}{llll}-3.297892 & -3.475492 & -2.751740\end{array}$

$\begin{array}{llll}-3.274261 & -2.515105 & -3.753459\end{array}$

$\begin{array}{llll}-10.523914 & -2.516612 & -2.729664\end{array}$

$\begin{array}{llll}-10.893705 & -2.175453 & -3.700185\end{array}$

$\begin{array}{llll}-10.961347 & -1.870281 & -1.963723\end{array}$

$\begin{array}{llll}-10.545922 & -2.734113 & 2.397509\end{array}$

$\begin{array}{llll}-10.935053 & -3.719578 & 2.129098\end{array}$

$\begin{array}{llll}-10.962669 & -2.012837 & 1.688985\end{array}$

$\begin{array}{lll}-10.471132 & 2.442970 & 2.624925\end{array}$

$\begin{array}{lll}-10.881737 & 2.125161 & 3.587055\end{array}$

$\begin{array}{lll}-10.898267 & 1.795717 & 1.854214\end{array}$

$\begin{array}{llll}-10.541523 & 2.605376 & -2.311611\end{array}$

$\begin{array}{llll}-10.919458 & 3.591244 & -2.029241\end{array}$

$\begin{array}{llll}-10.922180 & 1.886352 & -1.581164\end{array}$

$\begin{array}{llll}-10.959806 & 2.338207 & -3.285727\end{array}$

$\begin{array}{llll}-10.809801 & 3.462079 & 2.421660\end{array}$

$\begin{array}{llll}-10.917871 & -2.471724 & 3.391459\end{array}$

$\begin{array}{llll}-10.893654 & -3.529825 & -2.552436\end{array}$

$\begin{array}{lll}-1.877667 & -2.532692 & 3.061245\end{array}$

$\begin{array}{llll}-1.935956 & -4.604417 & 1.983800\end{array}$

$\begin{array}{llll}-1.893714 & -3.690907 & -2.261678\end{array}$

$\begin{array}{llll}-1.839806 & -2.113126 & -3.987957\end{array}$

$\begin{array}{lll}-1.869987 & 2.045660 & 4.170098\end{array}$

$\begin{array}{llll}-1.806154 & 3.885709 & 2.728066\end{array}$

$\begin{array}{llll}-2.001198 & 4.747596 & -2.356987\end{array}$

$\begin{array}{llll}-1.961151 & 2.541531 & -3.125988\end{array}$

$\begin{array}{llll}-1.106445 & -2.895085 & -3.078234\end{array}$

$\begin{array}{llll}-1.144916 & -3.564172 & 2.506002\end{array}$

$\begin{array}{lll}-1.078106 & 2.921760 & 3.407944\end{array}$

$\begin{array}{llll}-1.217555 & 3.621149 & -2.685070\end{array}$

$\begin{array}{llll}-1.594209 & 5.781698 & -1.888600\end{array}$

$\begin{array}{llll}-1.588818 & 1.394875 & -3.309494\end{array}$

$\begin{array}{llll}-1.382673 & -1.304831 & -4.759911\end{array}$ $\begin{array}{llll}-1.536266 & -4.371768 & -1.318976\end{array}$

$\begin{array}{llll}-1.532648 & -5.585548 & 1.408068\end{array}$

$\begin{array}{llll}-1.466696 & 1.132266 & 4.848476\end{array}$

$\begin{array}{llll}-1.483588 & -1.458486 & 3.479457\end{array}$

$\begin{array}{llll}-1.376608 & 4.715661 & 1.947332\end{array}$

$\begin{array}{llll}-0.216234 & 3.588167 & -2.486997\end{array}$

$\begin{array}{llll}-0.065012 & 2.790780 & 3.343128\end{array}$

$\begin{array}{llll}-0.127756 & -3.569806 & 2.408632\end{array}$

$\begin{array}{llll}-0.097566 & -2.780911 & -2.969168\end{array}$

$\begin{array}{llll}-4.379543 & -2.409315 & 3.532377\end{array}$

$\begin{array}{llll}-4.498976 & -4.809695 & 1.990365\end{array}$

$\begin{array}{llll}-4.419999 & 1.961591 & 4.350929\end{array}$

$\begin{array}{lll}-4.338290 & 4.219108 \quad 2.613649\end{array}$

$\begin{array}{llll}-4.416920 & -4.060792 & -2.318236\end{array}$

$\begin{array}{llll}-4.380618 & -2.047487 & -4.339331\end{array}$

$\begin{array}{llll}-4.559134 & 4.923735 & -2.326110\end{array}$

$\begin{array}{llll}-4.451964 & 2.387600 & -3.626650\end{array}$

$\begin{array}{llll}0.955652 & -0.763482 & 0.499553\end{array}$

$\begin{array}{llll}0.561420 & -1.736866 & 1.118910\end{array}$

$\begin{array}{llll}0.133901 & 0.170360 & -0.010375\end{array}$

$\begin{array}{llll}-1.256499 & 0.057521 & 0.371696\end{array}$

$\begin{array}{lll}-1.669001 & 1.028113 & 0.110261\end{array}$

$\begin{array}{llll}-1.311320 & -0.080186 & 1.454498\end{array}$

$\begin{array}{llll}-1.996214 & -1.062576 & -0.342868\end{array}$

$\begin{array}{llll}-1.582949 & -2.019369 & -0.023882\end{array}$

$\begin{array}{llll}-1.817257 & -0.961154 & -1.417244\end{array}$

$\begin{array}{llll}-3.501782 & -0.978253 & -0.050925\end{array}$

$\begin{array}{llll}-3.647271 & -0.722563 & 1.008953\end{array}$

$\begin{array}{llll}-3.962546 & -1.963322 & -0.180686\end{array}$

$\begin{array}{llll}-4.212584 & 0.058228 & -0.936978\end{array}$

$\begin{array}{llll}-4.451817 & -0.392437 & -1.907959\end{array}$

$\begin{array}{llll}-3.519259 & 0.869683 & -1.170713\end{array}$

$\begin{array}{llll}2.384575 & -0.466113 & 0.272635\end{array}$

$\begin{array}{llll}2.813633 & 0.656187 & -0.447727\end{array}$

$\begin{array}{llll}3.324824 & -1.340253 & 0.828209\end{array}$

$\begin{array}{llll}4.173116 & 0.878510 & -0.628387\end{array}$

$\begin{array}{llll}2.080642 & 1.335113 & -0.864721\end{array}$

$\begin{array}{llll}4.681791 & -1.101974 & 0.654700\end{array}$

$\begin{array}{llll}2.978096 & -2.188897 & 1.403973\end{array}$

$\begin{array}{llll}5.123613 & 0.005220 & -0.077483\end{array}$

$\begin{array}{llll}4.505526 & 1.739861 & -1.199866\end{array}$

$\begin{array}{lll}5.408875 & -1.777490 & 1.089782\end{array}$

$\begin{array}{llll}6.585199 & 0.274529 & -0.253308\end{array}$

$\begin{array}{llll}6.893182 & 1.136036 & 0.342982\end{array}$

$\begin{array}{llll}7.194322 & -0.576606 & 0.051335\end{array}$

$\begin{array}{llll}6.815065 & 0.505075 & -1.292570\end{array}$

$\begin{array}{llll}-5.487204 & 0.628179 & -0.314235\end{array}$

$\begin{array}{llll}-5.916008 & 1.381693 & -0.982646\end{array}$

$\begin{array}{llll}-5.240628 & 1.155548 & 0.616963\end{array}$

$\begin{array}{llll}-6.529060 & -0.445314 & -0.030309\end{array}$

$\begin{array}{llll}-6.203818 & -1.128959 & 0.758763\end{array}$

$\begin{array}{llll}-7.476754 & -0.007996 & 0.285622\end{array}$

$\begin{array}{llll}-6.719300 & -1.038305 & -0.925750\end{array}$

$\begin{array}{llll}-3.709764 & 3.187300 & 0.037133\end{array}$

$\begin{array}{llll}-2.609733 & 3.086397 & 0.035492\end{array}$

2c 
$\begin{array}{llll}6 & 0.406363 & 0.406554 & 1.142785\end{array}$

$\begin{array}{llll}8 & 0.104249 & -0.038414 & 2.238381\end{array}$

$\begin{array}{llll}7 & -0.537876 & 1.220114 & 0.461556\end{array}$

$\begin{array}{llll}7 & -0.094728 & 2.023015 & -0.547497\end{array}$

$\begin{array}{lllll}8 & -0.956275 & 2.709763 & -1.068154\end{array}$

$\begin{array}{lllll}6 & -1.933715 & 1.269174 & 0.938284\end{array}$

$\begin{array}{lllll}6 & 1.708904 & 0.107118 & 0.494692\end{array}$

$\begin{array}{lllll}6 & 1.886510 & -0.029213 & -0.888827\end{array}$

$\begin{array}{lllll}6 & 2.787655 & -0.160309 & 1.349983\end{array}$

$\begin{array}{lllll}6 & 3.124743 & -0.406245 & -1.397583\end{array}$

$\begin{array}{lllll}1 & 1.058557 & 0.136935 & -1.565709\end{array}$

$\begin{array}{lllll}6 & 4.025485 & -0.514991 & 0.830395\end{array}$

$\begin{array}{lllll}1 & 2.633601 & -0.087688 & 2.420673\end{array}$

$\begin{array}{lllll}6 & 4.216607 & -0.641077 & -0.552658\end{array}$

$\begin{array}{lllll}1 & 3.246092 & -0.522394 & -2.470662\end{array}$

$\begin{array}{lllll}1 & 4.855995 & -0.705881 & 1.504107\end{array}$

$\begin{array}{lllll}6 & 5.566880 & -1.001993 & -1.115569\end{array}$

$\begin{array}{lllll}1 & 6.124005 & -1.649666 & -0.432874\end{array}$

$\begin{array}{lllll}1 & 5.475384 & -1.513562 & -2.077492\end{array}$

$\begin{array}{lllll}1 & 6.172681 & -0.102415 & -1.279404\end{array}$

$\begin{array}{llll}6 & -2.763031 & 0.170419 & 0.316149\end{array}$

$\begin{array}{lllll}6 & -3.371942 & 0.361706 & -0.929440\end{array}$

$\begin{array}{lllll}6 & -2.892620 & -1.063660 & 0.962604\end{array}$

$\begin{array}{llll}6 & -4.110129 & -0.665461 & -1.515318\end{array}$

$\begin{array}{lllll}1 & -3.257938 & 1.315365 & -1.435214\end{array}$

$\begin{array}{llll}6 & -3.631060 & -2.090453 & 0.375166\end{array}$

$\begin{array}{llll}1 & -2.405029 & -1.215233 & 1.920607\end{array}$

$\begin{array}{llll}6 & -4.241892 & -1.892936 & -0.863840\end{array}$

$\begin{array}{llll}1 & -4.583390 & -0.507521 & -2.479722\end{array}$

$\begin{array}{llll}1 & -3.729721 & -3.043843 & 0.885251\end{array}$

$\begin{array}{llll}1 & -4.818477 & -2.691929 & -1.319963\end{array}$

$\begin{array}{lllll}1 & -2.310588 & 2.255085 & 0.667764\end{array}$

$\begin{array}{llll}1 & -1.889283 & 1.168251 & 2.021467\end{array}$

\section{2c'-TS}

$\begin{array}{llll}6 & 0.658122 & 1.264815 & 0.326177\end{array}$ $\begin{array}{lllll}8 & 0.558211 & 2.378030 & 0.825545\end{array}$

$\begin{array}{lllll}7 & -0.470738 & 0.557372 & -0.124722\end{array}$

$\begin{array}{lllll}7 & -0.309098 & -0.128756 & -1.494121\end{array}$

$\begin{array}{lllll}8 & -0.392706 & -1.302224 & -1.420378\end{array}$

$\begin{array}{lllll}6 & -1.729414 & 1.307920 & -0.203171\end{array}$

$\begin{array}{llll}1 & -1.803813 & 1.817972 & -1.174195\end{array}$

$\begin{array}{lllll}1 & -1.650431 & 2.092058 & 0.555657\end{array}$

$\begin{array}{llll}6 & 1.962948 & 0.559383 & 0.216529\end{array}$

$\begin{array}{lllll}6 & 2.085891 & -0.832618 & 0.316199\end{array}$

$\begin{array}{lllll}6 & 3.118822 & 1.339437 & 0.074257\end{array}$

$\begin{array}{lllll}6 & 3.342037 & -1.428173 & 0.248727\end{array}$

$\begin{array}{lllll}1 & 1.203222 & -1.441777 & 0.470316\end{array}$

$\begin{array}{lllll}6 & 4.366381 & 0.734666 & -0.006080\end{array}$

$\begin{array}{lllll}1 & 3.016201 & 2.418167 & 0.033045\end{array}$

$\begin{array}{lllll}6 & 4.499311 & -0.659254 & 0.074882\end{array}$

$\begin{array}{lllll}1 & 3.427337 & -2.507341 & 0.339439\end{array}$

$1 \quad 5.254668 \quad 1.348496 \quad-0.126613$

$\begin{array}{lllll}6 & 5.852859 & -1.312021 & -0.040437\end{array}$

$1 \quad 6.110706 \quad-1.491596 \quad-1.091338$

$\begin{array}{lllll}1 & 6.638318 & -0.679746 & 0.383180\end{array}$

$\begin{array}{lllll}1 & 5.876025 & -2.277863 & 0.471363\end{array}$

$\begin{array}{llll}6 & -2.933391 & 0.421672 & 0.017405\end{array}$

$\begin{array}{llll}6 & -2.910423 & -0.595765 & 0.977707\end{array}$

$\begin{array}{lllll}6 & -4.101154 & 0.631076 & -0.721513\end{array}$

$\begin{array}{llll}6 & -4.038789 & -1.382936 & 1.198696\end{array}$

$\begin{array}{llll}1 & -1.998198 & -0.771565 & 1.538093\end{array}$

$\begin{array}{llll}6 & -5.233595 & -0.152261 & -0.497839\end{array}$

$\begin{array}{lllll}1 & -4.123188 & 1.411327 & -1.477989\end{array}$

$\begin{array}{llll}6 & -5.204907 & -1.162183 & 0.463455\end{array}$

$\begin{array}{llll}1 & -4.007921 & -2.171244 & 1.944852\end{array}$

$\begin{array}{llll}1 & -6.133493 & 0.021220 & -1.080078\end{array}$

$\begin{array}{lllll}1 & -6.082833 & -1.777414 & 0.634866\end{array}$
$2 c^{\prime}$

$\begin{array}{llll}6 & -0.167589 & 1.143597 & -0.857563\end{array}$

$\begin{array}{lllll}8 & 0.201649 & 1.272989 & -2.010663\end{array}$

$\begin{array}{lllll}7 & 0.685292 & 1.653429 & 0.189582\end{array}$

$\begin{array}{lllll}7 & 0.284490 & 2.422788 & 1.252364\end{array}$

$\begin{array}{llll}8 & -0.911805 & 2.609000 & 1.358174\end{array}$

$\begin{array}{lllll}6 & 2.137381 & 1.624041 & -0.033548\end{array}$

$\begin{array}{lllll}1 & 2.563701 & 2.236748 & 0.763208\end{array}$

$\begin{array}{lllll}1 & 2.352180 & 2.093328 & -0.995723\end{array}$

$\begin{array}{lllll}6 & -1.383637 & 0.409069 & -0.463671\end{array}$

$\begin{array}{llll}6 & -1.502688 & -0.213754 & 0.785567\end{array}$

$\begin{array}{lllll}6 & -2.399440 & 0.248008 & -1.414147\end{array}$

$\begin{array}{llll}6 & -2.626548 & -0.975012 & 1.076932\end{array}$

$\begin{array}{llll}1 & -0.704989 & -0.119358 & 1.513342\end{array}$

$\begin{array}{llll}6 & -3.529502 & -0.498888 & -1.105935\end{array}$

$\begin{array}{lllll}1 & -2.284335 & 0.714690 & -2.386093\end{array}$

$\begin{array}{llll}6 & -3.663292 & -1.120000 & 0.143451\end{array}$

$\begin{array}{llll}1 & -2.705877 & -1.466144 & 2.042456\end{array}$

$\begin{array}{llll}1 & -4.318989 & -0.609608 & -1.843675\end{array}$

$\begin{array}{llll}6 & -4.901411 & -1.907143 & 0.486298\end{array}$

$\begin{array}{llll}1 & -5.625677 & -1.278694 & 1.018787\end{array}$

$\begin{array}{llll}1 & -5.395469 & -2.287250 & -0.411791\end{array}$

$\begin{array}{llll}1 & -4.668570 & -2.755495 & 1.136204\end{array}$

$\begin{array}{lllll}6 & 2.661579 & 0.208306 & 0.010998\end{array}$

$\begin{array}{lllll}6 & 2.948999 & -0.483455 & -1.168580\end{array}$

$\begin{array}{lllll}6 & 2.811240 & -0.442867 & 1.241123\end{array}$

$\begin{array}{lllll}6 & 3.390589 & -1.806253 & -1.119443\end{array}$

$\begin{array}{lllll}1 & 2.812023 & 0.013944 & -2.123688\end{array}$

$\begin{array}{lllll}6 & 3.251410 & -1.763496 & 1.291859\end{array}$

$\begin{array}{lllll}1 & 2.580451 & 0.091765 & 2.158999\end{array}$

$\begin{array}{lllll}6 & 3.542900 & -2.447550 & 0.109476\end{array}$

$\begin{array}{lllll}1 & 3.611902 & -2.335706 & -2.041029\end{array}$

$\begin{array}{lllll}1 & 3.369727 & -2.258628 & 2.250860\end{array}$

$\begin{array}{lllll}1 & 3.886753 & -3.476636 & 0.147533\end{array}$

\section{4c-TS}

$\begin{array}{llll}6 & 1.947248 & -1.364431 & 0.283066\end{array}$

$\begin{array}{lllll}8 & 2.588888 & -1.965590 & 1.081673\end{array}$

$\begin{array}{lllll}7 & 2.345578 & 0.447656 & 0.099658\end{array}$

$\begin{array}{lllll}7 & 2.870364 & 0.309991 & -1.039265\end{array}$

$\begin{array}{lllll}8 & 2.842335 & -0.951306 & -1.317141\end{array}$

$\begin{array}{lllll}6 & 2.015217 & 1.774525 & 0.617893\end{array}$

$\begin{array}{lllll}1 & 2.580057 & 2.518568 & 0.048016\end{array}$

$\begin{array}{lllll}1 & 2.338380 & 1.809901 & 1.662323\end{array}$

$\begin{array}{lllll}6 & 0.503980 & -1.483584 & -0.034829\end{array}$

$\begin{array}{lllll}6 & -0.099241 & -0.881075 & -1.144355\end{array}$

$\begin{array}{llll}6 & -0.286338 & -2.160479 & 0.898927\end{array}$

$\begin{array}{llll}6 & -1.476415 & -0.934878 & -1.294759\end{array}$

$\begin{array}{lllll}1 & 0.509173 & -0.370242 & -1.879170\end{array}$

$\begin{array}{llll}6 & -1.669167 & -2.205635 & 0.738611\end{array}$

$\begin{array}{lllll}1 & 0.192005 & -2.629914 & 1.751467\end{array}$

$\begin{array}{llll}6 & -2.286582 & -1.581593 & -0.349359\end{array}$

$\begin{array}{llll}1 & -1.937986 & -0.450853 & -2.150211\end{array}$

$\begin{array}{llll}1 & -2.277440 & -2.722981 & 1.475130\end{array}$

$\begin{array}{llll}6 & -3.785664 & -1.565725 & -0.495549\end{array}$

$\begin{array}{lllll}1 & -4.177783 & -0.559190 & -0.305753\end{array}$

$\begin{array}{llll}1 & -4.092235 & -1.844883 & -1.508620\end{array}$

$\begin{array}{llll}1 & -4.266492 & -2.250355 & 0.207569\end{array}$

$\begin{array}{llll}6 & 0.521468 & 1.999872 & 0.502836\end{array}$

$\begin{array}{llll}6 & -0.347073 & 1.448813 & 1.449932\end{array}$

$\begin{array}{lllll}6 & -0.009629 & 2.668848 & -0.603240\end{array}$

$\begin{array}{llll}6 & -1.726513 & 1.559214 & 1.291474\end{array}$

$\begin{array}{lllll}1 & 0.059660 & 0.911164 & 2.301446\end{array}$

$\begin{array}{lllll}6 & -1.390283 & 2.790301 & -0.758524\end{array}$

$\begin{array}{lllll}1 & 0.660921 & 3.086681 & -1.349137\end{array}$

$\begin{array}{lllll}6 & -2.250997 & 2.232454 & 0.187227\end{array}$

$\begin{array}{llll}1 & -2.390853 & 1.114718 & 2.025493\end{array}$

$\begin{array}{lllll}1 & -1.792753 & 3.316621 & -1.618579\end{array}$ $\begin{array}{llll}1 & -3.326104 & 2.322650 & 0.064905\end{array}$

\section{$4 \mathrm{c}$}

$\begin{array}{llll}6 & -1.369788 & -1.107062 & 0.099906\end{array}$

$\begin{array}{llll}8 & -1.149070 & -2.150115 & 0.665830\end{array}$

$\begin{array}{lllll}7 & 1.615998 & -0.309556 & -1.258178\end{array}$

$\begin{array}{lllll}7 & 0.843042 & -1.061944 & -0.676321\end{array}$

$\begin{array}{llll}8 & -0.427609 & -0.394600 & -0.616687\end{array}$

$\begin{array}{lllll}6 & 2.963086 & -0.914189 & -1.341413\end{array}$

$\begin{array}{lllll}1 & 3.281101 & -0.813030 & -2.382241\end{array}$

$\begin{array}{lllll}1 & 2.919823 & -1.977664 & -1.079846\end{array}$

$\begin{array}{llll}6 & -2.676504 & -0.402052 & 0.075970\end{array}$

$\begin{array}{lllll}6 & -2.876600 & 0.810478 & -0.596166\end{array}$

$\begin{array}{llll}6 & -3.743586 & -0.992326 & 0.765989\end{array}$

$\begin{array}{lllll}6 & -4.129180 & 1.415952 & -0.574767\end{array}$

$\begin{array}{lllll}1 & -2.052657 & 1.272656 & -1.126257\end{array}$

$\begin{array}{llll}6 & -4.989492 & -0.379318 & 0.780004\end{array}$

$\begin{array}{llll}1 & -3.570836 & -1.928484 & 1.284998\end{array}$

$\begin{array}{llll}6 & -5.204402 & 0.832876 & 0.107834\end{array}$

$\begin{array}{lllll}1 & -4.276731 & 2.357957 & -1.095279\end{array}$

$\begin{array}{llll}1 & -5.810636 & -0.842541 & 1.319992\end{array}$

$\begin{array}{llll}6 & -6.567085 & 1.476756 & 0.100297\end{array}$

$\begin{array}{lllll}1 & -7.201190 & 1.037646 & -0.679555\end{array}$

$\begin{array}{llll}1 & -7.081653 & 1.333140 & 1.054885\end{array}$

$\begin{array}{lllll}1 & -6.501647 & 2.550143 & -0.096239\end{array}$

$\begin{array}{lllll}6 & 3.906468 & -0.164591 & -0.425994\end{array}$

$\begin{array}{lllll}6 & 3.711321 & -0.215128 & 0.959099\end{array}$

$\begin{array}{lllll}6 & 4.970232 & 0.579782 & -0.940469\end{array}$

$\begin{array}{lllll}6 & 4.575238 & 0.462194 & 1.816237\end{array}$

$\begin{array}{lllll}1 & 2.877543 & -0.784113 & 1.360688\end{array}$

$\begin{array}{lllll}6 & 5.838310 & 1.256443 & -0.082777\end{array}$

$\begin{array}{lllll}1 & 5.121056 & 0.628108 & -2.015277\end{array}$

$\begin{array}{llll}6 & 5.641845 & 1.198905 & 1.296433\end{array}$

$\begin{array}{lllll}1 & 4.417100 & 0.415998 & 2.889256\end{array}$

$\begin{array}{lllll}1 & 6.664340 & 1.829503 & -0.492533\end{array}$

$\begin{array}{llll}1 & 6.315372 & 1.726580 & 1.964656\end{array}$

\section{4c'-TS}

$\begin{array}{llll}6 & -0.830530 & -1.093739 & -0.365094\end{array}$

$\begin{array}{lllll}8 & -0.155545 & -0.768536 & -1.368616\end{array}$

$\begin{array}{lllll}7 & 2.149604 & -2.084295 & 0.058035\end{array}$

$\begin{array}{lllll}7 & 1.250430 & -2.589798 & -0.416666\end{array}$

$\begin{array}{lllll}8 & -0.450772 & -1.937783 & 0.527144\end{array}$

$\begin{array}{llll}6 & 3.521804 & -1.601239 & 0.047301\end{array}$

$\begin{array}{lllll}1 & 3.987450 & -2.010741 & -0.857667\end{array}$

$\begin{array}{llll}6 & -2.180990 & -0.463068 & -0.145628\end{array}$

$\begin{array}{llll}6 & -2.976410 & -0.809194 & 0.951495\end{array}$

$\begin{array}{llll}6 & -2.650720 & 0.489145 & -1.055194\end{array}$

$\begin{array}{llll}6 & -4.219054 & -0.208979 & 1.133535\end{array}$

$\begin{array}{llll}1 & -2.607731 & -1.553385 & 1.648518\end{array}$

$\begin{array}{llll}6 & -3.894350 & 1.085205 & -0.868466\end{array}$

$\begin{array}{lllll}1 & -2.024665 & 0.739337 & -1.904535\end{array}$

$\begin{array}{llll}6 & -4.696994 & 0.749346 & 0.229922\end{array}$

$\begin{array}{llll}1 & -4.832143 & -0.487636 & 1.987103\end{array}$

$\begin{array}{lllll}1 & -4.253030 & 1.820506 & -1.584603\end{array}$

$\begin{array}{llll}6 & -6.029733 & 1.421277 & 0.449012\end{array}$

$\begin{array}{llll}1 & -6.473675 & 1.746806 & -0.496241\end{array}$

$\begin{array}{lllll}1 & -6.738287 & 0.751819 & 0.945748\end{array}$

$\begin{array}{lllll}1 & -5.924123 & 2.310596 & 1.082988\end{array}$

$\begin{array}{lllll}6 & 3.559440 & -0.089256 & 0.099974\end{array}$

$\begin{array}{lllll}6 & 2.547900 & 0.675645 & -0.489129\end{array}$

$\begin{array}{lllll}6 & 4.651119 & 0.527541 & 0.716966\end{array}$

$\begin{array}{lllll}6 & 2.643768 & 2.066674 & -0.454654\end{array}$

$\begin{array}{lllll}1 & 1.680013 & 0.201277 & -0.946230\end{array}$

$\begin{array}{llll}6 & 4.743246 & 1.918152 & 0.732549\end{array}$

$\begin{array}{lllll}1 & 5.427176 & -0.073227 & 1.184145\end{array}$

$\begin{array}{llll}6 & 3.737789 & 2.688876 & 0.147492\end{array}$

$\begin{array}{lllll}1 & 1.853093 & 2.661988 & -0.899562\end{array}$ 
$\begin{array}{llll}1 & 5.591624 & 2.396604 & 1.211272\end{array}$

$\begin{array}{llll}1 & 3.804231 & 3.772112 & 0.168427\end{array}$

$\begin{array}{lllll}1 & 4.002650 & -2.045159 & 0.922329\end{array}$

\section{4c-TS(2)}

$\begin{array}{llll}6 & 0.541248 & 1.100018 & 0.845226\end{array}$

$\begin{array}{llll}8 & -0.006529 & 1.131651 & 1.901412\end{array}$

$\begin{array}{lllll}7 & -0.589000 & 1.092129 & -0.643126\end{array}$

$\begin{array}{lllll}7 & -0.335044 & 2.291402 & -0.942708\end{array}$

$\begin{array}{lllll}8 & 0.527218 & 2.721433 & -0.085312\end{array}$

$\begin{array}{lllll}6 & -1.498696 & 0.305454 & -1.459770\end{array}$

$1-0.921275 \quad-0.517128 \quad-1.900550$

$\begin{array}{lllll}1 & -1.843496 & 0.942990 & -2.283322\end{array}$

$\begin{array}{lllll}6 & 1.784017 & 0.392363 & 0.467224\end{array}$

$\begin{array}{lllll}6 & 2.414583 & 0.551932 & -0.771220\end{array}$

$\begin{array}{lllll}6 & 2.327449 & -0.483984 & 1.413526\end{array}$

$\begin{array}{lllll}6 & 3.571325 & -0.162997 & -1.054513\end{array}$

$\begin{array}{lllll}1 & 2.011237 & 1.247465 & -1.497011\end{array}$

$6 \quad 3.485910 \quad-1.195596 \quad 1.117910$

$\begin{array}{lllll}1 & 1.833612 & -0.593441 & 2.372618\end{array}$

$6 \quad 4.124192-1.050886 \quad-0.119470$

$14.061164-0.027959-2.014646$

$\begin{array}{lllll}1 & 3.902548 & -1.872049 & 1.858678\end{array}$

$6 \quad 5.362096 \quad-1.844149 \quad-0.450914$

$1 \quad 5.104669-2.757558 \quad-1.000876$

$1 \quad 6.047857 \quad-1.269050 \quad-1.079579$

$\begin{array}{lllll}1 & 5.897005 & -2.146062 & 0.453320\end{array}$

$6 \begin{array}{llll}6 & -2.667302 & -0.254624 & -0.673339\end{array}$

$\begin{array}{lllll}6 & -3.508663 & -1.185317 & -1.294220\end{array}$

$\begin{array}{lllll}6 & -2.932216 & 0.138839 & 0.640479\end{array}$

$\begin{array}{lllll}6 & -4.606147 & -1.709330 & -0.615889\end{array}$

$1-3.303505 \quad-1.500038 \quad-2.314695$

$\begin{array}{lllll}6 & -4.029777 & -0.392680 & 1.320633\end{array}$

$\begin{array}{lllll}1 & -2.276245 & 0.843294 & 1.139592\end{array}$

$\begin{array}{lllll}6 & -4.870020 & -1.313023 & 0.696925\end{array}$

$1 \quad-5.252510 \quad-2.428792 \quad-1.109368$

$\begin{array}{lllll}1 & -4.223407 & -0.084152 & 2.343358\end{array}$

$\begin{array}{llll}1 & -5.722874 & -1.723037 & 1.228999\end{array}$

\section{$4 \mathrm{c}^{\prime}$}

$\begin{array}{llll}6 & -0.839485 & 0.526365 & 0.682994\end{array}$

$\begin{array}{llll}8 & -0.153811 & -0.197812 & 1.403758\end{array}$

$\begin{array}{llll}7 & 2.009444 & 2.052782 & 0.560655\end{array}$

$\begin{array}{llll}7 & 1.007325 & 2.203918 & 1.135185\end{array}$

$\begin{array}{llll}8 & -0.423928 & 1.666474 & 0.163360\end{array}$

$\begin{array}{lllll}6 & 2.287179 & 1.360343 & -0.724353\end{array}$

$\begin{array}{lllll}1 & 2.732839 & 2.118900 & -1.371219\end{array}$

$\begin{array}{llll}1 & 1.310050 & 1.061281 & -1.109095\end{array}$

$\begin{array}{llll}6 & -2.236848 & 0.158719 & 0.288742\end{array}$

$\begin{array}{lllll}6 & -3.060131 & 1.029600 & -0.433426\end{array}$

$\begin{array}{lllll}6 & -2.727326 & -1.097896 & 0.662122\end{array}$

$\begin{array}{lllll}6 & -4.353130 & 0.644129 & -0.775839\end{array}$

$\begin{array}{lllll}1 & -2.680987 & 2.005704 & -0.713059\end{array}$

$\begin{array}{llll}6 & -4.019254 & -1.475353 & 0.315035\end{array}$

$\begin{array}{llll}1 & -2.079323 & -1.756540 & 1.229414\end{array}$

$\begin{array}{llll}6 & -4.852204 & -0.613043 & -0.412075\end{array}$

$\begin{array}{lllll}1 & -4.988703 & 1.328900 & -1.330886\end{array}$

$\begin{array}{llll}1 & -4.393334 & -2.451446 & 0.612602\end{array}$

$\begin{array}{llll}6 & -6.241742 & -1.040251 & -0.812527\end{array}$

$\begin{array}{llll}1 & -6.221062 & -1.618377 & -1.744613\end{array}$

$\begin{array}{llll}1 & -6.700360 & -1.674619 & -0.048305\end{array}$

$\begin{array}{lllll}1 & -6.893406 & -0.177793 & -0.977353\end{array}$

$\begin{array}{llll}6 & 3.231790 & 0.209110 & -0.495974\end{array}$ $\begin{array}{llll}6 & 2.814704 & -0.893642 & 0.260580\end{array}$

$\begin{array}{lllll}6 & 4.517907 & 0.236254 & -1.042332\end{array}$

$\begin{array}{llll}6 & 3.687803 & -1.961418 & 0.458143\end{array}$

$\begin{array}{lllll}1 & 1.820076 & -0.899471 & 0.699055\end{array}$

$\begin{array}{lllll}6 & 5.383728 & -0.839453 & -0.849196\end{array}$

$\begin{array}{lllll}1 & 4.840607 & 1.096293 & -1.622778\end{array}$

$\begin{array}{lllll}6 & 4.968798 & -1.938788 & -0.097447\end{array}$

$\begin{array}{lllll}1 & 3.365518 & -2.814374 & 1.047004\end{array}$

$\begin{array}{lllll}1 & 6.379099 & -0.816758 & -1.281479\end{array}$

$\begin{array}{lllll}1 & 5.642788 & -2.775960 & 0.056209\end{array}$

\section{$3 c$}

$\begin{array}{llll}6 & 0.290955 & 0.908530 & -0.610061\end{array}$ $\begin{array}{lllll}8 & -0.114722 & 2.014033 & -0.299358\end{array}$ $\begin{array}{lllll}8 & -0.438805 & 0.012457 & -1.313167\end{array}$ $\begin{array}{lllll}6 & -1.793749 & 0.410802 & -1.653134\end{array}$ $\begin{array}{llll}1 & -2.007826 & -0.129813 & -2.576678\end{array}$ $\begin{array}{lllll}1 & -1.801966 & 1.485278 & -1.841899\end{array}$ $\begin{array}{lllll}6 & 1.641073 & 0.390378 & -0.271991\end{array}$ $\begin{array}{lllll}6 & 2.060044 & -0.899617 & -0.620196\end{array}$ $\begin{array}{lllll}6 & 2.513085 & 1.231430 & 0.429592\end{array}$ $\begin{array}{lllll}6 & 3.334669 & -1.333396 & -0.271029\end{array}$

$\begin{array}{lllll}1 & 1.382295 & -1.553965 & -1.154917\end{array}$ $\begin{array}{lllll}6 & 3.784754 & 0.788998 & 0.773265\end{array}$ $\begin{array}{lllll}1 & 2.170837 & 2.224638 & 0.697822\end{array}$ $\begin{array}{lllll}6 & 4.217892 & -0.498288 & 0.426281\end{array}$ $\begin{array}{lllll}1 & 3.652141 & -2.337010 & -0.540300\end{array}$ $\begin{array}{lllll}1 & 4.453977 & 1.447039 & 1.320567\end{array}$ $\begin{array}{lllll}6 & 5.609381 & -0.963723 & 0.772104\end{array}$

$\begin{array}{lllll}1 & 6.314654 & -0.716034 & -0.030733\end{array}$

$\begin{array}{lllll}1 & 5.975011 & -0.485932 & 1.685286\end{array}$

$\begin{array}{lllll}1 & 5.644857 & -2.047492 & 0.913803\end{array}$

$\begin{array}{lllll}6 & -2.765125 & 0.033888 & -0.566112\end{array}$

$\begin{array}{lllll}6 & -3.401451 & -1.211593 & -0.591731\end{array}$ $\begin{array}{llll}6 & -3.022793 & 0.911376 & 0.494583\end{array}$ $\begin{array}{llll}6 & -4.283842 & -1.578315 & 0.423513\end{array}$ $\begin{array}{llll}1 & -3.204009 & -1.895418 & -1.413112\end{array}$ $\begin{array}{llll}6 & -3.904606 & 0.545464 & 1.510180\end{array}$ $\begin{array}{lllll}1 & -2.514829 & 1.869208 & 0.520260\end{array}$ $\begin{array}{llll}6 & -4.536580 & -0.698782 & 1.476816\end{array}$ $\begin{array}{llll}1 & -4.774592 & -2.546346 & 0.391712\end{array}$ $\begin{array}{llll}1 & -4.099685 & 1.232287 & 2.328208\end{array}$ $\begin{array}{llll}1 & -5.224891 & -0.981266 & 2.267702\end{array}$

\section{$2 c @ 12$}

$\begin{array}{llll}6 & 8.905353 & 1.929994 & 3.027240\end{array}$

$\begin{array}{lllll}6 & 8.342121 & 2.669956 & 1.816948\end{array}$

$\begin{array}{lllll}6 & 8.312359 & 0.531075 & 3.145150\end{array}$

$1 \quad 8.567060 \quad 2.480406 \quad 3.905523$

$\begin{array}{lllll}6 & 8.880225 & 2.537493 & 0.535121\end{array}$

$\begin{array}{lllll}6 & 7.253737 & 3.537573 & 1.958127\end{array}$

$\begin{array}{lllll}6 & 8.881571 & -0.572554 & 2.500215\end{array}$

$\begin{array}{llll}6 & 7.167101 & 0.300519 & 3.917490\end{array}$

$\begin{array}{lllll}6 & 8.406627 & 3.254383 & -0.569363\end{array}$

$\begin{array}{llll}9.704590 & 1.849470 & 0.388129\end{array}$

$\begin{array}{llll}6.760229 & 4.293015 & 0.904624\end{array}$

$\begin{array}{llll}6.678452 & 3.671812 & 3.228097\end{array}$

$\begin{array}{llll}8.359297 & -1.863593 & 2.603909\end{array}$

$\begin{array}{llll}9.774421 & -0.420523 & 1.906121\end{array}$

$\begin{array}{llll}6.601449 & -0.962474 & 4.049142\end{array}$

$\begin{array}{llll}6.614522 & 1.369430 & 4.636347\end{array}$

$\begin{array}{llll}8.995903 & 3.060128 & -1.962186\end{array}$

$\begin{array}{llll}7.352794 & 4.147631 & -0.344958\end{array}$

$\begin{array}{llll}5.928253 & 4.970251 & 1.048672\end{array}$

$\begin{array}{llll}6 & 5.471466 & 3.078436 & 3.423428\end{array}$ $\begin{array}{llll}5.716533 & -1.115760 & 4.652529\end{array}$

$\begin{array}{lllll}6 & 5.432610 & 1.880808 & 4.197080\end{array}$

$\begin{array}{llll}8.695503 & 3.930114 & -2.546901\end{array}$

$\begin{array}{llll}8.370023 & 1.846504 & -2.646099\end{array}$

$\begin{array}{llll}6.874609 & 4.934128 & -1.406745\end{array}$

$\begin{array}{llll}8.389573 & -3.257813 & 0.521453\end{array}$

$\begin{array}{llll}8.681417 & -3.947926 & 2.494143\end{array}$

$\begin{array}{llll}6.697371 & -3.312222 & 3.507909\end{array}$

$\begin{array}{llll}8.907471 & 0.560345 & -2.552144\end{array}$

$\begin{array}{llll}7.211586 & 1.994996 & -3.418253\end{array}$

$\begin{array}{llll}5.630790 & 4.564677 & -1.833629\end{array}$

$\begin{array}{llll}3.248731 & 3.020911 & 3.263148\end{array}$

$\begin{array}{llll}8.882734 & -2.549110 & -0.580420\end{array}$

$\begin{array}{llll}7.322723 & -4.135427 & 0.289746\end{array}$

$\begin{array}{llll}5.535872 & -3.617143 & 2.875271\end{array}$

$\begin{array}{llll}3.203256 & 1.886371 & 4.060064\end{array}$

$\begin{array}{llll}8.353903 & -0.546487 & -3.206158\end{array}$

$\begin{array}{llll}9.799547 & 0.414769 & -1.955285\end{array}$

$\begin{array}{llll}6.637991 & 0.939109 & -4.114125\end{array}$

$\begin{array}{llll}6.678740 & 3.280348 & -3.538475\end{array}$

$\begin{array}{llll}5.537455 & 3.592699 & -2.870278\end{array}$

$\begin{array}{llll}8.362925 & -2.682808 & -1.869935\end{array}$

$\begin{array}{llll}9.712581 & -1.869698 & -0.425330\end{array}$

$\begin{array}{llll}6.752097 & -4.285357 & -0.968798\end{array}$

$\begin{array}{llll}6.825163 & -4.921771 & 1.342792\end{array}$

$\begin{array}{llll}5.593984 & -4.551509 & 1.802301\end{array}$

$\begin{array}{llll}8.945943 & -1.945876 & -3.074682\end{array}$

$\begin{array}{llll}7.218955 & -0.318986 & -3.995183\end{array}$

$\begin{array}{llll}5.757935 & 1.087866 & -4.725691\end{array}$

$3.406654 \quad 4.565628 \quad-1.654183$

$\begin{array}{llll}7.274800 & -3.549223 & -2.022858\end{array}$

$\begin{array}{llll}5.914961 & -4.954195 & -1.121288\end{array}$

$\begin{array}{llll}3.321574 & -3.563536 & 2.677872\end{array}$

$\begin{array}{llll}8.620977 & -2.499391 & -3.956017\end{array}$

$\begin{array}{llll}6.679384 & -1.390196 & -4.721619\end{array}$

$\begin{array}{llll}3.330712 & 3.526062 & -2.568765\end{array}$

$\begin{array}{llll}6.720202 & -3.691564 & -3.300708\end{array}$

$\begin{array}{llll}3.362209 & -4.564127 & 1.718810\end{array}$

$\begin{array}{llll}5.493450 & -1.886539 & -4.267941\end{array}$

$\begin{array}{llll}5.519901 & -3.079145 & -3.491851\end{array}$

$\begin{array}{llll}3.276975 & -1.798548 & -4.020886\end{array}$

$\begin{array}{llll}3.311647 & -2.935837 & -3.226664\end{array}$

$10.481918-1.946240 \quad-3.069827$

$\begin{array}{llll}10.854128 & -2.973717 & -3.049745\end{array}$

$10.907114-1.426845 \quad-2.206565$

$10.530747 \quad 3.015462 \quad-1.955289$

$\begin{array}{llll}10.909058 & 2.919417 & -2.976465\end{array}$

$\begin{array}{llll}10.932801 & 2.180748 & -1.374355\end{array}$

$\begin{array}{llll}10.441307 & 1.931137 & 3.047038\end{array}$

$\begin{array}{lll}10.812768 & 2.959048 & 3.037974\end{array}$

$\begin{array}{llll}10.880712 & 1.416493 & 2.188136\end{array}$

$10.517474 \quad-3.032281 \quad 1.907373$

$\begin{array}{llll}10.895646 & -2.953176 & 2.930062\end{array}$

$\begin{array}{llll}10.920601 & -2.188416 & 1.340662\end{array}$

$\begin{array}{llll}10.913530 & -3.947177 & 1.459337\end{array}$

$\begin{array}{lll}10.806007 & 1.436475 & 3.951170\end{array}$ 
$\begin{array}{lllll}1 & 10.927690 & 3.937077 & -1.522000\end{array}$

$1 \quad 10.860800 \quad-1.455677 \quad-3.970338$

$\begin{array}{lllll}6 & 2.005500 & 4.909753 & -1.217816\end{array}$

$\begin{array}{lllll}6 & 1.900961 & 3.081871 & -2.665265\end{array}$

$\begin{array}{lllll}6 & 1.854780 & -1.315948 & -4.087651\end{array}$

$\begin{array}{lllll}6 & 1.922704 & -3.221787 & -2.735030\end{array}$

$\begin{array}{lllll}6 & 1.846876 & 3.392266 & 2.876616\end{array}$

$\begin{array}{lllll}6 & 1.758298 & 1.506757 & 4.262655\end{array}$

$\begin{array}{lllll}6 & 1.900180 & -3.148891 & 2.879972\end{array}$

$\begin{array}{lllll}6 & 1.939419 & -4.909866 & 1.355720\end{array}$

$\begin{array}{lllll}7 & 1.141723 & -2.209248 & -3.277186\end{array}$

$\begin{array}{lllll}7 & 1.191888 & 3.987530 & -1.900945\end{array}$

$\begin{array}{lllll}7 & 1.039426 & 2.483163 & 3.549288\end{array}$

$\begin{array}{llll}7 & 1.153575 & -4.051720 & 2.157505\end{array}$

$\begin{array}{lllll}8 & 1.516514 & -2.177333 & 3.514286\end{array}$

$\begin{array}{llll}8 & 1.514428 & -5.726272 & 0.577312\end{array}$

$\begin{array}{lllll}8 & 1.562888 & -4.126286 & -2.008607\end{array}$

$\begin{array}{lllll}8 & 1.388989 & -0.374417 & -4.687371\end{array}$

$\begin{array}{lllll}8 & 1.484815 & 2.097228 & -3.248509\end{array}$

$\begin{array}{lllll}8 & 1.496453 & 4.263790 & 2.105560\end{array}$

$\begin{array}{lllll}8 & 1.624828 & 5.767560 & -0.458756\end{array}$

$\begin{array}{lllll}8 & 1.289959 & 0.578829 & 4.877616\end{array}$

$\begin{array}{lllll}1 & 0.146095 & -3.970228 & 1.994375\end{array}$

$\begin{array}{lllll}1 & 0.021776 & 2.483501 & 3.494019\end{array}$

$\begin{array}{lllll}1 & 0.175795 & 4.005932 & -1.795059\end{array}$

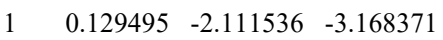

$\begin{array}{lllll}7 & 4.566326 & 5.086332 & -1.243893\end{array}$

$\begin{array}{lllll}7 & 4.389158 & 3.032889 & -3.215082\end{array}$

$\begin{array}{lllll}7 & 4.380956 & 3.641704 & 2.927226\end{array}$

$\begin{array}{lllll}7 & 4.298490 & 1.280234 & 4.528002\end{array}$

$\begin{array}{lllll}7 & 4.369258 & -1.245456 & -4.552182\end{array}$

$\begin{array}{lllll}7 & 4.430804 & -3.605546 & -2.953194\end{array}$

$\begin{array}{lllll}7 & 4.401349 & -3.080023 & 3.291520\end{array}$

$\begin{array}{lllll}7 & 4.507416 & -5.059480 & 1.238769\end{array}$

$\begin{array}{lllll}6 & -8.995772 & -3.603330 & -0.795440\end{array}$

$\begin{array}{lllll}6 & -8.393167 & -2.748255 & -1.906698\end{array}$

$\begin{array}{lllll}6 & -8.396815 & -3.233027 & 0.562918\end{array}$

$\begin{array}{lllll}1 & -8.684258 & -4.629383 & -0.995196\end{array}$

$\begin{array}{lllll}6 & -8.901232 & -1.479073 & -2.218940\end{array}$

$\begin{array}{lllll}6 & -7.283331 & -3.187883 & -2.640181\end{array}$

$\begin{array}{lllll}6 & -8.930447 & -2.242373 & 1.390805\end{array}$

$\begin{array}{lllll}6 & -7.256279 & -3.896009 & 1.033238\end{array}$

$\begin{array}{lllll}6 & -8.328490 & -0.644227 & -3.182067\end{array}$

$\begin{array}{lllll}1 & -9.774461 & -1.127761 & -1.682341\end{array}$

$\begin{array}{lllll}6 & -6.663613 & -2.387470 & -3.592495\end{array}$

$\begin{array}{lllll}8 & -6.771090 & -4.478730 & -2.433268\end{array}$

$\begin{array}{lllll}6 & -8.395453 & -1.921698 & 2.646474\end{array}$

$\begin{array}{llll}1 & -9.796221 & -1.690515 & 1.044746\end{array}$

$\begin{array}{lllll}6 & -6.708945 & -3.649299 & 2.282575\end{array}$

$\begin{array}{lllll}8 & -6.689056 & -4.875517 & 0.216887\end{array}$

$\begin{array}{lllll}6 & -8.894397 & 0.724871 & -3.559889\end{array}$

$\begin{array}{lllll}6 & -7.182186 & -1.124319 & -3.826169\end{array}$

$\begin{array}{lllll}1 & -5.788718 & -2.737771 & -4.123593\end{array}$

$\begin{array}{lllll}6 & -5.547356 & -4.497299 & -1.826048\end{array}$

$\begin{array}{lllll}6 & -8.967074 & -0.792628 & 3.498317\end{array}$

$\begin{array}{llll}6 & -7.293069 & -2.669831 & 3.078318\end{array}$

$\begin{array}{lllll}1 & -5.842645 & -4.197601 & 2.628594\end{array}$

$\begin{array}{lllll}6 & -5.517533 & -4.587526 & -0.407008\end{array}$
$1 \quad-8.551486 \quad 0.921440 \quad-4.576364$

$\begin{array}{lllll}6 & -8.316949 & 1.843845 & -2.696054\end{array}$

$8 \quad-6.570411 \quad-0.292047 \quad-4.765317$

$\begin{array}{lllll}6 & -8.377506 & 0.559563 & 3.092833\end{array}$

$\begin{array}{llll}-8.625731 & -0.977304 & 4.517643\end{array}$

$\begin{array}{llll}-6.750257 & -2.448403 & 4.354173\end{array}$

$\begin{array}{llll}-8.867530 & 2.165374 & -1.450422\end{array}$

$\begin{array}{llll}-7.225386 & 2.612679 & -3.120052\end{array}$

$\begin{array}{llll}-5.420444 & 0.324014 & -4.388326\end{array}$

$\begin{array}{llll}-3.322126 & -4.327796 & -1.845298\end{array}$

$\begin{array}{llll}-8.957516 & 1.390421 & 2.129334\end{array}$

$\begin{array}{llll}-7.210437 & 1.028568 & 3.708949\end{array}$

$\begin{array}{llll}-5.517362 & -1.858082 & 4.317323\end{array}$

$\begin{array}{llll}-3.310021 & -4.289127 & -0.458770\end{array}$

$\begin{array}{llll}-8.383593 & 3.192027 & -0.634746\end{array}$

$\begin{array}{llll}-9.718165 & 1.592435 & -1.101620\end{array}$

$\begin{array}{llll}-6.691068 & 3.632739 & -2.339210\end{array}$

$\begin{array}{llll}-6.651291 & 2.374179 & -4.379914\end{array}$

$\begin{array}{llll}-5.440682 & 1.744001 & -4.311472\end{array}$

$\begin{array}{llll}-8.442031 & 2.651770 & 1.807292\end{array}$

$\begin{array}{llll}-9.847349 & 1.047337 & 1.615143\end{array}$

$\begin{array}{lll}-6.680469 & 2.284431 & 3.458992\end{array}$

$\begin{array}{llll}-6.604489 & 0.211327 & 4.662757\end{array}$

$\begin{array}{llll}-5.458960 & -0.437224 & 4.330374\end{array}$

$\begin{array}{llll}-9.030855 & 3.529532 & 0.707794\end{array}$

$\begin{array}{llll}-7.267890 & 3.899513 & -1.103906\end{array}$

$\begin{array}{llll}-5.839770 & 4.204468 & -2.686498\end{array}$

$\begin{array}{llll}-3.251039 & 0.346117 & -3.885831\end{array}$

$\begin{array}{llll}-7.317324 & 3.082161 & 2.519041\end{array}$

$\begin{array}{llll}-5.794872 & 2.629183 & 3.975424\end{array}$

$\begin{array}{llll}-3.304080 & -1.923419 & 4.063238\end{array}$

$\begin{array}{llll}-8.739432 & 4.554483 & 0.939464\end{array}$

$\begin{array}{llll}-6.756982 & 4.948120 & -0.331463\end{array}$

$\begin{array}{llll}-3.240066 & 1.732031 & -3.952308\end{array}$

$\begin{array}{llll}-6.843349 & 4.378893 & 2.291378\end{array}$

$\begin{array}{lll}-3.270194 & -0.541621 & 3.920240\end{array}$

$\begin{array}{llll}-5.572519 & 4.743081 & 0.311646\end{array}$

$\begin{array}{llll}-5.618683 & 4.472262 & 1.707305\end{array}$

$\begin{array}{llll}-3.344578 & 4.672456 & 0.400116\end{array}$

$\begin{array}{llll}-3.390997 & 4.423267 & 1.764432\end{array}$

$\begin{array}{llll}-10.564165 & 3.484714 & 0.654445\end{array}$

$\begin{array}{llll}-10.979841 & 3.787903 & 1.618899\end{array}$

$\begin{array}{llll}-10.957241 & 2.490375 & 0.424675\end{array}$

$6 \quad-10.430248 \quad 0.736237 \quad-3.582876$

$\begin{array}{llll}-10.792092 & 1.710689 & -3.920792\end{array}$

$1 \quad-10.874659 \quad 0.542187 \quad-2.602755$

$\begin{array}{lllll}6 & -10.530742 & -3.579203 & -0.789161\end{array}$

$1 \quad-10.913793 \quad-3.906939-1.759012$

$1 \quad-10.943036 \quad-2.585886 \quad-0.591135$

$\begin{array}{lllll}6 & -10.502196 & -0.785288 & 3.520107\end{array}$

$\begin{array}{lllll}1 & -10.874900 & -1.746681 & 3.882901\end{array}$

$\begin{array}{llll}1 & -10.944216 & -0.612549 & 2.534886\end{array}$

$\begin{array}{lllll}1 & -10.864428 & 0.002416 & 4.185776\end{array}$

$\begin{array}{lllll}1 & -10.912897 & -4.252846 & -0.017840\end{array}$

$1 \quad-10.799852 \quad-0.030141 \quad-4.269093$

$1 \quad-10.933034 \quad 4.168783 \quad-0.114142$

$6 \quad-1.874249-0.124856 \quad-3.514820$

$\begin{array}{lllll}6 & -1.833505 & 2.203238 & -3.715180\end{array}$
6

6

$\begin{array}{llll}-1.902574 & 4.688568 & -0.027247\end{array}$

$\begin{array}{llll}-1.979153 & 4.283542 & 2.267517\end{array}$

$\begin{array}{llll}-1.896142 & -4.245721 & -2.326096\end{array}$

$\begin{array}{llll}-1.895200 & -4.087485 & 0.001760\end{array}$

$\begin{array}{llll}-1.900981 & -2.448423 & 3.955395\end{array}$

$\begin{array}{llll}-1.856738 & -0.134124 & 3.588790\end{array}$

$\begin{array}{llll}-1.181008 & 4.521217 & 1.146278\end{array}$

$\begin{array}{llll}-1.103883 & 1.025738 & -3.473338\end{array}$

$\begin{array}{llll}-1.133785 & -4.128078 & -1.146534\end{array}$

$\begin{array}{llll}-1.127614 & -1.313093 & 3.661732\end{array}$

$\begin{array}{llll}-1.487758 & -3.574938 & 4.092284\end{array}$

$\begin{array}{llll}-1.443569 & 0.962709 & 3.267705\end{array}$

$\begin{array}{llll}-1.590594 & 4.006943 & 3.381147\end{array}$

$\begin{array}{llll}-1.469481 & 4.783174 & -1.157880\end{array}$

$\begin{array}{llll}-1.395729 & 3.328863 & -3.709803\end{array}$

$\begin{array}{llll}-1.460139 & -4.273085 & -3.450570\end{array}$

$\begin{array}{llll}-1.531132 & -1.265028 & -3.262154\end{array}$

$\begin{array}{llll}-1.521903 & -3.894940 & 1.147437\end{array}$

$\begin{array}{llll}-0.106091 & -1.392106 & 3.620299\end{array}$

$\begin{array}{llll}-0.107961 & -4.082367 & -1.185751\end{array}$

$\begin{array}{llll}-0.085992 & 1.069988 & -3.389114\end{array}$

$\begin{array}{llll}-0.160917 & 4.471056 & 1.206899\end{array}$

$\begin{array}{llll}-4.337770 & -0.390372 & -4.120250\end{array}$

$\begin{array}{llll}-4.344043 & 2.462249 & -4.126692\end{array}$

$\begin{array}{llll}-4.449287 & -4.399489 & -2.559829\end{array}$

$\begin{array}{llll}-4.404191 & -4.432885 & 0.293046\end{array}$

$\begin{array}{llll}-4.432569 & 4.830208 & -0.358635\end{array}$

$\begin{array}{llll}-4.531042 & 4.310238 & 2.446535\end{array}$

$\begin{array}{llll}-4.436436 & -2.614040 & 4.224226\end{array}$

$\begin{array}{llll}-4.346986 & 0.234743 & 4.073199\end{array}$

$\begin{array}{llll}-4.788412 & 0.028986 & -0.060607\end{array}$

$\begin{array}{llll}-3.909903 & -0.907979 & 0.501330\end{array}$

$\begin{array}{llll}-2.535850 & -0.697356 & 0.499728\end{array}$

$\begin{array}{llll}-2.002039 & 0.475355 & -0.056488\end{array}$

$\begin{array}{llll}-2.876759 & 1.419235 & -0.617025\end{array}$

$\begin{array}{llll}-4.247142 & 1.192623 & -0.626287\end{array}$

$\begin{array}{llll}-4.307949 & -1.809541 & 0.957522\end{array}$

$\begin{array}{llll}-1.890683 & -1.432196 & 0.959258\end{array}$

$\begin{array}{llll}-2.467547 & 2.327400 & -1.041064\end{array}$

$\begin{array}{llll}-4.908597 & 1.930446 & -1.068924\end{array}$

$\begin{array}{llll}-6.268896 & -0.223216 & -0.074059\end{array}$

$\begin{array}{llll}-6.546194 & -0.828255 & -0.942800\end{array}$

$\begin{array}{llll}-6.588218 & -0.769450 & 0.815178\end{array}$

$\begin{array}{llll}-6.836872 & 0.707648 & -0.128011\end{array}$

$\begin{array}{llll}-0.575843 & 0.857736 & 0.051133\end{array}$

$\begin{array}{llll}-0.185047 & 2.010223 & -0.033298\end{array}$

$\begin{array}{llll}0.387757 & -0.152203 & 0.350231\end{array}$

$\begin{array}{llll}1.578195 & 0.168521 & 1.149927\end{array}$

$\begin{array}{llll}1.421751 & 1.202486 & 1.445662\end{array}$

$\begin{array}{llll}1.553669 & -0.460895 & 2.041563\end{array}$

$\begin{array}{llll}0.192763 & -1.379061 & -0.171135\end{array}$

$\begin{array}{llll}1.081461 & -2.175166 & 0.099352\end{array}$

$\begin{array}{llll}2.910002 & 0.023077 & 0.451930\end{array}$

$\begin{array}{llll}3.062058 & 0.303372 & -0.906474\end{array}$

$\begin{array}{llll}4.035789 & -0.315080 & 1.213622\end{array}$

$\begin{array}{llll}4.324803 & 0.253397 & -1.497860\end{array}$

$\begin{array}{llll}2.199170 & 0.566881 & -1.507308\end{array}$

$\begin{array}{llll}5.299923 & -0.356002 & 0.623008\end{array}$ 
$\begin{array}{llll}1 & 3.922674 & -0.550554 & 2.267893\end{array}$

$\begin{array}{lllll}6 & 5.448860 & -0.069811 & -0.735739\end{array}$

$1 \quad 4.422661 \quad 0.489965 \quad-2.549235$

$1 \quad 6.166816 \quad-0.612988 \quad 1.221513$

$\begin{array}{lllll}1 & 6.432179 & -0.095256 & -1.192407\end{array}$

\section{2c'-TS@12}

$\begin{array}{llll}6 & -8.957579 & 2.977195 & -2.014543\end{array}$

$\begin{array}{lllll}6 & -8.352680 & 3.168009 & -0.625900\end{array}$

$\begin{array}{lllll}6 & -8.366499 & 1.740895 & -2.682455\end{array}$

$\begin{array}{lllll}1 & -8.636375 & 3.832860 & -2.609108\end{array}$

$\begin{array}{lllll}6 & -8.846109 & 2.484231 & 0.488090\end{array}$

$\begin{array}{lllll}6 & -7.275025 & 4.037131 & -0.409734\end{array}$

$\begin{array}{lllll}6 & -8.936036 & 0.466619 & -2.568383\end{array}$

$\begin{array}{lllll}6 & -7.190379 & 1.856498 & -3.433929\end{array}$

$\begin{array}{lllll}6 & -8.342285 & 2.653744 & 1.781421\end{array}$

$\begin{array}{lllll}1 & -9.665745 & 1.790504 & 0.342588\end{array}$

$\begin{array}{lllll}6 & -6.741949 & 4.249710 & 0.856103\end{array}$

$8 \quad-6.741664 \quad 4.745588 \quad-1.490931$

$6 \quad-8.373418 \quad-0.667670 \quad-3.162921$

$\begin{array}{lllll}1 & -9.855129 & 0.358241 & -2.004862\end{array}$

$\begin{array}{lllll}6 & -6.582205 & 0.762846 & -4.033115\end{array}$

$8 \quad-6.670497 \quad 3.135583 \quad-3.641001$

$\begin{array}{lllll}6 & -8.932676 & 1.901171 & 2.969533\end{array}$

$\begin{array}{lllll}6 & -7.283930 & 3.557909 & 1.934013\end{array}$

$\begin{array}{lllll}1 & -5.915467 & 4.934751 & 0.998441\end{array}$

$\begin{array}{lllll}6 & -5.519549 & 4.332680 & -1.941068\end{array}$

$\begin{array}{lllll}6 & -8.988981 & -2.067268 & -3.070453\end{array}$

$6 \quad-7.184305 \quad-0.477606 \quad-3.875873$

$1 \quad-5.668011 \quad 0.874121 \quad-4.600188$

$\begin{array}{lllll}6 & -5.481425 & 3.468924 & -3.070162\end{array}$

$\begin{array}{lllll}1 & -8.612202 & 2.441636 & 3.861092\end{array}$

$\begin{array}{lllll}6 & -8.349897 & 0.495142 & 3.094912\end{array}$

$\begin{array}{lllll}8 & -6.747533 & 3.793988 & 3.211320\end{array}$

$\begin{array}{lllll}6 & -8.421282 & -2.834138 & -1.876644\end{array}$

$\begin{array}{lllll}1 & -8.661638 & -2.603791 & -3.961960\end{array}$

$8 \quad-6.628643 \quad-1.598451 \quad-4.493070$

$\begin{array}{lllll}6 & -8.918901 & -0.632040 & 2.497145\end{array}$

$\begin{array}{lllll}6 & -7.195782 & 0.288471 & 3.860358\end{array}$

$\begin{array}{lllll}6 & -5.519720 & 3.222578 & 3.390217\end{array}$

$6 \quad-3.288438 \quad 4.334526 \quad-1.892790$

$6 \begin{array}{llll}6 & -8.948450 & -2.671059 & -0.587220\end{array}$

$6 \quad-7.329155 \quad-3.702966 \quad-2.008856$

$\begin{array}{lllll}6 & -5.482937 & -2.105972 & -3.977424\end{array}$

$\begin{array}{lllll}6 & -3.251506 & 3.489046 & -2.994099\end{array}$

$\begin{array}{lllll}6 & -8.408169 & -1.926599 & 2.665842\end{array}$

$\begin{array}{lllll}1 & -9.796940 & -0.501863 & 1.876091\end{array}$

$\begin{array}{lllll}6 & -6.673744 & -0.972154 & 4.098995\end{array}$

$8 \quad-6.607514 \quad 1.405181 \quad 4.454332$

$\begin{array}{llll}6 & -5.461991 & 1.903576 & 3.923406\end{array}$

$\begin{array}{lllll}6 & -8.415411 & -3.290587 & 0.546012\end{array}$

$\begin{array}{lllll}1 & -9.806161 & -2.020519 & -0.464297\end{array}$

$6 \quad-6.746159 \quad-4.324360 \quad-0.908760$

$8 \quad-6.784198 \quad-3.978324 \quad-3.274172$

$6 \quad-5.544463 \quad-3.426554 \quad-3.454236$

$\begin{array}{lllll}6 & -9.010503 & -3.131023 & 1.946955\end{array}$

$6 \quad-7.299056 \quad-2.065264 \quad 3.509083$

$\begin{array}{llll}1 & -5.798648 & -1.103758 & 4.721417\end{array}$ $\begin{array}{lllll}6 & -3.303127 & 3.197116 & 3.147985\end{array}$

$\begin{array}{lllll}6 & -7.279450 & -4.085297 & 0.347375\end{array}$

$\begin{array}{lllll}1 & -5.883932 & -4.966339 & -1.032853\end{array}$

$\begin{array}{lllll}6 & -3.300808 & -2.061453 & -3.555108\end{array}$

$\begin{array}{lllll}1 & -8.698824 & -4.011253 & 2.510740\end{array}$

$\begin{array}{lllll}8 & -6.792357 & -3.346195 & 3.786939\end{array}$

$\begin{array}{lllll}6 & -3.268104 & 1.866487 & 3.539600\end{array}$

$8 \quad-6.696213 \quad-4.687291 \quad 1.465124$

$\begin{array}{lllll}6 & -3.329381 & -3.397908 & -3.182705\end{array}$

$\begin{array}{lllll}6 & -5.568998 & -3.571660 & 3.226918\end{array}$

$\begin{array}{lllll}6 & -5.532675 & -4.168857 & 1.935473\end{array}$

$\begin{array}{lllll}6 & -3.351391 & -3.344160 & 3.170408\end{array}$

$\begin{array}{lllll}6 & -3.333930 & -3.811536 & 1.864039\end{array}$

$\begin{array}{lllll}6 & -10.545768 & -3.109810 & 1.933737\end{array}$

$\begin{array}{lllll}1 & -10.929291 & -4.019056 & 1.463748\end{array}$

$\begin{array}{lllll}1 & -10.956930 & -2.257869 & 1.385170\end{array}$

$\begin{array}{lllll}6 & -10.468806 & 1.903845 & 2.956789\end{array}$

$\begin{array}{lllll}1 & -10.852785 & 1.407838 & 3.852092\end{array}$

$\begin{array}{llll}1 & -10.889599 & 1.390052 & 2.088071\end{array}$

$\begin{array}{lllll}6 & -10.492615 & 2.979271 & -1.991466\end{array}$

$\begin{array}{lllll}1 & -10.858463 & 3.915622 & -1.562492\end{array}$

$1 \quad-10.913866 \quad 2.161658-1.399909$

$6 \quad-10.523850 \quad-2.042789 \quad-3.082784$

$1 \quad-10.883588 \quad-1.533811 \quad-3.980801$

$\begin{array}{lllll}1 & -10.950779 & -1.526210 & -2.218571\end{array}$

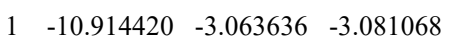

$\begin{array}{lllll}1 & -10.882686 & 2.886464 & -3.008488\end{array}$

$\begin{array}{lllll}1 & -10.839488 & 2.931877 & 2.941492\end{array}$

$\begin{array}{lllll}1 & -10.927756 & -3.058213 & 2.956545\end{array}$

$\begin{array}{lllll}6 & -1.898538 & 3.641370 & 2.847938\end{array}$

$\begin{array}{lllll}6 & -1.853268 & 1.375546 & 3.423259\end{array}$

$\begin{array}{lllll}6 & -1.936715 & -3.031740 & 3.578675\end{array}$

$\begin{array}{lllll}6 & -1.923567 & -3.746265 & 1.355610\end{array}$

$6 \quad-1.874253 \quad 4.619092 \quad-1.460161$

$\begin{array}{lllll}6 & -1.807248 & 3.214614 & -3.329361\end{array}$

$\begin{array}{lllll}6 & -1.910809 & -1.536095 & -3.361712\end{array}$

$\begin{array}{lllll}6 & -1.920191 & -3.821295 & -2.858589\end{array}$

$\begin{array}{lllll}7 & -1.171408 & -3.320617 & 2.431848\end{array}$

$\begin{array}{llll}7 & -1.113779 & 2.493781 & 3.078612\end{array}$

$\begin{array}{lllll}7 & -1.083429 & 3.973339 & -2.404914\end{array}$

$7 \quad-1.164846 \quad-2.637930 \quad-2.973754$

$8 \quad-1.551564 \quad-0.379303 \quad-3.461955$

$8 \quad-1.489526 \quad-4.911694 \quad-2.572154$

$\begin{array}{lllll}8 & -1.534874 & -3.987004 & 0.224039\end{array}$

$\begin{array}{lllll}8 & -1.504809 & -2.627798 & 4.630202\end{array}$

$\begin{array}{lllll}8 & -1.461730 & 0.229049 & 3.555239\end{array}$

$\begin{array}{lllll}8 & -1.504436 & 5.247453 & -0.491024\end{array}$

$\begin{array}{lllll}8 & -1.499937 & 4.719939 & 2.483862\end{array}$

$8 \quad-1.346446 \quad 2.504874 \quad-4.194115$

$\begin{array}{lllll}1 & -0.145523 & -2.657721 & -2.857178\end{array}$

$\begin{array}{lllll}1 & -0.074749 & 3.864522 & -2.312136\end{array}$

$\begin{array}{lllll}1 & -0.095739 & 2.558806 & 3.044022\end{array}$

$\begin{array}{lllll}1 & -0.148331 & -3.221700 & 2.444119\end{array}$

$\begin{array}{lllll}7 & -4.436986 & 3.890847 & 3.025628\end{array}$

$\begin{array}{llll}7 & -4.344436 & 1.192671 & 3.950515\end{array}$

$7 \quad-4.423532 \quad 4.766103 \quad-1.336589$

$7 \begin{array}{llll}7 & -4.348628 & 3.034921 & -3.604014\end{array}$

$\begin{array}{llll}7 & -4.478037 & -3.185671 & 3.869484\end{array}$
$7 \quad-4.420304 \quad-4.248320 \quad 1.221166$

$7 \quad-4.370774-1.390814 \quad-3.982758$

$7 \quad-4.464774 \quad-4.100009 \quad-3.088997$

$\begin{array}{lllll}6 & 9.017962 & -3.619424 & -0.817630\end{array}$

$\begin{array}{llll}8.436081 & -3.291497 & 0.553344\end{array}$

$\begin{array}{lllll}6.368020 & -2.745624 & -1.888507\end{array}$

$\begin{array}{llll}8.726674 & -4.646593 & -1.038976\end{array}$

$\begin{array}{llll}8.924521 & -2.229881 & 1.324689\end{array}$

$\begin{array}{llll}7.371168 & -4.029437 & 1.083941\end{array}$

$6 \quad 8.860818-1.487583-2.242238$

$\begin{array}{llll}7.221535 & -3.187288 & -2.559919\end{array}$

$\begin{array}{lllll}6 & 8.389348 & -1.880925 & 2.566966\end{array}$

$\begin{array}{llll}9.762693 & -1.659736 & 0.942350\end{array}$

$\begin{array}{llll}6.778184 & -3.700901 & 2.297440\end{array}$

$\begin{array}{llll}6.892566 & -5.151591 & 0.386554\end{array}$

$\begin{array}{llll}8.278289 & -0.687659 & -3.232670\end{array}$

$\begin{array}{llll}9.740617 & -1.114586 & -1.732058\end{array}$

$\begin{array}{llll}6.615067 & -2.446346 & -3.565244\end{array}$

$\begin{array}{llll}6.720461 & -4.448118 & -2.220407\end{array}$

$\begin{array}{llll}8.976118 & -0.774190 & 3.442571\end{array}$

$\begin{array}{llll}7.286763 & -2.622687 & 3.006170\end{array}$

$\begin{array}{llll}5.936468 & -4.266665 & 2.674499\end{array}$

$\begin{array}{llll}5.652887 & -4.984056 & -0.159095\end{array}$

$\begin{array}{llll}8.856353 & 0.676343 & -3.597466\end{array}$

$\begin{array}{llll}7.152586 & -1.203918 & -3.888177\end{array}$

$\begin{array}{llll}5.740561 & -2.819404 & -4.082160\end{array}$

$\begin{array}{llll}5.571065 & -4.506792 & -1.498392\end{array}$

$\begin{array}{llll}8.654831 & -0.992430 & 4.461451\end{array}$

$\begin{array}{llll}8.390390 & 0.590270 & 3.095111\end{array}$

$\begin{array}{llll}6.712321 & -2.275171 & 4.234604\end{array}$

$\begin{array}{llll}8.316505 & 1.786128 & -2.696184\end{array}$

$\begin{array}{llll}8.493119 & 0.899094 & -4.601244\end{array}$

$\begin{array}{llll}6.561745 & -0.475007 & -4.932482\end{array}$

$\begin{array}{llll}8.930388 & 1.393184 & 2.084679\end{array}$

$\begin{array}{llll}7.283084 & 1.097990 & 3.785026\end{array}$

$\begin{array}{llll}5.537816 & -1.594707 & 4.188755\end{array}$

$\begin{array}{llll}3.424704 & -4.922337 & -0.015738\end{array}$

$\begin{array}{llll}8.902072 & 2.142310 & -1.479139\end{array}$

$\begin{array}{llll}7.193369 & 2.520439 & -3.091250\end{array}$

$\begin{array}{llll}5.373046 & 0.120045 & -4.613265\end{array}$

$\begin{array}{llll}3.360762 & -4.336258 & -1.270970\end{array}$

$\begin{array}{llll}8.411091 & 2.644711 & 1.745887\end{array}$

$\begin{array}{llll}9.800129 & 1.031850 & 1.549820\end{array}$

$\begin{array}{llll}6.708363 & 2.324921 & 3.470008\end{array}$

$\begin{array}{llll}6.751805 & 0.355540 & 4.851736\end{array}$

$\begin{array}{llll}5.549193 & -0.228423 & 4.590827\end{array}$

$\begin{array}{llll}8.429220 & 3.197925 & -0.689818\end{array}$

$\begin{array}{llll}9.759603 & 1.579853 & -1.128946\end{array}$

$\begin{array}{llll}6.705317 & 3.598379 & -2.369661\end{array}$

$\begin{array}{llll}6.566334 & 2.149344 & -4.282765\end{array}$

$\begin{array}{llll}5.397050 & 1.468220 & -4.162216\end{array}$

$\begin{array}{llll}9.036936 & 3.526704 & 0.668191\end{array}$

$\begin{array}{llll}7.272597 & 3.069966 & 2.442686\end{array}$

$\begin{array}{llll}5.844404 & 2.688971 & 4.010566\end{array}$

$\begin{array}{llll}3.337574 & -1.458834 & 3.868391\end{array}$

$\begin{array}{llll}7.339576 & 3.926608 & -1.178781\end{array}$

$\begin{array}{llll}5.843864 & 4.154934 & -2.713453\end{array}$

$\begin{array}{lllll}6 & 3.167408 & 0.074211 & -4.278393\end{array}$ 
$\begin{array}{lllll}1 & 8.743108 & 4.550992 & 0.899645\end{array}$

$\begin{array}{lllll}8 & 6.750562 & 4.327673 & 2.136222\end{array}$

$\begin{array}{lrrrr}6 & 3.335200 & -0.154466 & 4.342590\end{array}$

$\begin{array}{lllll}8 & 6.878904 & 5.038422 & -0.456974\end{array}$

$\begin{array}{lllll}6 & 3.222482 & 1.335812 & -3.700947\end{array}$

$\begin{array}{lllll}6 & 5.579659 & 4.423691 & 1.451121\end{array}$

$\begin{array}{lllll}6 & 5.646505 & 4.888149 & 0.106357\end{array}$

$\begin{array}{lllll}6 & 3.353864 & 4.421899 & 1.312562\end{array}$

$\begin{array}{lllll}6 & 3.416706 & 4.946868 & 0.030198\end{array}$

$\begin{array}{lllll}6 & 10.571000 & 3.473769 & 0.668831\end{array}$

$\begin{array}{lllll}1 & 10.967065 & 4.172197 & -0.072870\end{array}$

$\begin{array}{lllll}1 & 10.966922 & 2.482836 & 0.429639\end{array}$

$\begin{array}{llll}6 & 10.512212 & -0.779100 & 3.434638\end{array}$

$\begin{array}{lllll}1 & 10.892567 & -0.017544 & 4.120432\end{array}$

$\begin{array}{lllll}1 & 10.936548 & -0.575313 & 2.447601\end{array}$

$\begin{array}{lllll}6 & 10.551605 & -3.556933 & -0.847026\end{array}$

$\begin{array}{lllll}1 & 10.966993 & -4.244655 & -0.105875\end{array}$

$\begin{array}{lllll}1 & 10.945064 & -2.560636 & -0.627182\end{array}$

$\begin{array}{lllll}6 & 10.391461 & 0.670461 & -3.655566\end{array}$

$\begin{array}{lllll}1 & 10.738093 & -0.095967 & -4.353714\end{array}$

$\begin{array}{lllll}1 & 10.856248 & 0.468717 & -2.686465\end{array}$

$\begin{array}{lllll}1 & 10.755066 & 1.643174 & -3.996824\end{array}$

$\begin{array}{lllll}1 & 10.919946 & -3.845481 & -1.834965\end{array}$

$\begin{array}{lllll}1 & 10.884187 & -1.755262 & 3.756344\end{array}$

$\begin{array}{lllll}1 & 10.957024 & 3.754233 & 1.652397\end{array}$

$\begin{array}{lllll}6 & 1.938547 & -1.817383 & 3.462711\end{array}$

$\begin{array}{lllll}6 & 1.924319 & 0.358528 & 4.303831\end{array}$

$\begin{array}{lllll}6 & 1.908898 & 4.221236 & 1.675152\end{array}$

$\begin{array}{lllll}6 & 2.007063 & 5.162069 & -0.460654\end{array}$

$\begin{array}{lllll}6 & 2.017076 & -5.050975 & 0.511972\end{array}$

$\begin{array}{lllll}6 & 1.941990 & -3.939162 & -1.540358\end{array}$

$\begin{array}{lllll}6 & 1.739532 & -0.394429 & -4.243247\end{array}$

$\begin{array}{lllll}6 & 1.858146 & 1.683823 & -3.174764\end{array}$

$\begin{array}{lllll}7 & 1.196732 & 4.749150 & 0.611015\end{array}$

$\begin{array}{lllll}7 & 1.174036 & -0.715532 & 3.804610\end{array}$

$\begin{array}{lllll}7 & 1.216329 & -4.464236 & -0.488481\end{array}$

$\begin{array}{lllll}7 & 1.054659 & 0.623454 & -3.556821\end{array}$

$\begin{array}{lllll}8 & 1.250290 & -1.404023 & -4.691618\end{array}$

$\begin{array}{lllll}8 & 1.539562 & 2.659959 & -2.520399\end{array}$

$\begin{array}{lllll}8 & 1.624121 & 5.575359 & -1.528855\end{array}$

$\begin{array}{lllll}8 & 1.475669 & 3.664438 & 2.666379\end{array}$

$\begin{array}{lllll}8 & 1.490690 & 1.449909 & 4.592861\end{array}$

$\begin{array}{lllll}8 & 1.616832 & -5.536612 & 1.540877\end{array}$

$\begin{array}{lllll}8 & 1.564306 & -2.837766 & 2.912022\end{array}$

$\begin{array}{lllll}8 & 1.539608 & -3.245665 & -2.458090\end{array}$

$\begin{array}{lllll}1 & 0.042544 & 0.563864 & -3.425711\end{array}$

$\begin{array}{lllll}1 & 0.208383 & -4.328878 & -0.359981\end{array}$

$\begin{array}{lllll}1 & 0.167441 & -0.608202 & 3.649809\end{array}$

$\begin{array}{lllll}1 & 0.177615 & 4.749204 & 0.535361\end{array}$

$\begin{array}{llll}7 & 4.435021 & -2.211431 & 3.790910\end{array}$

$\begin{array}{lllll}7 & 4.445501 & 0.496805 & 4.691076\end{array}$

$\begin{array}{lllll}7 & 4.580597 & -5.233630 & 0.577967\end{array}$

$\begin{array}{lllll}7 & 4.425584 & -4.141151 & -2.049914\end{array}$

$\begin{array}{lllll}7 & 4.432245 & 4.152268 & 2.054296\end{array}$

$\begin{array}{lllll}7 & 4.567996 & 5.170350 & -0.608986\end{array}$

$\begin{array}{lllll}7 & 4.250159 & -0.575355 & -4.713414\end{array}$

$\begin{array}{lllll}7 & 4.333183 & 2.070015 & -3.652554\end{array}$

$\begin{array}{llll}6 & -0.717434 & 1.255383 & 0.003327\end{array}$ $\begin{array}{llll}8 & -0.558560 & 2.470889 & -0.041109\end{array}$

$\begin{array}{llll}7 & 0.418281 & 0.430692 & 0.087120\end{array}$

$\begin{array}{lllll}7 & 0.315428 & -0.807955 & 0.929246\end{array}$

$\begin{array}{lllll}8 & 0.351336 & -1.793741 & 0.273459\end{array}$

$\begin{array}{lllll}6 & 1.641100 & 1.137813 & 0.516260\end{array}$

$\begin{array}{lllll}1 & 1.574054 & 1.405681 & 1.575591\end{array}$

$\begin{array}{lllll}1 & 1.616029 & 2.069900 & -0.050904\end{array}$

$\begin{array}{lllll}6 & -2.094648 & 0.689115 & -0.040856\end{array}$

$\begin{array}{lllll}6 & -2.443727 & -0.640422 & -0.315565\end{array}$

$\begin{array}{llll}6 & -3.125992 & 1.620558 & 0.182985\end{array}$

$\begin{array}{lllll}6 & -3.783897 & -1.022913 & -0.336143\end{array}$

$\begin{array}{lllll}1 & -1.694864 & -1.378644 & -0.557725\end{array}$

$\begin{array}{lllll}6 & -4.456841 & 1.233456 & 0.153510\end{array}$

$\begin{array}{lllll}1 & -2.858831 & 2.652373 & 0.369729\end{array}$

$\begin{array}{lllll}6 & -4.809736 & -0.100030 & -0.099117\end{array}$

$\begin{array}{lllll}1 & -4.035436 & -2.057376 & -0.550807\end{array}$

$\begin{array}{lllll}1 & -5.235741 & 1.970511 & 0.325461\end{array}$

$\begin{array}{lllll}6 & -6.251097 & -0.518109 & -0.103582\end{array}$

$\begin{array}{llll}1 & -6.618509 & -0.654321 & 0.918542\end{array}$

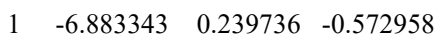

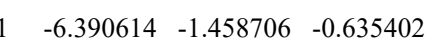

$\begin{array}{lllll}6 & 2.932633 & 0.411659 & 0.232396\end{array}$

$\begin{array}{lllll}6 & 3.068895 & -0.476595 & -0.837468\end{array}$

$\begin{array}{llll}6 & 4.060964 & 0.726626 & 1.004059\end{array}$

$\begin{array}{lllll}6 & 4.317858 & -1.013896 & -1.153579\end{array}$

$\begin{array}{lllll}1 & 2.196882 & -0.750262 & -1.420109\end{array}$

$\begin{array}{lllll}6 & 5.308045 & 0.183996 & 0.691250\end{array}$

$\begin{array}{llll}3.967950 & 1.415043 & 1.839749\end{array}$

$\begin{array}{lllll}6 & 5.441531 & -0.684923 & -0.394309\end{array}$

$1 \quad 4.404404 \quad-1.686264 \quad-1.997543$

$\begin{array}{lllll}1 & 6.175995 & 0.446480 & 1.286384\end{array}$

$\begin{array}{llll}1 & 6.412356 & -1.099315 & -0.644129\end{array}$

\section{2c'@12}

$\begin{array}{llll}6 & 8.911893 & 2.343183 & 2.799227\end{array}$

$\begin{array}{lllll}6 & 8.344078 & 2.892483 & 1.493053\end{array}$

$\begin{array}{lllll}6 & 8.317465 & 0.975844 & 3.117373\end{array}$

$\begin{array}{lllll}1 & 8.571851 & 3.015869 & 3.586903\end{array}$

$\begin{array}{lllll}6 & 8.881815 & 2.575465 & 0.243806\end{array}$

$\begin{array}{lllll}6 & 7.244896 & 3.757380 & 1.507367\end{array}$

$\begin{array}{lllll}6 & 8.888140 & -0.208738 & 2.637788\end{array}$

$\begin{array}{lllll}6 & 7.167064 & 0.858276 & 3.907540\end{array}$

$\begin{array}{lllll}6 & 8.391590 & 3.107257 & -0.953629\end{array}$

$\begin{array}{llll}1 & 9.719960 & 1.889619 & 0.200403\end{array}$

$\begin{array}{lllll}6 & 6.726664 & 4.326771 & 0.352847\end{array}$

$\begin{array}{lllll}6.685480 & 4.083155 & 2.748781\end{array}$

$\begin{array}{llll}6 & 8.359792 & -1.471740 & 2.913486\end{array}$

$\begin{array}{llll}9.787350 & -0.141728 & 2.037356\end{array}$

$\begin{array}{llll}6.589095 & -0.372569 & 4.197417\end{array}$

$\begin{array}{llll}6.620177 & 2.013664 & 4.481127\end{array}$

$\begin{array}{llll}8.992479 & 2.733205 & -2.304128\end{array}$

$\begin{array}{llll}7.315242 & 3.997566 & -0.862808\end{array}$

$\begin{array}{llll}5.882772 & 5.003328 & 0.395442\end{array}$

$\begin{array}{llll}5.476570 & 3.537642 & 3.043064\end{array}$

$\begin{array}{llll}8.995661 & -2.770922 & 2.420635\end{array}$

$\begin{array}{llll}7.189880 & -1.512939 & 3.681072\end{array}$

$\begin{array}{llll}5.695441 & -0.437014 & 4.803770\end{array}$

$\begin{array}{llll}6 & 5.436554 & 2.468615 & 3.985214\end{array}$ $\begin{array}{llll}8.686674 & 3.512878 & -3.002328\end{array}$

$\begin{array}{llll}8.389369 & 1.431378 & -2.825361\end{array}$

$\begin{array}{llll}6.824405 & 4.602027 & -2.031324\end{array}$

$\begin{array}{llll}8.426057 & -3.164474 & 1.061017\end{array}$

$\begin{array}{llll}8.687460 & -3.548602 & 3.120176\end{array}$

$\begin{array}{llll}6.660656 & -2.773003 & 3.964815\end{array}$

$\begin{array}{llll}8.925947 & 0.176358 & -2.529342\end{array}$

$\begin{array}{llll}7.256090 & 1.458733 & -3.646818\end{array}$

$\begin{array}{llll}5.599443 & 4.149423 & -2.428108\end{array}$

$\begin{array}{llll}3.252685 & 3.482430 & 2.917707\end{array}$

$\begin{array}{llll}8.924575 & -2.614082 & -0.126511\end{array}$

$\begin{array}{llll}7.371891 & -4.078877 & 0.945519\end{array}$

$\begin{array}{llll}5.528912 & -3.151925 & 3.319348\end{array}$

$\begin{array}{lll}3.205204 & 2.479718 \quad 3.875220\end{array}$

$\begin{array}{llll}8.400720 & -1.017254 & -3.038039\end{array}$

$\begin{array}{llll}9.793961 & 0.124213 & -1.882975\end{array}$

$\begin{array}{llll}6.710271 & 0.309351 & -4.202085\end{array}$

$\begin{array}{llll}6.712593 & 2.706686 & -3.959957\end{array}$

$\begin{array}{llll}5.547797 & 3.078673 & -3.365175\end{array}$

$\begin{array}{llll}8.408007 & -2.923762 & -1.386469\end{array}$

$\begin{array}{llll}9.753611 & -1.918790 & -0.064155\end{array}$

$\begin{array}{llll}6.798828 & -4.393359 & -0.281578\end{array}$

$\begin{array}{llll}6.862717 & -4.712359 & 2.093950\end{array}$

$\begin{array}{llll}5.617805 & -4.267090 & 2.438570\end{array}$

$\begin{array}{llll}8.997629 & -2.375090 & -2.684741\end{array}$

$\begin{array}{llll}7.293706 & -0.914090 & -3.890029\end{array}$

$\begin{array}{llll}5.848710 & 0.361563 & -4.854802\end{array}$

$\begin{array}{llll}3.370665 & 4.133463 & -2.312762\end{array}$

$\begin{array}{llll}7.313655 & -3.795576 & -1.421346\end{array}$

$\begin{array}{llll}5.961732 & -5.076309 & -0.342685\end{array}$

$\begin{array}{llll}3.340209 & -3.061390 & 2.938020\end{array}$

$\begin{array}{llll}8.684277 & -3.060274 & -3.472898\end{array}$

$6.772988-2.078336 \quad-4.475827$

$\begin{array}{llll}3.331236 & 3.018076 & -3.136212\end{array}$

$\begin{array}{llll}6.751175 & -4.089676 & -2.667424\end{array}$

$\begin{array}{llll}3.399029 & -4.233551 & 2.200244\end{array}$

$\begin{array}{llll}5.571222 & -2.486788 & -3.974629\end{array}$

$\begin{array}{llll}5.568649 & -3.486165 & -2.961620\end{array}$

$\begin{array}{llll}3.348970 & -2.351854 & -3.819622\end{array}$

$\begin{array}{llll}3.359786 & -3.256837 & -2.766564\end{array}$

$10.533546-2.363724 \quad-2.667726$

$10.911871-3.371616-2.478456$

$10.948815-1.707826-1.897693$

$10.527804 \quad 2.708904 \quad-2.284190$

$\begin{array}{llll}10.912617 & 2.485475 & -3.282763\end{array}$

$10.936459 \quad 1.961350 \quad-1.598779$

$\begin{array}{llll}10.447440 & 2.349871 & 2.819102\end{array}$

$\begin{array}{llll}10.816673 & 3.366037 & 2.658486\end{array}$

$\begin{array}{llll}10.889939 & 1.715548 & 2.046157\end{array}$

$10.530254 \quad-2.723136 \quad 2.430463$

$\begin{array}{llll}10.892202 & -2.485705 & 3.434351\end{array}$

$\begin{array}{llll}10.940704 & -1.975127 & 1.746656\end{array}$

$\begin{array}{lll}10.935140 & -3.694514 & 2.135235\end{array}$

$\begin{array}{llll}10.812547 & 1.995351 & 3.786643\end{array}$

$\begin{array}{llll}10.911911 & 3.683820 & -1.973110\end{array}$

$\begin{array}{llll}10.916879 & -2.023187 & -3.633171\end{array}$

$\begin{array}{llll}1.954761 & 4.501829 & -1.951068\end{array}$

$\begin{array}{llll}1.904735 & 2.559976 & -3.243767\end{array}$ 
$6 \quad 1.925842 \quad-1.932582 \quad-4.064829$

$\begin{array}{lllll}6 & 1.952700 & -3.421153 & -2.265197\end{array}$

$\begin{array}{lllll}6 & 1.851682 & 3.797809 & 2.483251\end{array}$

$\begin{array}{lllll}6 & 1.757339 & 2.146463 & 4.138949\end{array}$

$\begin{array}{lllll}6 & 1.931209 & -2.565577 & 2.939478\end{array}$

$\begin{array}{lllll}6 & 1.992037 & -4.599055 & 1.800514\end{array}$

$7 \quad 1.184625 \quad-2.629300 \quad-3.100605$

$\begin{array}{lllll}7 & 1.167012 & 3.541410 & -2.607991\end{array}$

$\begin{array}{llll}7 & 1.042442 & 3.017402 & 3.299144\end{array}$

$\begin{array}{lllll}7 & 1.202171 & -3.535397 & 2.291947\end{array}$

$\begin{array}{lllll}8 & 1.542656 & -1.502912 & 3.402796\end{array}$

$\begin{array}{lllll}8 & 1.578630 & -5.570265 & 1.219289\end{array}$

$8 \quad 1.572156 \quad-4.093787 \quad-1.324938$

$\begin{array}{lllll}8 & 1.476117 & -1.165367 & -4.884348\end{array}$

$\begin{array}{lllll}8 & 1.506649 & 1.515292 & -3.727156\end{array}$

$\begin{array}{lllll}8 & 1.505681 & 4.525823 & 1.574329\end{array}$

$8 \quad 1.555177 \quad 5.400756 \quad-1.250514$

$\begin{array}{lllll}8 & 1.285747 & 1.318481 & 4.881614\end{array}$

$\begin{array}{lllll}1 & 0.191275 & -3.499033 & 2.111380\end{array}$

$\begin{array}{llll}1 & 0.026110 & 3.004598 & 3.225738\end{array}$

$\begin{array}{lllll}1 & 0.150870 & 3.538927 & -2.529294\end{array}$

$1 \quad 0.165425 \quad-2.528143 \quad-3.053040$

$\begin{array}{lllll}7 & 4.510824 & 4.704307 & -1.916847\end{array}$

$\begin{array}{lllll}7 & 4.415398 & 2.476844 & -3.694755\end{array}$

$\begin{array}{lllll}7 & 4.385792 & 4.033860 & 2.481065\end{array}$

$\begin{array}{llll}4.300956 & 1.936855 & 4.415158\end{array}$

$\begin{array}{llll}4.458494 & -1.930230 & -4.429623\end{array}$

$\begin{array}{llll}4.466948 & -3.853444 & -2.324209\end{array}$

$\begin{array}{llll}4.394754 & -2.508279 & 3.537200\end{array}$

$\begin{array}{lllll}7 & 4.550733 & -4.845429 & 1.908469\end{array}$

$\begin{array}{lllll}6 & -9.036969 & -3.647782 & -0.174441\end{array}$

$6 \quad-8.437170 \quad-2.984368 \quad-1.410791$

$\begin{array}{lllll}6 & -8.413344 & -3.078362 & 1.100893\end{array}$

$\begin{array}{llll}-8.742452 & -4.697161 & -0.215749\end{array}$

$\begin{array}{lllll}6 & -8.918941 & -1.759335 & -1.891184\end{array}$

$\begin{array}{lllll}6 & -7.358383 & -3.556883 & -2.097154\end{array}$

$\begin{array}{lllll}6 & -8.935971 & -1.980110 & 1.788072\end{array}$

$6 \quad-7.257330 \quad-3.661189 \quad 1.634795$

$6 \quad-8.356738 \quad-1.090703 \quad-2.981304$

$\begin{array}{llll}-9.765394 & -1.307901 & -1.386815\end{array}$

$6 \quad-6.743348 \quad-2.916906 \quad-3.167868$

$8 \quad-6.874004 \quad-4.820485 \quad-1.719409$

$\begin{array}{lllll}6 & -8.377153 & -1.481974 & 2.973427\end{array}$

$\begin{array}{llll}-9.810924 & -1.483477 & 1.385588\end{array}$

$\begin{array}{llll}6 & -6.686530 & -3.238259 & 2.824735\end{array}$

$\begin{array}{llll}-6.714262 & -4.753028 & 0.958221\end{array}$

$\begin{array}{lllll}6 & -8.919370 & 0.214940 & -3.540326\end{array}$

$6 \quad-7.239028 \quad-1.688250 \quad-3.575631$

$\begin{array}{lllll}1 & -5.892597 & -3.364690 & -3.664781\end{array}$

$6 \quad-5.633324 \quad-4.776799 \quad-1.147831$

$\begin{array}{lllll}6 & -8.931050 & -0.239908 & 3.665456\end{array}$

$\begin{array}{lllll}6 & -7.263071 & -2.158652 & 3.485783\end{array}$

$1 \quad-5.811971 \quad-3.731180 \quad 3.228503$

$\begin{array}{llll}6 & -5.560639 & -4.593932 & 0.261519\end{array}$

$\begin{array}{llll}-8.586842 & 0.266494 & -4.577659\end{array}$

$\begin{array}{llll}-8.328609 & 1.439210 & -2.846640\end{array}$

$8 \quad-6.635719 \quad-1.027546 \quad-4.649761$

$\begin{array}{lllll}6 & -8.338325 & 1.035238 & 3.062390\end{array}$ $\begin{array}{llll}1 & -8.576388 & -0.281185 & 4.696049\end{array}$

$8 \quad-6.704703 \quad-1.757669 \quad 4.709465$

$\begin{array}{lllll}6 & -8.868825 & 1.951179 & -1.662575\end{array}$

$6 \quad-7.227437 \quad 2.117406 \quad-3.383085$

$6 \quad-5.461844 \quad-0.390495 \quad-4.397886$

$6 \quad-3.407243 \quad-4.680101 \quad-1.258046$

$\begin{array}{lllll}6 & -8.923010 & 1.716708 & 1.989826\end{array}$

$\begin{array}{lllll}6 & -7.162528 & 1.583311 & 3.590423\end{array}$

$6 \quad-5.470788 \quad-1.183874 \quad 4.578466$

$6 \quad-3.352372 \quad-4.355279 \quad 0.089801$

$\begin{array}{lllll}6 & -8.369832 & 3.086574 & -1.016871\end{array}$

$\begin{array}{llll}-9.721450 & 1.444463 & -1.226447\end{array}$

$6 \quad-6.683554 \quad 3.244648 \quad-2.777553$

$8 \quad-6.665360 \quad 1.665612 \quad-4.586826$

$6 \quad-5.465621 \quad 1.032239 \quad-4.444417$

$\begin{array}{lllll}6 & -8.403567 & 2.910901 & 1.476768\end{array}$

$\begin{array}{llll}-9.819356 & 1.306553 & 1.539321\end{array}$

$\begin{array}{lllll}6 & -6.623804 & 2.780293 & 3.144153\end{array}$

$\begin{array}{lllll}8 & -6.550883 & 0.918179 & 4.653518\end{array}$

$\begin{array}{lllll}6 & -5.408212 & 0.226062 & 4.408520\end{array}$

$\begin{array}{llll}-9.000835 & 3.623218 & 0.267104\end{array}$

$\begin{array}{llll}-7.256088 & 3.707618 & -1.598618\end{array}$

$\begin{array}{llll}-5.829070 & 3.748293 & -3.211575\end{array}$

$\begin{array}{llll}-3.270005 & -0.352372 & -3.999212\end{array}$

$\begin{array}{llll}-7.264808 & 3.431860 & 2.100495\end{array}$

$\begin{array}{llll}-5.728095 & 3.189571 & 3.591511\end{array}$

$\begin{array}{llll}-3.262513 & -1.295074 & 4.297038\end{array}$

$\begin{array}{llll}-8.701089 & 4.668809 & 0.343514\end{array}$

$\begin{array}{llll}-6.737387 & 4.863237 & -1.005930\end{array}$

$\begin{array}{llll}-3.258623 & 1.027949 & -4.128819\end{array}$

$\begin{array}{llll}-6.784006 & 4.672671 & 1.669941\end{array}$

$\begin{array}{llll}-3.225099 & 0.059657 & 3.987504\end{array}$

$\begin{array}{llll}-5.542305 & 4.755528 & -0.357524\end{array}$

$\begin{array}{llll}-5.567450 & 4.673551 & 1.061770\end{array}$

$\begin{array}{llll}-3.313032 & 4.707061 & -0.294836\end{array}$

$\begin{array}{lllll}6 & -3.339456 & 4.632741 & 1.090391\end{array}$

$\begin{array}{lllll}6 & -10.534832 & 3.581908 & 0.237023\end{array}$

$\begin{array}{lllll}1 & -10.938025 & 4.026655 & 1.150559\end{array}$

$\begin{array}{lllll}1 & -10.935588 & 2.567537 & 0.160476\end{array}$

$\begin{array}{lllll}6 & -10.455605 & 0.230828 & -3.548999\end{array}$

$1 \quad-10.817600 \quad 1.149445 \quad-4.017875$

$\begin{array}{lllll}1 & -10.889400 & 0.179387 & -2.546824\end{array}$

$\begin{array}{lllll}6 & -10.571190 & -3.597868 & -0.156838\end{array}$

$\begin{array}{lllll}1 & -10.970689 & -4.073065 & -1.056424\end{array}$

$\begin{array}{lllll}1 & -10.965519 & -2.578882 & -0.120702\end{array}$

$\begin{array}{lllll}6 & -10.465593 & -0.216452 & 3.706014\end{array}$

$1 \quad-10.839716 \quad-1.113698 \quad 4.205836$

$\begin{array}{lllll}1 & -10.920255 & -0.180217 & 2.712255\end{array}$

$\begin{array}{llll}1 & -10.812840 & 0.660392 & 4.258628\end{array}$

$\begin{array}{lllll}1 & -10.954093 & -4.130327 & 0.717638\end{array}$

$1 \quad-10.835202 \quad-0.623095 \quad-4.116020$

$\begin{array}{lllll}1 & -10.908812 & 4.148086 & -0.619921\end{array}$

$\begin{array}{lllll}6 & -1.874562 & -0.815042 & -3.695989\end{array}$

$\begin{array}{lllll}6 & -1.845826 & 1.506736 & -3.953954\end{array}$

$\begin{array}{lllll}6 & -1.876898 & 4.676910 & -0.743950\end{array}$

$\begin{array}{lllll}6 & -1.921160 & 4.564293 & 1.589452\end{array}$

$6 \quad-1.995044 \quad-4.734508 \quad-1.782826$

$\begin{array}{lllll}6 & -1.921772 & -4.083768 & 0.452888\end{array}$ $\begin{array}{llll}-1.867772 & -1.846064 & 4.199121\end{array}$

$\begin{array}{llll}-1.821183 & 0.408045 & 3.562262\end{array}$

$\begin{array}{llll}-1.138454 & 4.640974 & 0.436284\end{array}$

$\begin{array}{llll}-1.103230 & 0.340590 & -3.705860\end{array}$

$\begin{array}{llll}-1.194712 & -4.416954 & -0.667007\end{array}$

$\begin{array}{llll}-1.099516 & -0.759998 & 3.752553\end{array}$

$\begin{array}{llll}-1.457500 & -2.956107 & 4.440292\end{array}$

$\begin{array}{llll}-1.408406 & 1.451165 & 3.094430\end{array}$

$\begin{array}{llll}-1.526040 & 4.452598 & 2.729615\end{array}$

$\begin{array}{llll}-1.457753 & 4.651604 & -1.880860\end{array}$

$\begin{array}{llll}-1.418054 & 2.636387 & -3.995683\end{array}$

$\begin{array}{llll}-1.593295 & -4.989789 & -2.890701\end{array}$

$\begin{array}{llll}-1.514272 & -1.954272 & -3.471735\end{array}$

$\begin{array}{llll}-1.510513 & -3.629732 & 1.510664\end{array}$

$\begin{array}{llll}-0.088006 & -0.863643 & 3.612440\end{array}$

$\begin{array}{llll}-0.182124 & -4.271345 & -0.768995\end{array}$

$\begin{array}{llll}-0.083053 & 0.383946 & -3.686674\end{array}$

$\begin{array}{llll}-0.118638 & 4.630837 & 0.481873\end{array}$

$\begin{array}{llll}-4.370243 & -1.094104 & -4.136627\end{array}$

$\begin{array}{llll}-4.361176 & 1.753328 & -4.326512\end{array}$

$\begin{array}{llll}-4.557450 & -4.861291 & -1.914344\end{array}$

$\begin{array}{llll}-4.424731 & -4.323404 & 0.885850\end{array}$

$\begin{array}{llll}-4.413193 & 4.761399 & -1.050769\end{array}$

$\begin{array}{llll}-4.468812 & 4.608813 & 1.799439\end{array}$

$\begin{array}{llll}-4.394273 & -1.952419 & 4.563532\end{array}$

$\begin{array}{llll}-4.295897 & 0.854921 & 4.058986\end{array}$

$\begin{array}{llll}-4.790859 & 0.015225 & 0.020535\end{array}$

$\begin{array}{llll}-3.865357 & -0.792372 & 0.702443\end{array}$

$\begin{array}{llll}-2.502746 & -0.542115 & 0.624500\end{array}$

$\begin{array}{llll}-2.024824 & 0.536450 & -0.134615\end{array}$

$\begin{array}{llll}-2.942639 & 1.351481 & -0.812921\end{array}$

$\begin{array}{llll}-4.306224 & 1.088896 & -0.737914\end{array}$

$\begin{array}{llll}-4.218360 & -1.628383 & 1.299234\end{array}$

$\begin{array}{llll}-1.817676 & -1.181843 & 1.162663\end{array}$

$\begin{array}{llll}-2.575962 & 2.184452 & -1.399581\end{array}$

$\begin{array}{llll}-5.003471 & 1.725304 & -1.272678\end{array}$

$\begin{array}{llll}-6.259669 & -0.290212 & 0.087934\end{array}$

$\begin{array}{llll}-6.508057 & -1.130357 & -0.568513\end{array}$

$\begin{array}{llll}-6.558114 & -0.574497 & 1.099002\end{array}$

$\begin{array}{llll}-6.867054 & 0.561747 & -0.222987\end{array}$

$\begin{array}{llll}-0.603456 & 0.926674 & -0.180755\end{array}$

$\begin{array}{llll}-0.221388 & 2.010456 & -0.585626\end{array}$

$\begin{array}{llll}0.401345 & 0.034918 & 0.366013\end{array}$

$\begin{array}{lll}1.566768 & 0.615956 & 1.061205\end{array}$

$\begin{array}{lll}1.406300 & 1.691394 & 1.011235\end{array}$

$\begin{array}{llll}1.535246 & 0.301915 & 2.103581\end{array}$

$\begin{array}{llll}0.532393 & -1.264331 & 0.027637\end{array}$

$-0.300238-1.685906-0.764001$

$\begin{array}{llll}2.901620 & 0.264550 & 0.444744\end{array}$

$\begin{array}{llll}3.078061 & 0.271232 & -0.942714\end{array}$

$\begin{array}{llll}3.998446 & -0.006192 & 1.270743\end{array}$

$\begin{array}{llll}4.332597 & 0.023241 & -1.496695\end{array}$

$\begin{array}{llll}2.233539 & 0.465634 & -1.594011\end{array}$

$\begin{array}{llll}5.257655 & -0.250522 & 0.714644\end{array}$

$\begin{array}{llll}3.867423 & -0.043808 & 2.347918\end{array}$

$\begin{array}{llll}5.429579 & -0.233866 & -0.669910\end{array}$

$\begin{array}{llll}4.450131 & 0.053379 & -2.572624\end{array}$

$\begin{array}{llll}6.101282 & -0.459549 & 1.363322\end{array}$ 


\section{4c-TS@12}

$\begin{array}{lllll}6 & 8.985548 & -2.857120 & -2.363011\end{array}$

$\begin{array}{lllll}6 & 8.394882 & -3.244707 & -1.010743\end{array}$

$6 \quad 8.377612 \quad-1.534226 \quad-2.824945$

$\begin{array}{lllll}1 & 8.656170 & -3.615256 & -3.074251\end{array}$

$\begin{array}{lllll}6 & 8.886898 & -2.709709 & 0.186606\end{array}$

$6 \quad 7.297713 \quad-4.108907 \quad-0.919608$

$\begin{array}{lllll}6 & 8.965675 & -0.293837 & -2.550510\end{array}$

$\begin{array}{lllll}6 & 7.176792 & -1.530031 & -3.546123\end{array}$

$\begin{array}{lllll}6 & 8.319411 & -2.984505 & 1.433264\end{array}$

$\begin{array}{lllll}1 & 9.738243 & -2.040662 & 0.140445\end{array}$

$\begin{array}{lllll}6 & 6.689709 & -4.407712 & 0.294437\end{array}$

$8 \quad 6.791771 \quad-4.706260 \quad-2.080912$

$\begin{array}{lllll}6 & 8.410553 & 0.913642 & -2.983033\end{array}$

$\begin{array}{lllll}1 & 9.899726 & -0.270817 & -2.002004\end{array}$

$\begin{array}{lllll}6 & 6.573866 & -0.360154 & -3.986619\end{array}$

$8 \quad 6.640536 \quad-2.764761 \quad-3.918680$

$\begin{array}{lllll}6 & 8.865778 & -2.420513 & 2.742346\end{array}$

$6 \quad 7.197542 \quad-3.821384 \quad 1.443167$

$\begin{array}{lllll}1 & 5.827967 & -5.060622 & 0.335875\end{array}$

$\begin{array}{lllll}6 & 5.551411 & -4.278824 & -2.459475\end{array}$

$\begin{array}{lllll}6 & 9.043968 & 2.282700 & -2.728918\end{array}$

$\begin{array}{lllll}6 & 7.208333 & 0.838184 & -3.694602\end{array}$

$\begin{array}{lllll}1 & 5.647766 & -0.384300 & -4.544649\end{array}$

$\begin{array}{lllll}6 & 5.470338 & -3.205535 & -3.392531\end{array}$

$\begin{array}{lllll}1 & 8.530830 & -3.098963 & 3.527677\end{array}$

$\begin{array}{lllll}6 & 8.258535 & -1.059231 & 3.072348\end{array}$

$\begin{array}{lllll}8 & 6.592737 & -4.084278 & 2.675357\end{array}$

$\begin{array}{lllll}6 & 8.450408 & 2.919363 & -1.474244\end{array}$

$\begin{array}{lllll}1 & 8.748819 & 2.917700 & -3.565046\end{array}$

$\begin{array}{lllll}8 & 6.704466 & 2.039757 & -4.188357\end{array}$

$\begin{array}{lllll}6 & 8.789682 & 0.129654 & 2.560872\end{array}$

$\begin{array}{lllll}6 & 7.157735 & -0.942198 & 3.929953\end{array}$

$\begin{array}{lllll}6 & 5.417041 & -3.453759 & 2.929801\end{array}$

$6 \quad 3.323175 \quad-4.368246 \quad-2.394885$

$\begin{array}{lllll}6 & 8.909062 & 2.560084 & -0.200902\end{array}$

$6 \quad 7.412016 \quad 3.857734 \quad-1.529660$

$\begin{array}{lllll}6 & 5.566945 & 2.551568 & -3.665123\end{array}$

$\begin{array}{lllll}6 & 3.243344 & -3.298411 & -3.277264\end{array}$

$\begin{array}{lllll}6 & 8.280978 & 1.392146 & 2.877921\end{array}$

$\begin{array}{lllll}1 & 9.646188 & 0.069638 & 1.899655\end{array}$

$\begin{array}{lllll}6 & 6.601924 & 0.286848 & 4.263413\end{array}$

$\begin{array}{lllll}8 & 6.603850 & -2.100074 & 4.502022\end{array}$

$\begin{array}{lllll}6 & 5.408718 & -2.472547 & 3.961976\end{array}$

$\begin{array}{llll}6 & 8.363292 & 3.060212 & 0.983201\end{array}$

$\begin{array}{lllll}1 & 9.722420 & 1.847135 & -0.132803\end{array}$

$\begin{array}{lllll}6 & 6.821174 & 4.371469 & -0.378309\end{array}$

$\begin{array}{lllll}8 & 6.921412 & 4.305698 & -2.767709\end{array}$

$\begin{array}{lllll}6 & 5.667644 & 3.824844 & -3.037409\end{array}$

$\begin{array}{lllll}6 & 8.906326 & 2.681566 & 2.356878\end{array}$

$\begin{array}{llll}6 & 7.170446 & 1.434151 & 3.728295\end{array}$

$1 \quad 5.743541 \quad 0.346544 \quad 4.920012$

$\begin{array}{lllll}6 & 3.222704 & -3.172600 & 2.673866\end{array}$

$\begin{array}{llll}6 & 7.284615 & 3.945037 & 0.857000\end{array}$

$1 \quad 6.000106 \quad 5.073622 \quad-0.448604$

$\begin{array}{lllll}6 & 3.373993 & 2.581586 & -3.326678\end{array}$ $\begin{array}{llll}1 & 8.584851 & 3.470258 & 3.037728\end{array}$

$\begin{array}{lllll}8 & 6.641803 & 2.684811 & 4.077450\end{array}$

$\begin{array}{lllll}6 & 3.195392 & -2.298606 & 3.749912\end{array}$

$\begin{array}{lllll}8 & 6.683337 & 4.440404 & 2.020410\end{array}$

$\begin{array}{lllll}6 & 3.446785 & 3.881418 & -2.848741\end{array}$

$\begin{array}{lllll}6 & 5.458914 & 3.006081 & 3.483661\end{array}$

$\begin{array}{lllll}6 & 5.484116 & 3.898037 & 2.374022\end{array}$

$\begin{array}{llll}6 & 3.240305 & 2.859610 & 3.297268\end{array}$

$\begin{array}{lllll}6 & 3.267414 & 3.692982 & 2.188788\end{array}$

$\begin{array}{lllll}6 & 10.441899 & 2.643650 & 2.387876\end{array}$

$\begin{array}{llll}10.846948 & 3.608436 & 2.071597\end{array}$

$\begin{array}{llll}10.864205 & 1.878109 & 1.731269\end{array}$

$\begin{array}{llll}6 & 10.401599 & -2.401977 & 2.775465\end{array}$

$1 \quad 10.750540 \quad-2.056973 \quad 3.752146$

$\begin{array}{lllll}1 & 10.838395 & -1.744447 & 2.018890\end{array}$

$\begin{array}{lllll}6 & 10.519935 & -2.855967 & -2.364330\end{array}$

$\begin{array}{llll}10.893687 & -3.847092 & -2.094455\end{array}$

$\begin{array}{llll}10.949851 & -2.140828 & -1.657597\end{array}$

$\begin{array}{lllll}6 & 10.578066 & 2.241579 & -2.699360\end{array}$

$\begin{array}{lllll}1 & 10.960787 & 1.824044 & -3.634442\end{array}$

$\begin{array}{llll}10.975810 & 1.635763 & -1.880417\end{array}$

$\begin{array}{llll}10.975794 & 3.252735 & -2.581109\end{array}$

$\begin{array}{llll}10.894660 & -2.602221 & -3.359474\end{array}$

$\begin{array}{llll}10.791550 & -3.408267 & 2.601257\end{array}$

$\begin{array}{llll}1 & 10.790042 & 2.435208 & 3.402891\end{array}$

$\begin{array}{lllll}6 & 1.826064 & -3.318418 & 2.152033\end{array}$

$\begin{array}{lllll}6 & 1.766719 & -1.903228 & 4.001607\end{array}$

$\begin{array}{lllll}6 & 1.809255 & 2.494512 & 3.579842\end{array}$

$\begin{array}{lllll}6 & 1.856296 & 3.868431 & 1.694199\end{array}$

$\begin{array}{lllll}6 & 1.928079 & -4.796749 & -2.026236\end{array}$

$6 \quad \begin{array}{llll}6 & 1.786125 & -2.981070 & -3.512232\end{array}$

$\begin{array}{lllll}6 & 1.955833 & 2.114753 & -3.230985\end{array}$

$\begin{array}{lllll}6 & 2.044308 & 4.346343 & -2.554890\end{array}$

$\begin{array}{lllll}7 & 1.071162 & 3.181057 & 2.607108\end{array}$

$\begin{array}{llll}7 & 1.029659 & -2.644286 & 3.059457\end{array}$

$\begin{array}{lllll}7 & 1.099093 & -3.916011 & -2.735108\end{array}$

$\begin{array}{lllll}7 & 1.237952 & 3.225406 & -2.842417\end{array}$

$8 \quad 1.563785 \quad 0.973352 \quad-3.408409$

$\begin{array}{lllll}8 & 1.652598 & 5.419325 & -2.167544\end{array}$

$\begin{array}{lllll}8 & 1.492834 & 4.469370 & 0.700951\end{array}$

$\begin{array}{lllll}8 & 1.366744 & 1.774631 & 4.444846\end{array}$

$8 \quad 1.328945 \quad-1.115475 \quad 4.804126$

$8 \quad 1.569862 \quad-5.677301 \quad-1.279885$

$\begin{array}{lllll}8 & 1.470540 & -3.876258 & 1.126091\end{array}$

$8 \quad 1.300075 \quad-2.108889 \quad-4.197271$

$\begin{array}{lllll}1 & 0.222838 & 3.273488 & -2.721017\end{array}$

$1 \quad 0.083032 \quad-3.975112 \quad-2.673643$

$\begin{array}{llll}1 & 0.011010 & -2.646625 & 2.986367\end{array}$

$\begin{array}{llll}1 & 0.054502 & 3.088324 & 2.577987\end{array}$

$\begin{array}{llll}7 & 4.331205 & -3.777420 & 2.244310\end{array}$

$7 \quad 4.294430 \quad-1.903428 \quad 4.396170$

$\begin{array}{lllll}7 & 4.481259 & -4.873912 & -1.958633\end{array}$

$\begin{array}{lllll}7 & 4.316885 & -2.698041 & -3.801022\end{array}$

$\begin{array}{llll}7 & 4.336698 & 2.485565 & 3.958036\end{array}$

$\begin{array}{lllll}7 & 4.389450 & 4.230320 & 1.703443\end{array}$

$7 \quad 4.425554 \quad 1.894381 \quad-3.771332$

$7 \quad 4.607241 \quad 4.519680 \quad-2.658958$

$\begin{array}{lllll}6 & -9.043336 & 3.526943 & -0.578796\end{array}$ $\begin{array}{lllll}6 & -8.501387 & 3.060766 & 0.767057\end{array}$

$\begin{array}{lllll}6 & -8.352320 & 2.772153 & -1.711895\end{array}$

$\begin{array}{lllll}1 & -8.754345 & 4.572953 & -0.684622\end{array}$

$\begin{array}{llll}6 & -9.021889 & 1.946733 & 1.435696\end{array}$

$\begin{array}{lllll}6 & -7.429793 & 3.727690 & 1.371269\end{array}$

$\begin{array}{lllll}6 & -8.817877 & 1.542263 & -2.182796\end{array}$

$\begin{array}{lllll}6 & -7.208966 & 3.296318 & -2.329367\end{array}$

$\begin{array}{llll}6 & -8.507153 & 1.482449 & 2.648933\end{array}$

$\begin{array}{llll}-9.864677 & 1.425652 & 0.996734\end{array}$

$\begin{array}{lllll}6 & -6.860644 & 3.290503 & 2.560006\end{array}$

$\begin{array}{llll}-6.938645 & 4.895997 & 0.774156\end{array}$

$\begin{array}{llll}-8.223460 & 0.853066 & -3.245865\end{array}$

$\begin{array}{llll}-9.688014 & 1.104828 & -1.707625\end{array}$

$\begin{array}{llll}-6.589108 & 2.663399 & -3.399252\end{array}$

$\begin{array}{llll}-6.728615 & 4.532470 & -1.888556\end{array}$

$\begin{array}{lll}-9.109205 & 0.303972 & 3.414649\end{array}$

$\begin{array}{lll}-7.399626 & 2.164999 & 3.165301\end{array}$

$\begin{array}{llll}-6.016903 & 3.808170 & 2.996666\end{array}$

$\begin{array}{llll}-5.688800 & 4.809042 & 0.238719\end{array}$

$\begin{array}{llll}-8.771392 & -0.482078 & -3.739432\end{array}$

$\begin{array}{lllll}6 & -7.110984 & 1.453034 & -3.847842\end{array}$

$\begin{array}{llll}-5.720033 & 3.100180 & -3.874853\end{array}$

$\begin{array}{llll}-5.579529 & 4.560138 & -1.158643\end{array}$

$\begin{array}{llll}-8.812647 & 0.435065 & 4.455912\end{array}$

$\begin{array}{llll}6 & -8.502012 & -1.020354 & 2.961958\end{array}$

$\begin{array}{llll}-6.855911 & 1.707302 & 4.367152\end{array}$

$\begin{array}{lllll}6 & -8.230890 & -1.656687 & -2.924899\end{array}$

$\begin{array}{llll}-8.384361 & -0.613759 & -4.750712\end{array}$

$\begin{array}{llll}-6.503940 & 0.832421 & -4.951761\end{array}$

$\begin{array}{llll}-9.010664 & -1.729437 & 1.865906\end{array}$

$\begin{array}{llll}-7.402280 & -1.577589 & 3.627588\end{array}$

$\begin{array}{llll}-5.672300 & 1.042917 & 4.301365\end{array}$

$\begin{array}{llll}-3.462551 & 4.834670 & 0.398529\end{array}$

$\begin{array}{llll}-8.854586 & -2.146024 & -1.774556\end{array}$

$\begin{array}{llll}-7.065485 & -2.314511 & -3.336777\end{array}$

$\begin{array}{llll}-5.294064 & 0.272633 & -4.648550\end{array}$

$\begin{array}{llll}-3.359068 & 4.545339 & -0.954571\end{array}$

$\begin{array}{llll}-8.466466 & -2.935256 & 1.416924\end{array}$

$\begin{array}{llll}-9.868103 & -1.322740 & 1.343504\end{array}$

$\begin{array}{llll}-6.808925 & -2.763451 & 3.208023\end{array}$

$\begin{array}{llll}-6.898304 & -0.952251 & 4.776283\end{array}$

$\begin{array}{llll}-5.685634 & -0.342982 & 4.617500\end{array}$

$\begin{array}{llll}-8.389023 & -3.264383 & -1.069300\end{array}$

$\begin{array}{llll}-9.736117 & -1.635474 & -1.405716\end{array}$

$\begin{array}{llll}-6.593030 & -3.456735 & -2.712906\end{array}$

$\begin{array}{llll}-6.390392 & -1.817336 & -4.450553\end{array}$

$\begin{array}{lllll}6 & -5.262682 & -1.087235 & -4.236742\end{array}$

$\begin{array}{llll}-9.038267 & -3.720098 & 0.234463\end{array}$

$\begin{array}{llll}-7.342182 & -3.410069 & 2.102862\end{array}$

$\begin{array}{llll}-5.950698 & -3.165444 & 3.730046\end{array}$

$\begin{array}{lllll}6 & -3.456937 & 0.954481 & 4.081093\end{array}$

$6 \quad-7.271665 \quad-3.924645 \quad-1.593960$

$\begin{array}{llll}-5.706858 & -3.960036 & -3.074989\end{array}$

$\begin{array}{lllll}6 & -3.108990 & 0.420969 & -4.238517\end{array}$

$\begin{array}{llll}-8.739951 & -4.758667 & 0.382095\end{array}$

$\begin{array}{llll}-6.774834 & -4.611461 & 1.665297\end{array}$

$\begin{array}{llll}6 & -3.457508 & -0.365878 & 4.509676\end{array}$

$8 \quad-6.795655 \quad-5.094969 \quad-0.975065$ 
$6 \quad-3.112807 \quad-0.853902 \quad-3.684166$

$\begin{array}{lllll}6 & -5.590114 & -4.547128 & 1.008949\end{array}$

$6 \quad-5.587979 \quad-4.914119 \quad-0.367306$

$\begin{array}{lllll}6 & -3.389068 & -4.228795 & 0.896841\end{array}$

$\begin{array}{lllll}6 & -3.369818 & -4.683825 & -0.411595\end{array}$

$\begin{array}{lllll}6 & -10.572085 & -3.682081 & 0.183566\end{array}$

$\begin{array}{lllll}1 & -10.933767 & -4.305422 & -0.638225\end{array}$

$\begin{array}{lllll}1 & -10.971144 & -2.675291 & 0.032331\end{array}$

$\begin{array}{lllll}6 & -10.644315 & 0.298876 & 3.370338\end{array}$

$\begin{array}{lllll}1 & -11.033999 & -0.522809 & 3.976892\end{array}$

$\begin{array}{llll}1 & -11.043461 & 0.180272 & 2.359310\end{array}$

$\begin{array}{lllll}6 & -10.574437 & 3.459452 & -0.667452\end{array}$

$\begin{array}{lllll}1 & -11.020754 & 4.058503 & 0.130512\end{array}$

$\begin{array}{lllll}1 & -10.965323 & 2.442828 & -0.573477\end{array}$

$6 \begin{array}{llll}6 & -10.304824 & -0.491088 & -3.830158\end{array}$

$1 \quad-10.645538 \quad 0.323290 \quad-4.474675$

$\begin{array}{lllll}1 & -10.792338 & -0.368283 & -2.859181\end{array}$

$\begin{array}{lllll}1 & -10.649298 & -1.438119 & -4.253730\end{array}$

$\begin{array}{lllll}1 & -10.912234 & 3.853773 & -1.629380\end{array}$

$\begin{array}{lllll}1 & -11.032625 & 1.240080 & 3.767925\end{array}$

$\begin{array}{lllll}1 & -10.988348 & -4.063675 & 1.119493\end{array}$

$\begin{array}{lllll}6 & -2.037179 & 1.360325 & 3.793488\end{array}$

$\begin{array}{lllll}6 & -2.030469 & -0.834723 & 4.606953\end{array}$

$\begin{array}{lllll}6 & -2.000101 & -3.822180 & 1.275613\end{array}$

$6 \quad-1.949258 \quad-4.645522 \quad-0.905863$

$\begin{array}{lllll}6 & -2.068226 & 4.929411 & 0.966424\end{array}$

$\begin{array}{lllll}6 & -1.905115 & 4.397326 & -1.300056\end{array}$

$\begin{array}{lllll}6 & -1.721894 & 0.981859 & -4.138817\end{array}$

$6 \quad-1.743232 \quad-1.117676 \quad-3.102455$

$7 \quad-1.215334 \quad-4.131789 \quad 0.175871$

$\begin{array}{lllll}7 & -1.272768 & 0.278801 & 4.210144\end{array}$

$\begin{array}{lllll}7 & -1.225927 & 4.682231 & -0.130724\end{array}$

$7 \quad-0.999165 \quad-0.001123 \quad-3.454851$

$8 \quad-1.293662 \quad 2.041821 \quad-4.533142$

$\begin{array}{lllll}8 & -1.368595 & -2.056829 & -2.429069\end{array}$

$8 \quad-1.518984 \quad-4.969068 \quad-1.988225$

$8 \quad-1.642440 \quad-3.297941 \quad 2.315212$

$\begin{array}{lllll}8 & -1.610071 & -1.919959 & 4.930543\end{array}$

$\begin{array}{llll}8 & -1.710543 & 5.153458 & 2.097722\end{array}$

$\begin{array}{llll}8 & -1.652950 & 2.390827 & 3.275922\end{array}$

$\begin{array}{lllll}8 & -1.441041 & 4.048968 & -2.370133\end{array}$

$1 \quad 0.007358 \quad 0.127802 \quad-3.317351$

$1 \quad-0.208088 \quad 4.618103 \quad-0.014420$

$\begin{array}{llll}-0.254585 & 0.285652 & 4.207607\end{array}$

$\begin{array}{llll}-0.195510 & -4.069082 & 0.225789\end{array}$

$\begin{array}{llll}-4.563321 & 1.692680 & 3.980367\end{array}$

$\begin{array}{llll}-4.576432 & -1.054628 & 4.750198\end{array}$

$\begin{array}{llll}-4.633768 & 4.958602 & 1.026727\end{array}$

$\begin{array}{llll}-4.411720 & 4.400586 & -1.763043\end{array}$

$\begin{array}{llll}-4.493956 & -4.160457 & 1.642194\end{array}$

$\begin{array}{llll}-4.477284 & -5.013662 & -1.081274\end{array}$

$\begin{array}{llll}-4.207961 & 1.026012 & -4.691541\end{array}$

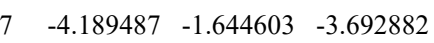

$\begin{array}{lllll}6 & -0.479265 & 0.981656 & 0.048412\end{array}$

$\begin{array}{lllll}8 & -0.005559 & 1.975865 & -0.408518\end{array}$

$\begin{array}{llll}0.813686 & -0.428259 & 0.034626\end{array}$

$\begin{array}{llll}1.045825 & -0.295125 & 1.262224\end{array}$

$\begin{array}{llll}8 & 0.173852 & 0.536561 & 1.734237\end{array}$ $\begin{array}{lllll}6 & -1.876901 & 0.518544 & 0.009366\end{array}$

$\begin{array}{lllll}6 & -2.371342 & -0.461997 & 0.875183\end{array}$

$\begin{array}{lllll}6 & -2.750749 & 1.164862 & -0.875631\end{array}$

$\begin{array}{lllll}6 & -3.727538 & -0.769468 & 0.873570\end{array}$

$\begin{array}{lllll}1 & -1.700994 & -0.957496 & 1.563363\end{array}$

$6 \quad-4.104186 \quad 0.841511 \quad-0.877934$

$\begin{array}{lllll}1 & -2.364995 & 1.912551 & -1.555930\end{array}$

$6 \begin{array}{llll}6 & -4.616889 & -0.123963 & -0.001498\end{array}$

$\begin{array}{llll}1 & -4.104740 & -1.510543 & 1.571688\end{array}$

$\begin{array}{lllll}1 & -4.772827 & 1.354151 & -1.563034\end{array}$

$\begin{array}{lllll}6 & -6.079404 & -0.478568 & -0.009760\end{array}$

$\begin{array}{lllll}1 & -6.677679 & 0.282470 & -0.516197\end{array}$

$1 \quad-6.242161 \quad-1.427340 \quad-0.531697$

$1 \quad-6.466901 \quad-0.598550 \quad 1.004628$

$\begin{array}{lllll}6 & 1.825683 & -1.088287 & -0.800318\end{array}$

$\begin{array}{lllll}1 & 1.784541 & -2.165268 & -0.621447\end{array}$

$\begin{array}{lllll}6 & 3.214141 & -0.538829 & -0.546135\end{array}$

$\begin{array}{lllll}6 & 3.421816 & 0.845367 & -0.476353\end{array}$

$\begin{array}{lllll}6 & 4.302499 & -1.399840 & -0.384359\end{array}$

$\begin{array}{lllll}6 & 4.701805 & 1.356860 & -0.277393\end{array}$

$\begin{array}{lllll}1 & 2.574453 & 1.520187 & -0.558299\end{array}$

$\begin{array}{lllll}6 & 5.586363 & -0.885859 & -0.183285\end{array}$

$\begin{array}{lllll}1 & 4.146819 & -2.474303 & -0.392650\end{array}$

$\begin{array}{lllll}6 & 5.790759 & 0.492111 & -0.135509\end{array}$

$\begin{array}{lllll}1 & 4.848578 & 2.429947 & -0.234143\end{array}$

$1 \quad 6.424186 \quad-1.559368 \quad-0.057564$

$\begin{array}{lllll}1 & 6.786588 & 0.888566 & 0.022465\end{array}$

$\begin{array}{lllll}1 & 1.525873 & -0.901951 & -1.832311\end{array}$

\section{4c@1 12}

$\begin{array}{lllll}6 & 8.943364 & -0.754319 & 3.687551\end{array}$

$\begin{array}{lllll}6 & 8.361016 & 0.589220 & 3.228391\end{array}$

$\begin{array}{lllll}6 & 8.391299 & -1.928648 & 2.879315\end{array}$

$\begin{array}{lllll}1 & 8.591058 & -0.897863 & 4.709608\end{array}$

$\begin{array}{llll}6 & 8.942419 & 1.409821 & 2.253920\end{array}$

$\begin{array}{lllll}6 & 7.190604 & 1.065966 & 3.829122\end{array}$

$\begin{array}{lllll}6 & 8.938068 & -2.259808 & 1.631252\end{array}$

$\begin{array}{lllll}6 & 7.295325 & -2.691462 & 3.312278\end{array}$

$\begin{array}{llll}6 & 8.410955 & 2.659336 & 1.899805\end{array}$

$\begin{array}{llll}1 & 9.842338 & 1.069179 & 1.754729\end{array}$

$\begin{array}{lllll}6 & 6.644434 & 2.308126 & 3.548157\end{array}$

$\begin{array}{lllll}8 & 6.587975 & 0.251105 & 4.786807\end{array}$

$\begin{array}{lllll}6 & 8.417317 & -3.253685 & 0.799232\end{array}$

$\begin{array}{llll}1 & 9.791674 & -1.692671 & 1.280511\end{array}$

$\begin{array}{llll}6 & 6.743912 & -3.698725 & 2.522555\end{array}$

$\begin{array}{lllll}8 & 6.688140 & -2.429013 & 4.552409\end{array}$

$\begin{array}{lllll}6 & 8.998528 & 3.538504 & 0.797983\end{array}$

$\begin{array}{llll}6 & 7.272953 & 3.089926 & 2.590402\end{array}$

$\begin{array}{llll}1 & 5.749303 & 2.651161 & 4.048398\end{array}$

$\begin{array}{lllll}6 & 5.445260 & -0.386681 & 4.428311\end{array}$

$\begin{array}{lllll}6 & 9.021806 & -3.589249 & -0.564292\end{array}$

$\begin{array}{lllll}6 & 7.285703 & -3.936718 & 1.267254\end{array}$

$\begin{array}{lllll}1 & 5.872827 & -4.243699 & 2.862355\end{array}$

$\begin{array}{lllll}6 & 5.469629 & -1.807865 & 4.442673\end{array}$

$\begin{array}{lllll}1 & 8.689172 & 4.559658 & 1.022863\end{array}$

$\begin{array}{lllll}6 & 8.382714 & 3.194041 & -0.558924\end{array}$

$\begin{array}{lllll}8 & 6.781906 & 4.375989 & 2.330314\end{array}$

$\begin{array}{lllll}6 & 8.421743 & -2.729922 & -1.676118\end{array}$ $\begin{array}{lllll}1 & 8.724950 & -4.615853 & -0.782264\end{array}$

$\begin{array}{lllll}8 & 6.713647 & -4.905834 & 0.437641\end{array}$

$\begin{array}{lllll}6 & 8.895737 & 2.165151 & -1.351492\end{array}$

$\begin{array}{lllll}6 & 7.278641 & 3.890570 & -1.070647\end{array}$

$\begin{array}{lllll}6 & 5.565300 & 4.452485 & 1.725957\end{array}$

$\begin{array}{lllll}6 & 3.287646 & -0.441335 & 3.885390\end{array}$

$\begin{array}{lllll}6 & 8.915772 & -1.456528 & -1.987997\end{array}$

$\begin{array}{lllll}6 & 7.355304 & -3.201266 & -2.450184\end{array}$

$\begin{array}{lllll}6 & 5.570942 & -4.599154 & -0.236083\end{array}$

$\begin{array}{lllll}6 & 3.267477 & -1.818583 & 4.066735\end{array}$

$\begin{array}{lllll}6 & 8.377538 & 1.823087 & -2.603327\end{array}$

$\begin{array}{lllll}1 & 9.739820 & 1.600627 & -0.974435\end{array}$

$\begin{array}{lllll}6 & 6.741950 & 3.612349 & -2.324865\end{array}$

$\begin{array}{lllll}8 & 6.723932 & 4.925083 & -0.308794\end{array}$

$\begin{array}{lllll}6 & 5.538006 & 4.688509 & 0.322293\end{array}$

$\begin{array}{lllll}6 & 8.381229 & -0.662964 & -3.006798\end{array}$

$\begin{array}{lllll}1 & 9.762716 & -1.078038 & -1.427758\end{array}$

$\begin{array}{lllll}6 & 6.755651 & -2.432427 & -3.442611\end{array}$

$\begin{array}{lllll}8 & 6.880037 & -4.507241 & -2.237166\end{array}$

$\begin{array}{lllll}6 & 5.645720 & -4.535366 & -1.656800\end{array}$

$\begin{array}{lllll}6 & 8.972923 & 0.683067 & -3.418840\end{array}$

$\begin{array}{lllll}6 & 7.291023 & 2.573417 & -3.070545\end{array}$

$1 \quad 5.896826 \quad 4.175609 \quad-2.700554$

$\begin{array}{lllll}6 & 3.333358 & 4.384082 & 1.752170\end{array}$

$\begin{array}{lllll}6 & 7.269025 & -1.168547 & -3.689439\end{array}$

$\begin{array}{lllll}1 & 5.914297 & -2.813439 & -4.006549\end{array}$

$\begin{array}{lllll}6 & 3.374296 & -4.225046 & -0.373043\end{array}$

$\begin{array}{lllll}1 & 8.660745 & 0.848521 & -4.450236\end{array}$

$\begin{array}{lllll}8 & 6.769943 & 2.303944 & -4.345901\end{array}$

$\begin{array}{lllll}6 & 3.308774 & 4.562774 & 0.376650\end{array}$

$\begin{array}{lllll}8 & 6.728546 & -0.382890 & -4.713045\end{array}$

$\begin{array}{lllll}6 & 3.427633 & -4.314014 & -1.756062\end{array}$

$\begin{array}{lllll}6 & 5.562531 & 1.669912 & -4.397679\end{array}$

$\begin{array}{lllll}6 & 5.544392 & 0.247947 & -4.510655\end{array}$

$\begin{array}{lllll}6 & 3.325896 & 1.674150 & -4.385157\end{array}$

$\begin{array}{lllll}6 & 3.314547 & 0.285629 & -4.397336\end{array}$

$\begin{array}{lllll}6 & 10.508855 & 0.680072 & -3.400395\end{array}$

$1 \quad 10.887824 \quad-0.110738-4.052936$

$\begin{array}{lllll}1 & 10.926184 & 0.511337 & -2.404001\end{array}$

$\begin{array}{lllll}6 & 10.533390 & 3.517069 & 0.768220\end{array}$

$\begin{array}{lllll}1 & 10.904394 & 4.201173 & 0.000667\end{array}$

$\begin{array}{lllll}1 & 10.944858 & 2.527007 & 0.552200\end{array}$

$\begin{array}{lllll}6 & 10.478612 & -0.741007 & 3.727027\end{array}$

$\begin{array}{lllll}1 & 10.829422 & 0.070315 & 4.370107\end{array}$

$\begin{array}{lllll}1 & 10.930836 & -0.598191 & 2.741652\end{array}$

$\begin{array}{lllll}6 & 10.557082 & -3.546515 & -0.551540\end{array}$

$\begin{array}{lllll}1 & 10.945571 & -4.221575 & 0.215500\end{array}$

$1 \quad 10.955960 \quad-2.549393 \quad-0.345064$

$1 \quad 10.946743 \quad-3.861288-1.523016$

$\begin{array}{lllll}1 & 10.851847 & -1.687905 & 4.125682\end{array}$

$\begin{array}{lllll}1 & 10.928851 & 3.832857 & 1.737144\end{array}$

$\begin{array}{lllll}1 & 10.889563 & 1.640089 & -3.758698\end{array}$

$\begin{array}{lllll}6 & 1.909748 & 4.264028 & 2.235968\end{array}$

$\begin{array}{lllll}6 & 1.881361 & 4.483385 & -0.086767\end{array}$

$\begin{array}{lllll}6 & 1.902037 & 2.158774 & -4.266028\end{array}$

$\begin{array}{lllll}6 & 1.893192 & -0.181202 & -4.219221\end{array}$

$\begin{array}{lllll}6 & 1.926635 & 0.001745 & 3.428296\end{array}$

$\begin{array}{lllll}6 & 1.853850 & -2.295887 & 3.862647\end{array}$ 
$\begin{array}{llll}6 & 1.965662 & -3.882881 & 0.019401\end{array}$

$\begin{array}{lllll}6 & 2.027958 & -4.168617 & -2.294315\end{array}$

$\begin{array}{lllll}7 & 1.135652 & 0.981038 & -4.211154\end{array}$

$\begin{array}{lllll}7 & 1.133629 & 4.375985 & 1.069041\end{array}$

$\begin{array}{lllll}7 & 1.155424 & -1.143748 & 3.450992\end{array}$

$\begin{array}{lllll}7 & 1.241657 & -3.926529 & -1.153332\end{array}$

$\begin{array}{lllll}8 & 1.562019 & -3.587857 & 1.132927\end{array}$

$\begin{array}{lllll}8 & 1.626322 & -4.233610 & -3.430255\end{array}$

$\begin{array}{lllll}8 & 1.506485 & -1.321518 & -4.050957\end{array}$

$\begin{array}{lllll}8 & 1.480264 & 3.287554 & -4.191167\end{array}$

$\begin{array}{lllll}8 & 1.486185 & 4.458582 & -1.239709\end{array}$

$\begin{array}{lllll}8 & 1.582675 & 1.120163 & 3.084491\end{array}$

$\begin{array}{lllll}8 & 1.486588 & 4.110719 & 3.355832\end{array}$

$\begin{array}{lllll}8 & 1.375092 & -3.391221 & 4.025002\end{array}$

$1 \quad 0.228571 \quad-3.785262-1.232642$

$\begin{array}{lllll}1 & 0.148070 & -1.173840 & 3.249616\end{array}$

$\begin{array}{lllll}1 & 0.124738 & 4.197940 & 1.092092\end{array}$

$1 \quad 0.130056 \quad 1.010187 \quad-4.036630$

$\begin{array}{lllll}7 & 4.466467 & 4.315110 & 2.454088\end{array}$

$\begin{array}{lllll}7 & 4.407544 & 4.719033 & -0.367858\end{array}$

$\begin{array}{lllll}7 & 4.368999 & 0.307928 & 4.097435\end{array}$

$\begin{array}{lllll}7 & 4.370602 & -2.534574 & 4.312036\end{array}$

$\begin{array}{lllll}7 & 4.452228 & 2.392543 & -4.353012\end{array}$

$\begin{array}{lllll}7 & 4.421937 & -0.455833 & -4.469249\end{array}$

$\begin{array}{lllll}7 & 4.437174 & -4.399859 & 0.417292\end{array}$

$7 \quad 4.573769-4.441110 \quad-2.427782$

$\begin{array}{lllll}6 & -9.048057 & -2.059374 & -2.901986\end{array}$

$\begin{array}{lllll}6 & -8.475411 & -0.655042 & -3.081866\end{array}$

$\begin{array}{lllll}6 & -8.430977 & -2.761797 & -1.689981\end{array}$

$\begin{array}{lllll}1 & -8.731497 & -2.631746 & -3.774846\end{array}$

$\begin{array}{lllll}6 & -9.023159 & 0.478108 & -2.466213\end{array}$

$\begin{array}{lllll}6 & -7.340609 & -0.458827 & -3.876830\end{array}$

$\begin{array}{lllll}6 & -8.955663 & -2.620508 & -0.401722\end{array}$

$\begin{array}{lllll}6 & -7.308609 & -3.592101 & -1.820332\end{array}$

$\begin{array}{lllll}6 & -8.460683 & 1.755088 & -2.580933\end{array}$

$\begin{array}{lllll}1 & -9.918411 & 0.359074 & -1.867484\end{array}$

$\begin{array}{lllll}6 & -6.725892 & 0.778593 & -4.004576\end{array}$

$\begin{array}{lllll}8 & -6.825499 & -1.547259 & -4.592624\end{array}$

$\begin{array}{lllll}6 & -8.438085 & -3.271082 & 0.726622\end{array}$

$\begin{array}{lllll}1 & -9.801954 & -1.958009 & -0.264036\end{array}$

$\begin{array}{lllll}6 & -6.787932 & -4.302692 & -0.747705\end{array}$

$\begin{array}{lllll}8 & -6.725016 & -3.754217 & -3.080509\end{array}$

$\begin{array}{lllll}6 & -9.066331 & 3.002583 & -1.928822\end{array}$

$\begin{array}{lllll}6 & -7.283629 & 1.856545 & -3.335222\end{array}$

$\begin{array}{lllll}1 & -5.833138 & 0.898036 & -4.603579\end{array}$

$\begin{array}{lllll}6 & -5.599220 & -1.977778 & -4.188690\end{array}$

$\begin{array}{llll}6 & -9.017380 & -3.020388 & 2.119639\end{array}$

$\begin{array}{lllll}6 & -7.360184 & -4.141399 & 0.510887\end{array}$

$\begin{array}{lllll}1 & -5.938907 & -4.960516 & -0.884545\end{array}$

$\begin{array}{llll}6 & -5.549887 & -3.108540 & -3.327074\end{array}$

$\begin{array}{lllll}1 & -8.748603 & 3.847666 & -2.541453\end{array}$

$\begin{array}{lllll}6 & -8.479934 & 3.242343 & -0.537402\end{array}$

$\begin{array}{lllll}8 & -6.699733 & 3.114086 & -3.470340\end{array}$

$\begin{array}{llll}6 & -8.411370 & -1.769069 & 2.764716\end{array}$

$\begin{array}{lllll}1 & -8.698271 & -3.862235 & 2.735975\end{array}$

$\begin{array}{lllll}8 & -6.820704 & -4.890093 & 1.571356\end{array}$

$\begin{array}{lllll}6 & -8.989875 & 2.605585 & 0.603179\end{array}$

$\begin{array}{lllll}6 & -7.378785 & 4.088585 & -0.350184\end{array}$ $\begin{array}{llll}6 & -5.543754 & 3.387769 & -2.811580\end{array}$

$\begin{array}{llll}6 & -3.378799 & -1.810272 & -4.085506\end{array}$

$\begin{array}{llll}6 & -8.976539 & -0.492525 & 2.682003\end{array}$

$\begin{array}{lllll}6 & -7.222292 & -1.880140 & 3.495351\end{array}$

$\begin{array}{lllll}6 & -5.578244 & -4.453767 & 1.946725\end{array}$

$\begin{array}{lllll}6 & -3.334565 & -2.873766 & -3.195424\end{array}$

$\begin{array}{lllll}6 & -8.425238 & 2.735257 & 1.876580\end{array}$

$\begin{array}{lllll}1 & -9.852015 & 1.959280 & 0.486334\end{array}$

$\begin{array}{lllll}6 & -6.771903 & 4.242431 & 0.890539\end{array}$

$\begin{array}{lllll}8 & -6.858095 & 4.821015 & -1.428356\end{array}$

$\begin{array}{lllll}6 & -5.615696 & 4.404590 & -1.820378\end{array}$

$\begin{array}{llll}6 & -8.411871 & 0.636850 & 3.295857\end{array}$

$\begin{array}{llll}1 & -9.884545 & -0.364329 & 2.104540\end{array}$

$\begin{array}{llll}6 & -6.645561 & -0.812545 & 4.162045\end{array}$

$\begin{array}{llll}8 & -6.648816 & -3.145850 & 3.593795\end{array}$

$\begin{array}{llll}6 & -5.509711 & -3.407159 & 2.907093\end{array}$

$\begin{array}{llll}6 & -8.994555 & 2.039876 & 3.118897\end{array}$

$\begin{array}{lllll}6 & -7.284559 & 3.543314 & 1.972518\end{array}$

$\begin{array}{lllll}1 & -5.906651 & 4.881994 & 1.006051\end{array}$

$\begin{array}{lllll}6 & -3.339819 & 3.263295 & -2.497288\end{array}$

$\begin{array}{llll}6 & -7.254688 & 0.431643 & 4.059906\end{array}$

$\begin{array}{lllll}1 & -5.738984 & -0.938968 & 4.738162\end{array}$

$\begin{array}{llll}6 & -3.364166 & -4.340536 & 1.698884\end{array}$

$\begin{array}{lllll}1 & -8.648226 & 2.622241 & 3.973926\end{array}$

$\begin{array}{lllll}8 & -6.660295 & 3.673372 & 3.215064\end{array}$

$\begin{array}{lllll}6 & -3.393776 & 4.354092 & -1.638764\end{array}$

$\begin{array}{llll}8 & -6.688366 & 1.502652 & 4.768464\end{array}$

$\begin{array}{lllll}6 & -3.332474 & -3.206045 & 2.498825\end{array}$

$\begin{array}{llll}6 & -5.480326 & 3.016577 & 3.386111\end{array}$

$\begin{array}{lllll}6 & -5.479925 & 1.920128 & 4.290092\end{array}$

$\begin{array}{llll}6 & -3.292387 & 2.725603 & 3.070835\end{array}$

$\begin{array}{llll}6 & -3.271237 & 1.725209 & 4.032467\end{array}$

$\begin{array}{llll}6 & -10.529650 & 2.053958 & 3.140388\end{array}$

$\begin{array}{lllll}1 & -10.895587 & 1.607708 & 4.068678\end{array}$

$\begin{array}{lllll}1 & -10.973322 & 1.497584 & 2.309994\end{array}$

$\begin{array}{lllll}6 & -10.601250 & 2.982341 & -1.929067\end{array}$

$\begin{array}{lllll}1 & -10.987221 & 3.921152 & -1.523791\end{array}$

$\begin{array}{lllll}1 & -11.018483 & 2.169397 & -1.328276\end{array}$

$\begin{array}{lllll}6 & -10.582949 & -2.079224 & -2.868069\end{array}$

$\begin{array}{lllll}1 & -10.982274 & -1.630722 & -3.781403\end{array}$

$\begin{array}{lllll}1 & -10.999366 & -1.527145 & -2.020906\end{array}$

$\begin{array}{llll}6 & -10.552540 & -3.001230 & 2.124228\end{array}$

$\begin{array}{lllll}1 & -10.939901 & -3.939621 & 1.719376\end{array}$

$\begin{array}{lllll}1 & -10.972193 & -2.187369 & 1.526416\end{array}$

$\begin{array}{lllll}1 & -10.922604 & -2.883335 & 3.145979\end{array}$

$\begin{array}{lllll}1 & -10.943834 & -3.108547 & -2.798449\end{array}$

$\begin{array}{lllll}1 & -10.975406 & 2.863029 & -2.949153\end{array}$

$\begin{array}{lllll}1 & -10.895903 & 3.081908 & 3.079282\end{array}$

$\begin{array}{lllll}6 & -1.904806 & 2.805536 & -2.592005\end{array}$

$\begin{array}{lllll}6 & -1.989181 & 4.717178 & -1.240482\end{array}$

$\begin{array}{llll}6 & -1.908300 & 2.870852 & 2.504116\end{array}$

$\begin{array}{lllll}6 & -1.847773 & 1.251623 & 4.185333\end{array}$

$\begin{array}{lllll}6 & -1.973685 & -1.321017 & -4.313194\end{array}$

$\begin{array}{lllll}6 & -1.905012 & -3.076365 & -2.773780\end{array}$

$\begin{array}{llll}6 & -1.956909 & -4.632918 & 1.242853\end{array}$

$\begin{array}{lllll}6 & -1.940881 & -2.645972 & 2.465194\end{array}$

$\begin{array}{llll}7 & -1.132257 & 1.975297 & 3.208829\end{array}$

$\begin{array}{llll}7 & -1.189696 & 3.733369 & -1.850396\end{array}$ $\begin{array}{lllll}7 & -1.182063 & -2.134008 & -3.486621\end{array}$

$\begin{array}{lllll}7 & -1.195428 & -3.569361 & 1.765207\end{array}$

$\begin{array}{lllll}8 & -1.533160 & -5.550375 & 0.584858\end{array}$

$\begin{array}{lllll}8 & -1.565867 & -1.575786 & 2.916749\end{array}$

$\begin{array}{llll}8 & -1.377969 & 0.457758 & 4.961541\end{array}$

$\begin{array}{lllll}8 & -1.562467 & 3.604163 & 1.592123\end{array}$

$\begin{array}{lllll}8 & -1.594984 & 5.638558 & -0.568434\end{array}$

$\begin{array}{lllll}8 & -1.576416 & -0.435490 & -5.032728\end{array}$

$8 \quad-1.471116 \quad 1.822509 \quad-3.160553$

$\begin{array}{lllll}8 & -1.484065 & -3.870341 & -1.953013\end{array}$

$\begin{array}{lllll}1 & -0.189514 & -3.477139 & 1.568795\end{array}$

$\begin{array}{lllll}1 & -0.162977 & -2.035193 & -3.486534\end{array}$

$\begin{array}{lllll}1 & -0.168272 & 3.790269 & -1.785826\end{array}$

$\begin{array}{llll}1 & -0.126682 & 1.821831 & 3.062441\end{array}$

$\begin{array}{llll}7 & -4.414331 & 2.770040 & -3.122345\end{array}$

$\begin{array}{lllll}7 & -4.540065 & 4.924027 & -1.252635\end{array}$

$\begin{array}{lllll}7 & -4.516444 & -1.329381 & -4.589694\end{array}$

$\begin{array}{lllll}7 & -4.417452 & -3.551191 & -2.800832\end{array}$

$\begin{array}{lllll}7 & -4.392666 & 3.405878 & 2.738426\end{array}$

$\begin{array}{lllll}7 & -4.373033 & 1.285737 & 4.647528\end{array}$

$\begin{array}{llll}7 & -4.501626 & -4.962596 & 1.369751\end{array}$

$\begin{array}{lllll}7 & -4.398466 & -2.729946 & 3.145756\end{array}$

$\begin{array}{lllll}6 & -1.040475 & -0.407872 & -0.257997\end{array}$

$\begin{array}{lllll}8 & -0.443404 & -1.450161 & -0.422518\end{array}$

$\begin{array}{lllll}7 & 1.606222 & 1.580495 & -0.392719\end{array}$

$\begin{array}{lllll}7 & 0.849390 & 0.739091 & -0.855207\end{array}$

$\begin{array}{lllll}8 & -0.396629 & 0.803718 & -0.154164\end{array}$

$\begin{array}{lllll}6 & 2.880521 & 1.505041 & -1.120061\end{array}$

$\begin{array}{lllll}1 & 3.224658 & 2.519331 & -1.316689\end{array}$

$\begin{array}{lllll}1 & 2.714358 & 1.007556 & -2.072314\end{array}$

$6 \quad-2.496877 \quad-0.251150 \quad-0.139273$

$\begin{array}{lllll}6 & -3.085943 & 1.009024 & 0.033381\end{array}$

$\begin{array}{lllll}6 & -3.300138 & -1.392279 & -0.230313\end{array}$

$\begin{array}{lllll}6 & -4.464140 & 1.115782 & 0.130488\end{array}$

$\begin{array}{lllll}1 & -2.460882 & 1.888765 & 0.094797\end{array}$

$6 \quad-4.680458 \quad-1.274380 \quad-0.137858$

$\begin{array}{lllll}1 & -2.831255 & -2.355977 & -0.379428\end{array}$

$\begin{array}{llll}6 & -5.278192 & -0.023142 & 0.050275\end{array}$

$\begin{array}{lllll}1 & -4.919941 & 2.090289 & 0.276258\end{array}$

$\begin{array}{lllll}1 & -5.304815 & -2.157585 & -0.210196\end{array}$

$\begin{array}{lllll}6 & -6.759086 & 0.117175 & 0.175699\end{array}$

$\begin{array}{lllll}1 & -7.150500 & 0.784965 & -0.591543\end{array}$

$\begin{array}{lllll}1 & -7.267853 & -0.839887 & 0.088177\end{array}$

$\begin{array}{lllll}1 & -7.016321 & 0.553720 & 1.140480\end{array}$

$\begin{array}{lllll}6 & 3.886292 & 0.709466 & -0.332271\end{array}$

$\begin{array}{lllll}6 & 3.514863 & -0.466744 & 0.328205\end{array}$

$\begin{array}{lllll}6 & 5.224925 & 1.100485 & -0.368474\end{array}$

$\begin{array}{lllll}6 & 4.492106 & -1.245795 & 0.940862\end{array}$

$\begin{array}{lllll}1 & 2.477046 & -0.781918 & 0.353255\end{array}$

$\begin{array}{lllll}6 & 6.201194 & 0.308689 & 0.226535\end{array}$

$\begin{array}{lllll}1 & 5.499763 & 2.007603 & -0.888035\end{array}$

$\begin{array}{lllll}6 & 5.830459 & -0.862436 & 0.878143\end{array}$

$\begin{array}{lllll}1 & 4.214968 & -2.159739 & 1.450931\end{array}$

$\begin{array}{lllll}1 & 7.241358 & 0.602639 & 0.183773\end{array}$

$\begin{array}{llll}1 & 6.586951 & -1.472039 & 1.332919\end{array}$

\section{4c'@12}

$\begin{array}{llll}6 & -8.976151 & 1.093263 & -3.304032\end{array}$ 
$\begin{array}{lllll}6 & -8.409481 & 2.142008 & -2.348660\end{array}$

$\begin{array}{lllll}6 & -8.381319 & -0.290410 & -3.062099\end{array}$

$\begin{array}{llll}-8.651831 & 1.390073 & -4.302097\end{array}$

$\begin{array}{lllll}6 & -8.950570 & 2.368359 & -1.082599\end{array}$

$\begin{array}{lllll}6 & -7.321430 & 2.943951 & -2.718509\end{array}$

$6 \quad-8.919384 \quad-1.192593 \quad-2.135535$

$6 \quad-7.271667 \quad-0.719320 \quad-3.799170$

$\begin{array}{lllll}6 & -8.480496 & 3.353389 & -0.204673\end{array}$

$\begin{array}{lllll}1 & -9.767374 & 1.736806 & -0.752900\end{array}$

$6 \quad-6.868425 \quad 3.988475 \quad-1.925692$

$\begin{array}{llll}-6.693432 & 2.686560 & -3.939350\end{array}$

$\begin{array}{llll}-8.394772 & -2.471944 & -1.926938\end{array}$

$\begin{array}{llll}-9.792919 & -0.891253 & -1.569011\end{array}$

$\begin{array}{llll}-6.692545 & -1.967882 & -3.610961\end{array}$

$\begin{array}{llll}-6.758298 & 0.120230 & -4.801001\end{array}$

$\begin{array}{llll}-9.051330 & 3.473792 & 1.205445\end{array}$

$\begin{array}{llll}-7.448862 & 4.179140 & -0.672791\end{array}$

$\begin{array}{llll}-6.032330 & 4.599134 & -2.241660\end{array}$

$\begin{array}{llll}-5.526887 & 1.982003 & -3.938319\end{array}$

$\begin{array}{lllll}6 & -9.017262 & -3.488494 & -0.966375\end{array}$

$\begin{array}{llll}-7.260712 & -2.817095 & -2.671525\end{array}$

$\begin{array}{llll}-5.826842 & -2.272690 & -4.184495\end{array}$

$\begin{array}{llll}-5.549075 & 0.680029 & -4.518131\end{array}$

$\begin{array}{llll}-8.748372 & 4.453273 & 1.577352\end{array}$

$\begin{array}{llll}-8.416576 & 2.445918 & 2.146492\end{array}$

$\begin{array}{llll}-6.923321 & 5.195423 & 0.146774\end{array}$

$\begin{array}{llll}-8.442926 & -3.339329 & 0.439456\end{array}$

$\begin{array}{llll}-8.705423 & -4.470893 & -1.322267\end{array}$

$\begin{array}{llll}-6.735950 & -4.100956 & -2.497300\end{array}$

$\begin{array}{llll}-8.950801 & 1.177803 & 2.397912\end{array}$

$\begin{array}{lll}-7.239614 & 2.778600 & 2.826599\end{array}$

$\begin{array}{llll}-5.672047 & 4.883058 & 0.613393\end{array}$

$\begin{array}{llll}-3.314980 & 1.798649 & -3.693690\end{array}$

$\begin{array}{llll}-8.948277 & -2.392521 & 1.339587\end{array}$

$\begin{array}{llll}-7.346863 & -4.102729 & 0.866434\end{array}$

$\begin{array}{llll}-5.555020 & -4.218424 & -1.836429\end{array}$

$\begin{array}{llll}-3.323105 & 0.597692 & -4.388184\end{array}$

$\begin{array}{llll}-8.382174 & 0.278868 & 3.312896\end{array}$

$\begin{array}{llll}-9.850604 & 0.878812 & 1.872600\end{array}$

$\begin{array}{llll}-6.657918 & 1.949477 & 3.771237\end{array}$

$\begin{array}{llll}-6.693832 & 4.038229 & 2.575590\end{array}$

$\begin{array}{llll}-5.573978 & 4.127791 & 1.816536\end{array}$

$\begin{array}{llll}-8.388870 & -2.153344 & 2.597996\end{array}$

$\begin{array}{llll}-9.801238 & -1.797843 & 1.034169\end{array}$

$\begin{array}{llll}-6.758119 & -3.911805 & 2.112248\end{array}$

$\begin{array}{llll}-6.806422 & -5.073934 & 0.012475\end{array}$

$\begin{array}{llll}-5.585327 & -4.780443 & -0.528866\end{array}$

$\begin{array}{llll}-8.958319 & -1.113870 & 3.566816\end{array}$

$\begin{array}{llll}-7.251935 & 0.716850 & 4.013219\end{array}$

$\begin{array}{llll}-5.765163 & 2.249254 & 4.302993\end{array}$

$\begin{array}{llll}-3.459493 & 4.718241 & 0.357694\end{array}$

$\begin{array}{llll}-7.263646 & -2.916510 & 2.937903\end{array}$

$\begin{array}{llll}-5.886562 & -4.483552 & 2.402440\end{array}$

$\begin{array}{llll}-3.331856 & -4.089175 & -1.714154\end{array}$

$\begin{array}{llll}-8.613028 & -1.406670 & 4.559184\end{array}$

$\begin{array}{llll}-6.705468 & -0.103276 & 5.015994\end{array}$

$\begin{array}{llll}-3.399353 & 3.841089 & 1.431794\end{array}$

$\begin{array}{llll}-6.631978 & -2.656197 & 4.157462\end{array}$
$6 \quad-3.352315 \quad-4.688199 \quad-0.461367$

$6 \quad-5.494643 \quad-0.636151 \quad 4.687217$

$\begin{array}{lllll}6 & -5.475399 & -1.939014 & 4.114526\end{array}$

$\begin{array}{lllll}6 & -3.294089 & -0.469392 & 4.360634\end{array}$

$\begin{array}{lllll}6 & -3.304381 & -1.677905 & 3.679098\end{array}$

$\begin{array}{lllll}6 & -10.494073 & -1.125027 & 3.591311\end{array}$

$\begin{array}{llll}-10.858104 & -2.124581 & 3.842508\end{array}$

$\begin{array}{llll}-10.939593 & -0.844563 & 2.632665\end{array}$

$\begin{array}{llll}-10.586061 & 3.425998 & 1.225458\end{array}$

$\begin{array}{llll}-10.952318 & 3.571250 & 2.245122\end{array}$

$\begin{array}{llll}-10.989380 & 2.476643 & 0.862337\end{array}$

$\begin{array}{llll}-10.512643 & 1.084024 & -3.304348\end{array}$

$\begin{array}{llll}-10.893630 & 2.078636 & -3.550062\end{array}$

$\begin{array}{llll}-10.939393 & 0.797480 & -2.339038\end{array}$

$\begin{array}{llll}-10.552183 & -3.454408 & -0.986673\end{array}$

$\begin{array}{llll}-10.916496 & -3.626709 & -2.002708\end{array}$

$\begin{array}{llll}-10.964323 & -2.500110 & -0.647206\end{array}$

$\begin{array}{llll}-10.952988 & -4.235956 & -0.335951\end{array}$

$\begin{array}{llll}-10.880822 & 0.375165 & -4.050496\end{array}$

$\begin{array}{llll}-10.993129 & 4.219034 & 0.593008\end{array}$

$\begin{array}{llll}-10.860705 & -0.421316 & 4.343067\end{array}$

$\begin{array}{llll}-2.063939 & 4.893608 & -0.183754\end{array}$

$\begin{array}{llll}-1.999464 & 3.305054 & 1.524518\end{array}$

$\begin{array}{llll}-1.888160 & 0.072063 & 4.342274\end{array}$

$\begin{array}{llll}-1.946831 & -1.888051 & 3.078557\end{array}$

$\begin{array}{llll}-1.896427 & 2.099726 & -3.298520\end{array}$

$\begin{array}{llll}-1.899633 & 0.141585 & -4.566542\end{array}$

$\begin{array}{llll}-1.905367 & -3.776675 & -2.076975\end{array}$

$\begin{array}{llll}-1.928248 & -4.830145 & 0.014583\end{array}$

$\begin{array}{llll}-1.177672 & -0.840372 & 3.532866\end{array}$

$\begin{array}{llll}-1.279971 & 4.002399 & 0.580470\end{array}$

$\begin{array}{llll}-1.131054 & 1.142613 & -3.937331\end{array}$

$\begin{array}{llll}-1.160146 & -4.218395 & -0.993519\end{array}$

$\begin{array}{llll}-1.498822 & -3.227906 & -3.082203\end{array}$

$\begin{array}{llll}-1.498317 & -5.350882 & 1.014910\end{array}$

$\begin{array}{llll}-1.616497 & -2.756895 & 2.283276\end{array}$

$\begin{array}{llll}-1.424931 & 1.044712 & 4.882303\end{array}$

$\begin{array}{llll}-1.604315 & 2.403315 & 2.249656\end{array}$

$\begin{array}{llll}-1.514266 & 2.972217 & -2.535517\end{array}$

$\begin{array}{llll}-1.661318 & 5.621367 & -1.055626\end{array}$

$\begin{array}{llll}-1.469171 & -0.847351 & -5.105830\end{array}$

$\begin{array}{llll}-0.135326 & -4.177933 & -0.927666\end{array}$

$\begin{array}{llll}-0.119893 & 1.024292 & -3.833156\end{array}$

$\begin{array}{llll}-0.261563 & 3.933504 & 0.440837\end{array}$

$\begin{array}{llll}-0.177641 & -0.709739 & 3.323383\end{array}$

$\begin{array}{llll}-4.610081 & 5.224893 & -0.098345\end{array}$

$\begin{array}{llll}-4.447960 & 3.550515 & 2.204288\end{array}$

$\begin{array}{llll}-4.414184 & 2.526328 & -3.468417\end{array}$

$\begin{array}{llll}-4.447068 & -0.004540 & -4.782020\end{array}$

$\begin{array}{llll}-4.399746 & 0.089014 & 4.857852\end{array}$

$\begin{array}{llll}-4.386884 & -2.451499 & 3.563792\end{array}$

$\begin{array}{llll}-4.431105 & -3.847308 & -2.430390\end{array}$

$\begin{array}{llll}-4.483864 & -5.031972 & 0.163527\end{array}$

$\begin{array}{llll}9.024951 & -3.229525 & 1.856232\end{array}$

$\begin{array}{llll}8.418501 & -2.092182 & 2.678795\end{array}$

$\begin{array}{llll}8.460992 & -3.248908 & 0.430271\end{array}$

$\begin{array}{llll}8.689638 & -4.155966 & 2.324651\end{array}$

$\begin{array}{llll}8.960312 & -0.796803 & 2.693884\end{array}$ $\begin{array}{llll}6 & 7.248272 & -2.299960 & 3.420162\end{array}$

$\begin{array}{llll}9.017904 & -2.502100 & -0.613173\end{array}$

$\begin{array}{llll}7.341260 & -4.028238 & 0.109441\end{array}$

$\begin{array}{llll}8.352908 & 0.276660 & 3.354770\end{array}$

$\begin{array}{llll}9.878578 & -0.617525 & 2.147035\end{array}$

$\begin{array}{llll}6.598683 & -1.267532 & 4.082379\end{array}$

$\begin{array}{llll}6.711819 & -3.592148 & 3.519462\end{array}$

$\begin{array}{llll}8.523878 & -2.514745 & -1.924825\end{array}$

$\begin{array}{llll}9.864147 & -1.862332 & -0.391455\end{array}$

$\begin{array}{llll}6.839423 & -4.115886 & -1.180879\end{array}$

$\begin{array}{llll}6.726066 & -4.771887 & 1.120765\end{array}$

$\begin{array}{llll}8.929313 & 1.698003 & 3.400482\end{array}$

$\begin{array}{llll}7.147536 & 0.001162 & 4.012061\end{array}$

$\begin{array}{llll}5.679864 & -1.447467 & 4.623461\end{array}$

$\begin{array}{llll}5.511776 & -3.761090 & 2.892417\end{array}$

$\begin{array}{llll}9.130097 & -1.639208 & -3.021540\end{array}$

$\begin{array}{llll}7.436793 & -3.360532 & -2.182969\end{array}$

$\begin{array}{llll}5.987591 & -4.746318 & -1.400793\end{array}$

$\begin{array}{llll}5.529942 & -4.312764 & 1.582848\end{array}$

$\begin{array}{llll}8.570280 & 2.136823 & 4.332887\end{array}$

$\begin{array}{llll}8.376196 & 2.580428 & 2.278628\end{array}$

$\begin{array}{llll}6.515880 & 1.055686 & 4.667035\end{array}$

$\begin{array}{llll}8.531069 & -0.229889 & -3.016357\end{array}$

$\begin{array}{llll}8.828596 & -2.090324 & -3.968066\end{array}$

$\begin{array}{llll}6.927548 & -3.483620 & -3.484458\end{array}$

$\begin{array}{llll}8.945845 & 2.570184 & 0.996544\end{array}$

$\begin{array}{llll}7.269096 & 3.420438 & 2.465671\end{array}$

$\begin{array}{llll}5.385756 & 1.574897 & 4.127026\end{array}$

$\begin{array}{llll}3.296755 & -3.527528 & 2.750535\end{array}$

$\begin{array}{llll}9.078940 & 0.839843 & -2.299037\end{array}$

$\begin{array}{llll}7.372565 & 0.038853 & -3.757341\end{array}$

$\begin{array}{llll}5.692104 & -2.920328 & -3.653831\end{array}$

$\begin{array}{llll}3.328836 & -3.981445 & 1.438880\end{array}$

$\begin{array}{llll}8.442049 & 3.301112 & -0.083975\end{array}$

$\begin{array}{llll}9.810355 & 1.938953 & 0.828370\end{array}$

$\begin{array}{llll}6.722353 & 4.159606 & 1.421185\end{array}$

$\begin{array}{llll}6.666222 & 3.548404 & 3.729433\end{array}$

$\begin{array}{llll}5.433909 & 2.953797 & 3.785064\end{array}$

$\begin{array}{llll}8.535141 & 2.131513 & -2.319684\end{array}$

$\begin{array}{llll}9.955293 & 0.658692 & -1.687996\end{array}$

$\begin{array}{llll}6.824082 & 1.307071 & -3.856560\end{array}$

$\begin{array}{llll}6.803345 & -1.008241 & -4.477682\end{array}$

$\begin{array}{llll}5.635665 & -1.556759 & -4.052086\end{array}$

$\begin{array}{llll}9.090717 & 3.274589 & -1.470945\end{array}$

$\begin{array}{llll}7.294494 & 4.067924 & 0.161170\end{array}$

$\begin{array}{llll}5.852469 & 4.782258 & 1.586788\end{array}$

$\begin{array}{llll}3.236383 & 1.528574 & 3.552978\end{array}$

$\begin{array}{llll}7.424547 & 2.337823 & -3.146455\end{array}$

$\begin{array}{llll}5.947965 & 1.487237 & -4.465060\end{array}$

$\begin{array}{llll}3.464612 & -2.954707 & -3.539329\end{array}$

$\begin{array}{llll}8.783948 & 4.197485 & -1.965012\end{array}$

$\begin{array}{llll}6.738633 & 4.806120 & -0.886771\end{array}$

$\begin{array}{llll}3.238542 & 2.908587 & 3.394230\end{array}$

$\begin{array}{llll}6.909985 & 3.634402 & -3.285538\end{array}$

$\begin{array}{llll}3.423286 & -1.588029 & -3.784823\end{array}$

$\begin{array}{llll}5.580926 & 4.355055 & -1.445676\end{array}$

$\begin{array}{llll}5.666885 & 3.810993 & -2.756328\end{array}$

$\begin{array}{lllll}6 & 3.370743 & 4.058410 & -1.498177\end{array}$ 
$\begin{array}{lllll}6 & 3.447710 & 3.600082 & -2.806537\end{array}$

$\begin{array}{lllll}6 & 10.624171 & 3.275560 & -1.400773\end{array}$

$\begin{array}{lllll}1 & 11.044127 & 3.323537 & -2.408835\end{array}$

$1 \quad 11.031140 \quad 2.383233 \quad-0.917132$

$\begin{array}{lllll}6 & 10.463912 & 1.705832 & 3.448913\end{array}$

$\begin{array}{llll}10.829963 & 2.730414 & 3.552740\end{array}$

$\begin{array}{llll}10.922185 & 1.282809 & 2.550685\end{array}$

$\begin{array}{lllll}6 & 10.559874 & -3.229391 & 1.876123\end{array}$

$\begin{array}{llll}10.920783 & -3.279723 & 2.906711\end{array}$

$\begin{array}{llll}10.993131 & -2.335713 & 1.418602\end{array}$

$\begin{array}{llll}10.664822 & -1.626987 & -2.984143\end{array}$

$\begin{array}{llll}11.048490 & -2.647534 & -3.060984\end{array}$

$\begin{array}{llll}11.066831 & -1.193471 & -2.064260\end{array}$

$\begin{array}{llll}11.056445 & -1.043389 & -3.821413\end{array}$

$10.942543 \quad-4.095433 \quad 1.330043$

$\begin{array}{llll}10.815173 & 1.121753 & 4.303534\end{array}$

$\begin{array}{llll}10.974089 & 4.143501 & -0.835931\end{array}$

$\begin{array}{llll}1.870747 & 1.015540 & 3.195906\end{array}$

$\begin{array}{llll}1.834674 & 3.347347 & 3.070084\end{array}$

$\begin{array}{llll}1.933513 & 3.991254 & -1.063078\end{array}$

$\begin{array}{llll}2.053306 & 3.280593 & -3.280228\end{array}$

$\begin{array}{llll}1.866403 & -3.215360 & 3.107312\end{array}$

$\begin{array}{llll}1.937386 & -3.905836 & 0.875083\end{array}$

$\begin{array}{llll}2.050956 & -3.456273 & -3.405979\end{array}$

$\begin{array}{llll}1.987168 & -1.134413 & -3.703787\end{array}$

$\begin{array}{llll}1.238451 & 3.557507 & -2.171970\end{array}$

$\begin{array}{llll}1.115071 & 2.140919 & 2.950806\end{array}$

$\begin{array}{llll}1.152351 & -3.453623 & 1.918131\end{array}$

$\begin{array}{llll}1.258581 & -2.302820 & -3.544350\end{array}$

$\begin{array}{llll}1.647322 & -4.580382 & -3.233133\end{array}$

$\begin{array}{llll}1.567679 & 0.007156 & -3.739869\end{array}$

$\begin{array}{llll}1.673687 & 2.879722 & -4.354410\end{array}$

$\begin{array}{llll}1.480251 & 4.236254 & 0.042211\end{array}$

$\begin{array}{llll}1.377420 & 4.456513 & 2.951856\end{array}$

$\begin{array}{llll}1.390466 & -2.861319 & 4.158051\end{array}$

$\begin{array}{llll}1.511259 & -0.148659 & 3.110662\end{array}$

$\begin{array}{llll}1.585634 & -4.165956 & -0.262612\end{array}$

$\begin{array}{llll}0.241621 & -2.354341 & -3.470728\end{array}$

$\begin{array}{llll}0.140391 & -3.307194 & 1.869653\end{array}$

$\begin{array}{llll}0.121985 & 2.130309 & 2.686681\end{array}$

$\begin{array}{llll}0.229316 & 3.366818 & -2.185248\end{array}$

$\begin{array}{llll}4.302478 & 0.833985 & 3.957643\end{array}$

$\begin{array}{llll}4.352193 & 3.645884 & 3.466797\end{array}$

$\begin{array}{llll}4.393734 & -3.391248 & 3.499669\end{array}$

$\begin{array}{llll}4.442285 & -4.399964 & 0.831737\end{array}$

$\begin{array}{llll}4.432810 & 4.458380 & -0.792642\end{array}$

$\begin{array}{llll}4.602439 & 3.452703 & -3.458362\end{array}$

$\begin{array}{llll}4.606043 & -3.642344 & -3.430110\end{array}$

$\begin{array}{llll}4.508314 & -0.862081 & -4.070688\end{array}$

$\begin{array}{llll}1.066649 & 0.139194 & -0.359689\end{array}$

$\begin{array}{llll}0.439942 & 1.201296 & -0.329975\end{array}$

$\begin{array}{llll}-1.773859 & -0.542848 & -1.355701\end{array}$

$\begin{array}{llll}-0.743264 & -0.817447 & -1.816743\end{array}$

$\begin{array}{llll}0.520252 & -1.044582 & -0.589089\end{array}$

$\begin{array}{llll}-2.140375 & -0.308940 & 0.057268\end{array}$

$\begin{array}{llll}-1.544980 & 0.567062 & 0.338168\end{array}$

$\begin{array}{llll}2.537169 & 0.072518 & -0.170411\end{array}$

$\begin{array}{llll}3.241235 & -1.097428 & -0.481697\end{array}$ $\begin{array}{llll}6 & 3.229253 & 1.204436 & 0.266246\end{array}$

$6 \quad 4.622110 \quad-1.127248 \quad-0.360819$

$\begin{array}{llll}2.695044 & -1.965929 & -0.823071\end{array}$

$\begin{array}{llll}6 & 4.612412 & 1.164834 & 0.394454\end{array}$

$\begin{array}{llll}2.671172 & 2.099317 & 0.505825\end{array}$

$\begin{array}{lllll}6 & 5.324202 & 0.001514 & 0.081782\end{array}$

$\begin{array}{lllll}1 & 5.166257 & -2.032348 & -0.610101\end{array}$

$\begin{array}{llll}1 & 5.149824 & 2.042873 & 0.737058\end{array}$

$\begin{array}{lllll}6 & 6.810310 & -0.054299 & 0.211800\end{array}$

$\begin{array}{llll}7.109071 & -0.909748 & 0.815097\end{array}$

$\begin{array}{llll}7.281593 & -0.171871 & -0.764539\end{array}$

$\begin{array}{llll}7.207609 & 0.845308 & 0.672686\end{array}$

$1 \quad-1.764114 \quad-1.180430 \quad 0.596176$

$\begin{array}{lllll}6 & -3.617443 & -0.120933 & 0.149475\end{array}$

$6 \quad-4.443053 \quad-1.185105 \quad 0.512478$

$6 \quad-4.181269 \quad 1.092964 \quad-0.250266$

$\begin{array}{lllll}6 & -5.826316 & -1.027807 & 0.480520\end{array}$

$\begin{array}{llll}-4.008529 & -2.130973 & 0.819604\end{array}$

$\begin{array}{lllll}6 & -5.563098 & 1.249552 & -0.260273\end{array}$

$1 \quad-3.537546 \quad 1.902870 \quad-0.578488$

$\begin{array}{lllll}6 & -6.388556 & 0.186676 & 0.101769\end{array}$

$1 \quad-6.461304 \quad-1.854970 \quad 0.738826$

$\begin{array}{lllll}1 & -5.990801 & 2.192128 & -0.557822\end{array}$

$\begin{array}{lllll}1 & -7.464018 & 0.302677 & 0.082238\end{array}$

\section{5:6c@1 $1_{2}$}

$\begin{array}{llll}6 & 9.023678 & -0.007022 & 3.640786\end{array}$ $\begin{array}{lllll}6 & 8.441499 & 1.211325 & 2.919192\end{array}$

$\begin{array}{lllll}6 & 8.418325 & -1.302440 & 3.107845\end{array}$

$1 \quad 8.705738 \quad 0.075524 \quad 4.680896$

$\begin{array}{llll}6 & 9.003212 & 1.772044 & 1.768291\end{array}$

$\begin{array}{lllll}6 & 7.293696 & 1.832613 & 3.424547\end{array}$

$\begin{array}{lllll}6 & 8.924491 & -1.918932 & 1.955415\end{array}$

$\begin{array}{lllll}6 & 7.317022 & -1.912654 & 3.722843\end{array}$

$\begin{array}{llll}6 & 8.488665 & 2.922572 & 1.155163\end{array}$

$\begin{array}{llll}1 & 9.880141 & 1.302824 & 1.337481\end{array}$

$\begin{array}{lllll}6 & 6.761582 & 2.989901 & 2.880754\end{array}$

$\begin{array}{lllll}8 & 6.709589 & 1.272917 & 4.561157\end{array}$

$\begin{array}{lllll}6 & 8.362156 & -3.064837 & 1.389591\end{array}$

$\begin{array}{lllll}1 & 9.789695 & -1.477683 & 1.474888\end{array}$

$\begin{array}{lllll}6 & 6.706587 & -3.042243 & 3.185491\end{array}$

$\begin{array}{lllll}8 & 6.794099 & -1.388797 & 4.918673\end{array}$

$\begin{array}{lllll}6 & 9.083427 & 3.507552 & -0.122839\end{array}$

$\begin{array}{lllll}6 & 7.378593 & 3.524023 & 1.757890\end{array}$

$\begin{array}{lllll}1 & 5.885919 & 3.457736 & 3.309652\end{array}$

$\begin{array}{lllll}6 & 5.548868 & 0.588790 & 4.413684\end{array}$

$\begin{array}{lllll}6 & 8.939740 & -3.752395 & 0.153777\end{array}$

$\begin{array}{lllll}6 & 7.219764 & -3.579304 & 2.014639\end{array}$

$\begin{array}{llll}5.839438 & -3.479054 & 3.663413\end{array}$

$\begin{array}{lllll}6 & 5.574055 & -0.792270 & 4.756365\end{array}$

$1 \quad 8.782343 \quad 4.555316 \quad-0.150239$

$\begin{array}{lllll}6 & 8.462178 & 2.850301 & -1.354306\end{array}$

$\begin{array}{lllll}8 & 6.887753 & 4.725915 & 1.227852\end{array}$

$6 \quad 8.353069 \quad-3.168462 \quad-1.127403$

$\begin{array}{llll}8.605406 & -4.789517 & 0.198400\end{array}$

$8 \quad 6.618096 \quad-4.705998 \quad 1.447167$

$\begin{array}{lllll}6 & 8.970633 & 1.671410 & -1.905879\end{array}$

$\begin{array}{lllll}6 & 7.335560 & 3.403000 & -1.977718\end{array}$
6

6

6

6

8

6

6

1

6

8

6

6

6

1

1

8

8

6

6

6

6

6

6

1

1

6

1

1

6

1

1

6

1

1

1

1

1 $\begin{array}{llll}5.662831 & 4.643280 & 0.639571\end{array}$

$\begin{array}{llll}3.364743 & 0.429133 & 4.022026\end{array}$

$\begin{array}{llll}8.887061 & -2.020622 & -1.726659\end{array}$

$7.253728 \quad-3.762854 \quad-1.757664$

$\begin{array}{llll}5.464311 & -4.530715 & 0.750916\end{array}$

$\begin{array}{llll}3.356628 & -0.865395 & 4.524791\end{array}$

$8.426838 \quad 1.056566 \quad-3.039093$

$\begin{array}{llll}9.826997 & 1.208614 & -1.429950\end{array}$

$6.775828 \quad 2.853659 \quad-3.123620$

$\begin{array}{llll}6.793405 & 4.578860 & -1.451131\end{array}$

$\begin{array}{llll}5.615967 & 4.491852 & -0.774598\end{array}$

$\begin{array}{llll}8.365733 & -1.458119 & -2.893898\end{array}$

$\begin{array}{llll}9.752158 & -1.554707 & -1.269189\end{array}$

$6.675402-3.224178 \quad-2.901160$

$6.717573-4.955800 \quad-1.239438$

$\begin{array}{llll}5.498665 & -4.790932 & -0.649331\end{array}$

$\begin{array}{llll}8.990209 & -0.248710 & -3.589788\end{array}$

$\begin{array}{llll}7.332264 & 1.689250 & -3.641073\end{array}$

$\begin{array}{llll}5.922166 & 3.318029 & -3.600588\end{array}$

$\begin{array}{llll}3.436544 & 4.561446 & 0.709950\end{array}$

$\begin{array}{llll}7.231619 & -2.072753 & -3.436628\end{array}$

$\begin{array}{llll}5.810294 & -3.688097 & -3.356322\end{array}$

$\begin{array}{llll}3.280706 & -4.118094 & 0.588581\end{array}$

$\begin{array}{llll}8.675483 & -0.302347 & -4.632526\end{array}$

$\begin{array}{llll}6.797702 & 1.161786 & -4.825775\end{array}$

$\begin{array}{llll}3.394450 & 4.341615 & -0.659095\end{array}$

$\begin{array}{llll}6.687827 & -1.518875 & -4.598963\end{array}$

$\begin{array}{llll}3.286385 & -4.509400 & -0.741546\end{array}$

$\begin{array}{llll}5.571939 & 0.575850 & -4.704499\end{array}$

$\begin{array}{llll}5.522854 & -0.831213 & -4.491478\end{array}$

$\begin{array}{llll}3.344242 & 0.651170 & -4.590221\end{array}$

$3.310023-0.700183-4.275765$

$10.525339-0.289334 \quad-3.570738$

$10.880191-1.212288-4.036532$

$\begin{array}{llll}10.942512 & -0.249987 & -2.561055\end{array}$

$\begin{array}{llll}10.618017 & 3.467320 & -0.142617\end{array}$

$10.994502 \quad 3.945250 \quad-1.050734$

$\begin{array}{llll}11.020239 & 2.451214 & -0.114929\end{array}$

$\begin{array}{llll}10.559125 & -0.020623 & 3.628731\end{array}$

$\begin{array}{llll}10.945179 & 0.900188 & 4.073616\end{array}$

$\begin{array}{llll}10.979015 & -0.100673 & 2.622403\end{array}$

$10.475555 \quad-3.764326 \quad 0.151635$

$10.848284 \quad-4.244471 \quad 1.060218$

$\begin{array}{llll}10.911723 & -2.762875 & 0.105828\end{array}$

$10.842911-4.322676 \quad-0.713246$

$\begin{array}{llll}10.930324 & -0.869754 & 4.208368\end{array}$

$\begin{array}{llll}11.016477 & 4.002406 & 0.723243\end{array}$

$\begin{array}{llll}10.929569 & 0.559887 & -4.127814\end{array}$

$\begin{array}{llll}2.024216 & 4.573174 & 1.228660\end{array}$

$\begin{array}{llll}1.958993 & 4.144359 & -1.057921\end{array}$

$\begin{array}{llll}1.929787 & 1.166804 & -4.600011\end{array}$

$\begin{array}{llll}1.887538 & -1.086780 & -3.997983\end{array}$

$\begin{array}{llll}1.993382 & 0.734667 & 3.499984\end{array}$

$\begin{array}{llll}1.940663 & -1.372633 & 4.497726\end{array}$

$\begin{array}{llll}1.902689 & -3.648392 & 0.939608\end{array}$

$\begin{array}{llll}1.873265 & -4.431448 & -1.258343\end{array}$

$\begin{array}{llll}1.143369 & 0.043694 & -4.293562\end{array}$

$\begin{array}{llll}1.229806 & 4.399535 & 0.090910\end{array}$ 
$\begin{array}{lllll}7 & 1.215097 & -0.341981 & 3.862804\end{array}$

$\begin{array}{lllll}7 & 1.139643 & -3.878479 & -0.185063\end{array}$

$\begin{array}{lllll}8 & 1.553251 & -3.123714 & 1.986808\end{array}$

$\begin{array}{lllll}8 & 1.417148 & -4.763476 & -2.323145\end{array}$

$\begin{array}{lllll}8 & 1.480390 & -2.145695 & -3.557481\end{array}$

$\begin{array}{lllll}8 & 1.517086 & 2.286809 & -4.788939\end{array}$

$\begin{array}{lllll}8 & 1.536288 & 3.782999 & -2.141727\end{array}$

$\begin{array}{lllll}8 & 1.657034 & 1.692790 & 2.812642\end{array}$

$\begin{array}{lllll}8 & 1.638582 & 4.680459 & 2.372115\end{array}$

$\begin{array}{lllll}8 & 1.481965 & -2.407749 & 4.911633\end{array}$

$\begin{array}{llll}0.129934 & -3.702351 & -0.271668\end{array}$

$\begin{array}{llll}0.192834 & -0.410128 & 3.756531\end{array}$

$\begin{array}{llll}0.214813 & 4.322710 & 0.140656\end{array}$

$\begin{array}{llll}0.131586 & 0.134130 & -4.147676\end{array}$

$\begin{array}{llll}4.574360 & 4.696702 & 1.392720\end{array}$

$\begin{array}{llll}4.481627 & 4.306790 & -1.433598\end{array}$

$\begin{array}{llll}4.455154 & 1.196637 & 3.983836\end{array}$

$\begin{array}{llll}4.472914 & -1.518256 & 4.863684\end{array}$

$\begin{array}{llll}4.482198 & 1.325331 & -4.779195\end{array}$

$\begin{array}{llll}4.395170 & -1.474314 & -4.230457\end{array}$

$\begin{array}{llll}4.360538 & -4.149047 & 1.372416\end{array}$

$\begin{array}{llll}4.405961 & -4.828586 & -1.396639\end{array}$

$\begin{array}{llll}-9.085539 & -2.798189 & -2.390716\end{array}$

$\begin{array}{lll}-8.489231 & -1.499213 & -2.933920\end{array}$

$\begin{array}{lll}-8.481299 & -3.168260 & -1.030746\end{array}$

$\begin{array}{llll}-8.778013 & -3.585223 & -3.080963\end{array}$

$\begin{array}{llll}-8.983867 & -0.243826 & -2.548799\end{array}$

$\begin{array}{llll}-7.400279 & -1.503658 & -3.815680\end{array}$

$\begin{array}{llll}-9.023631 & -2.764987 & 0.193484\end{array}$

$\begin{array}{llll}-7.312779 & -3.938173 & -0.971198\end{array}$

$\begin{array}{llll}-8.413452 & 0.962787 & -2.962068\end{array}$

$\begin{array}{llll}-9.843291 & -0.211513 & -1.889416\end{array}$

$\begin{array}{llll}-6.775828 & -0.330276 & -4.228302\end{array}$

$\begin{array}{llll}-6.890671 & -2.718356 & -4.309297\end{array}$

$\begin{array}{llll}-8.463161 & -3.108756 & 1.433557\end{array}$

$\begin{array}{llll}-9.902917 & -2.131706 & 0.186108\end{array}$

$\begin{array}{llll}-6.751864 & -4.360917 & 0.222380\end{array}$

$\begin{array}{llll}-6.724373 & -4.322491 & -2.175323\end{array}$

$\begin{array}{llll}-8.977601 & 2.335318 & -2.599164\end{array}$

$\begin{array}{lll}-7.274490 & 0.877597 & -3.772215\end{array}$

$\begin{array}{llll}-5.914122 & -0.364021 & -4.881809\end{array}$

$\begin{array}{llll}-5.650018 & -2.992079 & -3.802651\end{array}$

$\begin{array}{llll}-9.004710 & -2.550990 & 2.749883\end{array}$

$\begin{array}{lll}-7.339548 & -3.946563 & 1.411656\end{array}$

$\begin{array}{llll}-5.865651 & -4.981119 & 0.231288\end{array}$

$\begin{array}{llll}-5.583817 & -3.702667 & -2.572637\end{array}$

$\begin{array}{llll}-8.666371 & 3.008882 & -3.398603\end{array}$

$\begin{array}{llll}-8.360348 & 2.886104 & -1.317593\end{array}$

$\begin{array}{llll}-6.681807 & 2.071173 & -4.185873\end{array}$

$\begin{array}{lll}-8.363450 & -1.195469 & 3.069743\end{array}$

$\begin{array}{lll}-8.673280 & -3.235620 & 3.531605\end{array}$

$\begin{array}{llll}-6.780439 & -4.400591 & 2.615188\end{array}$

$\begin{array}{llll}-8.841239 & 2.510336 & -0.059497\end{array}$

$\begin{array}{lllll}6 & -7.301953 & 3.803246 & -1.340683\end{array}$

$\begin{array}{llll}6 & -5.520799 & 2.470288 & -3.604707\end{array}$

$\begin{array}{llll}6 & -3.439888 & -2.710122 & -3.738359\end{array}$

$\begin{array}{lllll}6 & -8.899084 & 0.024059 & 2.641008\end{array}$

$\begin{array}{lllll}6 & -7.186510 & -1.130497 & 3.824800\end{array}$ $\begin{array}{lllll}6 & -5.545455 & -3.890645 & 2.900964\end{array}$

$\begin{array}{lllll}6 & -3.402725 & -3.257322 & -2.462961\end{array}$

$\begin{array}{lllll}6 & -8.321764 & 3.000770 & 1.141032\end{array}$

$\begin{array}{llll}-9.662072 & 1.804180 & -0.013770\end{array}$

$\begin{array}{llll}-6.740293 & 4.313867 & -0.174446\end{array}$

$\begin{array}{llll}-6.777775 & 4.229511 & -2.572308\end{array}$

$\begin{array}{lll}-5.558258 & 3.680481 & -2.855829\end{array}$

$\begin{array}{lll}-8.314766 & 1.258545 & 2.946429\end{array}$

$\begin{array}{lll}-9.811071 & 0.013297 & 2.055935\end{array}$

$\begin{array}{lll}-6.560922 & 0.065335 & 4.152836\end{array}$

$\begin{array}{llll}-6.653516 & -2.330787 & 4.302791\end{array}$

$\begin{array}{lll}-5.489714 & -2.746052 & 3.741174\end{array}$

$\begin{array}{lll}-8.902119 & 2.589112 & 2.490400\end{array}$

$\begin{array}{lll}-7.245065 & 3.892051 & 1.049596\end{array}$

$\begin{array}{llll}-5.909855 & 5.007419 & -0.219225\end{array}$

$\begin{array}{lll}-3.317814 & 2.361608 & -3.279148\end{array}$

$\begin{array}{lll}-7.137593 & 1.243891 & 3.704672\end{array}$

$\begin{array}{lll}-5.653636 & 0.077113 & 4.741762\end{array}$

$\begin{array}{llll}-3.326669 & -3.804018 & 2.667827\end{array}$

$\begin{array}{lll}-8.564004 & 3.336368 & 3.208909\end{array}$

$\begin{array}{lll}-6.684416 & 4.404145 & 2.223607\end{array}$

$\begin{array}{lll}-3.338030 & 3.600738 & -2.654241\end{array}$

$\begin{array}{llll}-6.593930 & 2.469438 & 4.096313\end{array}$

$\begin{array}{llll}-3.291343 & -2.635083 & 3.416759\end{array}$

$\begin{array}{lll}-5.461040 & 3.917431 & 2.588120\end{array}$

$\begin{array}{lll}-5.411499 & 2.886030 & 3.569038\end{array}$

$\begin{array}{llll}-3.229705 & 3.943888 & 2.538257\end{array}$

$\begin{array}{lll}-3.178367 & 2.939568 & 3.496816\end{array}$

$\begin{array}{lll}-10.437449 & 2.604691 & 2.495997\end{array}$

$\begin{array}{lll}-10.810759 & 2.352488 & 3.492074\end{array}$

$\begin{array}{lll}-10.876577 & 1.895054 & 1.789354\end{array}$

$\begin{array}{llll}-10.513112 & 2.348032 & -2.557986\end{array}$

$\begin{array}{llll}-10.872903 & 3.362352 & -2.367347\end{array}$

$\begin{array}{llll}-10.925410 & 1.701560 & -1.778437\end{array}$

$\begin{array}{llll}-10.620362 & -2.778223 & -2.365306\end{array}$

$\begin{array}{lll}-11.008096 & -2.575888 & -3.366991\end{array}$

$\begin{array}{llll}-11.026850 & -2.015482 & -1.695362\end{array}$

$\begin{array}{llll}-10.538215 & -2.506038 & 2.793460\end{array}$

$\begin{array}{llll}-10.947124 & -3.504486 & 2.617887\end{array}$

$\begin{array}{llll}-10.969402 & -1.837844 & 2.042796\end{array}$

$\begin{array}{lll}-10.875299 & -2.159050 & 3.773616\end{array}$

$\begin{array}{llll}-11.003470 & -3.746302 & -2.032241\end{array}$

$\begin{array}{llll}-10.916973 & 2.009101 & -3.515619\end{array}$

$\begin{array}{lll}-10.802064 & 3.600086 & 2.229500\end{array}$

$\begin{array}{lll}-1.897692 & 1.866120 & -3.299490\end{array}$

$\begin{array}{llll}-1.924790 & 3.978695 & -2.305431\end{array}$

$\begin{array}{lll}-1.824563 & 4.321259 & 2.160712\end{array}$

$\begin{array}{lll}-1.726497 & 2.654964 & 3.812876\end{array}$

$\begin{array}{llll}-2.035878 & -2.343239 & -4.136078\end{array}$

$\begin{array}{llll}-1.991542 & -3.167256 & -1.952132\end{array}$

$\begin{array}{llll}-1.919595 & -4.124786 & 2.233392\end{array}$

$\begin{array}{llll}-1.886308 & -2.112456 & 3.429104\end{array}$

$\begin{array}{lll}-1.018604 & 3.535861 & 2.984046\end{array}$

$\begin{array}{lll}-1.150208 & 2.907103 & -2.786885\end{array}$

$\begin{array}{llll}-1.257763 & -2.682360 & -3.014298\end{array}$

$\begin{array}{lll}-1.154808 & -3.045640 & 2.712337\end{array}$

$\begin{array}{lll}-1.495164 & -5.076471 & 1.623826\end{array}$

$\begin{array}{llll}-1.486301 & -1.079517 & 3.930600\end{array}$ $\begin{array}{lll}-1.249533 & 1.870452 & 4.597940\end{array}$

$\begin{array}{lll}-1.451534 & 5.105976 & 1.315851\end{array}$

$\begin{array}{llll}-1.508169 & 4.961801 & -1.744352\end{array}$

$\begin{array}{llll}-1.622305 & -1.873312 & -5.167783\end{array}$

$\begin{array}{llll}-1.504273 & 0.772159 & -3.668930\end{array}$

$\begin{array}{llll}-1.591287 & -3.441327 & -0.831545\end{array}$

$\begin{array}{llll}-0.141660 & -2.993540 & 2.553362\end{array}$

$\begin{array}{llll}-0.242898 & -2.535794 & -3.019673\end{array}$

$\begin{array}{llll}-0.136822 & 2.932714 & -2.673913\end{array}$

$\begin{array}{lll}-0.002940 & 3.619956 & 2.966150\end{array}$

$\begin{array}{llll}-4.406268 & 1.775251 & -3.782613\end{array}$

$\begin{array}{llll}-4.464487 & 4.269268 & -2.398152\end{array}$

$\begin{array}{llll}-4.575219 & -2.530003 & -4.419458\end{array}$

$\begin{array}{llll}-4.470848 & -3.789078 & -1.860244\end{array}$

$\begin{array}{llll}-4.370157 & 4.446999 & 2.058297\end{array}$

$\begin{array}{llll}-4.272352 & 2.386301 & 4.027841\end{array}$

$\begin{array}{llll}-4.462612 & -4.447306 & 2.377922\end{array}$

$\begin{array}{llll}-4.366659 & -2.090274 & 3.984464\end{array}$

$\begin{array}{llll}-1.124351 & 1.336505 & 0.644945\end{array}$

$\begin{array}{lll}-0.407790 & 0.625517 & 1.517274\end{array}$

$\begin{array}{llll}1.043327 & -0.000605 & -1.190245\end{array}$

$\begin{array}{llll}0.104317 & -0.655635 & -1.151760\end{array}$

$\begin{array}{llll}-0.715776 & 2.367294 & 0.128337\end{array}$

$\begin{array}{llll}2.121046 & 0.717986 & -1.256914\end{array}$

$\begin{array}{llll}2.064851 & 1.588326 & -1.896223\end{array}$

$\begin{array}{llll}0.393238 & 1.144435 & 1.787155\end{array}$

$\begin{array}{llll}-2.481325 & 0.798450 & 0.414472\end{array}$

$\begin{array}{llll}-3.497671 & 1.705600 & 0.095369\end{array}$

$\begin{array}{llll}-2.773228 & -0.563593 & 0.545804\end{array}$

$\begin{array}{llll}-4.803509 & 1.256343 & -0.052755\end{array}$

$\begin{array}{lll}-3.258770 & 2.757840 & 0.001119\end{array}$

$\begin{array}{llll}-4.076922 & -1.008617 & 0.363115\end{array}$

$\begin{array}{llll}-1.973973 & -1.262069 & 0.755433\end{array}$

$\begin{array}{llll}-5.108807 & -0.103102 & 0.077928\end{array}$

$\begin{array}{llll}-5.598298 & 1.960293 & -0.268565\end{array}$

$\begin{array}{llll}-4.301166 & -2.068824 & 0.435568\end{array}$

$\begin{array}{llll}-6.510624 & -0.587004 & -0.119972\end{array}$

$\begin{array}{llll}-7.241530 & 0.194005 & 0.096655\end{array}$

$\begin{array}{llll}-6.662817 & -0.900356 & -1.155565\end{array}$

$\begin{array}{llll}-6.722946 & -1.443091 & 0.516890\end{array}$

$\begin{array}{llll}3.330242 & 0.348168 & -0.535435\end{array}$

$\begin{array}{llll}4.556981 & 0.900192 & -0.946199\end{array}$

$\begin{array}{llll}3.316232 & -0.555960 & 0.540155\end{array}$

$\begin{array}{llll}5.739446 & 0.542217 & -0.304350\end{array}$

$\begin{array}{llll}4.576877 & 1.601310 & -1.773608\end{array}$

$\begin{array}{lll}4.505620 & -0.916019 & 1.168071\end{array}$

$\begin{array}{llll}2.373726 & -0.960726 & 0.893750\end{array}$

$\begin{array}{llll}5.722777 & -0.375168 & 0.748186\end{array}$

$\begin{array}{llll}6.678563 & 0.969669 & -0.633651\end{array}$

$\begin{array}{llll}4.478657 & -1.629413 & 1.982983\end{array}$

$\begin{array}{lll}6.646621 & -0.660089 & 1.234175\end{array}$

\section{$7^{-}: 8 c^{+} @ 1_{2}$}

$\begin{array}{lllll}6 & 9.057277 & -2.822979 & 1.998055\end{array}$

$\begin{array}{lllll}6 & 8.370967 & -1.664114 & 2.713482\end{array}$

$\begin{array}{lllll}6 & 8.553739 & -3.000158 & 0.568264\end{array}$

$\begin{array}{lllll}1 & 8.764382 & -3.725315 & 2.535136\end{array}$

$\begin{array}{lllll}6 & 8.831530 & -0.349409 & 2.615940\end{array}$ 
$\begin{array}{lllll}6 & 7.255578 & -1.883560 & 3.531603\end{array}$

$\begin{array}{lllll}6 & 9.080357 & -2.283803 & -0.509171\end{array}$

$6 \quad 7.546676 \quad-3.928905 \quad 0.278315$

$\begin{array}{llll}6 & 8.250016 & 0.722134 & 3.302928\end{array}$

$\begin{array}{lllll}1 & 9.690632 & -0.149559 & 1.986793\end{array}$

$\begin{array}{lllll}6 & 6.642392 & -0.857570 & 4.241243\end{array}$

$\begin{array}{lllll}8 & 6.763434 & -3.191663 & 3.645997\end{array}$

$\begin{array}{lllll}6 & 8.668699 & -2.481137 & -1.832364\end{array}$

$\begin{array}{lllll}1 & 9.844407 & -1.540069 & -0.313504\end{array}$

$6 \quad 7.116339 \quad-4.179188 \quad-1.016802$

$\begin{array}{lllll}8 & 6.963934 & -4.631198 & 1.343198\end{array}$

$\begin{array}{lllll}6 & 8.813138 & 2.132978 & 3.179621\end{array}$

$\begin{array}{llll}6 & 7.146983 & 0.434239 & 4.118202\end{array}$

$1 \quad 5.785559 \quad-1.056879 \quad 4.872000$

$\begin{array}{lllll}6 & 5.629648 & -3.433011 & 2.926603\end{array}$

$\begin{array}{lllll}6 & 9.244339 & -1.654231 & -2.977242\end{array}$

$\begin{array}{lllll}6 & 7.689127 & -3.454033 & -2.056306\end{array}$

$1 \quad 6.341543 \quad-4.909040 \quad-1.212293$

$\begin{array}{lllll}6 & 5.735332 & -4.172384 & 1.715871\end{array}$

$1 \quad 8.433063 \quad 2.687638 \quad 4.038162$

$\begin{array}{llll}6 & 8.278284 & 2.848623 & 1.941662\end{array}$

$\begin{array}{lllll}8 & 6.539122 & 1.459936 & 4.863791\end{array}$

$\begin{array}{lllll}6 & 8.552874 & -0.295168 & -3.034726\end{array}$

$\begin{array}{lllll}1 & 8.981460 & -2.174915 & -3.898657\end{array}$

$\begin{array}{lllll}8 & 7.264601 & -3.722133 & -3.368694\end{array}$

$\begin{array}{lllll}6 & 8.885384 & 2.787234 & 0.685423\end{array}$

$\begin{array}{llll}6 & 7.124878 & 3.633800 & 2.046479\end{array}$

$\begin{array}{lllll}6 & 5.336610 & 1.876934 & 4.362339\end{array}$

$\begin{array}{lllll}6 & 3.447516 & -3.161405 & 2.548093\end{array}$

$\begin{array}{lllll}6 & 9.068376 & 0.844080 & -2.411047\end{array}$

$\begin{array}{lllll}6 & 7.348790 & -0.151628 & -3.737332\end{array}$

$\begin{array}{lllll}6 & 5.989312 & -3.308174 & -3.625232\end{array}$

$\begin{array}{lllll}6 & 3.551804 & -3.870913 & 1.360746\end{array}$

$\begin{array}{lllll}6 & 8.409523 & 3.500388 & -0.423794\end{array}$

$\begin{array}{lllll}1 & 9.759820 & 2.159237 & 0.559636\end{array}$

$\begin{array}{lllll}6 & 6.645360 & 4.404247 & 1.002439\end{array}$

$\begin{array}{llll}8 & 6.470271 & 3.654458 & 3.277567\end{array}$

$\begin{array}{lllll}6 & 5.330091 & 2.927916 & 3.402423\end{array}$

$\begin{array}{lllll}6 & 8.447912 & 2.094671 & -2.484050\end{array}$

$\begin{array}{llll}10.002289 & 0.760330 & -1.869463\end{array}$

$\begin{array}{lllll}6 & 6.690591 & 1.068760 & -3.848080\end{array}$

$8 \quad 6.890490 \quad-1.267806 \quad-4.435205$

$\begin{array}{lllll}6 & 5.788331 & -1.964027 & -4.060287\end{array}$

$\begin{array}{lllll}6 & 9.024882 & 3.339734 & -1.810816\end{array}$

$\begin{array}{lllll}6 & 7.305963 & 4.333879 & -0.217971\end{array}$

$\begin{array}{llll}5.767456 & 5.024004 & 1.125129\end{array}$

$\begin{array}{lllll}6 & 3.130067 & 1.664720 & 4.099672\end{array}$

$\begin{array}{lllll}6 & 7.251843 & 2.170436 & -3.209456\end{array}$

$1 \quad 5.778875 \quad 1.161458 \quad-4.423426$

$\begin{array}{lllll}6 & 3.779781 & -3.624970 & -3.632465\end{array}$

$\begin{array}{llll}8.691023 & 4.194012 & -2.400553\end{array}$

$\begin{array}{lllll}8 & 6.822287 & 5.121285 & -1.277589\end{array}$

$\begin{array}{lllll}6 & 3.165048 & 2.549888 & 3.029317\end{array}$

$8 \quad 6.680401 \quad 3.435894 \quad-3.363469$

$\begin{array}{lllll}6 & 3.584309 & -2.288850 & -3.948471\end{array}$

$\begin{array}{lllll}6 & 5.587358 & 4.730933 & -1.701023\end{array}$

$\begin{array}{lllll}6 & 5.520538 & 3.756107 & -2.740407\end{array}$

$\begin{array}{lllll}6 & 3.363710 & 4.697618 & -1.533646\end{array}$ $\begin{array}{lllll}6 & 3.310152 & 3.707345 & -2.500637\end{array}$

$\begin{array}{lllll}6 & 10.559177 & 3.362889 & -1.789703\end{array}$

$\begin{array}{lllll}1 & 10.952918 & 3.267464 & -2.805063\end{array}$

$1 \quad 10.992805 \quad 2.558505 \quad-1.189150$

$\begin{array}{lllll}6 & 10.347328 & 2.162406 & 3.255751\end{array}$

$\begin{array}{lllll}1 & 10.702369 & 3.195974 & 3.234447\end{array}$

$\begin{array}{llll}10.826804 & 1.631995 & 2.428549\end{array}$

$\begin{array}{lllll}6 & 10.589560 & -2.724734 & 2.064108\end{array}$

$\begin{array}{llll}10.917194 & -2.664041 & 3.105195\end{array}$

$\begin{array}{llll}10.983951 & -1.848755 & 1.541811\end{array}$

$\begin{array}{llll}10.775093 & -1.556555 & -2.921349\end{array}$

$\begin{array}{llll}11.211227 & -2.557630 & -2.965431\end{array}$

$\begin{array}{lllll}1 & 11.141480 & -1.081493 & -2.007158\end{array}$

$1 \quad 11.148796 \quad-0.977197 \quad-3.769920$

$1 \quad 11.039865 \quad-3.609984 \quad 1.607848$

$\begin{array}{lllll}1 & 10.684898 & 1.696811 & 4.185235\end{array}$

$1 \quad 10.909040 \quad 4.308870 \quad-1.368853$

$\begin{array}{lllll}6 & 1.709703 & 1.209940 & 4.285709\end{array}$

$\begin{array}{lllll}6 & 1.796452 & 2.585457 & 2.402718\end{array}$

$\begin{array}{lllll}6 & 1.955911 & 4.980436 & -1.070924\end{array}$

$\begin{array}{lllll}6 & 1.888743 & 3.295278 & -2.680267\end{array}$

$\begin{array}{lllll}6 & 2.052164 & -2.613810 & 2.635896\end{array}$

$6 \quad 2.212001 \quad-3.816965 \quad 0.677450$

$\begin{array}{lllll}6 & 2.431680 & -4.290371 & -3.519923\end{array}$

$\begin{array}{lllll}6 & 2.107418 & -2.012879 & -3.966856\end{array}$

$\begin{array}{lllll}7 & 1.156625 & 4.081987 & -1.819793\end{array}$

$\begin{array}{lllll}7 & 1.011332 & 1.799757 & 3.213085\end{array}$

$\begin{array}{lllll}7 & 1.388764 & -3.094763 & 1.520071\end{array}$

$\begin{array}{lllll}7 & 1.509793 & -3.249612 & -3.754911\end{array}$

$8 \quad 2.160810 \quad-5.442420 \quad-3.292171$

$8 \quad 1.551230 \quad-0.937070 \quad-4.074941$

$8 \quad 1.484717 \quad 2.405429 \quad-3.416456$

$\begin{array}{lllll}8 & 1.550430 & 5.775941 & -0.263809\end{array}$

$\begin{array}{llll}8 & 1.456282 & 3.167912 & 1.380695\end{array}$

$\begin{array}{lllll}8 & 1.616429 & -1.835773 & 3.465461\end{array}$

$\begin{array}{lllll}8 & 1.228667 & 0.510097 & 5.141862\end{array}$

$8 \quad 1.918619 \quad-4.262400 \quad-0.422106$

$\begin{array}{lllll}1 & 0.496930 & -3.389627 & -3.635279\end{array}$

$\begin{array}{lllll}1 & 0.378610 & -2.778303 & 1.263680\end{array}$

$\begin{array}{llll}0.001797 & 1.615750 & 3.094472\end{array}$

$\begin{array}{lllll}0.137139 & 4.023724 & -1.692262\end{array}$

$\begin{array}{llll}7 & 4.225812 & 1.275224 & 4.757260\end{array}$

$\begin{array}{llll}7 & 4.260988 & 3.225536 & 2.678583\end{array}$

$7 \quad 4.482576 \quad-2.922404 \quad 3.350820$

$7 \quad 4.696923 \quad-4.388411 \quad 0.918797$

$7 \quad 4.513389 \quad 5.224246 \quad-1.103641$

$7 \quad 4.378820 \quad 3.216404 \quad-3.133382$

$\begin{array}{lllll}7 & 4.990853 & -4.155611 & -3.438280\end{array}$

$7 \quad 4.583010 \quad-1.431176 \quad-4.184654$

$6 \quad-9.020846 \quad 0.821277 \quad-3.616139$

$\begin{array}{lllll}6 & -8.516070 & 1.990878 & -2.766232\end{array}$

$6 \quad-8.360528 \quad-0.502410 \quad-3.201495$

$1 \quad-8.683626 \quad 1.019387 \quad-4.634873$

$\begin{array}{lllll}6 & -9.064389 & 2.270747 & -1.504593\end{array}$

$\begin{array}{lllll}6 & -7.446979 & 2.798668 & -3.178372\end{array}$

$\begin{array}{lllll}6 & -8.928273 & -1.408351 & -2.296979\end{array}$

$6 \quad-7.120728 \quad-0.859902 \quad-3.745072$

$\begin{array}{lllll}6 & -8.553147 & 3.240100 & -0.636148\end{array}$ $\begin{array}{llll}-9.906692 & 1.676018 & -1.171219\end{array}$

$\begin{array}{llll}-6.889934 & 3.763354 & -2.344484\end{array}$

$\begin{array}{llll}-6.873579 & 2.644874 & -4.453093\end{array}$

$\begin{array}{llll}-8.309869 & -2.617488 & -1.952727\end{array}$

$\begin{array}{llll}-9.884396 & -1.166790 & -1.847680\end{array}$

$\begin{array}{llll}-6.472576 & -2.051173 & -3.455714\end{array}$

$\begin{array}{llll}-6.556436 & 0.010807 & -4.673035\end{array}$

$\begin{array}{llll}-9.153588 & 3.530564 & 0.742881\end{array}$

$\begin{array}{llll}-7.423433 & 3.941175 & -1.079574\end{array}$

$\begin{array}{llll}-6.038766 & 4.345581 & -2.672367\end{array}$

$\begin{array}{llll}-5.602314 & 2.137912 & -4.377986\end{array}$

$\begin{array}{llll}-8.874029 & -3.598639 & -0.930923\end{array}$

$\begin{array}{llll}-7.086419 & -2.914246 & -2.563280\end{array}$

$\begin{array}{llll}-5.521862 & -2.296002 & -3.908865\end{array}$

$\begin{array}{llll}-5.462213 & 0.725920 & -4.317997\end{array}$

$\begin{array}{llll}-8.858108 & 4.551035 & 0.991366\end{array}$

$\begin{array}{llll}-8.542235 & 2.636450 & 1.821726\end{array}$

$\begin{array}{llll}-6.842188 & 4.882252 & -0.223980\end{array}$

$\begin{array}{llll}-8.285536 & -3.309611 & 0.448889\end{array}$

$\begin{array}{llll}-8.515053 & -4.587584 & -1.217968\end{array}$

$\begin{array}{llll}-6.532278 & -4.166852 & -2.309691\end{array}$

$\begin{array}{llll}-9.036257 & 1.350833 & 2.092123\end{array}$

$\begin{array}{llll}-7.434972 & 3.058346 & 2.568585\end{array}$

$\begin{array}{llll}-5.670832 & 4.564974 & 0.388499\end{array}$

$\begin{array}{llll}-3.409619 & 2.339697 & -4.041277\end{array}$

$\begin{array}{llll}-8.815649 & -2.295189 & 1.251379\end{array}$

$\begin{array}{llll}-7.198763 & -4.026401 & 0.970410\end{array}$

$\begin{array}{llll}-5.342734 & -4.275496 & -1.664751\end{array}$

$\begin{array}{llll}-3.310776 & 0.972677 & -3.801058\end{array}$

$\begin{array}{llll}-8.452497 & 0.491389 & 3.026644\end{array}$

$\begin{array}{llll}-9.906150 & 1.007811 & 1.544873\end{array}$

$\begin{array}{llll}-6.806509 & 2.235861 & 3.496698\end{array}$

$\begin{array}{llll}-6.936232 & 4.362413 & 2.400074\end{array}$

$\begin{array}{llll}-5.710926 & 4.408211 & 1.803258\end{array}$

$\begin{array}{llll}-8.350359 & -1.996113 & 2.535012\end{array}$

$\begin{array}{llll}-9.637052 & -1.710279 & 0.854548\end{array}$

$\begin{array}{llll}-6.708267 & -3.781031 & 2.247941\end{array}$

$\begin{array}{llll}-6.600525 & -5.045488 & 0.218456\end{array}$

$\begin{array}{llll}-5.377989 & -4.770927 & -0.331103\end{array}$

$\begin{array}{llll}-8.997976 & -0.894545 & 3.372194\end{array}$

$\begin{array}{llll}-7.314668 & 0.962684 & 3.692915\end{array}$

$\begin{array}{llll}-5.938535 & 2.579432 & 4.043210\end{array}$

$\begin{array}{llll}-3.461987 & 4.241637 & 0.445778\end{array}$

$\begin{array}{llll}-7.287165 & -2.774236 & 3.013211\end{array}$

$\begin{array}{llll}-5.872728 & -4.351447 & 2.634782\end{array}$

$\begin{array}{llll}-3.114209 & -4.272105 & -1.596600\end{array}$

$\begin{array}{llll}-8.699107 & -1.085486 & 4.403774\end{array}$

$\begin{array}{llll}-6.733957 & 0.119871 & 4.638345\end{array}$

$\begin{array}{llll}-3.487960 & 4.207809 & 1.833298\end{array}$

$\begin{array}{llll}-6.772586 & -2.548600 & 4.299971\end{array}$

$\begin{array}{llll}-3.142991 & -4.776449 & -0.302712\end{array}$

$\begin{array}{llll}-5.557781 & -0.488389 & 4.337840\end{array}$

$\begin{array}{llll}-5.562472 & -1.909118 & 4.286388\end{array}$

$\begin{array}{llll}-3.360913 & -0.502549 & 3.992622\end{array}$

$\begin{array}{llll}-3.342571 & -1.888293 & 4.069855\end{array}$

$\begin{array}{llll}-10.532034 & -0.953446 & 3.319918\end{array}$

$\begin{array}{llll}-10.879242 & -1.938198 & 3.642809\end{array}$

$\begin{array}{llll}-10.932788 & -0.776799 & 2.317931\end{array}$ 
$\begin{array}{llll}6 & -10.688136 & 3.483080 & 0.732876\end{array}$

$\begin{array}{lllll}1 & -11.077555 & 3.756918 & 1.716819\end{array}$

$\begin{array}{lllll}1 & -11.083253 & 2.493736 & 0.485969\end{array}$

$\begin{array}{lllll}6 & -10.552853 & 0.734230 & -3.644992\end{array}$

$\begin{array}{llll}-10.973759 & 1.671759 & -4.017228\end{array}$

$\begin{array}{llll}-10.989041 & 0.547533 & -2.659459\end{array}$

$\begin{array}{llll}-10.407748 & -3.649625 & -0.914310\end{array}$

$\begin{array}{llll}-10.784902 & -3.904493 & -1.908418\end{array}$

$\begin{array}{llll}-10.865207 & -2.700846 & -0.620119\end{array}$

$\begin{array}{llll}-10.749052 & -4.409964 & -0.207221\end{array}$

$\begin{array}{llll}-10.871581 & -0.077053 & -4.304697\end{array}$

$\begin{array}{llll}-11.080353 & 4.186661 & -0.006025\end{array}$

$\begin{array}{llll}-10.959379 & -0.197648 & 3.984253\end{array}$

$\begin{array}{llll}-2.048396 & 3.995252 & -0.002443\end{array}$

$\begin{array}{llll}-2.076205 & 4.044662 & 2.333592\end{array}$

$\begin{array}{llll}-1.970958 & -0.023808 & 3.705678\end{array}$

$\begin{array}{llll}-1.915393 & -2.348737 & 3.926505\end{array}$

$\begin{array}{llll}-2.039650 & 2.944436 & -3.929203\end{array}$

$\begin{array}{llll}-1.896579 & 0.667680 & -3.374525\end{array}$

$\begin{array}{llll}-1.678450 & -4.152239 & -2.031622\end{array}$

$\begin{array}{llll}-1.723178 & -5.009130 & 0.146151\end{array}$

$\begin{array}{llll}-1.182822 & -1.155115 & 3.755466\end{array}$

$\begin{array}{llll}-1.308291 & 3.908429 & 1.156834\end{array}$

$\begin{array}{llll}-1.228213 & 1.876534 & -3.526220\end{array}$

$\begin{array}{llll}-0.944656 & -4.663251 & -0.979110\end{array}$

$\begin{array}{llll}-1.251122 & -3.710207 & -3.085731\end{array}$

$\begin{array}{llll}-1.302277 & -5.424631 & 1.195456\end{array}$

$\begin{array}{llll}-1.458851 & -3.463557 & 3.946294\end{array}$

$\begin{array}{llll}-1.634249 & 1.119069 & 3.443077\end{array}$

$\begin{array}{llll}-1.645538 & 4.046663 & 3.458521\end{array}$

$\begin{array}{llll}-1.670599 & 4.073715 & -4.151055\end{array}$

$\begin{array}{llll}-1.633454 & 3.867327 & -1.145173\end{array}$

$\begin{array}{llll}-1.419883 & -0.365834 & -2.951729\end{array}$

$\begin{array}{llll}0.075187 & -4.617622 & -0.922891\end{array}$

$\begin{array}{llll}-0.211143 & 1.981507 & -3.456309\end{array}$

$\begin{array}{llll}-0.301401 & 3.706038 & 1.177424\end{array}$

$\begin{array}{llll}-0.170234 & -1.182157 & 3.609426\end{array}$

$\begin{array}{llll}-4.549204 & 4.443163 & -0.306661\end{array}$

$\begin{array}{llll}-4.619674 & 4.269334 & 2.539851\end{array}$

$\begin{array}{llll}-4.569383 & 2.957054 & -4.291722\end{array}$

$\begin{array}{llll}-4.334263 & 0.130135 & -3.965863\end{array}$

$\begin{array}{llll}-4.463839 & 0.231006 & 4.143497\end{array}$

$\begin{array}{llll}-4.451973 & -2.622820 & 4.184555\end{array}$

$\begin{array}{llll}-4.214438 & -4.012670 & -2.306587\end{array}$

$\begin{array}{llll}-4.277580 & -5.026007 & 0.360645\end{array}$

$\begin{array}{llll}-1.139647 & -1.046526 & 0.762821\end{array}$

$\begin{array}{llll}-0.224399 & -0.179851 & 0.813066\end{array}$

$\begin{array}{llll}0.848580 & -0.959428 & -1.236578\end{array}$

$\begin{array}{llll}0.466031 & -1.987781 & -1.381537\end{array}$

$\begin{array}{llll}-0.952792 & -2.305068 & 0.848075\end{array}$

$\begin{array}{llll}1.559870 & 0.327296 & -1.255141\end{array}$

$\begin{array}{llll}0.883488 & 1.004181 & -0.734487\end{array}$

$\begin{array}{llll}-2.549179 & -0.625984 & 0.519805\end{array}$

$\begin{array}{llll}-3.594010 & -1.509162 & 0.809176\end{array}$

$\begin{array}{llll}-2.830090 & 0.618700 & -0.048666\end{array}$

$\begin{array}{llll}-4.907965 & -1.141784 & 0.548122\end{array}$

$\begin{array}{llll}-3.360322 & -2.475459 & 1.237913\end{array}$

$\begin{array}{lll}-4.141820 & 0.963762 & -0.348387\end{array}$
$1 \quad-2.006119 \quad 1.282940 \quad-0.278967$

$\begin{array}{lllll}6 & -5.195843 & 0.092509 & -0.040809\end{array}$

$\begin{array}{lllll}1 & -5.720977 & -1.815881 & 0.788106\end{array}$

$1 \quad-4.360413 \quad 1.910166 \quad-0.833351$

$6 \quad-6.607842 \quad 0.491752 \quad-0.323186$

$1 \quad-6.695990 \quad 0.930611 \quad-1.315856$

$\begin{array}{lllll}1 & -7.290400 & -0.356483 & -0.261971\end{array}$

$\begin{array}{lllll}1 & -6.944417 & 1.241122 & 0.394498\end{array}$

$1 \quad 1.640551 \quad 0.547318 \quad-2.321866$

$\begin{array}{lllll}6 & 2.897786 & 0.162849 & -0.571836\end{array}$

$\begin{array}{lllll}6 & 2.952872 & -0.062332 & 0.808986\end{array}$

$\begin{array}{lllll}6 & 4.072886 & 0.261128 & -1.324659\end{array}$

$\begin{array}{lllll}6 & 4.192658 & -0.188685 & 1.433882\end{array}$

$\begin{array}{lllll}1 & 2.023542 & -0.148473 & 1.362005\end{array}$

$6 \quad 5.310215 \quad 0.146240 \quad-0.685438$

$1 \quad 4.022847 \quad 0.433750 \quad-2.394682$

$\begin{array}{lllll}6 & 5.371320 & -0.082704 & 0.689968\end{array}$

$\begin{array}{lllll}1 & 4.239222 & -0.375445 & 2.499607\end{array}$

$\begin{array}{lllll}1 & 6.223728 & 0.230502 & -1.261973\end{array}$

$\begin{array}{llll}1 & 6.334315 & -0.176643 & 1.181411\end{array}$

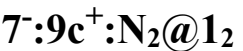

$\begin{array}{lllll}6 & 8.907822 & -1.997647 & 3.091156\end{array}$

$\begin{array}{lllll}6 & 8.303841 & -0.603591 & 3.250219\end{array}$

$\begin{array}{lllll}6 & 8.332921 & -2.715285 & 1.874159\end{array}$

$\begin{array}{lllll}1 & 8.585957 & -2.568535 & 3.962481\end{array}$

$\begin{array}{lllll}6 & 8.839678 & 0.530881 & 2.637668\end{array}$

$\begin{array}{lllll}6 & 7.172051 & -0.413258 & 4.053836\end{array}$

$\begin{array}{lllll}6 & 8.856793 & -2.523082 & 0.590861\end{array}$

$\begin{array}{lllll}6 & 7.257279 & -3.603832 & 1.997967\end{array}$

$\begin{array}{lllll}6 & 8.317379 & 1.817900 & 2.818264\end{array}$

$\begin{array}{llll}9.706743 & 0.412891 & 1.998935\end{array}$

$6 \quad \begin{array}{llll}6.630733 & 0.843361 & 4.294933\end{array}$

$8 \quad 6.613444 \quad-1.541701 \quad 4.655055$

$\begin{array}{lllll}6 & 8.353827 & -3.167356 & -0.541891\end{array}$

$\begin{array}{lllll}1 & 9.701908 & -1.854930 & 0.473699\end{array}$

$\begin{array}{lllll}6 & 6.698311 & -4.246069 & 0.897704\end{array}$

$\begin{array}{lllll}8 & 6.736918 & -3.877337 & 3.272919\end{array}$

$\begin{array}{lllll}6 & 8.917972 & 3.025655 & 2.106039\end{array}$

$\begin{array}{lllll}6 & 7.212230 & 1.945806 & 3.672470\end{array}$

$\begin{array}{lllll}1 & 5.762529 & 0.962386 & 4.929833\end{array}$

$\begin{array}{lllll}6 & 5.463905 & -2.035273 & 4.116978\end{array}$

$\begin{array}{lllll}6 & 8.970719 & -2.995898 & -1.928638\end{array}$

$6 \quad 7.248998 \quad-4.006623 \quad-0.354050$

$\begin{array}{lllll}1 & 5.854859 & -4.914318 & 1.015694\end{array}$

$\begin{array}{lllll}6 & 5.511838 & -3.314558 & 3.498100\end{array}$

$\begin{array}{lllll}1 & 8.589814 & 3.903492 & 2.663246\end{array}$

$\begin{array}{lllll}6 & 8.347158 & 3.179786 & 0.696151\end{array}$

$\begin{array}{lllll}8 & 6.672615 & 3.217074 & 3.916047\end{array}$

$\begin{array}{lllll}6 & 8.383550 & -1.778597 & -2.633354\end{array}$

$1 \quad 8.662854 \quad-3.865376 \quad-2.509746$

$8 \quad 6.730506 \quad-4.665516 \quad-1.474191$

$\begin{array}{lllll}6 & 8.895110 & 2.555217 & -0.427568\end{array}$

$\begin{array}{lllll}6 & 7.231811 & 3.998673 & 0.480836\end{array}$

$\begin{array}{lllll}6 & 5.471395 & 3.455005 & 3.305111\end{array}$

$\begin{array}{lllll}6 & 3.273094 & -1.954920 & 3.716053\end{array}$

$\begin{array}{lllll}6 & 8.918112 & -0.496439 & -2.467352\end{array}$

$\begin{array}{lllll}6 & 7.272521 & -1.904775 & -3.476793\end{array}$
$6 \quad 5.540402 \quad-4.235775 \quad-1.970108$

$\begin{array}{lllll}6 & 3.294733 & -3.253968 & 3.232587\end{array}$

$\begin{array}{lllll}6 & 8.401528 & 2.750555 & -1.724548\end{array}$

$\begin{array}{llll}9.748596 & 1.901002 & -0.293426\end{array}$

$\begin{array}{llll}6.718496 & 4.248881 & -0.783373\end{array}$

$\begin{array}{llll}6.658074 & 4.611162 & 1.595747\end{array}$

$\begin{array}{llll}5.476810 & 4.104925 & 2.040352\end{array}$

$\begin{array}{llll}8.381301 & 0.638477 & -3.080667\end{array}$

$\begin{array}{llll}9.796111 & -0.381218 & -1.843448\end{array}$

$\begin{array}{llll}6.691237 & -0.806163 & -4.103422\end{array}$

$\begin{array}{llll}6.767350 & -3.183551 & -3.733507\end{array}$

$\begin{array}{llll}5.557075 & -3.496155 & -3.187693\end{array}$

$\begin{array}{llll}8.995733 & 2.029080 & -2.929474\end{array}$

$\begin{array}{llll}7.321712 & 3.629487 & -1.872017\end{array}$

$\begin{array}{llll}5.868671 & 4.905124 & -0.918539\end{array}$

$\begin{array}{llll}3.248736 & 3.269397 & 3.173939\end{array}$

$\begin{array}{llll}7.238184 & 0.450185 & -3.868700\end{array}$

$\begin{array}{llll}5.830755 & -0.927194 & -4.749219\end{array}$

$\begin{array}{llll}3.310286 & -4.149142 & -1.969785\end{array}$

$\begin{array}{llll}8.689307 & 2.595835 & -3.809227\end{array}$

$\begin{array}{llll}6.841767 & 3.921813 & -3.158489\end{array}$

$\begin{array}{llll}3.272785 & 3.801064 & 1.891913\end{array}$

$\begin{array}{llll}6.688201 & 1.573857 & -4.487196\end{array}$

$\begin{array}{llll}3.324170 & -3.486039 & -3.188582\end{array}$

$\begin{array}{llll}5.606755 & 3.405843 & -3.424325\end{array}$

$\begin{array}{llll}5.535282 & 2.107321 & -4.004159\end{array}$

$\begin{array}{llll}3.377519 & 3.471682 & -3.330467\end{array}$

$\begin{array}{llll}3.318156 & 2.158078 & -3.769401\end{array}$

$\begin{array}{lll}10.530880 & 2.000823 & -2.909443\end{array}$

$\begin{array}{llll}10.909839 & 1.517138 & -3.813428\end{array}$

$\begin{array}{llll}10.939082 & 1.460990 & -2.050563\end{array}$

$\begin{array}{llll}10.454016 & 3.017342 & 2.118984\end{array}$

$\begin{array}{llll}10.836102 & 3.932531 & 1.659617\end{array}$

$\begin{array}{llll}10.882617 & 2.171878 & 1.573700\end{array}$

$\begin{array}{llll}10.443835 & -1.976555 & 3.094641\end{array}$

$\begin{array}{llll}10.810665 & -1.507416 & 4.011257\end{array}$

$\begin{array}{llll}10.867117 & -1.425620 & 2.250083\end{array}$

$\begin{array}{llll}10.506038 & -2.984791 & -1.893405\end{array}$

$10.874438-3.907504 \quad-1.438154$

$\begin{array}{llll}10.916841 & -2.149625 & -1.319312\end{array}$

$\begin{array}{llll}10.903844 & -2.914830 & -2.909147\end{array}$

$\begin{array}{llll}10.830008 & -2.997863 & 3.047517\end{array}$

$\begin{array}{llll}10.819161 & 2.965336 & 3.147827\end{array}$

$\begin{array}{llll}10.920672 & 3.021113 & -2.871231\end{array}$

$\begin{array}{llll}1.816634 & 2.962996 & 3.526220\end{array}$

$\begin{array}{llll}1.874205 & 3.783544 & 1.336241\end{array}$

$\begin{array}{llll}1.965691 & 3.968010 & -3.135539\end{array}$

$\begin{array}{llll}1.881393 & 1.725031 & -3.782547\end{array}$

$\begin{array}{llll}1.886195 & -1.407768 & 3.553399\end{array}$

$\begin{array}{llll}1.881119 & -3.631131 & 2.860544\end{array}$

$\begin{array}{llll}1.876219 & -4.296662 & -1.528973\end{array}$

$\begin{array}{llll}1.896349 & -3.206646 & -3.588797\end{array}$

$\begin{array}{llll}1.158180 & 2.869093 & -3.494544\end{array}$

$\begin{array}{llll}1.092951 & 3.284850 & 2.363367\end{array}$

$\begin{array}{llll}1.131441 & -2.468630 & 3.106566\end{array}$

$\begin{array}{llll}1.126652 & -3.789138 & -2.582815\end{array}$

$\begin{array}{llll}1.481957 & -4.732944 & -0.469377\end{array}$

$\begin{array}{llll}1.499891 & -2.557718 & -4.531835\end{array}$ 
$\begin{array}{lllll}8 & 1.467268 & 0.592885 & -3.957052\end{array}$

$\begin{array}{lllll}8 & 1.587684 & 5.046264 & -2.751201\end{array}$

$\begin{array}{lllll}8 & 1.530073 & 4.114401 & 0.217235\end{array}$

$\begin{array}{lllll}8 & 1.553112 & -0.248858 & 3.734666\end{array}$

$\begin{array}{lllll}8 & 1.358680 & 2.550757 & 4.563276\end{array}$

$\begin{array}{lllll}8 & 1.458222 & -4.679127 & 2.437149\end{array}$

$\begin{array}{lllll}1 & 0.111827 & -3.659710 & -2.559393\end{array}$

$\begin{array}{lllll}1 & 0.129529 & -2.440708 & 2.890920\end{array}$

$\begin{array}{lllll}1 & 0.069446 & 3.191411 & 2.358809\end{array}$

$\begin{array}{lllll}1 & 0.136836 & 2.906325 & -3.478766\end{array}$

$\begin{array}{lllll}7 & 4.353589 & 3.055612 & 3.894012\end{array}$

$\begin{array}{lllll}7 & 4.384442 & 4.243418 & 1.303501\end{array}$

$7 \quad 4.349604 \quad-1.324575 \quad 4.186858$

$\begin{array}{lllll}7 & 4.424698 & -3.949540 & 3.084092\end{array}$

$7 \begin{array}{llll}7 & 4.529886 & 4.110777 & -3.112305\end{array}$

$\begin{array}{lllll}7 & 4.392335 & 1.448290 & -4.126607\end{array}$

$\begin{array}{llll}4.417610 & -4.537962 & -1.334024\end{array}$

$\begin{array}{lllll}7 & 4.449243 & -3.116964 & -3.808109\end{array}$

$\begin{array}{lllll}6 & -8.923327 & -0.580018 & -3.511352\end{array}$

$\begin{array}{lllll}6 & -8.345903 & 0.768601 & -3.089299\end{array}$

$\begin{array}{lllll}6 & -8.342865 & -1.728619 & -2.688815\end{array}$

$\begin{array}{llll}-8.591326 & -0.744443 & -4.536898\end{array}$

$\begin{array}{lllll}6 & -8.908232 & 1.561147 & -2.084021\end{array}$

$\begin{array}{lllll}6 & -7.201767 & 1.266125 & -3.722670\end{array}$

$\begin{array}{lllll}6 & -8.870673 & -2.081928 & -1.443738\end{array}$

$\begin{array}{lllll}6 & -7.263609 & -2.491681 & -3.153820\end{array}$

$\begin{array}{lllll}6 & -8.382518 & 2.803948 & -1.712903\end{array}$

$\begin{array}{llll}-9.790856 & 1.198500 & -1.570725\end{array}$

$\begin{array}{lllll}6 & -6.641498 & 2.494047 & -3.401434\end{array}$

$\begin{array}{lllll}8 & -6.640920 & 0.501468 & -4.751754\end{array}$

$\begin{array}{lllll}6 & -8.395209 & -3.152073 & -0.679122\end{array}$

$\begin{array}{llll}-9.692464 & -1.496718 & -1.048932\end{array}$

$\begin{array}{lllll}6 & -6.761509 & -3.574436 & -2.442204\end{array}$

$\begin{array}{llll}6 & -6.682105 & -2.172848 & -4.390044\end{array}$

$\begin{array}{llll}6 & -8.989816 & 3.649452 & -0.595011\end{array}$

$\begin{array}{lllll}6 & -7.244067 & 3.243899 & -2.399677\end{array}$

$\begin{array}{llll}-5.758286 & 2.855491 & -3.911189\end{array}$

$\begin{array}{llll}6 & -5.463177 & -0.118091 & -4.473968\end{array}$

$\begin{array}{lllll}6 & -9.007570 & -3.493352 & 0.676368\end{array}$

$\begin{array}{llll}6 & -7.334155 & -3.892874 & -1.215173\end{array}$

$\begin{array}{llll}-5.930133 & -4.150569 & -2.828520\end{array}$

$\begin{array}{lllll}6 & -5.479165 & -1.531531 & -4.313521\end{array}$

$\begin{array}{llll}-8.674723 & 4.677006 & -0.779826\end{array}$

$\begin{array}{llll}6 & -8.389465 & 3.250281 & 0.751455\end{array}$

$\begin{array}{llll}8 & -6.732686 & 4.511742 & -2.102460\end{array}$

$\begin{array}{lllll}6 & -8.399573 & -2.638898 & 1.786842\end{array}$

$\begin{array}{lllll}1 & -8.718365 & -4.522819 & 0.890694\end{array}$

$\begin{array}{lllll}8 & -6.840967 & -5.007711 & -0.520704\end{array}$

$\begin{array}{lllll}6 & -8.924586 & 2.219275 & 1.532963\end{array}$

$\begin{array}{lllll}6 & -7.249299 & 3.897174 & 1.244776\end{array}$

$\begin{array}{lllll}6 & -5.526523 & 4.574049 & -1.474852\end{array}$

$\begin{array}{lllll}6 & -3.260153 & -0.118063 & -4.142092\end{array}$

$\begin{array}{lllll}6 & -8.927019 & -1.409489 & 2.187107\end{array}$

$\begin{array}{lllll}6 & -7.257493 & -3.086614 & 2.461215\end{array}$

$\begin{array}{lllll}6 & -5.613840 & -4.821867 & 0.055226\end{array}$

$6 \quad-3.268246 \quad-1.499116 \quad-4.016849$

$\begin{array}{lllll}6 & -8.370701 & 1.823715 & 2.754032\end{array}$

$\begin{array}{llll}1 & -9.808815 & 1.706158 & 1.174633\end{array}$ $\begin{array}{lllll}6 & -6.650330 & 3.535047 & 2.444677\end{array}$

$8 \quad-6.732853 \quad 4.988054 \quad 0.536406$

$6 \quad-5.526066 \quad 4.831483 \quad-0.075251$

$6 \quad-8.380528 \quad-0.646890 \quad 3.227954$

$\begin{array}{llll}-9.798908 & -1.025441 & 1.671312\end{array}$

$\begin{array}{llll}-6.684548 & -2.383988 & 3.510019\end{array}$

$\begin{array}{llll}-6.737108 & -4.324920 & 2.085310\end{array}$

$\begin{array}{llll}-5.565518 & -4.359299 & 1.398447\end{array}$

$\begin{array}{llll}-8.962138 & 0.709754 & 3.618269\end{array}$

$\begin{array}{llll}-7.217676 & 2.497999 & 3.171863\end{array}$

$\begin{array}{llll}-5.766216 & 4.047200 & 2.800413\end{array}$

$\begin{array}{llll}-3.295663 & 4.579046 & -1.458968\end{array}$

$\begin{array}{llll}-7.261951 & -1.175826 & 3.885070\end{array}$

$\begin{array}{llll}-5.808756 & -2.760964 & 4.021453\end{array}$

$\begin{array}{llll}-3.384445 & -4.775208 & -0.017429\end{array}$

$\begin{array}{llll}-8.628005 & 0.903968 & 4.637998\end{array}$

$\begin{array}{llll}-6.658303 & 2.130633 & 4.398322\end{array}$

$\begin{array}{llll}-3.293987 & 4.838611 & -0.093971\end{array}$

$\begin{array}{llll}-6.717828 & -0.493199 & 4.984785\end{array}$

$\begin{array}{llll}-3.349243 & -4.213047 & 1.251866\end{array}$

$\begin{array}{llll}-5.482012 & 1.450444 \quad 4.364076\end{array}$

$\begin{array}{llll}-5.502337 & 0.083506 & 4.753689\end{array}$

$\begin{array}{llll}-3.281212 & 1.309874 & 4.062532\end{array}$

$\begin{array}{llll}-3.282765 & 0.004376 & 4.533108\end{array}$

$\begin{array}{llll}-10.497299 & 0.719709 & 3.626467\end{array}$

$\begin{array}{llll}-10.873092 & -0.053820 & 4.301266\end{array}$

$\begin{array}{llll}-10.932863 & 0.535404 & 2.640448\end{array}$

$\begin{array}{llll}-10.524377 & 3.629519 & -0.596747\end{array}$

$\begin{array}{llll}-10.908551 & 4.289516 & 0.185462\end{array}$

$\begin{array}{llll}-10.941193 & 2.634041 & -0.420309\end{array}$

$\begin{array}{llll}-10.459344 & -0.589377 & -3.518549\end{array}$

$\begin{array}{llll}-10.835641 & 0.205789 & -4.167596\end{array}$

$\begin{array}{llll}-10.892847 & -0.437630 & -2.526272\end{array}$

$\begin{array}{llll}-10.542133 & -3.439241 & 0.659327\end{array}$

$\begin{array}{llll}-10.931471 & -4.116199 & -0.105536\end{array}$

$\begin{array}{llll}-10.933504 & -2.440982 & 0.443950\end{array}$

$\begin{array}{llll}-10.938723 & -3.745606 & 1.630924\end{array}$

$\begin{array}{llll}-10.825981 & -1.548522 & -3.893385\end{array}$

$\begin{array}{llll}-10.901911 & 3.976738 & -1.562107\end{array}$

$\begin{array}{llll}-10.863532 & 1.690567 & 3.970605\end{array}$

$\begin{array}{llll}-1.869650 & 4.487702 & -1.932730\end{array}$

$\begin{array}{llll}-1.863740 & 4.952105 & 0.366404\end{array}$

$\begin{array}{llll}-1.893341 & 1.656058 & 3.615129\end{array}$

$\begin{array}{llll}-1.861808 & -0.497136 & 4.509588\end{array}$

$\begin{array}{llll}-1.849563 & 0.368105 & -3.971288\end{array}$

$\begin{array}{llll}-1.860510 & -1.960449 & -3.762556\end{array}$

$\begin{array}{llll}-1.968485 & -4.889291 & -0.518807\end{array}$

$\begin{array}{llll}-1.924616 & -3.869477 & 1.579879\end{array}$

$\begin{array}{llll}-1.126559 & 0.558838 & 3.942340\end{array}$

$\begin{array}{llll}-1.108748 & 4.761692 & -0.798179\end{array}$

$\begin{array}{llll}-1.099992 & -0.793055 & -3.773974\end{array}$

$\begin{array}{llll}-1.186702 & -4.366699 & 0.519369\end{array}$

$\begin{array}{llll}-1.561652 & -5.311451 & -1.576512\end{array}$

$\begin{array}{llll}-1.518951 & -3.250215 & 2.544079\end{array}$

$\begin{array}{llll}-1.406675 & -1.548036 & 4.891545\end{array}$

$\begin{array}{llll}-1.550274 & 2.668400 & 3.027120\end{array}$

$\begin{array}{llll}-1.424880 & 5.165829 & 1.472626\end{array}$

$\begin{array}{llll}-1.440188 & 1.507855 & -3.995204\end{array}$
$8 \quad-1.457674 \quad 4.215632 \quad-3.039626$

$\begin{array}{llll}-1.472401 & -3.093803 & -3.577273\end{array}$

$\begin{array}{llll}-0.172948 & -4.298815 & 0.421840\end{array}$

$\begin{array}{llll}-0.089612 & -0.769249 & -3.694797\end{array}$

$\begin{array}{llll}-0.092306 & 4.708484 & -0.761882\end{array}$

$\begin{array}{llll}-0.129067 & 0.443201 & 3.732327\end{array}$

$\begin{array}{llll}-4.412124 & 4.439096 & -2.178112\end{array}$

$\begin{array}{llll}-4.411049 & 4.965400 & 0.627978\end{array}$

$\begin{array}{llll}-4.355832 & 0.602798 & -4.376411\end{array}$

$\begin{array}{llll}-4.378931 & -2.235214 & -4.088909\end{array}$

$\begin{array}{llll}-4.374621 & 2.067829 & 3.983711\end{array}$

$\begin{array}{llll}-4.400153 & -0.643180 & 4.873180\end{array}$

$\begin{array}{llll}-4.521575 & -5.061980 & -0.655454\end{array}$

$\begin{array}{llll}-4.436049 & -4.010153 & 1.995770\end{array}$

$\begin{array}{llll}-0.561179 & -1.120203 & 0.053326\end{array}$

$\begin{array}{llll}-0.109029 & -1.819554 & -0.906330\end{array}$

$\begin{array}{llll}0.435893 & 1.562104 & -0.894715\end{array}$

$\begin{array}{llll}-0.623913 & 1.854958 & -1.002895\end{array}$

$\begin{array}{llll}0.122374 & -0.613001 & 0.984147\end{array}$

$\begin{array}{llll}2.499527 & -0.804818 & -1.689848\end{array}$

$\begin{array}{llll}2.770354 & -0.937396 & -2.731831\end{array}$

$\begin{array}{llll}1.471867 & -1.063900 & -1.373007\end{array}$

$\begin{array}{llll}-2.046094 & -0.822966 & 0.047996\end{array}$

$\begin{array}{llll}-2.526705 & 0.282378 & 0.757688\end{array}$

$\begin{array}{llll}-2.955746 & -1.583022 & -0.692693\end{array}$

$\begin{array}{llll}-3.876296 & 0.618640 & 0.735759\end{array}$

$\begin{array}{llll}-1.808429 & 0.888141 & 1.289615\end{array}$

$\begin{array}{llll}-4.315665 & -1.270633 & -0.684182\end{array}$

$\begin{array}{llll}-2.587654 & -2.413968 & -1.280617\end{array}$

$\begin{array}{llll}-4.798552 & -0.165323 & 0.028125\end{array}$

$\begin{array}{llll}-4.219952 & 1.501951 & 1.268224\end{array}$

$\begin{array}{llll}-5.011229 & -1.883866 & -1.248391\end{array}$

$\begin{array}{llll}-6.263698 & 0.177499 & 0.046499\end{array}$

$\begin{array}{llll}-6.418752 & 1.257199 & -0.028962\end{array}$

$\begin{array}{llll}-6.801582 & -0.298952 & -0.774162\end{array}$

$\begin{array}{lll}-6.733202 & -0.145952 & 0.980925\end{array}$

$\begin{array}{llll}3.452718 & -0.360913 & -0.793725\end{array}$

$\begin{array}{llll}3.082228 & -0.091612 & 0.565273\end{array}$

$\begin{array}{llll}4.804761 & -0.166315 & -1.227003\end{array}$

$\begin{array}{llll}4.052293 & 0.369276 & 1.439984\end{array}$

$\begin{array}{llll}2.035921 & -0.255917 & 0.855500\end{array}$

$\begin{array}{llll}5.755709 & 0.274692 & -0.335043\end{array}$

$\begin{array}{llll}5.054985 & -0.377377 & -2.258554\end{array}$

$\begin{array}{lllll}6 & 5.372481 & 0.538413 & 0.993812\end{array}$

$\begin{array}{llll}3.802645 & 0.577313 & 2.473230\end{array}$

$\begin{array}{llll}6.784712 & 0.419340 & -0.641571\end{array}$

$1 \quad 6.126852 \quad 0.880535 \quad 1.690495$

\section{5:9c $\mathbf{c}^{+} @ \mathbf{1}^{-}$}

$\begin{array}{llll}6 & -9.021661 & -3.541290 & 0.234997\end{array}$

$6 \begin{array}{llll}6 & -8.426021 & -3.028269 & -1.072108\end{array}$

$\begin{array}{lllll}6 & -8.408120 & -2.844454 & 1.445310\end{array}$

$\begin{array}{lllll}1 & -8.734720 & -4.590748 & 0.309288\end{array}$

$\begin{array}{lllll}6 & -8.898652 & -1.858875 & -1.675563\end{array}$

$\begin{array}{lllll}6 & -7.405060 & -3.719330 & -1.737322\end{array}$

$\begin{array}{lllll}6 & -8.922854 & -1.668984 & 1.995294\end{array}$

$\begin{array}{lllll}6 & -7.276229 & -3.390095 & 2.062920\end{array}$

$\begin{array}{llll}6 & -8.423529 & -1.373682 & -2.897477\end{array}$ 
$1 \quad-9.682844 \quad-1.304008 \quad-1.173751$

$6 \quad-6.891203 \quad-3.276416 \quad-2.951729$

$8 \quad-6.898935 \quad-4.912168 \quad-1.190657$

$\begin{array}{lllll}6 & -8.376832 & -1.048338 & 3.127170\end{array}$

$\begin{array}{lllll}1 & -9.782649 & -1.209915 & 1.521704\end{array}$

$6 \quad-6.724626 \quad-2.848412 \quad 3.214190$

$\begin{array}{lllll}8 & -6.743586 & -4.555461 & 1.505689\end{array}$

$\begin{array}{llll}6 & -9.017699 & -0.124683 & -3.546596\end{array}$

$6 \begin{array}{llll}6 & -7.405264 & -2.112133 & -3.513569\end{array}$

$1 \quad-6.095470 \quad-3.821701 \quad-3.442826$

$6 \begin{array}{llll}6 & -5.668078 & -4.798638 & -0.606538\end{array}$

$\begin{array}{lllll}6 & -8.938905 & 0.271874 & 3.650662\end{array}$

$\begin{array}{lllll}6 & -7.284061 & -1.682845 & 3.733992\end{array}$

$1 \quad-5.863656 \quad-3.302209 \quad 3.687601$

$\begin{array}{lllll}6 & -5.597417 & -4.477065 & 0.781089\end{array}$

$1 \quad-8.714060 \quad-0.155345 \quad-4.593458$

$\begin{array}{lllll}6 & -8.432964 & 1.164934 & -2.973812\end{array}$

$\begin{array}{lllll}8 & -6.858109 & -1.650973 & -4.732081\end{array}$

$\begin{array}{llll}6 & -8.351114 & 1.466968 & 2.894737\end{array}$

$\begin{array}{lllll}1 & -8.592351 & 0.364668 & 4.680554\end{array}$

$8 \quad-6.714839 \quad-1.142975 \quad 4.898217$

$\begin{array}{lllll}6 & -8.981738 & 1.837886 & -1.877685\end{array}$

$\begin{array}{lllll}6 & -7.305747 & 1.743494 & -3.566477\end{array}$

$6 \begin{array}{llll}6 & -5.648280 & -1.049536 & -4.530432\end{array}$

$\begin{array}{lllll}6 & -3.434326 & -4.732469 & -0.709218\end{array}$

$\begin{array}{llll}6 & -8.935920 & 2.029412 & 1.756912\end{array}$

$\begin{array}{lllll}6 & -7.174061 & 2.065875 & 3.357219\end{array}$

$\begin{array}{lllll}6 & -5.488513 & -0.570188 & 4.687278\end{array}$

$6 \quad-3.382546 \quad-4.251937 \quad 0.591038$

$\begin{array}{lllll}6 & -8.458213 & 3.038308 & -1.381506\end{array}$

$\begin{array}{lllll}1 & -9.851552 & 1.414659 & -1.389232\end{array}$

$6 \quad-6.757036 \quad 2.940695 \quad-3.132443$

$8 \quad-6.727638 \quad 1.070173 \quad-4.653066$

$6 \quad-5.608068 \quad 0.362451 \quad-4.363940$

$\begin{array}{llll}6 & -8.408230 & 3.154201 & 1.109089\end{array}$

$\begin{array}{llll}1 & -9.843436 & 1.584544 & 1.365827\end{array}$

$\begin{array}{llll}6 & -6.612781 & 3.187385 & 2.764969\end{array}$

$8 \quad-6.591975 \quad 1.518681 \quad 4.499396$

$\begin{array}{lllll}6 & -5.446561 & 0.808662 & 4.345061\end{array}$

$\begin{array}{lllll}6 & -9.039191 & 3.735478 & -0.153571\end{array}$

$\begin{array}{lllll}6 & -7.341972 & 3.568447 & -2.039913\end{array}$

$\begin{array}{lllll}1 & -5.886692 & 3.364866 & -3.613594\end{array}$

$6 \quad-3.511010 \quad-1.168052 \quad-3.895432$

$\begin{array}{lllll}6 & -7.244780 & 3.717480 & 1.648506\end{array}$

$\begin{array}{lllll}1 & -5.706509 & 3.630485 & 3.155213\end{array}$

$\begin{array}{lllll}6 & -3.281419 & -0.686054 & 4.390038\end{array}$

$\begin{array}{lllll}1 & -8.720447 & 4.776675 & -0.208695\end{array}$

$8 \quad-6.821770 \quad 4.791911 \quad-1.601601$

$\begin{array}{lllll}6 & -3.517694 & 0.192667 & -3.610557\end{array}$

$8 \quad-6.741240 \quad 4.894211 \quad 1.077450$

$\begin{array}{lllll}6 & -3.277070 & 0.615446 & 3.906964\end{array}$

$\begin{array}{lllll}6 & -5.597762 & 4.773958 & -1.005371\end{array}$

$\begin{array}{lllll}6 & -5.553895 & 4.838760 & 0.417647\end{array}$

$6 \begin{array}{lllll}6 & -3.366001 & 4.764684 & -1.053962\end{array}$

$\begin{array}{lllll}6 & -3.320697 & 4.860313 & 0.330921\end{array}$

$\begin{array}{lllll}6 & -10.573421 & 3.721385 & -0.126081\end{array}$

$\begin{array}{lllll}1 & -10.936986 & 4.270930 & 0.746049\end{array}$

$\begin{array}{llll}1 & -10.993048 & 2.713042 & -0.077829\end{array}$ $\begin{array}{lllll}6 & -10.554207 & -0.137441 & -3.514086\end{array}$

$\begin{array}{lllll}1 & -10.948503 & 0.737114 & -4.038266\end{array}$

$\begin{array}{lllll}1 & -10.958660 & -0.125722 & -2.498579\end{array}$

$\begin{array}{lllll}6 & -10.557111 & -3.484216 & 0.236728\end{array}$

$\begin{array}{lllll}1 & -10.952409 & -4.057247 & -0.605849\end{array}$

$\begin{array}{lllll}1 & -10.947148 & -2.466336 & 0.153343\end{array}$

$\begin{array}{llll}6 & -10.474228 & 0.288837 & 3.678812\end{array}$

$\begin{array}{llll}1 & -10.846490 & -0.543323 & 4.281876\end{array}$

$\begin{array}{llll}1 & -10.922915 & 0.201242 & 2.685480\end{array}$

$\begin{array}{llll}1 & -10.830026 & 1.224089 & 4.118844\end{array}$

$\begin{array}{llll}1 & -10.947321 & -3.913019 & 1.163508\end{array}$

$\begin{array}{lllll}1 & -10.929985 & -1.037910 & -4.006446\end{array}$

$\begin{array}{lllll}1 & -10.968220 & 4.198327 & -1.026949\end{array}$

$\begin{array}{lllll}6 & -2.233038 & -1.716060 & -3.314198\end{array}$

$\begin{array}{lllll}6 & -2.357247 & 0.410121 & -2.704817\end{array}$

$\begin{array}{lllll}6 & -1.959512 & 4.662178 & -1.573904\end{array}$

$\begin{array}{lllll}6 & -1.871564 & 4.905433 & 0.759554\end{array}$

$\begin{array}{lllll}6 & -2.019877 & -4.862394 & -1.214140\end{array}$

$\begin{array}{lllll}6 & -1.956956 & -3.887012 & 0.909155\end{array}$

$\begin{array}{lllll}6 & -1.876307 & -1.217033 & 4.310104\end{array}$

$\begin{array}{lllll}6 & -1.907277 & 0.927161 & 3.387323\end{array}$

$\begin{array}{lllll}7 & -1.153840 & 4.788540 & -0.446996\end{array}$

$\begin{array}{lllll}7 & -1.671984 & -0.685775 & -2.512838\end{array}$

$7 \quad-1.230091 \quad-4.301871 \quad-0.190945$

$\begin{array}{lllll}7 & -1.147543 & -0.182572 & 3.691215\end{array}$

$\begin{array}{lllll}8 & -1.428337 & -2.276005 & 4.676591\end{array}$

$\begin{array}{lllll}8 & -1.564032 & 1.933512 & 2.786240\end{array}$

$\begin{array}{lllll}8 & -1.400245 & 5.014187 & 1.863883\end{array}$

$8 \quad-1.590842 \quad 4.442893 \quad-2.711652$

$\begin{array}{lllll}8 & -2.210912 & 1.530154 & -2.049212\end{array}$

$\begin{array}{lllll}8 & -1.609078 & -5.344396 & -2.240753\end{array}$

$8 \quad-1.751750 \quad-2.815781 \quad-3.461553$

$\begin{array}{lllll}8 & -1.543707 & -3.311213 & 1.900713\end{array}$

$1 \quad-0.135940 \quad-0.284901 \quad 3.548861$

$1 \quad-0.208920 \quad-4.222574 \quad-0.291311$

$1 \quad-0.197516 \quad-0.798750 \quad-1.792686$

$\begin{array}{lllll}1 & -0.133689 & 4.794357 & -0.509101\end{array}$

$7 \quad-4.580926 \quad-1.817910 \quad-4.343639$

$\begin{array}{lllll}7 & -4.550799 & 0.989225 & -3.860861\end{array}$

$7 \quad-4.584108 \quad-4.978306 \quad-1.346170$

$\begin{array}{llll}7 & -4.459179 & -4.151137 & 1.373699\end{array}$

$7 \quad-4.505230 \quad 4.721270 \quad-1.751790$

$\begin{array}{llll}7 & -4.416539 & 4.891036 & 1.095783\end{array}$

$\begin{array}{lllll}7 & -4.399082 & -1.318926 & 4.758085\end{array}$

$\begin{array}{lllll}7 & -4.350765 & 1.403092 & 3.902955\end{array}$

$\begin{array}{lllll}6 & 8.969514 & 2.850240 & 2.397824\end{array}$

$\begin{array}{llll}6 & 8.382839 & 3.175059 & 1.015001\end{array}$

$\begin{array}{lllll}6 & 8.411846 & 1.545767 & 2.976775\end{array}$

$\begin{array}{llll}8.622450 & 3.644336 & 3.060926\end{array}$

$\begin{array}{lllll}6 & 8.945224 & 2.746516 & -0.194853\end{array}$

$\begin{array}{llll}6 & 7.211166 & 3.936315 & 0.918910\end{array}$

$\begin{array}{llll}6 & 8.985021 & 0.301058 & 2.674769\end{array}$

$\begin{array}{lllll}6 & 7.283318 & 1.531627 & 3.809916\end{array}$

$\begin{array}{lllll}6 & 8.387309 & 3.049919 & -1.446140\end{array}$

$\begin{array}{llll}9.834582 & 2.127421 & -0.162335\end{array}$

$\begin{array}{lllll}6 & 6.655460 & 4.320070 & -0.290087\end{array}$

$\begin{array}{lllll}8 & 6.590007 & 4.337732 & 2.099970\end{array}$

$\begin{array}{llll}6 & 8.456567 & -0.920410 & 3.105239\end{array}$ $\begin{array}{llll}1 & 9.866729 & 0.284615 & 2.045140\end{array}$

$\begin{array}{lllll}6 & 6.713979 & 0.343851 & 4.259074\end{array}$

$8 \quad 6.691513 \quad 2.733912 \quad 4.230856$

$\begin{array}{lllll}6 & 8.933812 & 2.500948 & -2.762578\end{array}$

$\begin{array}{lllll}6 & 7.255002 & 3.871885 & -1.456507\end{array}$

$\begin{array}{lllll}1 & 5.760344 & 4.925511 & -0.322362\end{array}$

$\begin{array}{lllll}6 & 5.441196 & 3.696106 & 2.443175\end{array}$

$\begin{array}{lllll}6 & 9.084375 & -2.274165 & 2.753030\end{array}$

$\begin{array}{lllll}6 & 7.287392 & -0.859097 & 3.876380\end{array}$

$\begin{array}{lllll}1 & 5.830560 & 0.360556 & 4.884256\end{array}$

$\begin{array}{lllll}6 & 5.468714 & 2.965718 & 3.659224\end{array}$

$1 \quad 8.589299 \quad 3.182391 \quad-3.541614$

$\begin{array}{lllll}6 & 8.324011 & 1.136254 & -3.096702\end{array}$

$8 \quad 6.705164 \quad 4.263574 \quad-2.685154$

$\begin{array}{lllll}6 & 8.502007 & -2.838047 & 1.455703\end{array}$

$\begin{array}{lllll}1 & 8.781723 & -2.961352 & 3.544494\end{array}$

$\begin{array}{lllll}8 & 6.727751 & -2.056012 & 4.328836\end{array}$

$\begin{array}{lllll}6 & 8.890056 & -0.058283 & -2.640732\end{array}$

$\begin{array}{lllll}6 & 7.170013 & 1.019156 & -3.885595\end{array}$

$\begin{array}{lllll}6 & 5.467914 & 3.749053 & -2.939583\end{array}$

$\begin{array}{llll}6 & 3.279463 & 3.201816 & 2.211313\end{array}$

$\begin{array}{lllll}6 & 9.009527 & -2.490506 & 0.194197\end{array}$

$\begin{array}{lllll}6 & 7.405781 & -3.709485 & 1.476340\end{array}$

$\begin{array}{llll}6 & 5.573276 & -2.505563 & 3.766884\end{array}$

$\begin{array}{llll}6 & 3.268325 & 2.639676 & 3.483516\end{array}$

$\begin{array}{lllll}6 & 8.385327 & -1.325832 & -2.954412\end{array}$

$\begin{array}{lllll}1 & 9.762292 & 0.000113 & -2.000820\end{array}$

$\begin{array}{lllll}6 & 6.658399 & -0.212796 & -4.270911\end{array}$

$\begin{array}{lllll}8 & 6.544379 & 2.176894 & -4.353201\end{array}$

$\begin{array}{lllll}6 & 5.391085 & 2.582498 & -3.748935\end{array}$

$\begin{array}{lllll}6 & 8.444973 & -2.933829 & -1.005892\end{array}$

$\begin{array}{lllll}1 & 9.870947 & -1.834228 & 0.150116\end{array}$

$\begin{array}{lllll}6 & 6.790215 & -4.156036 & 0.312983\end{array}$

$\begin{array}{lllll}8 & 6.907046 & -4.171018 & 2.704852\end{array}$

$\begin{array}{lllll}6 & 5.659888 & -3.698842 & 2.996902\end{array}$

$\begin{array}{lllll}6 & 9.012673 & -2.596720 & -2.386870\end{array}$

$\begin{array}{lllll}6 & 7.272898 & -1.370006 & -3.804921\end{array}$

$1 \quad 5.784068 \quad-0.270734 \quad-4.906899$

$\begin{array}{lllll}6 & 3.252224 & 3.707337 & -2.691973\end{array}$

$\begin{array}{lllll}6 & 7.304542 & -3.740109 & -0.903432\end{array}$

$\begin{array}{lllll}1 & 5.927792 & -4.807937 & 0.357388\end{array}$

$\begin{array}{lllll}6 & 3.369171 & -2.486766 & 3.430609\end{array}$

$18.701699 \quad-3.408446 \quad-3.045990$

$\begin{array}{lllll}8 & 6.765411 & -2.604377 & -4.234313\end{array}$

$\begin{array}{lllll}6 & 3.182439 & 2.520489 & -3.410875\end{array}$

$\begin{array}{lllll}8 & 6.727548 & -4.199768 & -2.085639\end{array}$

$\begin{array}{lllll}6 & 3.440894 & -3.702405 & 2.766663\end{array}$

$\begin{array}{lllll}6 & 5.550711 & -2.943822 & -3.707922\end{array}$

$\begin{array}{lllll}6 & 5.537389 & -3.692509 & -2.497907\end{array}$

$\begin{array}{lllll}6 & 3.319774 & -2.920413 & -3.693873\end{array}$

$\begin{array}{lllll}6 & 3.315652 & -3.539018 & -2.450980\end{array}$

$\begin{array}{lllll}6 & 10.547418 & -2.556259 & -2.398123\end{array}$

$\begin{array}{lllll}1 & 10.949630 & -3.511609 & -2.051175\end{array}$

$\begin{array}{lllll}1 & 10.960573 & -1.773563 & -1.755938\end{array}$

$\begin{array}{lllll}6 & 10.468359 & 2.484599 & -2.808611\end{array}$

$\begin{array}{lllll}1 & 10.813194 & 2.133853 & -3.784750\end{array}$

$\begin{array}{lllll}1 & 10.912725 & 1.834971 & -2.049454\end{array}$

$\begin{array}{llll}6 & 10.504485 & 2.885266 & 2.398166\end{array}$ 
$\begin{array}{llll}1 & 10.857009 & 3.860910 & 2.053819\end{array}$

$\begin{array}{llll}1 & 10.949187 & 2.127523 & 1.746931\end{array}$

$\begin{array}{llll}6 & 10.618594 & -2.227777 & 2.741835\end{array}$

$\begin{array}{lllll}1 & 10.991782 & -1.877310 & 3.707623\end{array}$

$\begin{array}{lllll}1 & 11.021039 & -1.562730 & 1.972656\end{array}$

$\begin{array}{lllll}1 & 11.020548 & -3.226794 & 2.554450\end{array}$

$\begin{array}{lllll}1 & 10.883778 & 2.714015 & 3.408898\end{array}$

$\begin{array}{lllll}1 & 10.855559 & 3.493453 & -2.643873\end{array}$

$\begin{array}{lllll}1 & 10.909522 & -2.373744 & -3.413192\end{array}$

$\begin{array}{lllll}6 & 1.860306 & 4.128762 & -2.317209\end{array}$

$\begin{array}{lllll}6 & 1.734656 & 2.094779 & -3.485162\end{array}$

$\begin{array}{lllll}6 & 1.891027 & -2.731682 & -4.135063\end{array}$

$\begin{array}{lllll}6 & 1.888702 & -3.681391 & -2.001108\end{array}$

$\begin{array}{llll}6 & 1.902843 & 3.045322 & 1.612705\end{array}$

$\begin{array}{llll}6 & 1.856111 & 2.230808 & 3.808123\end{array}$

$\begin{array}{lllll}6 & 1.945632 & -2.012425 & 3.393331\end{array}$

$\begin{array}{lllll}6 & 2.045678 & -4.098351 & 2.351235\end{array}$

$\begin{array}{lllll}7 & 1.130043 & -3.287918 & -3.086516\end{array}$

$\begin{array}{lllll}7 & 1.042198 & 3.113399 & -2.813017\end{array}$

$\begin{array}{llll}7 & 1.144099 & 2.487655 & 2.627863\end{array}$

$\begin{array}{lllll}7 & 1.242400 & -3.012509 & 2.752920\end{array}$

$\begin{array}{lllll}8 & 1.521898 & -0.944752 & 3.801284\end{array}$

$\begin{array}{lllll}8 & 1.657741 & -5.103666 & 1.810056\end{array}$

$\begin{array}{lllll}8 & 1.490934 & -4.039179 & -0.905793\end{array}$

$\begin{array}{lllll}8 & 1.455966 & -2.242333 & -5.146658\end{array}$

$\begin{array}{lllll}8 & 1.244939 & 1.117774 & -4.001514\end{array}$

$\begin{array}{lllll}8 & 1.520277 & 3.312791 & 0.490491\end{array}$

$\begin{array}{lllll}8 & 1.495755 & 5.127904 & -1.734418\end{array}$

$\begin{array}{lllll}8 & 1.396354 & 1.795067 & 4.836705\end{array}$

$\begin{array}{lllll}1 & 0.236910 & -2.988182 & 2.559017\end{array}$

$\begin{array}{lllll}1 & 0.133212 & 2.309579 & 2.554829\end{array}$

$\begin{array}{lllll}1 & 0.029635 & 3.225415 & -2.832563\end{array}$

$\begin{array}{lllll}1 & 0.109449 & -3.240429 & -3.085357\end{array}$

$\begin{array}{lllll}7 & 4.399195 & 4.334931 & -2.424308\end{array}$

$\begin{array}{lllll}7 & 4.252792 & 1.938927 & -3.964662\end{array}$

$\begin{array}{lllll}7 & 4.362741 & 3.771574 & 1.677771\end{array}$

$\begin{array}{llll}7 & 4.373875 & 2.473322 & 4.216730\end{array}$

$7 \quad 4.442681 \quad-2.576590 \quad-4.332374$

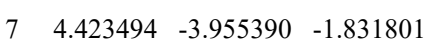

$\begin{array}{lllll}7 & 4.428748 & -1.869398 & 3.962727\end{array}$

$\begin{array}{lllll}7 & 4.596176 & -4.325852 & 2.518761\end{array}$

$\begin{array}{lllll}6 & 1.119550 & -0.526662 & -0.361784\end{array}$

$\begin{array}{lllll}8 & 0.321637 & -0.500095 & 0.572592\end{array}$

$\begin{array}{lllll}8 & 0.789379 & -0.752012 & -1.626513\end{array}$

$\begin{array}{lllll}6 & -1.975069 & 1.383711 & -0.584875\end{array}$

$\begin{array}{lllll}1 & -1.101189 & 0.767922 & -0.394926\end{array}$

$\begin{array}{lllll}6 & 2.572667 & -0.299676 & -0.223780\end{array}$

$\begin{array}{lllll}6 & 3.403676 & -0.309836 & -1.350618\end{array}$

$\begin{array}{lllll}6 & 3.113740 & -0.068456 & 1.043202\end{array}$

$\begin{array}{lllll}6 & 4.771907 & -0.141584 & -1.194744\end{array}$

$\begin{array}{lllll}1 & 2.972399 & -0.432116 & -2.335369\end{array}$

$\begin{array}{lllll}6 & 4.482013 & 0.104693 & 1.189207\end{array}$

$\begin{array}{lllll}1 & 2.447816 & -0.021950 & 1.892907\end{array}$

$\begin{array}{lllll}6 & 5.327687 & 0.050064 & 0.073143\end{array}$

$\begin{array}{lllll}1 & 5.419156 & -0.153137 & -2.062421\end{array}$

$\begin{array}{lllll}1 & 4.903725 & 0.272398 & 2.174599\end{array}$

$\begin{array}{lllll}6 & 6.802771 & 0.181421 & 0.244775\end{array}$

$\begin{array}{lllll}1 & 7.307975 & 0.344261 & -0.704306\end{array}$ $\begin{array}{lllll}1 & 7.041198 & 1.006093 & 0.910556\end{array}$

$\begin{array}{llll}1 & 7.213657 & -0.724215 & 0.691237\end{array}$

$\begin{array}{lllll}1 & -1.772469 & 2.398078 & -0.258856\end{array}$

$\begin{array}{lllll}6 & -3.226707 & 0.790059 & -0.006134\end{array}$

$\begin{array}{lllll}6 & -3.160562 & -0.373591 & 0.762218\end{array}$

$\begin{array}{lllll}6 & -4.473577 & 1.351555 & -0.315980\end{array}$

$\begin{array}{llll}6 & -4.337427 & -0.951351 & 1.244049\end{array}$

$\begin{array}{lllll}1 & -2.193673 & -0.818000 & 0.975884\end{array}$

$\begin{array}{llll}6 & -5.645531 & 0.757274 & 0.140234\end{array}$

$\begin{array}{lllll}1 & -4.521743 & 2.222466 & -0.957619\end{array}$

$\begin{array}{llll}6 & -5.576298 & -0.395898 & 0.924995\end{array}$

$\begin{array}{lllll}1 & -4.283197 & -1.842900 & 1.855350\end{array}$

$\begin{array}{lllll}1 & -6.608758 & 1.185594 & -0.114709\end{array}$

$\begin{array}{llll}1 & -6.487888 & -0.860618 & 1.273339\end{array}$

\section{$3 c @ 12$}

$\begin{array}{llll}6 & -8.991806 & -3.186015 & 1.552314\end{array}$

$\begin{array}{lllll}6 & -8.372072 & -3.180408 & 0.156491\end{array}$

$\begin{array}{lllll}6 & -8.398502 & -2.068945 & 2.402051\end{array}$

$\begin{array}{lllll}1 & -8.686602 & -4.123450 & 2.017966\end{array}$

$\begin{array}{lllll}6 & -8.874634 & -2.382599 & -0.874205\end{array}$

$\begin{array}{llll}-7.269805 & -3.989336 & -0.150900\end{array}$

$\begin{array}{llll}-8.915766 & -0.767788 & 2.409208\end{array}$

$\begin{array}{llll}-7.275012 & -2.315690 & 3.198179\end{array}$

$\begin{array}{lllll}6 & -8.364599 & -2.394621 & -2.177353\end{array}$

$\begin{array}{llll}-9.707210 & -1.724475 & -0.655391\end{array}$

$\begin{array}{llll}-6.738916 & -4.058266 & -1.431922\end{array}$

$\begin{array}{llll}-6.726207 & -4.796621 & 0.854014\end{array}$

$\begin{array}{lll}-8.342166 & 0.269336 & 3.150281\end{array}$

$\begin{array}{lll}-9.801434 & -0.558629 & 1.820960\end{array}$

$\begin{array}{llll}-6.647154 & -1.316266 & 3.928063\end{array}$

$\begin{array}{llll}-6.787738 & -3.627084 & 3.279252\end{array}$

$\begin{array}{llll}-8.951507 & -1.500322 & -3.265830\end{array}$

$\begin{array}{llll}-7.301064 & -3.269533 & -2.431649\end{array}$

$\begin{array}{llll}-5.902778 & -4.710733 & -1.649436\end{array}$

$\begin{array}{lll}-5.540396 & -4.410350 & 1.403072\end{array}$

$\begin{array}{llll}-8.928278 & 1.680711 & 3.214088\end{array}$

$\begin{array}{llll}-7.182777 & -0.038925 & 3.871924\end{array}$

$\begin{array}{llll}-5.762573 & -1.524942 & 4.514389\end{array}$

$\begin{array}{llll}-5.571422 & -3.838771 & 2.706400\end{array}$

$\begin{array}{llll}-8.633406 & -1.930456 & -4.216341\end{array}$

$\begin{array}{llll}-8.356751 & -0.092740 & -3.216646\end{array}$

$\begin{array}{llll}-6.773092 & -3.377331 & -3.730355\end{array}$

$\begin{array}{lll}-8.351114 & 2.569586 & 2.116400\end{array}$

$\begin{array}{llll}-8.590418 & 2.106939 & 4.159551\end{array}$

$\begin{array}{llll}-6.587329 & 0.985061 & 4.607157\end{array}$

$\begin{array}{llll}-8.913316 & 0.959559 & -2.485231\end{array}$

$\begin{array}{llll}-7.196667 & 0.192817 & -3.945923\end{array}$

$\begin{array}{llll}-5.540074 & -2.796593 & -3.842022\end{array}$

$\begin{array}{lll}-3.314610 & -4.254455 & 1.417102\end{array}$

$\begin{array}{lll}-8.887116 & 2.579162 & 0.821470\end{array}$

$\begin{array}{lll}-7.248315 & 3.400270 & 2.355426\end{array}$

$\begin{array}{llll}-5.432942 & 1.524639 & 4.138269\end{array}$

$\begin{array}{llll}-3.344206 & -3.734572 & 2.702937\end{array}$

$\begin{array}{llll}-8.380101 & 2.256337 & -2.487945\end{array}$

$\begin{array}{llll}-9.795829 & 0.766331 & -1.887122\end{array}$

$\begin{array}{llll}-6.651803 & 1.464209 & -4.022440\end{array}$

$\begin{array}{llll}-6.616621 & -0.854007 & -4.661908\end{array}$ $\begin{array}{llll}-5.480487 & -1.415082 & -4.177681\end{array}$

$\begin{array}{llll}-8.360556 & 3.347337 & -0.219967\end{array}$

$\begin{array}{lll}-9.747954 & 1.953372 & 0.617605\end{array}$

$\begin{array}{lll}-6.673390 & 4.167879 & 1.347929\end{array}$

$\begin{array}{llll}-6.701409 & 3.490198 & 3.645172\end{array}$

$\begin{array}{lll}-5.474857 & 2.895707 & 3.765187\end{array}$

$\begin{array}{llll}-8.964450 & 3.371923 & -1.625520\end{array}$

$\begin{array}{llll}-7.261701 & 2.483276 & -3.300048\end{array}$

$\begin{array}{llll}-5.767952 & 1.657643 & -4.615192\end{array}$

$\begin{array}{llll}-3.329333 & -2.798829 & -3.553198\end{array}$

$\begin{array}{llll}-7.226302 & 4.112410 & 0.077142\end{array}$

$\begin{array}{llll}-5.808097 & 4.784909 & 1.551545\end{array}$

$\begin{array}{llll}-3.240072 & 1.500741 & 3.742541\end{array}$

$\begin{array}{llll}-8.642190 & 4.310006 & -2.078816\end{array}$

$\begin{array}{llll}-6.734866 & 3.780828 & -3.408587\end{array}$

$\begin{array}{llll}-3.304754 & -1.420164 & -3.711036\end{array}$

$\begin{array}{llll}-6.659409 & 4.870971 & -0.951866\end{array}$

$\begin{array}{llll}-3.256382 & 2.874915 & 3.534685\end{array}$

$\begin{array}{llll}-5.515938 & 3.935176 & -2.814744\end{array}$

$\begin{array}{llll}-5.490053 & 4.420900 & -1.477491\end{array}$

$\begin{array}{llll}-3.299424 & 3.674664 & -2.750848\end{array}$

$\begin{array}{llll}-3.294396 & 4.050268 & -1.415514\end{array}$

$\begin{array}{llll}-10.499615 & 3.371263 & -1.605821\end{array}$

$\begin{array}{llll}-10.866985 & 4.223888 & -1.028890\end{array}$

$\begin{array}{llll}-10.922065 & 2.466777 & -1.159830\end{array}$

$\begin{array}{llll}-10.487221 & -1.492129 & -3.255377\end{array}$

$\begin{array}{llll}-10.864704 & -0.892092 & -4.087525\end{array}$

$\begin{array}{llll}-10.907220 & -1.079394 & -2.334113\end{array}$

$\begin{array}{llll}-10.526491 & -3.165779 & 1.516058\end{array}$

$\begin{array}{llll}-10.899467 & -4.016660 & 0.940127\end{array}$

$\begin{array}{llll}-10.933082 & -2.258180 & 1.061744\end{array}$

$\begin{array}{llll}-10.463707 & 1.681773 & 3.230573\end{array}$

$\begin{array}{llll}-10.830039 & 1.082375 & 4.068078\end{array}$

$\begin{array}{lll}-10.902690 & 1.272899 & 2.316391\end{array}$

$\begin{array}{llll}-10.835263 & 2.703378 & 3.344785\end{array}$

$\begin{array}{llll}-10.924385 & -3.231984 & 2.532051\end{array}$

$\begin{array}{lll}-10.866490 & -2.511720 & -3.361049\end{array}$

$\begin{array}{llll}-10.886943 & 3.447728 & -2.625118\end{array}$

$\begin{array}{llll}-1.926515 & -3.264578 & -3.277483\end{array}$

$\begin{array}{llll}-1.915472 & -0.936264 & -3.420218\end{array}$

$\begin{array}{llll}-1.883490 & 3.341890 & -3.148384\end{array}$

$\begin{array}{llll}-1.906198 & 3.886308 & -0.879535\end{array}$

$\begin{array}{llll}-1.882062 & -4.318039 & 0.964787\end{array}$

$\begin{array}{llll}-1.928957 & -3.471774 & 3.140379\end{array}$

$\begin{array}{llll}-1.830928 & 1.005395 & 3.542193\end{array}$

$\begin{array}{llll}-1.841648 & 3.337349 & 3.320204\end{array}$

$\begin{array}{llll}-1.145341 & 3.484894 & -1.954568\end{array}$

$\begin{array}{llll}-1.167450 & -2.079591 & -3.231729\end{array}$

$\begin{array}{lll}-1.144446 & -3.861632 & 2.047083\end{array}$

$\begin{array}{llll}-1.085756 & 2.154021 & 3.330548\end{array}$

$\begin{array}{lll}-1.431430 & -0.142216 & 3.526016\end{array}$

$\begin{array}{lll}-1.407753 & 4.455563 & 3.179306\end{array}$

$\begin{array}{llll}-1.544842 & 4.031176 & 0.278221\end{array}$

$\begin{array}{llll}-1.432405 & 3.031431 & -4.222350\end{array}$

$\begin{array}{llll}-1.550355 & 0.224105 & -3.321301\end{array}$

$\begin{array}{llll}-1.469404 & -4.659992 & -0.126158\end{array}$

$\begin{array}{llll}-1.501772 & -4.382291 & -3.114591\end{array}$

$\begin{array}{llll}-1.525447 & -3.020682 & 4.187982\end{array}$ 
$\begin{array}{lll}-0.067562 & 2.187161 & 3.209661\end{array}$

$\begin{array}{lll}-0.123735 & -3.822990 & 2.094565\end{array}$

$\begin{array}{llll}-0.153643 & -2.132029 & -3.073053\end{array}$

$\begin{array}{llll}-0.136152 & 3.284809 & -1.911398\end{array}$

$\begin{array}{llll}-4.459632 & -3.510642 & -3.571006\end{array}$

$\begin{array}{llll}-4.374248 & -0.699144 & -4.052416\end{array}$

$\begin{array}{llll}-4.410533 & -4.603026 & 0.737784\end{array}$

$\begin{array}{llll}-4.475133 & -3.504895 & 3.371246\end{array}$

$\begin{array}{lll}-4.419594 & 3.588954 & -3.473519\end{array}$

$\begin{array}{lll}-4.382480 & 4.446293 & -0.753197\end{array}$

$\begin{array}{lll}-4.326547 & 0.799598 & 4.074532\end{array}$

$\begin{array}{llll}-4.382334 & 3.594234 & 3.501754\end{array}$

$\begin{array}{llll}8.994342 & 3.537635 & 1.112656\end{array}$

$\begin{array}{llll}8.394254 & 3.385572 & -0.281787\end{array}$

$\begin{array}{llll}8.378323 & 2.517320 & 2.069765\end{array}$

$\begin{array}{llll}8.689684 & 4.520257 & 1.474086\end{array}$

$\begin{array}{llll}8.884094 & 2.443743 & -1.196801\end{array}$

$\begin{array}{llll}7.306208 & 4.166130 & -0.691071\end{array}$

$\begin{array}{llll}8.902651 & 1.236191 & 2.256112\end{array}$

$\begin{array}{lll}7.234496 & 2.843708 & 2.808191\end{array}$

$\begin{array}{llll}8.316193 & 2.241659 & -2.456912\end{array}$

$\begin{array}{llll}9.744371 & 1.848050 & -0.913988\end{array}$

$\begin{array}{llll}6.682092 & 3.978007 & -1.918208\end{array}$

$\begin{array}{llll}6.812866 & 5.175104 & 0.154765\end{array}$

$\begin{array}{llll}8.353179 & 0.310724 & 3.150332\end{array}$

$\begin{array}{llll}9.782469 & 0.948966 & 1.692845\end{array}$

$\begin{array}{lll}6.652776 & 1.969825 & 3.717590\end{array}$

$\begin{array}{llll}6.702097 & 4.123883 & 2.635361\end{array}$

$\begin{array}{llll}8.882060 & 1.268923 & -3.491745\end{array}$

$\begin{array}{llll}7.182690 & 3.002477 & -2.763852\end{array}$

$\begin{array}{llll}5.815528 & 4.563569 & -2.194005\end{array}$

$\begin{array}{llll}5.585126 & 4.887009 & 0.678576\end{array}$

$\begin{array}{llll}8.952001 & -1.077328 & 3.341984\end{array}$

$\begin{array}{llll}7.224821 & 0.712967 & 3.875705\end{array}$

$\begin{array}{llll}5.776837 & 2.256021 & 4.284433\end{array}$

$\begin{array}{llll}5.540986 & 4.228327 & 1.939078\end{array}$

$\begin{array}{llll}8.532226 & 1.622814 & -4.462199\end{array}$

$\begin{array}{llll}8.308165 & -0.133075 & -3.317965\end{array}$

$\begin{array}{llll}6.563535 & 2.771119 & -3.994196\end{array}$

$\begin{array}{llll}8.376919 & -2.069335 & 2.334691\end{array}$

$\begin{array}{llll}8.625604 & -1.417568 & 4.325088\end{array}$

$\begin{array}{llll}6.693522 & -0.154095 & 4.840545\end{array}$

$\begin{array}{llll}8.864919 & -1.041580 & -2.409097\end{array}$

$\begin{array}{llll}7.203082 & -0.570536 & -4.060111\end{array}$

$\begin{array}{llll}5.417144 & 2.043394 & -3.977439\end{array}$

$\begin{array}{llll}3.363225 & 4.730511 & 0.534110\end{array}$

$\begin{array}{llll}8.908486 & -2.240080 & 1.054904\end{array}$

$\begin{array}{llll}7.281373 & -2.869774 & 2.676343\end{array}$

$\begin{array}{llll}5.500726 & -0.747983 & 4.558480\end{array}$

$\begin{array}{llll}3.342893 & 3.984390 & 1.702315\end{array}$

$\begin{array}{llll}8.361348 & -2.328181 & -2.209539\end{array}$

$\begin{array}{llll}9.733826 & -0.732175 & -1.840610\end{array}$

$\begin{array}{llll}6.647490 & -1.834040 & -3.880124\end{array}$

$\begin{array}{llll}6.635923 & 0.273328 & -5.032003\end{array}$

$\begin{array}{llll}5.428418 & 0.794021 & -4.658207\end{array}$

$\begin{array}{llll}8.422275 & -3.188573 & 0.148544\end{array}$

$\begin{array}{llll}9.739632 & -1.613768 & 0.752090\end{array}$

$\begin{array}{llll}6.774491 & -3.843761 & 1.828400\end{array}$ $\begin{array}{lllll}8 & 6.722745 & -2.702728 & 3.948460\end{array}$

$\begin{array}{llll}6 & 5.521969 & -2.073252 & 4.032944\end{array}$

$\begin{array}{lllll}6 & 9.003381 & -3.331450 & -1.253562\end{array}$

$\begin{array}{lllll}6 & 7.223117 & -2.681887 & -2.944226\end{array}$

$\begin{array}{llll}5.783066 & -2.144144 & -4.452680\end{array}$

$\begin{array}{lllll}6 & 3.257643 & 1.750222 & -3.523342\end{array}$

$\begin{array}{lllll}6 & 7.363381 & -3.996981 & 0.578136\end{array}$

$\begin{array}{lllll}1 & 5.938847 & -4.462961 & 2.128182\end{array}$

$\begin{array}{llll}6 & 3.267815 & -0.781271 & 4.502413\end{array}$

$1 \quad 8.711615 \quad-4.320392 \quad-1.608216$

$\begin{array}{lllll}8 & 6.705883 & -3.966034 & -2.757805\end{array}$

$\begin{array}{lllll}6 & 3.227289 & 0.624759 & -4.336052\end{array}$

$\begin{array}{lllll}8 & 6.886757 & -5.011558 & -0.268777\end{array}$

$\begin{array}{lllll}6 & 3.296326 & -2.040365 & 3.920135\end{array}$

$\begin{array}{lllll}6 & 5.558868 & -4.109266 & -2.042543\end{array}$

$\begin{array}{lllll}6 & 5.646543 & -4.766242 & -0.782462\end{array}$

$\begin{array}{lllll}6 & 3.347662 & -3.989292 & -1.794125\end{array}$

$\begin{array}{lllll}6 & 3.420903 & -4.734338 & -0.626433\end{array}$

$\begin{array}{lllll}6 & 10.537458 & -3.268354 & -1.271333\end{array}$

$\begin{array}{lllll}1 & 10.949750 & -4.046848 & -0.624227\end{array}$

$\begin{array}{llll}10.931385 & -2.309596 & -0.923202\end{array}$

$\begin{array}{lllll}6 & 10.417871 & 1.280364 & -3.523183\end{array}$

$\begin{array}{llll}10.781436 & 0.618351 & -4.313400\end{array}$

$\begin{array}{llll}10.867764 & 0.949768 & -2.582901\end{array}$

$\begin{array}{llll}6 & 10.529005 & 3.500023 & 1.111020\end{array}$

$\begin{array}{llll}10.920535 & 4.285341 & 0.459291\end{array}$

$\begin{array}{lll}10.934504 & 2.547831 & 0.758081\end{array}$

$\begin{array}{lllll}6 & 10.487915 & -1.070072 & 3.344192\end{array}$

$\begin{array}{llll}10.858774 & -0.386562 & 4.112541\end{array}$

$\begin{array}{llll}1 & 10.916999 & -0.756783 & 2.388417\end{array}$

$\begin{array}{lllll}1 & 10.864775 & -2.073361 & 3.559098\end{array}$

$\begin{array}{lllll}1 & 10.909717 & 3.665160 & 2.122377\end{array}$

$\begin{array}{lllll}1 & 10.779902 & 2.292363 & -3.722332\end{array}$

$\begin{array}{lllll}1 & 10.908220 & -3.427635 & -2.287330\end{array}$

$\begin{array}{lllll}6 & 1.902650 & 1.926327 & -2.906388\end{array}$

$\begin{array}{lllll}6 & 1.818697 & 0.100388 & -4.359988\end{array}$

$\begin{array}{lllll}6 & 1.915704 & -3.605767 & -2.020946\end{array}$

$\begin{array}{lllll}6 & 2.017770 & -4.948459 & -0.119930\end{array}$

$\begin{array}{lllll}6 & 1.943676 & 4.860969 & 0.043665\end{array}$

$\begin{array}{lllll}6 & 1.946384 & 3.514067 & 1.949033\end{array}$

$\begin{array}{lllll}6 & 1.825771 & -0.376469 & 4.681586\end{array}$

$\begin{array}{lllll}6 & 1.886139 & -2.464240 & 3.630084\end{array}$

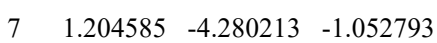

$\begin{array}{lllll}7 & 1.123719 & 0.931830 & -3.459922\end{array}$

$\begin{array}{lllll}7 & 1.187932 & 4.090772 & 0.954917\end{array}$

$\begin{array}{lllll}7 & 1.093586 & -1.469254 & 4.182719\end{array}$

$\begin{array}{lllll}8 & 1.364750 & 0.644297 & 5.132442\end{array}$

$\begin{array}{lllll}8 & 1.523264 & -3.431704 & 2.987884\end{array}$

$\begin{array}{lllll}8 & 1.633469 & -5.540617 & 0.859285\end{array}$

$\begin{array}{lllll}8 & 1.498809 & -2.817479 & -2.854142\end{array}$

$\begin{array}{lllll}8 & 1.339493 & -0.811727 & -4.988123\end{array}$

$\begin{array}{lllll}8 & 1.499447 & 5.479268 & -0.891081\end{array}$

$\begin{array}{lllll}8 & 1.562798 & 2.758909 & -2.080631\end{array}$

$\begin{array}{lllll}8 & 1.587754 & 2.747996 & 2.830647\end{array}$

$\begin{array}{llll}0.076715 & -1.452899 & 4.109841\end{array}$

$\begin{array}{llll}0.168544 & 4.007442 & 0.857572\end{array}$

$\begin{array}{llll}0.115698 & 0.800617 & -3.302085\end{array}$

$\begin{array}{lllll}1 & 0.193579 & -4.218426 & -0.908163\end{array}$ $\begin{array}{lllll}7 & 4.343606 & 2.504302 & -3.354970\end{array}$

$\begin{array}{lllll}7 & 4.324037 & 0.101276 & -4.889548\end{array}$

$7 \quad 4.495172 \quad 5.178536 \quad-0.016107$

$\begin{array}{llll}4.422536 & 3.734123 & 2.442495\end{array}$

$\begin{array}{llll}4.409978 & -3.677545 & -2.538642\end{array}$

$\begin{array}{llll}4.578457 & -5.114339 & -0.080736\end{array}$

$\begin{array}{llll}4.374035 & -0.097491 & 4.811105\end{array}$

$\begin{array}{llll}4.420699 & -2.715798 & 3.676241\end{array}$

$\begin{array}{llll}-0.461757 & -0.721027 & 0.136439\end{array}$

$\begin{array}{llll}-0.018714 & -1.839804 & -0.069721\end{array}$

$\begin{array}{llll}0.311166 & 0.306061 & 0.556100\end{array}$

$\begin{array}{llll}-1.898599 & -0.377969 & 0.013250\end{array}$

$\begin{array}{llll}-2.399617 & 0.882012 & 0.356077\end{array}$

$\begin{array}{llll}-2.791122 & -1.389832 & -0.365509\end{array}$

$\begin{array}{llll}-3.771535 & 1.118352 & 0.330708\end{array}$

$\begin{array}{llll}-1.714901 & 1.660318 & 0.662241\end{array}$

$\begin{array}{llll}-4.159250 & -1.146328 & -0.392108\end{array}$

$\begin{array}{llll}-2.397646 & -2.363386 & -0.625236\end{array}$

$\begin{array}{llll}-4.673582 & 0.109949 & -0.039978\end{array}$

$\begin{array}{llll}-4.150146 & 2.095778 & 0.615070\end{array}$

$\begin{array}{llll}-4.840322 & -1.942789 & -0.678434\end{array}$

$\begin{array}{llll}-6.153652 & 0.372822 & -0.077437\end{array}$

$\begin{array}{llll}-6.457087 & 0.727317 & -1.067661\end{array}$

$\begin{array}{llll}-6.729340 & -0.531891 & 0.134197\end{array}$

$\begin{array}{llll}-6.441647 & 1.137740 & 0.645500\end{array}$

$\begin{array}{llll}2.815294 & 0.061450 & 0.469258\end{array}$

$\begin{array}{llll}2.979293 & -0.537192 & -0.784943\end{array}$

$\begin{array}{llll}3.922171 & 0.655145 & 1.090910\end{array}$

$\begin{array}{llll}4.232092 & -0.553519 & -1.398179\end{array}$

$\begin{array}{llll}2.130578 & -1.007663 & -1.265564\end{array}$

$\begin{array}{llll}5.176040 & 0.640847 & 0.474292\end{array}$

$\begin{array}{llll}3.808319 & 1.117383 & 2.066789\end{array}$

$\begin{array}{llll}5.335515 & 0.031374 & -0.770690\end{array}$

$\begin{array}{llll}4.348575 & -1.051535 & -2.352981\end{array}$

$\begin{array}{llll}6.026826 & 1.095151 & 0.971205\end{array}$

$\begin{array}{llll}6.311645 & 0.003459 & -1.243942\end{array}$

$\begin{array}{llll}1.533548 & -0.073858 & 1.246396\end{array}$

$\begin{array}{llll}1.412142 & -1.115238 & 1.542610\end{array}$

$\begin{array}{llll}1.557693 & 0.566553 & 2.125333\end{array}$ 